\title{
A Target Signature Synthesis Program
}

by

Milan Patel

A thesis submitted to the Faculty of Graduate and Postdoctoral Affairs in partial fulfillment of the requirements for the degree of

Master of Applied Science

in

Electrical and Computer Engineering

Carleton University

Ottawa, Ontario

(C) 2021

Milan Patel 


\begin{abstract}
This thesis presents the design of a software program and digital system to synthesize target return signals for Electronic Warfare (EW) applications. The synthesized target echo returns are used to counter high resolution radar systems. Synthesizing a realistic target echo return requires knowledge of the motion and electromagnetic properties of the simulated target. The software program implements a realistic motion algorithm for Naval targets in a variety of sea conditions. Another algorithm is designed to create the target model used in the software. Within the software application a user interface is used to define simulation scenarios for producing synthesized target return signals. The digital system design utilizes parameters from the software to synthesize the false target echo signal. Simulations performed in MATLAB verify the viability of the motion algorithm, software program and digital system design.
\end{abstract}




\section{Acknowledgements}

Firstly, I would like to thank my supervisor Jim Wight for the invaluable guidance he has provided me throughout my research. I would also like to thank the technical and administrative staff who supported me throughout my program. Whether it was technical challenges, or paperwork they always provided me with the right tools and guidance. I would like to thank Scott Bruce and Stephen McLaurin. Without their consistent support and communication, I would not have been able to achieve progress in my simulation work.

I also want to thank Trevor and Scott for giving me the opportunity to pursue this research topic, as well as the patience and support they gave throughout the course of this thesis. To the rest of the staff at MC-CM I thank you for the support and advice, you guys made this process more enjoyable.

Finally, I want to thank my parents for the support and encouragement they gave me throughout my education and life, and without them I would not have been able to come this far. I want to thank my brother for being a true critic of my work and pointing out opportunities for me to improve my written communication in this thesis. Last, but not least I want to thank the love of my life, Manali. Your patience, understanding, support and unwavering belief in me was more than I ever could have asked for. 


\section{Table of Contents}

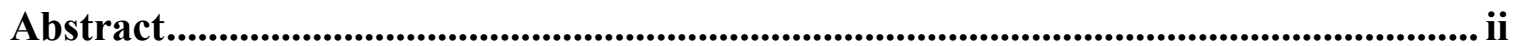

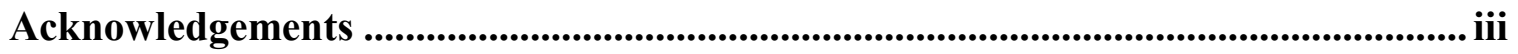

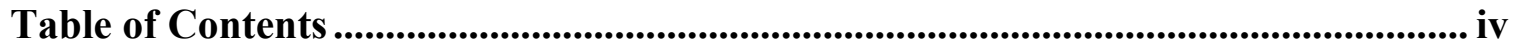

List of Tables ............................................................................................................. viii

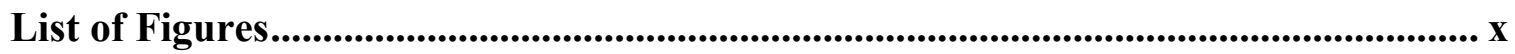

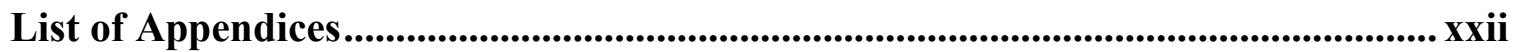

Chapter 1: Introduction ............................................................................................... 1

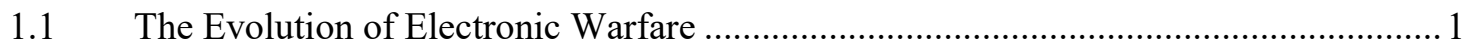

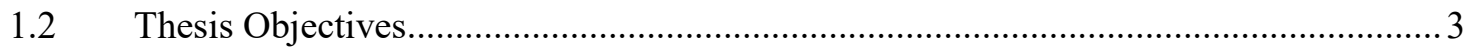

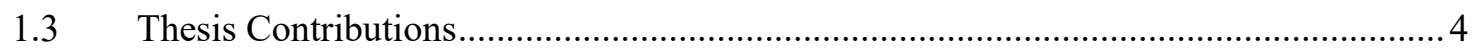

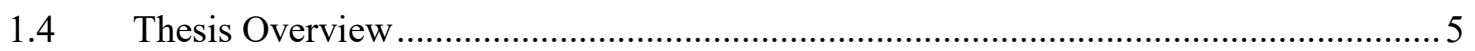

Chapter 2: Principles of High-Resolution Radar.................................................... 7

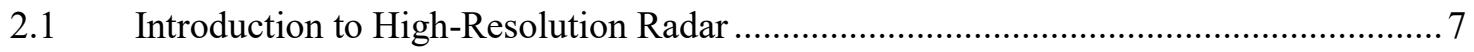

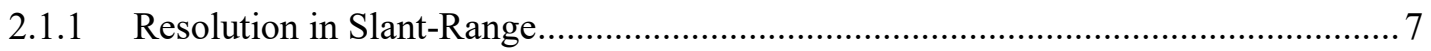

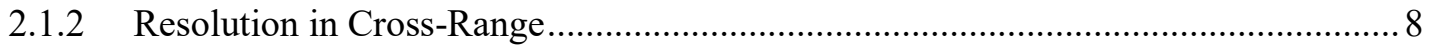

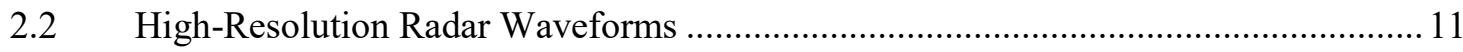

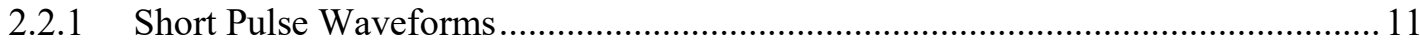

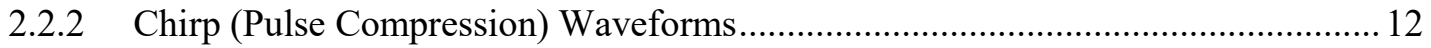

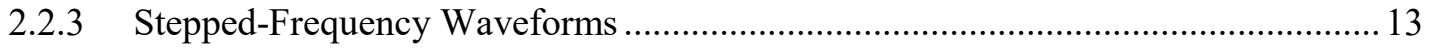

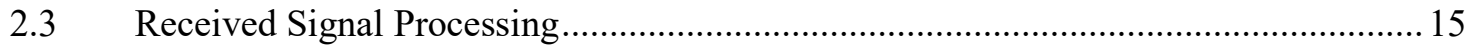

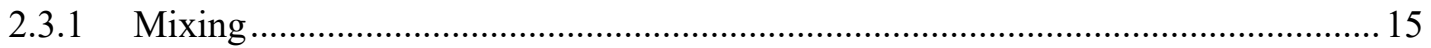

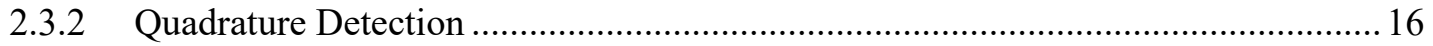

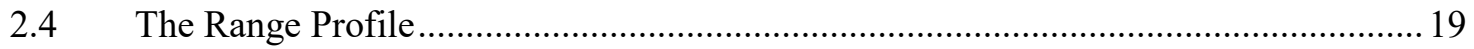

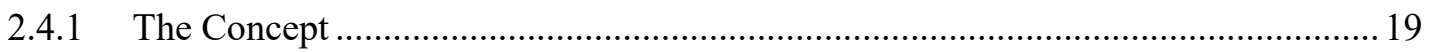




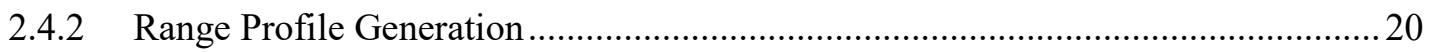

2.4.3 Real HRRP Generation \& Time-Domain Sampling .........................................2

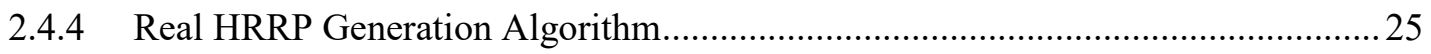

2.4.5 Synthetic HRRP Generation \& Frequency-Domain Sampling ..............................22

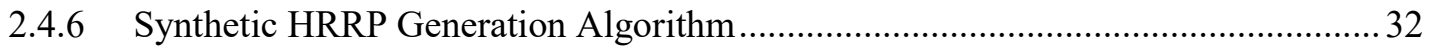

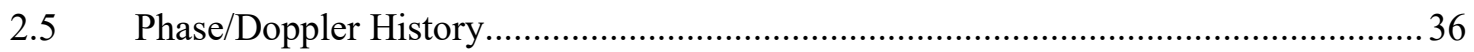

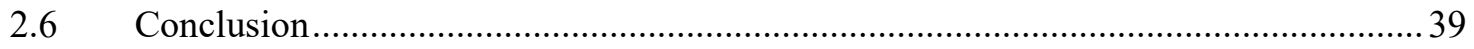

Chapter 3: Range-Doppler Imaging...................................................................................... 40

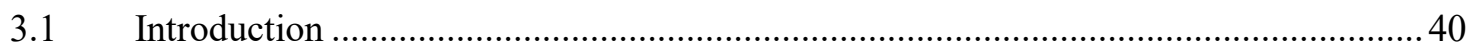

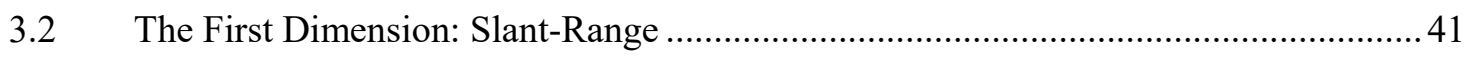

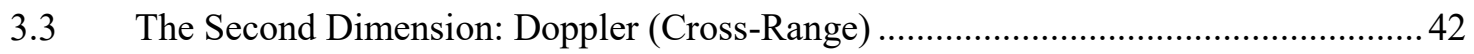

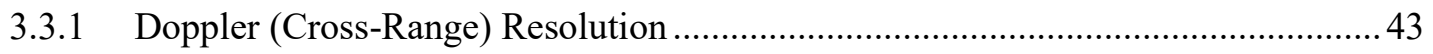

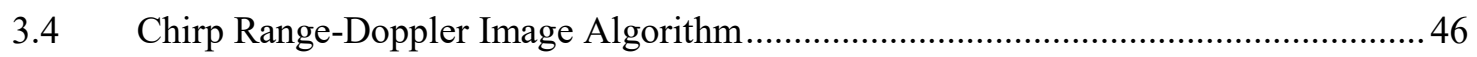

3.5 Stepped-Frequency Range-Doppler Image Algorithm ........................................... 49

3.6 Effects of Motion on Range-Doppler Image .................................................... 51

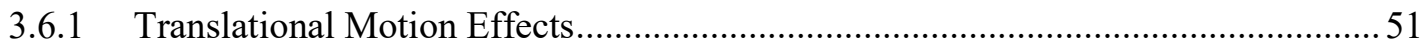

3.6.2 Translational Motion Compensation (TMC) Algorithms .....................................52

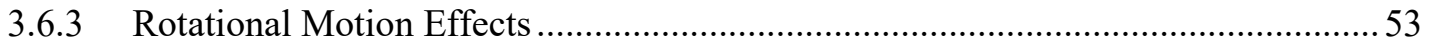

3.6.4 Rotational Motion Compensation (RMC) Algorithms ...........................................55

3.6.5 Motion Compensation \& Automatic Image Focus Algorithms ..............................56

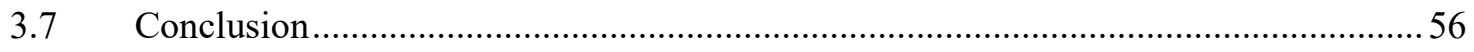

Chapter 4: Target Motion Algorithm .................................................................................... 57

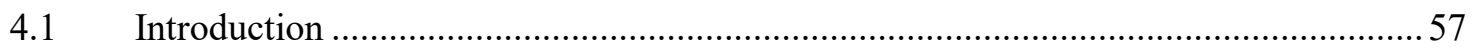

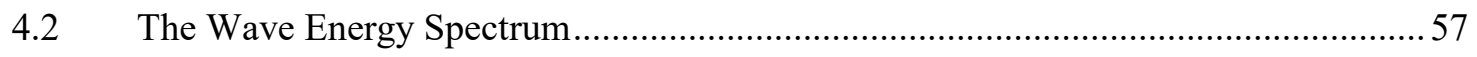

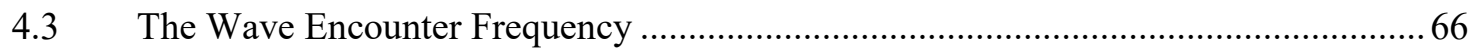

4.4 Ship Dynamics, Transfer Functions \& Response Amplitude Operators (RAOs)......... 72 


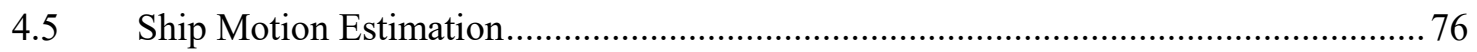

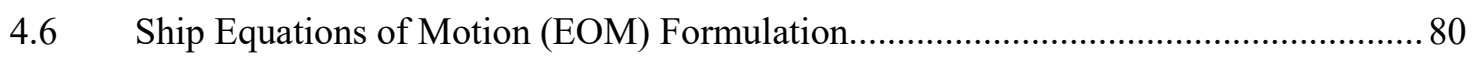

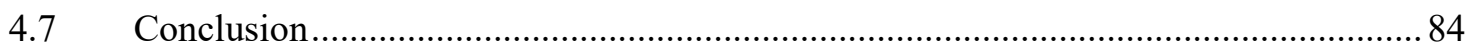

Chapter 5: Target Signature Synthesis Software ........................................................ 85

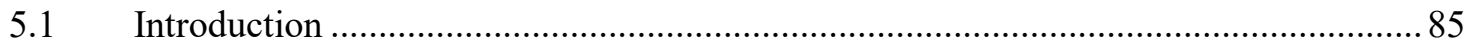

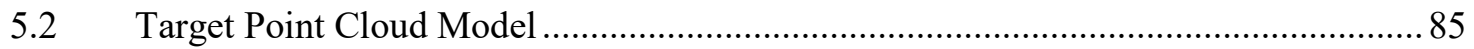

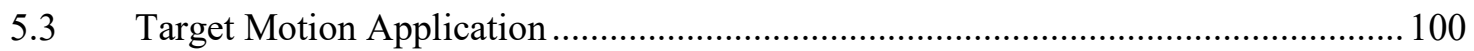

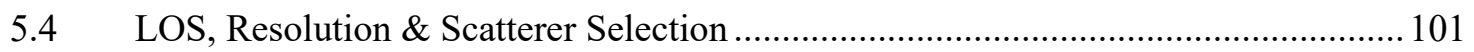

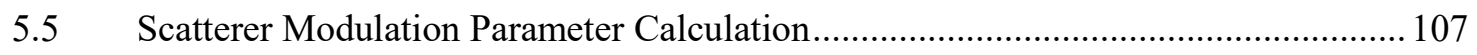

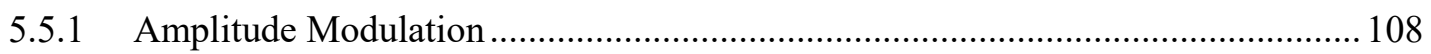

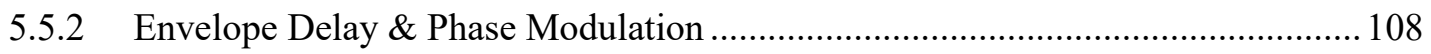

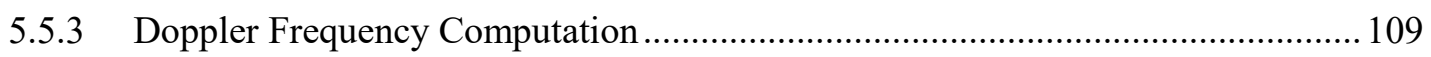

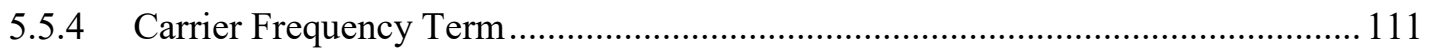

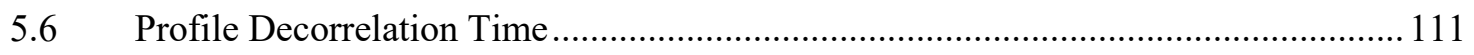

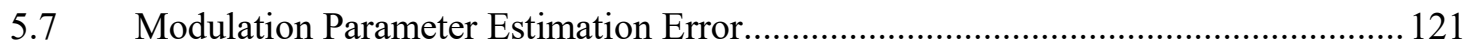

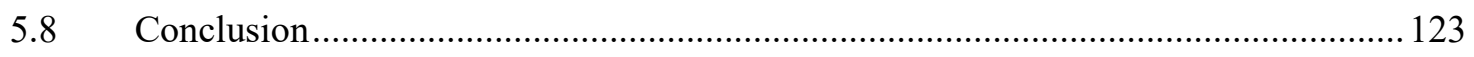

Chapter 6: Digital Profile Synthesis System Design .................................................... 124

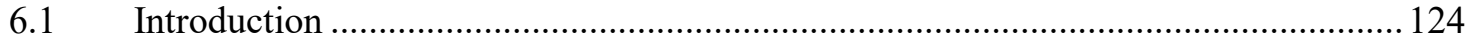

6.2 Digital Quadrature Demodulation System Design .............................................. 125

6.2.1 Sampling Rate \& Digital LO Frequency Selection ............................................. 126

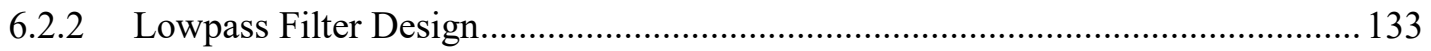

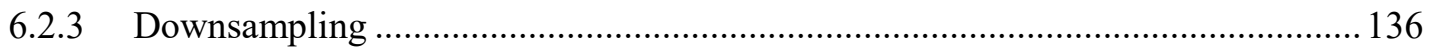

6.3 Digital Signal Modulation Application ............................................................ 148

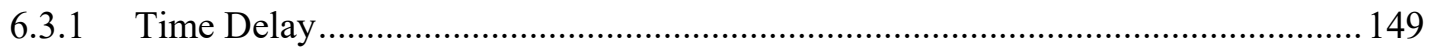

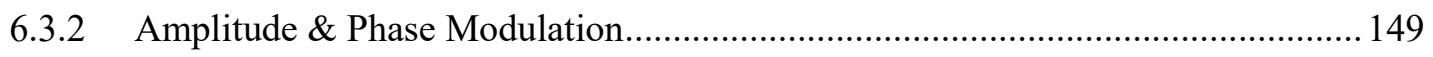

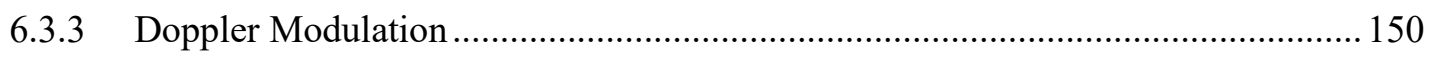


6.4 Conclusion

Chapter 7: Target Signature Synthesis System Simulation ...................................... 156

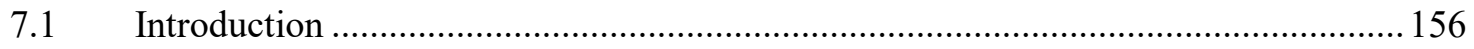

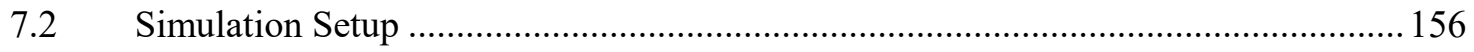

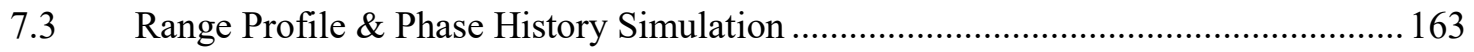

7.3.1 Countering Stepped-Frequency Radar Systems ...................................................... 163

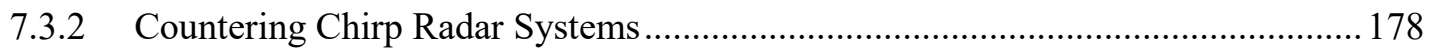

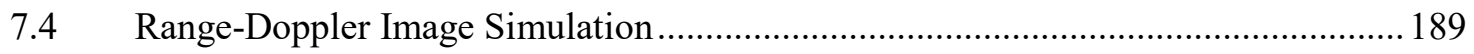

7.4.1 Image Generation from Stepped-Frequency Data............................................ 189

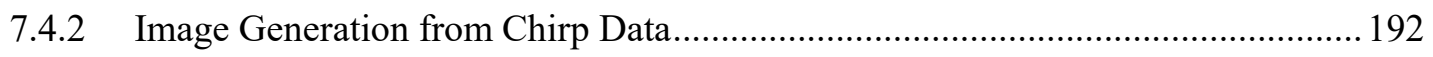

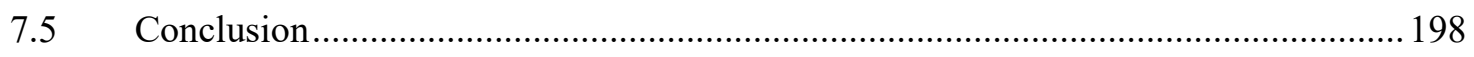

Chapter 8: Conclusions and Contributions .......................................................... 199

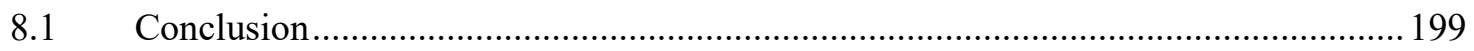

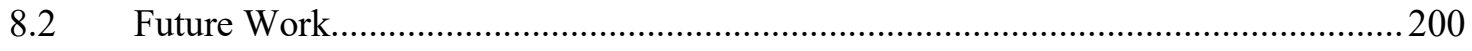

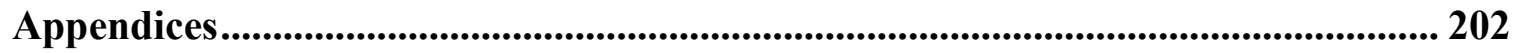

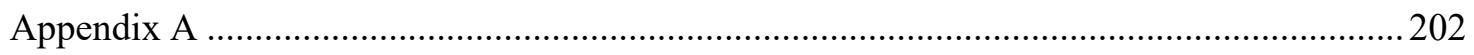

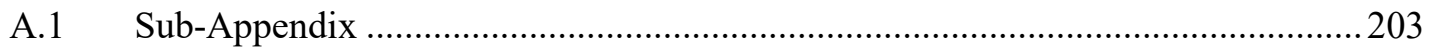

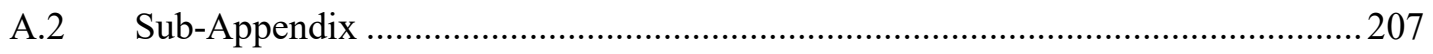

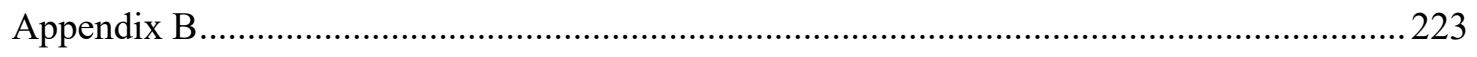

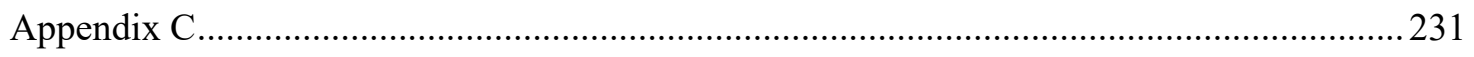

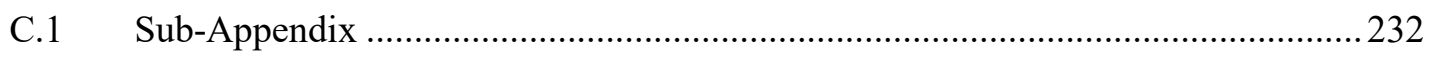

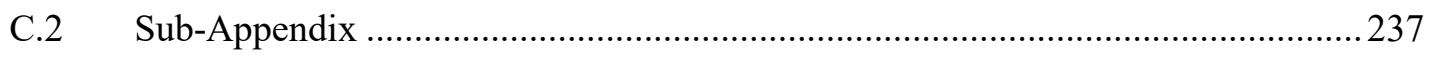

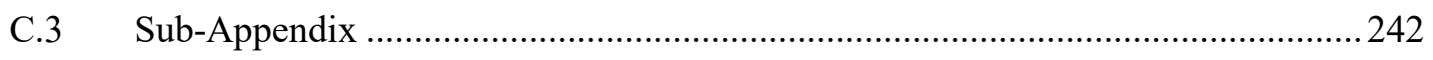

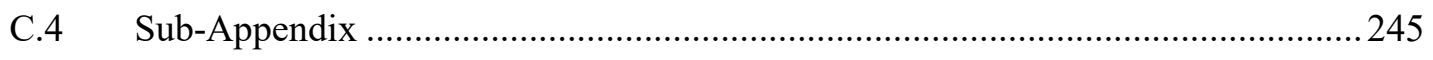

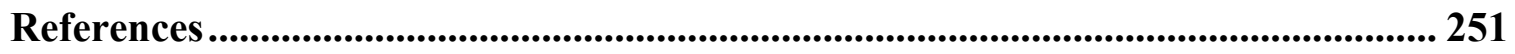




\section{List of Tables}

Table 4.1: NATO Sea State Table with Significant Wave Height and Modal Wave

Period. 61

Table 4.2: Wave Encounter Frequency relations as a function of $\alpha$, from [10].............. 68

Table 4.3: Motion equations for each of the six degrees of movement [3] ................... 82

Table 4.4: Rotation matrices for the three angular motions, based on [1] ..................... 83

Table 5.1: Approximation of number of data points in target cloud model based on rounding precision. 96

Table 5.2: Number of Data Points in the Target Cloud Model for the various options of decimal precision.

Table 5.3: The slopes of lines of the range and Doppler resolution cells according to LOS Azimuth relative to the target.

Table 5.4: Unit vector definition of hostile radar LOS, along principle axes of cartesian coordinate system 110

Table 5.5: Number of values which can be selected for each variable contributing to the target profile synthesis

Table 5.6: Maximum decorrelation time for four test cases

Table 5.7: Spectral resolution of Hann and Hamming windows for practical PRFs and FFT sizes

Table 5.8: Average time for a percentage change in the Doppler of a Profile. The change in Doppler is computed for various maximum Doppler frequencies. 121

Table 6.1: The maximum frequency component for varying downsampling factors..... 128 Table 6.2: The digital mixing products for sampling rate 3 GSPS and LO of $1 \mathrm{GHz} \ldots .134$ 
Table 6.3: Filter specifications for FIR Type 1 LPF designed in MATLAB.

Table 6.4: The continuous and discrete frequencies of a received signal through the stages

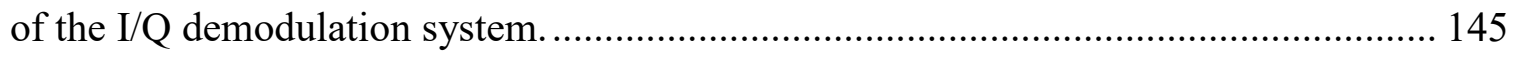

Table 7.1: Description of MATLAB scripts used in system simulation....................... 162

Table 7.2: Meaning of variables used in simulation scenarios. .................................. 163

Table 7.3: Simulation scenarios for countering a Stepped Frequency Radar System. ... 164

Table 7.4: Simulation scenarios for countering a Chirp Radar System........................ 178 


\section{List of Figures}

Figure 1.1: Three categories of Electronic Warfare (EW) [76] ........................................ 1

Figure 1.2: A conceptual diagram of a marine radar system providing a false target to a hostile radar, the real target return is also shown............................................................. 4

Figure 2.1: Traditional SAR systems observe a target as the radar platform undergoes

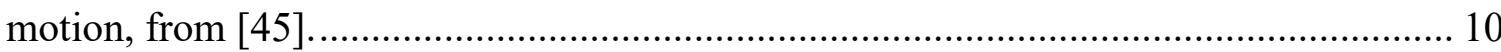

Figure 2.2: An ISAR system. For example, a coastal radar is observing a ship target, from [45].

Figure 2.3: Block diagram of a pulse compression system, from [53] 12

Figure 2.4: Chirp radar waveform; (a) pulse envelope, (b) pulse frequency as a function of pulse duration, (c) chirp waveform observed in time. 13

Figure 2.5: The stepped frequency transmitted waveform as a function of time. 14 Figure 2.6: Mixing (a) analog mixer low order outputs, (b) Superheterodyne mixer, from [45][53]. 16

Figure 2.7: Quadrature detection system, implemented in analog or digital hardware. ... 17 Figure 2.8: $(\mathrm{a}, \mathrm{b})$ Quadrature detection of received target signal. (a) Ideal coherent radar system receiver. (b) Quadrature detection waveforms. From [45][53]. 18 Figure 2.9: Practical quadrature detection in coherent radar receiver, from [45][53]..... 19 Figure 2.10: Range profile of a collection of arbitrarily spaced scatterers. 20 Figure 2.11: (a) Target echo signal, (b) Received spectrum at RF, (c) Received baseband spectrum, (d) Sampled received target echo signal. 22 Figure 2.12: Digital pulse compression system using a matched filter, from [45].......... 24 
Figure 2.13: Process diagram of the HRRP generation algorithm for processing chirp signals. 26

Figure 2.14: Block diagram of the synthetic HRRP generation algorithm.................... 33

Figure 2.15: Slow-Time history of a single sample position in a range profile [45]....... 37

Figure 2.16: Discrete Doppler Spectrum of a single range cell, sampled at the radar PRI,

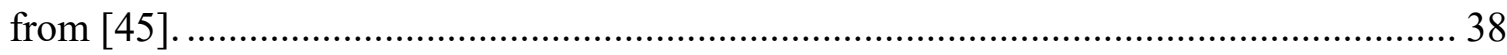

Figure 3.1: The slant-range and cross-range (Doppler) dimensions when imaging a target,

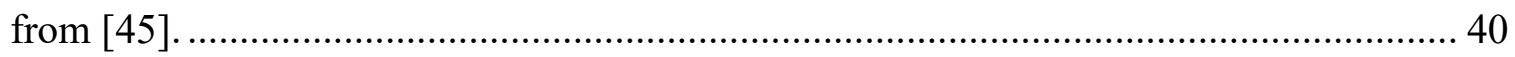

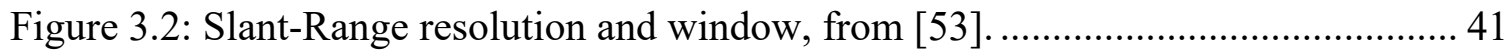

Figure 3.3: Illustration of the phase/Doppler dimension processing, from [53]............. 43

Figure 3.4: Angular velocity of a scattering at a cross-range position, from [53].......... 43

Figure 3.5: Logical diagram of ISAR Image generation for pulse compression systems

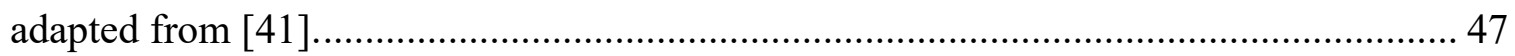

Figure 3.6: ISAR Image Processing for Chirp Pulse Data........................................... 48

Figure 3.7: ISAR Image processing steps for Stepped-Frequency Data. ....................... 50

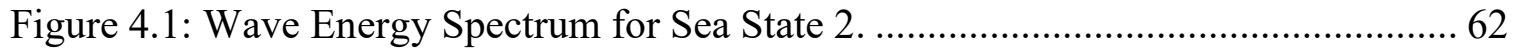

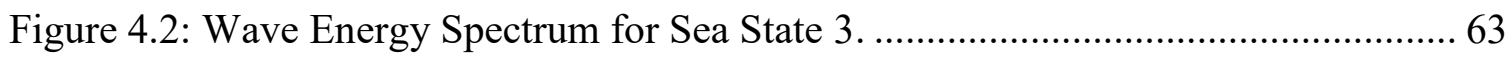

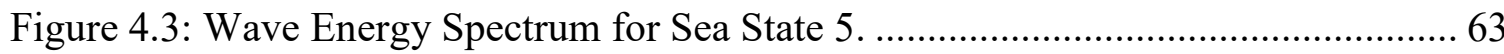

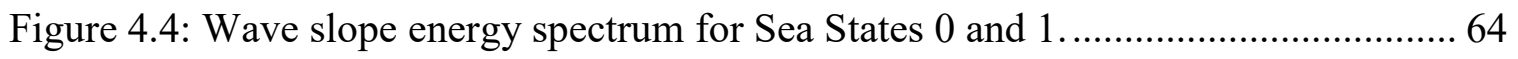

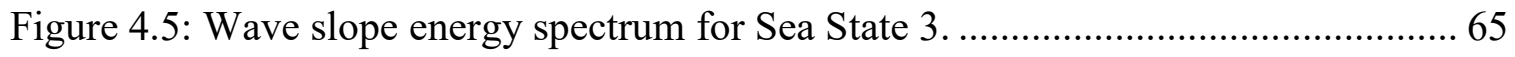

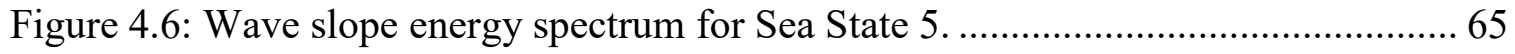

Figure 4.7: A graphical representation of Equation 4.12, for various values of $\alpha$, from

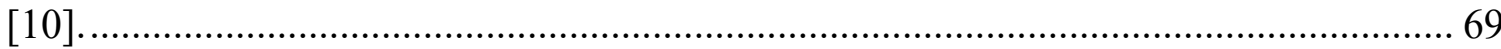


Figure 4.8: Mapping of the wave energy spectrum to the wave encounter energy spectrum, from [13][17] 70

Figure 4.9: The Wave Energy Spectrum for Sea State 5, as a function of encounter frequency, for a ship velocity of 5 knots, and a wave heading (relative to the ship stern) of 225 degrees. 71

Figure 4.10: The Wave Slope Energy Spectrum for Sea State 5, as a function of encounter frequency, for a ship velocity of 5 knots, and a wave heading (relative to the ship stern) of 225 degrees. 72

Figure 4.11: The six degrees of movement experienced by a ship, from [26]. .............. 72

Figure 4.12: A sample transfer function for ship roll, taken from [16] ....................... 75

Figure 4.13: A representation of the filter analogy to obtain a ship motion energy

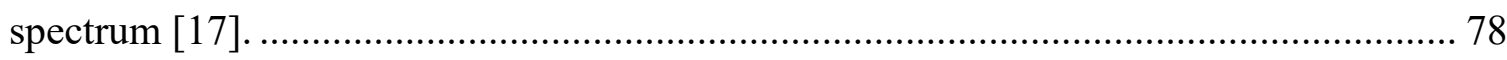

Figure 4.14: The inertial and reference coordinate systems of the ship, as adapted from $[3]$. 81

Figure 5.1: CAD model of the USS Spruance (DD-963) Destroyer class ship [58]. ...... 86 Figure 5.2: ISAR Contour of USS Spruance, LOS incident upon bow, target undergoing Yaw motion. Square resolution (Range \& Cross-Range resolution) of 0.5 meters. 88 Figure 5.3: ISAR Contour of USS Spruance, LOS incident upon aft, target undergoing Yaw motion. Square resolution (Range \& Cross-Range resolution) of 0.5 meters. 88 Figure 5.4: ISAR Contour of USS Spruance, LOS incident upon broadside, target undergoing Yaw motion. Square resolution (Range \& Cross-Range resolution) of 0.5 meters. 89 
Figure 5.5: ISAR Contour of USS Spruance, LOS incident upon stern, target undergoing Pitch motion. Square resolution (Range \& Cross-Range resolution) of 0.5 meters........ 90 Figure 5.6: ISAR Contour of USS Spruance, LOS incident upon top of target undergoing Pitch motion. Square resolution (Range \& Cross-Range resolution) of 0.5 meters. 90

Figure 5.7: ISAR Contour of USS Spruance, LOS incident upon Bow of target undergoing Pitch motion. Square resolution (Range \& Cross-Range resolution) of 0.5 meters. 91

Figure 5.8: DD963 USS Spruance target point cloud model generated using point matching algorithm on the ISAR image contour data obtained from ANSYS HFSS. .... 93 Figure 5.9: Refined/Filtered DD963 USS Spruance target point cloud model, with rounding to a single decimal precision in all dimensions. 94 Figure 5.10: Refined/Filtered DD963 USS Spruance target point cloud model, with rounding to a zero-decimal precision (i.e. nearest integer) in all dimensions. 97 Figure 5.11: Refined/Filtered point cloud model, with rounding to a zero-decimal precision (i.e., nearest integer) in the $\mathrm{X}$ and $\mathrm{Z}$ dimensions, and single decimal precision in the $\mathrm{Y}$ dimension. 98 Figure 5.12: Refined/Filtered target point cloud model, with zero-decimal precision in the $\mathrm{X}$ dimension, and single decimal precision in the $\mathrm{Y}$ and $\mathrm{Z}$ dimensions. ....................... 98 Figure 5.13: Target Signature Synthesis Software User Interface. 100 Figure 5.14: Radar LOS Azimuth is set to $0^{\circ}$. Cross-Range cells exist orthogonal to the LOS. Highlighted is the 3rd Cross-Range cell when the Cross-Range resolution is 4 bins. 103 
Figure 5.15: Radar LOS Azimuth is set to $90^{\circ}$. Cross-Range cells exist orthogonal to the LOS. Highlighted is the 13th Cross-Range cell when the Cross-Range resolution is 16 bins. 103

Figure 5.16: Radar LOS Azimuth is set to $130^{\circ}$. Cross-Range cells exist orthogonal to the LOS. Highlighted is the 55th Cross-Range cell when the Cross-Range resolution is 64 bins. 104

Figure 5.17: Radar LOS Azimuth is set to $0^{\circ}$. Cross-Range cells exist orthogonal to the LOS, and Range cells are parallel to the LOS. Highlighted is the 1st Cross-Range cell when the Cross-Range resolution is 2 bins, and the 1st range cell for a range resolution of 4 bins. 104

Figure 5.18: Radar LOS Azimuth is set to $90^{\circ}$. Highlighted is the 2nd Cross-Range cell when the Cross-Range resolution is 8 bins, and the 14th range cell for a range resolution of 16 bins 105 Figure 5.19: Radar LOS Azimuth is set to $40^{\circ}$. Highlighted is the 12th Cross-Range cell when the Cross-Range resolution is 32 bins, and the 14th range cell for a range resolution of 64 bins 105

Figure 5.20: Dominant scatterers along a resolution slice of the target are highlighted in white. These points are selected in the Target Signature Synthesis software. 107 Figure 5.21: Case 1, 4x4 resolution cell, percent error in Doppler for each resolution cell.

Figure 5.22: Case 2, 16x16 resolution cell, percent error in Doppler for each resolution cell. Only shown for 6 scatterers. 115 
Figure 5.23: Set 4, 64x64 resolution cell, percent error in Doppler for each resolution cell. Only shown for 6 scatterers.

Figure 5.24: The average percentage difference in Doppler for all scatterers in Case 1, using $4 \times 4$ resolution cell.

Figure 5.25: The average percentage difference in Doppler for all scatterers in Case 1, using $16 \times 16$ resolution cell.

Figure 5.26: The average percentage difference in Doppler for all scatterers in Case 1, using $64 \times 64$ resolution cell.

Figure 5.27: Frequency resolution for Hann/Hamming window in $\mathrm{Hz}$, for varying window size, and sampling frequency (i.e., PRF).

Figure 5.28: The percentage error in wavelength and modulation parameter as the frequency deviates from a $10 \mathrm{GHz}$ carrier frequency.

Figure 5.29: Wavelength as a function of frequency.

Figure 6.1: System diagram of a I/Q demodulation system.

Figure 6.2: The number of samples as a function of sampling rate for a pulse width of

1microsecond.

Figure 6.3: The digital frequency of the maximum and minimum IF following

downconversion, as a function of digital LO and sampling rate.

Figure 6.4: The number of samples as a function of sampling frequency for three options

of downsampling factor; 2,3 and 4.

Figure 6.5: The digital frequency LO and sampling rate combinations to achieve a downsampling factor of four. 
Figure 6.6: The digital frequency LO and sampling rate combinations for the minimum analog IF are seen in blue.

131

Figure 6.7: The digital frequency LO and sampling rate combinations for the minimum analog IF are seen in blue. 132

Figure 6.8: Order of Windowed LPF to achieve the minimum transition width required to isolate difference products from digital mixing. 133 Figure 6.9: Magnitude response of Kaiser Window FIR Type 1 LPF. 136

Figure 6.10: Phase response of Kaiser window FIR Type 1 LPF. 136 Figure 6.11: Magnitude spectrum of received (RF) $10.15 \mathrm{GHz}$, (IF) $1.35 \mathrm{GHz}, 1 \mu \mathrm{s}$ pulse, sampled at 3 GSPS 137

Figure 6.12: Phase spectrum of received (IF) $1.35 \mathrm{GHz}, 1 \mu$ s pulse, sampled at 3 GSPS.

Figure 6.13: Magnitude spectrum of received (IF) $1.35 \mathrm{GHz}, 1 \mu$ s pulse, sampled at 3 GSPS, mixed with the in-phase digital LO at $1 \mathrm{GHz}$ to create the in-phase (I) channel. 139 Figure 6.14: Phase spectrum of received (IF) $1.35 \mathrm{GHz}, 1 \mu$ s pulse, sampled at 3 GSPS, mixed with the in-phase digital $\mathrm{LO}$ at $1 \mathrm{GHz}$ to create the in-phase (I) channel. 139 Figure 6.15: Magnitude spectrum of received (IF) $1.35 \mathrm{GHz}, 1 \mu$ s pulse, sampled at 3 GSPS, mixed with the quadrature digital $\mathrm{LO}$ at $1 \mathrm{GHz}$ to create the quadrature (Q) channel. 140

Figure 6.16: Phase spectrum of received (IF) $1.35 \mathrm{GHz}, 1 \mu$ s pulse, sampled at 3 GSPS, mixed with the quadrature digital $\mathrm{LO}$ at $1 \mathrm{GHz}$ to create the quadrature (Q) channel... 140 
Figure 6.17: Magnitude spectrum of received (IF) $1.35 \mathrm{GHz}, 1 \mu$ s pulse, sampled at 3 GSPS, mixed with the in-phase digital $\mathrm{LO}$ at $1 \mathrm{GHz}$ to create the in-phase (I) channel. Following filtering using the FIR Type 1 Kaiser Windowed LPF. 141

Figure 6.18: Phase spectrum of received (IF) $1.35 \mathrm{GHz}, 1 \mu$ s pulse, sampled at $3 \mathrm{GSPS}$, mixed with the in-phase digital $\mathrm{LO}$ at $1 \mathrm{GHz}$ to create the in-phase (I) channel. Following filtering using the FIR Type 1 Kaiser Windowed LPF. 142 Figure 6.19: Magnitude spectrum of received (IF) $1.35 \mathrm{GHz}, 1 \mu$ s pulse, sampled at 3 GSPS, mixed with the quadrature digital $\mathrm{LO}$ at $1 \mathrm{GHz}$ to create the quadrature(Q) channel. Following filtering using the FIR Type 1 Kaiser Windowed LPF. 143 Figure 6.20: Phase spectrum of received (IF) $1.35 \mathrm{GHz}, 1 \mu$ s pulse, sampled at 3 GSPS, mixed with the quadrature digital $\mathrm{LO}$ at $1 \mathrm{GHz}$ to create the quadrature(Q) channel. Following filtering using the FIR Type 1 Kaiser Windowed LPF. 144 Figure 6.21: Filtered Magnitude spectrum of received (IF) $1.35 \mathrm{GHz}, 1 \mu$ s pulse, sampled at 3 GSPS, mixed with the in-phase digital $\mathrm{LO}$ at $1 \mathrm{GHz}$ to create the in-phase (I) channel. The signal has been downsampled by a factor of 4 . 146 Figure 6.22: Filtered Phase spectrum of received (IF) $1.35 \mathrm{GHz}, 1 \mu$ s pulse, sampled at 3 GSPS, mixed with the in-phase digital LO at $1 \mathrm{GHz}$ to create the in-phase (I) channel. The signal has been downsampled by a factor of 4 . 146 Figure 6.23: Filtered Magnitude spectrum of received (IF) $1.35 \mathrm{GHz}, 1 \mu$ s pulse, sampled at $3 \mathrm{GSPS}$, mixed with the quadrature digital $\mathrm{LO}$ at $1 \mathrm{GHz}$ to create the quadrature (Q) channel. The signal has been downsampled by a factor of 4 . 147 
Figure 6.24: Filtered Phase spectrum of received (IF) $1.35 \mathrm{GHz}, 1 \mu$ s pulse, sampled at 3 GSPS, mixed with the quadrature digital $\mathrm{LO}$ at $1 \mathrm{GHz}$ to create the quadrature $(\mathrm{Q})$ channel. The signal has been downsampled by a factor of 4 .

Figure 6.25: Doppler Modulation Signal for a single scatterer overlayed with 60 PRIs of the received hostile radar signal...................................................................... 152 Figure 6.26: Subsection of Doppler Modulation Signal for a single scatterer overlayed with two PRIs of the received hostile radar signal. 153 Figure 6.27: Baseband complex samples of slow-time sequence of a scattering location with a Doppler frequency of $3.5 \mathrm{kHz}$. 154

Figure 6.28: DFT of slow-time complex samples in figure 6.27. Note the peaks at the Doppler frequency of $3.5 \mathrm{kHz}$. 154

Figure 7.1: Top-Level Block Diagram of Target Signature Synthesis System. 157 Figure 7.2: System Block Diagram of Target Signature Synthesis Digital System Design up to the modulation block. 158 Figure 7.3: System Block Diagram of Signal Modulation Scheme in the In-Phase channel as implemented in MATLAB simulations. The same block diagram is applicable to the Quadrature channel.

Figure 7.4: Flow Chart of MATLAB simulation program setup. 161

Figure 7.5: The synthetic range profile of the target in the I channel. 166

Figure 7.6: The synthetic range profile of the target in the Q channel. 166 Figure 7.7: The complex valued synthetic high-resolution range profile for simulation case 1 167 
Figure 7.8: Target model perspective for which the range profile is produced in case one.

Figure 7.9: Target point cloud model with dominant scattering locations highlighted as chosen in the TSS program for case one. 169

Figure 7.10: Waterfall plot of the magnitude of the synthetic range profile generated from each burst. 170

Figure 7.11: A bird's eye view of the waterfall plot of the magnitude of the synthetic range profile generated from each burst.

Figure 7.12: A bird's eye view of the waterfall plot of the phase of the synthetic range profile generated from each burst.

Figure 7.13: The complex valued synthetic high-resolution range profile for simulation case four. 173

Figure 7.14: Target model perspective for which the range profile is produced in case four.

Figure 7.15: Simulation Case 4 Complex baseband magnitude range profile history.... 174 Figure 7.16: Simulation Case 4 Complex baseband phase history 174 Figure 7.17: The complex valued synthetic high-resolution range profile for simulation case 5 . 175

Figure 7.18: The complex valued synthetic high-resolution range profile for simulation case 5 , corrected for range aliasing. 176 Figure 7.19: Target model perspective for which the range profile is produced in case five. 176 Figure 7.20: Simulation Case 5 Complex baseband magnitude range profile history.... 177 
Figure 7.21: Simulation Case 5 Complex baseband phase history. 177

Figure 7.22: Chirp Radar simulation case $1 \mathrm{a} / 1 \mathrm{~b}$, complex baseband range profile. 181 Figure 7.23: Chirp Radar simulation case 1a/1b, complex baseband range profile, zoomed in. 181

Figure 7.24: Simulation case 1a, magnitude of complex baseband range profile history. 182

Figure 7.25: Simulation case 1a complex baseband phase history. 182

Figure 7.26: Simulation case 1b, magnitude of complex baseband range profile history. 183

Figure 7.27: Simulation case 1b complex baseband phase history................................ 183

Figure 7.28: Complex baseband range profile Simulation Case 4............................... 184

Figure 7.29: Complex baseband range profile Simulation Case 4, zoomed in............... 185

Figure 7.30: Chirp Radar simulation case 4, magnitude of complex baseband range profile history 185

Figure 7.31: Chirp Radar simulation case 4, magnitude of complex baseband range profile history. 186

Figure 7.32: Chirp Radar simulation case 4, complex baseband phase history. 187

Figure 7.33: Complex baseband range profile Simulation Case 5. 187

Figure 7.34: Complex baseband range profile Simulation Case 5, zoomed in. 188 Figure 7.35: Chirp Radar simulation case 5, magnitude of complex baseband range profile history 188

Figure 7.36: Chirp Radar simulation case 5, complex baseband phase history. 189 
Figure 7.37: Simulation Case 1 Range Doppler image, uncompensated complex baseband data. 190

Figure 7.38: Simulation Case 1 Range Doppler image, motion compensated complex baseband data. 190

Figure 7.39: Simulation Case 2 Range Doppler image, uncompensated complex baseband data. 191

Figure 7.40: Simulation Case 2 Range Doppler image, range and Doppler tracked complex baseband data. 191

Figure 7.41: Uncompensated Range Doppler image for Simulation case 1a. 193

Figure 7.42: Motion compensated Range-Doppler image for Simulation case 1a.... 193

Figure 7.43: Range-Doppler image for case 1a, Doppler corrected by $1200 \mathrm{~Hz}$. 194

Figure 7.44: Uncompensated Range Doppler image for Simulation case 1b. 194

Figure 7.45: Motion compensated Range-Doppler image for Simulation case 1b. 195

Figure 7.46: Range-Doppler image for case 1b, Doppler corrected by $500 \mathrm{~Hz}$. 195

Figure 7.47: Uncompensated Range-Doppler image for Simulation case 4. 196

Figure 7.48: Motion compensated Range-Doppler image for Simulation case 4. 196

Figure 7.49: Range-Doppler image for case 4, Doppler corrected by $4800 \mathrm{~Hz}$. 197 


\section{List of Appendices}

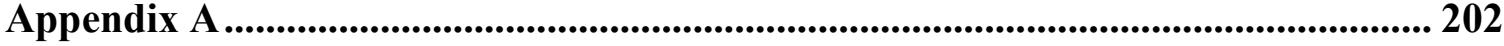

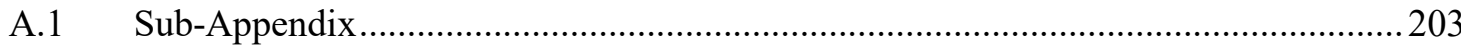

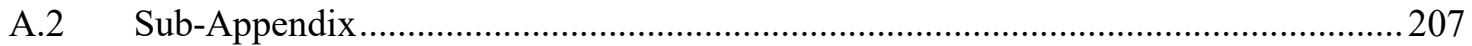

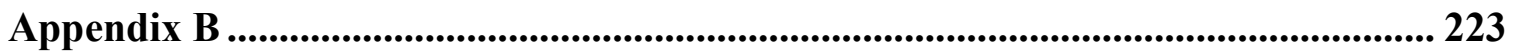

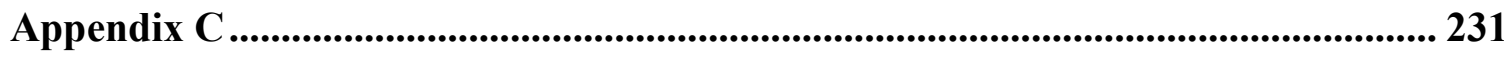

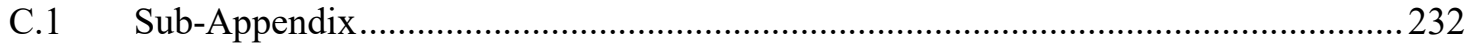

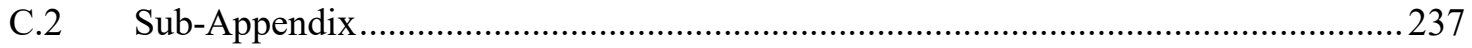

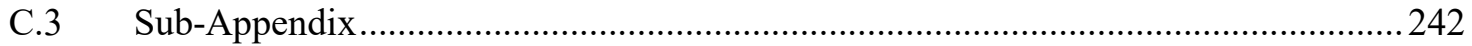

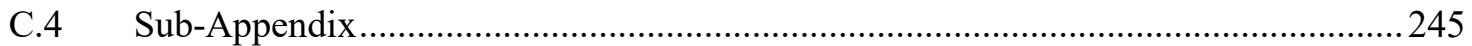




\section{Chapter 1: Introduction}

\subsection{The Evolution of Electronic Warfare}

Increasing demand in intense fields of use such as military applications, healthcare, and communications have driven the evolution of technology. Military applications have used technology to evolve a relatively new form of warfare unique to the $20^{\text {th }}$ and $21^{\text {st }}$ century known as Electronic Warfare (EW). In modern warfare applications, EW systems are used to ensure the safety of personnel and can determine the success of an operation [76]. EW encompasses operations in the Electromagnetic (EM) spectrum to perform communication, navigation, positioning, timing, weapons control, and other tactical actions [64][76]. EW refers to tactics and actions which aim to interfere with, attack, deceive, or in general take advantage of the enemy's use of the EM spectrum [63][67].

EW is a broad term in which tactics and technologies can be identified into three categories shown in figure 1.1 [63][76].

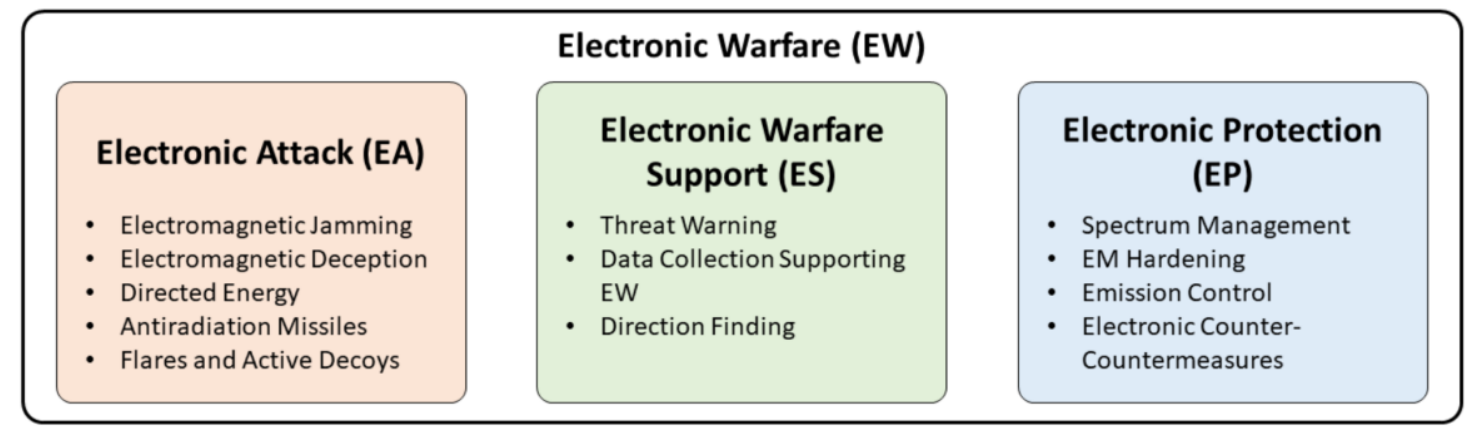

Figure 1.1: Three categories of Electronic Warfare (EW) [76].

Electronic Attack (EA) tactics are used to degrade the quality of enemy EW systems [63][76]. Electronic Support (ES) tactics provide supplementary data, such as missile warning systems, to aid in tactical operations [63][76]. Electronic Protection (EP) measures are used to protect systems from hostile and friendly EA tactics [63][76]. The categories of EW are deeply interconnected and there is continuous interest from government and private industry in developing and advancing technologies that enable and enhance EW. 
As the battlespace becomes increasingly complex, the cost of engaging in warfare also increases. The cost of building naval battleships is significant, averaging approximately $\$ 1$ billion per ship, depending on the class of battleship [77]. Naval ships operate in a fleet comprised of multiple ships which perform unique functions [78][79]. Generally, there are no more than one to two types of a single ship within a fleet, due to the high cost for each unique ship and the need to have ships to perform various functions [78][79]. Thus, protecting each ship within a fleet is of utmost importance because of the monetary investment, and more critically the protection of the personnel aboard and the tactical capabilities of each battleship [79]. The protection of assets begins through EW with the development of EP capabilities, such as radar signature reduction, to reduce the probability of a ship being detected by a hostile radar system. EA tactics are utilized as well, such as jamming or deception technologies, developed in radar systems to reduce the effectiveness of hostile EW tactics [76].

EW engineers aim to collect information about parameters of hostile systems, such as frequency, bandwidth, and possibly modulation techniques, to design countermeasures [50][67]. However, the evolution of technology has increased the challenges of performing EW activities which are continually effective against adaptive systems which can change parameters in real-time.

The advances in digital sampling technology alone have greatly increased the flexibility of systems to perform wideband signal processing applications that can reduce effectiveness of protective EW tactics [43][67]. The advancement of digital hardware technology following Moore's law has led to the development of high-speed processing systems, such as field programmable gate arrays (FPGAs) and software defined radios (SDRs), which can perform a greater variety of signal processing operations which were unavailable to EW engineers in decades past [43][50]. The significant risk associated with the increased flexibility of systems is the capability to perform EA actions may outpace EP or ES tactics [43][49][67]. Systems can now adapt to new environments and user requirements in real-time, such as real-time waveform optimization to counter background clutter and hostile interference in radar systems [49][76]. EW technologies which can perform target classification, identification and tracking operations of 
hundreds of unique targets simultaneously in real-time are deployed in almost all Naval operations [49][63][67].

Research into cognitive and adaptive technology has also enabled the design of systems which can determine, and then optimize the effectiveness of the EW tactics in real time [63][64]. EW operations have had to evolve beyond traditional tactics such as wideband noise or barrage jamming, into more complicated deception jamming technologies [50][64][67]. Noise jamming involves flooding the hostile radar receiver with significant interference to mask the presence of a friendly target [50]. Deception jamming can involve the deployment of false targets or other tactics to deceive the hostile radar into seeing multiple targets or misidentifying a target [50]. Academic research into deception technologies has also led to a great advance in the field of EW from a theoretical exercise standpoint. Research now consists of design and development of deception jamming technology, as well as countermeasures (ECM) to this technology and counter-countermeasures (ECCM) [1]-[9][27]-[56][63][64]. This has led to the innovation of technology which can identify deception jamming techniques to differentiate false targets from real targets [35]. Thus, EW continues to evolve as the predominant and primary method of warfare in today's technologically driven world.

\subsection{Thesis Objectives}

The primary objective of this thesis is to develop a realistic target motion algorithm and software application which can be used to generate false targets in Naval deception jamming and EA operations. This research has been performed in collaboration with MC Countermeasures Inc., as a proof-of-concept to be used in future systems developments. Figure 1.2 is a basic illustration of the desired application of the target. 

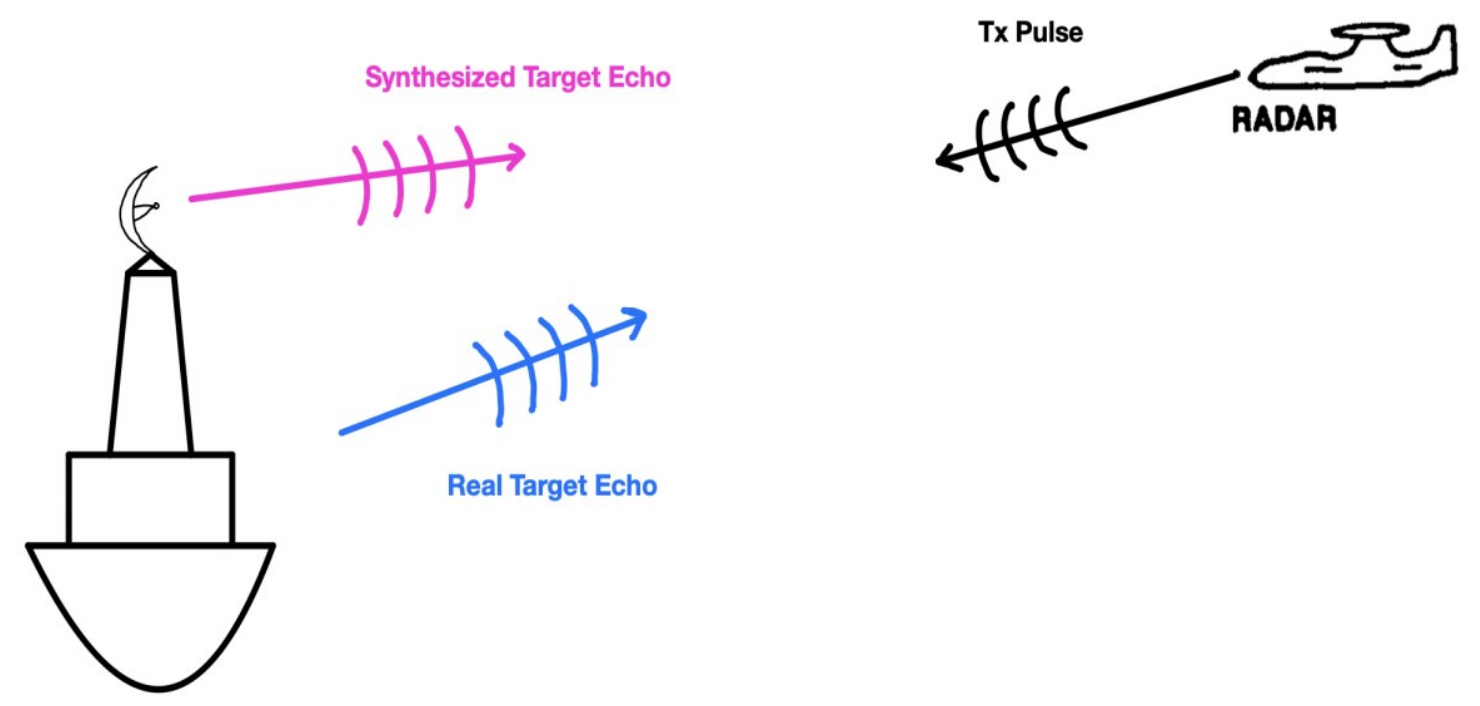

Figure 1.2: A conceptual diagram of a marine radar system providing a false target to a hostile radar, the real target return is also shown.

A practical false target synthesis system would require the design of an entire radar system including all digital and analog hardware components involved. Design of such a system is a complex process that requires the collaboration of many specialized engineers and technicians. This is beyond the scope of this research, which will only focus on the software development of a program and algorithm to create a realistic false target, as well as simulations to verify the effectiveness of the design.

\subsection{Thesis Contributions}

This research contributes a realistic ship motion algorithm developed from the principles of ship motion estimation used in the field of Ocean Engineering and Naval Ship Architecture design. When the prerequisite ship and ocean wave data are available, the presented motion algorithm is designed to be flexible to provide realistic motion parameters for a large variety of ships in different sea environments. There is significant research in the field of Naval Architecture and Ocean engineering to ensure that prerequisite ship and ocean wave data already exists in a large volume. The algorithm is 
implemented in an application programmed in the language $\mathrm{CH}$, which creates a false target and provides the parameters to synthesize a false target signal.

Also developed as part of this research is a digital system design, which is yet to be implemented in digital hardware to create a deception jamming system. This system would capture a hostile signal and modulate the captured signal to simulate the interaction of the pulse with the false target generated in the C\# application. In other words, the goal of this design is to synthesize a digital false target signal which would be used to counter wideband high-resolution radar systems. The system design is done purely as a theoretical proof-of-concept and the effectiveness of the motion algorithm and false target generation program is verified through simulations in MATLAB.

\subsection{Thesis Overview}

The remainder of this thesis describes the necessary background information and the research conducted to create the program and system design. Chapter Two focuses on the basic principles of high-resolution radar systems, the generation of high-resolution range profiles, and phase histories of a target. This chapter focuses on the functionality and processing steps of these radar systems as discussed in literature.

Chapter Three addresses the development of Range-Doppler images based on Inverse Synthetic Aperture Radar (ISAR) principles discussed in literature. This chapter begins with discussion on the algorithmic processing steps to create the Range-Doppler image, then addresses motion compensation algorithms used to improve target image quality.

In Chapter Four, a detailed description of the realistic target motion algorithm is provided. The chapter begins with a discussion on ocean wave statistics as measured and analyzed in the field of ocean engineering. Principles of naval architecture are used to show how the motion of waves is coupled to the motion of a ship. This is then described in a mathematical algorithmic process which is used to perform motion simulation of a radar false target. 
In Chapter Five, the implementation of the motion algorithm and the development of a false target generation software is discussed in detail. First, a unique algorithm is discussed to generate point target models used to simulate a radar target. The application of the motion algorithm to the target model in the $\mathrm{C} \#$ program is also shown. The software is designed to communicate with a digital hardware system to synthesize a false target signal. The programmatic method to obtain parameters to synthesize a false radar target is presented.

In Chapter Six, the conceptual digital system design is presented in detail. This chapter includes assumptions of system design parameters provided by the collaborating company MC Countermeasures Inc. The system is designed using digital signal processing (DSP) principles to utilize the data from the program discussed in Chapter Five to synthesize a digital representation of a realistic target echo signal. This system is a proof-of-concept and would require more careful design to implement in digital hardware such as an FPGA. The effectiveness of the motion algorithm, software application, and digital system are tested through simulations done in MATLAB, which is discussed in Chapter Seven.

Chapter Eight contains a summary of the research and design, as well as recommendations for future work to progress this research. 


\section{Chapter 2: Principles of High-Resolution Radar}

\subsection{Introduction to High-Resolution Radar}

Radar has been used in a variety of applications since its conception in the early 1900s [50][63]-[67]. The evolution of technology following Moore's Law has significantly increased the capabilities of radar systems beyond simple detection and ranging [50][63]-[67]. High-Resolution Radar has been a field of interest, particularly in military applications since the 1970s [50][63]-[67]. The term high-resolution radar is used to refer to radar systems which have the capability to identify unique targets within a group of targets and resolve individual scattering centers on a target [45][50][63]-[67]. These systems can perform target classification, identification, and imaging when using high-resolution antenna and signal processing techniques [45][50][63]-[67].

Radars operate with resolution in two primary dimensions: slant-range and crossrange [45][53]. High-resolution radar systems provide fine resolution in one or both dimensions. In traditional radar systems the resolution is equivalent to the range window of a pulse and is determined by the pulse duration, as shown in equation 2.1.

$$
R=\frac{c \tau}{2}
$$

In equation (2.1) the constant $c$ is the speed of the pulse propagation, which is the speed of light. The variable $\tau$ represents the pulse duration and is generally on the order of microseconds [45][50]. Observation of equation 2.1 shows the range window increases, or the resolution decreases, as the pulse duration increases. High-resolution radar systems decouple the resolution from the pulse duration [45][50]. In slant-range the resolution is increased by increasing the waveform bandwidth, and in cross-range the resolution is increased by using very narrow antenna beamwidths or Synthetic Aperture Radar (SAR) processing techniques [45][50][53].

\subsubsection{Resolution in Slant-Range}

The slant-range resolution is proportional to the time domain resolution of the radar pulse signal [45][49][50][53]. The time domain resolution is enhanced by increasing the radar signal bandwidth [45][49][50][53]. In traditional radar, the pulse bandwidth is the 
reciprocal of the pulse duration. Decoupling the signal bandwidth from the pulse duration is done by using wideband waveforms such as [45][49][50][53]:

- Short Pulse Waveforms

- Chirp (Pulse Compression) Waveforms

- Stepped-Frequency Waveforms

When using wideband waveforms, the range resolution is related to the bandwidth as shown in equation 2.2. Here $\Delta r_{s}$ is the slant-range resolution and $\beta$ is the waveform bandwidth.

$$
\Delta r_{s}=\frac{c}{2 \beta}
$$

Other waveform types are also used in high-resolution radar systems. However, the three waveforms mentioned above are the focus of the research conducted in this thesis.

\subsubsection{Resolution in Cross-Range}

The cross-range resolution is limited by the antenna beamwidth. A small 3-dB beamwidth, such as a pencil-beam radar, results in a fine resolution in cross-range [45][49][50]. Equation 2.3 represents the approximate 3-dB beamwidth of a parabolic reflector antenna commonly used in radar applications.

$$
\theta_{3 d B} \approx \frac{\lambda}{d}
$$

Here, $\lambda$ is the wavelength of the radar signal, and $d$ is the size of the antenna aperture [53]. Increasing the cross-range resolution requires a reduction in wavelength, equivalently an increase in frequency. This comes at the cost of reduced radar range, since the atmospheric attenuation loss of a propagating wave increases with frequency [45][49][50][53]. Another option is to increase the antenna aperture size which also has some associated cost such as increased power requirements and excessive weight. The antenna aperture size is also limited by practical physical dimension constraints depending on the application. For example, antenna limitations on size and weight on aircraft radar systems [45][49][50][53]. 
The innovation of synthetic aperture processing techniques allows the use of wide antenna beamwidths, combined with appropriate radar design parameters and signal processing techniques to achieve high resolution in cross-range [45][49][50][53]. Synthetic aperture radar systems rely on the relative motion between the target and the radar system. The relative motion results in a change of velocity when observing targets, which manifests as a Doppler shift in the target return data [45][49][50][53]. Synthetic aperture processing is broadly separated into two categories of; Synthetic Aperture Radar (SAR) and Inverse Synthetic Aperture Radar (ISAR).

Conventional SAR systems observe stationary targets from a moving platform [45][49][50][53]. The velocity of the radar is known, and signal processing techniques are applied to generate images of the target with varying slant-range and cross-range resolution. An example of this is visible in the images seen in navigation applications, such as Google Earth, where satellites orbiting around the world use SAR processing on portions of the earth to produce images of the terrain.

ISAR systems observe moving targets from a moving or stationary platform. The target being observed has both translational and rotational motion. The motions create unique Doppler shifts which are observed in the target echo response. Figure 2.1 and 2.2 show the difference between SAR and ISAR systems. 


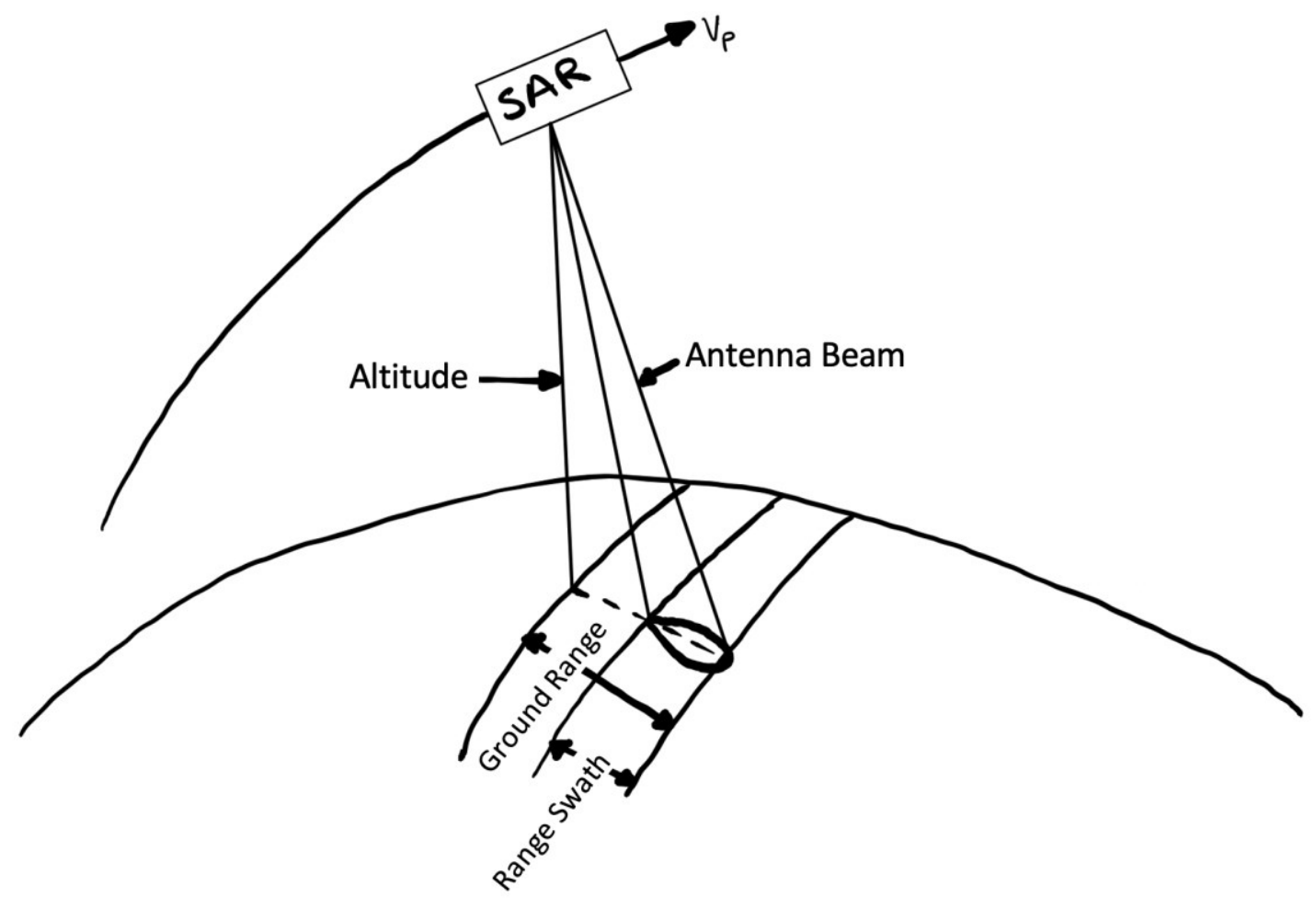

Figure 2.1: Traditional SAR systems observe a target as the radar platform undergoes motion.

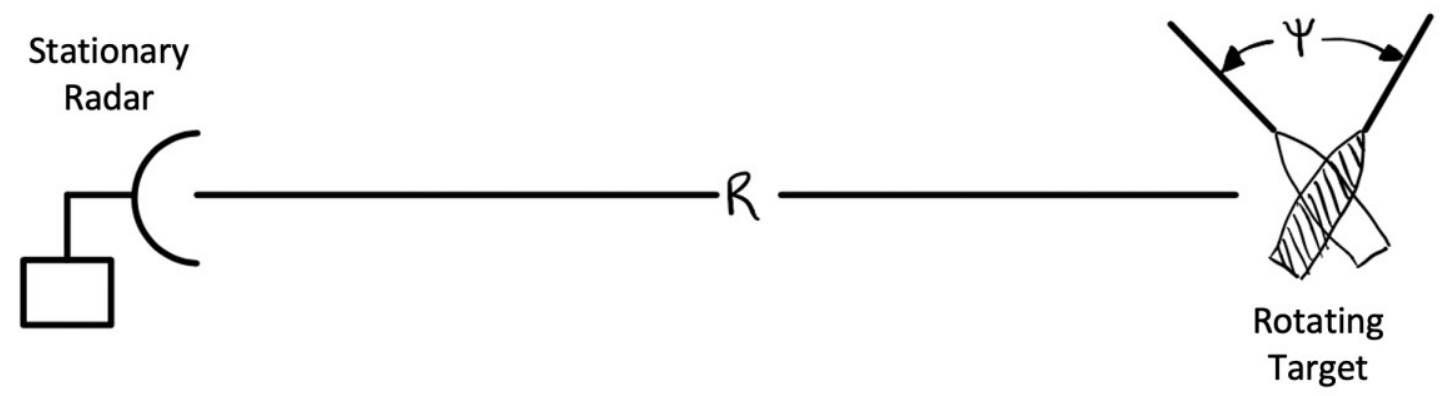

Figure 2.2: An ISAR system. For example, a coastal radar is observing a ship target.

The two main factors in determining the cross-range resolution in SAR/ISAR processing are the coherent integration time, and the degree of rotational motion of the target or platform [45][49][50][53]. The coherent integration time refers to the total time over which the target is observed by the radar and the number of target echoes which are obtained from the target prior to processing. The degree of rotational motion is dependent on the target. In SAR systems, the radar system is undergoing motion which is known, 
and thus the motion can be compensated in data processing. In ISAR systems, the target is undergoing rotational motion, which is not known or measured, thus, ISAR processing is often referred to as a blind process [45][49][50][53].

In ISAR processing, the control variable for cross-range resolution is the Coherent Integration Time. Intuition would dictate that increasing the Coherent Integration Time would result in finer cross-range resolution since a larger dataset is obtained prior to processing. However, increasing the Coherent Integration Time does come at the potential cost of data corruption in the form of blurring or smearing of the target image, especially when observing fast moving targets [45][49][50][53]. Additionally, as the observed target undergoes motion, the aspect angle of observation also changes with time, and large aspect angle changes can result in blurring or smearing of target data as the target image decorrelates with aspect angle change [45][49][50][53].

The focus of this research is to develop a realistic target motion model for Naval targets to counter high-resolution radar systems. Since the target is undergoing motion, it is assumed ISAR processing techniques are used to achieve fine cross-range resolution. For further information on SAR and ISAR processing the reader is referred to [45][49][50], and the ever-growing literature on the topics.

\subsection{High-Resolution Radar Waveforms}

\subsubsection{Short Pulse Waveforms}

Equation 2.1 represents the range resolution as a function of pulse duration; as the pulse duration decreases, the resolution increases. There are two significant issues with reduction of pulse duration. As the pulse duration decreases, the amount of power in the pulse also decreases, and in principle this reduces the effectiveness of the radar system [45][50]. The received power from a target is proportional to the transmitted pulse power and the Radar Cross-Section (RCS) of the target. As the transmitted power decreases, the power in the echo return also decreases, and this can result in a target echo signal being masked by the noise in the receiving system.

Additionally, digital processing of short pulses requires high sampling rates [53]. For short pulses on the order of microseconds to nanoseconds, the sampling rate required 
is on the order or mega-samples to giga-samples per second [53]. Sampling at these rates requires increasingly expensive hardware, and the resolution in the number of bits also decreases with an increase in sampling rate [49][53][46][47].

Short pulse waveforms are not commonly used in high-resolution applications for the reasons mentioned above. Thus, the bandwidth of the waveform is decoupled from the pulse duration by applying wideband modulation methods. Many wideband modulation methods exist, the methods focused on in this research are the chirp and stepped-frequency waveforms, which are described in further detail in the following subsections.

\subsubsection{Chirp (Pulse Compression) Waveforms}

Pulse compression waveforms spread the energy of a high bandwidth signal over time [53]. Pulse compression waveforms are named accordingly because on reception of the echo signal, the pulse is compressed through a compression filter, also referred to as a matched filter, to provide fine resolution in time [45][53]. A block diagram of a pulse compression system is shown in figure 2.3.

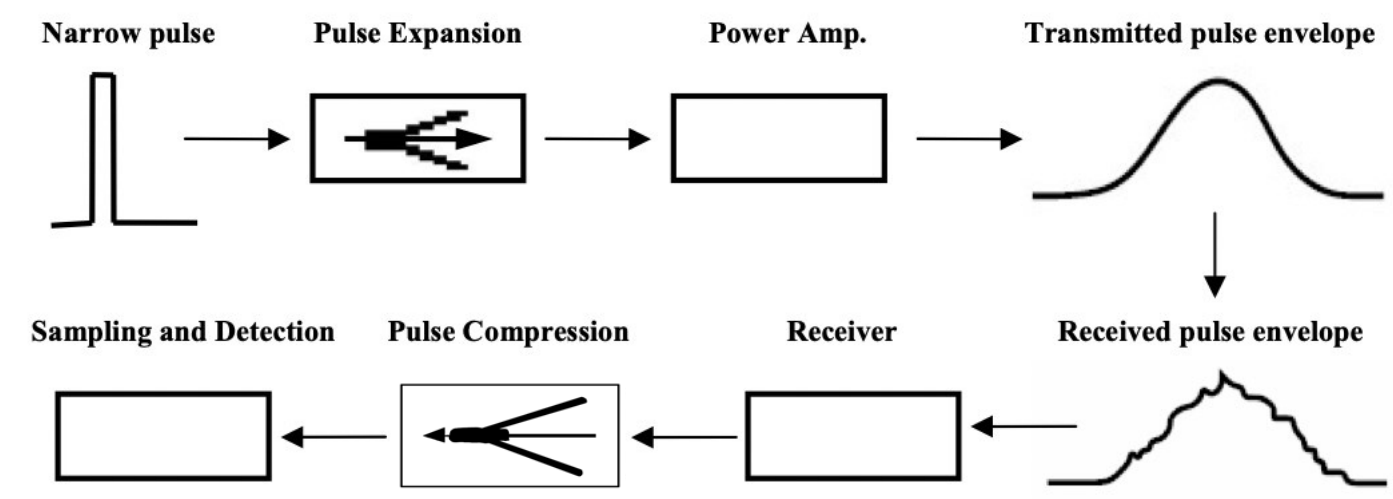

Figure 2.3: Block diagram of a pulse compression system, adapted from [53].

There are many types of pulse compressions waveforms. A common waveform used in high-resolution radar applications is the chirp pulse [45][50]. In a chirp waveform, the frequency is linearly increased across a specified bandwidth over the pulse duration [45][50][53]. This is illustrated in figure 2.4. 

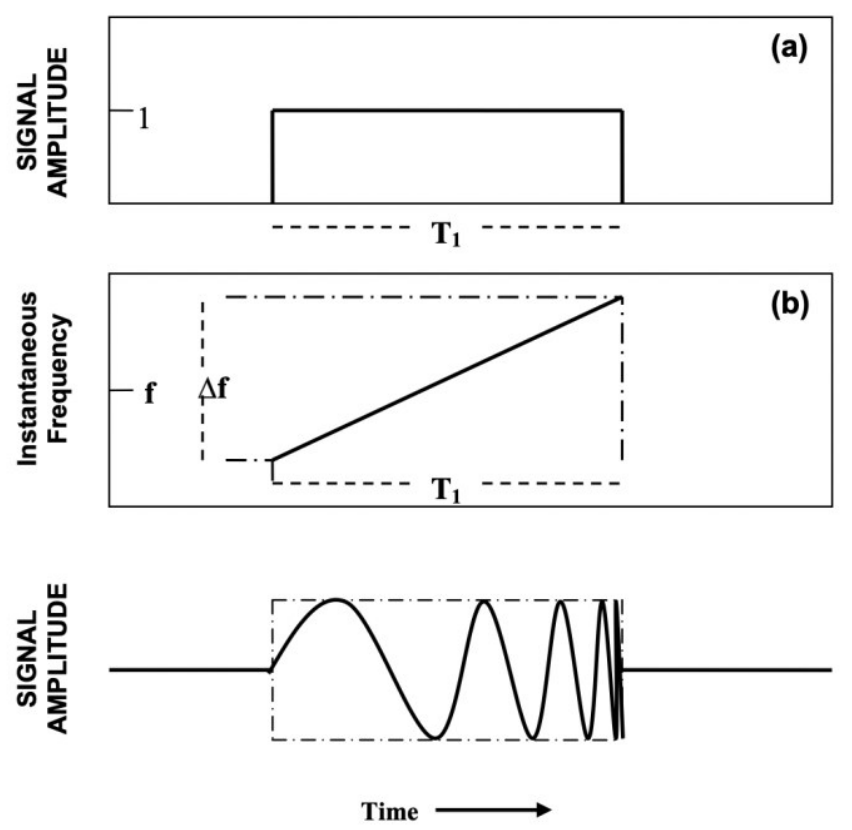

Figure 2.4: Chirp radar waveform; (a) pulse envelope, (b) pulse frequency as a function of pulse duration, (c) chirp waveform observed in time.

The chirp waveform is used because of the implementation of fast digital frequency synthesizers [43][45][50][53]. The advancement of digital technology has made creation of frequency synthesizers an economical choice in terms money as well as size, power and flexibility of technology required to create wideband waveforms $[43][45][50][53]$.

\subsubsection{Stepped-Frequency Waveforms}

Stepped Frequency radar systems create a synthetic wideband signal by using a collection of pulses, each at a single fixed frequency within a specified bandwidth. The collection of pulses is referred to as a burst [43][44][45]. A burst is comprised of $n$ pulses, each at discrete frequency, and the spacing between the frequency of each consecutive pulse is determined by the transmission bandwidth and the number of pulses in the burst, as shown in equation 2.4.

$$
\Delta f=n \beta
$$


Here $\beta$ represents the total effective transmitted bandwidth over the burst. It is an effective bandwidth because not all frequencies of the bandwidth are transmitted, only the select frequencies corresponding to the number of pulses in the burst. $\Delta f$ represents the frequency step between each consecutive pulse in a linear sequenced burst.

This waveform is referred to as a synthetic wideband signal because the return from a target for each pulse is treated as a discrete frequency signature of the target. For a total bandwidth $\beta$, and $n$ pulses in a burst, there are $n$ discrete frequency signatures of the target [43][44][45]. These signatures are treated as frequency domain samples of the target, rather than time domain samples. The frequency domain samples are then transformed into a synthetic high-resolution range profile using the Inverse Discrete Fourier Transform (IDFT) operation on the frequency samples of the target return [43][44][45].

Figure 2.5 on the following page illustrates the stepped frequency waveform. The time between each pulse is the pulse repetition interval (PRI), each pulse has a duration denoted by $T_{p}$, and the duration of a burst of $n$ pulses is denoted the burst interval $T_{\text {burst }}$.

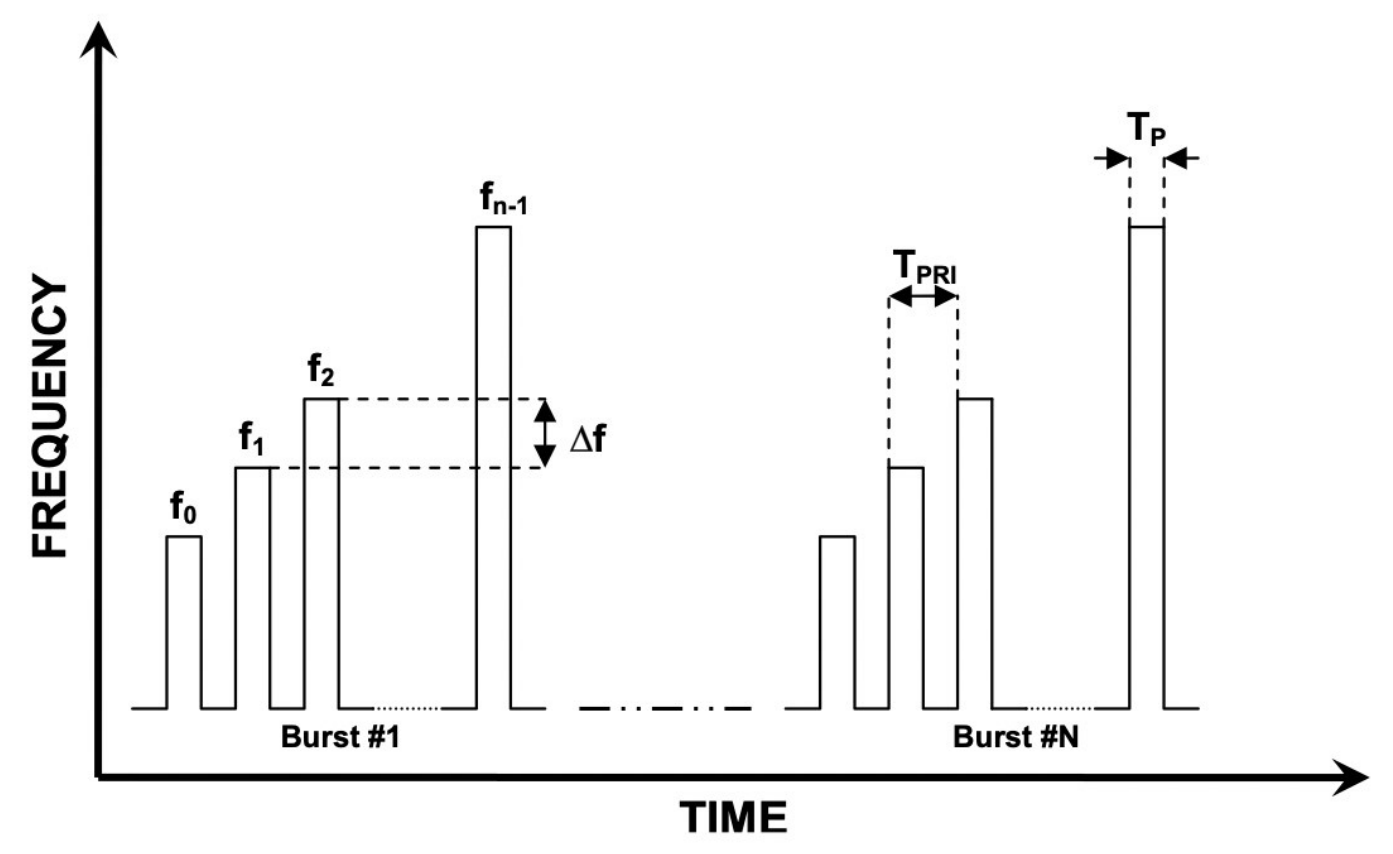

Figure 2.5: The stepped frequency transmitted waveform as a function of time. 
In practice, stepped frequency waveforms are more commonly used in research applications, such as RCS instrumentation ranges [45][53]. This is due to the extra time required to collect a dataset using this waveform. Pulse compression systems achieve similar resolution results in a fraction of the time and allow for greater flexibility in PRF and other radar system parameters [43][44][45].

The stepped frequency and the chirp radar waveforms are used in this research to verify the effectiveness of the target modelling program created. The design parameters of the radar systems and the methods of simulation are discussed later in this thesis. This chapter will focus on the principles of processing these waveforms to produce highresolution range profiles, phase histories and Range-Doppler images.

Processing of target return data from pulse compression waveforms has minor differences from processing of stepped frequency waveforms. However, a general mixing and quadrature detection system is used for initial processing of target return data. The mixing and quadrature receiving system is described in the following subsection, along with the variations in the system when processing chirp signals as opposed to steppedfrequency signals.

\subsection{Received Signal Processing}

The radar system receiver is comprised of many components, analog and digital. There are many variations of the design architecture based on the application of the radar system. The focus of this section will be on the mixing and quadrature detection of the received target echo response signal. There are many other components, with specific functions, such as noise rejection, low noise amplification, error compensation and other technologies that are beyond the scope of this research.

\subsubsection{Mixing}

Mixing of the received signal is performed in a superheterodyne system to convert signals at the radio frequency (RF) band down to an intermediate frequency (IF) region [43][44][45]. Mixing is performed using analog or digital mixer components, with analog components being more common in the early stages of the receiver system. This is 
illustrated in figure 2.6. The difference frequency product is isolated using a low-pass or bandpass filter centered at the desired IF frequency. In some systems the IF signal is also amplified.

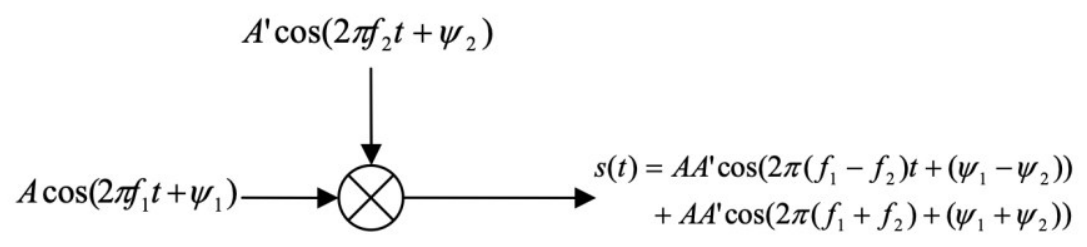

(a)

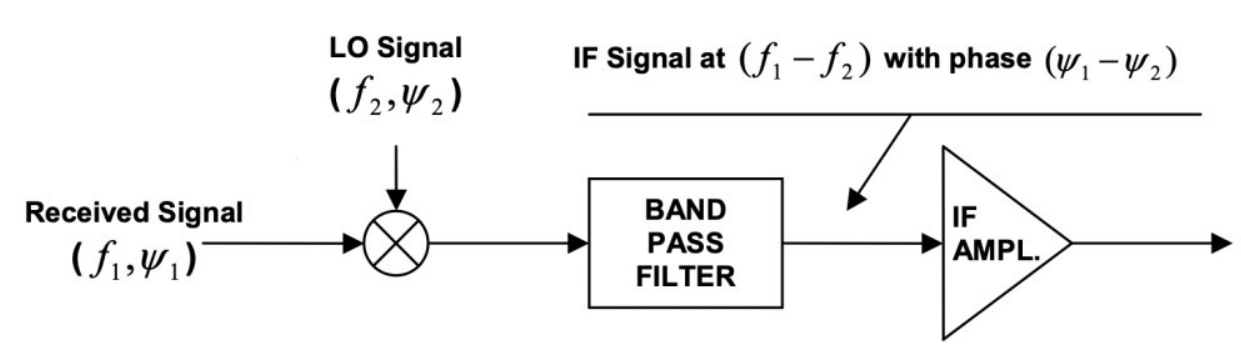

(b)

Figure 2.6: Mixing (a) analog mixer low order outputs, (b) Superheterodyne mixer, from [45][53].

Generally, the low frequency output of the mixer is isolated to achieve the Nyquist requirement for sampling in the digital stage of the receiver [43][45]. Some systems may require multiple superheterodyne stages to mix the received target echo signal to an IF which satisfies the sampling rate restrictions defined by the analog-todigital converter (ADC).

\subsubsection{Quadrature Detection}

Following the mixing operations, most coherent radar systems process the received target echo signal through a quadrature detector [43][44][45]. Coherent radar receivers preserve the phase of the transmitted radar signal, whereas non-coherent receivers do not preserve phase and are designed to maximize the amplitude of the received signal. Preservation of phase information allows for implementation of high- 
resolution processing techniques such as obtaining the phase history and generating Range-Doppler, also called ISAR, images from the return signals [43][45][53].

Quadrature detection involves mixing the signal to baseband, or an exceptionally low IF and decomposing the signal into the in-phase (I) and quadrature (Q) components to recover signal phase and amplitude information [43][44][45][53]. Quadrature systems can be implemented in analog or digital hardware. With advancements in digital signal processing (DSP) technology, quadrature systems are often implemented in digital hardware such as field-programmable gate-arrays (FPGAs) [43][44][45]. Figure 2.7 shows a block diagram of a generic quadrature detection system, where the input signal has a unique phase signature denoted $\Psi_{1}$. The phase signature represents unique Doppler and range information for the target return signal.

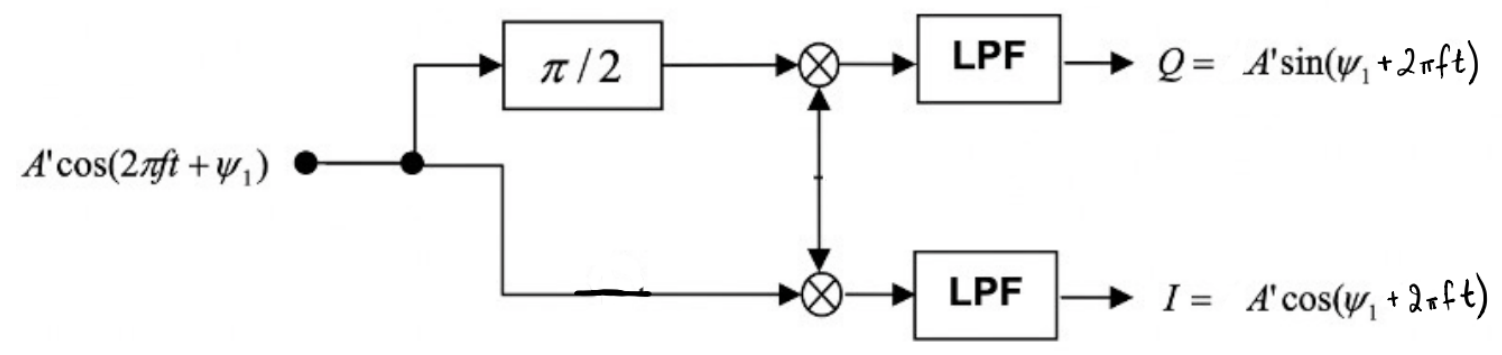

Figure 2.7: Quadrature detection system, implemented in analog or digital hardware.

Figure 2.8 below shows the processing of a received target echo signal in a quadrature detection system. 


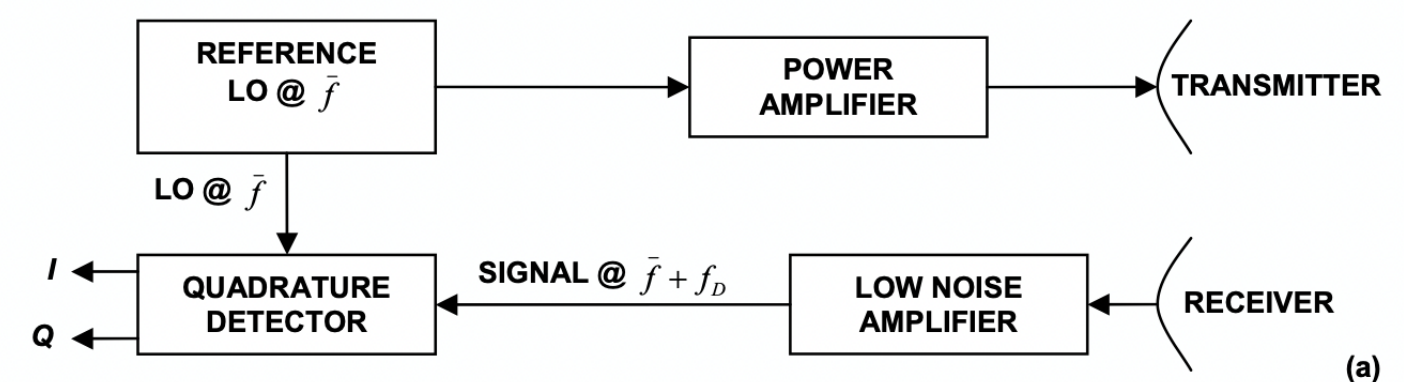

(a)

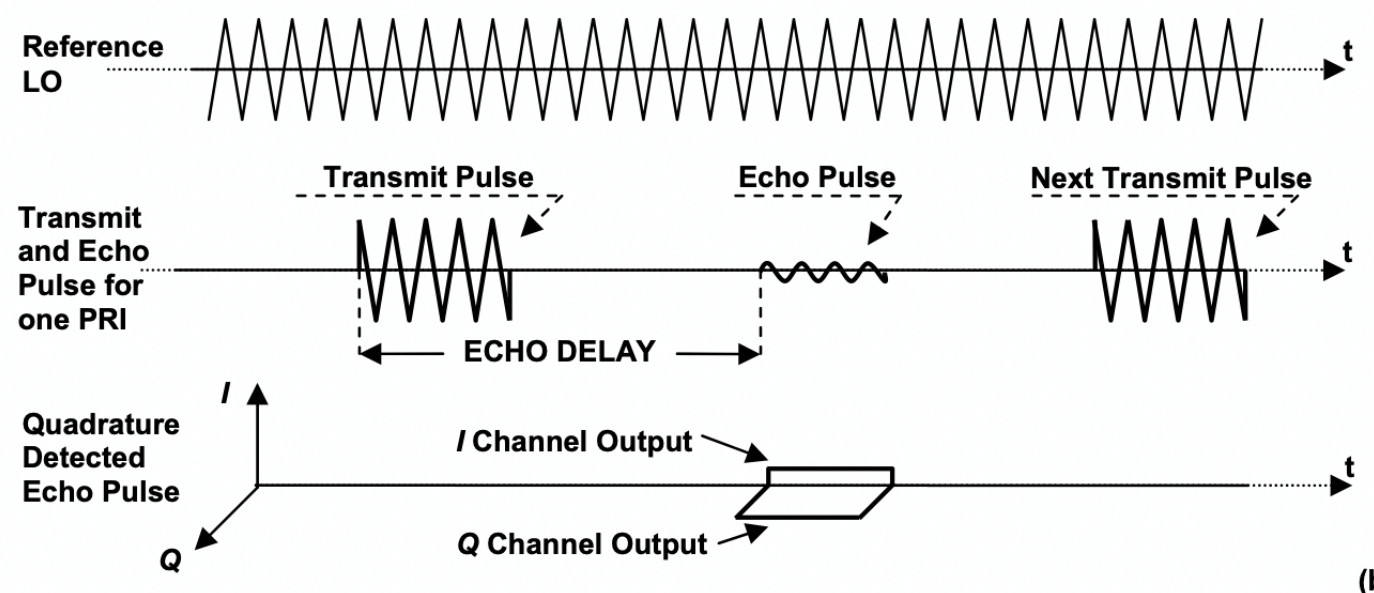

(b)

Figure 2.8: (a, b) Quadrature detection of received target signal. (a) Ideal coherent radar system receiver. (b) Quadrature detection waveforms. From [45][53].

The two channel outputs (I/Q) of the quadrature system are collectively referred to as the baseband signal [53]. These channels are produced by mixing the received signal with a LO frequency which matches the down converted IF of the received signal. In the I channel, the target echo signal is directly mixed with the LO signal. In the Q channel, the LO signal is shifted by a phase of $\pi / 2$ prior to mixing with the target echo signal. In an ideal quadrature system, a received signal with a Doppler frequency shift of $f_{D}$, on the carrier frequency, would produce a baseband signal output in the I and Q channels at the Doppler frequency only [43][44][45][53]. The carrier and IF frequencies are all removed, and the remaining frequency data will only contain modulations caused by the complex electromagnetic interaction between the pulse and the target. Figure 2.9 shows an idealized version of a practical quadrature detection system in a coherent radar receiver. 


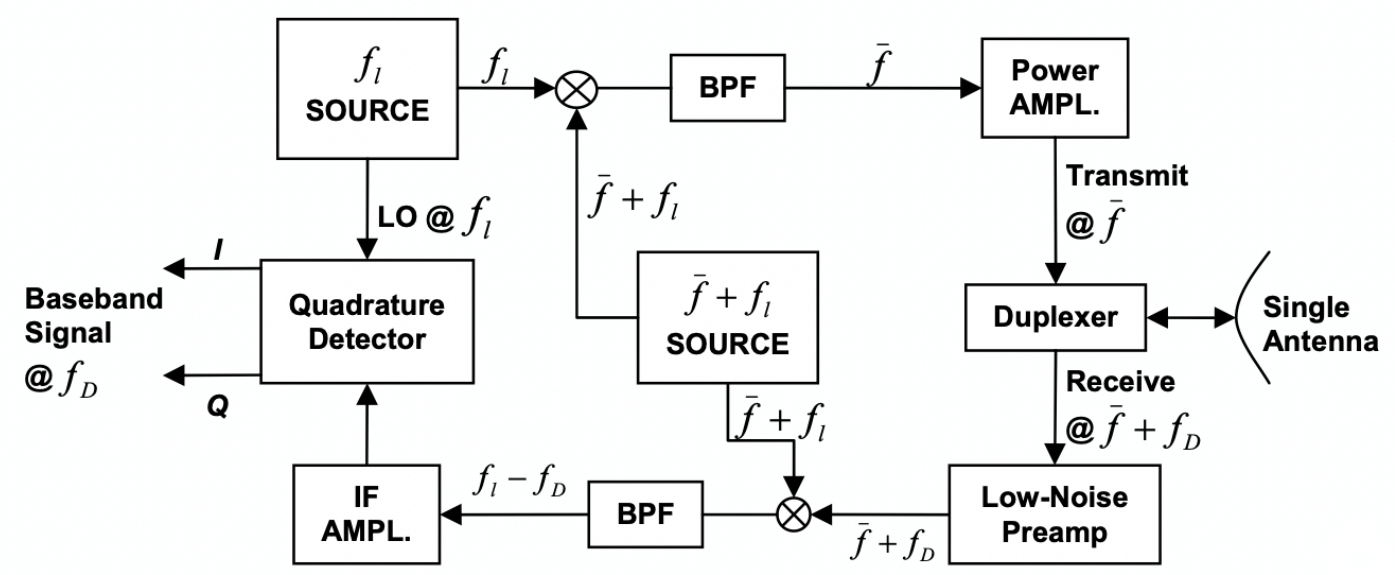

Figure 2.9: Practical quadrature detection in coherent radar receiver, from [45][53].

\subsection{The Range Profile}

\subsubsection{The Concept}

The range profile of a target is a projection of the complex electromagnetic response onto a single dimension along the radar line of sight (LOS) [53]. In highresolution applications, the terminology High-Resolution Range Profile (HRRP) is used in literature [44][45][53].

Range profiles provide information on the slant-range separation between resolved scattering locations [45][53]. The ability to resolve scattering locations in a range profile depends on the system resolution. A slant-range profile is comprised of resolution cells, or bins, defined in equation 2.2. When large complex targets are observed, multiple scattering locations exist within a single slant-range resolution cell, or bin. The returns from these scattering locations coherently combine, constructively or destructively, in a particular range cell within the profile. Range profiles do not provide an exact image of all the target scatterers projected along the LOS. Nevertheless, they are still useful in identifying basic target characteristics such as location of ship masts, weapons, and target dimensions [43][44][45][50]. 
In coherent radar systems, range profile data is a complex signal as the phase information is preserved in the quadrature detection block [45][53]. Range profile data is plotted as the magnitude of the complex baseband data to represent the peaks from scattering locations on the target. The phase information is used to generate the phase histories and Range-Doppler images. Figure 2.10 shows a basic diagram of a range profile generated from an arbitrary collection of randomly spaced scattering locations. The distance to each scattering location is denoted by $d_{n}$ and the amplitude of each scattering location is denoted $a_{n}$ [53]. On a real target, the scattering location amplitude is proportional to the radar cross-section (RCS) statistics, frequency of operation and aspect angle of the LOS [43][44][45][50].

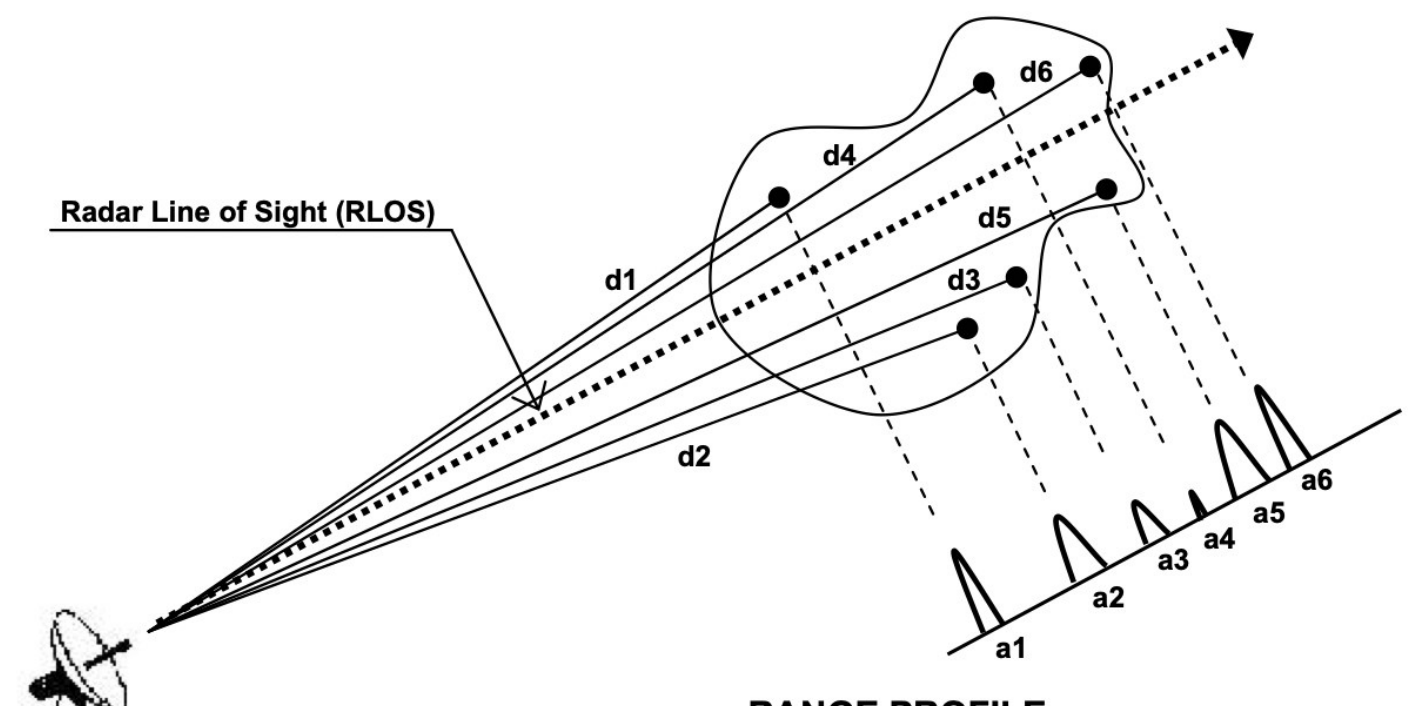

RANGE PROFILE

Figure 2.10: Range profile of a collection of arbitrarily spaced scatterers.

\subsubsection{Range Profile Generation}

Range profile generation is described in two general methods, depending on the waveform used in the radar system. These are:

- Synthetic High-Resolution Range Profile (HRRP) Generation

- 'Real' High-Resolution Range Profile (HRRP) Generation 
The term 'Real' HRRP is used in the context of this research to distinguish from Synthetic HRRP. In formal literature, the term 'Real' is not utilized, instead just highresolution range profile generation is used to refer to any method of profile generation not following the synthetic processing steps [45][53].

Synthetic HRRP generation refers to the processing steps utilized when the radar system applies stepped-frequency waveforms. The use of the term Synthetic refers to the processing of frequency samples and is not related to the terminology used in Synthetic Aperture Radar (SAR) or Inverse Synthetic Aperture Radar (ISAR) [53].

Generation of HRRPs is defined by a choice in the sampling type of the target echo return data. Radar systems applying stepped-frequency waveforms perform frequency domain sampling and processing to produce Synthetic HRRPs. Systems which utilize chirp, short pulse or other pulse compression waveforms perform time domain sampling and processing to produce HRRPs [45][53]. The following subsections describe in detail the processing method for the specific waveform and sampling type.

\subsubsection{Real HRRP Generation \& Time-Domain Sampling}

In modern systems, HRRP generation occurs in digital processing systems due to the speed and flexibility of digital hardware [43][44][45]. Current limitations in digital sampling technology mean that transmitted waveforms at the RF band cannot be directly sampled based on the Nyquist theorem [45][53]. Thus, signals are mixed to an IF or baseband on reception to achieve a frequency region which can be readily sampled by the technology available. Also performed in receiver systems, is direct baseband sampling of the received signal since the transmitted bandwidth is known [43][45].

A chirp waveform is transmitted with a bandwidth, $\beta$, corresponding to the resolution specifications of the system. The maximum frequency content of the signal is at the RF carrier frequency plus half of the total chirp bandwidth. The target response received will have approximately the same bandwidth as the transmitted bandwidth [45]. Upon reception, the echo signal is mixed to an IF band, following which the processing steps are dependent on the system receiver design. Some systems directly sample the IF target response following the Nyquist theorem or the bandpass sampling theorem [43][50]. Following sampling, the data is processed through a digital quadrature 
demodulation system which creates the complex baseband samples of the target response signal. An alternate system design implements an analog quadrature demodulation system to mix the signal to baseband prior to sampling. At this point, the maximum bandwidth of the signal content is $\beta / 2$. The data is then filtered and sampling at a rate equivalent to the bandwidth of the transmitted signal $\beta$, satisfying the Nyquist sampling requirement. This is illustrated in Figure 2.11.
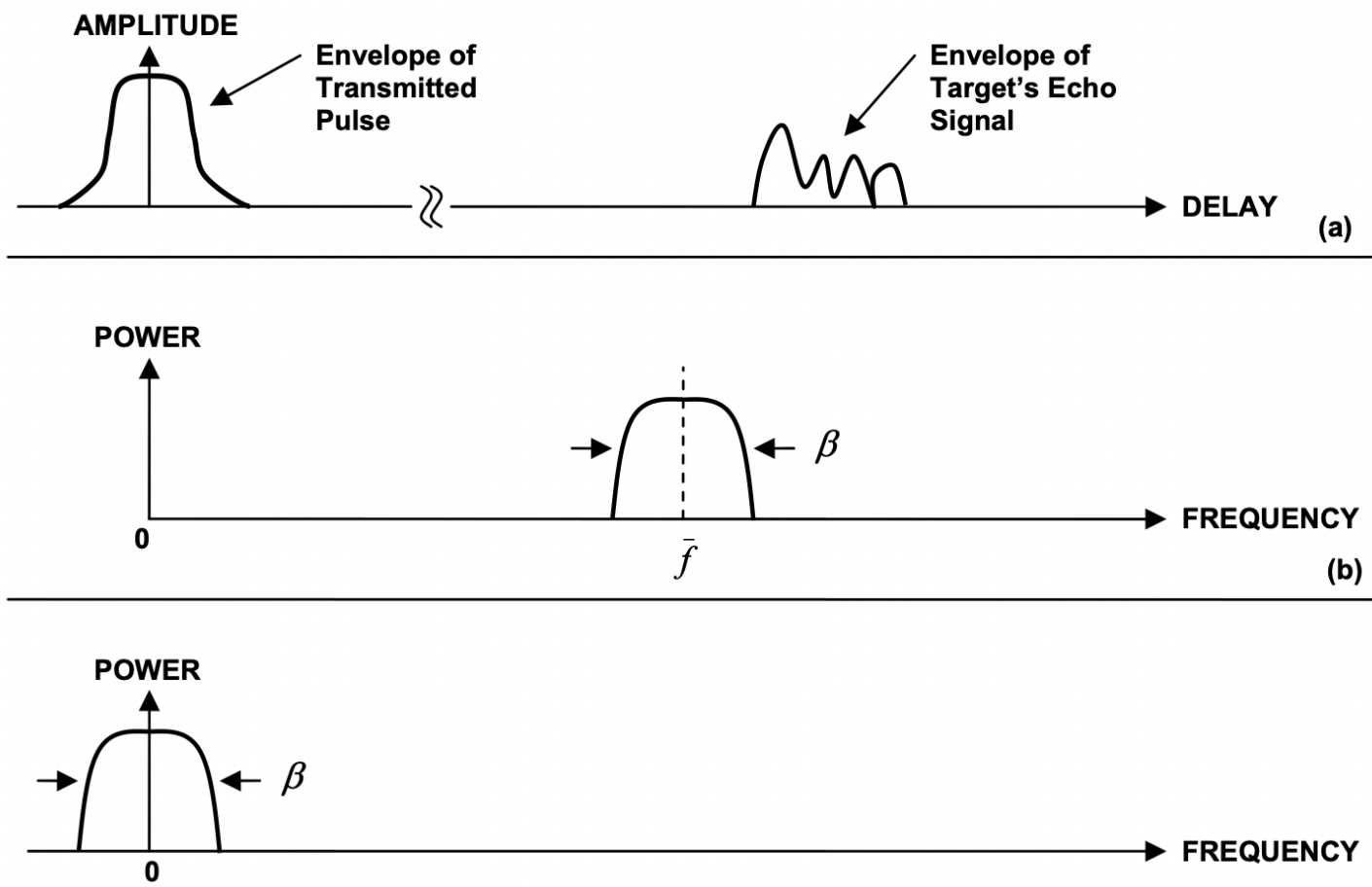

(c)

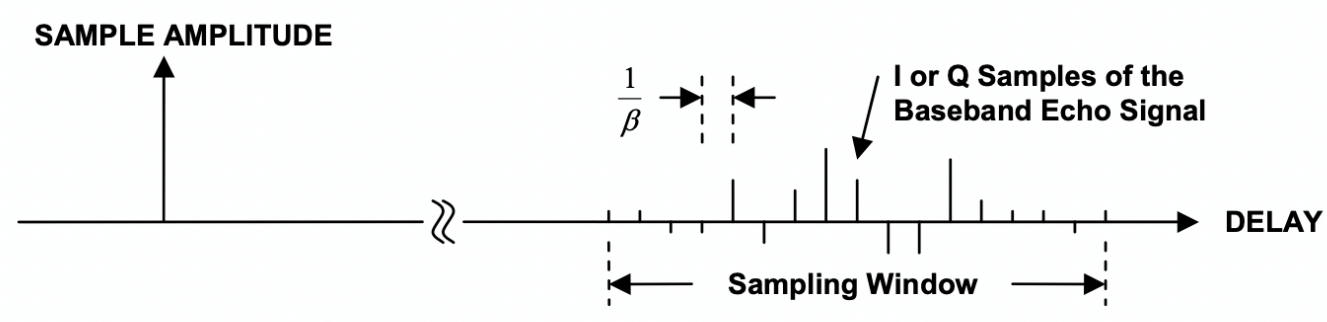

(d)

Figure 2.11: (a) Target echo signal, (b) Received spectrum at RF, (c) Received baseband spectrum, (d) Sampled received target echo signal. 
Combining the baseband samples in the in-phase (I) and quadrature (Q) channels creates the complex baseband target response, which is used to generate the range profile. The complex baseband data is represented in equation 2.5.

$$
B B=I+j Q
$$

In pulse compression systems, the complex baseband data is processed through a pulse compression filter to produce the complex HRRP. The pulse compression filter is the time-reversed complex conjugate of the transmitted waveform complex baseband envelope response [43]-[50]. An ideal transmitted baseband complex envelope response can be represented by the function $x(t)$. The corresponding pulse compression impulse response $z(t)$ is shown in equations 2.6 and 2.7, as per [43]-[50].

$$
\begin{aligned}
& z(t)=x^{*}(-t) \\
& Z(f)=X^{*}(f)
\end{aligned}
$$

The convolution of a target response signal with the matched filter of the transmitted waveform maximizes the signal-to-noise ratio (SNR) in the target response [43]-[50]. The theoretical matched filter response produces an output centered at the $0^{\text {th }}$ time sample position. In a digital system or simulation, this is made into a causal filter by delaying the matched filter response by the pulse duration [43][45]. The convolution operation creates an output signal which has a frequency vs. delay response which is reverse from that of the transmitted pulse [43]. This is amplitude modulated by the square root of the time bandwidth product which is the signal processing gain. It is also amplitude modulated by a Sinc function which has a Rayleigh resolution, in time, of approximately the reciprocal of the chirp bandwidth [43][45]. The Sinc function is the time response of the waveform which, in the frequency domain, is restricted to a rectangular shape by the bandwidth[43]. The Rayleigh resolution of this response is the duration of the output pulse [45]. Each scattering position contributes to the Sinc response due to the delay they introduce. The relative delay and Doppler of scattering locations reduces the peak amplitude response of the matched filter output and increases the response in the sidelobes of the Sinc function, as seen in the ambiguity function of the linear frequency modulated waveform [43][45]. The reduction in peak is due to energy dispersion in time, because of the mismatch, in delay and Doppler, between the received 
waveform and matched filter response [43]. This increases the amount of the noise in the profile and makes scattering location peaks more difficult to see. Each scattering location, within a range bin is resolved with possible error in peak location and loss of amplitude due to Range-Doppler coupling [43][45]. Since the matched filter introduces a delay equal to the pulse duration, the true range to a scattering location is found by using equation 2.8

$$
R_{d}=\frac{c\left(n_{i}-\tau_{s}\right)}{2}
$$

Here $n_{i}$ indicates the sample position of interest in the profile, and $\tau_{s}$ represents the number of samples required to represent the original pulse duration.

The effectiveness of the matched filter for a pulse compression waveform is evaluated using the ambiguity function [43][44][45]. This determines the maximum unambiguous bounds of the range and Doppler resolution of the waveform. Thus, the matched filter operation equation for a waveform is equivalent to the ambiguity function of the same waveform [45]. Pulse compression filters, equivalently matched filters, can be implemented in analog hardware generally following IF down conversion, or in the digital system following quadrature baseband demodulation [45]. Figure 2.12 shows the implementation of a matched filter in a digital system.

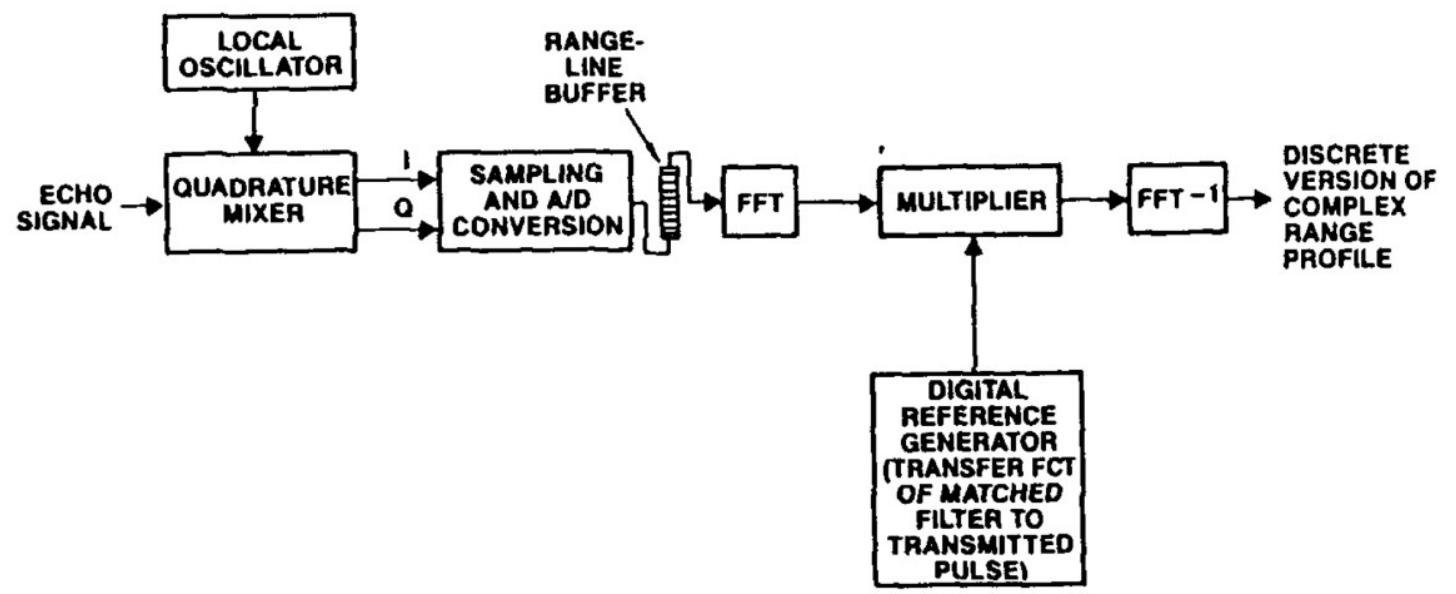

Figure 2.12: Digital pulse compression system using a matched filter, from [45].

For further information on the matched filtering operation, and HRRP generation for pulse compression waveforms, the reader is referred to [43]-[50]. 


\subsubsection{Real HRRP Generation Algorithm}

This section describes the algorithm implemented to create complex HRRPs which are then used to obtain phase histories and Range-Doppler images. The processing steps to produce a complex HRRP when processing a chirp target return signal can be implemented in a variety of methods. The major difference between methods is whether the matched filter and quadrature detection systems are implemented in analog or digital hardware. In this thesis, the focus was to develop a target model in a digital system, thus, the simulation of the chirp target return processing was done in MATLAB implying the use of digital signal processing principles. Figure 2.13 on the following page describes the algorithm implemented as a block diagram. 


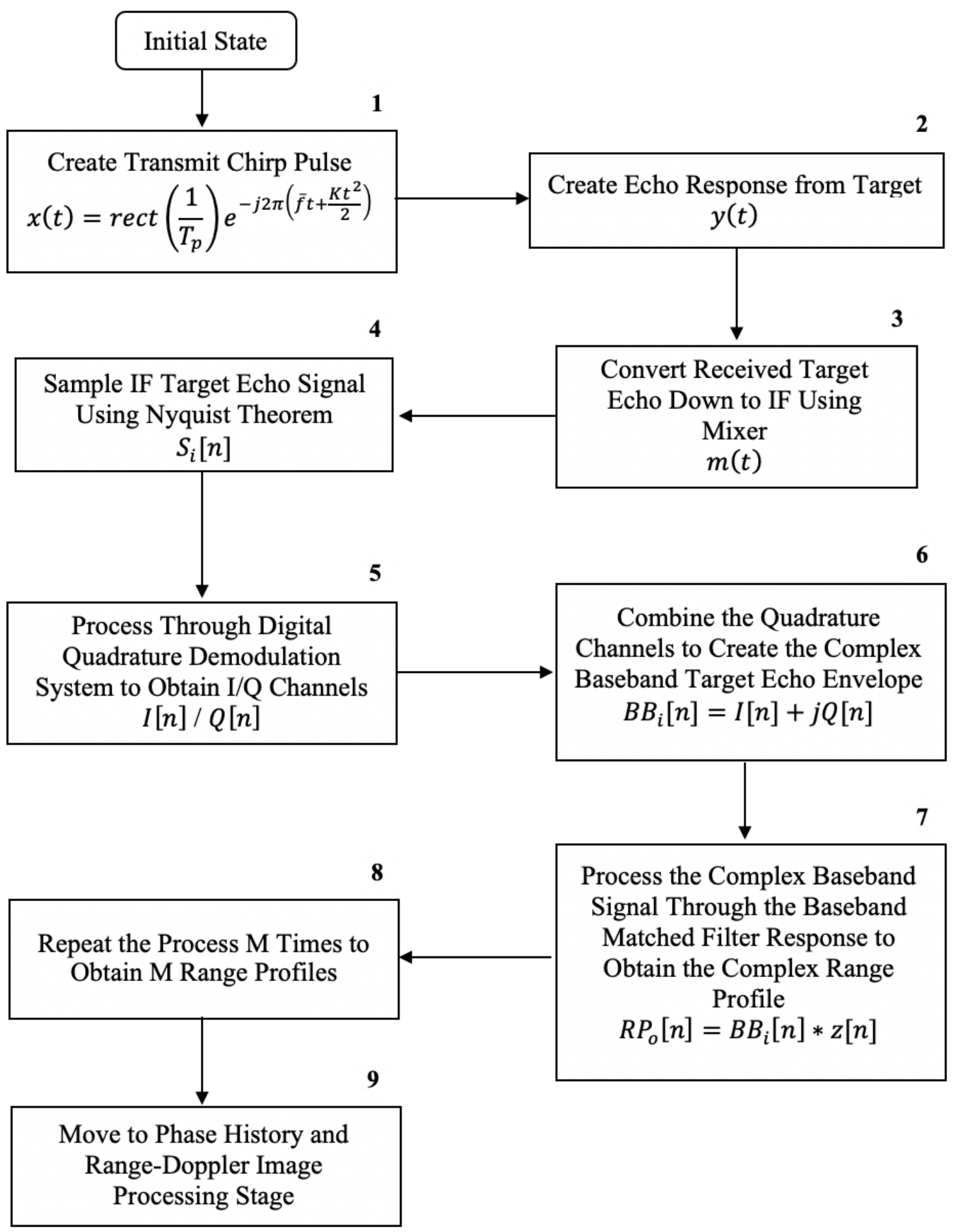

Figure 2.13: Process diagram of the HRRP generation algorithm for processing chirp signals. 
Equations for the steps in the block diagram are described in further detail with the assumption of processing a return from a point target. The initial step requires construction of a transmitted chirp waveform at a defined RF carrier frequency, and a bandwidth proportional to the defined resolution of the HRRP. Equation 2.9 represents the transmitted waveform. Here, the pulse is amplitude modulated by a rectangle function to restrict the chirp duration to $T_{p}$.

$$
x(t)=A_{i} \operatorname{rect}\left(\frac{1}{T_{p}}\right) \cos \left(-j 2 \pi\left(f_{c, R F} t+\frac{K t^{2}}{2}\right)\right)
$$

Here, $f_{c, R F}$ is the carrier frequency at the RF band and $A_{i}$ is the initial amplitude of the pulse. The value $K$ is the chirp rate which is defined below.

$$
K=\frac{\beta}{T_{p}}\left[\frac{H z}{s}\right]
$$

The transmitted waveform is modulated by the target response. The determination and application of modulation parameters for a target is shown in subsequent chapters of this thesis. The target return echo signal from a point target is described in equation 2.11.

$$
y(t)=A_{r}(t) \operatorname{rect}\left(\frac{1}{\tau}\right) \cos \left(-j 2 \pi\left(f_{c, R F} t+\frac{K t^{2}}{2}+\tau(t)\right)\right)
$$

The term $A_{r}$ is the amplitude modulation on the envelope. The pulse duration is also modified to a value $\tau$, because of the pulse interaction with the target. $\tau(t)$ is a function which represents the phase modulation factors, as described in equation 2.12.

$$
\tau(t)=\frac{2[R-v t]}{c}
$$

Where $R$ represents the range of the point target, and $v$ is the velocity of the target which produces the Doppler shift.

Following the mixing operation, the signal frequency content is reduced to an IF band as shown in equation 2.13 .

$$
m(t)=A_{r}(t) \operatorname{rect}\left(\frac{1}{\tau}\right) \cos \left(-j 2 \pi\left(f_{c, I F} t+\frac{K t^{2}}{2}+\tau(t)\right)\right)
$$


The downconverted IF signal is sampled and processed through a digital quadrature demodulation system to produce the I and Q channel signals shown in equations 2.14 and 2.15 .

$$
\begin{aligned}
& I[n]=A_{r}(n) \operatorname{rect}\left(\frac{1}{\tau}\right) \cos \left(-j 2 \pi\left(\frac{K n^{2}}{2}+\tau[n]\right)\right) \\
& Q[n]=A_{r}(n) \operatorname{rect}\left(\frac{1}{\tau}\right) \sin \left(-j 2 \pi\left(\frac{K n^{2}}{2}+\tau[n]\right)\right)
\end{aligned}
$$

The quadrature channels are combined to create the complex baseband envelope of the target echo signal. Observe, in the quadrature channel signals, that the carrier frequency terms are removed, leaving only the chirp and target modulation components of the signal. The complex baseband envelope signal is shown in equation 2.16. Here the subscript $i$ indicates the profile number which corresponds to the number of pulses received.

$$
B B_{i}[n]=I[n]+j Q[n]
$$

The matched filter impulse response is stored in digital memory, as shown in equation 2.17. This equation is based on the formulation of a matched filter impulse response for a chirp waveform as discussed in [45].

$$
z[n]=A_{i} \operatorname{rect}\left(\frac{1}{T_{p}}\right) \sqrt{j P} \cos \left(-j 2 \pi\left(\frac{K n^{2}}{2}\right)\right)
$$

Here, the term $P$ is the inverse of $K$ (i.e., $P=1 / K$ ). The HRRP of the target echo signal is produced through a convolution operation of the complex baseband echo response, with the matched filter impulse response as shown in equation 2.18.

$$
R P_{o}[n]=B B_{i}[n] * z[n]
$$

This process is repeated for a total of $M$ transmitted chirp pulses to produce $M$ complex HRRPs of the target. 


\subsubsection{Synthetic HRRP Generation \& Frequency-Domain Sampling}

Generation of HRRPs using stepped-frequency waveforms utilizes the principles of the time-frequency transformations described in the discrete Fourier transform. For a series of pulses stepped in frequency intervals over a bandwidth, $\beta$, the target echo response is a time-domain pulse which is amplitude and phase modulated. In steppedfrequency waveforms the effective bandwidth, for resolution, is distributed across a series of pulses. Thus, time-domain processing of a single target echo pulse is equivalent to processing a single discrete frequency signature response [43][45][53].

A collection of $n$ target echo pulses which contains $n$ discrete frequency steps over a bandwidth, $\beta$, is observed to be $n$ discrete frequency samples of the target signature [45]. A collection of time domain signals is treated as a collection of synthetic discrete frequency samples. Performing the Inverse Discrete Fourier Transform (IDFT) operation on the frequency samples, produces a time domain range profile of the target [43][45]. The time values are then converted to a range value based on the number of samples (i.e., the number of pulses in a burst, $n$ ), and the defined bandwidth, as shown in equation 2.19. Here the constant $c$ is the speed of light, $n_{i}$ indicates the sample position, or pulse number in the burst, and $\beta$ is the total bandwidth.

$$
R_{i}=\frac{c n_{i}}{2 \beta}
$$

Since the time-domain, or range profile, is generated from the discrete frequency samples, it is referred to as a synthetic profile. In a practical system, the received pulse data is processed through a quadrature demodulation system to create I and Q samples for the target return from each discrete frequency pulse in the burst [43][45]. An I and Q sample pair is selected from a defined range-delay position of the total response and this represents a single complex valued discrete frequency sample of the target return [53]. Collecting the complex baseband data at the same range-delay position for all pulses in the burst, creates the discrete frequency spectrum consisting of the samples to create the range profile. A key assumption in stepped-frequency processing is that the change in target reflectivity, aspect angle and motion are not significant over the duration of a burst $[45][53]$. 
In stepped-frequency waveform systems, four key design parameters determine the performance of the system. These are the bandwidth, frequency step size, pulse duration and PRF [45][53]. The bandwidth determines the slant-range resolution and is defined as previously shown in equation 2.2. The frequency step-size determines the total number of pulses required in a burst to achieve the desired bandwidth and slant-range resolution. Treating the target response from each discrete frequency pulse as a frequency sample, the Nyquist theorem in the frequency domain is used to analyze the frequency sample spacing required to resolve the target without aliasing or ambiguity [45][53]. The Nyquist theorem dictates that the spectrum of a signal is determined without ambiguity when the samples are separated by $1 / 2 \delta t \mathrm{~Hz}$, and the signal is only non-zero during the interval $\delta t[43][45][53]$. For any range-extended target, a target which is greater than a single point scatterer, the signal must be non-zero for the length of the target. The target dimension in range is related to the time duration of the signal in equations 2.20 and 2.21.

$$
\begin{gathered}
2 l_{\text {target }}=c \delta t \\
\delta t=\frac{2 l_{\text {target }}}{c}
\end{gathered}
$$

Here, the term $l_{\text {target }}$ refers to the length of the target along the radar LOS, and the factor two is included to account for the round-trip propagation path delay [45][50][53]. Using the Nyquist theorem, the frequency spacing to resolve the target without ambiguity is determined in equation 2.22 for real samples of the target.

$$
\Delta f \leq \frac{1}{2 \delta t}=\frac{c}{4 l_{\text {target }}}
$$

When using a quadrature demodulation system, the I-Q discrete complex frequency samples can be taken at $\Delta f=c / 2 l_{\text {target }}$ [45][53]. For example, the maximum frequency step size for unambiguous complex sampling of a $200 \mathrm{~m}$ target is $750 \mathrm{kHz}$. To achieve a resolution of $0.5 \mathrm{~m}$, a total bandwidth of $300 \mathrm{MHz}$ is required. Thus, a total of 400 complex frequency samples, or 400 pulses in a burst, are required to generate the HRRP.

Since only a single sample of the time domain samples of a target return pulse is taken to represent the discrete frequency sample, it is crucial that the entire target 
response is contained within a single pulse [45][53]. If the target response is not captured within a single pulse range, ambiguity will result and there can be aliasing and errors in the generated profile. The pulse duration is determined using equation 2.1 as it would be for a short pulse radar system. The minimum range window of the pulse corresponds to the length of the target along the LOS. For example, the minimum pulse duration for a $200 \mathrm{~m}$ target is $1.33 \mu \mathrm{s}$.

The final design parameter is the PRF of the system. Since synthetic range profile generation requires multiple pulses to achieve the same resolution as a pulse compression system, a major area of concern is the total time taken to produce a single range profile. When observing a target, it is expected that the target motion will produce aspect angle changes which would change the HRRP generated. Significant motion or aspect change in the target during a burst, would result in distortion of the HRRP. The total time for a burst must be minimized to less than the time after which the target motion or aspect angle change is significant, also referred to as the decorrelation time. When the burst time is minimized, the PRF increases to ensure that all pulses in a burst can be transmitted within the required time frame to generate the HRRP. High PRFs often result in rangeambiguity. However, since each pulse is at a unique frequency, the receiver system can identify each pulse by frequency, even if the return is beyond the unambiguous range [50]. Selection of an appropriate PRF has significant impact on the quality of HRRP generated. To produce a HRRP the PRF is computed by equation 2.23, where $n$ is the number of pulses in the burst and $T_{\text {Burst }}$ is the burst period.

$$
\text { PRF }=\frac{n}{T_{\text {Burst }}}
$$

For example, assuming a target profile decorrelates, meaning the motion creates significant aspect angle change, after 0.01 seconds the maximum burst time is 0.01 seconds. Imaging a $200 \mathrm{~m}$ target with $0.5 \mathrm{~m}$ resolution requires 400 discrete frequency pulses, within the burst period, to produce a HRRP which is not distorted. Thus, the PRF for the system is computed to be $40 \mathrm{kHz}$ using equation 2.23 . 


\subsubsection{Synthetic HRRP Generation Algorithm}

This subsection presents the algorithm to generate the synthetic HRRP. Variations of the algorithm exist, dependent on the implementation of components in analog or digital hardware. This algorithm implements the digital quadrature demodulation system described in the Real HRRP generation algorithm. Figure 2.14 shows a block diagram of the synthetic HRRP generation algorithm. 


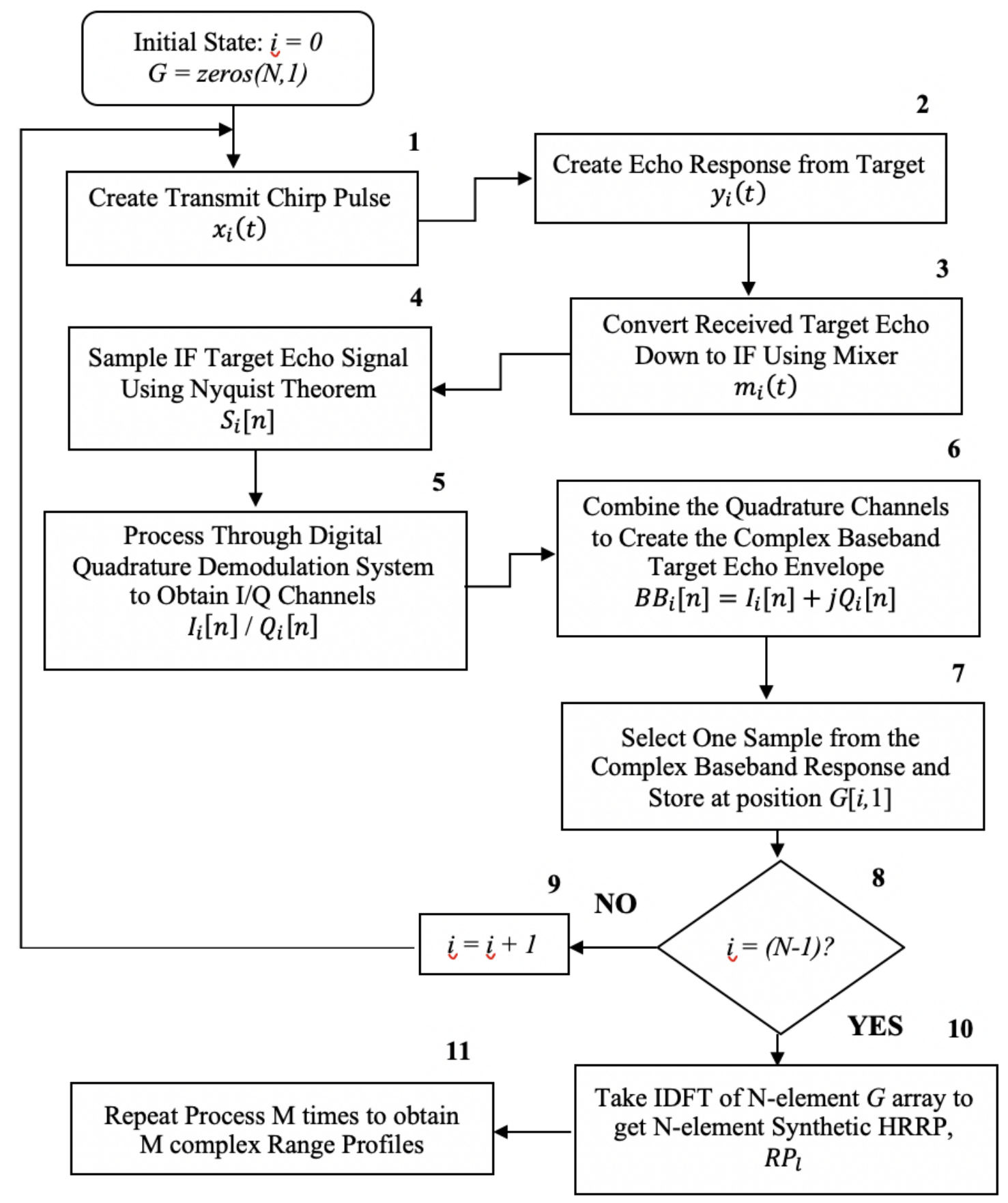

Figure 2.14: Block diagram of the synthetic HRRP generation algorithm.

Effective generation of range profiles is dependent on the reliability of the discrete frequency signature data collected. The data for a single burst, of $N$ pulses is stored in the array denoted $G$. The discrete frequency signature samples are obtained from a system which satisfies the following conditions [45][53]: 
1. The burst time is short relative to significant target aspect change, or profile decorrelation time.

2. The baseband echo response duration is greater than the target range-delay.

3. The frequency step size is less than the reciprocal of twice the target range-delay extent.

The processing steps are described using equations assuming processing of return from a point target as done for the real HRRP generation algorithm. The first step is to synthesize the transmitted signal $x_{i}(t)$. The frequency of the signal is determined by $f_{i}$, which is the frequency of the $i^{\text {th }}$ pulse in the burst where the frequency step sizes are determined by the bandwidth and equation 2.22 .

$$
x_{i}(t)=\left\{\begin{array}{c}
A_{i} \cos \left(2 \pi f_{i} t+\theta_{i}\right), i T_{P R I} \leq t \leq i T_{P R I}+T_{P} \quad i=0 \text { to }(N-1) \\
0, \text { otherwise }
\end{array}\right.
$$

Here, $T_{P R I}$ is the pulse repetition interval (PRI). The value $T_{P}$ is the pulse duration, $A_{i}$ is the pulse amplitude, and $\theta_{i}$ is the initial phase on transmission for the $i^{\text {th }}$ pulse. The frequency of the transmitted pulse is determined by equation 2.25 .

$$
f_{i}=f_{0}+(i \times \Delta f)
$$

The target echo response signal is described below.

$$
y_{i}(t)=\left\{\begin{array}{c}
A_{r} \cos \left\{2 \pi f_{i}[t-\tau(t)]+\theta_{i}\right\}, i T_{P R I}+\tau(t) \leq t \leq i T_{P R I}+T_{P}+\tau(t) \\
0, \text { otherwise }
\end{array}\right.
$$

Note that the modulation $\tau(t)$ is the same as shown in the real HRRP generation algorithm in equation 2.12. The received signal is downconverted to an IF prior to sampling, represented in equation 2.27 .

$$
m_{i}(t)=\left\{\begin{array}{c}
A_{r} \cos \left\{2 \pi f_{i, I F}[t-\tau(t)]+\theta_{i}\right\}, i T_{P R I}+\tau(t) \leq t \leq i T_{P R I}+T_{P}+\tau(t) \\
0, \text { otherwise }
\end{array}\right.
$$

Following sampling, the target echo response is processed through the digital quadrature demodulation system and mixed to baseband. The in-phase and quadrature channel representations of the response to a point target are shown in equations 2.28 and 2.29 . 


$$
\begin{aligned}
& I[n]=A_{r} \cos \left\{2 \pi f_{i} \tau[n]+\theta_{i}\right\} \\
& Q[n]=A_{r} \sin \left\{2 \pi f_{i} \tau[n]+\theta_{i}\right\}
\end{aligned}
$$

The I and Q channels are combined to create the complex baseband envelope response from the target shown in equation 2.30 .

$$
B B_{i}[n]=I_{i}[n]+j Q_{i}[n]
$$

From the complex baseband response, a single sample is taken as the discrete frequency signature sample for the $i^{\text {th }}$ pulse in the burst. Generally, to obtain a steadystate complex sample from the echo, the sample at the center of the baseband response is chosen [45][53]. The initial portion of the pulse may not contain response characteristics from the back end of a range-extended target. Similarly, samples near the tail end of the response may not contain modulation characteristics from the front of a range-extended target. The sampling position $S x_{i}$ is defined as,

$$
S x_{i}=i T_{P R I}+\frac{2 R}{c}+\tau_{r}
$$

Where the value $\tau_{r}$ is equivalent to half the echo pulse duration in time, or half the number of samples used to represent the target echo signal. $S x_{i}$ is advanced with each pulse in the burst to ensure the sample position selected is consistent for all target echo responses in the burst.

The sample is stored in the array denoted $G$, in the index position corresponding to the pulse number in the burst. Thus, the elements $G_{0}, \ldots \ldots, G_{N-1}$ are the complex discrete frequency signature samples of the target [45][53]. Performing an N-point IDFT of these samples produces the synthetic HRRP of the target, as shown in equation 2.32.

$$
R P_{l}=\frac{1}{N} \sum_{i=0}^{N-1} G_{i} e^{j\left(\frac{2 \pi}{N}\right) i}, 0 \leq l \leq M-1
$$

Here, the subscript $l$ denotes the profile number. Each burst is repeated a total of $M$ times to generate $M$ complex range profiles. With $M$ complex range profiles generated using the real or synthetic HRRP algorithm, the phase history is generated as discussed in the following subsection. Generating the Range-Doppler image is discussed in the following chapter. 


\subsection{Phase/Doppler History}

The phase history of a target is used to observe the change in the target motion and aspect characteristics over time. It is also referred to as the Doppler history of the target in some literature [43][44][45]. The processing method for obtaining the phase history is applicable to the complex range profile data which is obtained using either the real or synthetic HRRP algorithms.

Over an observation period $M$ complex range profiles are generated, referred to as the range profile history. Producing $M$ profiles in the stepped-frequency system requires a longer observation time than the system using chirp waveforms. The range profile history for the chirp system will be different than the history generated by the steppedfrequency system, even if both systems are observing the same target. Nevertheless, the algorithmic process to analyze the phase history is the same for both range profile histories.

The phase history is an observation of the change in a single range cell over each profile in the history [45]. For the defined resolution, each sample of the range profile is observed across all range profiles as the complex value of each sample changes as a function of time. The major contributing factor to the change in value of a resolution cell over time is the Doppler shift of the target [43][45]. To observe the Doppler modulation created by a scattering location, the radar pulse must be long enough to encompass at least one period of the Doppler signal [43][45][49][50]. In marine radar applications, Doppler periods are often longer than the pulse duration, which does not allow for observation of the Doppler contribution from a scattering location. The coherent combination of multiple scattering locations also causes overlapping Doppler signals to interfere constructively and destructively resulting in inaccurate Doppler measurements [43][45][49]. A more common method of observing Doppler modulation is by observing a sample location across multiple PRIs, as performed in pulsed radar, and moving target indicator (MTI) radar [43][45][49][50]. In a complex range profile, a single range cell, or sample, is observed through the slow-time history. The slow-time history is the observation of samples over multiple PRIs. This is shown in Figure 2.15, from [45]. 


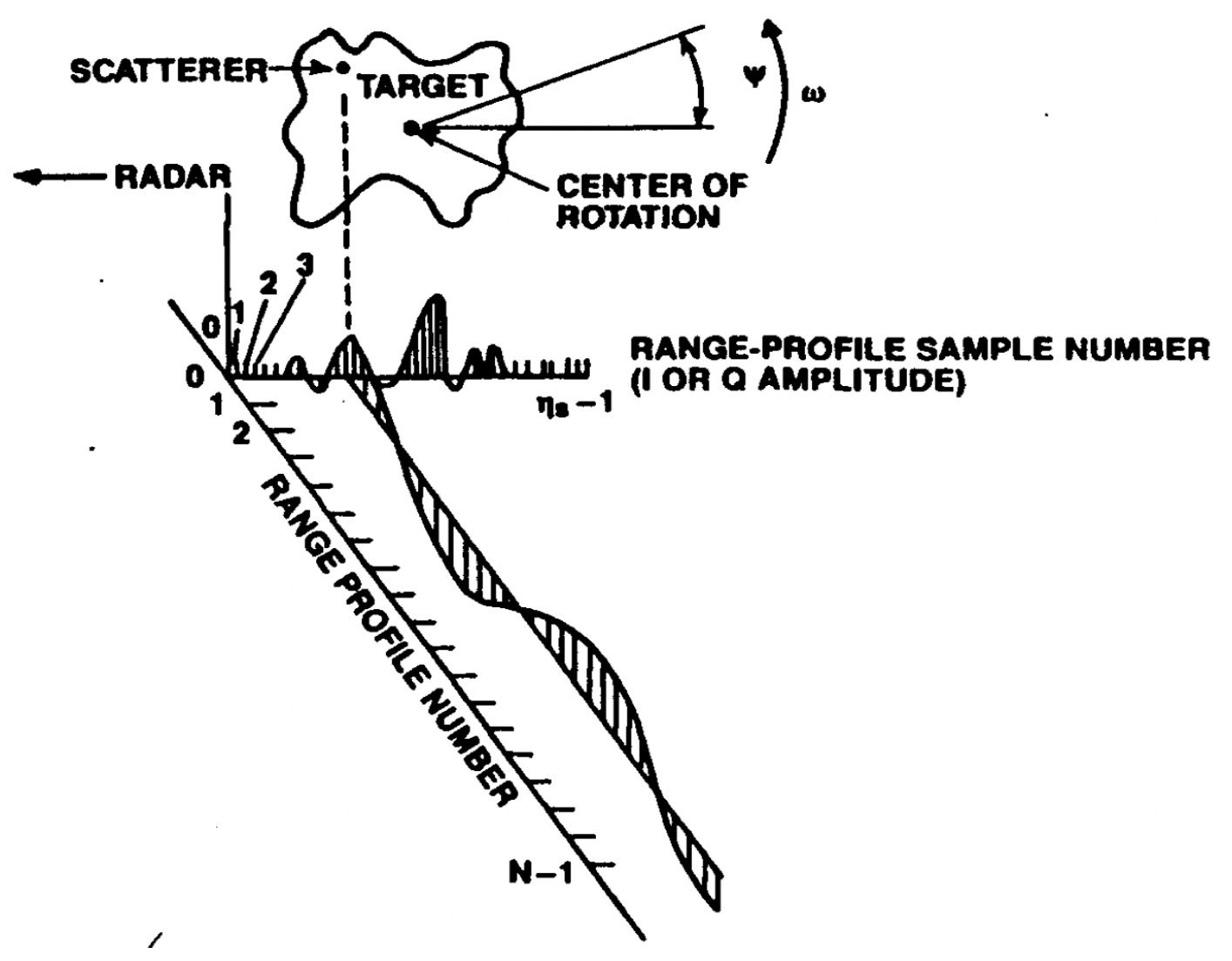

Figure 2.15: Slow-Time history of a single sample position in a range profile [45].

Assuming a scattering location maintains a constant Doppler shift over an observation period. Collecting the slow-time sequence of complex samples is equivalent to sampling a signal at the rate of the PRF for chirp systems or burst interval for stepped frequency systems. Performing the DFT of this sequence will indicate the Doppler shift of the selected range bin [43][45]. The resolution of the phase history is improved by windowing the range profile history of each resolution cell [45]. Common windows applied are Hann and Hamming windows, however, the choice of window implementation is left to the system designer [43][45][50]. The underlying assumption is the PRF (which is the sampling rate), satisfies the Nyquist theorem requirement for the Doppler signal being sampled [43][45][49]. That is, the maximum Doppler shift half of the PRF. If this condition is not satisfied, aliasing of the Doppler spectrum will result, which creates errors in the target phase history, and spectral spreading or fold-over in Range-Doppler imaging of the target [43][45][49]. 

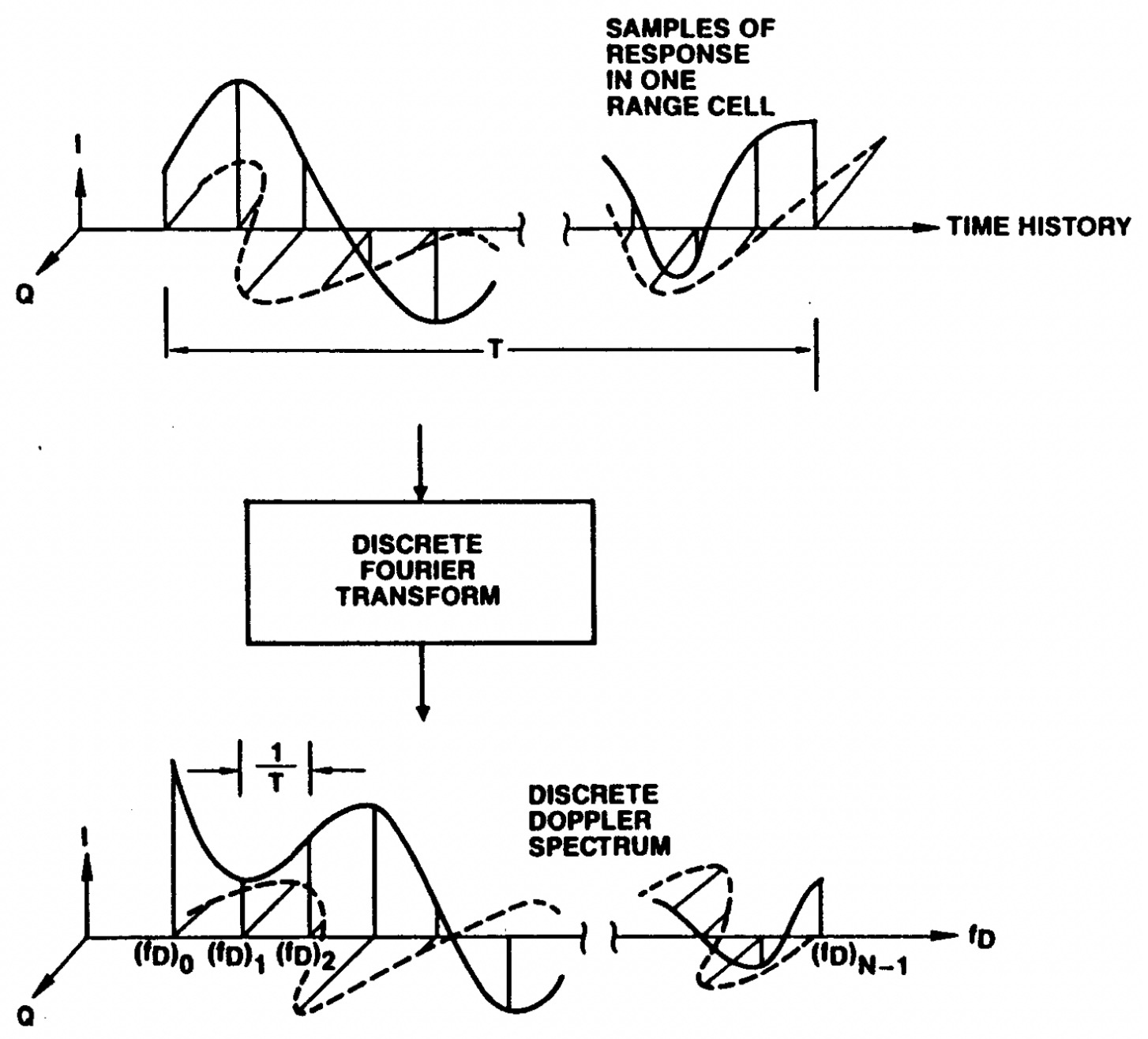

Figure 2.16: Discrete Doppler Spectrum of a single range cell, sampled at the radar PRI, from [45].

In practice, the Doppler frequency of a single range cell is not constant during the entire observation period. This is due to the complex random motion experienced by the ship target. The maximum time a range cell maintains a Doppler shift is determined by the profile decorrelation time, which is addressed in chapter five. Often, the target profile decorrelation time is shorter than the period of a single Doppler signal, resulting in energy being spread over multiple frequencies in the Doppler spectrum for a single range bin. Reduction of spectral energy spreading, and noise is performed using Doppler tracking and motion compensation algorithms in Range-Doppler image processing. 


\subsection{Conclusion}

This chapter provided an in-depth explanation of the principles of high-resolution radar, beginning with the explanation of the principles that define the resolution of a target. The generation of a range profile using the chirp and stepped-frequency waveforms is discussed. Also presented is the generation of a phase or Doppler history of the target being observed by the radar. The following chapter will address the generation of a Range-Doppler image, building on the principles discussed in this chapter. 


\section{Chapter 3: Range-Doppler Imaging}

\subsection{Introduction}

The Range-Doppler image is comprised of resolution cells which contain estimates of the magnitude and position of key scattering locations on a target in the slant-range and Doppler, or cross-range, dimensions [41][42]. The orientation of the image is determined by the relative rotation of the target to the radar system performing the ISAR imaging [41][42]. As discussed, the slant-range dimension is along the radar LOS, and the cross-range dimension is orthogonal to the plane of the slant-range dimension as illustrated in Figure 3.1.

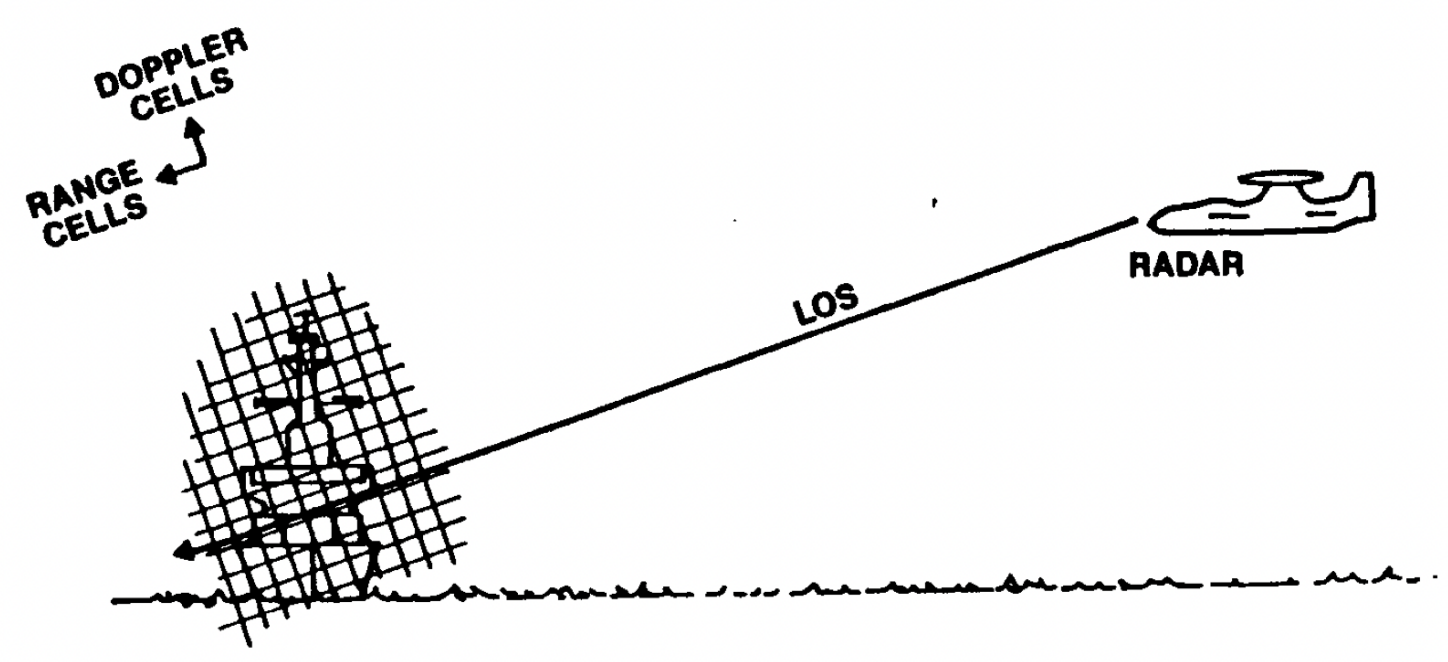

Figure 3.1: The slant-range and cross-range (Doppler) dimensions when imaging a target, from [45].

The basic dimensions and resolution of the image are defined by the radar system operator. Choice of these parameters is addressed in the first subsection of this chapter. The algorithm to generate a Range-Doppler image varies depending on whether a pulse compression or a stepped-frequency waveform is used. Both algorithms will also be addressed in this chapter. The final subsection will discuss motion compensation algorithms which improve image resolution. There are many motion compensation algorithms which can be applied, but discussion and evaluation of all algorithms is beyond the scope of this research, instead only two are presented in detail. 


\subsection{The First Dimension: Slant-Range}

As mentioned, the slant-range resolution is determined by the bandwidth of the transmitted waveform. Decoupling the bandwidth from the pulse duration allows for an increased resolution, evident in Equation 2.2. The slant-range window generated in the image is dependent on the bandwidth and the number of samples of the profile. As the resolution becomes finer, the number of samples within a pulse window also increases, which results in an increased bandwidth; however, the window size remains the same. This is illustrated in Figure 3.2 and shown in Equation 3.1 where $N$ is the number of samples in a chirp waveform, or the number of pulses in a burst in a stepped-frequency waveform.

$$
w_{s}=N \Delta r_{s}=N \frac{c}{2 \beta}
$$

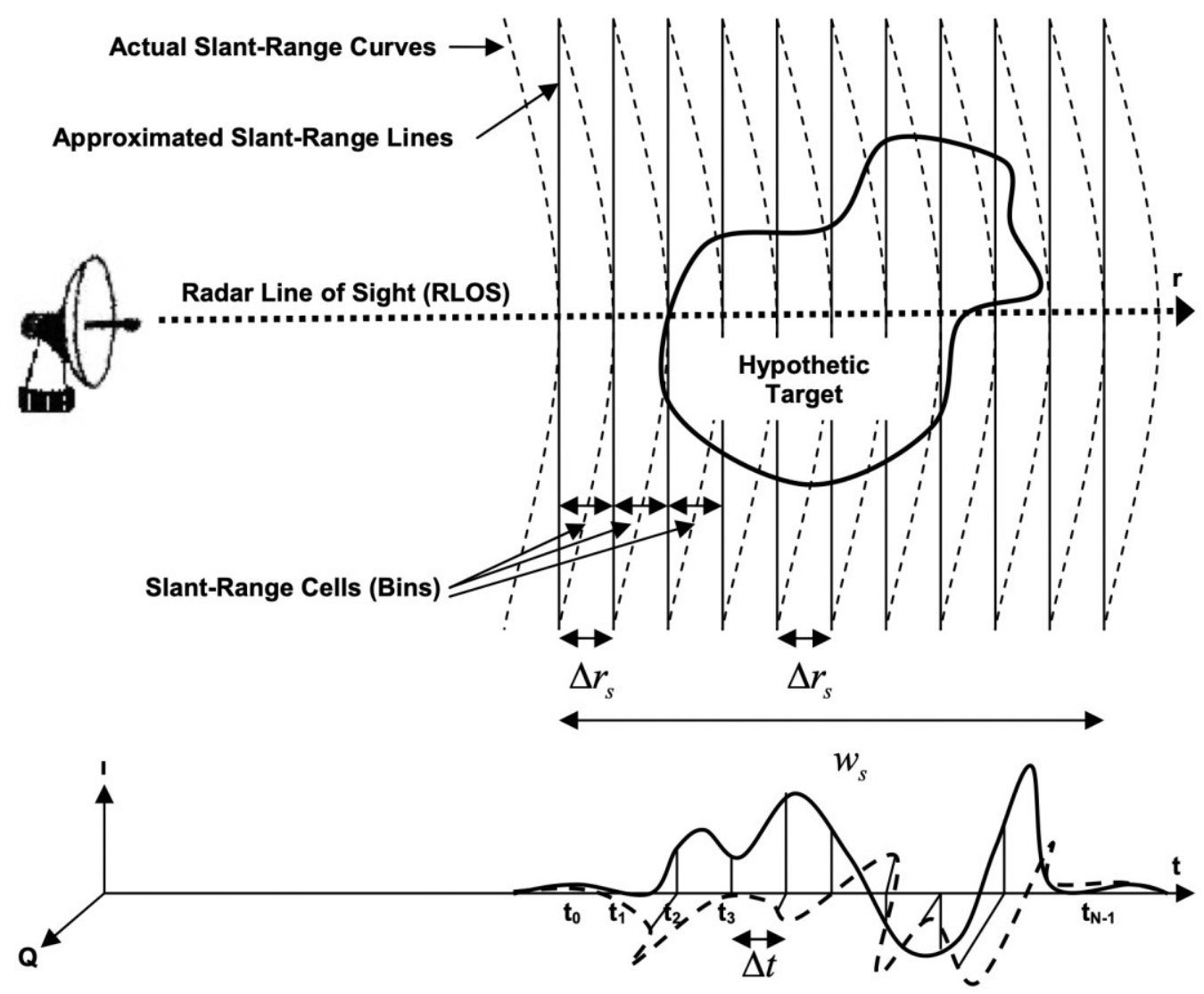

Figure 3.2: Slant-Range resolution and window, from [53]. 
In practice, the radar system is observing a large range in which the target to be imaged covers only a small portion of the entire range window. To isolate the target into a smaller range window the response from a target is tracked with each transmission. Thus, the window begins when the target echo pulse is received, and ends when the target echo pulse duration is completed [43][45]. The samples of the target echo signal within this duration comprise the range resolution cells within the window. The slant-range data is processed using the methods discussed in the previous chapter to generate the complex range profile history of the target.

\subsection{The Second Dimension: Doppler (Cross-Range)}

As introduced in the previous chapter, the cross-range resolution is dependent on either the antenna beamwidth or is generated by performing Synthetic Aperture processing. In ISAR processing, the antenna beamwidth is not of major concern in producing a cross-range window size or resolution. The cross-range data is produced by coherently processing multiple range-profiles along each range resolution cell [45][53]. Target scattering locations within a single slant-range resolution cell are distributed to the appropriate Doppler shift by performing the DFT operation. This operation occurs for each slant-range resolution cell across the profile history, as discussed in chapter 2.6. Another illustration of this process is presented in Figure 3.3. 


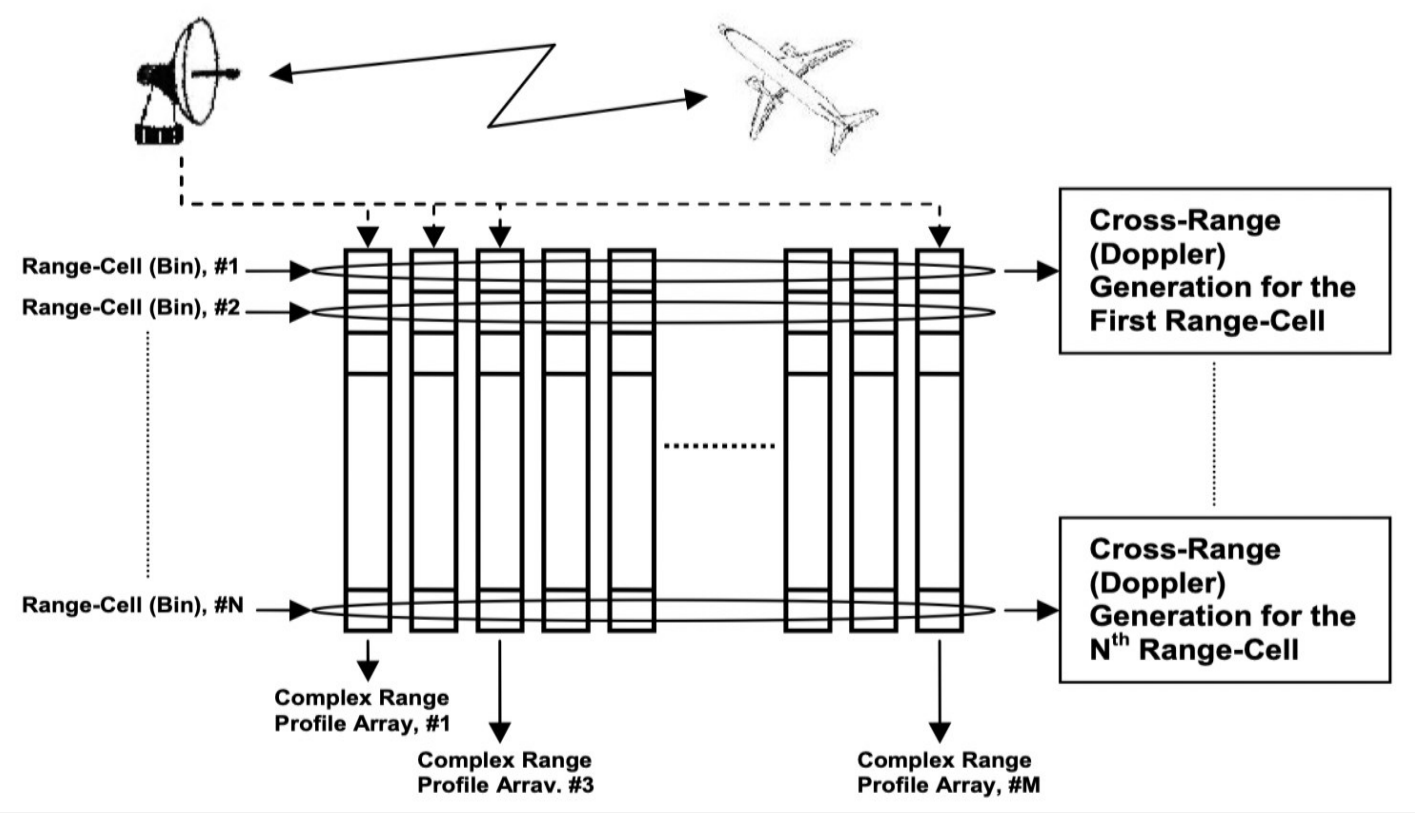

Figure 3.3: Illustration of the phase/Doppler dimension processing, from [53].

\subsubsection{Doppler (Cross-Range) Resolution}

The cross-range dimension of a target is predominantly determined by the angular rotation rate of the target relative to the radar LOS [45]. Consider a range-extended target, with a scatterer located at position with distance $r_{c}$, from the LOS. The target is rotating with an angular velocity, $\omega$, towards the radar as illustrated in Figure 3.4.

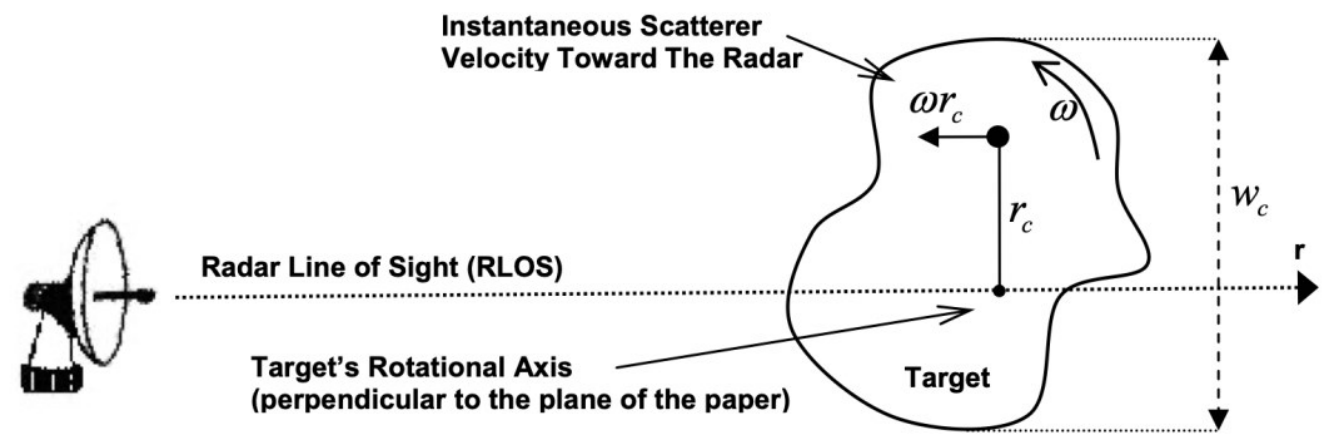

Figure 3.4: Angular velocity of a scattering at a cross-range position, from [53]. 
The instantaneous Doppler shift of the scattering location is determined below.

$$
f_{D}=\frac{2 \omega r_{C}}{\lambda}
$$

If there are at least two scattering locations within the same slant-range resolution cell, but they are separated in cross-range by a distance $\delta r_{c}$ the difference in the Doppler shift produced by the scatterers is:

$$
\delta f_{D}=\frac{2 \omega \delta r_{c}}{\lambda}
$$

Re-arranging this equation, the cross-range resolution of two scattering locations is determined by the Doppler frequency difference of the scatterers.

$$
\delta r_{c}=\frac{\lambda \delta f_{D}}{2 \omega}
$$

Thus, the Doppler resolution of the radar system determines the capability to resolve scattering locations separated in cross-range. The Doppler resolution in ISAR processing is proportional to the coherent integration time of the frame $[41][45][53]$. The frame is determined by the total number of range profiles used to create the RangeDoppler image. Assuming $M$ range profiles are used when processing either pulse compression or stepped-frequency waveforms, the coherent integration time for both systems is:

$$
\begin{aligned}
& T_{C I}=M \times T_{P R I}(\text { Pulse Compression Waveforms }) \\
& T_{C I}=M \times T_{B u r s t} \\
& \quad=M \times N \times T_{P R I}(\text { Stepped }- \text { Frequency Waveforms })
\end{aligned}
$$

Here, $T_{C I}$ is the coherent integration time to produce an image. The $T_{P R I}$ is the pulse repetition interval, and $T_{B u r s t}$ is the single burst interval. A singe burst consists of $N$ total pulses which are transmitted at the pulse repetition interval rate. It is observed that the coherent integration time is a function of the time taken to produce a single HRRP. The Doppler resolution is shown in equation 3.7.

$$
\Delta f_{D}=\frac{1}{T_{C I}}
$$

Over the coherent integration time, the target is expected to rotate with some unknown angular velocity. Thus, equation 3.4 can be rewritten as, 


$$
\Delta r_{c}=\frac{\lambda}{2 \omega T_{C I}}=\frac{\lambda}{2 \Delta \psi}
$$

Where $\Delta \psi=\omega T_{C I}$, is the aspect angle change in the target over the coherent integration time frame [45][53]. As mentioned in previous chapters, a large coherent integration time can lead to significant aspect angle change in the target, which will cause blurring of the target image. Selection of an appropriate coherent integration time is crucial to improved image resolution, as well as resolution in cross-range or Doppler.

Consider a target with a cross-range extent $w_{t c}$, rotating at a constant angular rate $\omega$. The total Doppler shift bandwidth from the target is expressed in equation 3.9.

$$
\beta_{D, \text { target }}=\frac{2 \omega w_{t c}}{\lambda}
$$

Since the Doppler history of the target is obtained by coherent processing of each range resolution cell across the range profile history, it can be considered that each range profile is a collection of samples of the Doppler spectrum of the target. In the case of pulse compression radar, the profiles are sampled at a rate equivalent to the PRF, which is the maximum Doppler bandwidth of the system. In a stepped-frequency system, the profiles are sampled at a rate equivalent to the burst rate. The Doppler resolution for both systems is presented in equation 3.10 and 3.11 .

$$
\begin{aligned}
\beta_{D, \text { system }} & =\frac{1}{M \times T_{P R I}}(\text { Pulse Compression Waveforms }) \\
\beta_{D, \text { system }}= & \frac{1}{M \times T_{\text {Burst }}} \\
& =\frac{1}{M \times N \times T_{P R I}}(\text { Stepped }- \text { Frequency Waveforms })
\end{aligned}
$$

To resolve the entire target, the Doppler bandwidth of the system must be greater than the Doppler bandwidth of the target. For unambiguous resolution of the Doppler dimension, the system bandwidth must meet the Nyquist sampling requirement. However, depending on the target Doppler bandwidth, achieving system parameters to perform imaging without ambiguity may not be possible, since the PRF may have to be increased to unreasonable values. The common term in Doppler resolution of the system 
is the PRI, which is the reciprocal of the PRF. Thus, resolving the entire Doppler bandwidth would require the system PRF to meet the following conditions:

$$
\begin{gathered}
P R F=\frac{1}{T_{P R I}} \geq \frac{2 \omega w_{c}}{\lambda}(\text { Pulse Compression Waveforms }) \\
P R F=\frac{1}{T_{P R I}} \geq \frac{2 N \omega w_{c}}{\lambda}(\text { Stepped }- \text { Frequency Waveforms })
\end{gathered}
$$

The cross-range window of the target image is then determined using equation 3.14 , shown below.

$$
w_{c}=M \Delta r_{c}=\frac{\lambda}{2 \omega T_{C I}}
$$

\subsection{Chirp Range-Doppler Image Algorithm}

The first step is to generate the complex range profile history of target as described in Chapter 2. The data is stored in digital memory as a matrix, in which each row of data is a single complex range profile. The columns contain the sample data for each slant-range resolution cell.

Phase history processing is performed on each column of data in the matrix. The columns are multiplied by a windowing function, then a column-wise DFT is performed to create the Range-Doppler image. Figure 3.5 illustrates the process of generating a Range-Doppler image in a chirp radar system. 


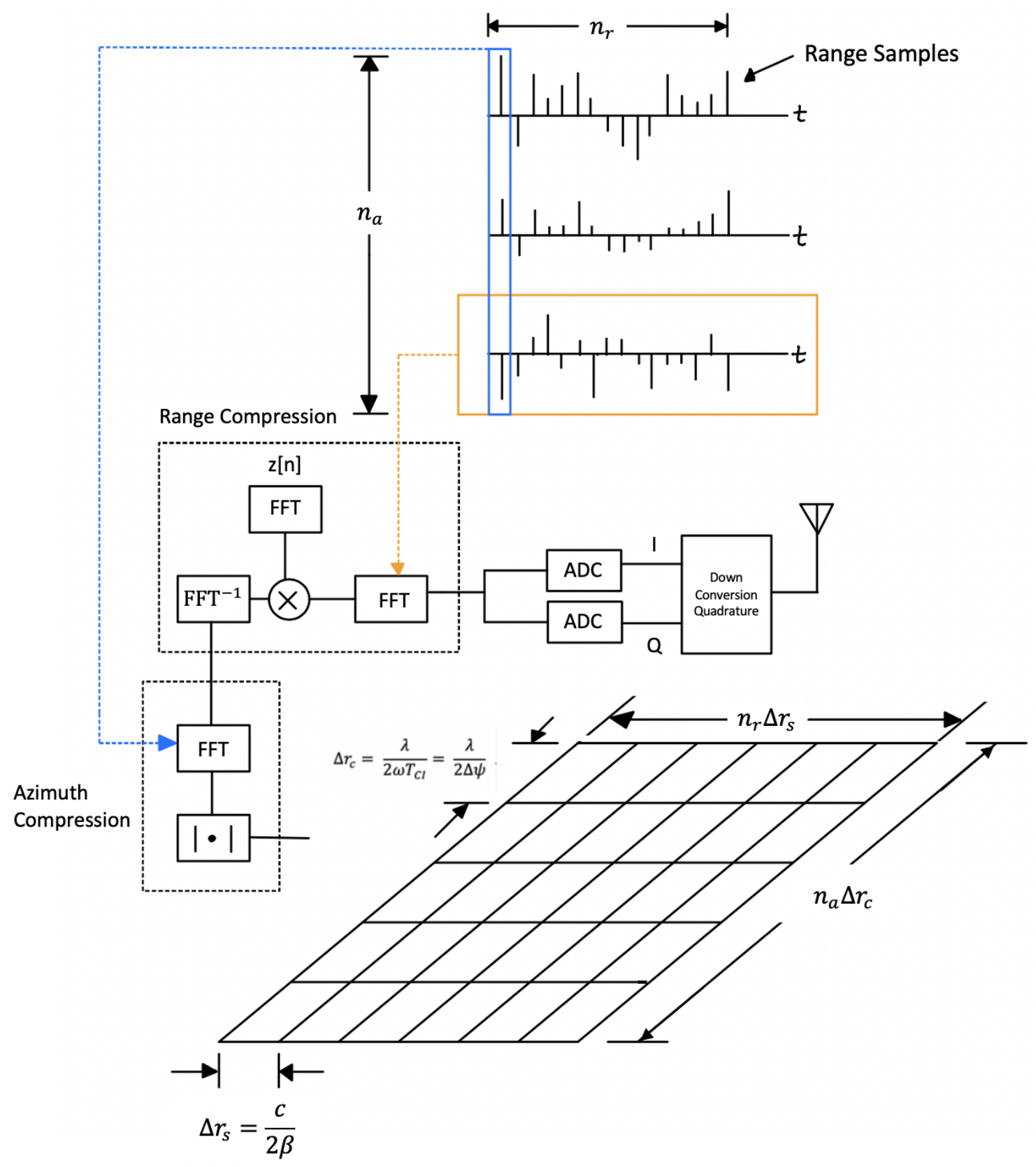

Figure 3.5: Logical diagram of ISAR Image generation for pulse compression systems.

This process is also illustrated in terms of the data matrix at each step in Figure 3.6 on the following page. 


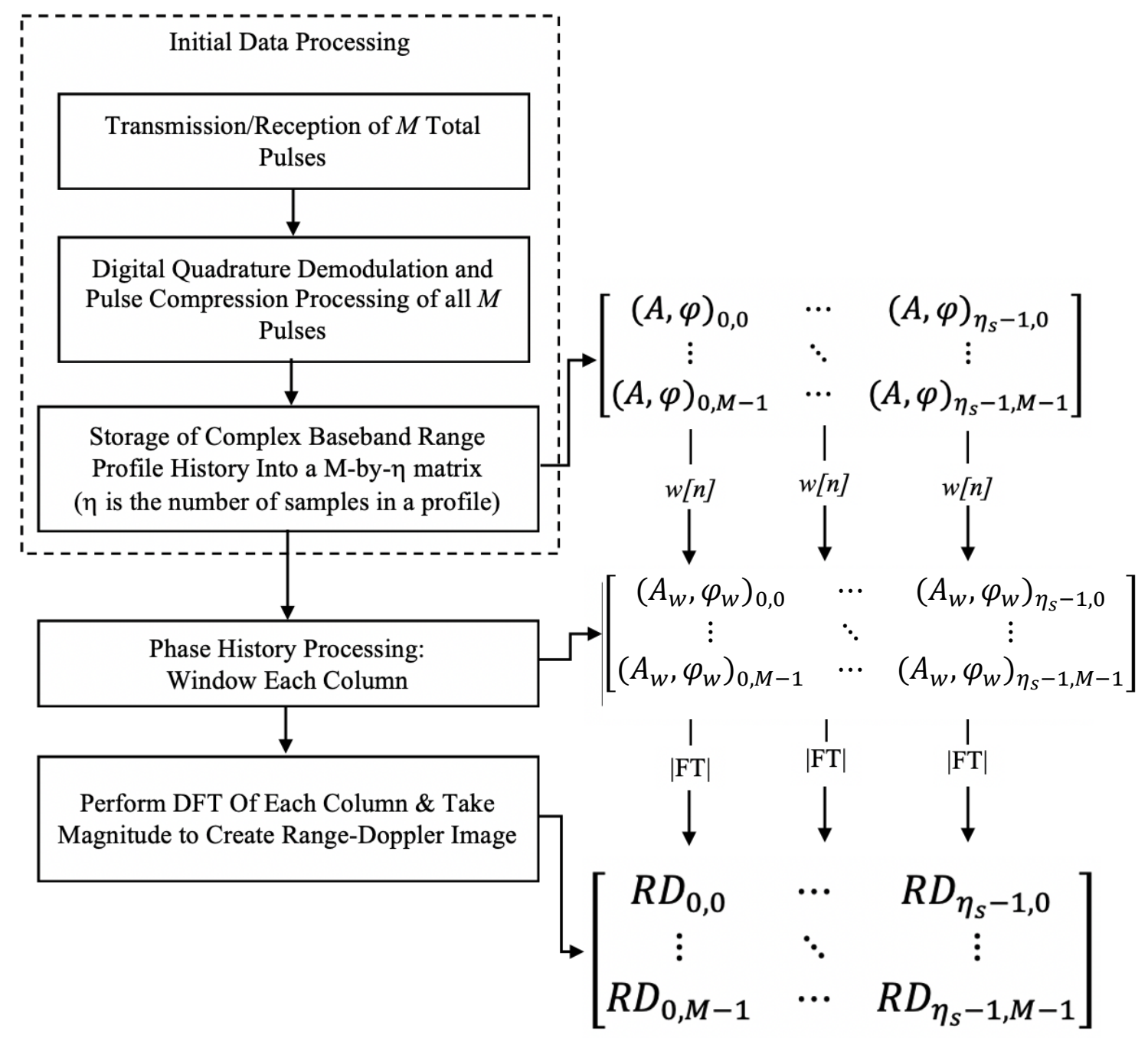

\begin{tabular}{|c|l|}
\hline Symbol & Definition \\
\hline$(A, \varphi)_{i, k}$ & $\begin{array}{l}\text { Amplitude and phase in the } \mathrm{i}^{\text {th }} \text { range cell for the } \mathrm{k}^{\text {th }} \text { range profile. There is a total of } \\
M \text { range profiles, and } \eta_{S} \text { range samples. }\end{array}$ \\
\hline$D_{i, k}$ & Magnitude in $\mathrm{i}^{\text {th }}$ range cell for the $\mathrm{k}^{\text {th }}$ Doppler cell in the Range-Doppler image. \\
\hline
\end{tabular}

Figure 3.6: ISAR Image Processing for Chirp Pulse Data. 


\subsection{Stepped-Frequency Range-Doppler Image Algorithm}

The process is remarkably similar to the algorithm for processing pulse compression data. The significant difference is in the generation of the range-profile history of the target. The first step is to obtain $M$ total range profiles by transmitting a total of $M$ times $N$ pulses, where $N$ is the total number of pulses in a single burst. The processing steps described in chapter 2 are used to generate the complex range profile history of the target. The complex range profiles are stored in digital memory as a N-by$\mathrm{M}$ matrix, or alternatively an M-by-N matrix. The system designer determines matrix dimension organization, and ensures the DFT operations occur for the appropriate matrix dimensions accordingly.

The phase processing steps of windowing each range resolution cell across the profile history is performed. The DFT for each range cell across is the history is computed, and the magnitude of each complex data point is computed to create the Range-Doppler image. This process is illustrated in Figure 3.7 on the following page. 
Initial Data Processing Stages

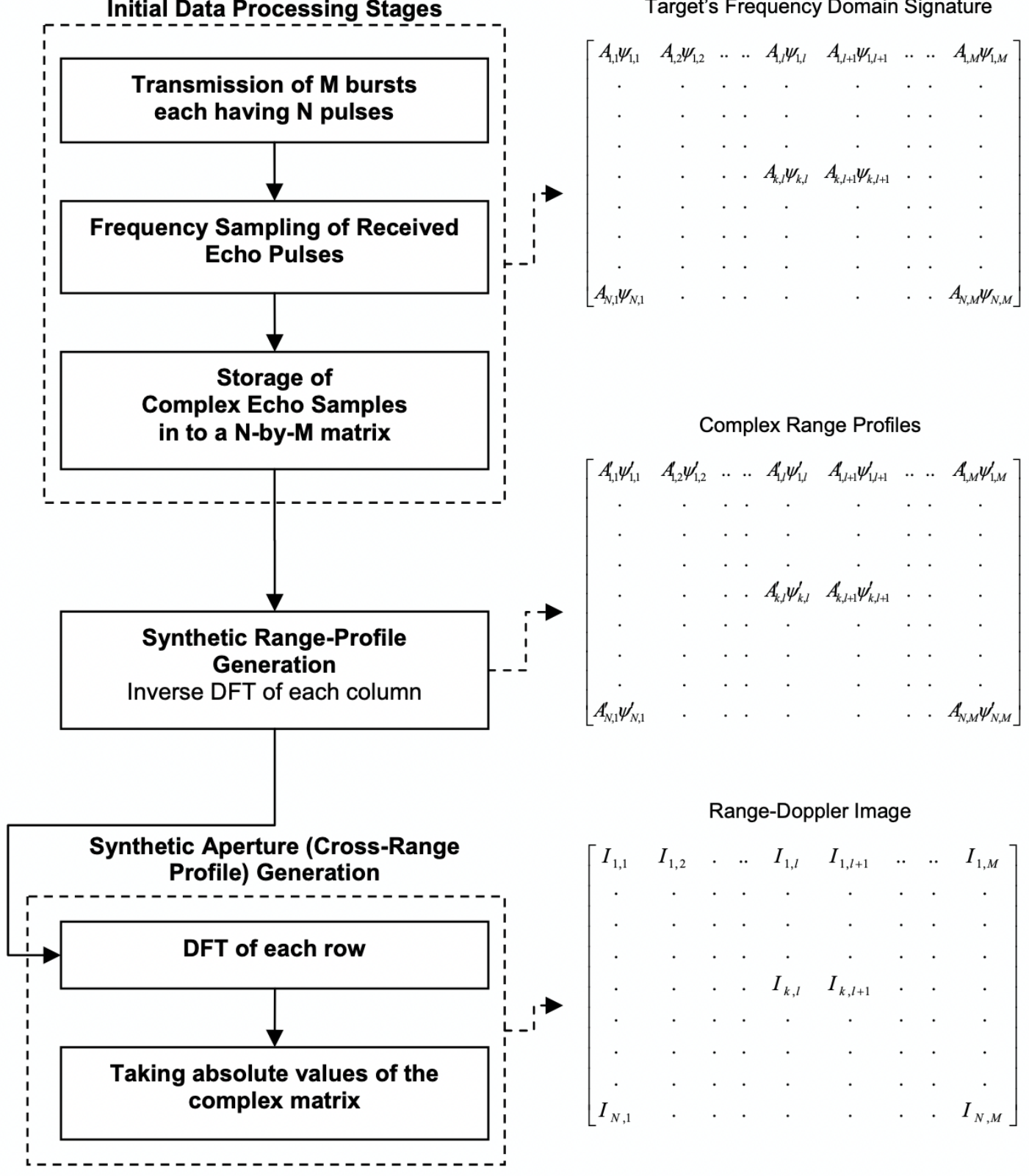

Figure 3.7: ISAR Image processing steps for Stepped-Frequency Data. 


\subsection{Effects of Motion on Range-Doppler Image}

The motion of a target is classified into translational and rotational motion [45]. Translational motion refers to motion of the entire target structure, in a given direction. Rotational motion is of particular interest in maritime ISAR imaging applications as it is this velocity which is used to identify unique scattering locations in the Range-Doppler image. Rotational motion occurs about a rotational axis on the target. The rotational axis is fixed to the target and moves with the target as a target undergoes translational motion. The magnitude and direction of the rotational axis are complex functions of time, dependent on target motion physics.

The velocity of a target is classified into radial and angular velocity [45]. Radial velocity is aligned with the radar LOS. For example, a ship moving directly towards a radar at $5 \mathrm{~km} / \mathrm{h}$ would have a radial velocity of $5 \mathrm{~km} / \mathrm{h}$. However, if the same ship were moving perpendicular to the radar LOS with the same velocity the radial velocity of the target would be $0 \mathrm{~km} / \mathrm{h}$. Angular velocity is motion of the target about the axis of the LOS intersecting with the target as illustrated in Figure 3.4. Both velocities contribute to the Doppler shift created by a scattering location on the target.

In practical ISAR systems, the motion characteristics of a target are unknown. This results in a degradation of resolution of the Range-Doppler image of the target. Motion compensation algorithms have been a topic of research for decades, to improve the quality of Range-Doppler images of target for use in Automatic Target Recognition (ATR) algorithms and other functions [27]-[40][53][55]. The effect of motion and compensation algorithms are discussed in the following subsections, beginning with translational motion effects.

\subsubsection{Translational Motion Effects}

The radial velocity of a target undergoing translational, or radial, motion can have a significant impact on the quality of ISAR image produced. Generally, the effects of radial motion create two types of change to the profiles used in image generation. These are the range walk and range offset in a profile [45]. As a target undergoes motion, the range position of the scattering locations change in the radar measurement systems for 
each pulse transmitted. The change in range position of a target between each profile generation is referred to as the range walk of the target [45]. In chirp radar systems, this profile-to-profile range change can be corrected by adjusting the sampling position of the profile to align the profiles within a range window rather than over the entire scan range of the radar [45]. In stepped-frequency radar systems, since the HRRPs are generated using synthetic processing, the techniques to account for range walk are different. These methods will be discussed in the following subsection regarding translational motion compensation algorithms.

Range offset is the change in range position of scattering locations within a profile. This occurs due to the range-doppler coupling characteristics of chirp and linear stepped frequency waveforms [43][45]. The range-Doppler coupling characteristics show that as the target Doppler shift increases, the range position of the scattering location shifts proportional to the chirp, or frequency variation, rate of the waveform [43][45]. Since Doppler resolution of a single pulse is inversely proportional to the pulse duration, it is determined that for short pulse applications, on the order of microseconds, the maximum unambiguous Doppler is on the order of Megahertz [43][45]. Ship targets will not demonstrate Doppler shifts this large, meaning the distortion seen within a profile due to motion of scatterers is not significant [45].

\subsubsection{Translational Motion Compensation (TMC) Algorithms}

Translational Motion Compensation (TMC) algorithms have been an area of research since the development of synthetic aperture imaging technology in the 1960s1970s [43][45]. These algorithms are often categorized as non-iterative or iterative. Basic non-iterative algorithms involve defining a tracking window for a target, enabling the digital processing system to track the target within the range window and perform alignment of multiple profiles prior to image generation. The general technique involves measurement of the phase difference between each profile to obtain a phase compensation factor [45]. This phase compensation factor is then multiplied to each profile to align the profiles in the range dimension. Some algorithms used to obtain the phase correction factor are: 
- Cross-Correlation Method [7][45]

- Prominent Point Processing [7][45][53]

- Target Centroid Tracking [7][45][53]

This thesis implements the cross-correlation TMC algorithm in the simulated ISAR image processing shown in chapter seven.

The cross-correlation method is used to obtain a phase correction factor for each range-cell within a HRRP [45][53]. HRRPs generated from chirp or synthetic processing contain complex data samples which identify the range position of unique scattering locations on the target. As the target undergoes motion, the location of these scattering centers can change and migrate between range cells or possibly become masked by new scattering locations. In ISAR imaging, keeping the observation time relatively small ensures the target aspect does not significantly change. During a longer observation time scattering locations can become masked or disappear from the collected range profiles. With extremely fine resolution systems, it is highly likely that scattering locations can migrate to adjacent range cells over time. A HRRP is selected as a reference profile, generally the first profile is the reference, and the remaining are cross correlated with the reference profile [53]. The maximum lag, in number of samples, from the result of the cross-correlation operation, indicates the average translation of scattering location in range cells [45][53]. The number of samples is then converted to a range value, which is proportional to a phase constant [53]. The phase constant is the correction term which is multiplied into both quadrature channels to correct the received target echoes for the translational motion errors [45][53]. The corrected quadrature data is reprocessed to generate the HRRPs, which are now range-aligned prior to any image processing operations.

\subsubsection{Rotational Motion Effects}

Rotational, or angular, motion creates the phase gradient from the various scattering locations; this makes Range-Doppler imaging of the target possible [45]. Rotational motion of scattering centers mean they follow curved paths of motion, rather 
than the linear motion paths observed by radial, or translational, motion [45]. The change in phase of scattering locations is also non-linear, resulting in distortion of the RangeDoppler image. As the resolution of the system increases, the distortion becomes increasingly severe.

Non-linear phase effects of rotational motion are modelled using quadratic phase correction factors in marine radar applications [45]. This is because the angular motions of a ship; pitch, roll, yaw, are all considered sinusoidal motion characteristics. In the duration that a ship is observed by a radar, only a portion of the sinusoidal motion characteristic contributes to the phase gradient observed in each profile [45][53]. This small portion of the sinusoidal motion characteristics, is modelled using a quadratic phase approximation [45].

In addition to non-linear phase distortion, the angular motion of scattering locations can result in the scatters moving across range resolution cells over time [45]. This generally occurs when the coherent integration time of the system is large enough that the scattering positions at the ends of the target can move multiple range cells over the observation period [8][45]. An estimate of angular motion is used to create phase correction factors which are multiplied into the quadrature data used to generate the HRRP history [8][31][33]. Migration of scattering locations occurs in slant-range cells and in cross-range resolution cells. This is of significance when considering that the target observed is experiencing angular motion, but the image produced is a rectangular formatted image. The target is observed over a small angular region, where the crossrange distance between sample points increases as the slant-range position increases [45][53]. Range-Doppler imaging algorithms process the data as if the spacing of crossrange scattering locations is constant, regardless of the slant-range position. This leads to blurring of the target image. To correct this, the polar-reformatting algorithm is applied [43][45][49]. This repositions cross-range scattering locations and creates the rectangular image grid by using frequency-space and adaptive beamforming principles $[4][6][7][8][31][33]$. There are many different types of polar reformatting algorithms, an investigation of each one is beyond the scope of this research. 


\subsubsection{Rotational Motion Compensation (RMC) Algorithms}

Many compensation algorithms have been researched and applied in industry from the 1980s to now. A few of these algorithms are listed below. Systems can apply one more of these algorithms in conjunction with one another to improve performance.

- Dominant Scatterer Algorithm [68][74]

- Polar Reformatting Algorithm [43][45][8]

- Cross-Range Centroid Tracking [75]

- Phase-Gradient Autofocus Technique [71]

Evaluation and implementation of all the above-mentioned algorithms is beyond the scope of this research. Many RMC algorithms also require assumptions or knowledge of the antenna characteristics used by the radar system. In the scope of this research application, antenna parameters are unknown since the system designed in this research is meant to counter a wide variety of wideband high-resolution radar systems. The algorithm selected for use in simulations in this research is the Dominant Scatterer Algorithm (DSA), due to its relative simplicity of application and the lack of requirement for knowledge of antenna parameters.

The principle of the DSA is to select a dominant scattering sample from the HRRP and use it as a phase synchronizing source [53][68][74]. A scattering location is considered the dominant contributor if it has the largest phasor contribution within a range cell [53][68][74]. In a HRRP, each resolution cell is dominated by a single scattering location on a complex range-extended target. The DSA can be described in the following steps based on interpretation from [53][68][74].

1. The amplitude variance in each range resolution cell (or each sample) of the HRRP is found across the total history of range profiles collected.

2. The range cell (or sample number) with the minimum amplitude variance across the history is defined as the reference phase synchronizing source. A reference HRRP from the history is also selected as the reference profile, with the first profile generally chosen as the reference profile. 
3. For the selected reference range cell (or sample number), the phase difference between the cell in the reference profile and the reference cell in all subsequent profiles is computed. The phase difference, from the reference, for each profile is stored as the correction factor for the specific profile number in the history.

4. The quadrature data for each profile following the reference profile is multiplied by the corresponding phase correction factor.

5. The corrected data is used to regenerate the HRRP history and then compute the Range-Doppler image.

\subsubsection{Motion Compensation \& Automatic Image Focus Algorithms}

The TMC and RMC algorithms discussed above have been enhanced because of extensive research over the past 40 years. Many motion algorithms can now perform both TMC and RMC combination techniques to greatly improve image focus, such as the Minimum Entropy Algorithm and many others found in various literature. For further information on these motion compensation or image focusing algorithms the reader is referred to $[4][6][7][8][31][33][43][45][49][53][68]-[75]$.

\subsection{Conclusion}

This chapter presented the principles of Range-Doppler imaging discussed in literature and industry. The processing methods used to create Range-Doppler images when processing data for chirp and stepped-frequency waveforms is discussed in detail. Also discussed is the application of motion compensation algorithms to improve image quality, and the algorithms implemented in this research are described in detail. The next chapter discusses the development of a realistic target motion algorithm using Ocean Engineering and Naval Architecture principles. 


\section{Chapter 4: Target Motion Algorithm}

\subsection{Introduction}

Radar target motion in a Naval environment has been a topic of study since the development of Doppler imaging processes [1]. The assumption of sinusoidal motion undergone by a ship has been exploited in research pertaining to optimization of ISAR imaging and target classification [2]-[9]. These sinusoidal motion approximations are used, by radar engineers, to simplify the complex motion experienced by a ship in the ocean. The study of ship motion has been further investigated by Naval Architects and Marine Engineers in application to ship design and seakeeping principles. This includes statistical modelling of ocean waves, and the corresponding motion response undergone by a ship to maintain stability while carrying out its objective. An algorithmic implementation of the target motion model is developed to hybridize the sinusoidal approximations developed by radar engineers, and the motion parameters obtained through research in naval architecture. The inclusion of environmental conditions contributing to the ship motion, as well as the variety of classes of targets capable of being simulated highlights the flexibility of the motion algorithm implemented.

This chapter presents an overview of the statistical modelling used to describe the ocean state. This is followed by a description of ship responses, and the extraction of valuable motion parameters in relation to ocean wave statistics as presented in literature. The final section of this chapter addresses mathematical application of the ship motion model to simulated targets.

\subsection{The Wave Energy Spectrum}

Oceanographers and naval architects have been using mathematical descriptions to model the seaway since before the 1950's [10]. The first models applied the 'regular wave' approximation, which assumed waves could be represented by a single sinusoid as a function of space and time. These models are effective in small environments such as wave tanks to observe the behavior of simple waves [15]. However, in the open sea the regular wave model is inaccurate requiring the development of the irregular waves model. 
The irregular waves model is based on the regular wave model, in that a single wave at a specific frequency can be described by a sinusoidal function [15]. By applying the principle of superposition of many regular waves, all at different frequencies, the irregular waves model is created [15]. Theoretically the number of individual waves combining in an open seaway can be infinite, thus, the time domain representation of the waves is an infinite summation of individual waves of unique amplitudes, frequencies, and relative phase shifts [15][22]. The representation of the waves in a time-domain based model is not feasible with a near infinite summation. Rather waves are described using statistical models [15][22]. When measured, the histogram of water elevation follows a Gaussian distribution [12][15][22]. The wave height histogram distribution is much better represented by a Rayleigh distribution [12][15][22][23]. The wave height or elevation data do not provide the information as to how the waves interaction with the ship hull produces the motion effect. A parameter of value that is extracted from these histograms is the Significant Wave Height, which is defined as the average of the highest $1 / 3^{\text {rd }}$ of the measured wave heights [10][12][15][22][23]. This is the value closest to the wave height, generally given by experienced sailors, through visual observation [10]. To determine the impact of waves on ships, the energy in the waves must be transferred to the ship's kinetic energy [12][15].

Waves are comprised of mainly two forms of energy: potential energy, and kinetic energy [12]. The kinetic energy stored in a wave comes from the velocity of the wave. The potential energy in a wave is stored underneath the water surface and manifests in the form of wave amplitude or height [12][15]. To determine the amount of potential energy in a two dimensional wave the vertical segment of a wave of height, $\zeta$, is examined [15]. The center of gravity of this wave is located at height $\zeta / 2$, and has a mass $\rho g \delta x$, where $\rho$ is the density of water [15]. The term $\delta x$ accounts for the length of the wave and often described in terms of wavelength. Thus, the potential energy of the wave is described by equation 4.1, from [15], as:

$$
E_{P E}=\frac{\left(\rho g \zeta^{2} \delta x\right)}{2}
$$


By integrating this portion of energy over the entire wavelength the potential energy per unit width is defined in equation 4.2 , as per [15]:

$$
E_{P E}=\frac{\left(\rho g \lambda \zeta_{0}^{2}\right)}{4}
$$

In Equation 4.2, the density of the water is represented by $\rho$, the term $g$ is the gravitational acceleration constant, $\lambda$ is the wavelength, and $\zeta_{0}^{2}$ is the wave height. The kinetic energy of a two-dimensional segment of the wave is defined in equation 4.3, as per [15]:

$$
E_{K E}=\frac{\left(\rho q(\lambda)^{2} \delta x \delta z\right)}{2}
$$

Where $q$, is the velocity of the specific wave segment, and it is a function of the wavelength. The velocity of a two dimensional wave can be described by the expression $q=\sqrt{\frac{g \lambda}{2 \pi}}$, where $g$ is the gravitational acceleration constant. By integrating this energy over the wavelength in the $\mathrm{x}$ dimension and the height of the wave in the $\mathrm{z}$ dimension, the per unit kinetic energy is the same as the per unit potential energy of the wave, as seen in equation 4.4. Thus, from [15], the total energy in a wave of single frequency is:

$$
E_{\text {TOT }}=\frac{\left(\rho g \zeta_{0}^{2}\right)}{2}
$$

By using the principle of superposition to generate a dataset for the wave energies as a function of wave frequency, a spectrum is created [22]. This frequency spectrum is also referred to as the wave energy spectrum [10][15][22][23]. This allows all the wave height data from a time history to be effectively represented by a summation of many individual waves of varying amplitudes for which the energy can be calculated [22]. Using the Fourier transform, the energy for each wave is mapped to the appropriate frequency range in the wave energy spectrum [10][15]. Both the wave energy spectrum, and the time history data of the wave elevation are statistically the same. This is verified by observing the spectral moments of the energy spectrum [15]. The first spectral moment is the variance defined as the total area of the spectrum [15].

$$
m_{0}=\int_{0}^{\infty} S_{\zeta}(\omega) d \omega
$$


This is also the variance of the wave elevation histogram which can be used to generate the spectrum [15]. Higher order spectral moments are also used to generate data such as mean frequency, mean period, mean peak period, and mean zero-crossing period [15][22]. The spectral moments are also used to compute the bandwidth parameter, in equation 4.6, of the energy spectrum [15].

$$
\epsilon=\sqrt{1-\frac{\bar{T}_{p}^{2}}{\bar{T}_{z}^{2}}}
$$

In equation $4.6, \bar{T}_{p}^{2}$ is the mean peak period, $\bar{T}_{z}^{2}$ is the mean zero crossing period, and $\epsilon$ is the bandwidth parameter [15]. As the bandwidth parameter approaches zero, it describes a narrowband spectrum [15]. Open ocean waves are assumed to be comprised of an almost infinite number of various frequency waves, creating a wideband spectrum for irregular wave patterns. The significant wave height data can also be extracted from the energy spectrum as seen in equation 4.7 from [15].

$$
\bar{H}_{1 / 3}=4 \sqrt{m_{0}} \sqrt{1-\frac{\epsilon^{2}}{2}}
$$

Due to the vastness of the ocean and the constantly changing environment, it is not feasible to generate wave amplitude or elevation time histories [10]. However, observational data has shown that a sea state can be considered to be steady for a time span of about 20 to 30 minutes, following which the energy spectrum of the seaway will demonstrate significant change [10][20]. The solution is to then create energy spectra which represent the energy within the moving seaway at a specific sea state. This spectral representation of the wave energy will be valid in the scenario that a ship is being tracked by a radar target for an extended period, such as 20 to 30 minutes [20].

Idealized wave energy spectra have been proposed by many different researchers and are all uniquely described by parameters such as wind velocity, significant wave height, modal wave period and more [10][12][15]. A representation of the wave energy spectrum defined in the ITTC (International Tank and Towing Conference) is the Bretschneider Spectrum for fully developed ocean waves created in 1973 [15]. Fully developed waves have enough space and time to reach maximum amplitude and then slowly degrade, unlike waves at the beach which crash into the shoreline. The 
Bretschneider spectrum is based on two parameters: the square of the significant wave height, $\bar{H}_{\frac{1}{3}}^{2}$, and the modal wave period, $\omega_{0}$. It is shown in equation 4.7 on the following page, as per [15].

$$
S_{\zeta}(\omega)=\frac{1.25}{4}\left(\frac{\omega_{0}}{\omega}\right)^{4}\left(\frac{\bar{H}_{1}^{2}}{\frac{\overline{3}}{\omega}}\right) e^{-1.25\left(\frac{\omega_{0}}{\omega}\right)^{4}}
$$

There are potentially an infinite number of combinations of modal wave period and significant wave height used to describe the wave energy spectrum, however, not all combinations would represent a realistic wave pattern. The North Atlantic Treaty Organization (NATO) has created categories named sea states to describe the state of the sea in terms of modal wave period and significant wave height [18][19], this is seen in Table 4.1. For a low Sea State number, the waves are slower and lower amplitude. As the Sea State increases so does the corresponding significant wave height and modal period.

Table 4.1: NATO Sea State Table with Significant Wave Height and Modal Wave Period.

\begin{tabular}{|c|c|c|c|c|c|c|c|}
\hline \multirow{3}{*}{$\begin{array}{l}\text { Sea State } \\
\text { Number }\end{array}$} & \multirow{2}{*}{\multicolumn{2}{|c|}{$\begin{array}{c}\text { Significant Wave } \\
\text { Height }(\mathrm{ft})\end{array}$}} & \multirow{2}{*}{\multicolumn{2}{|c|}{$\begin{array}{l}\text { Sustained Wind } \\
\text { Speed (Kts) }\end{array}$}} & \multirow{3}{*}{$\begin{array}{l}\text { Percentage } \\
\text { Probability } \\
\text { of Sea State }\end{array}$} & \multicolumn{2}{|c|}{ Modal Wave Period (s) } \\
\hline & & & & & & \multirow{2}{*}{ Range } & \multirow{2}{*}{$\begin{array}{c}\text { Most } \\
\text { Probable }\end{array}$} \\
\hline & Range & Mean & Range & Mean & & & \\
\hline $0-1$ & $0-0.3$ & 0.2 & $0-6$ & 3 & 0 & - & - \\
\hline 2 & $0.3-1.5$ & 1.0 & $7-10$ & 8.5 & 7.2 & $3.3-12.8$ & 7.5 \\
\hline 3 & $1.5-4$ & 2.9 & $11-16$ & 13.5 & 22.4 & $5.0-14.8$ & 7.5 \\
\hline 4 & $4-8$ & 6.2 & $17-21$ & 19 & 28.7 & $6.1-15.2$ & 8.8 \\
\hline 5 & $8-13$ & 10.7 & $22-27$ & 24.5 & 15.5 & $8.3-15.5$ & 9.7 \\
\hline 6 & $13-20$ & 16.4 & $28-47$ & 37.5 & 18.7 & $9.8-16.2$ & 12.4 \\
\hline 7 & $20-30$ & 24.6 & $48-55$ & 51.5 & 6.1 & $11.8-18.5$ & 15.0 \\
\hline 8 & $30-45$ & 37.7 & $56-63$ & 59.5 & 1.2 & $14.2-18.6$ & 16.4 \\
\hline$>8$ & $>45$ & $>45$ & $>63$ & $>63$ & $<0.05$ & $15.7-23.7$ & 20.0 \\
\hline
\end{tabular}

The mean significant wave height, and most probable modal wave period are used in the Bretschneider equation to generate the wave energy spectrum for each Sea State 
[12][15]. The modal wave period is defined as twice the average time interval between consecutive zero height readings in a wave record [10]. The modal wave period is generally larger than the periodicity of wave as observed by the eye [10]. If only observational data were to be used the measured observational wave period would not correspond to the frequency at which most energy in the waves is concentrated [10]. The energy spectrum development begins at the high frequency end, representing a collection of lower amplitude high frequency waves [10]. As the waves travel, the energy from smaller waves collects over a wide range, generating larger but slower travelling waves [10]. This results in more energy being focused into larger wave periods, correspondingly lower wave frequencies. Figures 4.1 to 4.3 show the Bretschneider wave energy spectrum for three different sea states.

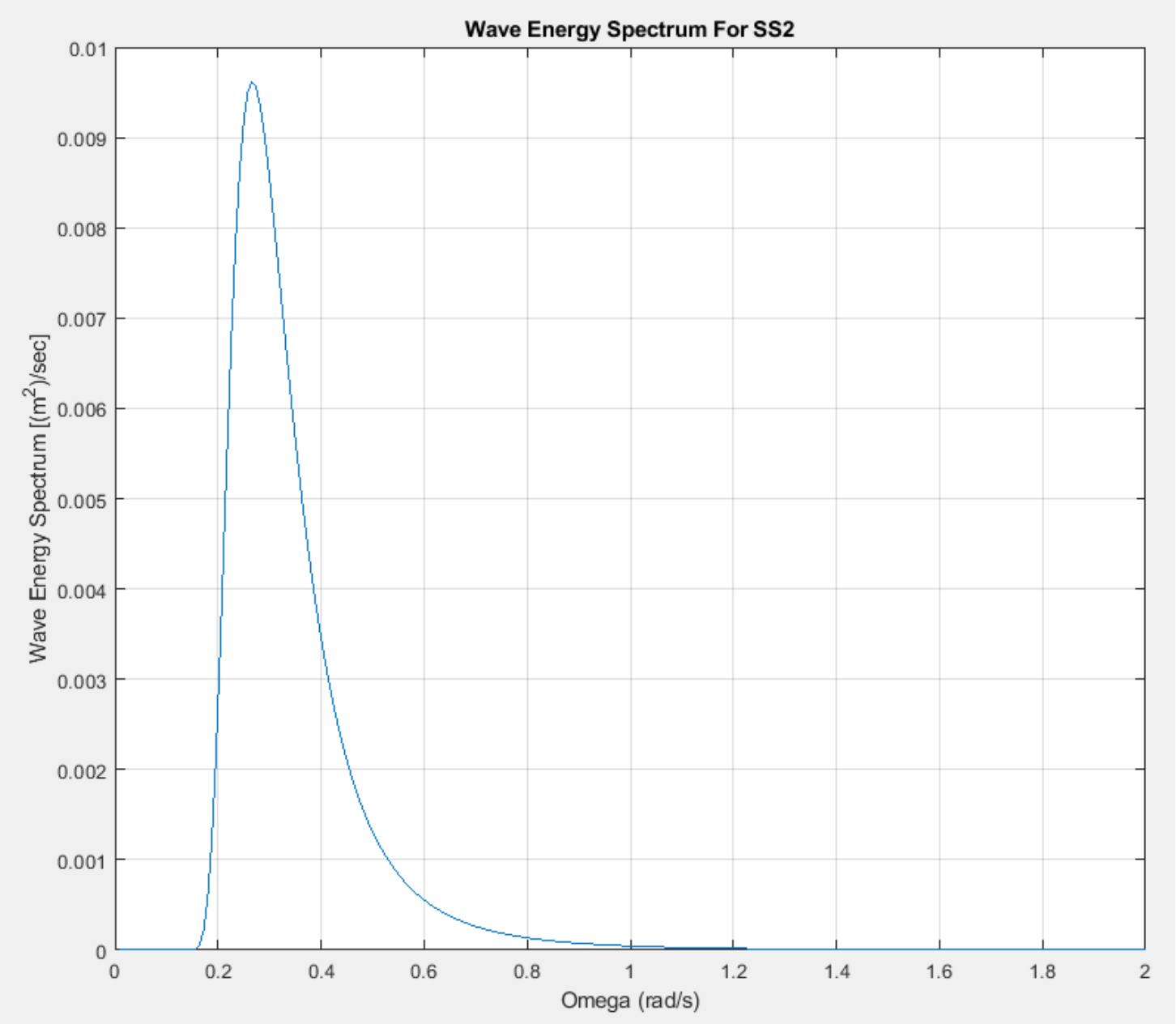

Figure 4.1: Wave Energy Spectrum for Sea State 2. 


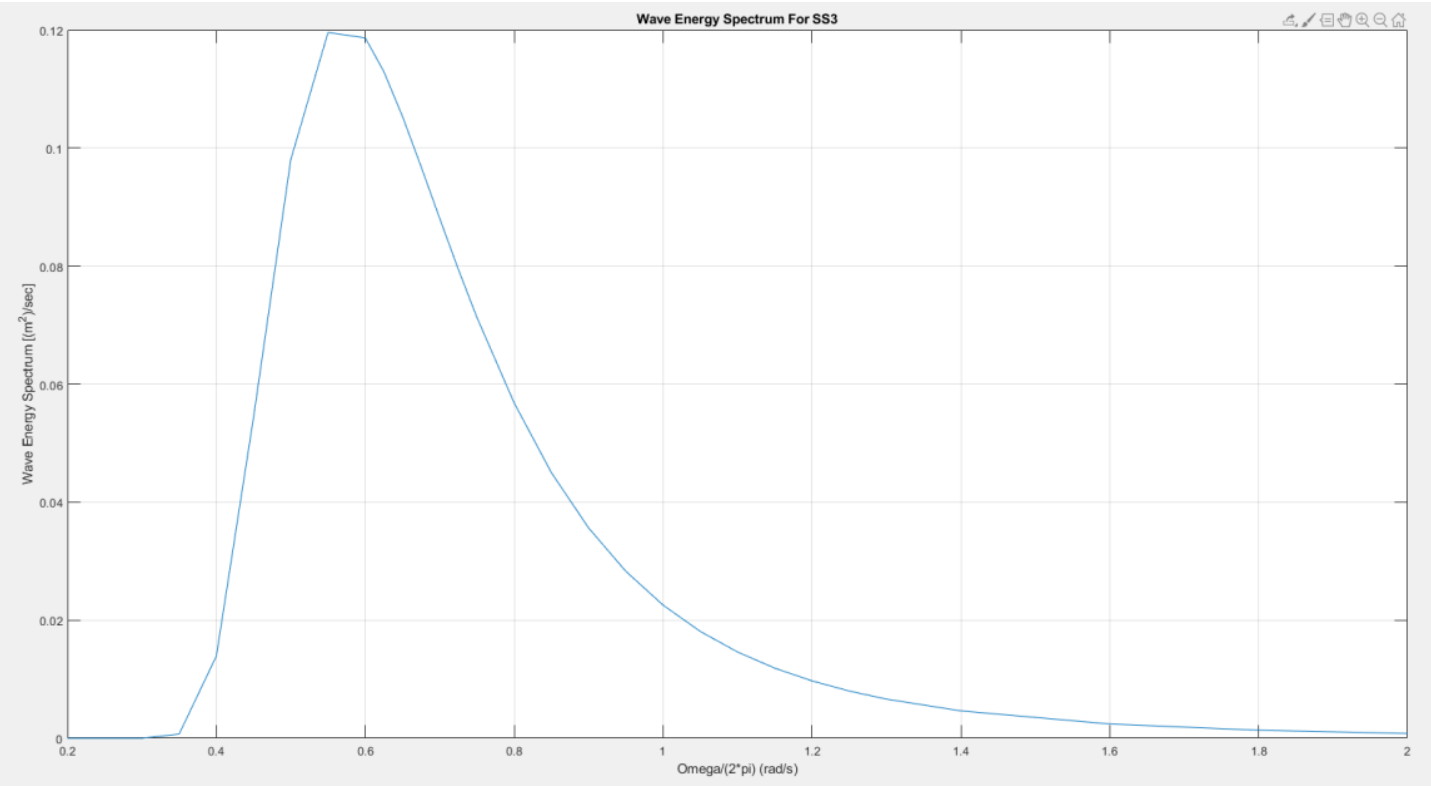

Figure 4.2: Wave Energy Spectrum for Sea State 3.

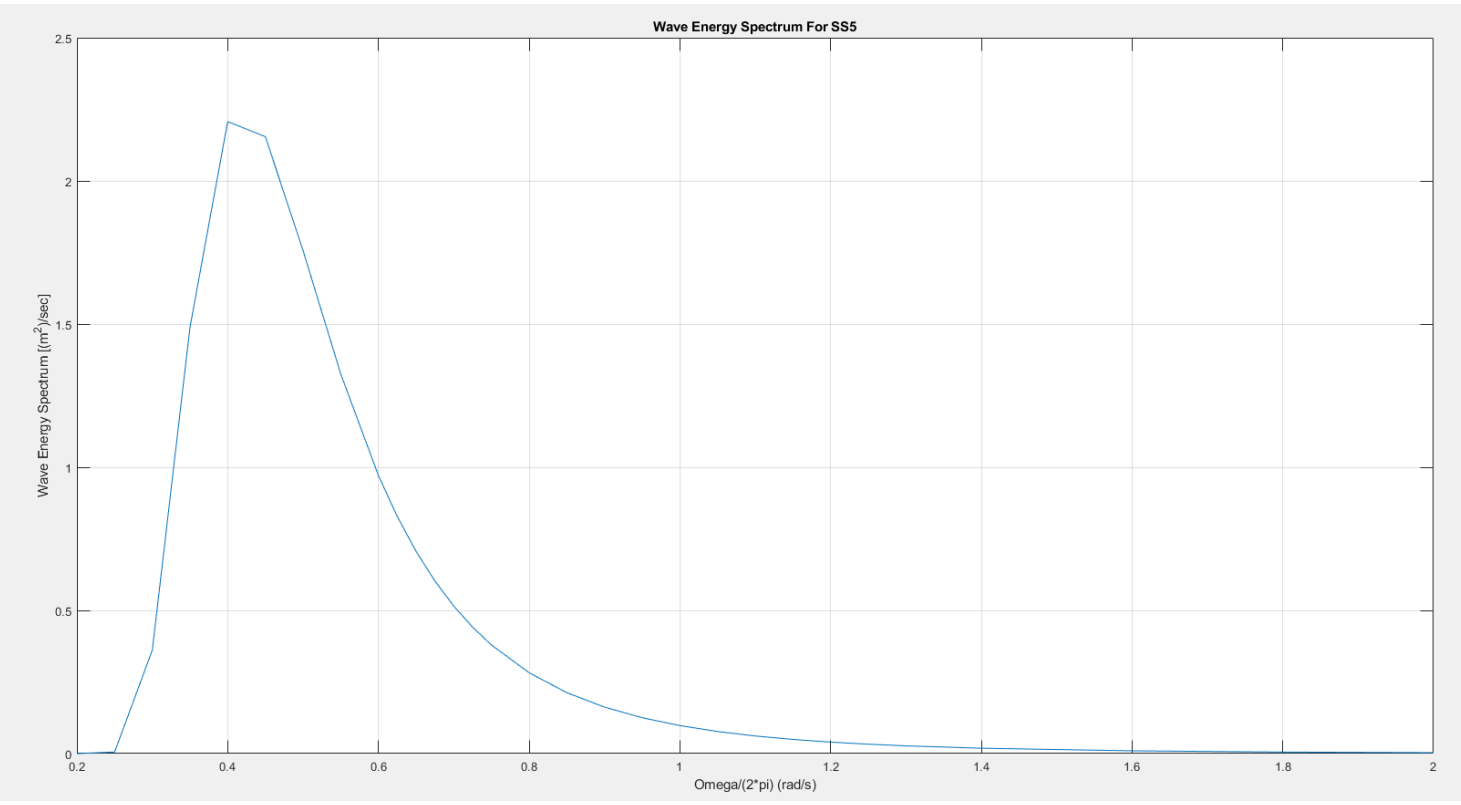

Figure 4.3: Wave Energy Spectrum for Sea State 5.

Between Sea State three and five, it is clearly visible that the maximum and total energy in sea state five is higher. Additionally, the modal frequency of Sea State five is lower than that of sea states two and three. The reduction in modal frequency, conversely increase in modal period, is due to the amount of time taken for a wave to develop to larger heights with more potential and kinetic energy. Smaller waves at lower sea states 
rise and fall quicker, and thus contain less energy than the larger, slower waves of higher sea states.

Another measure of value is the wave slope. The wave slope is the angle between a wave and the water surface [15]. A steep wave is an indicator of a high amplitude wave, thus a high potential energy wave [15]. The wave slope is defined in [15] as:

$$
\alpha_{0}=\frac{2 \pi}{\lambda} \zeta_{0}
$$

A wave slope energy spectrum is constructed using superposition and the Fourier Transform, in the same manner as the wave energy spectrum. The wave slope energy spectrum is also directly derived from the wave energy spectrum in equation 4.9, as per [10][12][17].

$$
S_{\alpha}(\omega)=\frac{\omega^{4}}{g^{2}} S_{\zeta}(\omega)
$$

The wave slope energy spectra for different Sea States are plotted in figure 4.4 to 4.6.

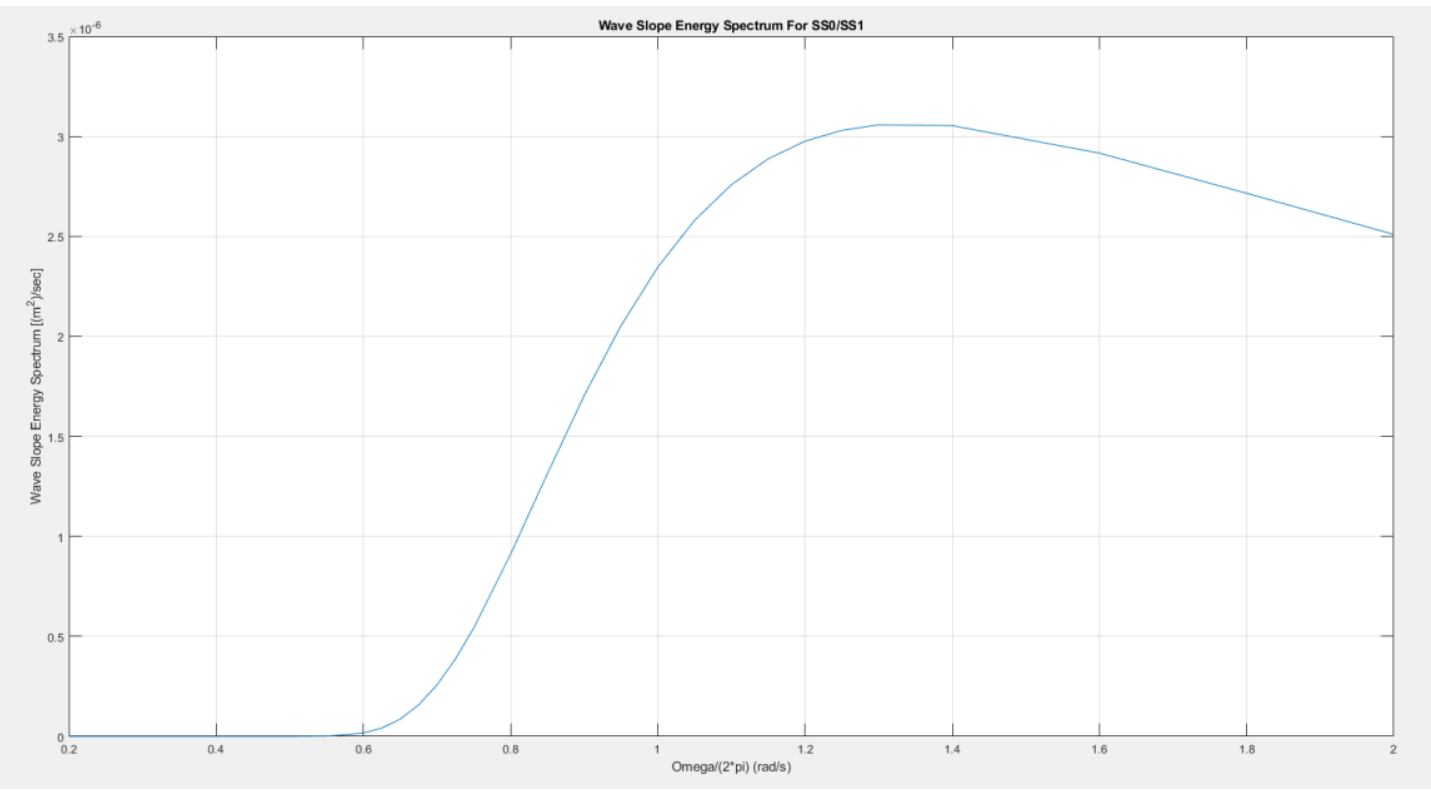

Figure 4.4: Wave slope energy spectrum for Sea States 0 and 1. 


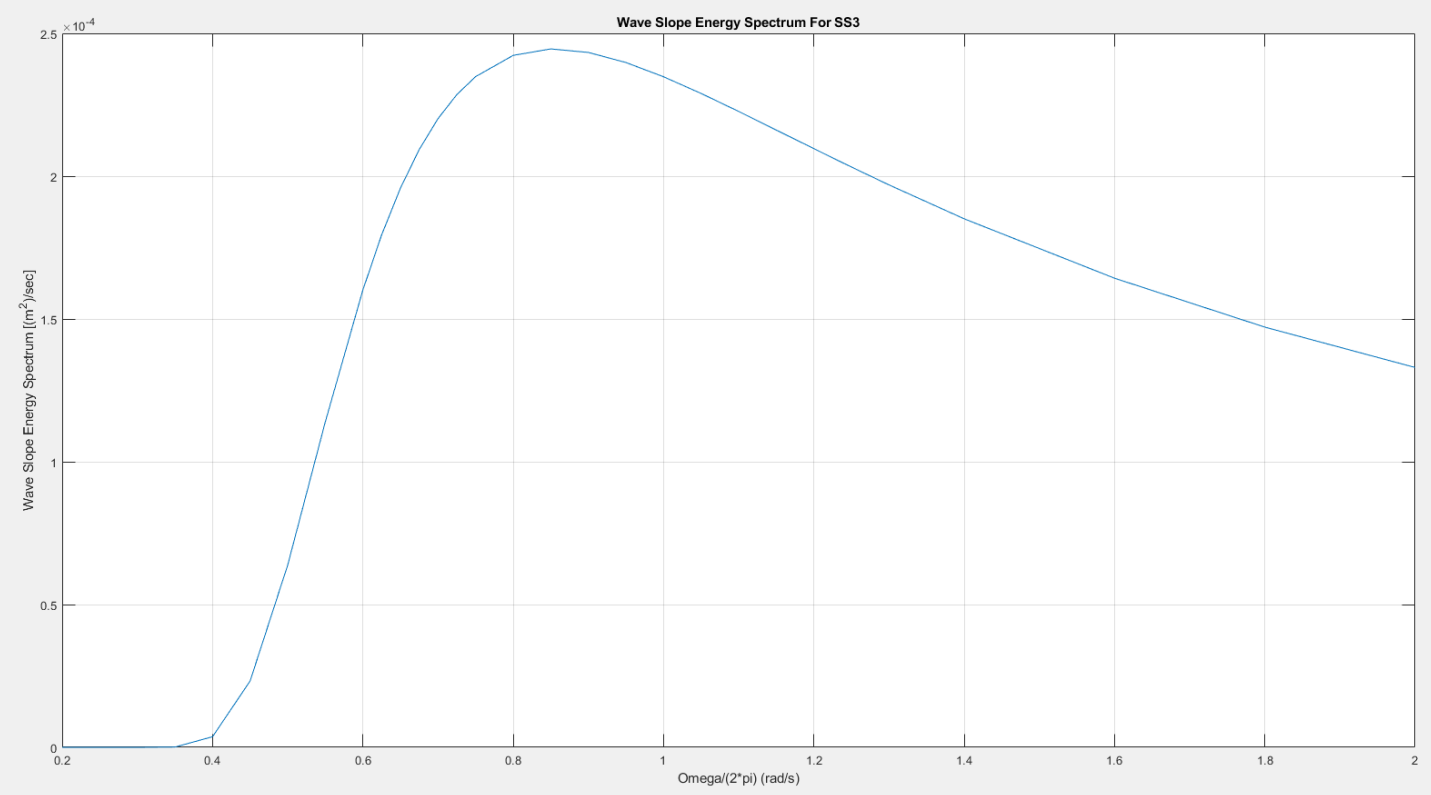

Figure 4.5: Wave slope energy spectrum for Sea State 3.

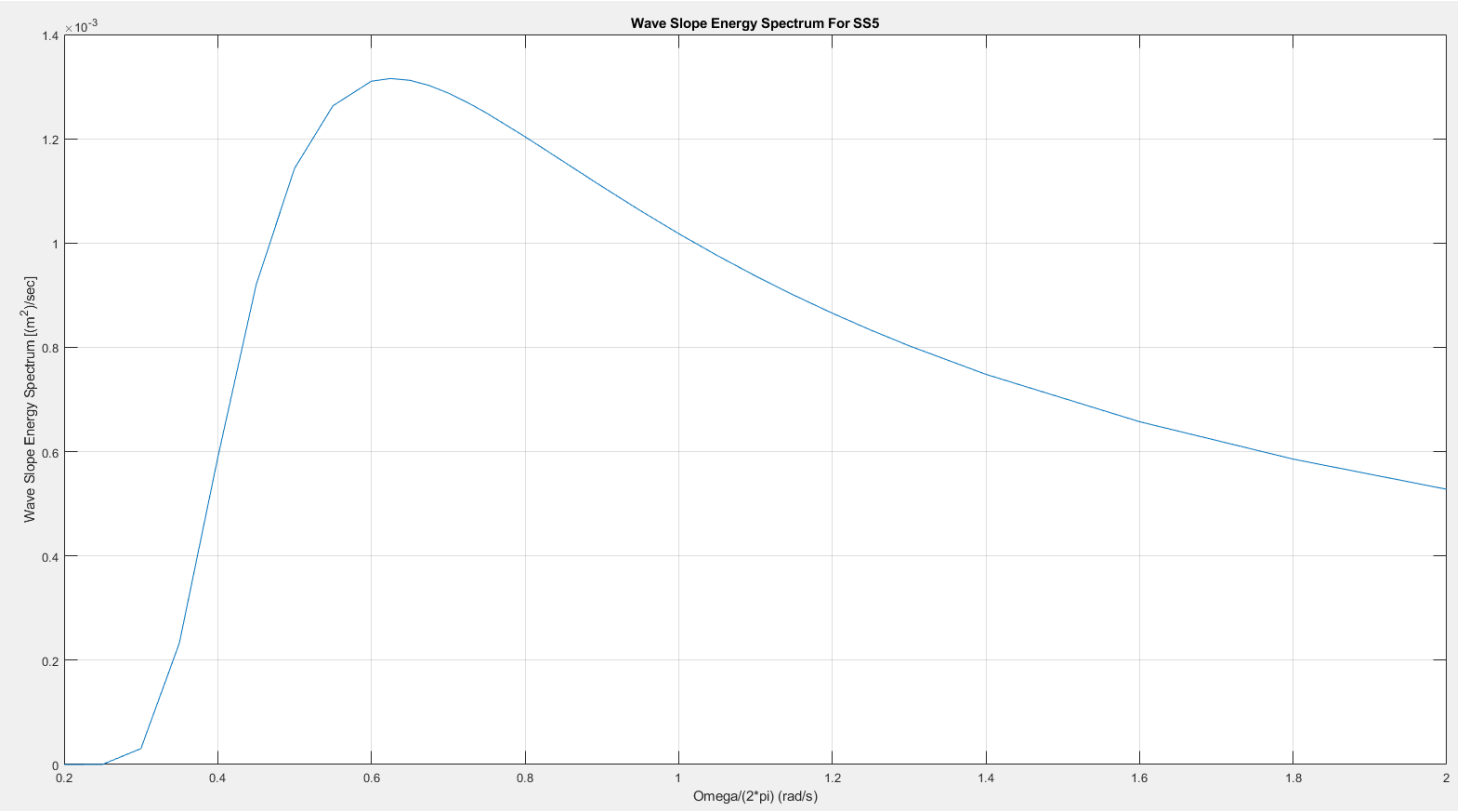

Figure 4.6: Wave slope energy spectrum for Sea State 5.

The concentration of energy at the peak moves to lower frequencies for increasing Sea State. This is because the spectra represent fully developed seas and do not account for wind in the development of waves. The wave energy spectra shape is like that of the Rayleigh Probability Density Function (PDF). Whereas the wave slope energy spectra 
have a much higher tail at the end of the distribution, like the Weibull PDF. However, determination of the order of the Weibull PDF is dependent on the Sea State.

The wave energy spectra formed from the Bretschneider equation are for longcrested waves [12][15]. This is not a completely accurate representation of the true sea surface, as the sea surface is a complex combination of waves generated from various origin positions [10]. This results in waves from different spatial angles combining in a constructive and destructive manner. The sea surface waves would then be described as a combination of long-crested and short-crested waves [10]. Short-crested waves can be described as a superposition of long-crested systems at various origin locations, with varying wave velocity direction [10]. This results in some energy dispersion among the frequencies in the wave energy spectrum. However, ship responses to higher wave frequencies are generally smaller due to rapid wave variation and lower wave energy content $[10][12][16]$. At exceptionally high wave frequencies, the dynamic motion effects experienced by the ship are almost negligible [16]. Thus, a single long-crested wave energy spectrum can be used to effectively model the sea state and ship motion responses for certain sea conditions and locations [10][20][21][24].

\subsection{The Wave Encounter Frequency}

The wave energy spectrum is defined in accordance with a global reference coordinate system. The origin of the system is an arbitrarily selected point, and the waves travel outward from the origin in a somewhat circular pattern, analogous to the propagation of waves from an ideal isotropic antenna. In relation to an entire seaway, a ship is small and will experience waves generally all travelling in a single angular direction, denoted $\chi$, with reference to the origin. This assumption simplifies the complex interaction of waves at different angular headings and is useful in generating simplified motion approximation models for simulated radar targets. In naval architecture, the complex interaction of waves at different heading angles is included in motion computations to determine ship stability and motion response characteristics crucial to operation [12]-[25].

The motion experienced by a naval vessel is a complex function of the ship dimensions, loading conditions, velocity, and directional heading [12]-[25]. In particular, 
the relationship between the velocity heading of a ship, with the angle of the sea waves relative to the ship determines the amount of wave energy which induces motion. In other terms, the frequency at which a naval vessel encounters waves in the seaway determines the amount of wave energy which couples to the ship to induce motion [12]-[25]. The encounter wave frequency is defined in equation 4.10, as per [12][16] and [17].

$$
\omega_{e} \triangleq \omega-\left(\frac{\omega^{2}}{g} U \times \cos \chi\right)
$$

In equation $4.10, \omega$ is the wave frequency, $g$ is the gravitational acceleration constant, $U$ is the ship velocity, and $\chi$ is the angle of the wave heading relative to the ship. The wave heading relative to the vessel is taken with reference to the ship stern, which is the rear of the ship [10][12][16]. Thus, for waves incident upon the stern $\chi$ is 0 degrees. For a range of $90^{\circ} \leq \chi \leq 270^{\circ}$, waves generally approach from the ship bow, and for the range $-90^{\circ} \leq \chi \leq 90^{\circ}$, waves approach from the ship stern [10]. When waves approach from the bow, the encounter frequency is always positive, as evident in observation of the cosine term in equation 4.10. Waves approaching from the stern are classified into three distinct scenarios dependent on the ship velocity, wave velocity and wave heading [10]. The second term of equation 4.10 is expanded in equation 4.11 [10].

$$
\alpha \triangleq \omega \times\left(\frac{\omega}{g} U \times \cos \chi\right)
$$

Here, $U \times \cos \chi$, represents the effective ship velocity in the direction of the waves [10]. The $\frac{\omega}{g}$ term is defined as the effective wave velocity [10][15]. Equation 4.10 is now rewritten in a simplified manner in equation 4.12 [10].

$$
\omega_{e}=\omega(1-\alpha)
$$

The three distinct scenarios of wave encounter frequency, as a function of $\alpha$, which itself is a function of $\omega, U$ and $\chi$, are outlined in Table 4.2. 
Table 4.2: Wave Encounter Frequency relations as a function of $\alpha$, from [10].

\begin{tabular}{|c|c|l|}
\hline $\boldsymbol{\alpha}$ & $\boldsymbol{\omega}_{\boldsymbol{e}}$ & \multicolumn{1}{c|}{ Description } \\
\hline$\alpha<1$ & $\omega_{e}>0$ & $\begin{array}{l}\text { The waves travel faster than the ship. Thus, waves from } \\
\text { the stern will overtake the ship over time. }\end{array}$ \\
\hline$\alpha=1$ & $\omega_{e}=0$ & $\begin{array}{l}\text { The ship remains in the same position relative to the } \\
\text { waves. }\end{array}$ \\
\hline$\alpha>1$ & $\omega_{e}<0$ & $\begin{array}{l}\text { The ship is moving faster than the waves. For waves } \\
\text { that are coming from the ship stern toward the bow, an } \\
\text { observer on the ship would see that the waves approach } \\
\text { from the bow and move toward the stern instead. }\end{array}$ \\
\hline
\end{tabular}

The negative encounter frequency only occurs in mathematical derivation and indicates that the wave motion is opposite to the original observation angle [10]. Thus, if a negative encounter frequency is generated this can be substituted by equation 4.13 as per [10].

$$
\chi_{e}=\chi+\pi
$$

The encounter frequency formulation is in essence a frequency mapping operation in which the wave frequency is converted into a frequency relative to a coordinate system centered at the ship, which moves with the ship [10][16][17]. For further details on the frequency mapping process the reader is referred to [10]. A visual representation of the encounter wave frequency is seen in Figure 4.7, from [10]. 

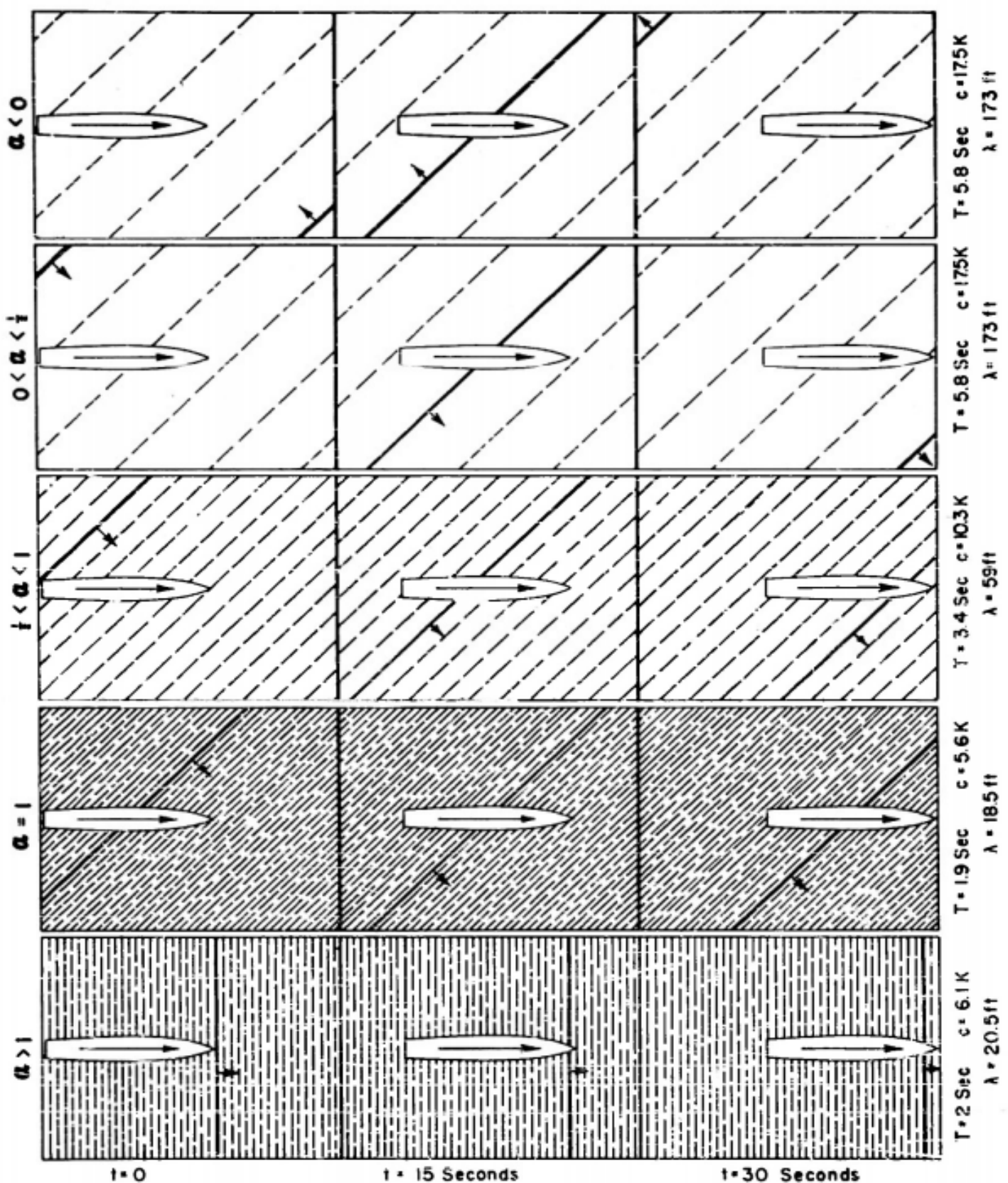

$V=7.9$ Knots

$L=430$ teet

Figure 4.7: A graphical representation of Equation 4.12, for various values of $\alpha$, from [10].

The wave energy spectrum will not be able to directly determine the amount of energy coupled to the ship to induce motion. The wave encounter energy spectrum must be generated through the frequency mapping described in equation 4.12 , to describe the 
wave energy with reference to the frame of the ship. The relationship between the frequency spacing on both energy spectra is described in [17] as:

$$
\delta \omega_{e}=\left(1-\left(\frac{2 \omega}{g} U * \cos \chi\right)\right) \delta \omega
$$

The area of the wave energy spectrum in a slice $\delta \omega$, must be equal to the area of the wave energy spectrum in the frequency mapped slice $\delta \omega_{e}$, as stated in equation 4.13 $[13][17]$. This is visually represented in figure 4.8 .

$$
S_{\zeta}\left(\omega_{e}\right) \delta \omega_{e}=S_{\zeta}(\omega) \delta \omega
$$

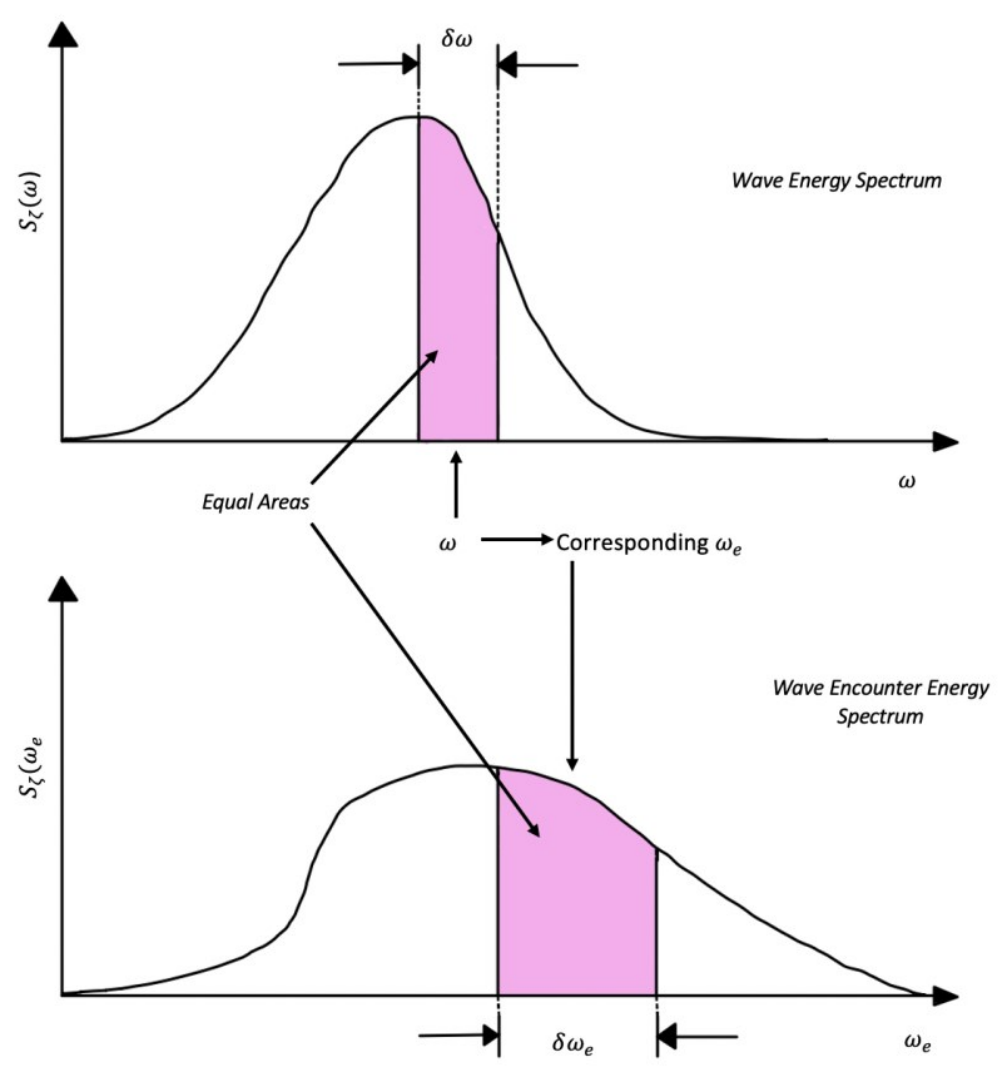

Figure 4.8: Mapping of the wave energy spectrum to the wave encounter energy spectrum, from [13][17].

Rearranging and substituting terms, the wave encounter energy spectrum can be found using equation 4.15 [13][17]. 


$$
S_{\zeta}\left(\omega_{e}\right)=S_{\zeta}(\omega)\left(\frac{g}{g-2 \omega U \cos \chi}\right)
$$

Similarly, the wave slope encounter energy spectrum is also determined as in equation 4.16 [13][17].

$$
S_{\alpha}\left(\omega_{e}\right)=S_{\alpha}(\omega)\left(\frac{g}{g-2 \omega U \cos \chi}\right)
$$

Figures 4.9 and 4.10 show the wave energy and wave slope energy spectra functions of encounter frequency. These figures are for Sea State 5 conditions, where the ship is travelling at a velocity of 5 Knots, and the wave heading is 225 degrees relative to the ship stern.

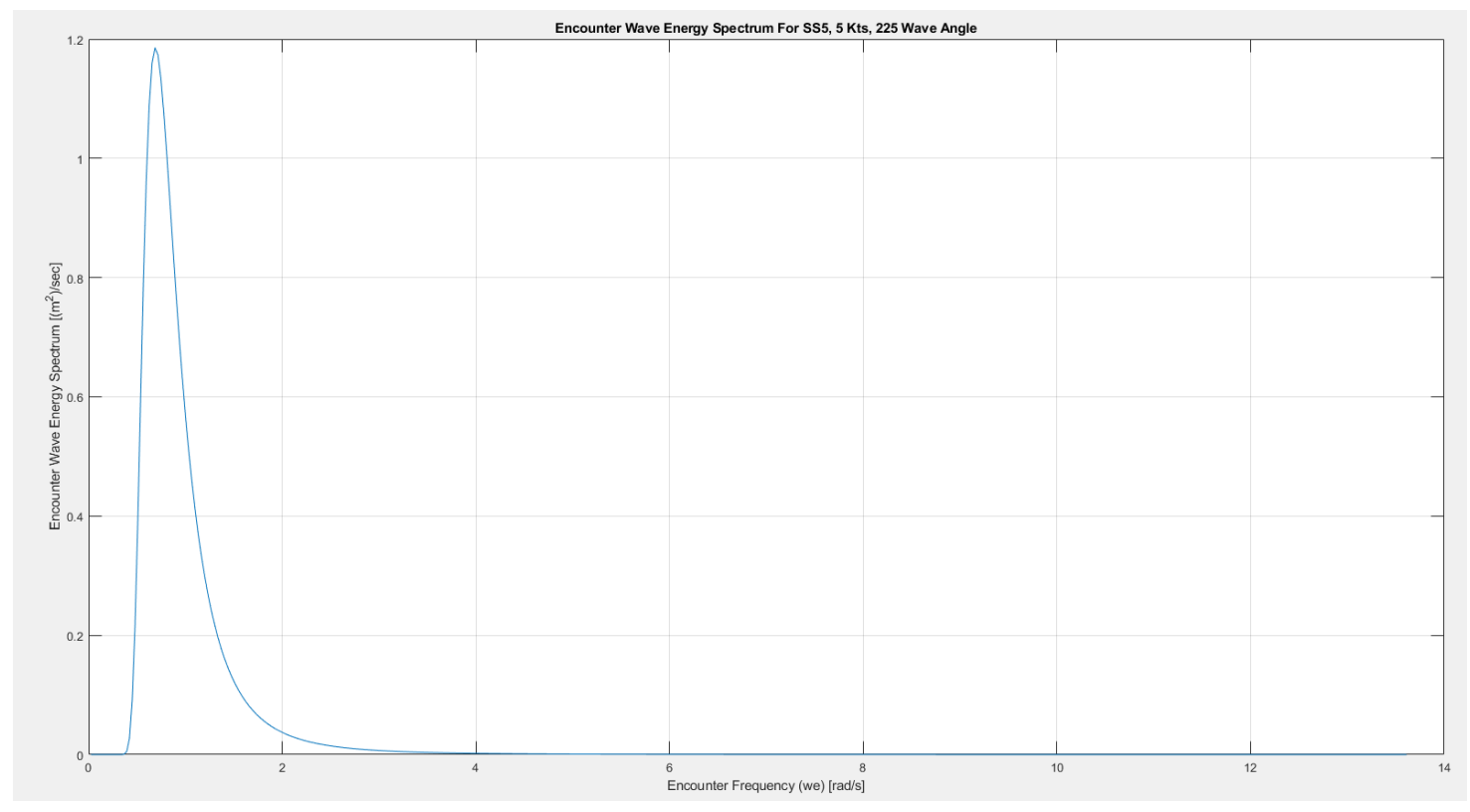

Figure 4.9: The Wave Energy Spectrum for Sea State 5, as a function of encounter frequency, for a ship velocity of $5 \mathrm{knots}$, and a wave heading (relative to the ship stern) of 225 degrees. 


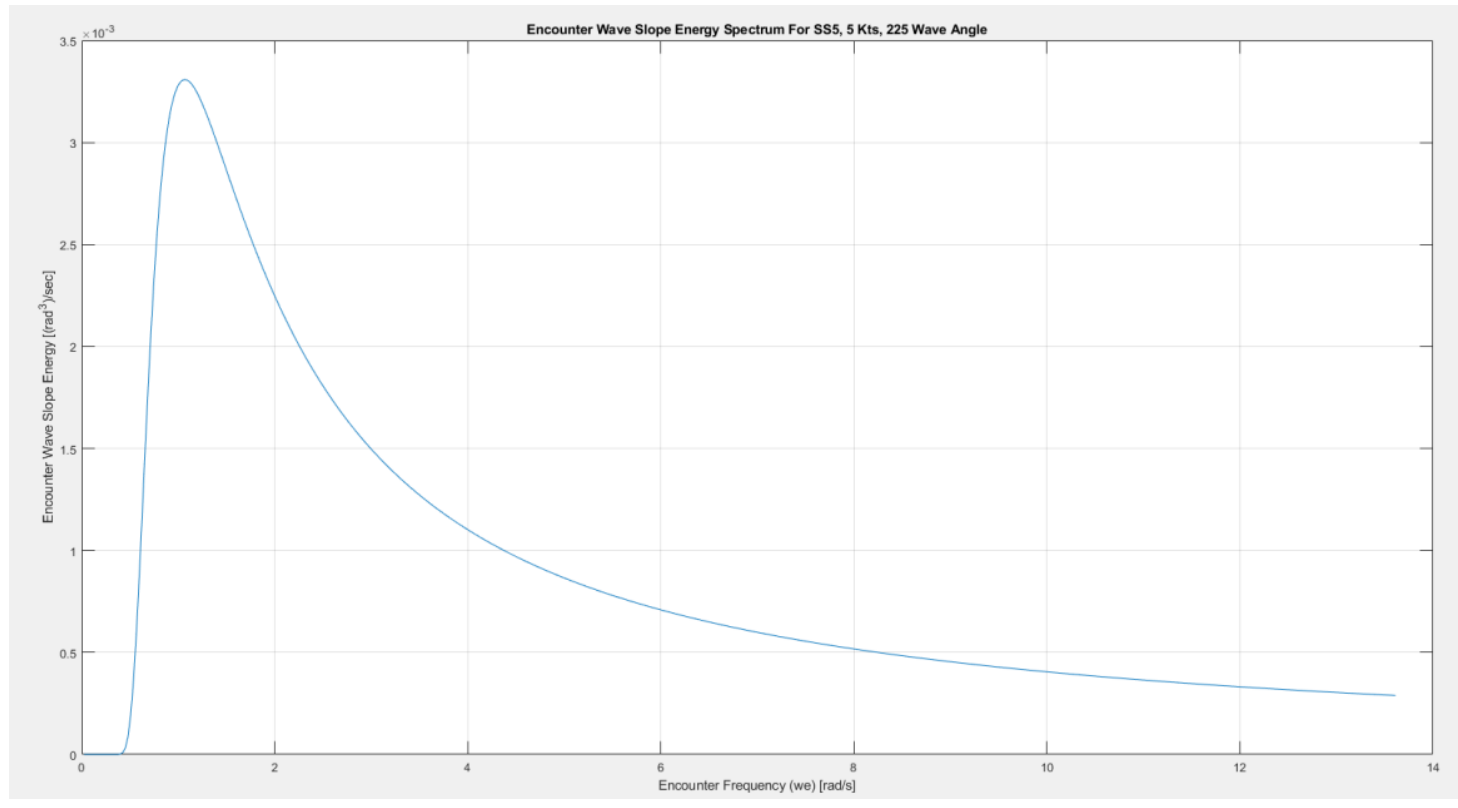

Figure 4.10: The Wave Slope Energy Spectrum for Sea State 5, as a function of encounter frequency, for a ship velocity of $5 \mathrm{knots}$, and a wave heading (relative to the ship stern) of 225 degrees.

\subsection{Ship Dynamics, Transfer Functions \& Response Amplitude Operators (RAOs)}

Ship motion is categorized into six degrees of freedom [12][13][16][25]. There are three rectilinear motions: surge, sway, and heave, and three angular motions: roll, pitch, and yaw [1]-[25]. These are illustrated in Figure 4.11.

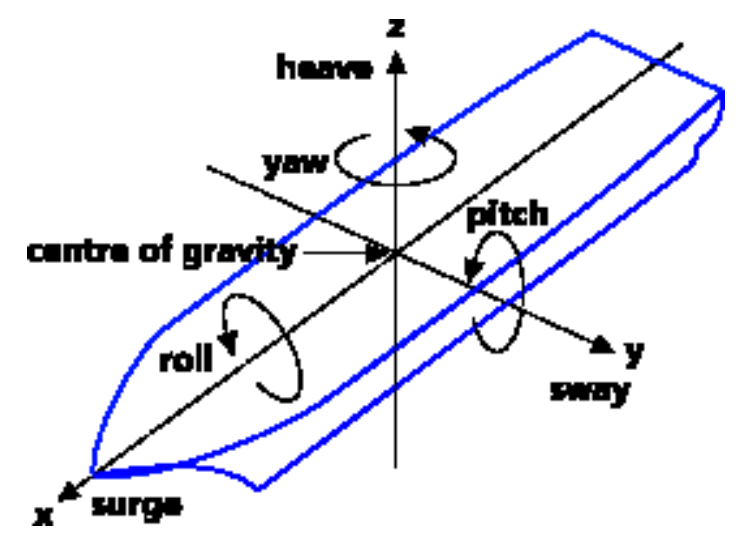

Figure 4.11: The six degrees of movement experienced by a ship, from [26]. 
Rectilinear motion estimations are derived from the wave energy spectrum, and angular motion estimations are derived from the wave slope energy spectra $[10][12][16][17]$.

Ship motion is governed by the laws of dynamics, specifically Newton's second law [16]. This states that for any force which induces motion on a ship there is balancing counterforce, Buoyant force, which aims to restore the ship to its original position [16]. The force acting upon the ship is a result of wave interaction with the ship hull. Equation 4.17 describes the wave force which generates heave motion expressed in the form of Newton's second law, as obtained from [16].

$$
F_{0} \sin \left(\omega_{e} t\right)=m \ddot{x}+b \dot{x}+c x
$$

Buoyant force is described by a sinusoidal equation with amplitude relative to wave height, $F_{0}$, and frequency equal to the encounter frequency of the wave, $\omega_{e}[16]$. The coefficients on the right side of the equation represent mass, $m$, damping coefficient, $b$, and stiffness of the hull coefficient, $c$ [16]. Solving equation 4.17 for the variable $x$ will produce an equation describing the position of the ship [16]. The first and second derivatives of the position will provide the velocity and acceleration of the ship in response to the excitation force [16]. The form of equation 4.17 describes the harmonic motion of the ship. Through linear systems analysis parameters such as the natural frequency, damping factor and tuning factor of ship motion are obtained [10][16]. The natural frequency of the response indicates the frequency at which the system oscillates when disturbed from equilibrium. The tuning factor is the ratio of the encounter frequency to the natural frequency [16]. The motion response amplitude and phase are obtained as shown in equations 4.18 and 4.19 , based on [16].

$$
\begin{gathered}
X_{0}=\frac{\frac{F_{o}}{c}}{\sqrt{\left(1-\Lambda^{2}\right)^{2}+\left(2 \eta \Lambda^{2}\right)^{2}}} \\
\tan \phi=\frac{2 \eta \Lambda}{1-\Lambda^{2}}
\end{gathered}
$$

Where $\eta$ is the damping factor, and $\Lambda$ is the tuning factor [16]. For each degree of freedom of motion there is a corresponding natural frequency for the motion oscillation [16]. 
Motion equation coefficients are not as simple as previously described. Ship mass is influenced by the added mass during motion, which includes the amount of water accelerated by the ship as it undergoes motion [16]. The damping coefficient is a complex function of hydrodynamics and is dependent on the viscosity and density of the water [16]. The stiffness coefficient is dependent on hull shape, material, and other naval architectural design parameters [16]. The coefficients are generally found using experiments which measure ship motion responses to waves in controlled environments such as a large wave tank used in the U.S. Naval Research Center [16]. Determining motion equations for each of the six degrees of movement in time domain solutions can be increasingly exhaustive, and unique to specific ships based on their dimensions.

Developing ship transfer functions based on the motion equations is a more common method of determining ship motion characteristics [10]-[25]. The prediction of ship motion responses as a function of excitation amplitude and frequency is seen in equation 4.18. The denominator of this equation is referred to as the Dynamic Magnification Factor (DMF) [10]-[15]. Using the DMF the transfer function of the ship response, normalized to wave amplitude, is plotted as a function of frequency; specifically, the tuning factor ratio of encounter frequency normalized by natural frequency [16]. Figure 4.12 below shows a sample ship transfer function for roll motion, as taken from [16]. 


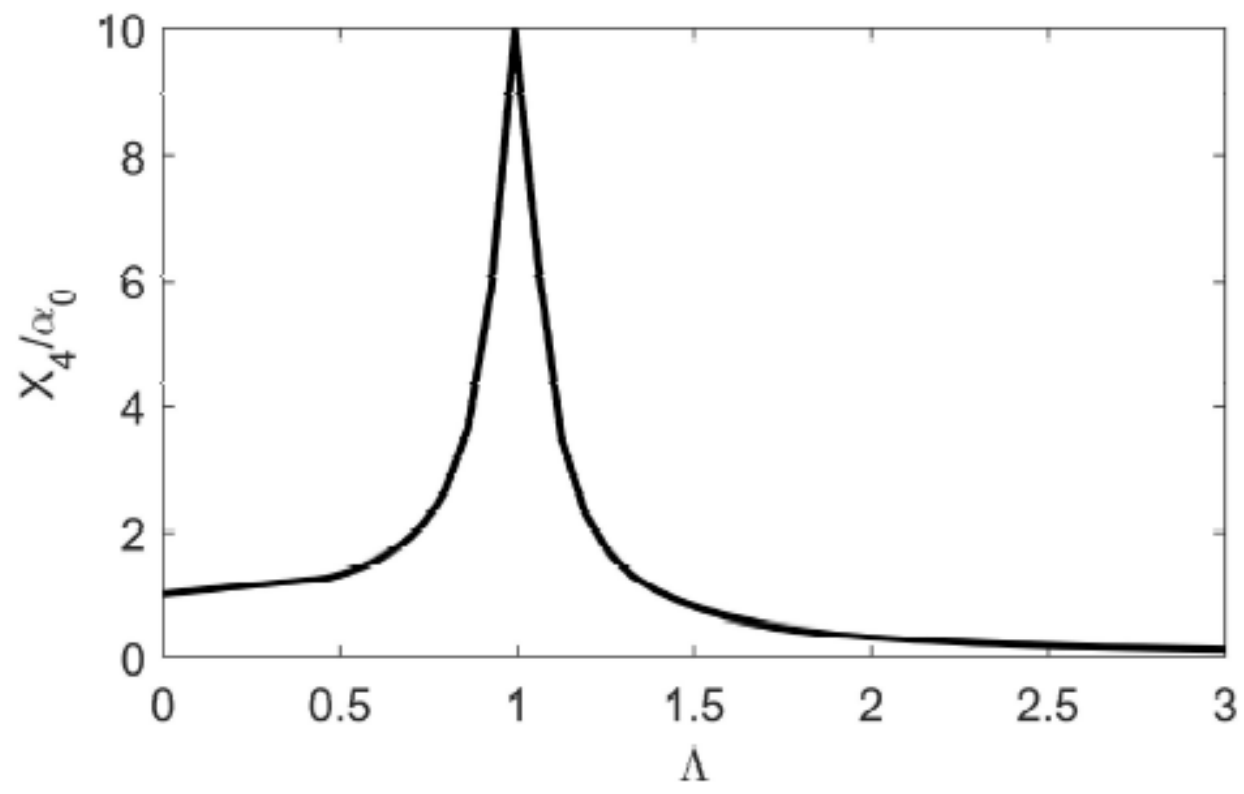

Figure 4.12: A sample transfer function for ship roll, taken from [16].

Observed in figure 4.12, the peak motion response occurs when the tuning factor is a value of one. This corresponds to when the encounter frequency is equal to the natural frequency for the specific degree of motion [16]. The ship transfer function can be obtained computationally, with known hydrodynamic coefficients [10]-[25]. An efficient method of computing transfer functions is developed using Strip Theory, in which the ship is segmented into two-dimensional strips, and the motion response of each strip is summed to determine the total response [10]-[25]. The transfer function can also be obtained experimentally using various methods such as the Knife Edge Method, the Lamboley Method, the Bifilar Suspension Method, and other experimental methods [16]. Determination of ship transfer functions is a vast area of research and the reader is referenced to [10]-[25] for further detail on methods. Ship transfer functions have both magnitude and phase responses [10][16]. The magnitude response determines the amount of motion, normalized by the wave amplitude [16]. The phase response is unique as it relates to the synchronization of the ship motion to the encountered waves. For a phase response where the transfer function phase matches the phase response of the encountered waves, the ship motion is maximized; conversely, when the phase response is not synchronized to the encounter wave frequency, the motion is highly damped [10][16]. 
As previously mentioned, waves are not generally described by amplitude, but by energy spectra. The ship transfer function, which is a function of wave amplitude, cannot be directly used with wave energy spectra to determine motion responses for the ship. Rather, the square of the magnitude of the transfer function is used [10][16][17]. This is also referred to as the Response Amplitude Operator (RAO) [10]-[25]. RAOs are used to predict ship motion responses in irregular waves, which are represented by the wave energy spectra, and more accurately the wave encounter energy spectra for the ship $[10][16][17]$. RAOs are derived for rectilinear motion as functions of the wave encounter frequency, and for angular motion as functions of the wave slope encounter frequency $[12][16][17]$.

\subsection{Ship Motion Estimation}

Computing estimation of ship motion has been related to the application of electronic filtering by St. Denis and Pierson [17]. An electronic filter, symbolized by a 'black box', receives an input, and produces an output assuming linear processing [17]. The transfer function of ship motion represents the 'black box' filter, and the waves are the input with the ship motion response as the output [17]. The ship transfer function is dependent on wave amplitude; however, waves are described by the energy spectral representation. The square of the transfer function is defined as the RAO, which is the energy representation of the ship motion transfer function [10][16][17]. Multiplying the wave energy spectrum by the RAO produces an energy spectrum at the output, defined as the ship motion energy spectrum [10][16][17]. The ship motion energy spectrum does not provide an exact prediction of the ship response but is indicative of the amount of kinetic energy coupled to the ship from interaction with waves. This is described in equations 4.20 and 4.21 as per [10][12][13][16] and [17].

$$
\begin{gathered}
S_{x \#}=S_{\zeta}\left(\omega_{e}\right) R A O_{x \#} \\
S_{x \#}=S_{\alpha}\left(\omega_{e}\right) R A O_{x \#}
\end{gathered}
$$

Where $S_{x \#}$ represents the motion energy spectrum and the number sign indicates which of the six degrees of freedom the spectrum represents [10][17]. Similarly, the RAO is also dependent on the degree of motion, and the ship wave encounter energy spectrum 
is dependent on ship velocity, and relative wave angle [10][17]. This is shown in Figure 4.13, as taken from [17]. The motion parameters determined are per unit responses of the ship motion interaction with waves [10][17][25]. These responses must be multiplied by the significant wave height for a specific sea state to obtain the actual motion response in units of feet, meters, degrees, or radians [10][17][25].

Determination of the spectral moments of the ship motion energy spectrum provides useful results such as the variance and RMS of the motion, the mean motion period, peak motion period and zero-crossing period, as done for the wave energy spectrum [15][17]. The location of the peak motion energy provides the information on the frequency of the motion parameter when represented as sinusoidal motion [10][17]. The representation of motion using sinusoidal functions is consistent with the restoring forces acting upon the ship to maintain stability. 

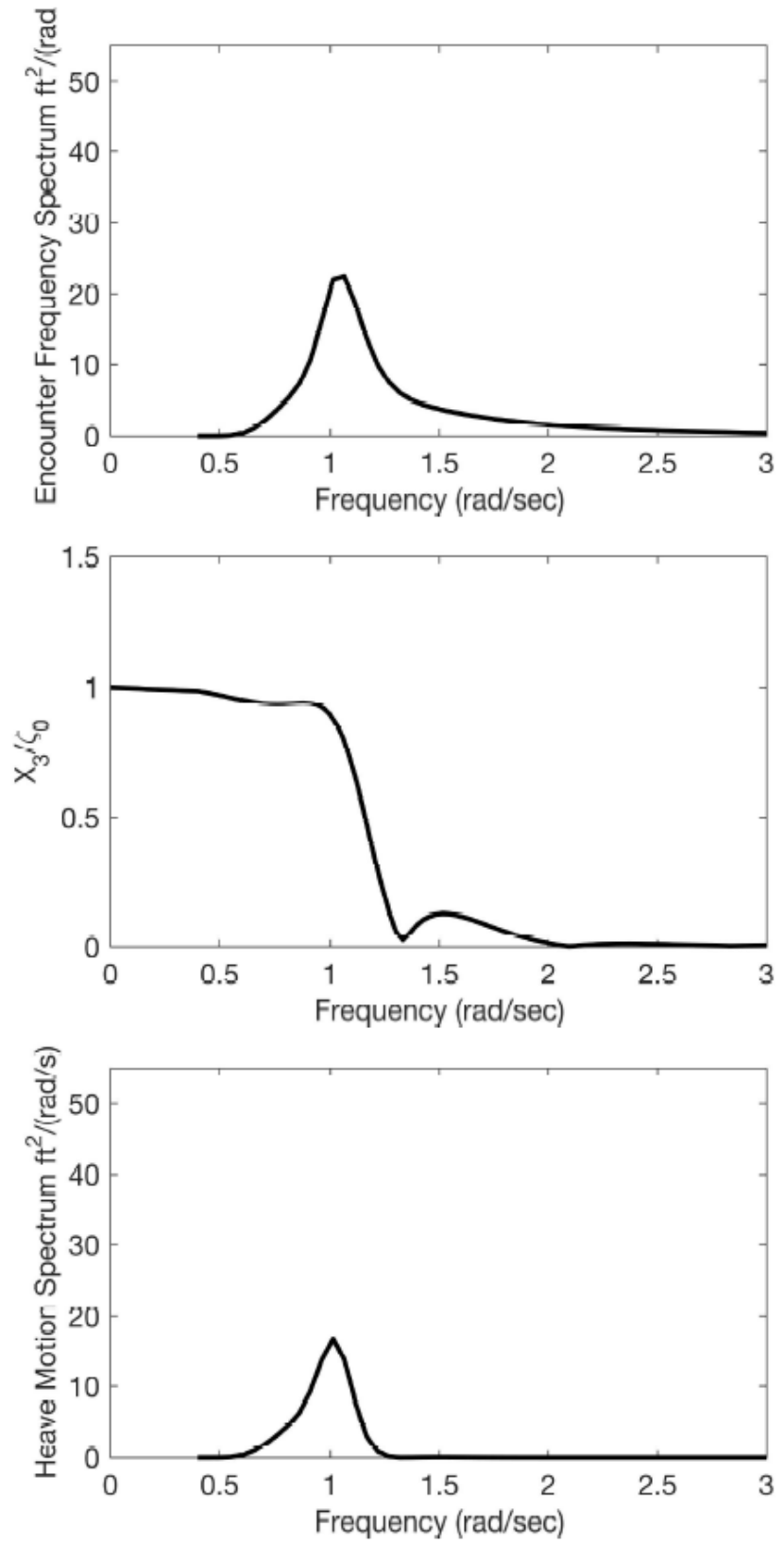

Figure 4.13: A representation of the filter analogy to obtain a ship motion energy spectrum [17]. 
The process of determining the ship motion responses has been described in a categorized manner in [10][17] and [25] and is briefly outlined below.

1. Build a wave energy spectrum and a wave slope energy spectrum. This can be done using the Bretschneider equation or other wave energy spectrum equations.

2. Perform a frequency transformation of the wave energy, and wave slope energy spectra to functions of encounter frequency.

3. Determine the ship RAOs by taking the square of the ship transfer functions for all six degrees of movement.

4. Find the ship motion energy spectrum by multiplying the wave energy, or wave slope energy spectra by the corresponding RAOs.

5. Compute the spectral moments of the motion energy spectrum to determine RMS motion. The RMS motion parameter multiplied by significant wave height for the specific sea state provides the actual motion response in specified units.

6. Determine the modal frequency response of the ship motion by locating the peak of the motion response.

As previously mentioned, the wave elevation histogram data followed a Rayleigh distribution. This allows for the use of Rayleigh statistics in the description of the wave energy spectrum since the energy spectrum is dependent on the linear superposition and Fourier analysis of wave amplitude data [15][17]. The Rayleigh statistics have also been applied to the ship motion energy spectrum by many Naval Architects [10][17]. This proves useful in determining probabilities of extreme ship motion in given sea conditions. The RMS motion parameter is the value of motion which is exceeded approximately $60 \%$ of the time [10][17]. To compute the extreme motion possibilities a probability of exceedance which is lower than the RMS probability is required [17]. The motion responses derived are not exact representations of the ship motion but provide useful estimates of the range of motion undergone by a ship in varying sea conditions. These estimates are utilized in simplified ship motion equation formulations to simulate ship motion over a period in which the sea state is assumed to be relatively constant. 


\subsection{Ship Equations of Motion (EOM) Formulation}

The equations of motion generated for simulating radar targets are simplified in comparison to the ones developed for naval architecture. This is to effectively model the motion of radar scatterer locations on the ship target without concern for stability conditions of the ship as it undergoes motion. There are many variations of the ship motion simulations based on assumptions of motion characteristics and motion amplitudes, these can be found in [1]-[9],[24]-[30]. The motion formulation utilized in this application is a hybridization of the methods used in [1],[3] and [7]. These equations of motion can be applied to any ship model for any sea state without loss of generality.

Two coordinate systems are defined to develop an effective motion model. The first is a non-accelerating inertial coordinate system with the origin located at the center

of gravity of the ship [3]. The positive x-axis is toward the starboard side of the ship, the positive y-axis points to the ship bow, and the positive z-axis points upwards [3]. This coordinate system travels with the ship; however, the axes are fixed in place as the ship undergoes angular motions [3]. The second coordinate system is the ship reference system. The origin is located at the center of gravity, and the axes are oriented the same as in the inertial coordinate system. However, the axes move with the ship as the ship undergoes angular motions [3]. An illustration of these coordinate systems is shown in Figure 4.14 from [3]. 


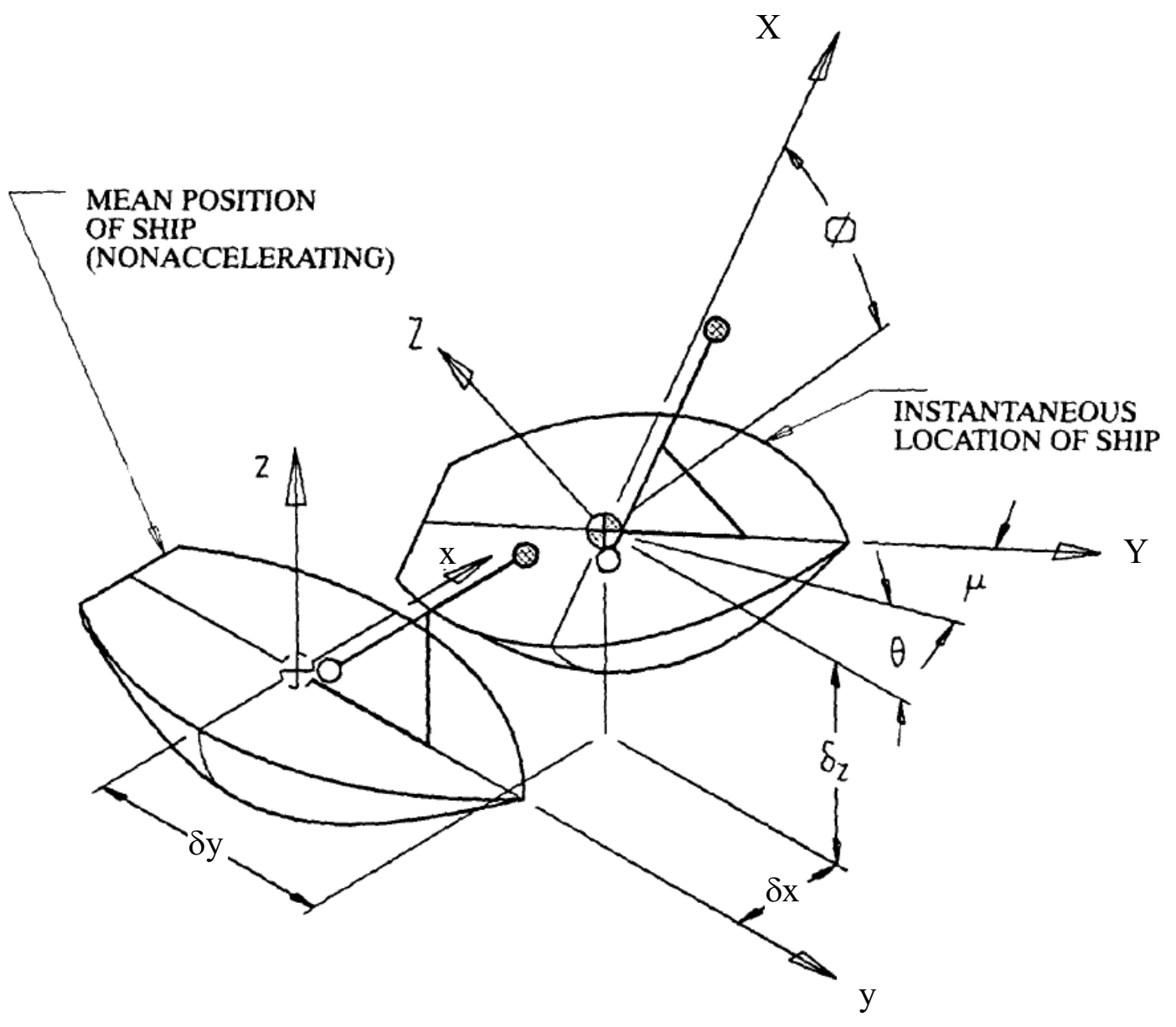

Figure 4.14: The inertial and reference coordinate systems of the ship, as adapted from [3].

The axes of the inertial reference system are labelled with the lowercase ' $x, y, z$ ', and the ship reference system axes are denoted with capital ' $\mathrm{X}, \mathrm{Y}, \mathrm{Z}$ '.

It is assumed that rectilinear motions can be represented in the inertial reference system, and the angular motions represented in the ship reference system [3]. This assumption allows for greater simplification of the rectilinear motions. A transformation of coordinates is performed on angular motions to translate results from the ship reference system to the inertial coordinate system [1][3].

The balancing counterforce of buoyancy enables the assumption of sinusoidal motion patterns for all six degrees of movement on the ship [1]-[9][24]-[30]. There are two possible choices for the sinusoidal motion experienced, the use of a sine function implies that at the initial time of 0 seconds the ship is stationary. A cosine function 
implies that at the initial time the ship is at peak motion along all six degrees of movement. The application of this motion model is to develop realistic ship motion to create radar range profile signatures, which would be impacted by the Doppler returns from scattering locations on the ship. Thus, the cosine function is used to represent the motion, since the ship is experiencing maximum motion at the initial time. However, use of either of the trigonometric functions in the formulation will produce the same motion effect. The equations of motion for each of six degrees of movement are taken from [3], shown in Table 4.3 below.

Table 4.3: Motion equations for each of the six degrees of movement [3].

\begin{tabular}{|l|c|l|r|}
\hline \multicolumn{2}{|l|}{ Rectilinear Motions } & \multicolumn{2}{l|}{ Angular Motions } \\
\hline Sway & $\delta_{x}(t)=\delta_{x} \cos \left(\omega_{x} t\right)$ & Roll & $\phi(t)=\phi_{0} \cos \left(\omega_{\phi} t\right)$ \\
\hline Surge & $\delta_{y}(t)=\delta_{y} \cos \left(\omega_{y} t\right)$ & Pitch & $\theta(t)=\theta_{0} \cos \left(\omega_{\theta} t\right)$ \\
\hline Heave & $\delta_{z}(t)=\delta_{z} \cos \left(\omega_{z} t\right)$ & Yaw & $\mu(t)=\mu_{0} \cos \left(\omega_{\mu} t\right)$ \\
\hline
\end{tabular}

The amplitudes of the motion equations are determined from the RMS motion value taken from the ship motion energy spectrum, multiplied by the significant wave height of the sea state. The motion frequencies are determined from the frequency location of the peak motion response in the ship motion energy spectrum.

In the application of equations to large ships the approximation is that the surge, and yaw motion equations are minimal, and can be set to zero [1]-[9][25]-[30]. Surge is along the $y$-axis and is in the same direction as ship forward motion. The forward motion of the ship is much greater than the surge motion validating the approximation of the motion to zero. The yaw motion for large ships is negligible due to controlled rudder systems, and the ship design parameters [1][3][25].

The equations in Table 4.3 describe the position of locations on the ship for each of the degrees of motion. The velocities and acceleration equations are also derived by taking the first and second derivatives, respectively, of the motion equations. To determine the motion of radar scattering locations on the ship, the order of motion equation application is vital [1][3]. The rectilinear motions are applied first in the inertial 
coordinate system. This does not result in a change in location of the scatterer locations as the inertial system moves with the ship; however, each radar scatterer location does have a corresponding velocity and acceleration along the $\hat{x}$ and $\hat{z}$ directions. The order of angular motion equation application is: Roll, Pitch then Yaw [1]. The exact order can be interchanged arbitrarily in the initial formulation, but in application of the equations the order must be kept consistent. This is to avoid accumulation of error in the noncommutative nature of matrix multiplications operations used to compute motion $[1][3][7]$. The angular motions translate fixed scatterer locations on the ship to the inertial reference system using rotation matrices developed in [1] and [7]. The rotation matrices for all three angular motions are listed in Table 4.4 [1], noting that in this application, the yaw rotation matrix will reduce to the identity diagonal matrix.

Table 4.4: Rotation matrices for the three angular motions, based on [1].

\begin{tabular}{|c|c|}
\hline $\begin{array}{c}\text { Roll } \\
R_{\text {roll }}\end{array}$ & {$\left[\begin{array}{ccc}1 & 0 & 0 \\
0 & \cos \phi(t) & -\sin \phi(t) \\
0 & \sin \phi(t) & \cos \phi(t)\end{array}\right]$} \\
\hline $\begin{array}{c}\text { Pitch } \\
R_{\text {pitch }}\end{array}$ & {$\left[\begin{array}{ccc}\cos \theta(t) & 0 & \sin \theta(t) \\
0 & 1 & 0 \\
-\sin \theta(t) & 0 & \cos \theta(t)\end{array}\right]$} \\
\hline Yaw & {$\left[\begin{array}{ccc}\cos \mu(t) & -\sin \mu(t) & 0 \\
\sin \mu(t) & \cos \mu(t) & 0 \\
0 & 0 & 1\end{array}\right]$} \\
\hline
\end{tabular}

The initial location of a radar scattering point on the ship is $\left(X_{0}, Y_{0}, Z_{0}\right)$ in the ship reference system. Following the application of the motion equations, the location of the scatterer in the inertial coordinate system is defined by $\left(x_{s}, y_{s}, z_{s}\right)$. Equation 4.22, from [7], describes the motion equation utilized. The yaw rotation matrix has been excluded as it is negligible.

$$
\left[\begin{array}{l}
x_{s}(t) \\
y_{s}(t) \\
z_{S}(t)
\end{array}\right]=R_{\text {roll }} \cdot R_{\text {pitch }} \cdot\left[\begin{array}{c}
X_{0} \\
Y_{0} \\
Z_{0}
\end{array}\right]+\left[\begin{array}{c}
0 \\
\delta_{y}(t) \\
\delta_{z}(t)
\end{array}\right]
$$


The multiplication of the roll and pitch rotation matrices is expanded and shown in equation 4.23 .

$$
R=R_{\text {roll }} \cdot R_{\text {pitch }}=\left[\begin{array}{ccc}
\cos \theta(t) & 0 & \sin \theta(t) \\
\cos \phi(t) \cdot \sin \theta(t) & \cos \phi(t) & -\sin \phi(t) \cdot \cos \theta(t) \\
\cos \phi(t) \cdot-\sin \theta(t) & \sin \phi(t) & \cos \theta(t)
\end{array}\right]
$$

Equation 4.23 can be used iteratively for all radar scatterer locations on the ship to simulate contiguous motion of the entire ship model. The absolute scatterer locations are known in the ship reference system. The positions of scatterers in the inertial reference systems are functions of time, allowing the use of a single target model to simulate motion over an extended period without repeated implementation of coordinate transformations. Taking the derivative, with respect to time, of the position equations provides the velocity equations for motion of scatterers along the principle axes [1]. Thus, the position and velocity of all scattering locations on the target are computed using the equations of motion developed above.

\subsection{Conclusion}

This chapter showed how the ship motion parameters are computed. The initial step begins with developing the ocean wave energy spectrum, which can be done for any sea environment if the appropriate data is provided. The relationship between how wave energy is converted into ship motion energy is described. This process shows that if the appropriate ship information is given, the motion parameters for any ship can be computed. Finally, the equations of motion are generated which are used to derive the Doppler and phase shift parameters of scatterers to synthesize a target echo signal. The motion formulation and the extraction of target modulation parameters for signature synthesis is implemented in software and is addressed in the next chapter. 


\section{Chapter 5: Target Signature Synthesis Software}

\subsection{Introduction}

The software stage, also referred to as the slow-processing stage or offline stage, stores the target model and performs motion simulation. In the software motion parameters, hostile radar approximate position, and simulated target resolution parameters are defined by a user. These parameters simulate realistic target motion and pose, following which, extraction of the modulation parameters required to synthesize the false target signal in hardware is performed.

This chapter addresses the software implementation stage of the target signature synthesis process performed in a program named Target Signature Synthesis (TSS). The first section addresses procurement of an effective target model. The application of motion equations to the target model is then visually presented. Subsequently, the method for selection of scattering locations in resolution cells is discussed. The computation of signal modulation parameters for each scattering location is presented. Finally, the motion decorrelation time and error sources are examined.

\subsection{Target Point Cloud Model}

Effective target modelling is performed using computational electromagnetic software to simulate physical interaction between an electromagnetic pulse and the complex target structure. Complex targets, such as a ship, are comprised of multiple scattering locations which vary in intensity of return, based on pulse frequency and angle of arrival (AOA) [34][54]. Investigation into generation of point cloud models to simulate ISAR images of complex targets for use in radar operator training, and radar target recognition algorithm development has been performed since the 1980s [34]. Many different electromagnetic prediction software programs have been developed by various organizations to address this topic, such as FEKO, ANSYS HFSS, GSC, and ISARLAB [34][52][54]. These applications apply principles of electromagnetics to generate ISAR images of a target at varying target pose and aspect angle. Interferometric techniques, often used in LIDAR applications, are utilized to generate three-dimensional point cloud models which are comprised of the main scattering locations on a complex target 
[32][34][52][54]. The software utilized to generate the target point cloud model in this application is ANSYS HFSS, with the ACT Extensions; RadarPre and RadarPost [56][57]. The target modelled is the USS Spruance (DD963) Destroyer Class ship, for which a three-dimensional CAD model was purchased from [58].

The electromagnetic approximations utilized in ANSYS HFSS for this simulation is the SBR+ solver. This tool implements shooting and bouncing rays, which consists of ray tracing algorithms to simulate the interaction between a propagating radar pulse and the target model [56][57]. The material of the hull is selected to be steel, and the superstructures aboard the ship are all assumed to be composed of aluminum [59]. The boundary condition applied to the target was the perfect electric conductor boundary. A true military target is often designed in a manner to reduce the radar cross-section (RCS) of the target. This is achieved by using radar absorbing materials and deflectors placed aboard ships [45]. The locations and designs of RCS reduction materials is classified data, and thus are not included in the target model. Figure 5.1 shows the CAD model of the target used.

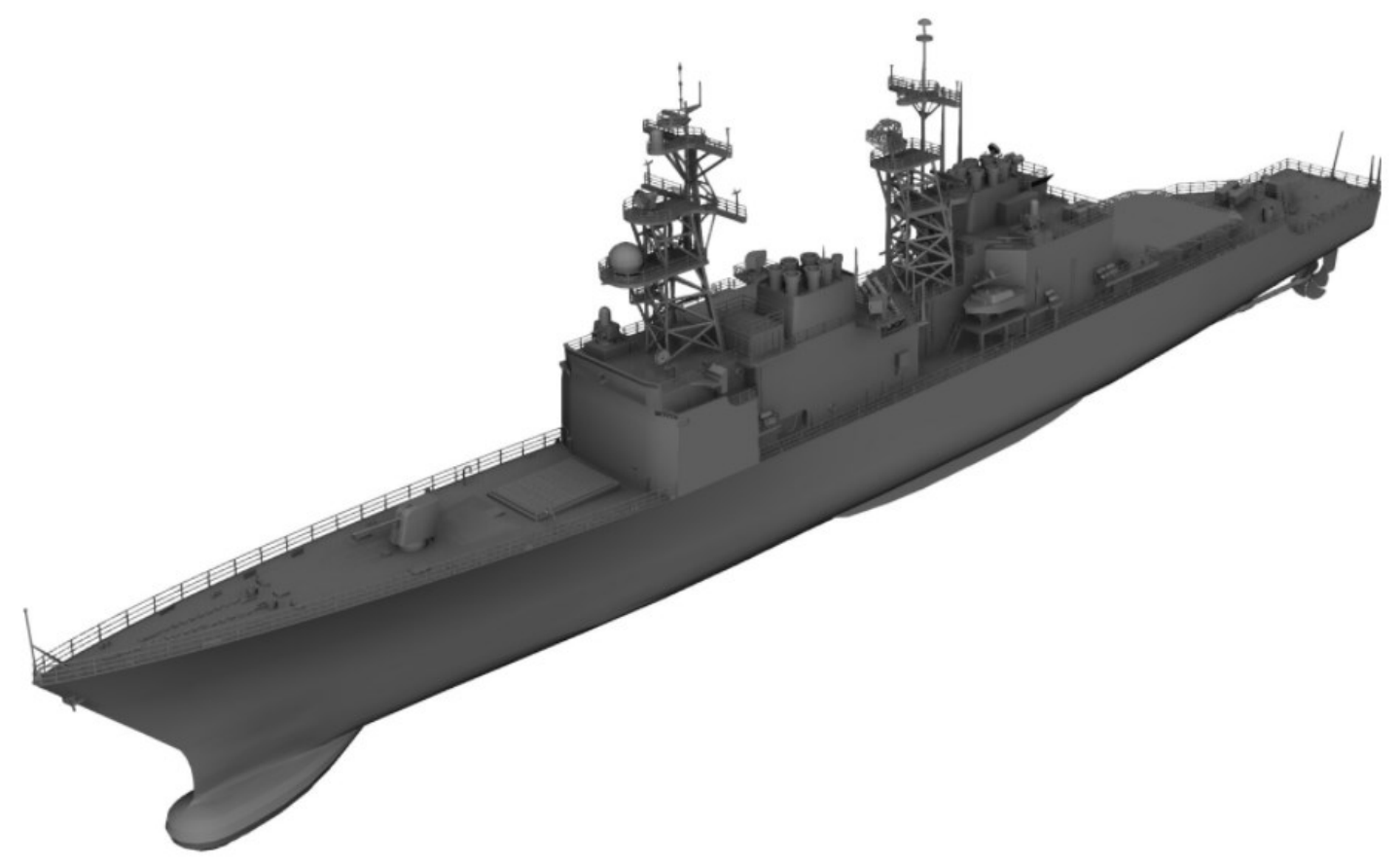

Figure 5.1: CAD model of the USS Spruance (DD-963) Destroyer class ship [58]. 
The assumed radar band of operation is X-Band, the $8-12 \mathrm{GHz}$ range, which is commonly used in marine radar applications [50]. The specific frequency in generation of ISAR images for the ship target is $10 \mathrm{GHz}$. The target center of gravity, obtained from [25], is placed at the origin of the simulation space. The simulation parameters for the RadarPre toolkit are defined by angle of arrival of pulse, radar frequency, size of image and resolution in range and cross-range dimensions. Obtaining a high-fidelity point cloud model requires simulation at exceptionally fine resolutions. The chosen resolution is 0.5 meters in both range and cross-range. The ISAR image size is determined based upon the selected AOA of the radar pulse. In the case of AOA incident upon the ship bow, the range dimension is chosen to be at least 180 meters, and the cross-range dimension is at least 30 meters, allowing for an image space larger than the ship. An AOA incident upon the ship broadside switches the range and cross-range window lengths for the image.

The RadarPre toolkit automatically determines the frequency bandwidth and cross-range sampling angles required to achieve the specified resolution [56]. These values are computed based on the ISAR principles discussed in chapters two and three and in [43]-[45][49]. The RadarPost toolkit implements the ISAR algorithms to produce a two-dimensional contour image of the target [57]. The contour indicates the magnitude, proportional to the RCS, of scattering locations on the ship as a function of space [57]. RadarPost also outputs range profile data for the target at a specific aspect angle [57]. Electromagnetic simulation software includes physical effects such as shadowing and multi-bounce interactions of rays. These effects can result in an ISAR image misidentifying or missing scattering locations on the target that would otherwise be visible from a different aspect angle. Simulation is performed over varying target poses and aspect angles to produce multiple ISAR images of the target. The target pose and motion determines the ISAR image produced. Figure 5.2 shows the ISAR image of the USS Spruance, with the assumption that the radar wave is incident upon the ship bow, and the ship is only undergoing yaw motion. Figure 5.3 is similar to Figure 5.2 except the aspect angle is changed such that the radar pulse is incident upon the ship stern. Figure 5.4 is the ISAR image of the ship with the pulses incident upon the broadside while simulating yaw motion. 


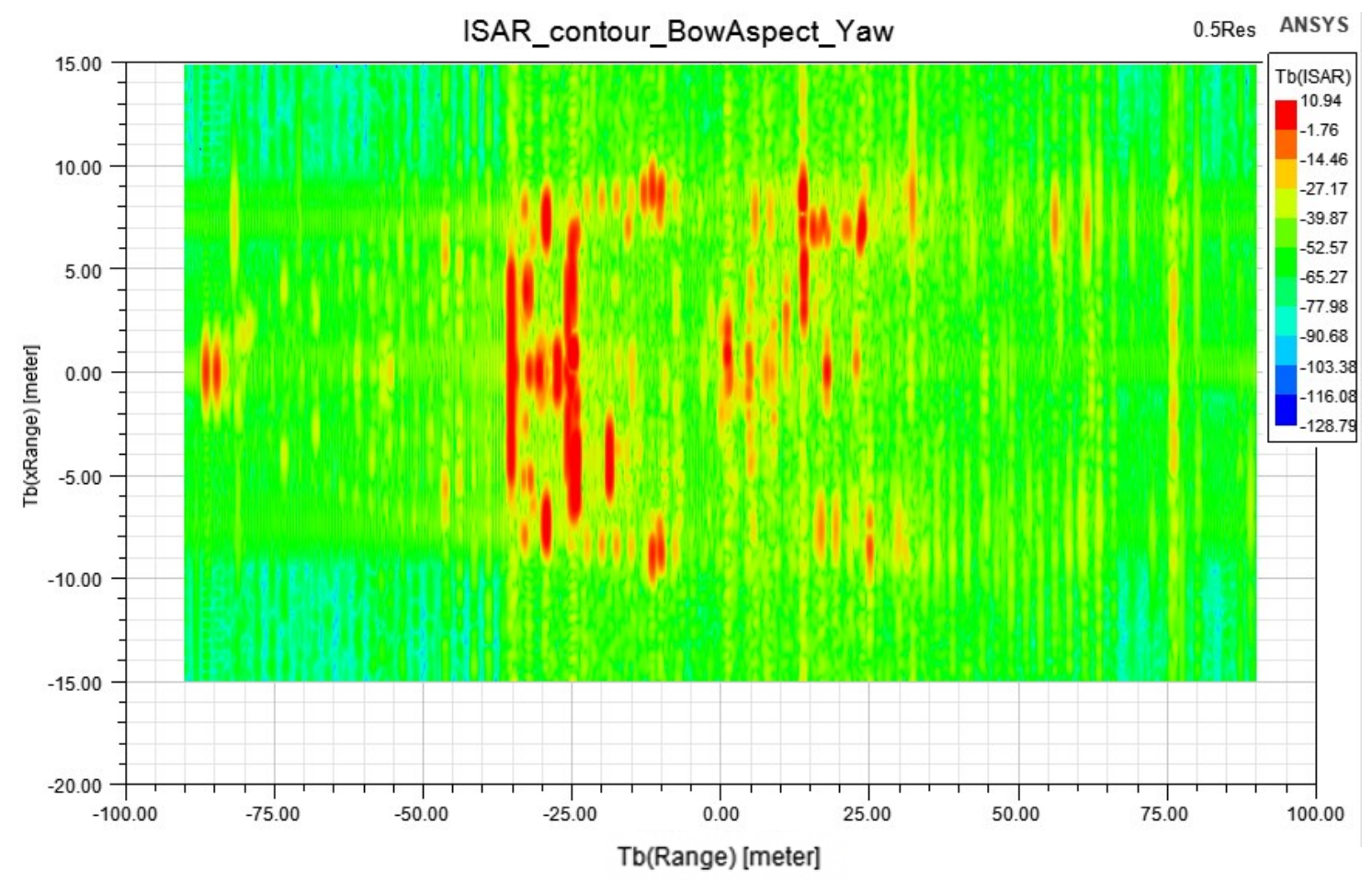

Figure 5.2: ISAR Contour of USS Spruance, LOS incident upon bow, target undergoing Yaw motion. Square resolution (Range \& Cross-Range resolution) of 0.5 meters.

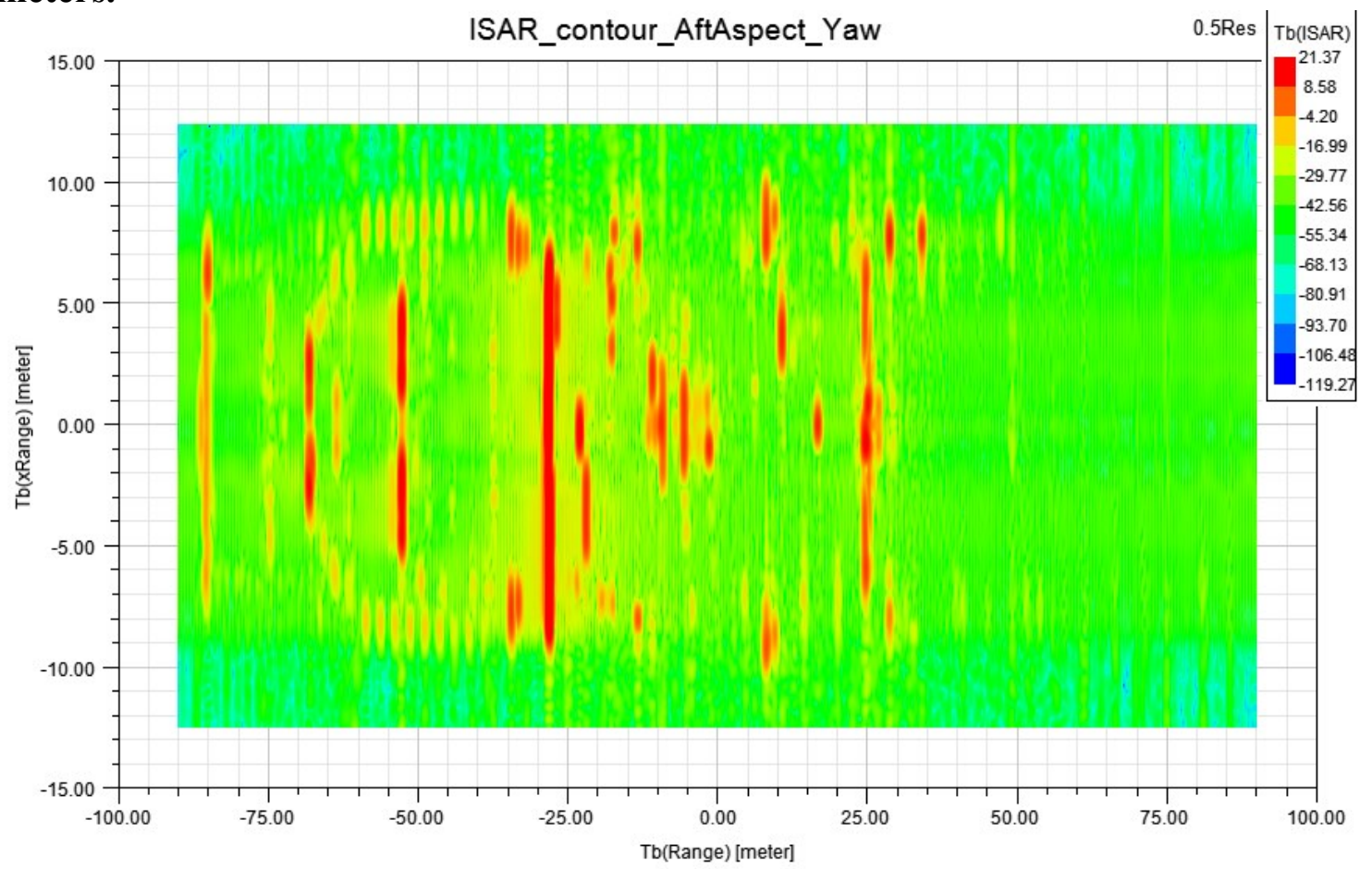

Figure 5.3: ISAR Contour of USS Spruance, LOS incident upon aft, target undergoing Yaw motion. Square resolution (Range \& Cross-Range resolution) of 0.5 meters. 


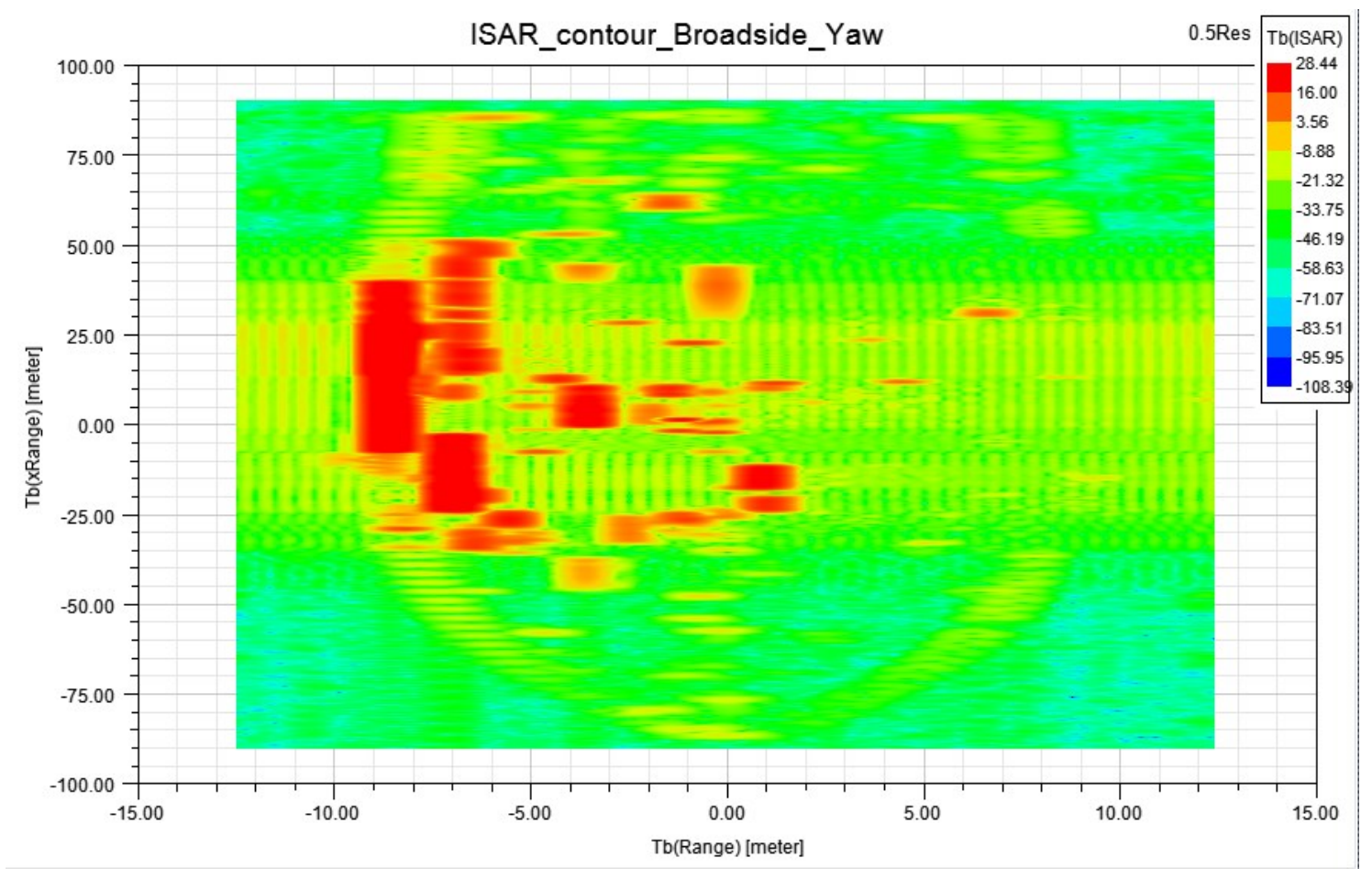

Figure 5.4: ISAR Contour of USS Spruance, LOS incident upon broadside, target undergoing Yaw motion. Square resolution (Range \& Cross-Range resolution) of 0.5 meters.

Observed from Figures 5.2 to 5.4, the change in aspect angle changes the scattering locations visible in the ISAR image, which can greatly be attributed to the effects of shadowing in ray tracing computations.

Figure 5.5 is the ISAR image of the ship for a radar pulse incident upon the bow when the ship is undergoing pitch motion only. Figure 5.6 is similar, with the aspect angle changed such that the radar pulse is incident upon the ship stern. Figure 5.7 is the ISAR image of the ship from an aspect angle above the ship while simulating pitch motion. 


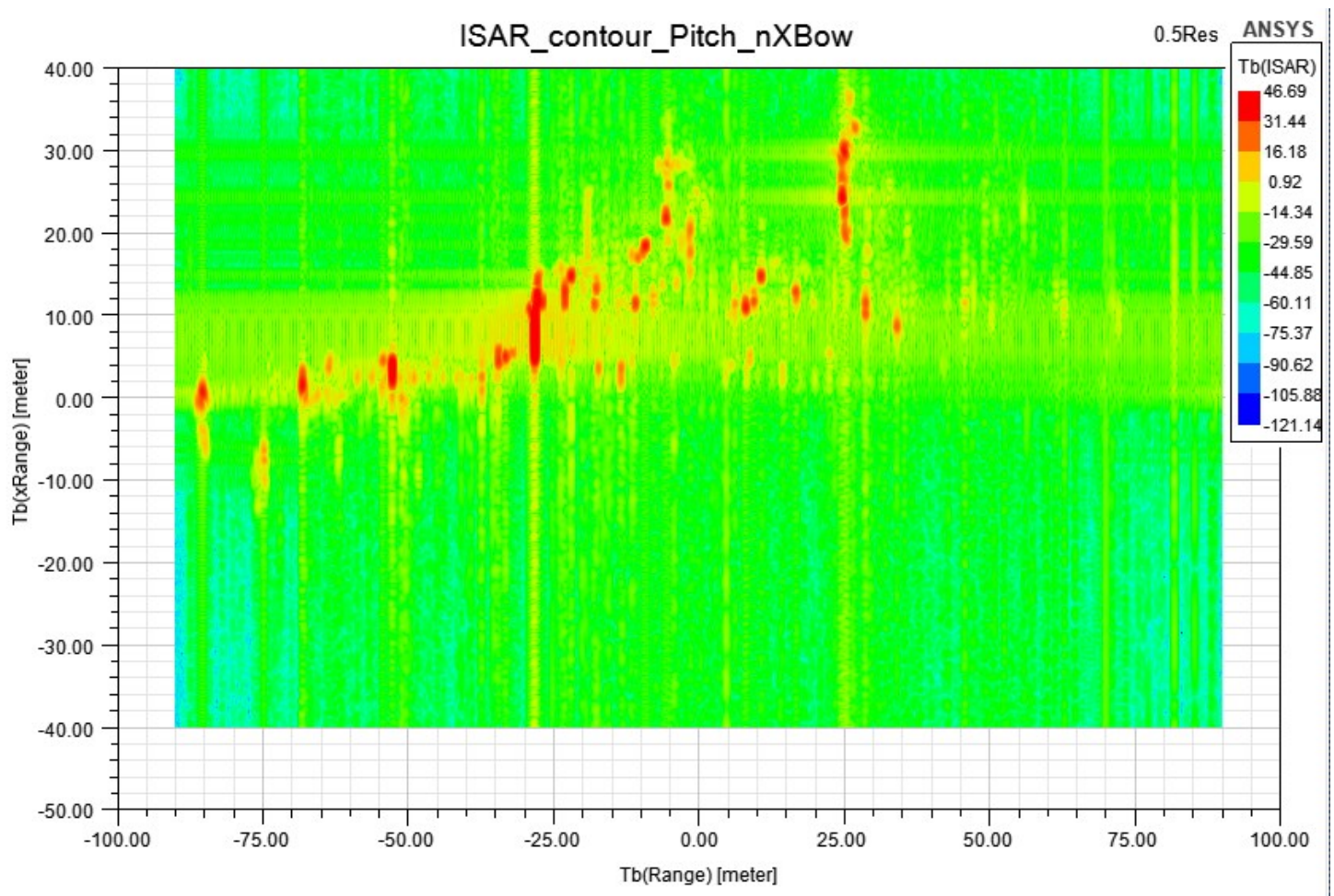

Figure 5.5: ISAR Contour of USS Spruance, LOS incident upon stern, target undergoing Pitch motion. Square resolution (Range \& Cross-Range resolution) of 0.5 meters.

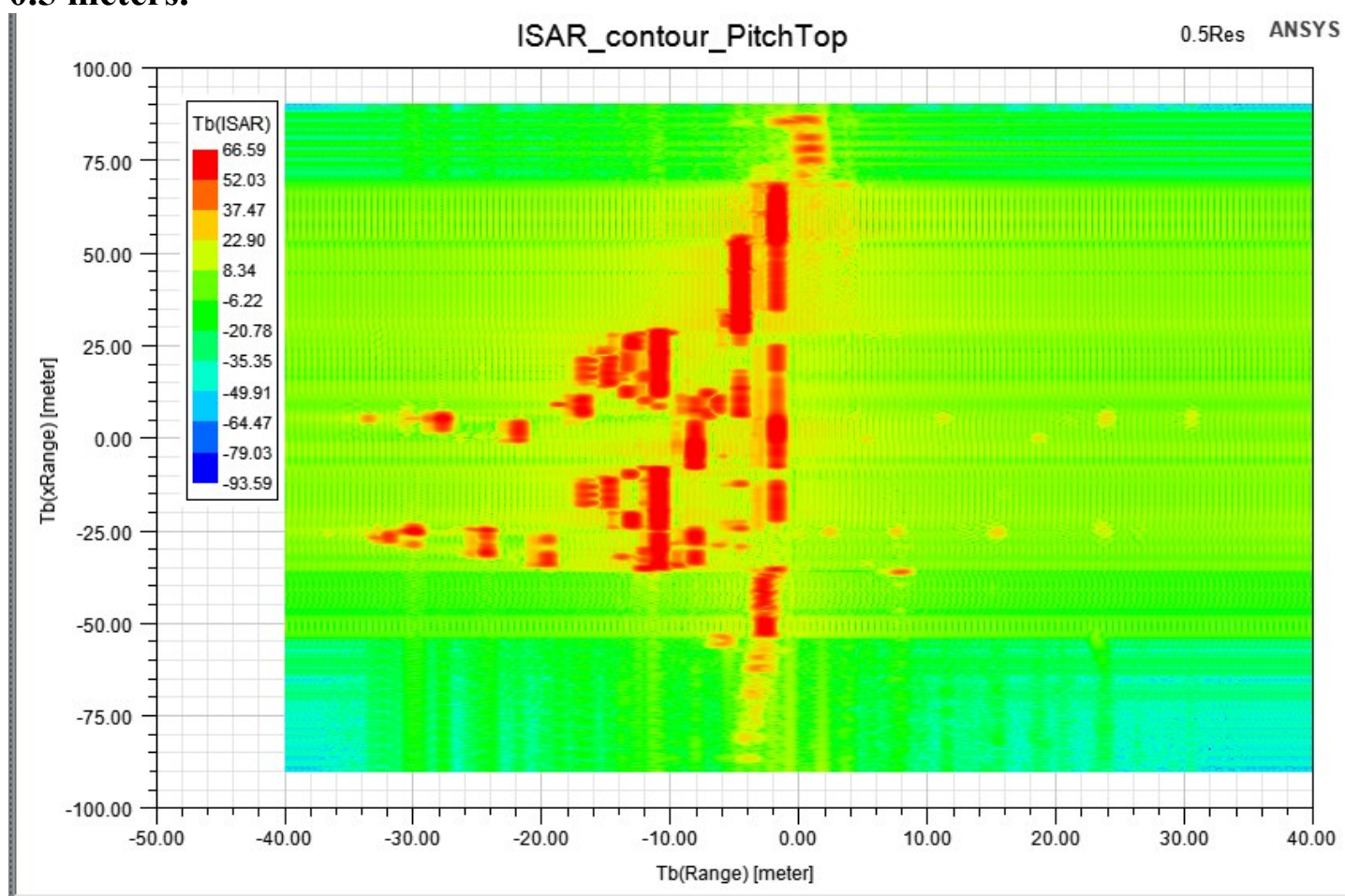

Figure 5.6: ISAR Contour of USS Spruance, LOS incident upon top of target undergoing Pitch motion. Square resolution (Range \& Cross-Range resolution) of 0.5 meters. 


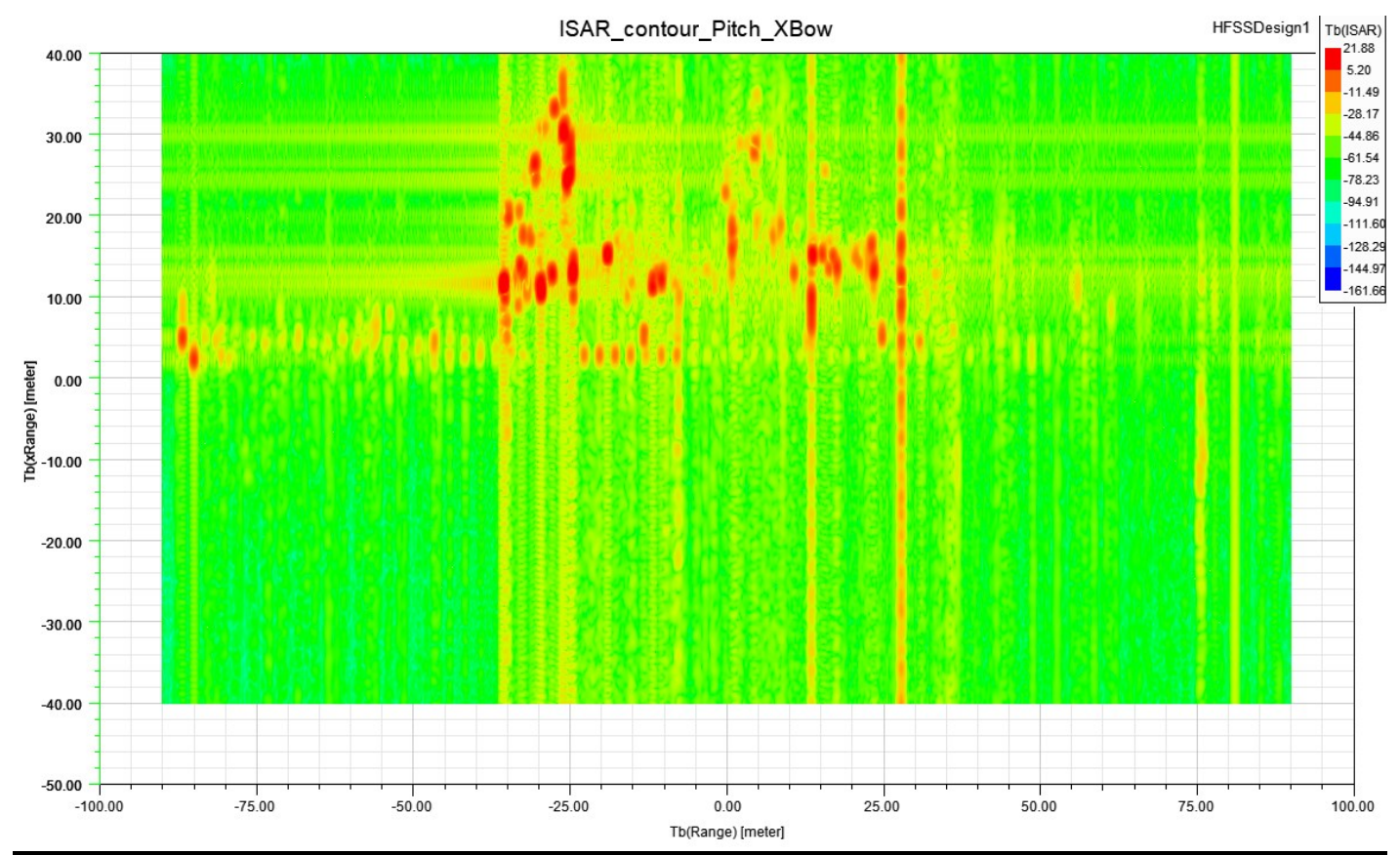

Figure 5.7: ISAR Contour of USS Spruance, LOS incident upon Bow of target undergoing Pitch motion. Square resolution (Range \& Cross-Range resolution) of 0.5 meters.

The ISAR image data is exported into a comma-separated values (csv) file type, which contains the range and cross-range position, as well as the scattering intensity power. Each image contour is incorporated into a custom point matching algorithm to generate a point cloud model of the target which contains all the scattering locations. The point matching algorithm first rotates or flips all images such that the range and crossrange dimensions are aligned. Initial processing is performed on the images produced from simulating yaw motion in which the range dimension represents the x-axis, and the cross-range dimension is the y-axis data. A threshold is defined for each image to determine the number of data points to include in the point matching algorithm. The threshold is determined uniquely for each image, and aims to include scattering locations of high intensity, as well as some surrounding noise. This threshold is defined by the algorithm designer after visual inspection of all the simulated ISAR images. The magnitude scaling shown in the legend of the figures indicates the region of values which are used in threshold definition.

The bow and aft aspect images are spliced at the halfway point of the image, such that the dominant scatterers for each aspect angle are combined into a single image. The 
broadside aspect image of Figure 5.4 is exploited using target symmetry, such that the dominant scatters are duplicated about the 0 -range position line in the figure. The point matching algorithm then matches locations of the scatterers for the spliced bow/aft image to the broadside contour image, and the data is output to a file. This produces the XY plane points of the target scatterer model.

The same process is repeated for the ISAR contour images of the ship undergoing pitch motion, with the range dimension representing the $\mathrm{x}$-axis, and the cross-range dimension now representative of the z-axis or height dimension. The point matching algorithm produces the $\mathrm{XZ}$ cross-sectional image of the target scattering locations. In both plane images, the point match algorithm identifies locations at the same range position (x-coordinate). Height information for each scattering location is extracted from the $\mathrm{XZ}$ cross-section image, and combined with the $(\mathrm{x}, \mathrm{y})$-coordinate of the scatterer, from the XY cross-section plane image, to define a three-dimensional location for each scattering location. The largest amplitude value of scattering intensity from all compiled images is assigned to this location. Thus, a three-dimensional point cloud model of the target is generated, with assigned scattering intensity at each location. The locations of scatterers are defined referenced to the center of gravity of the ship fixed at the origin $(0,0)$ position of the ISAR contour images and cloud model. This algorithm effectively creates the ship reference coordinate system discussed in the section regarding ship motion estimation. Figure 5.8 shows the generated point cloud model of the target following implementation of the custom point matching algorithm.

The initial resolution from the ISAR images obtained from ANSYS HFSS is defined to have a range and cross-range resolution of $0.5 \mathrm{~m}$ to image the target. However, the ISAR image contours that are created in the software provide datapoints on the target at a much finer precision. Thus, in point cloud creation, the number of datapoints is significant. The first point cloud model generated purely from the ISAR data and a point matching algorithm contains over 5.25 million data points. 


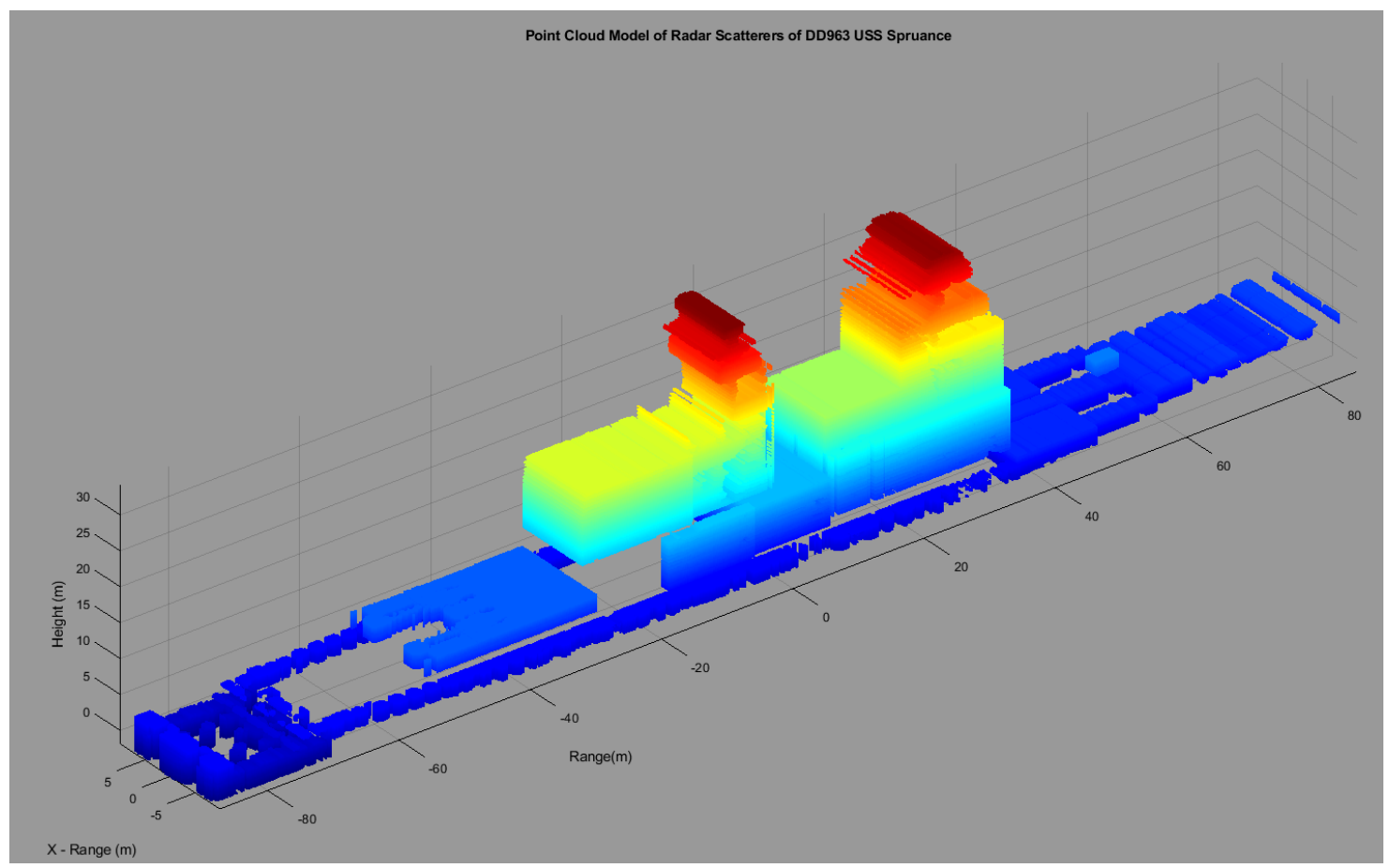

Figure 5.8: DD963 USS Spruance target point cloud model generated using point
matching algorithm on the ISAR image contour data obtained from ANSYS HFSS.

With over 5.25 million data points, the processing time required to compute modulation parameters, in the Target Signature Synthesis software, increases significantly. The speed of processing is dependent on the computer running the software, it is on the order of multiple hours of processing to produce a relatively small number of modulation profiles to process a file with 5.25 million data points. To reduce the computation time, the point cloud model is 'refined' or filtered. This filtering operation is done by rounding the data points in the cloud model to specific decimal places and then removing overlapping data points. This reduces the density of the point cloud model based on the number of decimal places chosen.

The principle for determining the number of decimal places for data rounding is understood by considering a 1 cubic meter volume in the Cartesian coordinate system. If the two decimal places are kept in each dimension (i.e. X, Y and Z) there are 100 values for each dimension (i.e. $100 \mathrm{X}$ values, $100 \mathrm{Y}$ values and $100 \mathrm{Z}$ values). With this many data values, the maximum total number of data points in one cubic meter is $100^{3}$, which is one million data points. Since the target dimensions and volume are significantly larger 
than 1 cubic meter it is evident that a resolution of two decimal places is too fine to effectively reduce the number of data points in the cloud.

If rounding is done to a single decimal place, there are still 10 possible values for a point in each dimension. In a cubic meter volume, the maximum total number of data points is $10^{3}=1000$ data points. The reduction in precision of a single decimal place results in a reduction of the total number of data points by a factor of 1000 . However, even with rounding to a single decimal place the total number of points in a cubic meter indicates that for the entire target model the number of points is still large enough to cause significant latency when processing the cloud in Target Signature Synthesis Software. Rounding the data to the nearest integer value results in a maximum of two data points in each dimension in a cubic meter volume. Thus, a total of 8 data points in a cubic meter volume. This significant reduction in resolution will speed up processing time but could impact the ability to counter wideband high-resolution radar with resolution finer than 1 meter.

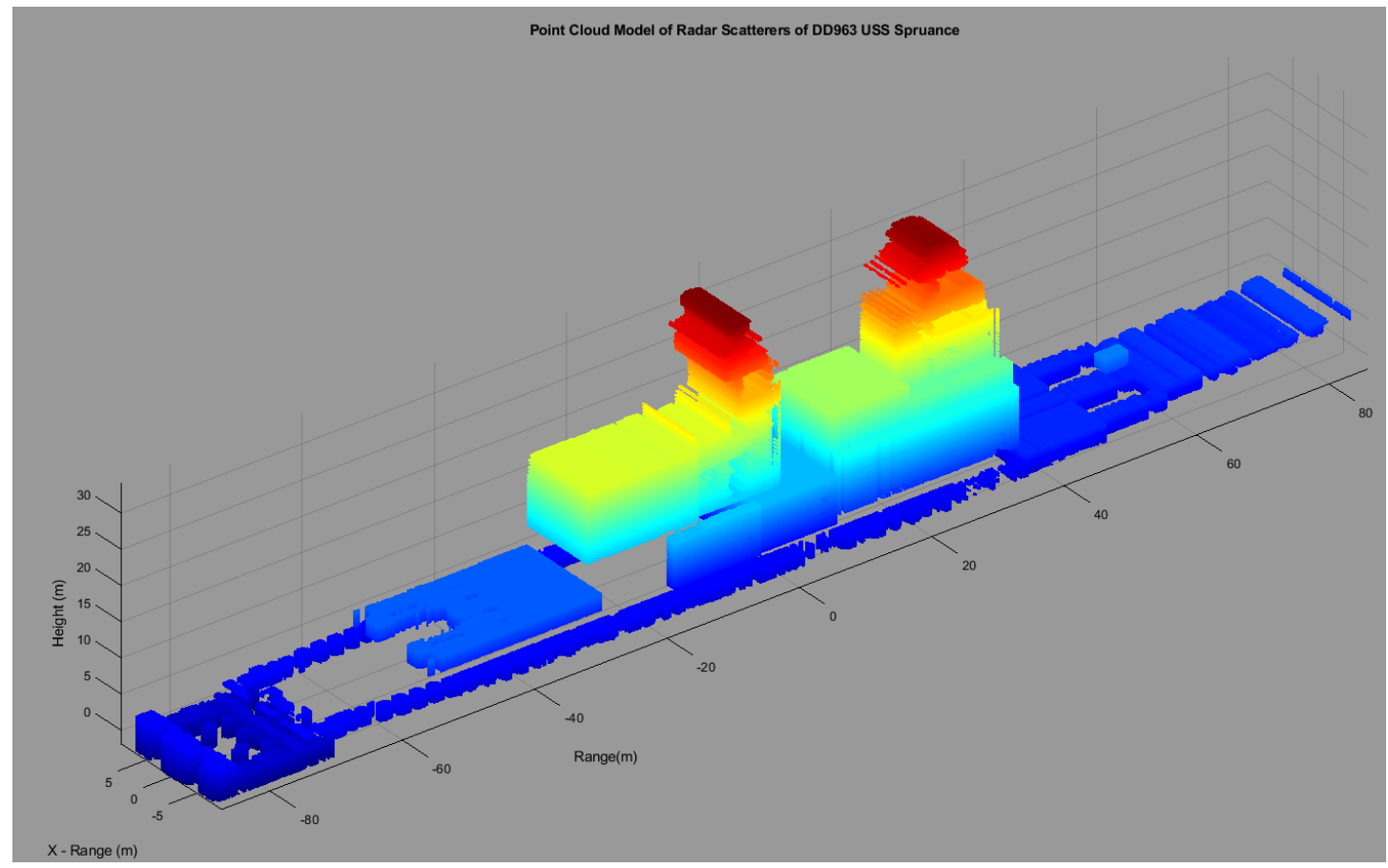

Figure 5.9: Refined/Filtered DD963 USS Spruance target point cloud model, with rounding to a single decimal precision in all dimensions.

This results in a designer choice in selection of decimal precision of data points. The largest dimension is the range dimension (i.e., $\mathrm{X}$ axis). Thus, to effectively reduce 
the total number of data points, the decimal precision in the $\mathrm{X}$ dimension is reduced to zero (i.e., rounding to the nearest integer). The target is approximately 170 meters in length, meaning a decimal precision of 0 places results in approximately 171 data points along the $\mathrm{X}$-axis. If the resolution were increased to even a single decimal place, the number of data points would increase to a maximum of approximately $(170 \times 10)=1700$ data points along the $\mathrm{X}$ dimension. With the inclusion of $\mathrm{Y}$ and $\mathrm{Z}$ coordinate data, this would result in far too many data points for efficient processing.

The $\mathrm{Y}$ and $\mathrm{Z}$ dimensions of the target are much smaller relative to the $\mathrm{X}$ dimension. A finer decimal precision can be utilized without significant increase in the number of data points. The maximum size in the $\mathrm{Y}$ dimension is approximately 20 meters, and the maximum target size in the $\mathrm{Z}$ dimension is approximately 45 meters. Table 5.1 on the following page shows the number of data points along each dimension depending on the decimal precision selected. 
Table 5.1: Approximation of number of data points in target cloud model based on rounding precision.

\begin{tabular}{|c|c|c|c|c|}
\hline \multirow[b]{2}{*}{$\begin{array}{l}\text { Target } \\
\text { Dimension/ } \\
\text { Maximum } \\
\text { Target Size in } \\
\text { The } \\
\text { Dimension }\end{array}$} & \multicolumn{4}{|c|}{$\begin{array}{l}\text { Approximate Number of Datapoints in a Dimension for Selected } \\
\text { Decimal Precision }\end{array}$} \\
\hline & $\begin{array}{l}\text { 0 (i.e., } \\
\text { Nearest } \\
\text { Integer) }\end{array}$ & $\begin{array}{l}1 \text { Decimal } \\
\text { Point } \\
\text { Precision } \\
\text { (i.e., } 10 \text { data } \\
\text { points in each } \\
\text { dimension) }\end{array}$ & $\begin{array}{l}2 \text { Decimal } \\
\text { Point } \\
\text { Precision } \\
\text { (i.e., } 100 \text { data } \\
\text { points in each } \\
\text { dimension) }\end{array}$ & $\begin{array}{l}3 \text { Decimal } \\
\text { Point } \\
\text { Precision } \\
\text { (i.e., } 1000 \text { data } \\
\text { points in each } \\
\text { dimension) }\end{array}$ \\
\hline $\begin{array}{l}\mathrm{X} \text { (Range)/ } \\
200 \mathrm{~m}\end{array}$ & 200 & 2,000 & 20,000 & 200,000 \\
\hline $\begin{array}{l}\text { Y (Cross- } \\
\text { Range)/ 20m }\end{array}$ & 20 & 200 & 2,000 & 20,000 \\
\hline $\begin{array}{l}\text { Z (Height)/ } \\
45 \mathrm{~m}\end{array}$ & 45 & 450 & 4,500 & 45,000 \\
\hline $\begin{array}{l}\text { Maximum } \\
\text { Total Number } \\
\text { of Data Points } \\
\text { in the Cloud } \\
\text { (Ignoring } \\
\text { Target Shape) } \\
(\mathrm{X} * \mathrm{Y} * \mathrm{Z})\end{array}$ & 180,000 & $180,000,000$ & $1.8 \times 10^{11}$ & $1.8 \times 10^{14}$ \\
\hline
\end{tabular}

From the table, it is evident that increasing the precision by a single decimal place increases the total number of points in the cloud by a maximum factor of 1,000. Three possible options are selected to minimize the total number of data points in the cloud.

1. Point position data is rounded to the nearest integer (i.e., 0 decimal places) for all target dimensions.

2. Point position data is rounded to the nearest integer (i.e., 0 decimal places) for the $\mathrm{X}$ and $\mathrm{Z}$ dimension, and the $\mathrm{Y}$ dimension data is rounded to a single decimal place. 
3. Point position data is rounded to the nearest integer (i.e., 0 decimal places) for the $\mathrm{X}$ dimension, and the $\mathrm{Y}$ and $\mathrm{Z}$ dimension data are rounded to a single decimal place.

The point cloud model for all three possible options is shown in Figure 5.10 to 5.12.

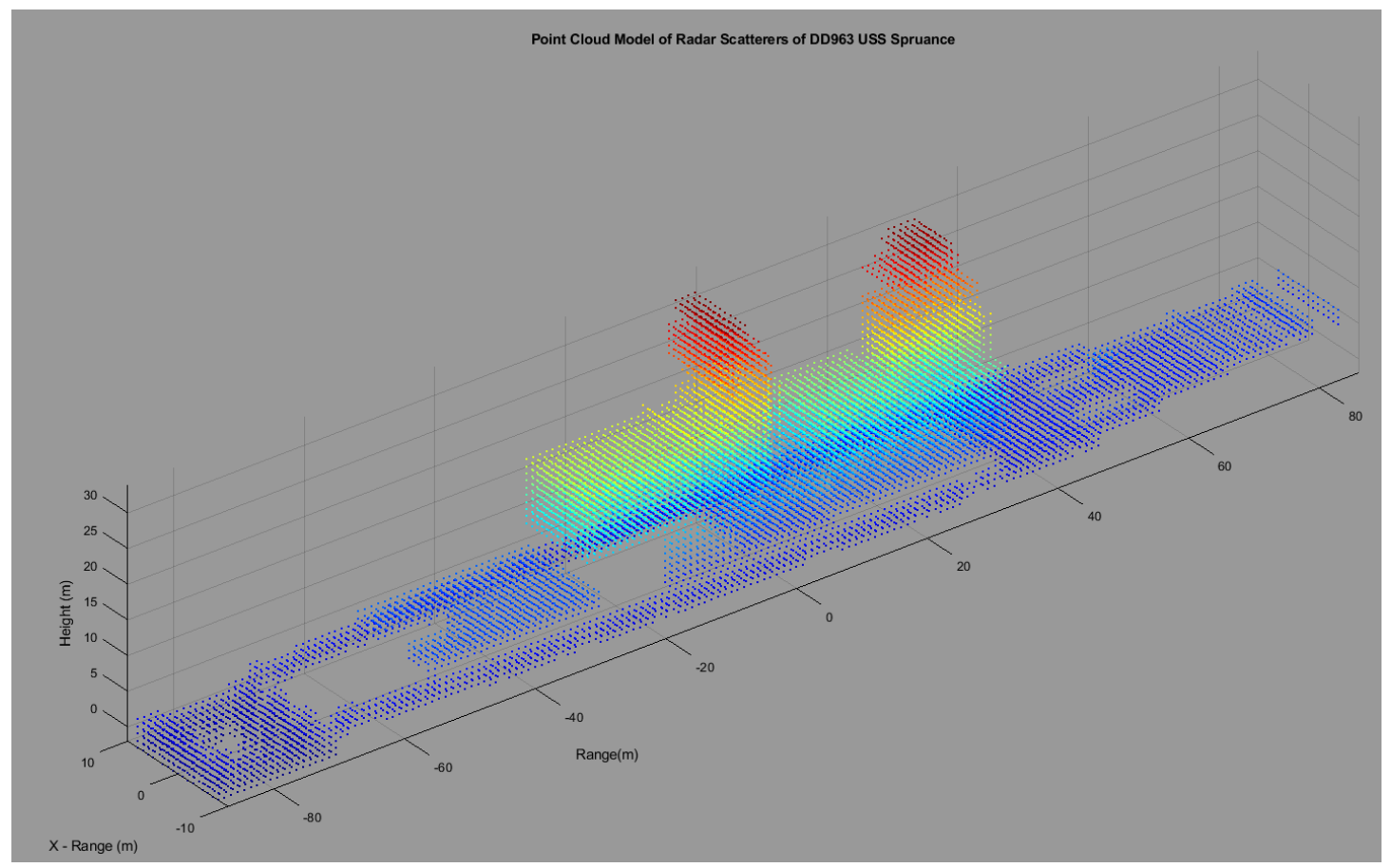

Figure 5.10: Refined/Filtered DD963 USS Spruance target point cloud model, with rounding to a zero-decimal precision (i.e. nearest integer) in all dimensions.

From option one, it is evident the point cloud model density has significantly decreased. However, with such a small density, the ability to counter a radar system with sub-meter resolution may be difficult. 


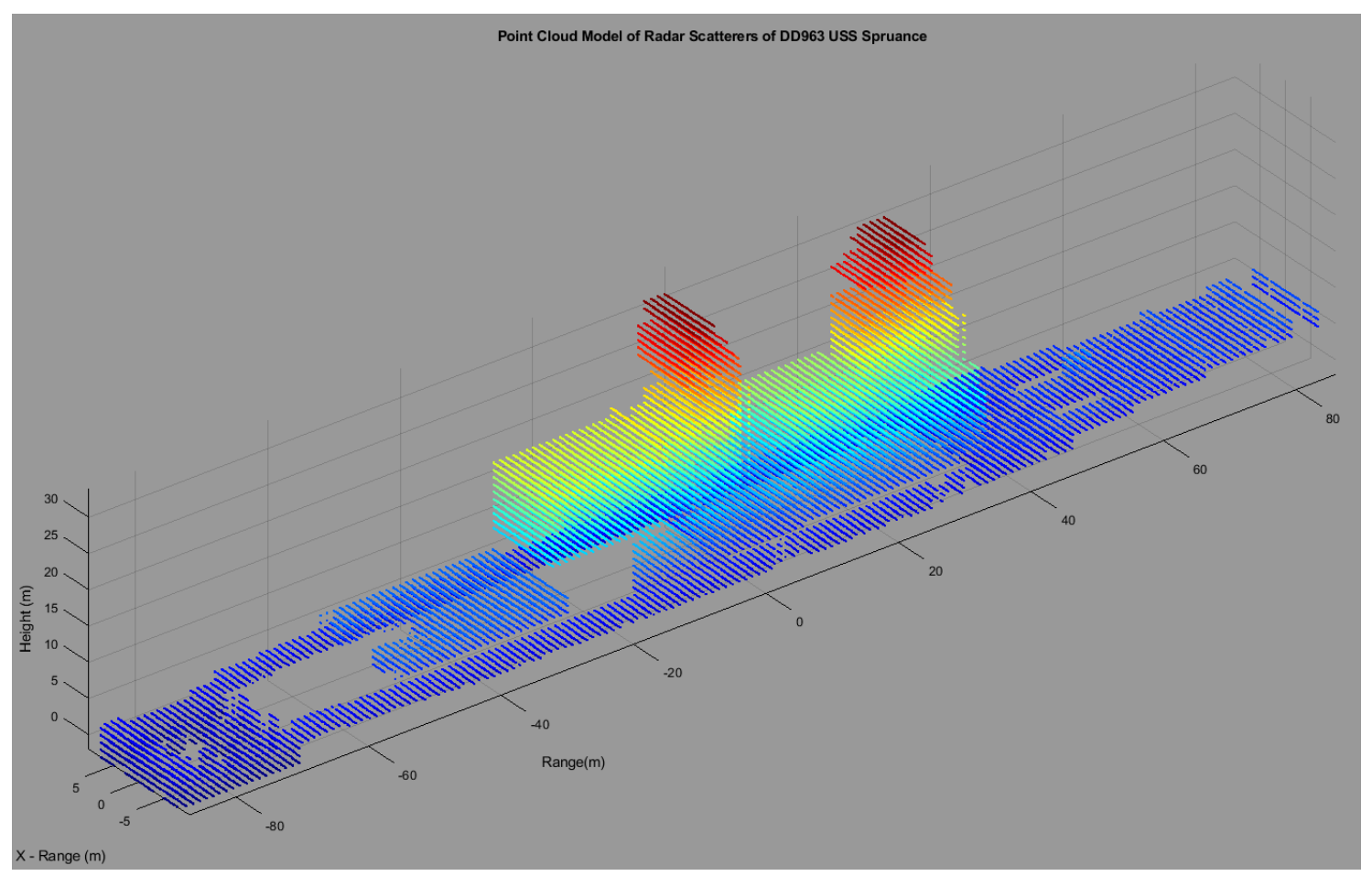

Figure 5.11: Refined/Filtered point cloud model, with rounding to a zero-decimal precision (i.e., nearest integer) in the $X$ and $Z$ dimensions, and single decimal precision in the $\mathrm{Y}$ dimension.

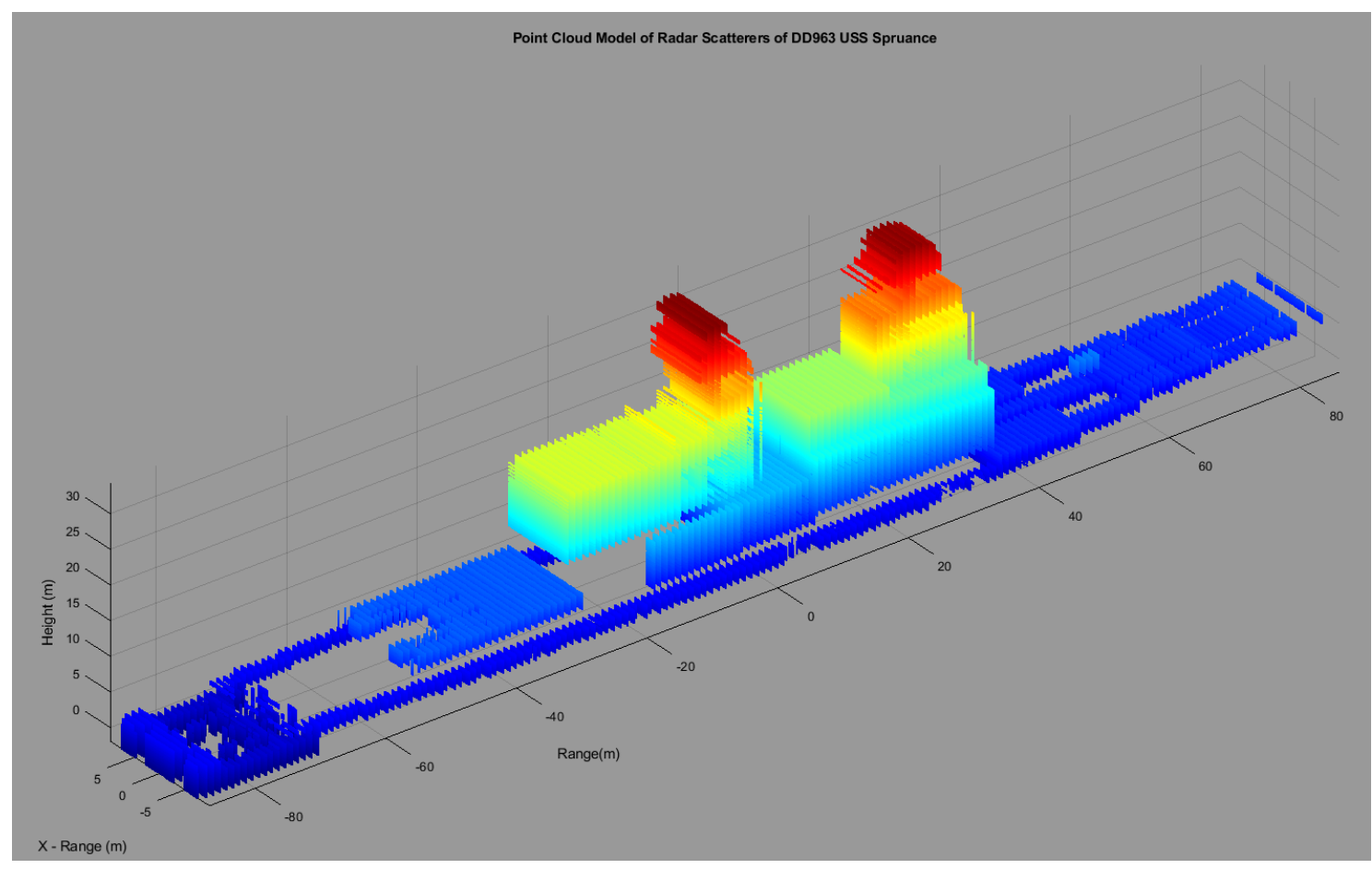

Figure 5.12: Refined/Filtered target point cloud model, with zero-decimal precision in the $\mathrm{X}$ dimension, and single decimal precision in the $\mathrm{Y}$ and $\mathrm{Z}$ dimensions. 
From all three options and the corresponding figures, it is evident the density of the cloud model increases with increasing decimal precision in a single dimension. The total number of data points in the cloud model for all three options is listed in Table 5.2 below.

Table 5.2: Number of Data Points in the Target Cloud Model for the various options of decimal precision.

\begin{tabular}{|c|c|}
\hline Option/Case Number & $\begin{array}{l}\text { Number of Data } \\
\text { Points in the Point } \\
\text { Cloud Model }\end{array}$ \\
\hline 0a (No refining/filtering) & $5,462,016$ \\
\hline $\begin{array}{l}\text { 0b (Rounding to Single Decimal Precision in All } \\
\text { Dimensions) }\end{array}$ & $5,215,508$ \\
\hline $\begin{array}{l}\text { 1. Point position data is rounded to the nearest integer } \\
\text { (i.e., } 0 \text { decimal places) for all target dimensions. }\end{array}$ & 19,153 \\
\hline $\begin{array}{l}\text { 2. Point position data is rounded to the nearest integer } \\
\text { (i.e., } 0 \text { decimal places) for the } \mathrm{X} \text { and } \mathrm{Z} \text { dimension, } \\
\text { and the } \mathrm{Y} \text { dimension data is rounded to a single } \\
\text { decimal place. }\end{array}$ & 133,560 \\
\hline $\begin{array}{l}\text { 3. Point position data is rounded to the nearest integer } \\
\text { (i.e., } 0 \text { decimal places) for the } \mathrm{X} \text { dimension, and the } \\
\mathrm{Y} \text { and } \mathrm{Z} \text { dimension data are rounded to a single } \\
\text { decimal place. }\end{array}$ & 811,366 \\
\hline
\end{tabular}

Selection of the cloud model is dependent on the specifications for processing speed in Target Signature Synthesis software. Reduction of processing time comes at the cost of reduced cloud model density and thus reduced resolution. The selection of a model is left to the user/designer; in this research model, case three is chosen. Multiple models with varying decimal precision can be stored and used in the TSS software as required.

Scatterer intensity is a function of frequency and aspect angle. The point cloud model implemented will not account for these variables in target signature synthesis 
operations. Inclusion of shadowing effects, frequency, and aspect angle dependance as well as other electromagnetic interaction phenomena are beyond the scope of this application, which focuses on the motion modelling effects on Doppler and phase modulation for target signature synthesis. The reader is referred to [32][34][52] and [54] for further information on scatterer modelling with physical electromagnetic effects.

\subsection{Target Motion Application}

The point cloud target generated contains all absolute locations of radar scatterers referenced to the center of gravity. These locations are utilized in equation 4.22 to simulate motion of the target point cloud model for a specific sea state, wave heading relative to ship bow, and ship velocity. The figures in Appendix A show effective implementation of the motion equations to produce angular motion of the simulated target. In the Target Signature Synthesis software, the user defines the motion conditions for the simulated target; the software applies the motion equations to the target point cloud object to compute the new positions, and velocities of scattering locations. Figure 5.13 shows the user interface of the software. The user-defined inputs for motion are Sea State, Wave heading, ship speed, and motion direction.

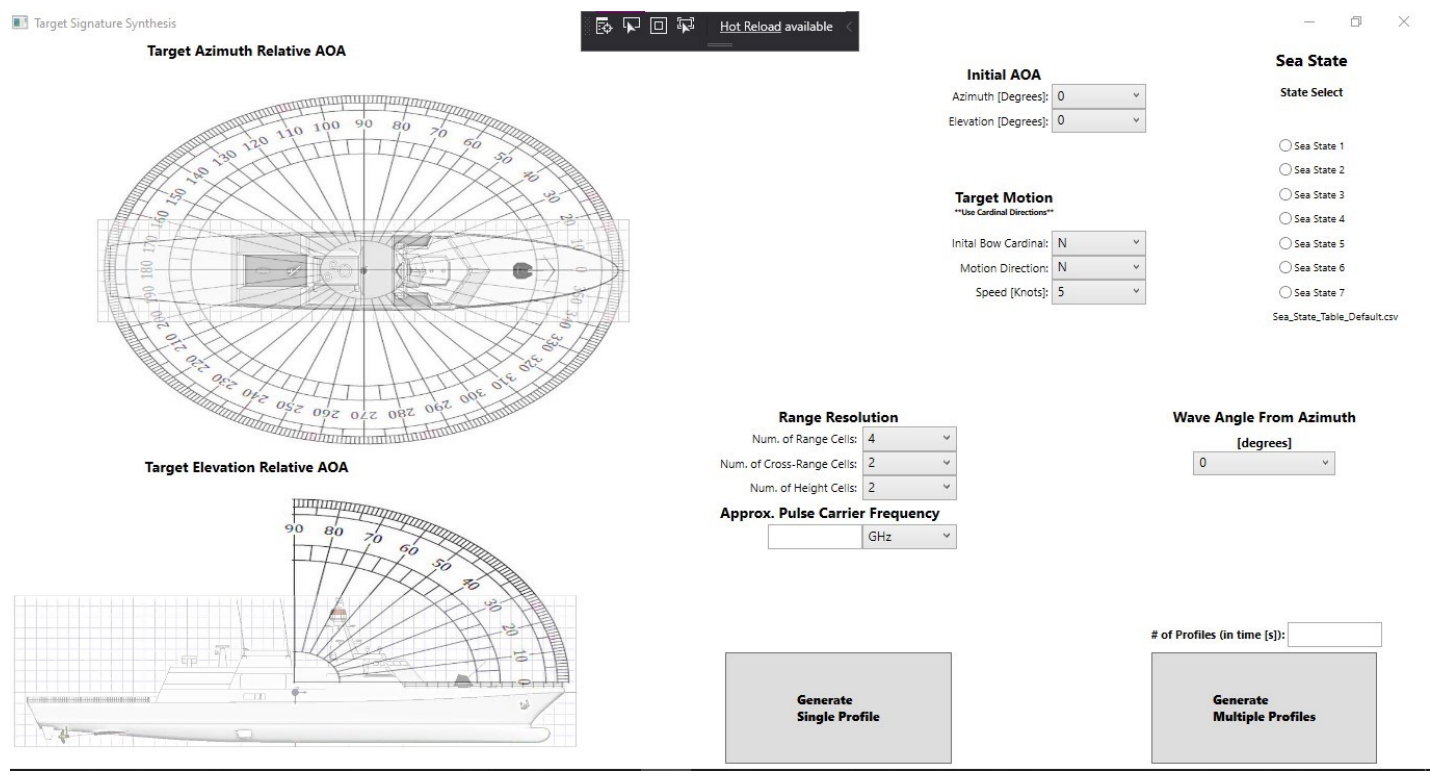

Figure 5.13: Target Signature Synthesis Software User Interface. 


\subsection{LOS, Resolution \& Scatterer Selection}

The software provides options for selection of the estimated hostile radar position in terms of azimuth and elevation angle positions. These are labeled 'Initial Angle of Arrival (AOA),' which indicates the line of sight (LOS) of the hostile radar. Selection tools are available for the simulated target resolution in range, cross-range, and height. This is to counter radar systems that have the capability to change operating modes and apply signal processing techniques for finer resolution target tracking, identification or imaging [45].

Definition of a resolution cell is dependent on assumptions of the antenna pattern of the hostile radar. The hostile radar is assumed to be at a sufficient distance such that the entire simulated target is within the main lobe of the antenna beam. Resolution cell formulation for hostile radars employing a narrower beam or monopulse techniques is left for future research. This application's maximum possible resolution will be 128 range bins, by 128 cross-range bins by 128 height bins. However, increasing resolution cell options is trivial to program. Increased resolution comes at the cost of increased computation time.

Range resolution cells are along the radar LOS; the lines that bound these cells are orthogonal to the LOS. Doppler resolution cells are orthogonal to the radar LOS, the lines that bound these cells are parallel to the LOS. Height resolution cells are fixed, and the input resolution determines the size of cells.

The grid observed in Figure 3.1 extends in three-dimensions to form a grid cube on the target. The dominant scatterer in each resolution cell contributes to the target echo. The resolution grid is three-dimension; however, range profiles or ISAR images produce only two-dimensional image representations of the target [45]. The Target Signature Synthesis software applies a three-dimensional grid applied in resolution cell formulation. The primary selection criteria of range and cross-range resolution determines the grid definition in the XY plane of the target. The user-defined Azimuth angle is used to create the line boundaries for the range and cross-range resolution cells. The height grid segments the $\mathrm{Z}$ dimension of the target into the appropriate number of resolution cells as defined by the user, regardless of hostile radar LOS angles. 
Cross-range and range resolution cells are bound by lines with a slope dependent on the LOS. The conditions for calculating the slope of the resolution cell lines are outlined in Table 5.3.

Table 5.3: The slopes of lines of the range and Doppler resolution cells according to LOS Azimuth relative to the target.

\begin{tabular}{|c|c|c|}
\hline $\begin{array}{c}\text { Slope of Range Bin } \\
\text { Lines }\end{array}$ & $\begin{array}{c}\text { Slope of Cross-Range Cell } \\
\text { Lines }\end{array}$ & $\begin{array}{c}\text { Radar LOS AZ Relative to } \\
\text { the Target }\end{array}$ \\
\hline$-\frac{1}{\tan (A Z)}$ & $\tan (A Z)$ & $0^{\circ}<\mathrm{AZ}<90^{\circ}$ \\
\hline$-\frac{1}{\tan (A Z)}$ & $\tan (A Z)$ & $90^{\circ}<\mathrm{AZ}<180^{\circ}$ \\
\hline$-\frac{1}{\tan (A Z)}$ & $\tan ($ AZ) & $180^{\circ}<\mathrm{AZ}<270^{\circ}$ \\
\hline$-\frac{1}{\tan (\text { AZ) }}$ & $\tan ($ AZ) & $270^{\circ}<\mathrm{AZ}<360^{\circ}$ \\
\hline$\infty($ Vertical Line $)$ & 0 & $\mathrm{AZ}=0^{\circ} \mathrm{OR} \mathrm{AZ}=180^{\circ}$ \\
\hline 0 & $\infty($ Vertical Line $)$ & $\mathrm{AZ}=90^{\circ} \mathrm{OR} \mathrm{AZ}=270^{\circ}$ \\
\hline
\end{tabular}

The number of lines is equivalent to one more than the number of resolution cells. The number of chosen resolution cells determines the spacing of the cell boundary lines along the length of the ship. For the case of eight resolution cells in range or cross-range, the length of the ship is segmented into eight sections along the X-axis. If the LOS is incident upon the front or back of the ship the range, the range cells split the length of the ship, and cross-range cells segment the width of the ship. The converse is true for a radar LOS incident upon either broadside of the target. Figures 5.14 to 5.16 show the segmentation of the target into range and cross-range resolution grids and cells. 


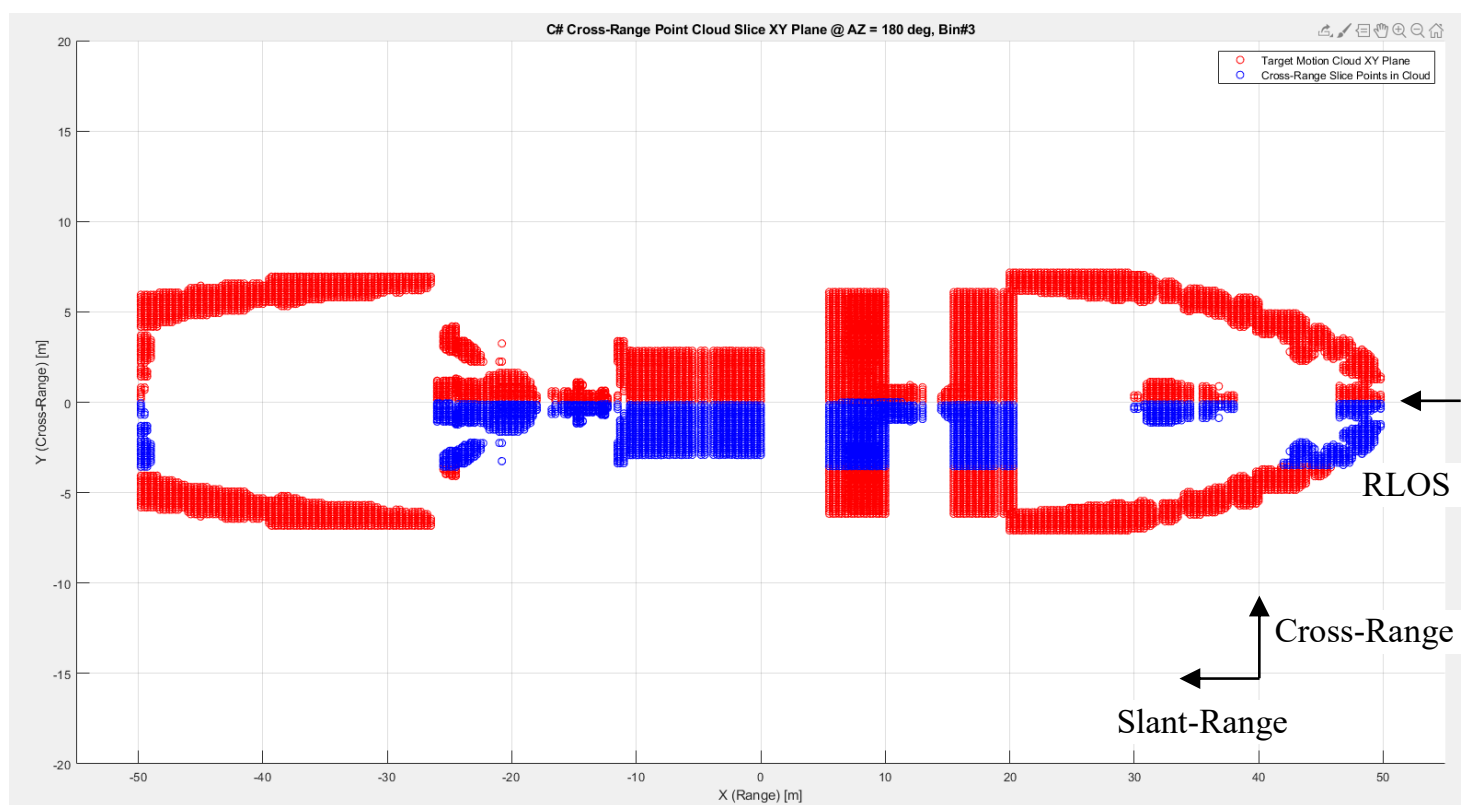

Figure 5.14: Radar LOS Azimuth is set to $0^{\circ}$. Cross-Range cells exist orthogonal to the LOS. Highlighted blue is the 3rd Cross-Range cell when the Cross-Range resolution is 4 bins.

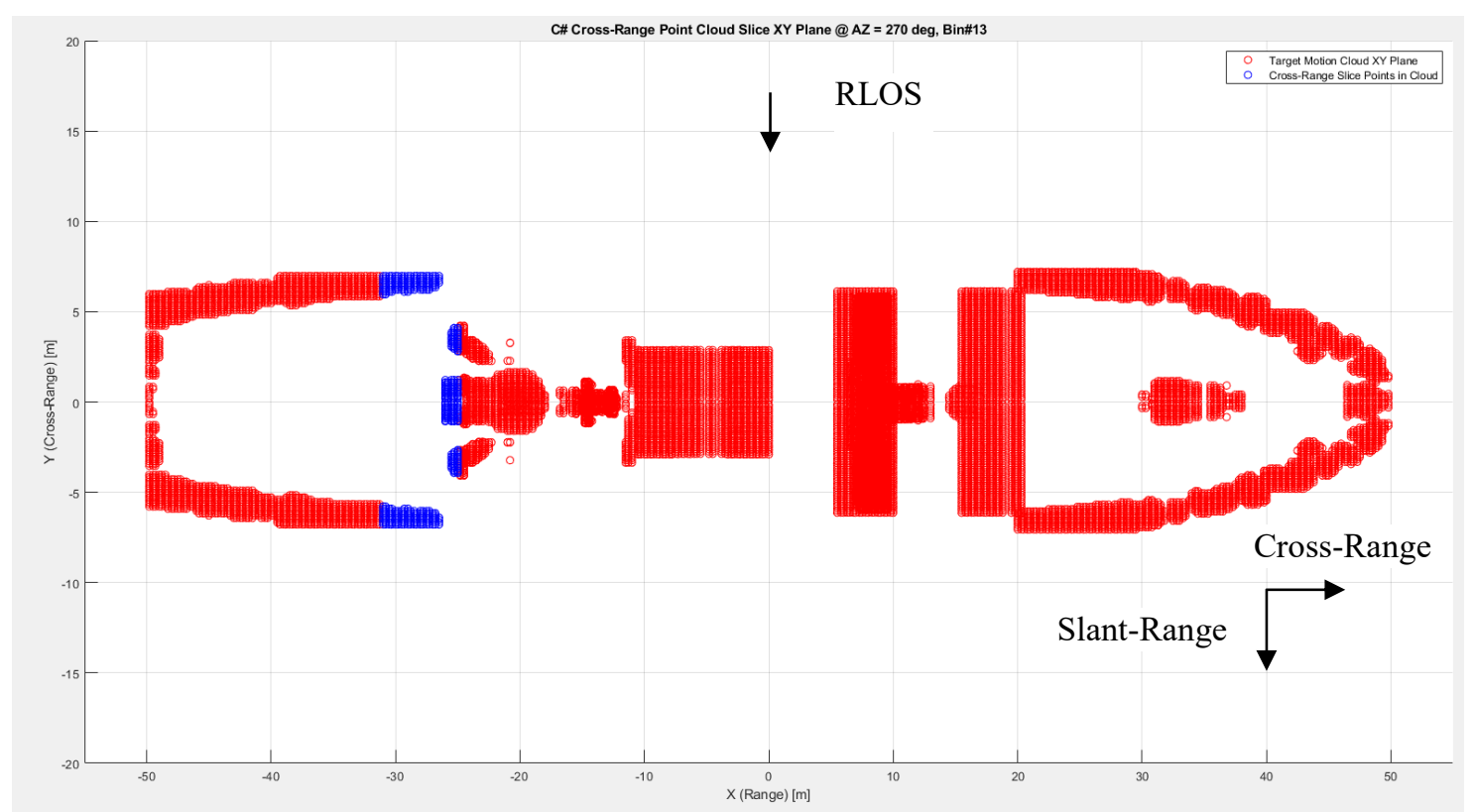

Figure 5.15: Radar LOS Azimuth is set to $90^{\circ}$. Cross-Range cells exist orthogonal to the LOS. Highlighted in blue is the 13th Cross-Range cell when the Cross-Range resolution is 16 bins. 


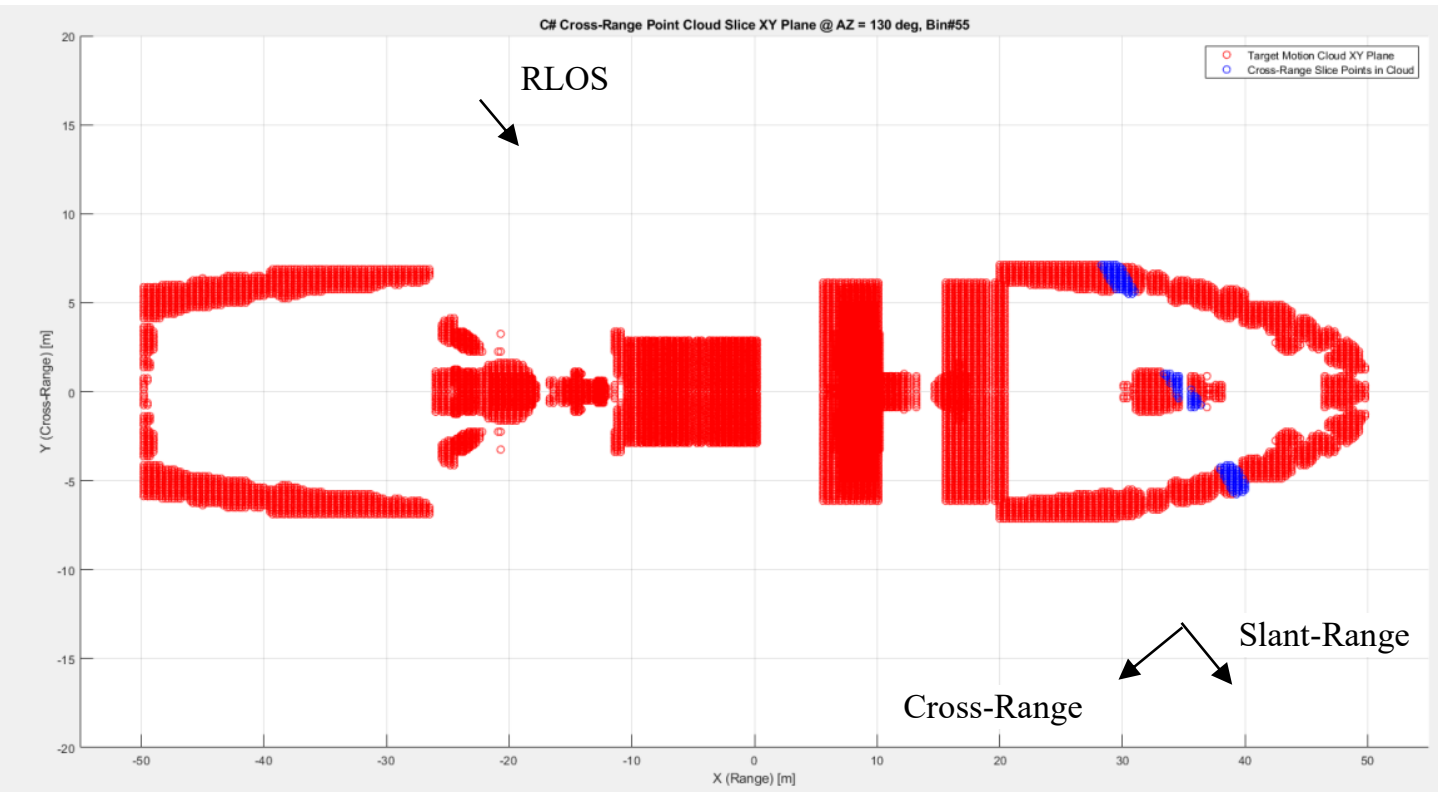

Figure 5.16: Radar LOS Azimuth is set to $130^{\circ}$. Cross-Range cells exist orthogonal to the LOS. Highlighted in blue is the 55th Cross-Range cell when the Cross-Range resolution is 64 bins.

The Cross-Range cells are represented as a slice of the target point cloud model until range resolution cells are implemented. Application of range resolution cells is shown in Figures 5.17 to 5.19 .

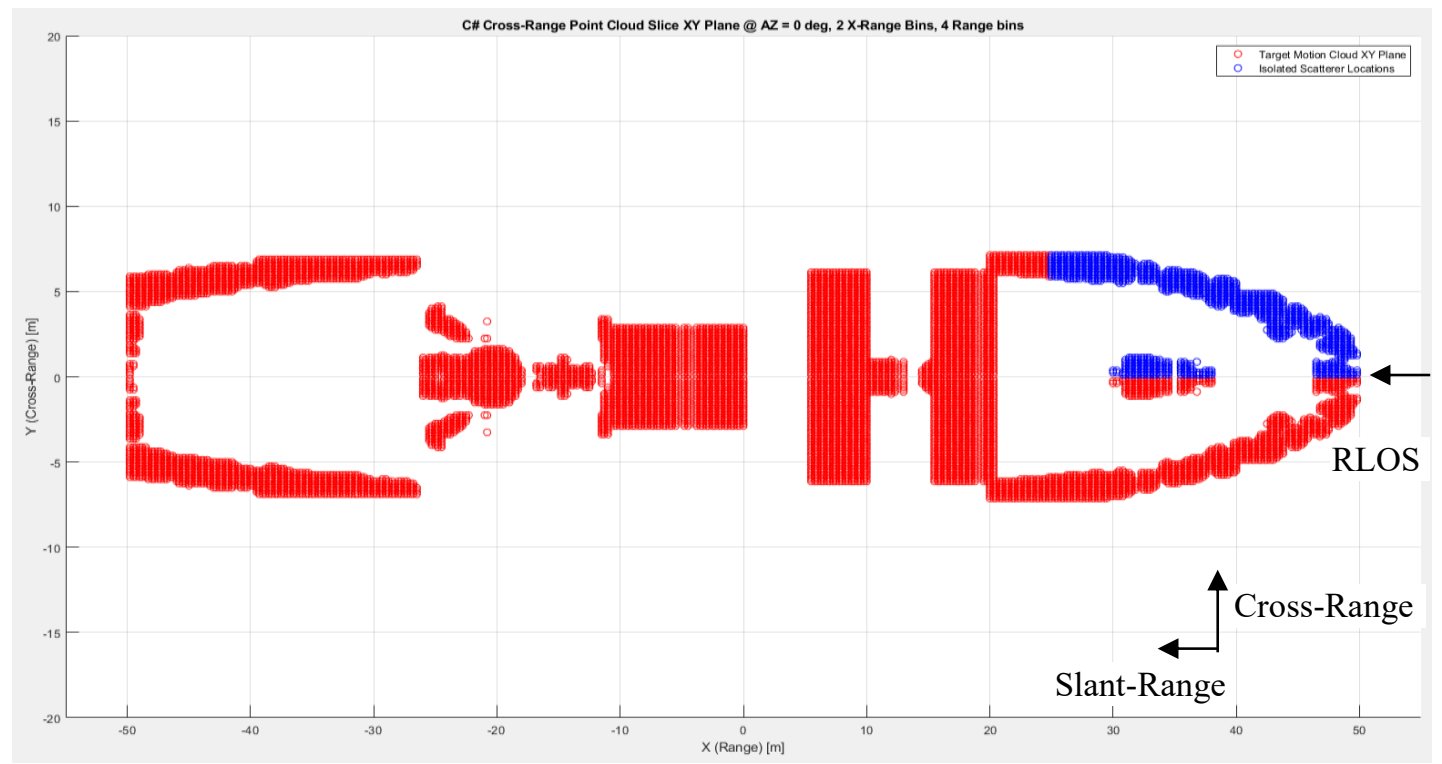

Figure 5.17: Radar LOS Azimuth is set to $0^{\circ}$. Cross-Range cells exist orthogonal to the LOS, and Range cells are parallel to the LOS. Highlighted in blue is the 1st Cross-Range cell when the Cross-Range resolution is 2 bins, and the 1st range cell for a range resolution of 4 bins. 


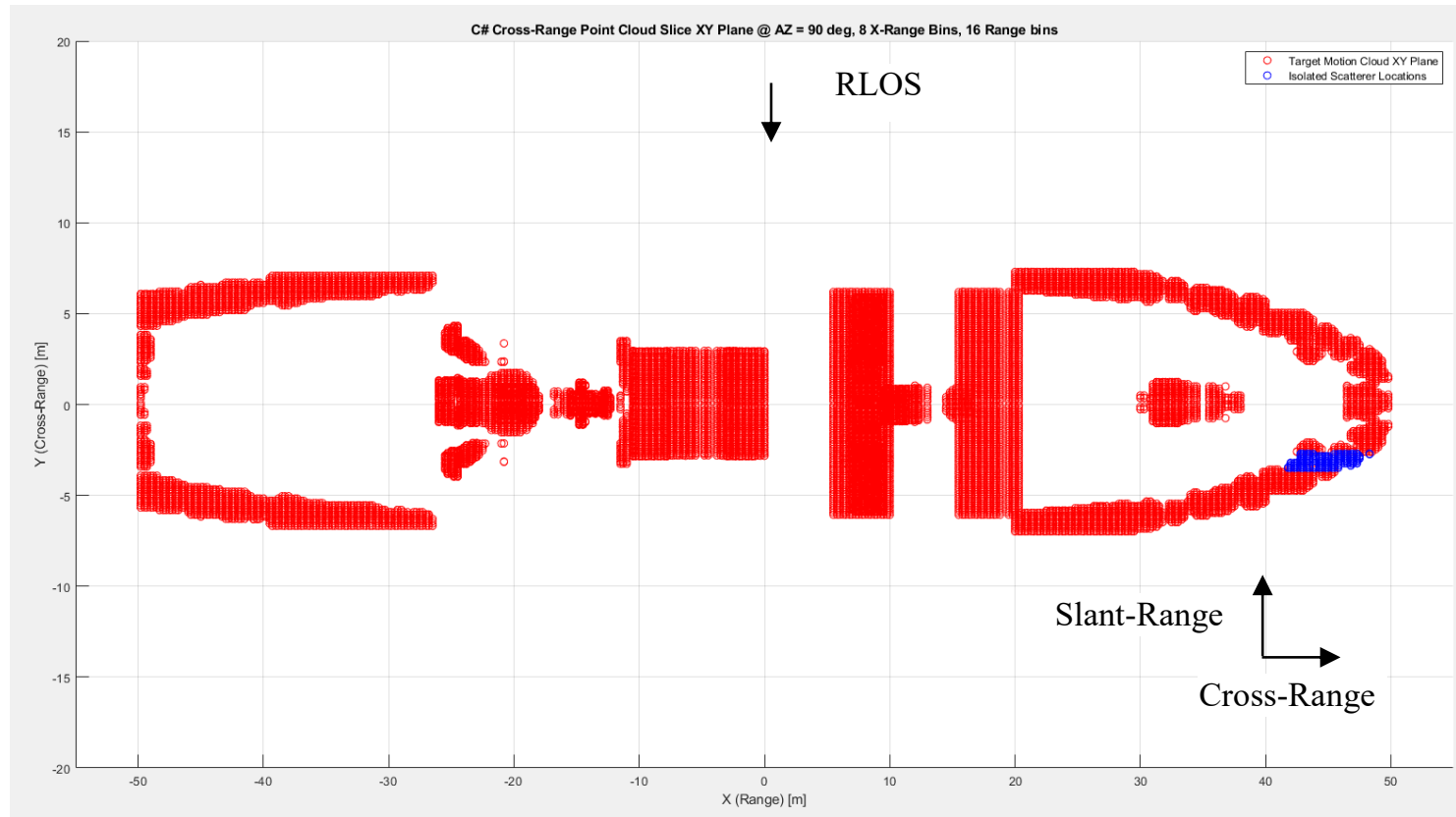

Figure 5.18: Radar LOS Azimuth is set to $90^{\circ}$. Highlighted in blue is the 2nd CrossRange cell when the Cross-Range resolution is 8 bins, and the 14th range cell for a range resolution of 16 bins.

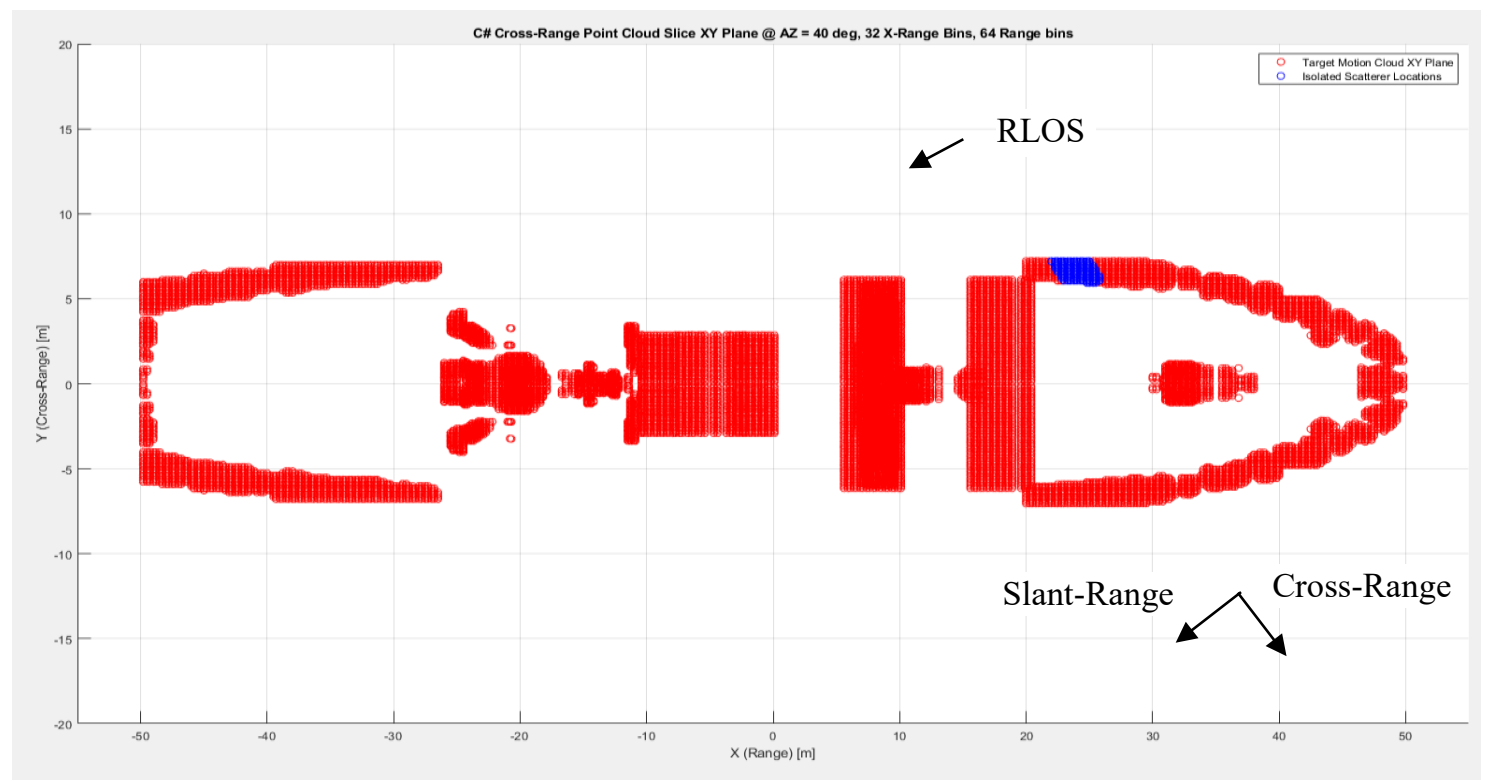

Figure 5.19: Radar LOS Azimuth is set to $40^{\circ}$. Highlighted in blue is the 12th CrossRange cell when the Cross-Range resolution is 32 bins, and the 14th range cell for a range resolution of 64 bins.

Selecting a fine resolution can result in cells that do not contain any scattering locations. The point cloud model contains the scattering locations present at the finest resolution with high-density sampling in range and cross-range dimensions. The 
simulated echoes will not achieve this resolution, as radars in application will not perform techniques to achieve as fine resolution as utilized in ANSYS to create the point cloud model. The necessary locations exist in the model to synthesize a high-resolution target echo, accounting for target locations that produce minimal backscatter.

Within a resolution cell, there are many scattering points that all contribute to the echo signal. Not all points are used in computation for echo signal synthesis, instead the dominant scattering location in each resolution cell is selected. The contribution of a scatterer to the amplitude of the echo signal is modelled by a target reflectivity function. This reflectivity function could be statistically defined for the target based on a Swerling case. The function would also be dependent on range, and aspect viewing angle, to include shadowing effects. However, the complexity of creating such a function model for all the scatterers in the model is extremely high and would require significant research and incorporation of Computational EM algorithms. Additional information on Radar Signature masking and/or minimization techniques used on the target is also required to accurately model the reflectivity. Without a scattering reflectivity function, the ship model is assumed to be comprised of simple PEC (Perfect Electric Conducting) materials; the scattering location amplitudes were determined using ISAR image simulation in ANSYS HFSS. Since the ship material and superstructures are all made of Steel, Aluminum, and other metal composite alloys, the PEC assumption is valid as a theoretical exercise only. In the range profiles generated, the effects of shadowing are not included, which can result in larger amplitude echoes for scattering locations that would otherwise be masked. This can also result in multiple scattering locations overlapping in the echo synthesis, which creates aliasing of the Doppler signature from a single dominant scattering location.

The dominant scatterer position is chosen as the highest position scattering point in the local resolution cell. Based on the assumption that the entire ship target is illuminated, the first contact point of an electromagnetic wave with a target will be at the locally highest locations on the ship structures. The amplitude of these locations is determined by summing the amplitude of all scattering locations at the same (X, Y) position as the selected dominant scatterer. The current implementation places taller structures of higher dominance, as the metal superstructures on the target will contribute 
the larger RCS in the echo signal. Once the dominant scatterers are selected, the amplitude, Doppler, phase, and delay to each scattering location contributing to the synthesis of the echo signal is computed. This data is exported to a CSV file format. In Figure 5.20, the dominant scatterers of the target model are shown for a specific simulation scenario. The white dots mark the selected dominant scatterer in each resolution cell in the point cloud.

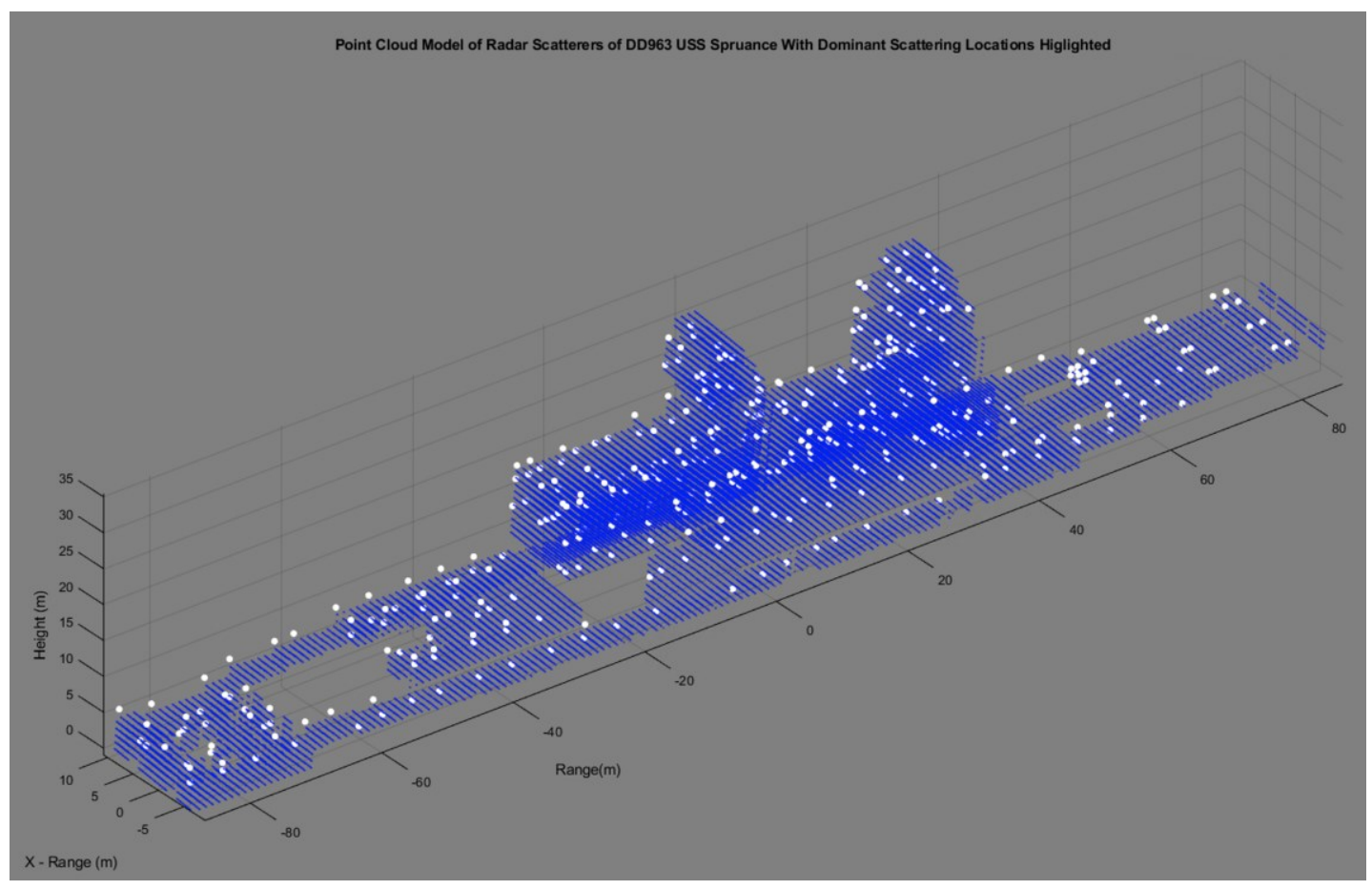

Figure 5.20: Dominant scatterers along a resolution slice of the target are highlighted in white. These points are selected in the Target Signature Synthesis software.

\subsection{Scatterer Modulation Parameter Calculation}

The target is a rigid body structure, allowing for the assumption of coherent scattering [43]. In coherent scattering, the contribution of each scatterer is summed to form a composite echo signal. The echo from a single scatterer, with the inclusion of time delay, phase modulation, Doppler shift and amplitude modulation, is described by equation 5.1 from [43].

$$
\begin{aligned}
\operatorname{Echo}(t)=k & \times A\left(t-\frac{2 R_{0,}}{c}\right) \times \exp \left(-j\left(\frac{4 \pi}{\lambda}\right) R_{0}\right) \times \exp \left(+j 2 \pi\left(\frac{2 v}{\lambda}\right) t\right) \\
& \times \exp \left(j\left(2 \pi F_{c} t+\varphi_{0}\right)\right)
\end{aligned}
$$


Equation 5.1 implicitly applies the stop-and-hop approximation of target motion [43]. In the stop-and-hop approximation, the target undergoes standard motion over time. The time frame in which a radar electromagnetic pulse interacts with the ship target is often on the order of microseconds. In such a small time frame, the motion of the target is extremely minimal. In essence, the target is frozen in time with scattering locations having position, velocity and acceleration fixed for the fraction of a second that the target is illuminated by a radar pulse. This would be analogous to taking a picture of the ship target undergoing motion at different times such that the ship is still in the photograph but still undergoing regular motion as a function of time.

\subsubsection{Amplitude Modulation}

The $k$ term in equation 5.1 compensates for the scatterer range reflectivity function and other amplitude effects in the radar range equation. This is not implemented, instead, the amplitudes are directly taken from ISAR simulation values from ANSYS HFSS. Developing a complex scatterer range reflectivity function is a topic for future research work.

\subsubsection{Envelope Delay \& Phase Modulation}

The second term $A\left(t-\frac{2 R_{0}}{c}\right)$, delays the envelope of the echo signal in time based on the range to the scatterer. In application, $R_{0}$ represents the distance from the hostile radar to the target. In this thesis $R_{0}$ is substituted with $r_{i}$, which represents the distance from the point of incidence on the target to the location of the $i^{\text {th }}$ scatterer. The scatterer closest to the LOS position is taken as the first scattering location in the profile, which is the point of incidence. This scatterer has no time or phase delay and is considered the reference scattering location. All other scattering locations have time delays, and phase modulation parameters computed with reference to the first scatterer position.

The third term $\exp \left(-j\left(\frac{4 \pi}{\lambda}\right) R_{0}\right)$, represents the phase shift induced on the radar pulse signal due to the range of the target. $R_{0}$ is replaced by $r_{i}$, to account for the range to 
each dominant scatterer. The received hostile radar signal is phase shifted relative to its transmission time and the distance between the hostile radar and the antenna of the simulated target. Thus, the incident scattering location has the phase shift and envelope delay already accounted for in the received waveform; however, the specific value of phase shift on the received waveform is unknown. All other scattering locations have a phase delay and envelope delay corresponding to the delay from the reference scattering location. Thus, the total range information in phase delay is preserved in the waveform. The phase delay and envelope delay from scattering locations on the target is only dependent on model dimensions and not the range to the hostile radar.

\subsubsection{Doppler Frequency Computation}

The fourth term $\exp \left(+j 2 \pi\left(\frac{2 v}{\lambda}\right) t\right)$, represents the Doppler shift induced on a complex radar pulse due to velocity of the scattering component. The motion is a complex function of time, as discussed in the equation of motion formulation. The stopand-hop approximation creates timestamps for which the dominant scatterer velocities and Doppler shifts are computed.

For a single scattering location, the Doppler frequency shift produced is a combination of the scatterer radial velocity and the rotational velocity normal to the hostile radar LOS. This is expressed in equation 5.2, as shown in [45].

$$
f_{D}=\frac{2[\boldsymbol{v}(t) \times \widehat{\boldsymbol{R}}(t)]}{c}+\frac{2 v_{r}}{c}
$$

The radial velocity, $v_{r}$, is the component of the scattering location velocity, which is along the hostile radar LOS. This can be considered to be the dot-product between the scattering location velocity and the LOS. The first term $\frac{2[v(t) \times \widehat{\mathbf{R}}(t)]}{c} f$, represents the Doppler shift produced by scattering location velocity, which is orthogonal to the radar LOS. The cross-product of the scattering location velocity with the radar LOS is used to compute this component of Doppler. The combined scattering velocity is a function of the effective target rotation vector and the distance of the scattering location in crossrange. [45]. 
In the Target Signature Synthesis program, the velocity of scattering locations is computed along each principle axis in the cartesian coordinate system. As per the equation of motion formulation, the scattering location velocity accounts for the crossrange position of the scattering location relative to the target center of gravity. The assumption of uniform target illumination implies the radar LOS intersects the target center of gravity. Thus, cross-range positions of scatterers relative to the LOS do not need to be recomputed in Doppler formulation. With a user-defined approximation of the hostile radar LOS angles, the unit vectors in the cartesian coordinate system are defined in Table 5.4.

Table 5.4: Unit vector definition of hostile radar LOS, along principle axes of cartesian coordinate system.

\begin{tabular}{|c|c|}
\hline Unit Vector Definition & Special Conditions \\
\hline$\widehat{\boldsymbol{R}}_{x}=\frac{1}{\cos \left(A Z^{\circ}\right)}$ & $A Z^{\circ}=0^{\circ}$ OR $180^{\circ}$ \\
$\widehat{\boldsymbol{R}}_{\boldsymbol{x}}=0$ \\
\hline$\widehat{\boldsymbol{R}}_{y}=\frac{1}{\sin \left(A Z^{\circ}\right)}$ & $A Z^{\circ}=90^{\circ}$ OR $270^{\circ}$ \\
\hline$\widehat{\boldsymbol{R}}_{z}=\frac{1}{\cos \left(E L^{\circ}\right)}$ & $E L^{\circ}=0$ \\
\hline & $\widehat{\boldsymbol{R}}_{z}=0$ \\
\hline
\end{tabular}

Following definition of the unit vectors for the radar LOS, the total Doppler of a scattering location is computed in the TSS program. The radial velocity of the scattering location is computed using trigonometric relations between the velocities in the principle axes and the radar LOS. The velocities of a scattering location along the principle axes are then used in a cross-product computation with the radar LOS unit vectors, according to the equation in [62]. Thus, the radial and the rotational velocities of the scattering locations are obtained and multiplied by the conversion factor of $2 / \lambda$, to obtain the Doppler frequency shift. 


\subsubsection{Carrier Frequency Term}

The final term $\exp \left(j\left(2 \pi F_{c} t+\varphi_{0}\right)\right)$, is the complex representation of the incoming carrier pulse at a carrier frequency of $F_{c}$. The phase shift can take on any constant value from 0 to $2 \pi$. Equation 5.3 represents the coherent combination of dominant scatterers to synthesize an echo signal.

$$
\begin{aligned}
& \operatorname{Echo}(t)=\sum_{i=1}^{N} A_{i}\left(t-\frac{2 r_{i}}{c}\right) \times \exp \left(-j\left(\frac{4 \pi}{\lambda}\right) r_{i}\right) \times \exp \left(+j 2 \pi\left(\frac{2 v_{i}}{\lambda}\right) t\right) \\
& \times \exp \left(j\left(2 \pi F_{c} t+\varphi_{0}\right)\right)
\end{aligned}
$$

\subsection{Profile Decorrelation Time}

The stop-and-hop approximation for profile synthesis is utilized so new profile modulation parameters are not required to be computed for every received hostile radar pulse. However, the Doppler and range delay of dominant scatterer positions change as functions of time. The hostile radar resolution limits the decorrelation time to the total time taken for a dominant scatterer to migrate to a new resolution cell. The Doppler history is generated for each scattering location across multiple profiles to determine the maximum decorrelation time. The decorrelation time determines the minimum time-step required between computation of modulation parameters for profile synthesis. At integer multiples of the decorrelation time, the stop-and-hop approximation is implemented to compute new modulation parameters for echo signal synthesis, which are utilized for all pulses received within the decorrelation timeframe.

Doppler history of a target is dependent on multiple factors: Sea State, Ship Velocity, Wave Heading relative to ship, the LOS Azimuth and Elevation Angle and the resolution in both slant-range, cross-range, and height. The total number of possible combinations for testing all cases is exceptionally large. For all possible combinations, a total of 39,328,380 unique target profiles would have to be simulated, and a Doppler history generated. 
Table 5.5: Number of values which can be selected for each variable contributing to the target profile synthesis.

\begin{tabular}{|l|l|l|}
\hline Parameter & $\begin{array}{l}\text { Number of Potential } \\
\text { Variable Values }\end{array}$ & Variable Values \\
\hline Sea State & 7 & $1,2,3,4,5,6,7$ \\
\hline Ship Velocity (kts) & 5 & $5,10,15,20,25$ \\
\hline Wave Heading Relative to & 13 & $15,30,45,60,75,90$, \\
Ship (deg) & & $105,120,135,150,165,180$ \\
\hline LOS Azimuth Angle & 36 & $0,10,20,30,40,50,60,70,80$, \\
& & $90,100,110,120,130,140$, \\
& & $150,160,170,180$ \\
\hline LOS Elevation Angle (deg) & 7 & $0,10,20,30,40,50,60$ \\
\hline Range Resolution & 7 & $2,4,8,16,32,64,128$ \\
\hline Cross-Range Resolution & 7 & $2,4,8,16,32,64,128$ \\
\hline Height Resolution & 7 & $2,4,8,16,32,64,128$ \\
\hline
\end{tabular}

Simulating the target experiencing maximum motion, in velocity and Sea State, the minimum de-correlation time for Doppler history can be found. However, there are still a possible 12,348 test cases based on LOS angle and resolution combinations alone. Cube resolution is assumed, the cases being $4 \times 4 \times 4,16 \times 16 \times 16$ and $64 \times 64 \times 64$, in range and Doppler resolution. With this resolution, the maximum number of scatterers coherently contributing to the synthesized echo is 262,144 . Four cases of LOS angle combinations are chosen. Two simulation cases will have a non-zero elevation angle. In each case pair, one profile is generated from a 'front' view and the other from a 'rear/side' view.

When a scatterer changes resolution cells or the number of total scatterers in the profile changes, the corresponding time stamp is taken as a maximum decorrelation time for the simulation case. Doppler history for each scattering location will only be generated for the period prior to the maximum decorrelation time.

The percentage of Doppler change for each scattering location is found as a function of time. The scattering location for the first timestamp is the reference Doppler frequency. In subsequent profiles, the amount of Doppler change, as a percentage, is found relative to the reference Doppler frequency for the corresponding scattering location. Equations 5.4 and 5.5 show the computation of percentage Doppler change for a 
single scatterer over a period of $\mathrm{n}$ timestamps. Timestamps are incremented by 0.005 seconds. This is equivalent to the period of a $200 \mathrm{~Hz}$ Doppler signal or a dominant scatterer velocity of $3 \mathrm{~m} / \mathrm{s}$.

$$
\begin{gathered}
\Delta f_{d, n}=\left|f_{d, n}-f_{d, 0}\right| \\
\% \Delta f_{d, n}=\Delta P f_{d, n}=\left(\frac{\Delta f_{d, n}}{f_{d, 0}}\right) * 100
\end{gathered}
$$

The percentage change in Doppler will be plotted for each unique scatterer as a function of time. The number of data points significantly increases as the resolution increases. To collect this data into a more readable format, the average percentage change in Doppler for each timestamp will be computed. The average percentage change in Doppler for the profile is found as a function of time, as seen in equation 5.6.

$$
\Delta P f_{d, a v g}=\frac{1}{N_{\text {scat }}} \sum_{n=1}^{N_{\text {scat }}} \Delta P f_{d, n}
$$

Four unique test cases are created with a combination of motion, resolution, and LOS conditions. For all cases, the maximum decorrelation time is found as the time at which the number of dominant scatterers in the profile changes. The minimum decorrelation time is computed from the Doppler history of each case. The test cases are outlined in Table 5.6. 
Table 5.6: Maximum decorrelation time for four test cases.

\begin{tabular}{|c|c|c|c|c|}
\hline $\begin{array}{c}\text { Case } \\
\text { Number }\end{array}$ & $\begin{array}{l}\text { Motion and LOS } \\
\text { Conditions }\end{array}$ & $\begin{array}{l}\text { Resolution } \\
\text { Size (Slant- } \\
\text { range bins } x \\
\text { cross-range } \\
\text { bins) }\end{array}$ & $\begin{array}{c}\text { Initial } \\
\text { Number } \\
\text { of } \\
\text { Scatterers } \\
\text { in Profile }\end{array}$ & $\begin{array}{l}\text { Time of Change } \\
\text { for Number of } \\
\text { Scatterers in } \\
\text { Profile [seconds] } \\
\text { (i.e., Maximum } \\
\text { Decorrelation } \\
\text { Time) }\end{array}$ \\
\hline \multirow[t]{3}{*}{1} & \multirow{3}{*}{$\begin{array}{l}\text { Sea State: } 7 \\
\text { Ship Velocity: } 25 \mathrm{Kts} \\
\text { Wave Heading Relative } \\
\text { to Ship: } 60 \mathrm{deg}(120 \\
\text { input) } \\
\text { LOS AZ: } 0 \mathrm{deg} \\
\text { LOS EL: } 30 \mathrm{deg}\end{array}$} & $4 \times 4$ & 12 & 1.07 \\
\hline & & $16 \times 16$ & 134 & 0.22 \\
\hline & & $64 \times 64$ & 1242 & 0.02 \\
\hline \multirow[t]{3}{*}{2} & \multirow{3}{*}{$\begin{array}{l}\text { Sea State: } 7 \\
\text { Ship Velocity: } 25 \mathrm{Kts} \\
\text { Wave Heading Relative } \\
\text { to Ship: } 60 \mathrm{deg}(120 \\
\text { input) } \\
\text { LOS AZ: } 0 \mathrm{deg} \\
\text { LOS EL: } 10 \mathrm{deg}\end{array}$} & $4 \times 4$ & 9 & 0.875 \\
\hline & & $16 \times 16$ & 71 & 0.415 \\
\hline & & $64 \times 64$ & 556 & 0.025 \\
\hline \multirow[t]{3}{*}{3} & \multirow{3}{*}{$\begin{array}{l}\text { Sea State: } 7 \\
\text { Ship Velocity: } 25 \mathrm{Kts} \\
\text { Wave Heading Relative } \\
\text { to Ship: } 60 \mathrm{deg}(120 \\
\text { input) } \\
\text { LOS AZ: } 150 \mathrm{deg} \\
\text { LOS EL: } 0 \mathrm{deg}\end{array}$} & $4 \times 4$ & 12 & 1 \\
\hline & & $16 \times 16$ & 134 & 0.22 \\
\hline & & $64 \times 64$ & 1242 & 0.02 \\
\hline \multirow[t]{3}{*}{4} & \multirow{3}{*}{$\begin{array}{l}\text { Sea State: } 7 \\
\text { Ship Velocity: } 25 \mathrm{Kts} \\
\text { Wave Heading Relative } \\
\text { to Ship: } 60 \mathrm{deg}(120 \\
\text { input) } \\
\text { LOS AZ: } 40 \mathrm{deg} \\
\text { LOS EL: } 0 \mathrm{deg}\end{array}$} & $4 \times 4$ & 9 & 0.95 \\
\hline & & $16 \times 16$ & 65 & 0.455 \\
\hline & & $64 \times 64$ & 516 & 0.03 \\
\hline
\end{tabular}


Figure 5.21 to 5.23 on the following pages show the percentage change in Doppler plotted for individual scatterers.

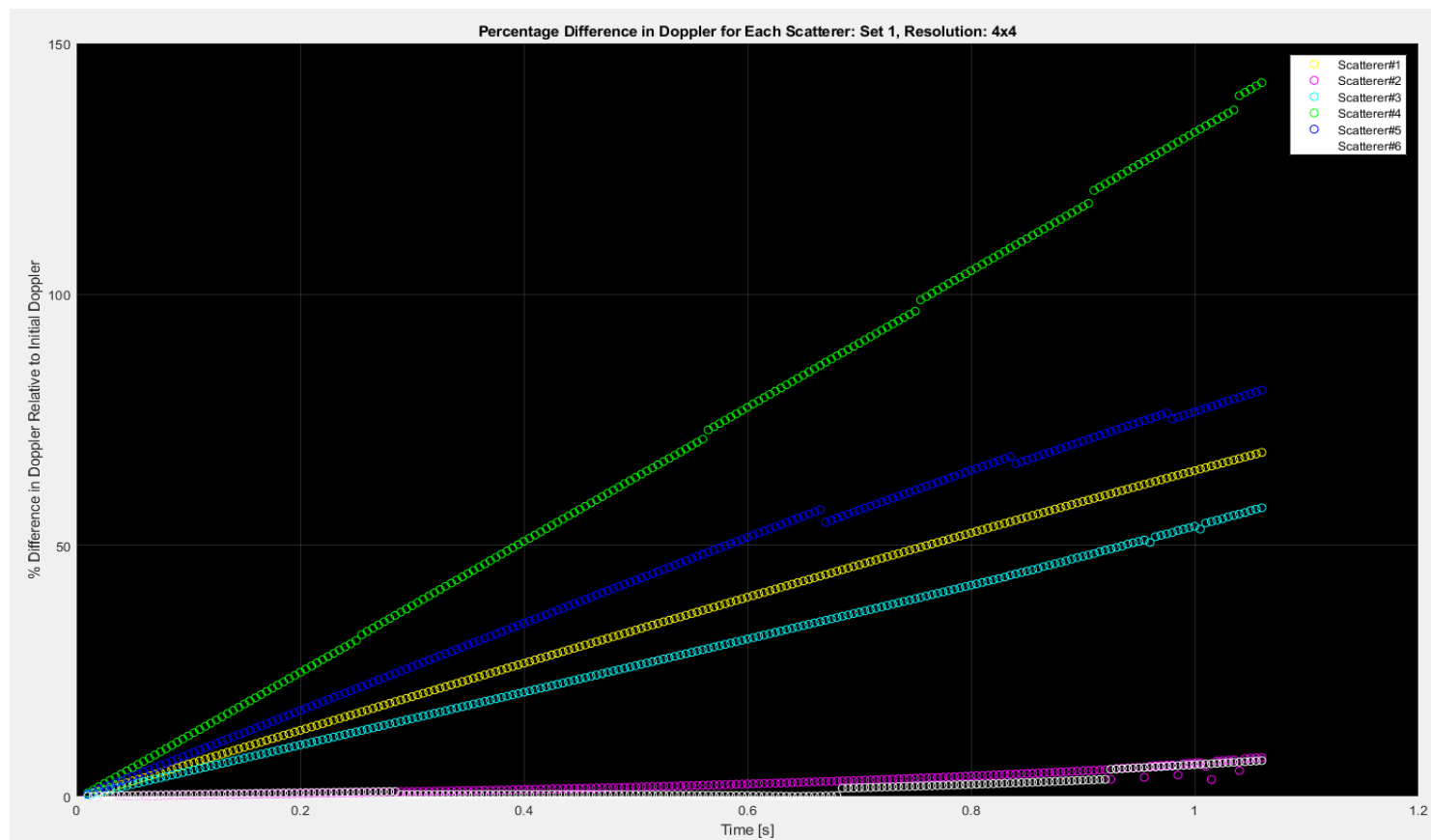

Figure 5.21: Case 1, 4x4 resolution cell, percent error in Doppler for each resolution cell.

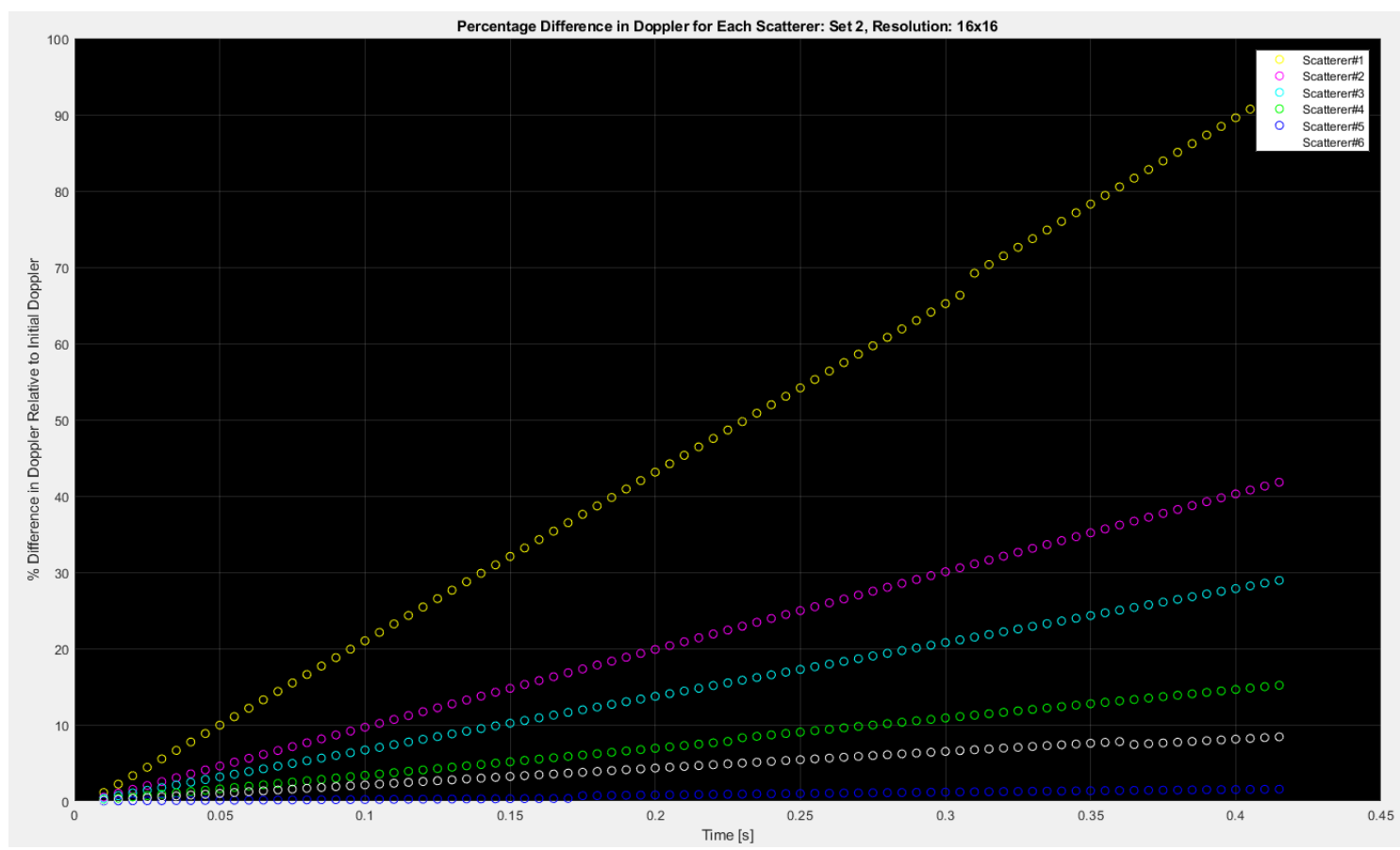

Figure 5.22: Case 2, 16x16 resolution cell, percent error in Doppler for each resolution cell. Only shown for 6 scatterers. 


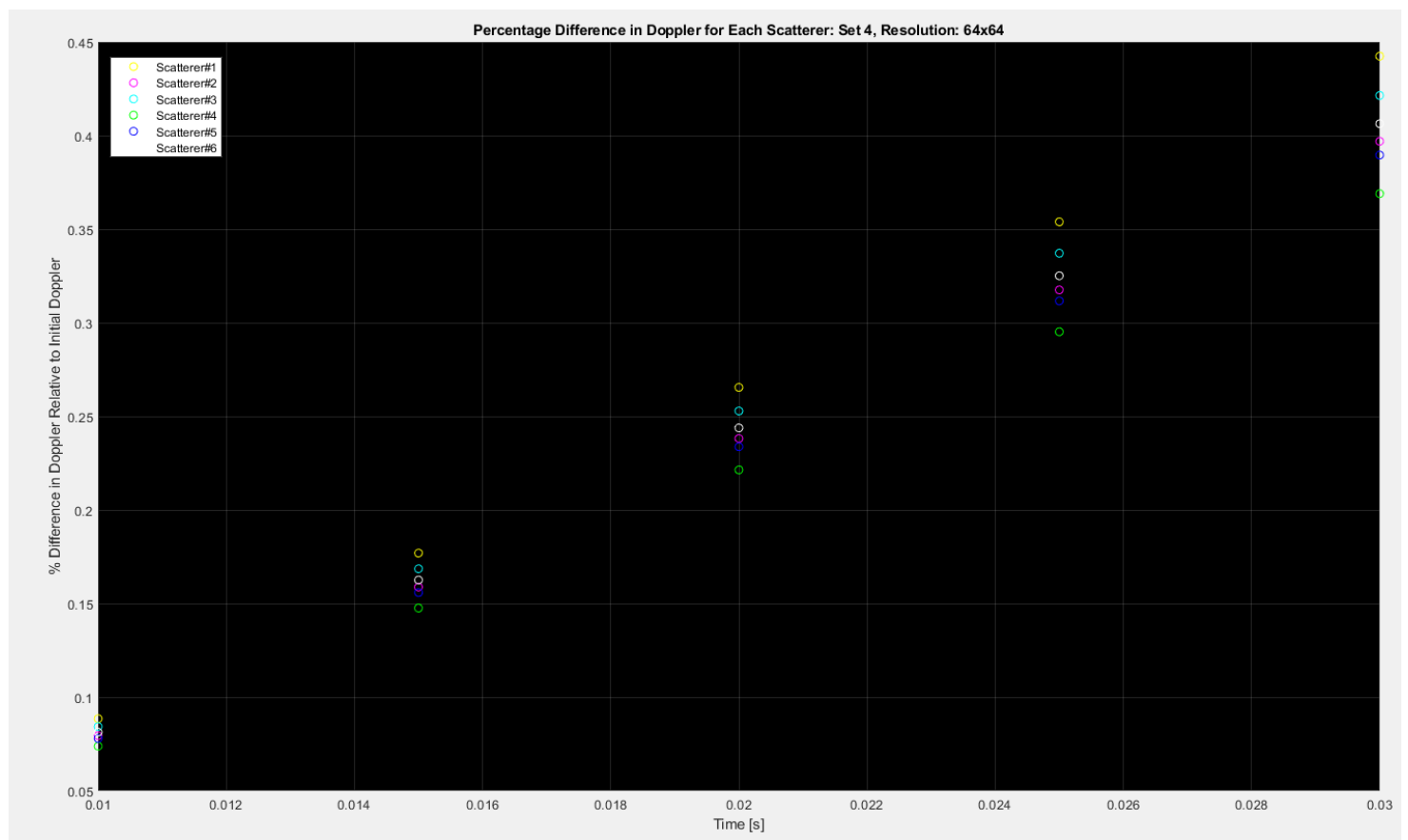

Figure 5.23: Set 4, 64x64 resolution cell, percent error in Doppler for each resolution cell. Only shown for 6 scatterers.

The average percentage Doppler changes, including all scatterers, for each test case is plotted in Figure 5.24 to 5.26 for case one.

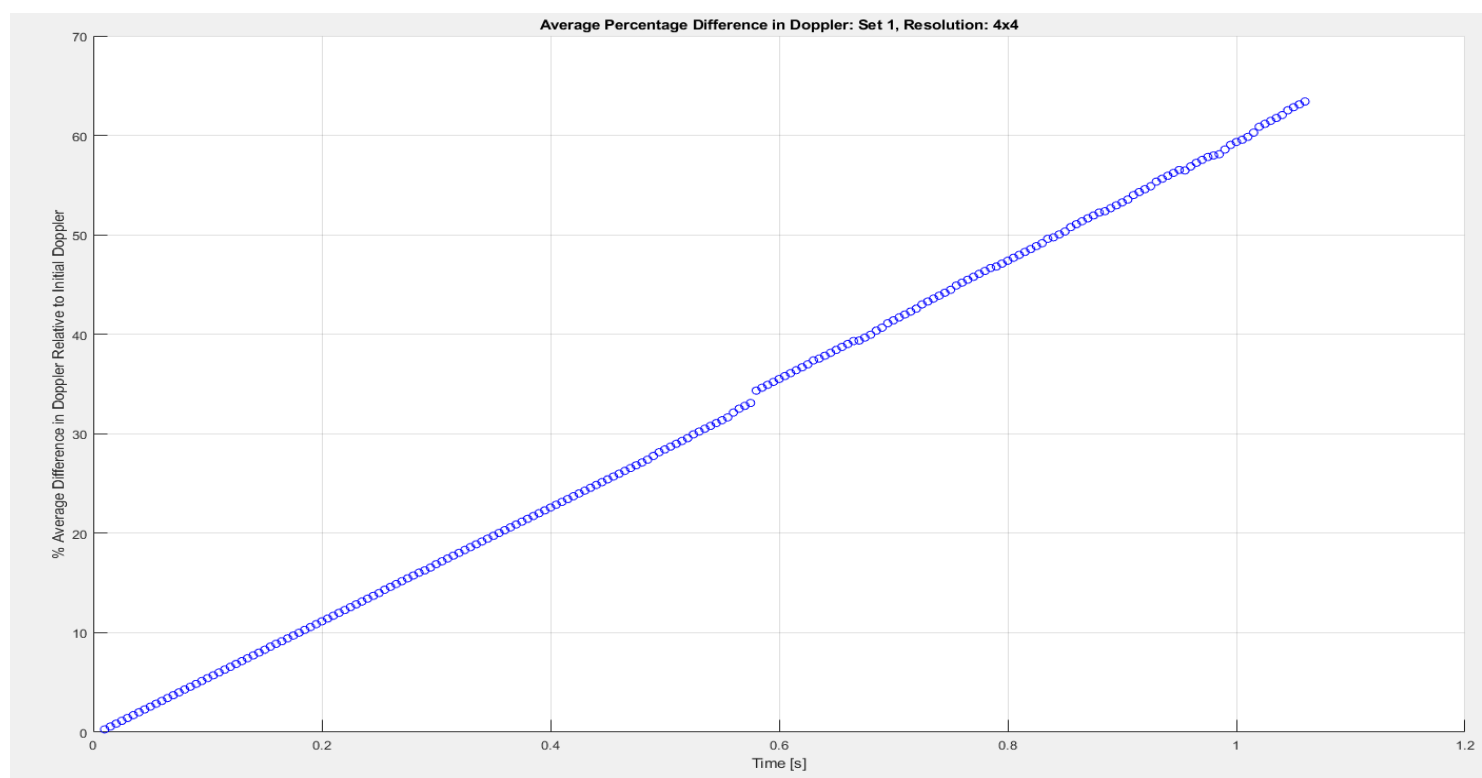

Figure 5.24: The average percentage difference in Doppler for all scatterers in Case 1 , using $4 \times 4$ resolution cell. 


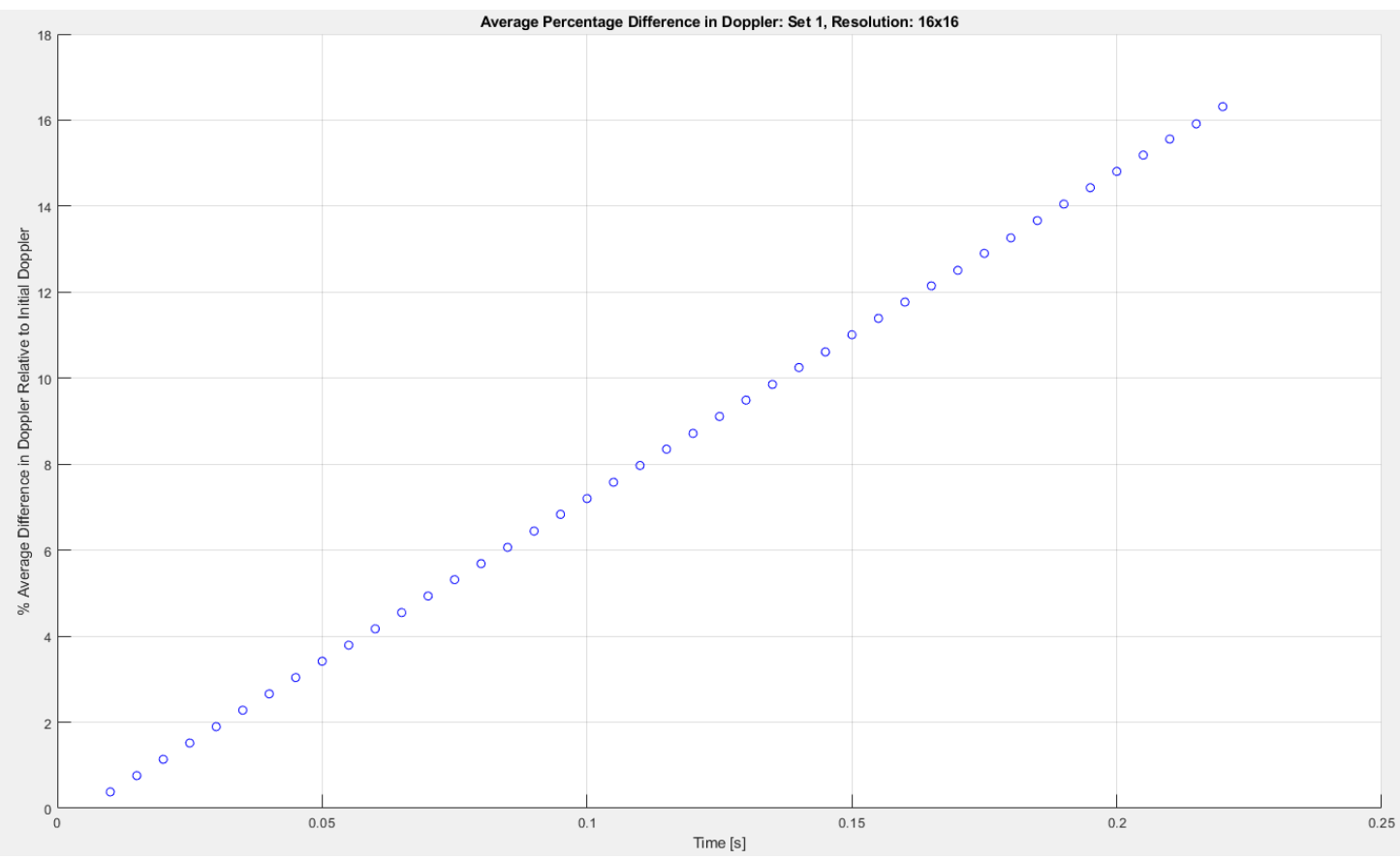

Figure 5.25: The average percentage difference in Doppler for all scatterers in Case 1 , using 16x16 resolution cell.

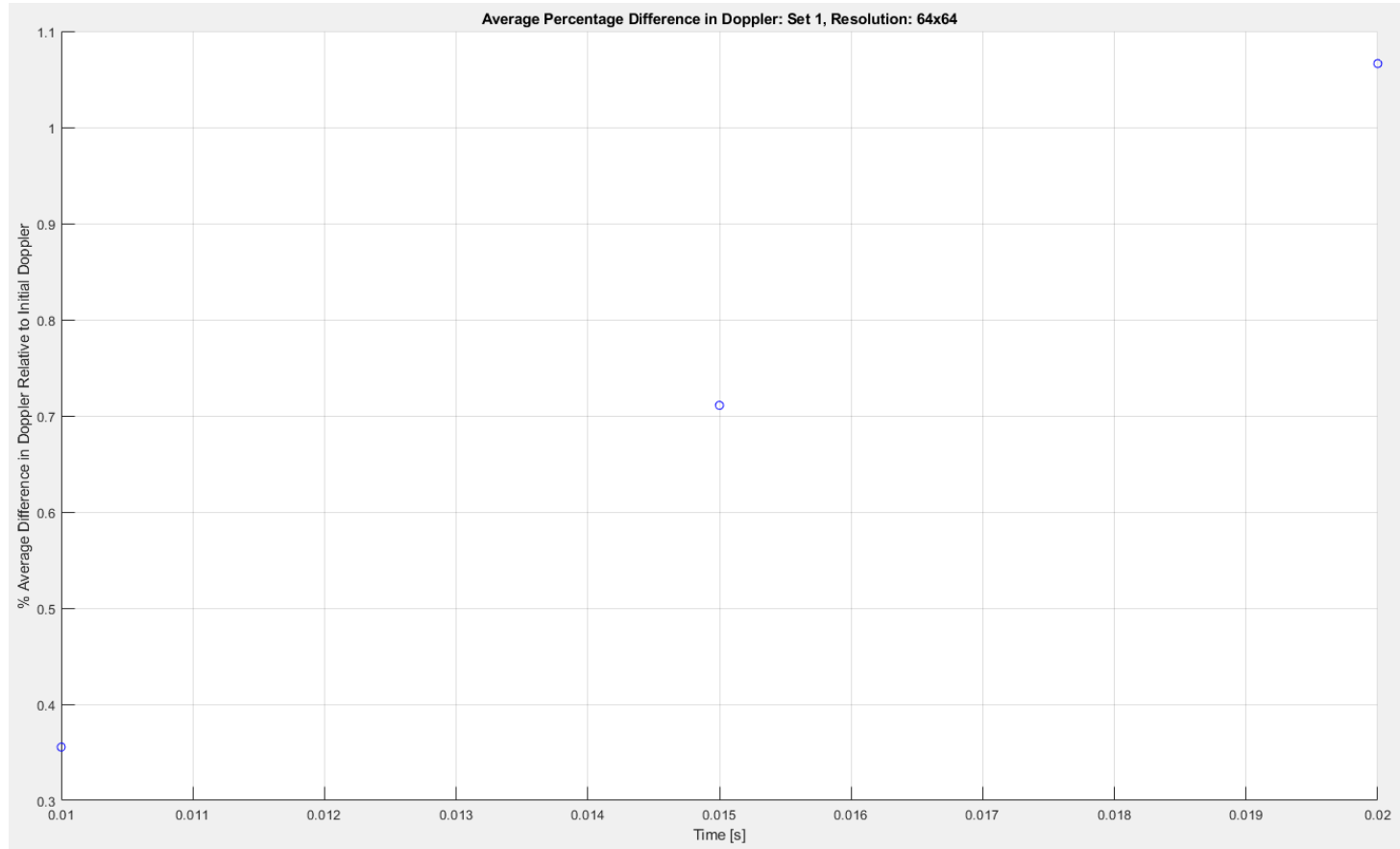

Figure 5.26: The average percentage difference in Doppler for all scatterers in Case 1 , using 64x64 resolution cell.

The decorrelation time of a profile is highly dependent on resolution. As the resolution increases, the decorrelation time decreases. The minimum decorrelation time is 
dependent on the change in Doppler as a function of time. The change in Doppler is measured through a DFT of the received pulses in slow-time, that is, across each PRI. The ability to define Doppler changes depends on the size of the DFT, determining computational resolution, and the window used prior to computing the DFT, determining spectral resolution [46][47]. Common windows in radar applications are the Hann and Hamming windows [47]. The spectral resolution of these windows is dependent on the main-lobe width, and the order of the window. Hamming and Hann windows have a resolution of $\frac{8 \pi}{M}$, where $\mathrm{M}$ is the number of points in the Window/DFT used.

The spectral resolution in frequency will depend on the sampling frequency used. In target Doppler identification, the sampling frequency matches the PRF of the hostile radar. Higher PRF results in finer resolution to a diminishing point. Equation 5.7 defines the spectral frequency resolution in Hertz based on number of points and the sampling rate.

$$
\Delta f_{H z}(M, P R F)=\Delta \omega_{\text {res }} *\left(\frac{P R F}{2 \pi}\right)=\left(\frac{8 \pi}{M}\right) *\left(\frac{P R F}{2 \pi}\right)=\frac{4 * P R F}{M}
$$

Figure 5.27 shows the spectral resolution for both windows as a function of sampling rate, equivalently PRF. As the number of data points used in the window increases, the resolution increases to a point of diminishing return. 


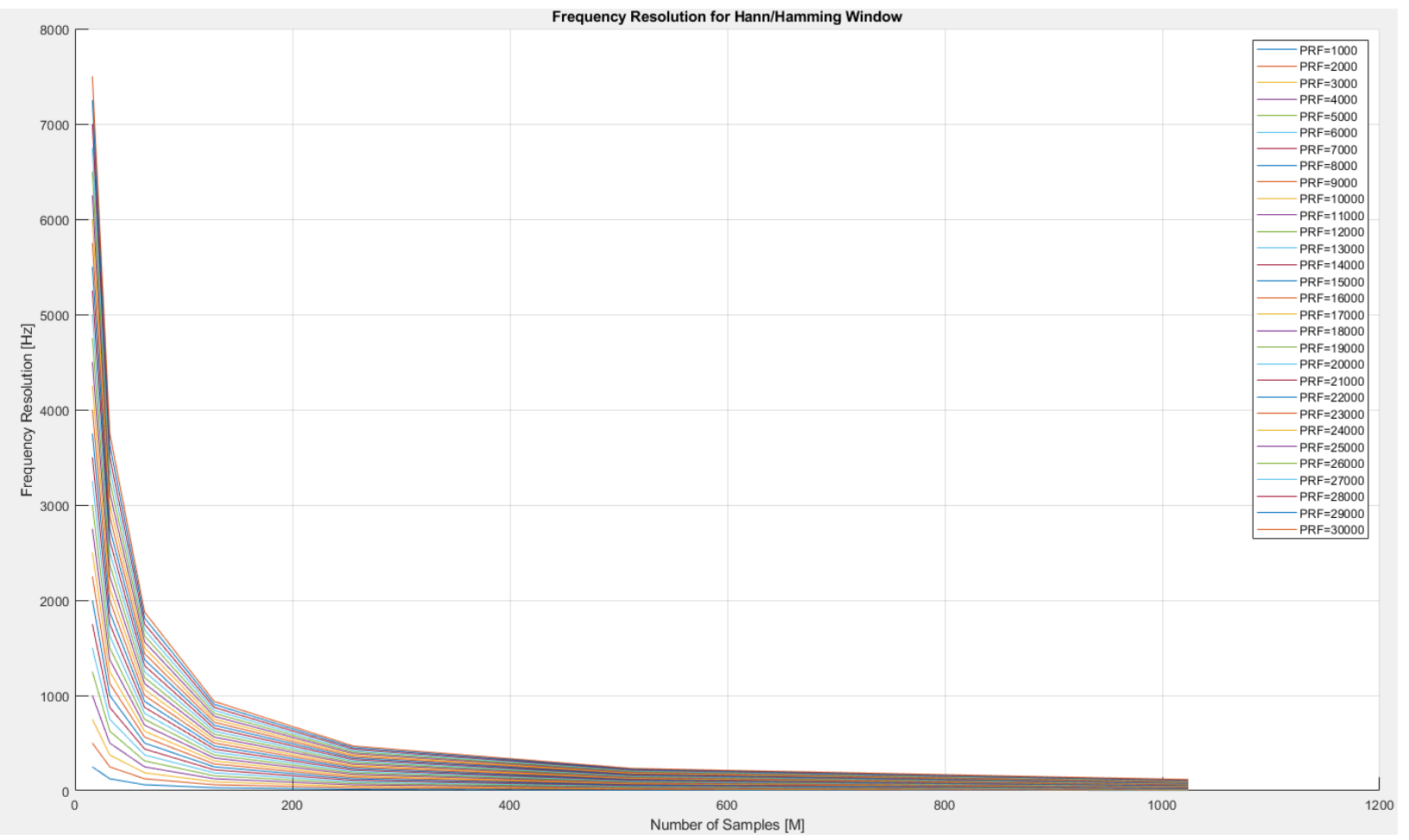

Figure 5.27: Frequency resolution for Hann/Hamming window in $\mathrm{Hz}$, for varying window size, and sampling frequency (i.e., PRF).

In marine radar applications, the PRF is on the order of kilohertz [45][49]. The maximum Doppler frequency produced by the simulated target is approximately $10 \mathrm{kHz}$. To observe this Doppler frequency without spectral aliasing would require a PRF of at least $20 \mathrm{kHz}$ or higher.

The number of points used in a DFT computation is rounded to a power of two to utilize FFT algorithms. The maximum number of points used in application is 512 data points, including possible zero-padding [43][45]. Using more data points increases the computation time and is avoided in radar processing which is a time-restricted environment. The spectral resolution for the Hann and Hamming windows at the operating PRFs and various FFT sizes are computed in Table 5.7. 
Table 5.7: Spectral resolution of Hann and Hamming windows for practical PRFs and FFT sizes.

\begin{tabular}{|c|c|c|c|c|c|}
\hline $\begin{array}{l}\text { Number } \\
\text { of Points } \\
\text { in } \\
\text { Window/D } \\
\text { FT }\end{array}$ & $\begin{array}{l}\text { Hann/Ham } \\
\text { ming } \\
\text { Window } \\
\text { Spectral } \\
\text { Resolution } \\
\text { [rad/sample] }\end{array}$ & $\begin{array}{l}\text { Hann/Ham } \\
\text { ming } \\
\text { Window } \\
\text { Spectral } \\
\text { Resolution } \\
\text { [Hz] for } \\
\text { sampling } \\
\text { rate (PRF) } \\
\text { of 5kHz }\end{array}$ & $\begin{array}{l}\text { Hann/Ham } \\
\text { ming } \\
\text { Window } \\
\text { Spectral } \\
\text { Resolution } \\
\text { [Hz] for } \\
\text { sampling } \\
\text { rate (PRF) } \\
\text { of } 10 \mathrm{kHz}\end{array}$ & $\begin{array}{l}\text { Hann/Ham } \\
\text { ming } \\
\text { Window } \\
\text { Spectral } \\
\text { Resolution } \\
\text { [Hz] for } \\
\text { sampling } \\
\text { rate (PRF) } \\
\text { of } 15 \mathrm{kHz}\end{array}$ & $\begin{array}{l}\text { Hann/Ham } \\
\text { ming } \\
\text { Window } \\
\text { Spectral } \\
\text { Resolution } \\
\text { [Hz] for } \\
\text { sampling } \\
\text { rate (PRF) } \\
\text { of } 20 \mathrm{kHz}\end{array}$ \\
\hline 32 & $0.25 \pi$ & 625 & 1250 & 1875 & 2500 \\
\hline 64 & $0.125 \pi$ & 312.5 & 625 & 937.5 & 1250 \\
\hline 128 & $0.0625 \pi$ & 156.25 & 312.5 & 468.75 & 625 \\
\hline 256 & $0.03125 \pi$ & 78.125 & 156.25 & 234.375 & 312.5 \\
\hline 512 & $0.015625 \pi$ & 39.0625 & 78.125 & 117.1875 & 156.25 \\
\hline
\end{tabular}

The average percentage change in Doppler frequency is dependent on the maximum Doppler frequency in the synthesized profile. As the Doppler frequency increases, the percentage change increases as well. If the change in Doppler frequency is greater than the presumed resolution of the hostile radar, new dominant scatterers and modulation parameters are computed. For all cases, the average time for varying percentage changes in Doppler is computed. The average percent change in Doppler is found as a value in $\mathrm{Hz}$ of the possible maximum Doppler frequency in the profile. This is shown in Table 5.8. 
Table 5.8: Average time for a percentage change in the Doppler of a Profile. The change in Doppler is computed for various maximum Doppler frequencies.

\begin{tabular}{|c|c|c|c|c|c|}
\hline $\begin{array}{l}\text { Average } \\
\text { Percent } \\
\text { Difference } \\
\text { in Doppler } \\
\text { All } \\
\text { scatterers, } \\
\text { All Cases, } \\
\text { All LOS } \\
\text { angles }\end{array}$ & $\begin{array}{l}\text { Average } \\
\text { Time for } \\
\text { All Cases } \\
\text { [seconds] }\end{array}$ & $\begin{array}{l}\text { Maximum } \\
\text { Doppler } \\
\text { Frequency } \\
\text { is 5kHz. } \\
\text { Maximum } \\
\text { percentage } \\
\text { Doppler } \\
\text { shift [Hz]. }\end{array}$ & $\begin{array}{l}\text { Maximum } \\
\text { Doppler } \\
\text { Frequency } \\
\text { is 10kHz. } \\
\text { Maximum } \\
\text { percentage } \\
\text { Doppler } \\
\text { shift [Hz]. }\end{array}$ & $\begin{array}{l}\text { Maximum } \\
\text { Doppler } \\
\text { Frequency } \\
\text { is 15kHz. } \\
\text { Maximum } \\
\text { percentage } \\
\text { Doppler } \\
\text { shift [Hz]. }\end{array}$ & $\begin{array}{l}\text { Maximum } \\
\text { Doppler } \\
\text { Frequency } \\
\text { is } 20 \mathrm{kHz} . \\
\text { Maximum } \\
\text { percentage } \\
\text { Doppler } \\
\text { shift [Hz]. }\end{array}$ \\
\hline $0.5 \%$ & 0.0112 & 25 & 50 & 75 & 100 \\
\hline $1 \%$ & 0.0158 & 50 & 100 & 150 & 200 \\
\hline $1.5 \%$ & 0.0192 & 75 & 150 & 225 & 300 \\
\hline $2 \%$ & 0.0225 & 100 & 200 & 300 & 400 \\
\hline $2.5 \%$ & 0.0258 & 125 & 250 & 375 & 500 \\
\hline
\end{tabular}

Assuming exceptionally fine resolution, the minimum decorrelation time can be determined to be approximately 0.01 seconds. As the resolution decreases, the minimum decorrelation time increases to approximately 0.025 seconds. In practice, the resolution achieved by a hostile radar is not known. It is appropriate to assume the hostile radar system can achieve fine resolution and resolve any aliasing present in the Doppler spectrum. The decorrelation time is selected to be 0.01 seconds. Thus, for a time frame of 0.01 seconds, the stop-and-hop approximation is utilized to compute the synthesized profile's modulation parameters. All pulses received within this time frame will be modulated by the same parameters of amplitude, phase, delay, and Doppler. New modulation parameters are computed in time steps of 0.01 seconds to counter highresolution hostile radars with longer dwell or coherent integration times.

\subsection{Modulation Parameter Estimation Error}

As previously mentioned, radars can utilize larger bandwidths to achieve fine resolution of targets. The frequency bandwidth of the hostile radar is not known, although general operating parameters of marine radars limit these to X-band and average transmission bandwidths of $300 \mathrm{MHz}$ [45][49][50]. 
Modulation parameters for scatterers are dependent on the radar frequency. The amplitude is proportional to the RCS of the scatterer [45][49][50]. Doppler, phase, and time delay equations are direct functions of the radar wavelength [45][49][50]. As the hostile radar changes frequency, an ideal false target simulator system would compute modulation parameters based on the received frequency. In practice, the software takes a user-defined estimate of the hostile radar frequency and uses this to compute the modulation parameters. This is because the time taken to perform spectral estimation and recompute modulation parameters is longer than the PRF of the hostile radar.

The difference between received frequency and the wavelength used to compute the modulation parameters is a source of error in the computation of modulation parameters. The assumed center frequency of operation is $10 \mathrm{GHz}$. Figure 5.28 shows the percentage error in wavelength relative to a $10 \mathrm{GHz}$ carrier over a bandwidth of $500 \mathrm{MHz}$

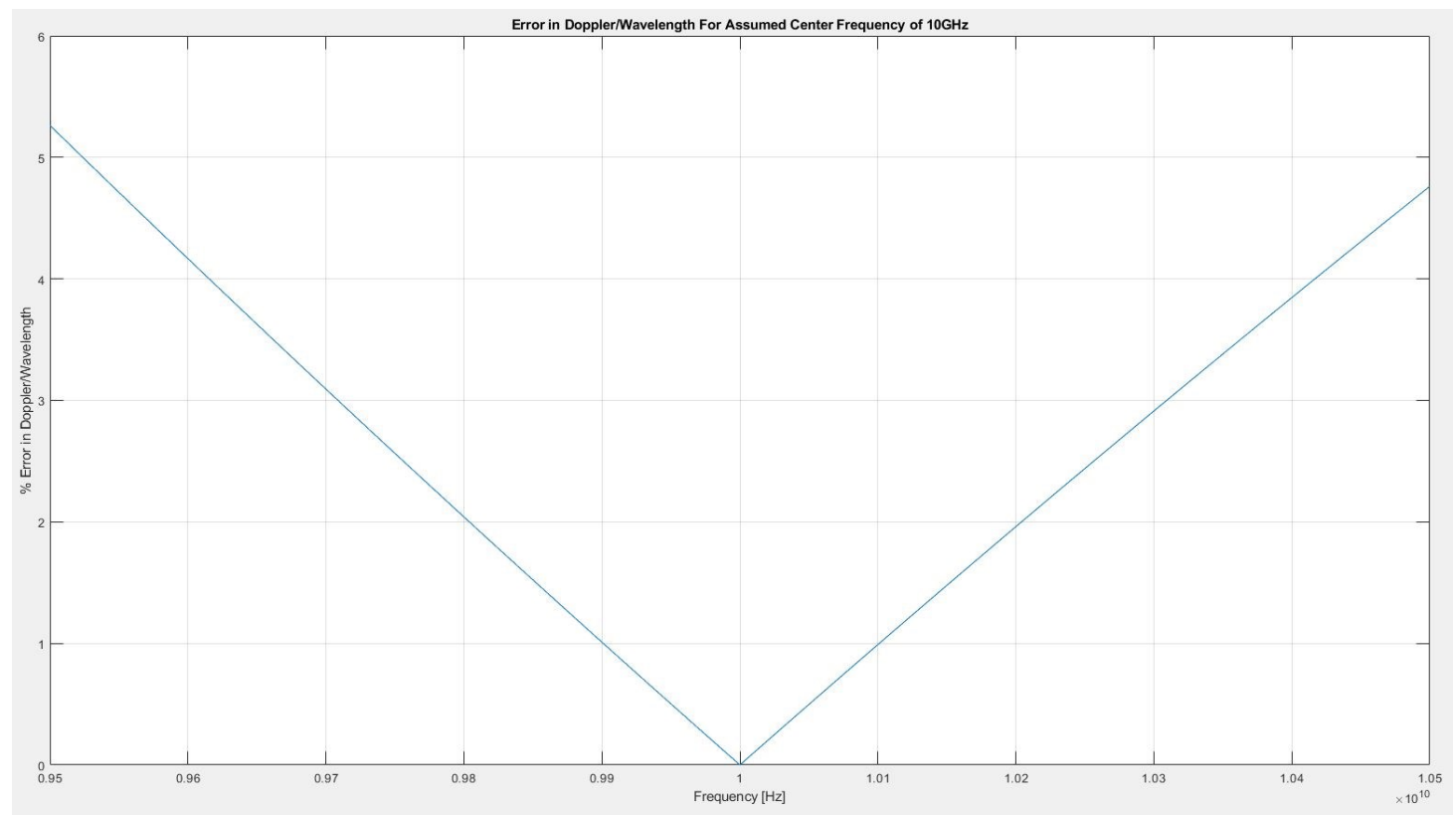

Figure 5.28: The percentage error in wavelength and modulation parameter as the frequency deviates from a $10 \mathrm{GHz}$ carrier frequency.

Observe that error increases linearly for wavelengths differing from the center frequency. The slope of error is steeper for decreasing frequency or increasing wavelength. The error is smaller for the maximum frequency in the bandwidth. Wavelength decays exponentially as frequency increases; thus the error will be larger for lower frequencies than for higher frequencies. This is illustrated in Figure 5.29. 
Definition of an approximate carrier frequency introduces a maximum error of $5.1 \%$ in wavelength for a bandwidth of $1 \mathrm{GHz}$, centered at the carrier. For a practical bandwidth of $300 \mathrm{MHz}$, the maximum error is $1.5 \%$. The target Doppler approaches a maximum of $10 \mathrm{kHz}$, for which the maximum error is $150 \mathrm{~Hz}$. Dependent on Doppler resolution, this error is significant. The maximum phase error is about $5.4^{\circ}$. The error in Doppler and other modulation parameters is small enough to be negligible relative to the cost of increased system latency in spectral estimation and recalculating modulation parameters.

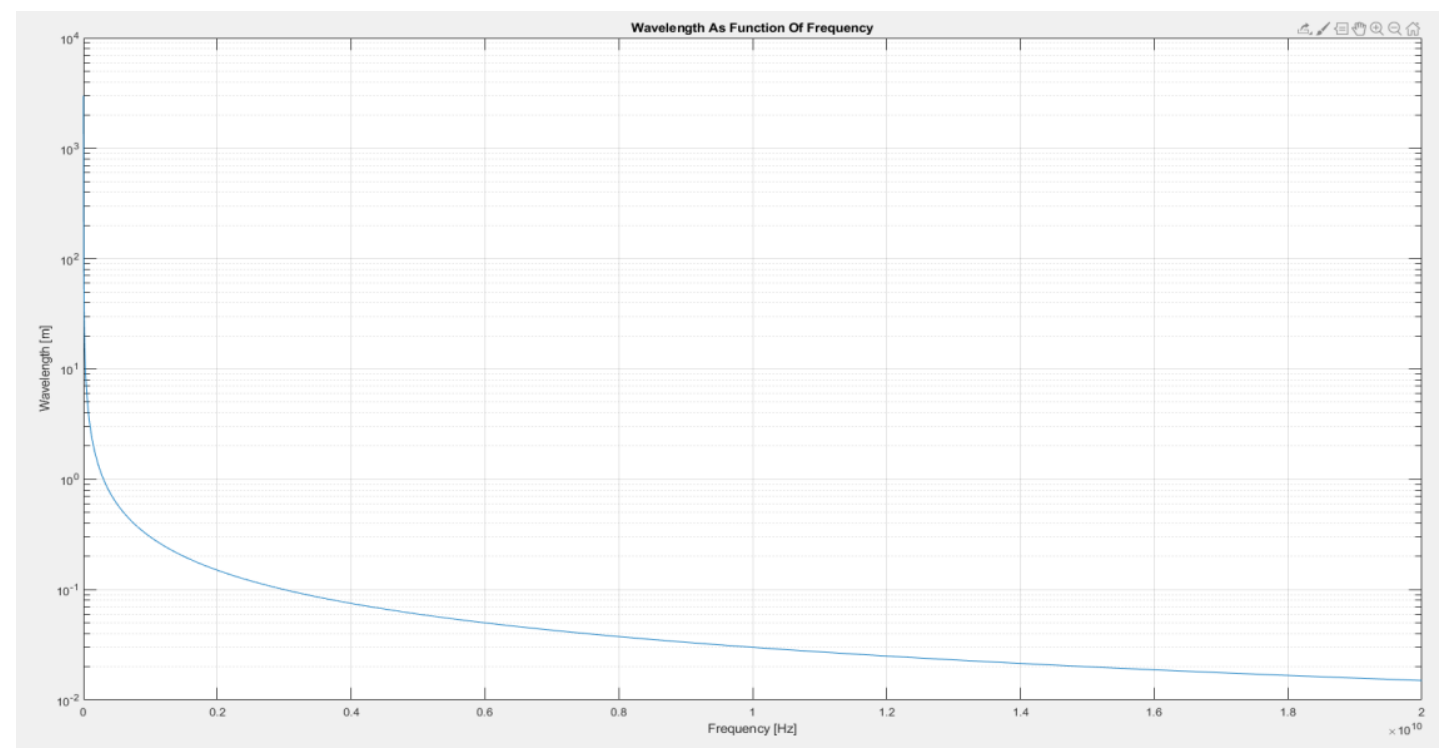

Figure 5.29: Wavelength as a function of frequency.

\subsection{Conclusion}

This chapter presents the Target Signature Synthesis software application developed in the language $\mathrm{C \#}$. The initial step involves the formation of a target point cloud model using ISAR image data generated in the ANSYS HFSS software. The application of EOM, from chapter four, to the point cloud model is shown for specific cases in Appendix A. The computation of modulation parameters used in profile synthesis is addressed, and the profile decorrelation time and error sources. The following chapter discusses the design of a digital system that synthesizes the false target echo signal using the modulation parameters computed earlier in this chapter. 


\section{Chapter 6: Digital Profile Synthesis System Design}

\subsection{Introduction}

Synthesis of a target signature in a practical environment requires high speed digital signal processing operations performed on a radar signal received from a hostile radar. To effectively reduce the hardware computation time the target signature synthesis process is segmented into two stages. The first stage, also called the offline stage, is addressed in the Target Signature Synthesis Software chapter. The second stage, also called the online or real-time stage, is comprised of the digital system designed to synthesize a target echo. The design is based on digital signal processing principles.

Pulsed, frequency stepped, and chirp radar systems often operate within the Xband frequency allocation (i.e., 8-12 GHz), and implement horizontal polarization in Naval applications [41]-[46][49][50]. The selected frequencies and design parameters of the radar systems are determined from common parameters used in the literature in combination with typical system parameters given by an industry consultant. These specifications are listed below.

- Radar system center-band radio frequency $(\mathrm{RF}): F_{c}=10 \mathrm{GHz}$

- Maximum Bandwidth (Centered at $F_{c}$ ): $\beta=300 \mathrm{MHz}$

- Minimum Pulse Width: $\tau=1 \mu \mathrm{s}$

- Maximum Pulse Width: $\tau=100 \mu \mathrm{s}$

- Pulse Repetition Frequency Range: $1 \mathrm{kHz} \leq P R F \leq 1 \mathrm{MHz}$

- Analog Mixing and Bandpass Filtering

○ $f_{L O}=8.8 \mathrm{GHz}$

- $f_{I F, \text { center }}=1.2 \mathrm{GHz}$

- $\beta_{I F}\left(\right.$ centerd at $\left.f_{I F, \text { center }}\right)=2 \mathrm{GHz}$

The first section of this chapter discusses design of a DSP system which will synthesize the target echo from the simulated target modulation parameters. The DSP system is based on a quadrature (I/Q) demodulation system. Design of this system includes the choice of sampling rate, digital local oscillator (LO) frequency, filtering and downsampling operations. The second section addresses the application of the scattering 
modulation parameters obtained from the TSS program. The system design is done as a proof-of-concept and is implemented and simulated in MATLAB as presented in chapter seven. Design of the system for hardware implementation is future research work.

\subsection{Digital Quadrature Demodulation System Design}

This system is designed to counter narrowband signals where the carrier frequency is significantly larger than the bandwidth of the pulse [43]-[49]. The signal modulation envelope is deconstructed into two lowpass signals, defined as the in-phase and quadrature components, which combine to create the complex baseband envelope of the pulse [43]-[49]. Deconstruction into lowpass signals requires mixing the received signal with a local-oscillator frequency (LO) close to or matching the carrier frequency [43]-[49]. The LO signal is Hilbert transformed to create an in-phase LO and a quadrature LO, both of which are mixed with the received narrowband signal [43]-[49]. This operation removes the carrier and isolates the positive and negative spectral images of the bandpass envelope modulation at a baseband frequency [43]-[49]. Decomposition into I/Q channels can be performed in analog hardware, digital hardware, or a hybrid combination [43]-[49]. This application focuses on design of a digital I/Q system. A system diagram for an I/Q system is shown in Figure 6.1.

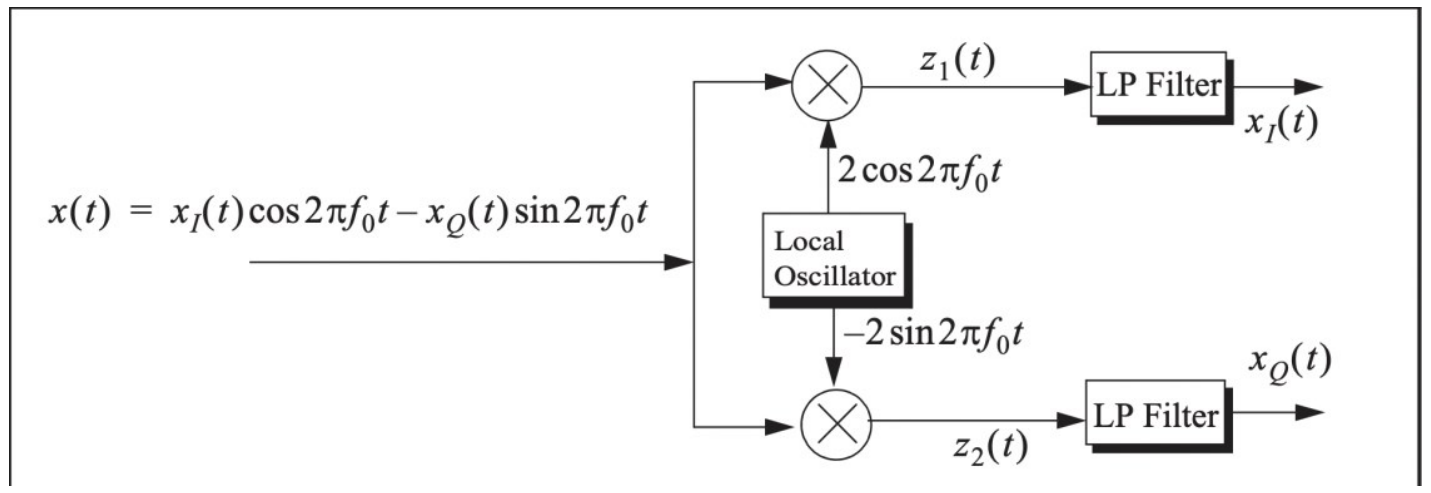

Figure 6.1: System diagram of a I/Q demodulation system.

In a digital I/Q system the input signal is the sampled received radar signal. The local oscillator frequency must be selected, a Hilbert transformer designed and appropriate lowpass filters are designed. Radar receivers attempt to completely remove the carrier component of the narrowband signal. This is achieved since the transmitted 
carrier frequency of the pulse is known a priori. In this application the received pulse frequency is not known, but the bandwidth of operation is known. Thus, the selection of LO will not remove the carrier, instead the signals will be mixed to a low IF band.

Digital I/Q systems do not demand the requirement of a Hilbert transformer [43][49]. Hilbert transformers shift the phase of the LO signal by $90^{\circ}$ to create the quadrature LO signal. Implementing a Hilbert transformer requires design of an all-pass system with the appropriate phase response [46]-[48]. This introduces latency into the system dependent on the order of the designed transformer. Hilbert transformers are useful when the $\mathrm{LO}$ frequency is changing to match each pulse frequency to remove the carrier frequency component. In this application the LO frequency is fixed. Thus, a Hilbert transform is not implemented, and it is assumed that the in-phase and quadrature LO signals are stored in memory and do not have to be computed.

\subsubsection{Sampling Rate \& Digital LO Frequency Selection}

The sampling rate choice is defined by the digital RF memory system employed. The chosen model for this application is the Xilinx RFSoc development board EK-U1ZCU111-G. The analog-to-digital converter on this board has a 12-bit resolution and is capable of speeds up to 4.096 GSPS (Giga-Samples Per Second). With a signal at maximum intermediate frequency (IF) of $1.35 \mathrm{GHz}$ the Nyquist sampling requirement is 2.7 GSPS. An increase in sampling rate produces more samples, which increases system latency in processing. Figure 6.2 shows the range of the number of samples obtained for the smallest pulse width of $1 \mu \mathrm{s}$. 


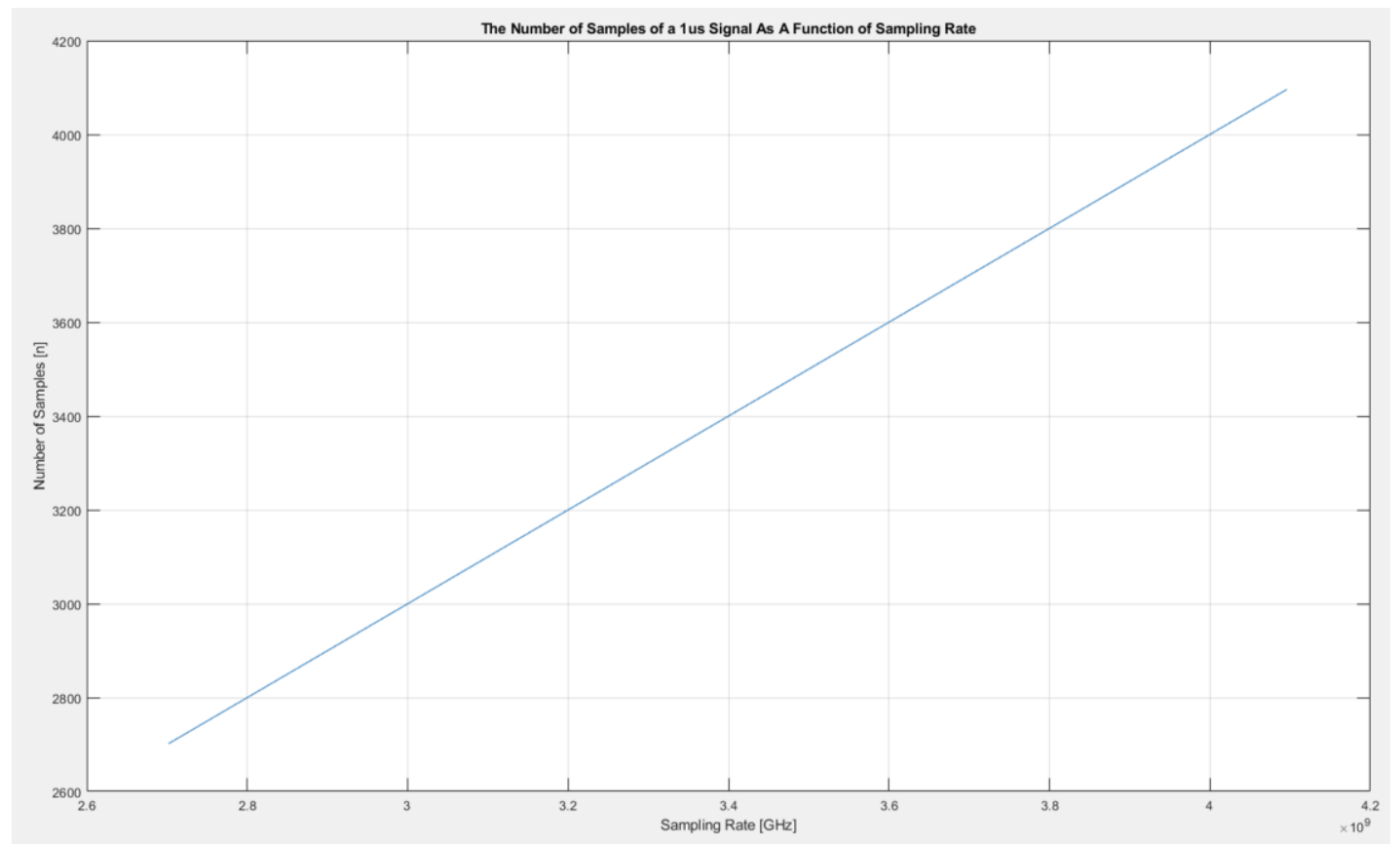

Figure 6.2: The number of samples as a function of sampling rate for a pulse width of 1 microsecond.

The minimum number of samples obtained is approximately 2700, at the Nyquist sampling rate. The number of samples determines the number of multiplications required per scatterer to apply the modulation parameters. Demodulation to I/Q baseband in addition to downsampling the signal can reduce the total number of operations required. Downsampling effectively discards samples of the signal while preserving spectral information and reducing the sampling bandwidth [46]-[49].

Achieving the minimum number of samples in design, while preserving the spectral information, minimizes the total latency of the system by reducing the number of processing operations per scattering location. Implementing an I/Q demodulation system requires selection of a digital LO frequency which can reduce the sampled frequency content to a range where downsampling can be applied without aliasing. The cut-off frequency of the lowpass filter (LPF) dictates the downsampling factor which can be used [46]-[49]. Table 6.1 shows the lowpass cut-off digital frequency based on the downsampling factor [46]-[49]. 
Table 6.1: The maximum frequency component for varying downsampling factors.

\begin{tabular}{|l|l|}
\hline Downsampling Factor & $\begin{array}{l}\text { LPF Cut-off Frequency } \\
\text { [rad/sample] }\end{array}$ \\
\hline 2 & $\pi / 2$ \\
\hline 3 & $\pi / 3$ \\
\hline 4 & $\pi / 4$ \\
\hline 5 & $\pi / 5$ \\
\hline
\end{tabular}

The selection of a downsampling factor requires reduction of signal bandwidth to digital frequencies below the lowpass cut-off points. Selection of sampling rate, digital LO frequency and downsampling factor are all interdependent. The method of parameter selection is user defined based on system or design limitations.

Figure 6.3 shows a surface plot combining the possible combinations of downconverted digital IF normalized by pi [ $\mathrm{rad} / \mathrm{sample}]$. The data is plotted as a function of digital LO frequency chosen and the sampling rate. The red planes on the plot indicate the frequency cut-off limits for lowpass filtering prior to downsampling.

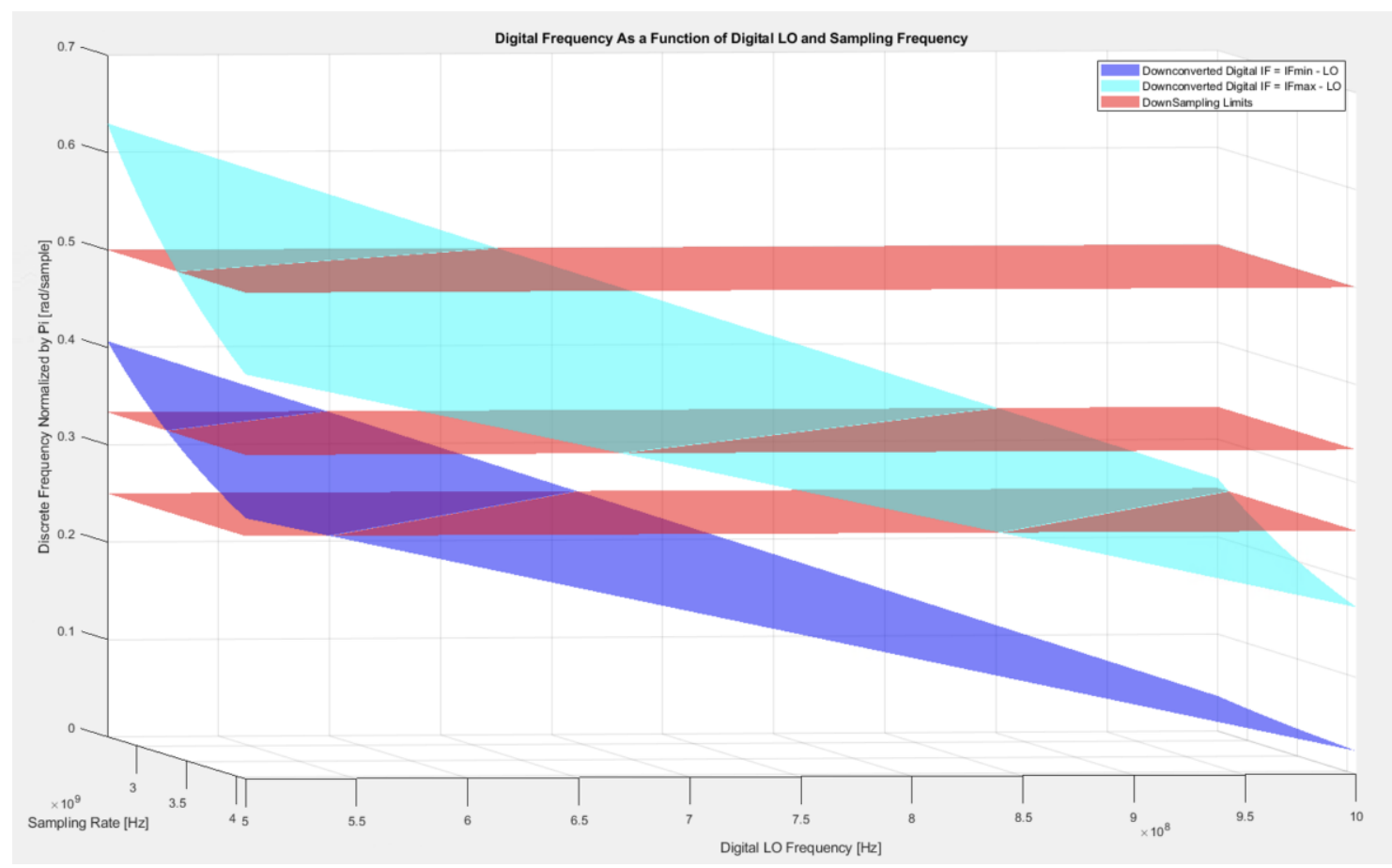

Figure 6.3: The digital frequency of the maximum and minimum IF following downconversion, as a function of digital LO and sampling rate. 
The blue and cyan mesh represent the difference products of mixing the lowest IF and highest IF with the digital LO, respectively. The red lines indicate digital frequency limits for downsampling by factors 2,3 and 4 .

Figure 6.4 shows the number of samples for each channel following downsampling as a function of sampling frequency.

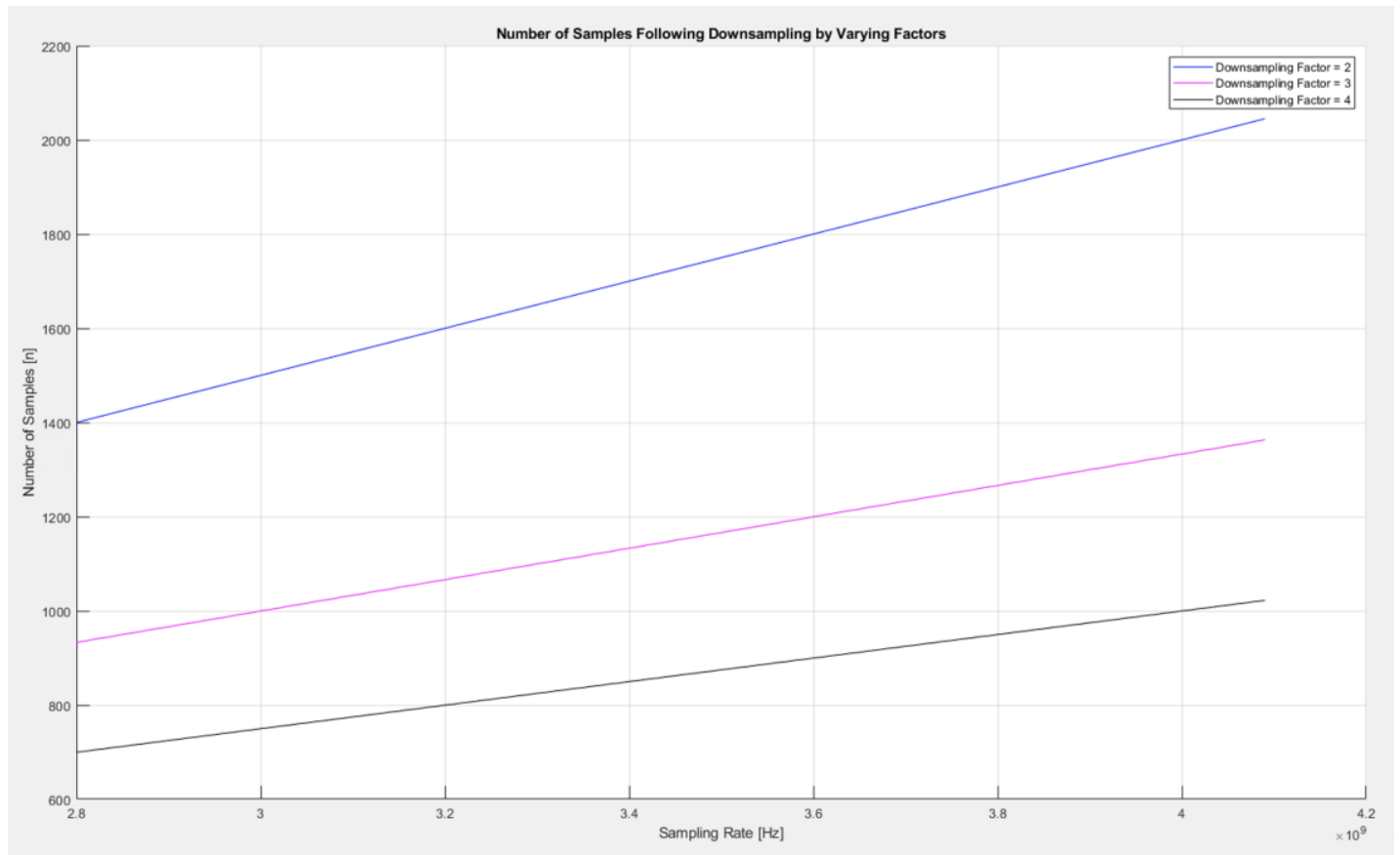

Figure 6.4: The number of samples as a function of sampling frequency for three options of downsampling factor; 2,3 and 4.

From Figure 6.4 it is observed that to downsample by a factor of four the maximum number of samples is slightly more than 1000 . The minimum number of samples for downsampling by a factor of three is approximately 930 samples. Defining this as the maximum number of samples a sampling rate is selected with a downsampling factor of four that achieves the lowest possible number of samples. The maximum sampling rate is then defined as 3.75 GSPS.

Figure 6.5 shows a clear region of acceptable digital $\mathrm{LO}$ and sampling rate combinations which can be selected. 


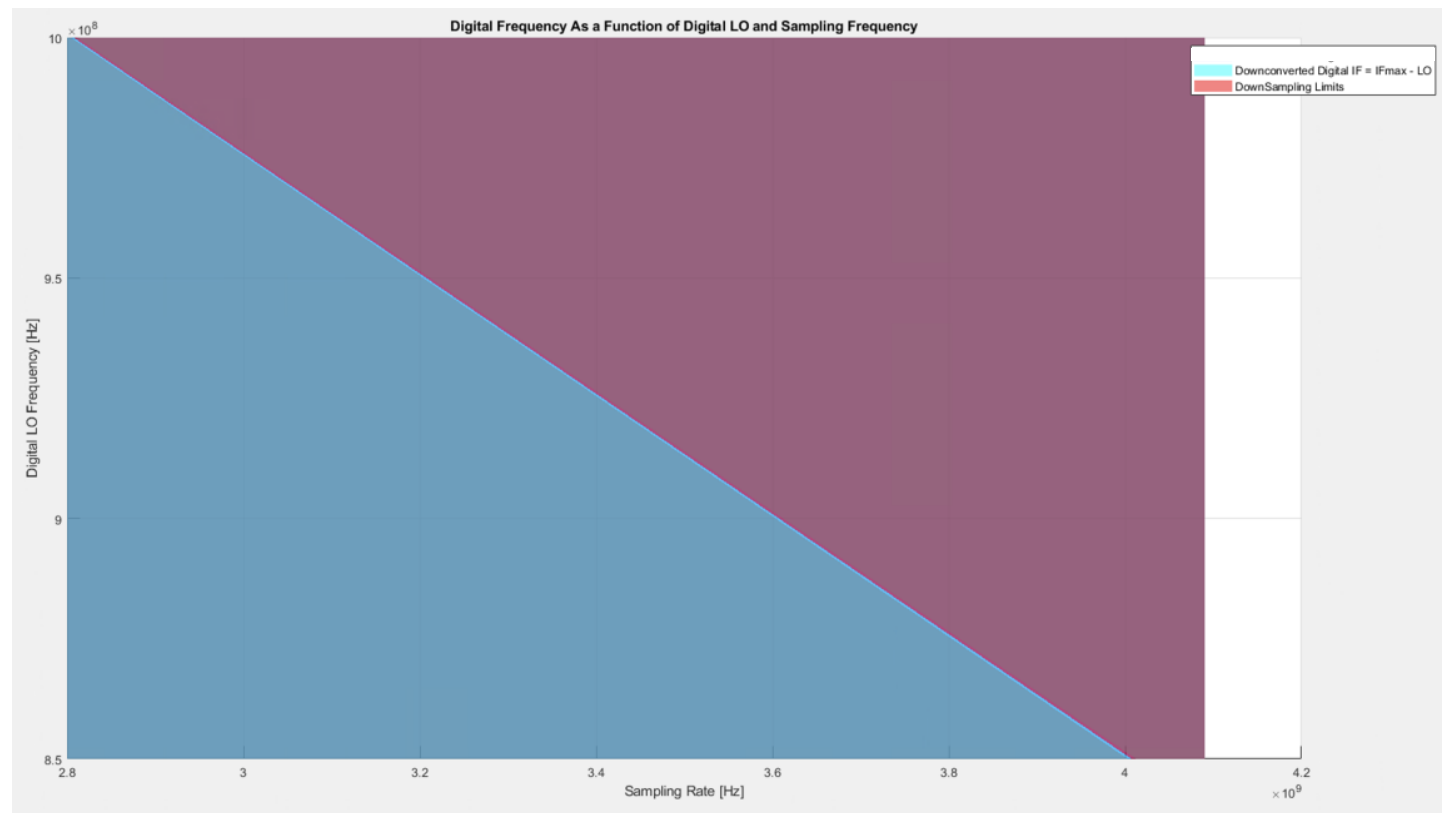

Figure 6.5: The digital frequency LO and sampling rate combinations to achieve a downsampling factor of four.

In Figure 6.5 the region highlighted in red indicates the region of selection for sampling rate and digital LO combinations. The minimum sampling rate to downsample by a factor of four is 2.81 GSPS. An optimal range for sampling rate selection is between 2.81 GSPS and 3.75 GSPS. The acceptable choice of digital LO frequencies for this sampling rate is approximately $882 \mathrm{MHz}$ to $1 \mathrm{GHz}$.

The choice of digital LO frequency and sampling rate determines if the downconverted signals fit within the required downsampling region of 0 to $(\pi / 4)$ [rad/sample]. As the digital LO frequency decreases the minimum downconverted digital IF increases. As the sampling rate increases the maximum downconverted digital IF decreases. Figure 6.6 shows the intersection of the plane of choices for sampling rate and digital LO for the maximum analog IF and minimum analog IF. 


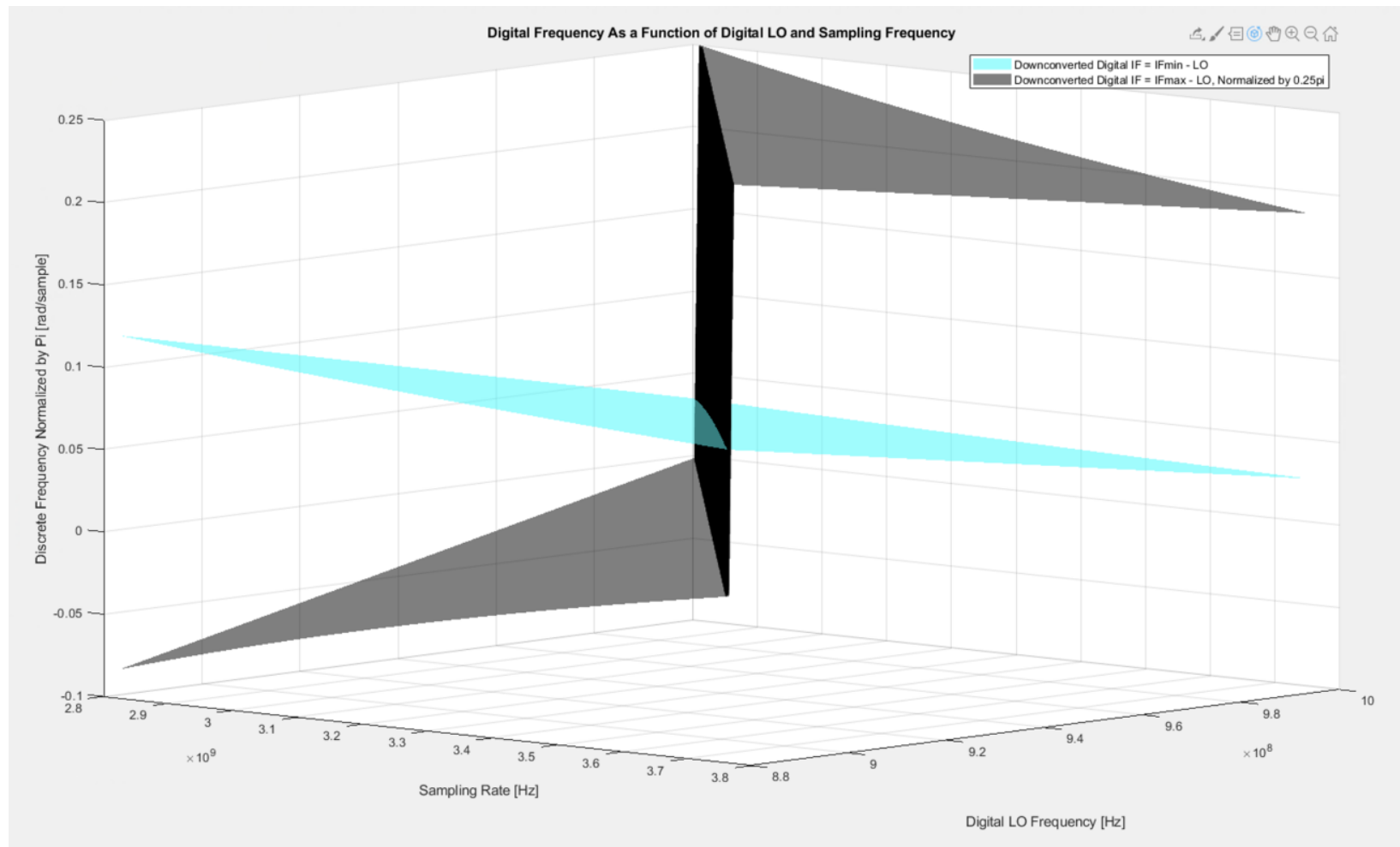

Figure 6.6: The digital frequency LO and sampling rate combinations for the minimum analog IF are seen in blue.

The black plane contains the frequency from downconverting the maximum analog IF, this frequency is subtracted from the downsampling limit of $0.25 \pi$ [rad/sample]. Negative frequency values in Figure 6.6, as well as regions where the black plane is lower than the blue plane indicate regions of sampling rate and digital LO which cannot be selected due to aliasing. Selection of the parameters in this region results in a maximum digital frequency component greater than the downsampling limit. Figure 6.7 shows the same plot with the sum products of mixing displayed. 


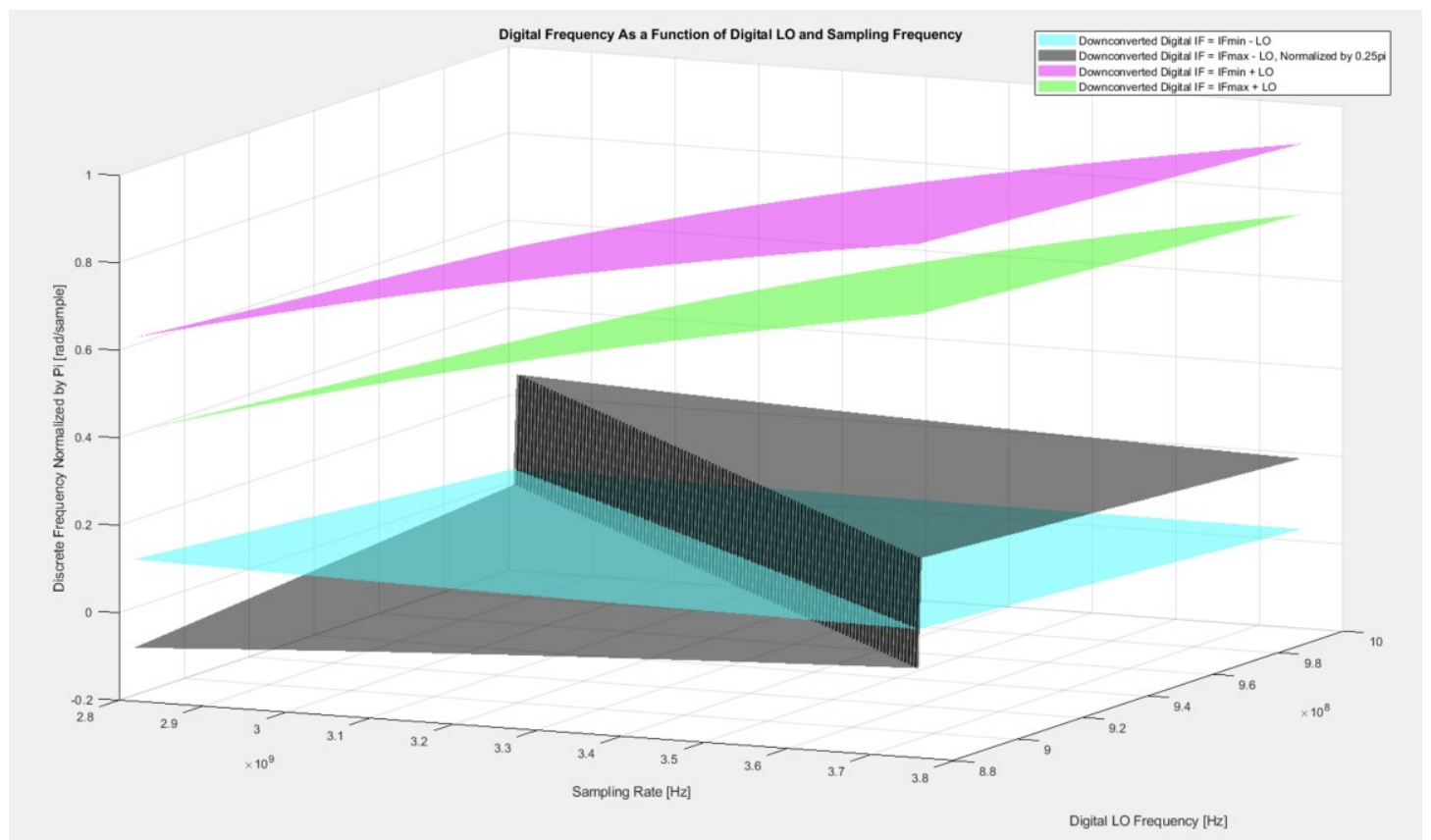

Figure 6.7: The digital frequency LO and sampling rate combinations for the minimum analog IF are seen in blue.

The magenta plane contains the sum frequency from mixing the minimum analog IF. The green plane contains the sum frequency from mixing the maximum analog IF. Observing Figure 6.7, it is seen that the frequency spacing between the maximum difference mixer product, and minimum sum mixer product increases with increasing sampling rate. There is also a subtle decrease of this spacing as the digital LO frequency increases.

Selection of a digital LO and sampling rate which is near the boundary of the shaded black intersection region in Figure 6.7 is problematic. Quantization and noise effects in hardware implementation can have detrimental effects on mixing products staying within tolerance for downsampling requirements. Additionally, as the sampling rate increases, the order of lowpass filter decreases. The decrease in filter order is due to the downconverted signal being closer to a baseband frequency. This increases the separation between the desired frequency band and the image frequency. With an increased separation the required window transition width for the LPF is wider, which allows a lower order filter implementation. 


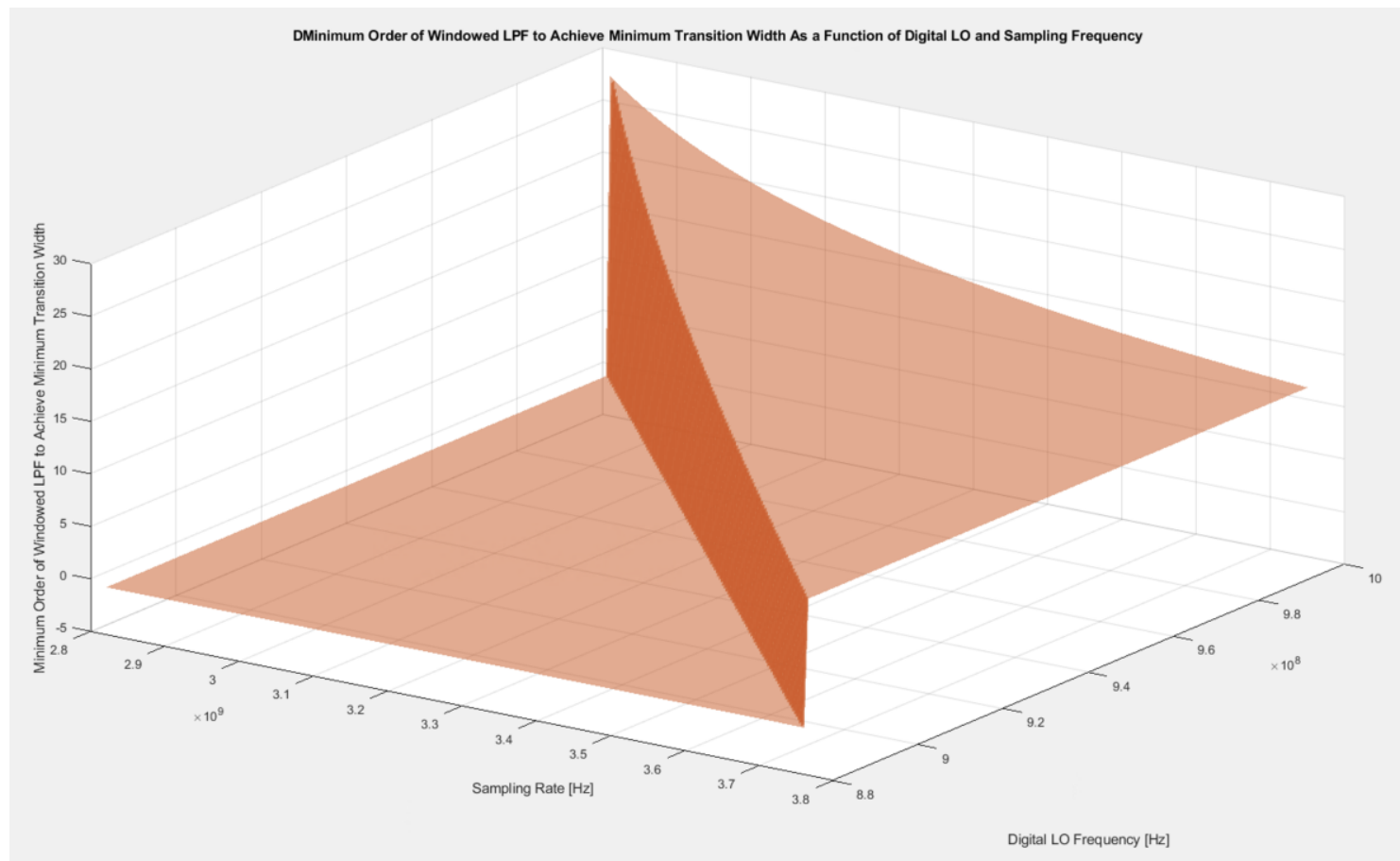

Figure 6.8: Order of Windowed LPF to achieve the minimum transition width required to isolate difference products from digital mixing.

From Figure 6.8, observe the minimal change in filter order requirements as digital LO frequency increases. Conversely the number of samples obtained as sampling rates increases is much larger than the change in filter order. Thus, a lower sampling rate is optimal even with a slightly higher order filter, since reducing the total number of samples is of higher value in latency reduction.

In this application, the chosen sampling rate to operate the ADC is $3.0 \mathrm{GSPS}$, and a digital local oscillator with a frequency equivalent of $1 \mathrm{GHz}$. With these specifications, the minimum number of samples obtained prior to downsampling is 3000 . Following demodulation and downsampling, each of the quadrature channels containing 750 samples each. The minimum windowed lowpass filter order required to achieve the transition width is $\mathrm{M}=40$ for a Hann or Hamming window.

\subsubsection{Lowpass Filter Design}

Digital LPFs can be implemented as finite impulse response (FIR) or infinite impulse response (IIR) systems. FIR Type 1 filter implementations provide linear phase, which is exploited to keep the delay in the I/Q channels the same. There are many 
methods for designing FIR lowpass filters outlined in the literature [46]-[49]. The selection of a design method is dependent on user choice, and quantization effects on the filter response.

The key specifications in filter design are the transition width and the stopband attenuation. The transition width is defined between the edge of the passband region and the edge of the stopband region. These frequencies are determined from the digital mixing products, outlined in Table 6.2.

Table 6.2: The digital mixing products for sampling rate 3 GSPS and LO of $1 \mathrm{GHz}$.

\begin{tabular}{|c|c|c|c|}
\hline $\begin{array}{l}f_{A, I F, \min }=1.05 G H z \\
f_{A, I F, \max }=1.35 \mathrm{GHz}\end{array}$ & $\begin{array}{l}\text { Difference } \\
\text { Frequency }\end{array}$ & & Sum Frequency \\
\hline $\begin{aligned} f_{D, I F, \min }= & f_{A, I F, \min } \\
& -f_{D, L O}\end{aligned}$ & $50 \mathrm{MHz}$ & $\begin{aligned} f_{D, I F, \min }= & f_{A, I F, \min } \\
& +f_{D, L O}\end{aligned}$ & $2.05 \mathrm{GHz}$ \\
\hline $\begin{array}{l}\omega_{D, I F, \min } \\
=\frac{2\left(f_{A, I F, \min }-f_{D, L O}\right) \pi}{F_{S}}\end{array}$ & $\frac{\pi}{30}\left[\frac{\text { rad }}{\text { sample }}\right]$ & $\begin{array}{l}\omega_{D, I F, \min } \\
=\frac{2\left(f_{A, I F, \min }+f_{D, L O}\right) \pi}{F_{S}}\end{array}$ & $\begin{array}{l}1.3667 \pi\left[\frac{\text { rad }}{\text { sample }}\right] \\
\text { Alias: } \frac{19 \pi}{30}\left[\frac{\text { rad }}{\text { sample }}\right]\end{array}$ \\
\hline $\begin{array}{r}f_{D, I F, \max }=f_{A, I F, \max } \\
-f_{D, L O}\end{array}$ & $350 \mathrm{MHz}$ & $\begin{array}{r}f_{D, I F, \max }=f_{A, I F, \max } \\
+f_{D, L O}\end{array}$ & $2.35 \mathrm{GHz}$ \\
\hline $\begin{array}{l}\omega_{D, I F, \max } \\
=\frac{2\left(f_{A, I F, \max }-f_{D, L O}\right) \pi}{F_{S}}\end{array}$ & $\frac{7 \pi}{30}\left[\frac{\text { rad }}{\text { sample }}\right]$ & $\begin{array}{l}\omega_{D, I F, \max } \\
=\frac{2\left(f_{A, I F, \max }+f_{D, L O}\right) \pi}{F_{S}}\end{array}$ & $\begin{array}{l}1.5667 \pi\left[\frac{\text { rad }}{\text { sample }}\right] \\
\text { Alias: } \frac{13 \pi}{30}\left[\frac{\text { rad }}{\text { sample }}\right]\end{array}$ \\
\hline
\end{tabular}

Table 6.2 shows the minimum undesired frequency content following mixing is aliased to $\frac{13 \pi}{30}\left[\frac{\mathrm{rad}}{\text { sample }}\right]$. The maximum desired frequency content is at a digital frequency of $\frac{7 \pi}{30}\left[\frac{\mathrm{rad}}{\text { sample }}\right]$. The stopband edge and passband edge frequencies have been defined, 
respectively. The transition band region is $\frac{\pi}{5}\left[\frac{\mathrm{rad}}{\text { sample }}\right]$. The stopband attenuation is variable, with the ideal target set to a minimum of $40 \mathrm{~dB}$. The acceptable ripple in the passband region is 0.01 . A larger ripple reduces the filter order at the cost of reduced signal energy in the desired passband. Also, a significant ripple in the passband causes distortion of wideband chirp signals in the digital system.

MATLAB provides a FilterDesigner tool which can create FIR lowpass filter implementations using a variety of design methods including windowing, equiripple and least constrained squares [61]. In this system design the highest value metric is processing time. Designing a filter which meets the specifications with the minimum order of coefficients is the priority. The equiripple FIR Type 1 filter can achieve a lower order for the band design specifications outlined in Table 6.3. However, the equiripple magnitude response creates magnitude distortion in processing of wideband chirp signals. Thus, a Kaiser windowed Type 1 FIR LPF is selected with the band, order and Beta value specifications outlined in Table 6.3.

Table 6.3: Filter specifications for FIR Type 1 LPF designed in MATLAB.

\begin{tabular}{|c|c|}
\hline Parameter & Value \\
\hline$\omega_{\text {pass }}$ & $0.25 \pi\left[\frac{\text { rad }}{\text { sample }}\right]$ \\
\hline$\omega_{\text {stop }}$ & $0.4 \pi\left[\frac{\text { rad }}{\text { sample }}\right]$ \\
\hline$\delta_{\text {stop }}$ & $40 d B$ \\
\hline$\delta_{\text {pass }}$ & 0.01 \\
\hline$M$ & 30 \\
\hline$\beta$ & 3.3953 \\
\hline
\end{tabular}

The magnitude and phase response of the FIR LPF are displayed in Figure 6.9 and 6.10 . 


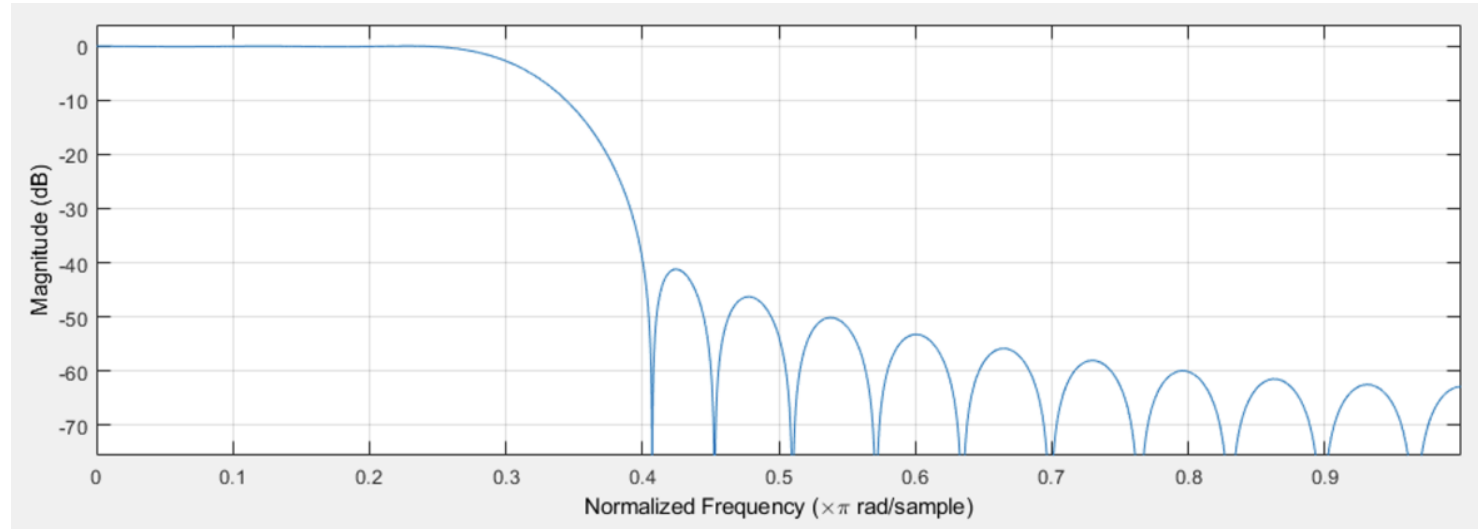

Figure 6.9: Magnitude response of Kaiser Window FIR Type 1 LPF $(\beta=3.3953)$.

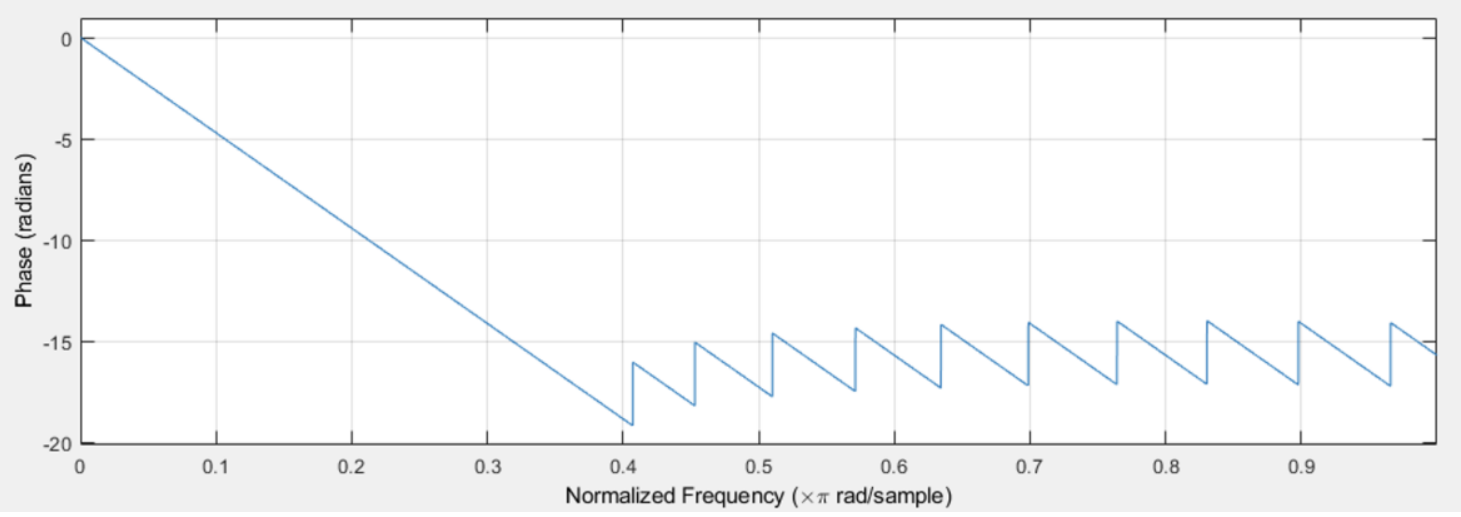

Figure 6.10: Phase response of Kaiser window FIR Type 1 LPF $(\beta=3.3953)$.

The linear phase response within the passband region introduces a group delay of fifteen samples. This filter is utilized in both the I and Q channel of the digital system. Thus, introducing a latency of fifteen samples in both channels, avoiding any latency mismatches.

\subsubsection{Downsampling}

The downsample factor is decided to be four. Digital signal processing theory dictates that to downsample by this factor 4 LPF with a cut-off located at $\pi / 4$ [rad/sample] [46]-[49]. This restriction is imposed to ensure no signal content exists above the downsampling cutoff rate. Resulting from mixing operations and received bandwidth specifications, previously defined, the maximum signal content in the system is at a digital frequency of $\frac{7 \pi}{30}$ or 0.2333 [rad/sample]. The maximum signal content is within the downsampling rate cut-off. Downsampling the I/Q channels is performed by 
discarding every four samples between consecutive samples [46]-[49]. This results in a sampling bandwidth reduction by a factor of four, such that the sampled spectrum bandwidth is $750 \mathrm{MHz}$. The maximum frequency content represented in the downsampled signal is $375 \mathrm{MHz}$.

Figures 6.11 to 6.20 show the demodulation of a received (RF) $10.15 \mathrm{GHz}$, (IF) $1.35 \mathrm{GHz}, 1 \mu \mathrm{s}$ pulse into I/Q channels. The spectral magnitude and phase of both channels is shown after sampling through the stages of demodulation, following mixing, post-filtering and post-downsampling.

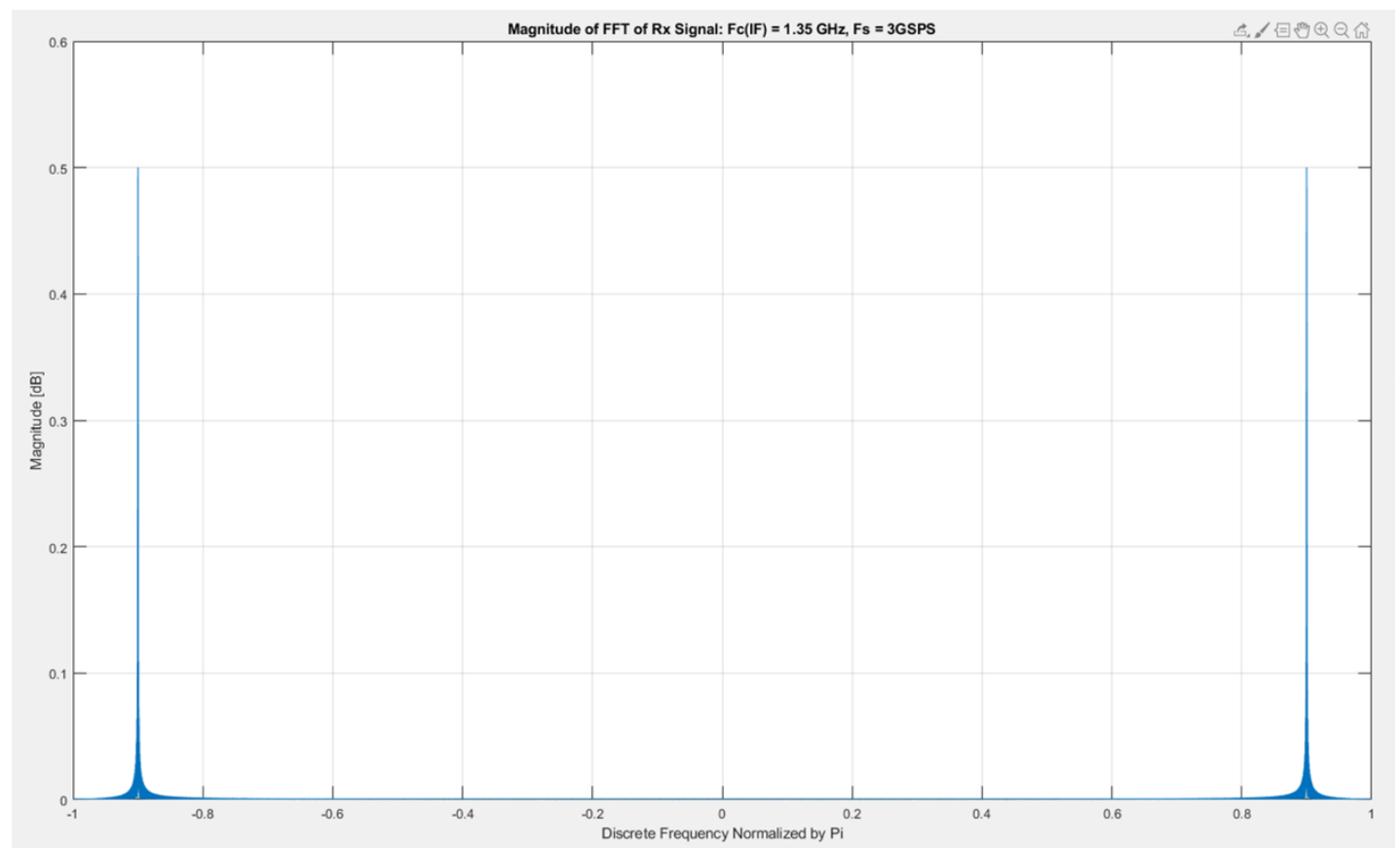

Figure 6.11: Magnitude spectrum of received (RF) $10.15 \mathrm{GHz}$, (IF) $1.35 \mathrm{GHz}, 1 \mu \mathrm{s}$ pulse, sampled at 3 GSPS. 


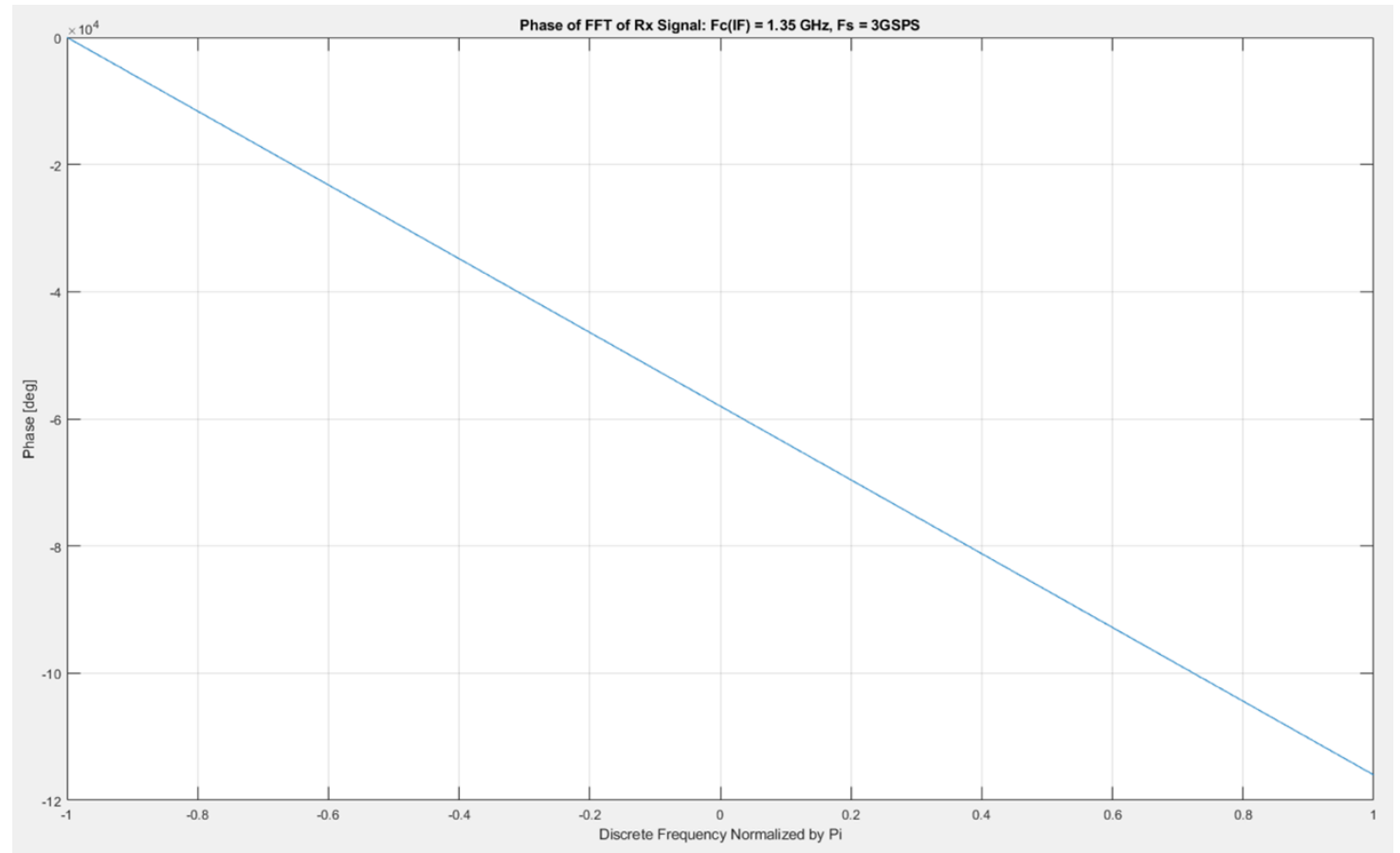

Figure 6.12: Phase spectrum of received (IF) $1.35 \mathrm{GHz}, 1 \mu$ s pulse, sampled at 3

\section{GSPS.}

The received signal is mixed with the digital $\mathrm{LO}$ at a frequency of $1 \mathrm{GHz}$. The mixing operation produces sum and difference frequency products in both the negative and positive portions of the spectrum. This is visible in both the I and Q channels of the system. The spectral magnitude of both the I and Q channel is consistent. The phase response of the channels highlights the difference between the in-phase channel and quadrature channels. 


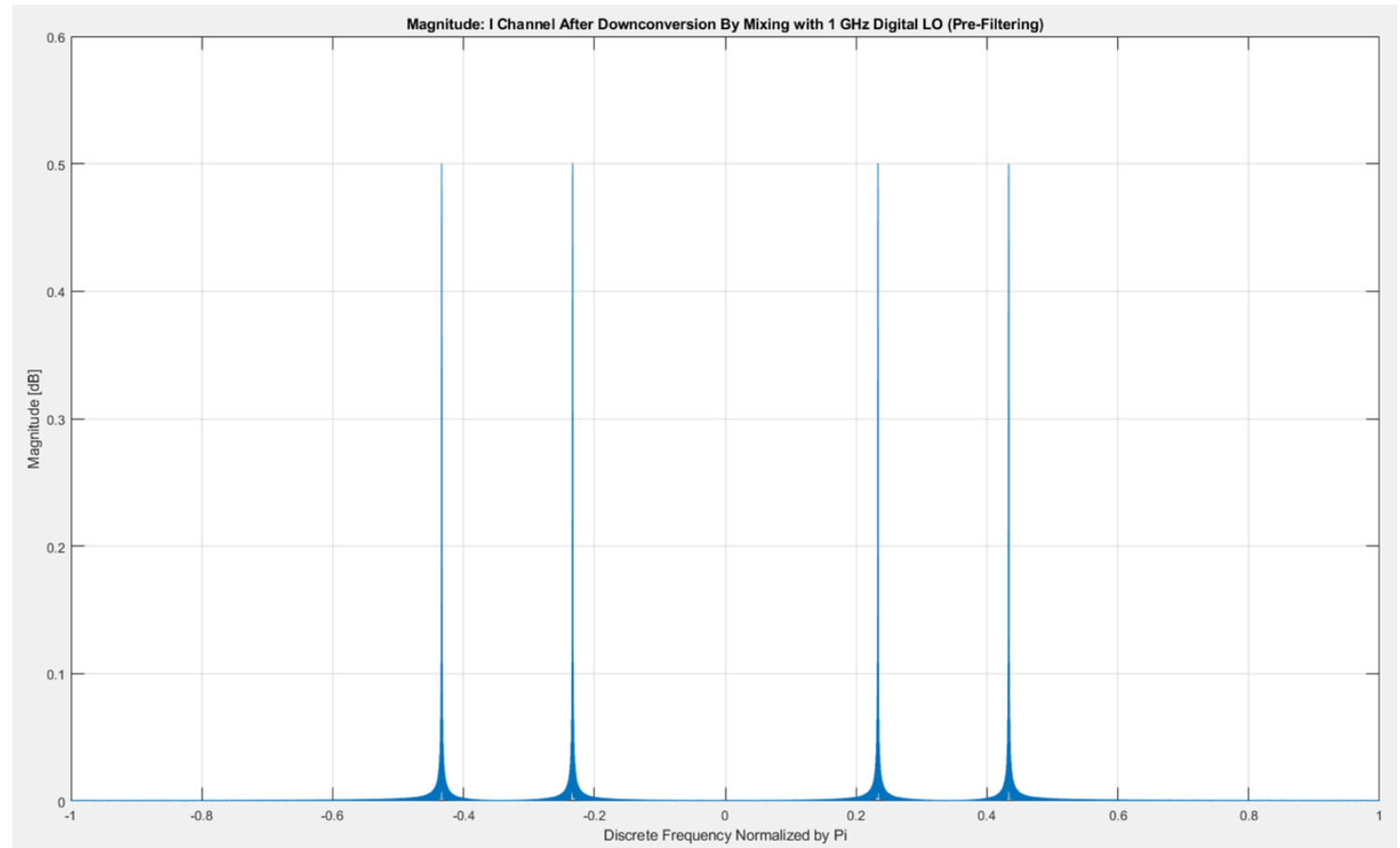

Figure 6.13: Magnitude spectrum of received (IF) $1.35 \mathrm{GHz}, 1 \mu$ s pulse, sampled at 3 GSPS, mixed with the in-phase digital LO at $1 \mathrm{GHz}$ to create the in-phase (I) channel.

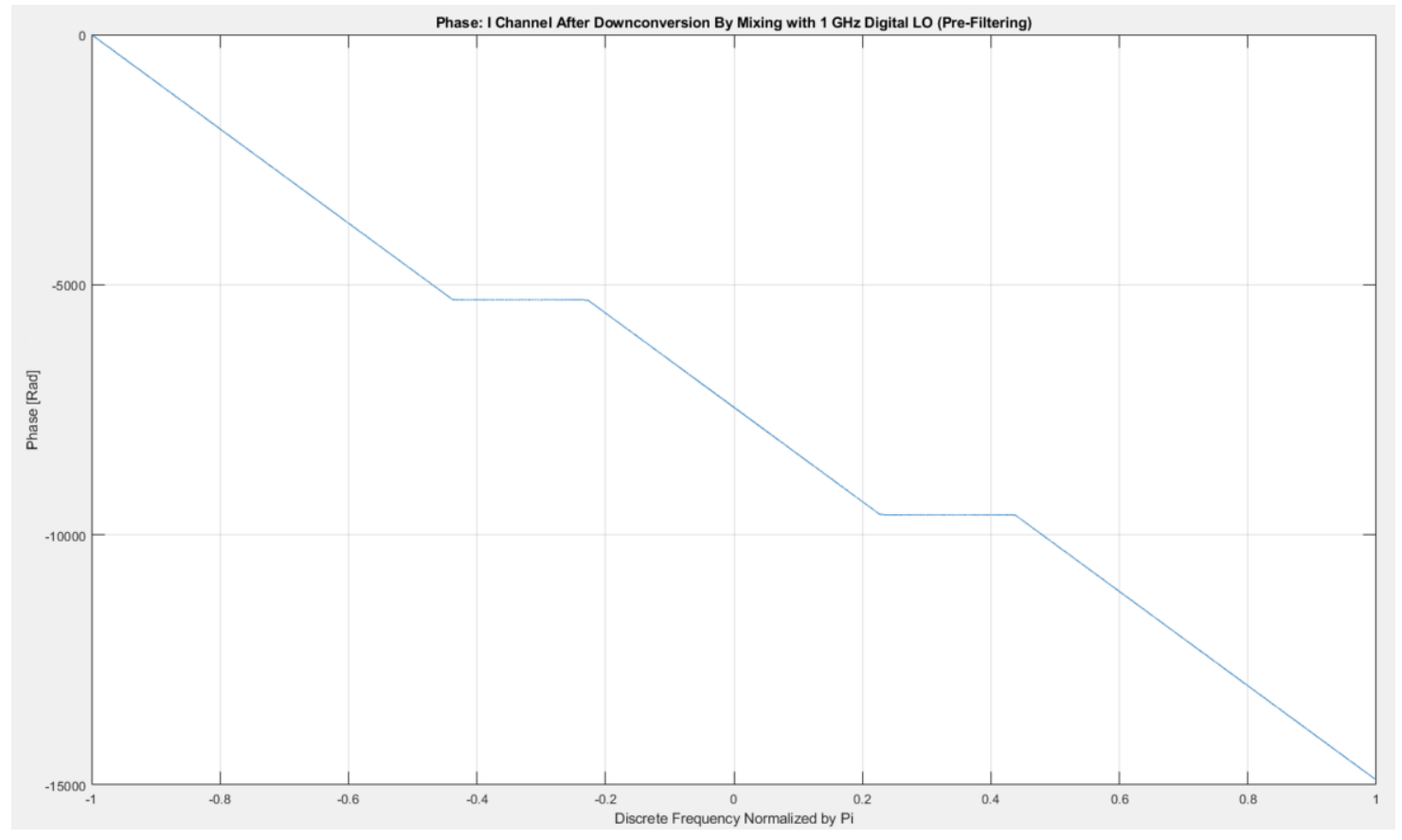

Figure 6.14: Phase spectrum of received (IF) $1.35 \mathrm{GHz}, 1 \mu$ s pulse, sampled at 3 GSPS, mixed with the in-phase digital LO at $1 \mathrm{GHz}$ to create the in-phase (I) channel. 


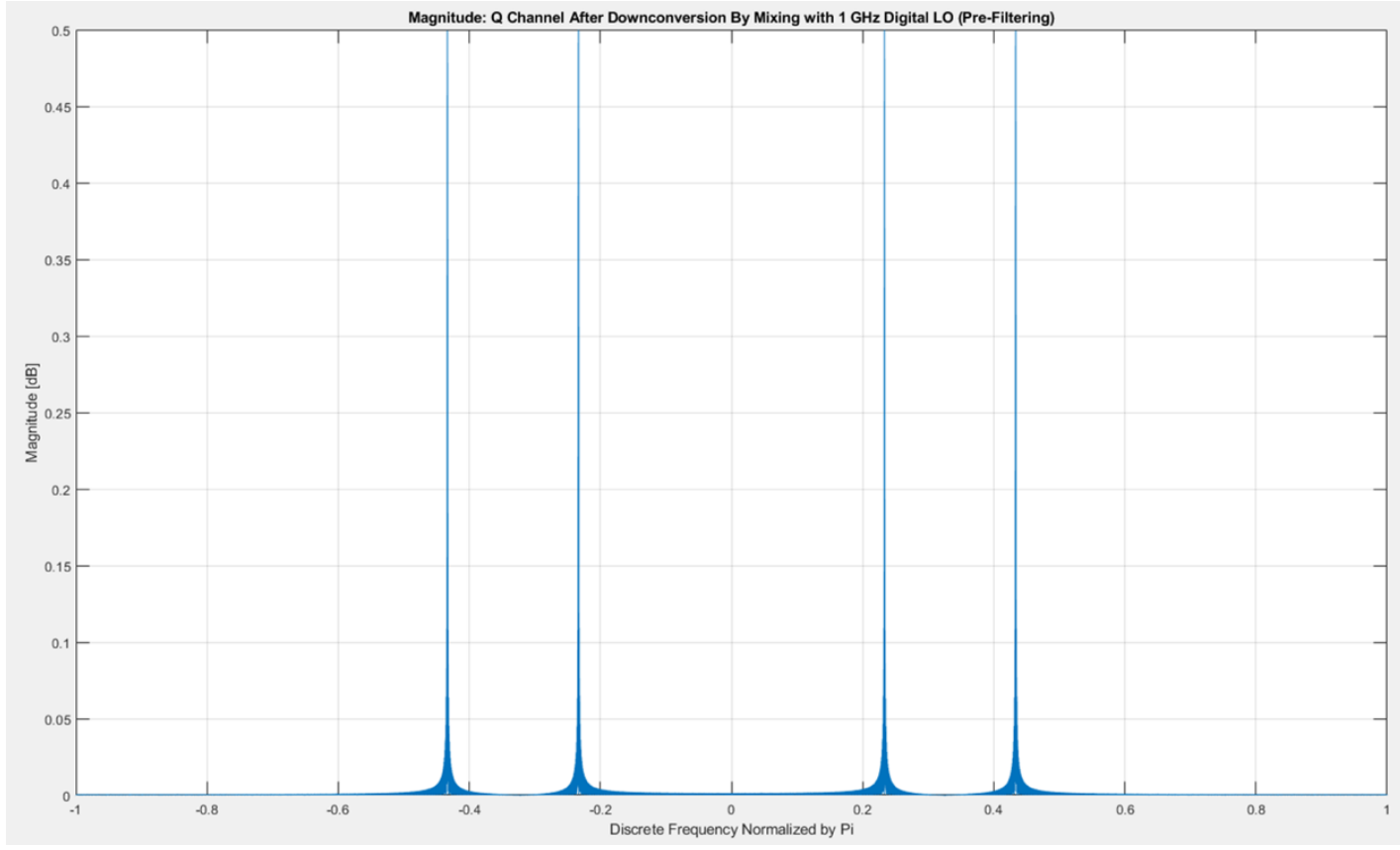

Figure 6.15: Magnitude spectrum of received (IF) $1.35 \mathrm{GHz}, 1 \mu$ s pulse, sampled at 3 GSPS, mixed with the quadrature digital LO at $1 \mathrm{GHz}$ to create the quadrature (Q) channel.

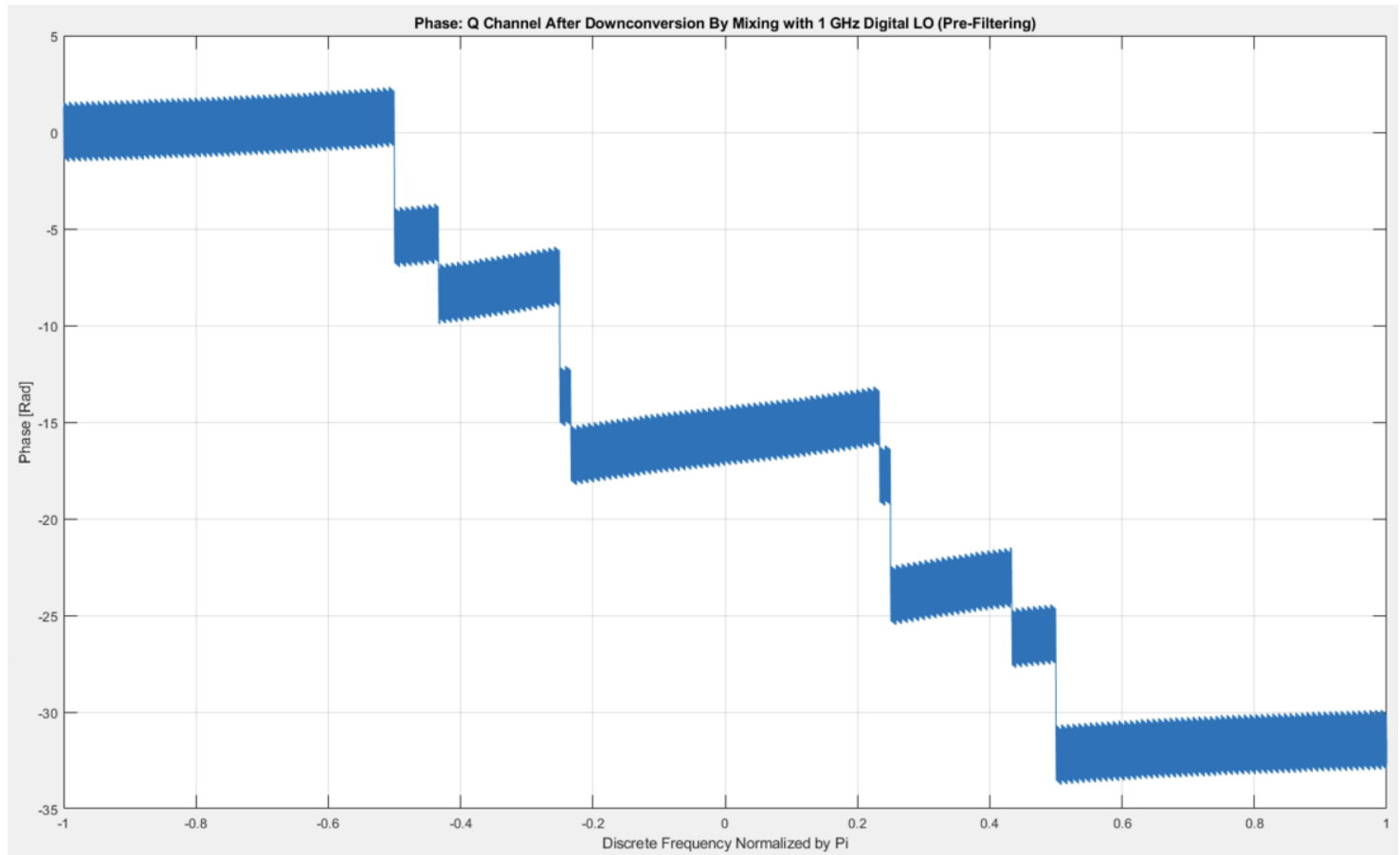

Figure 6.16: Phase spectrum of received (IF) $1.35 \mathrm{GHz}, 1 \mu$ s pulse, sampled at 3 GSPS, mixed with the quadrature digital $\mathrm{LO}$ at $1 \mathrm{GHz}$ to create the quadrature (Q) channel. 
The undesired spectral images are the sum products of the filtering operation. These are filtered using the Kaiser Windowed FIR Type 1 LPF designed previously.

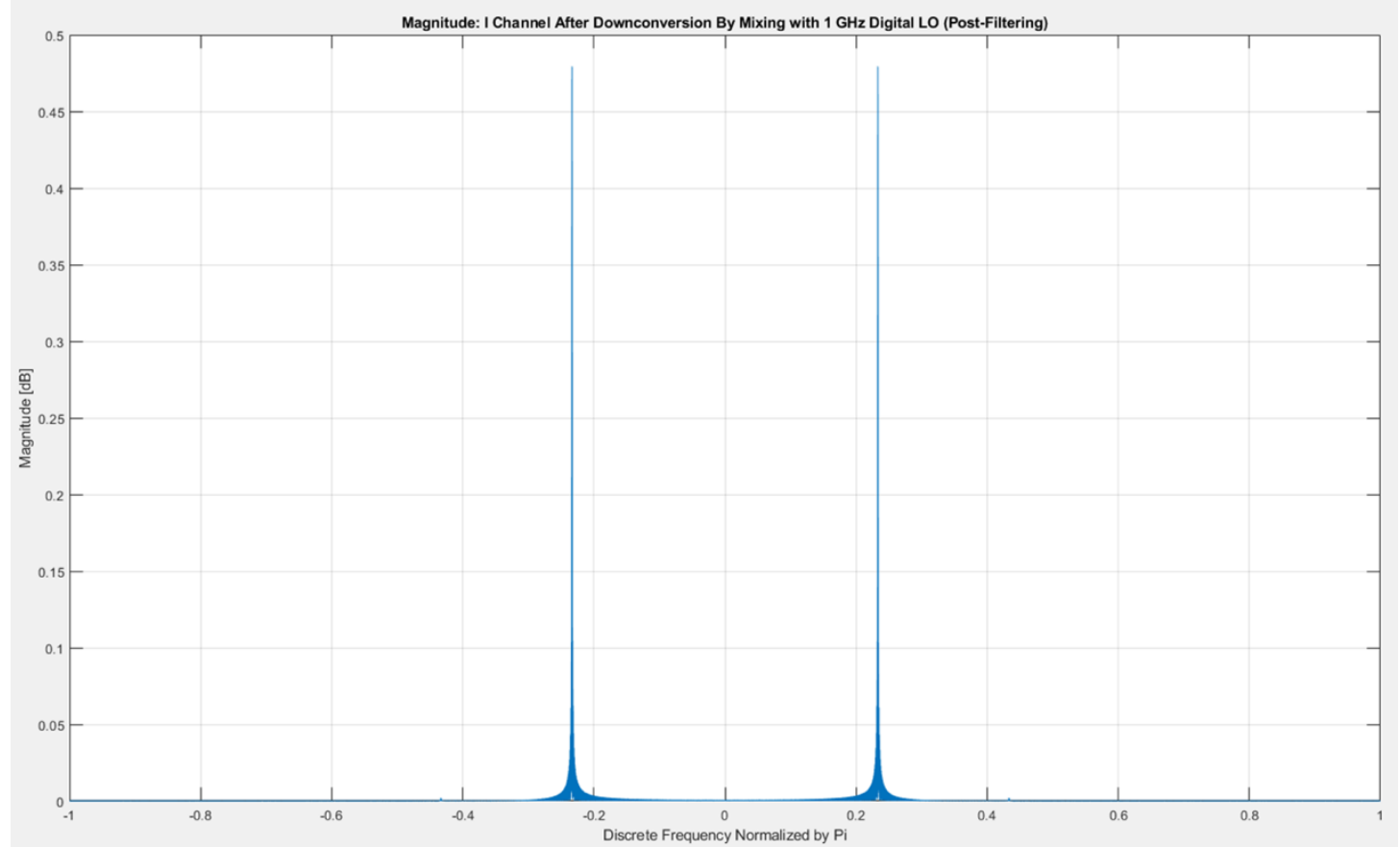

Figure 6.17: Magnitude spectrum of received (IF) $1.35 \mathrm{GHz}, 1 \mu$ s pulse, sampled at 3 GSPS, mixed with the in-phase digital LO at $1 \mathrm{GHz}$ to create the in-phase (I) channel. Following filtering using the FIR Type 1 Kaiser Windowed LPF $(\beta=$ 3.3953). 


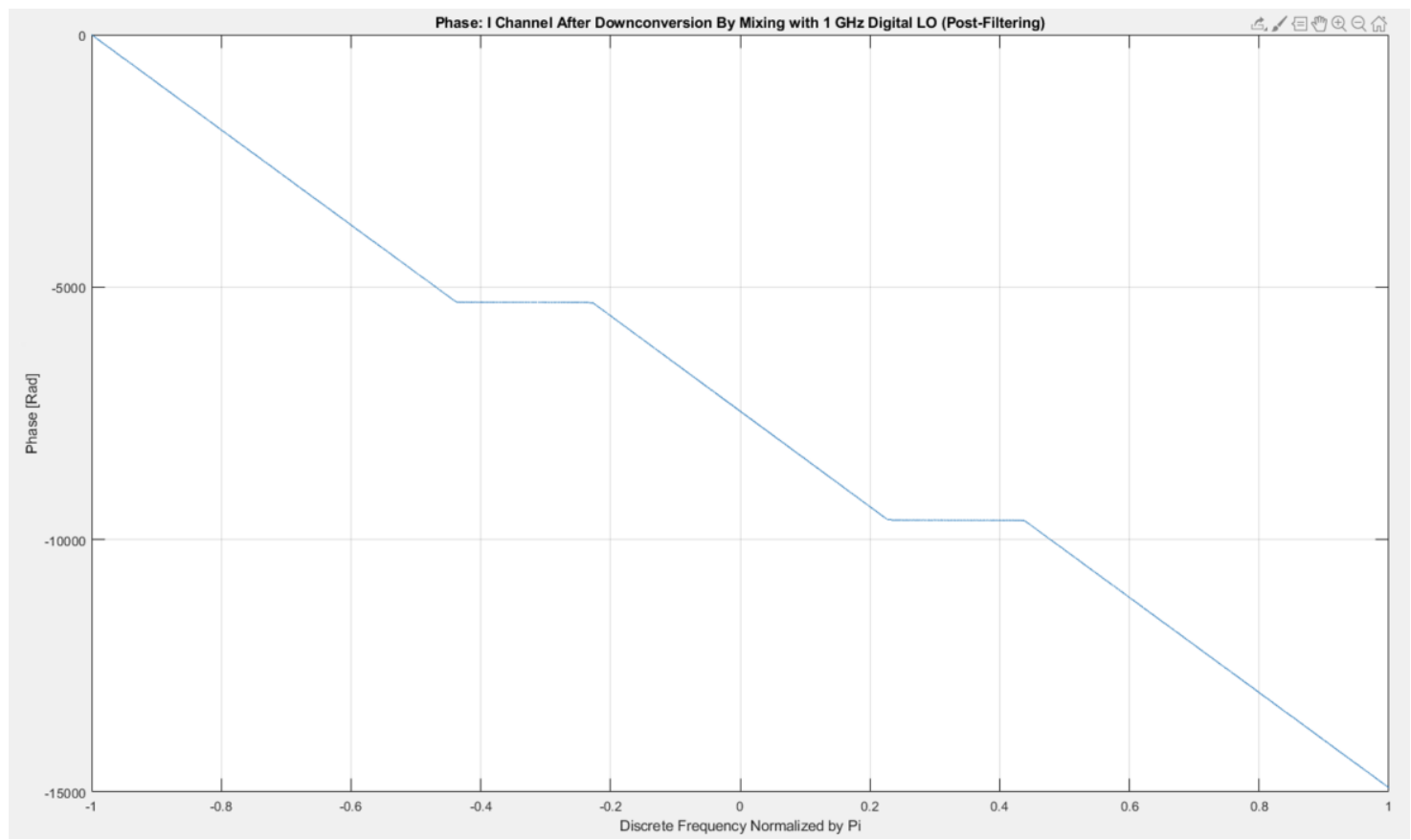

Figure 6.18: Phase spectrum of received (IF) $1.35 \mathrm{GHz}, 1 \mu$ s pulse, sampled at 3 GSPS, mixed with the in-phase digital LO at $1 \mathrm{GHz}$ to create the in-phase (I) channel. Following filtering using the FIR Type 1 Kaiser Windowed LPF ( $\beta=$ 3.3953). 


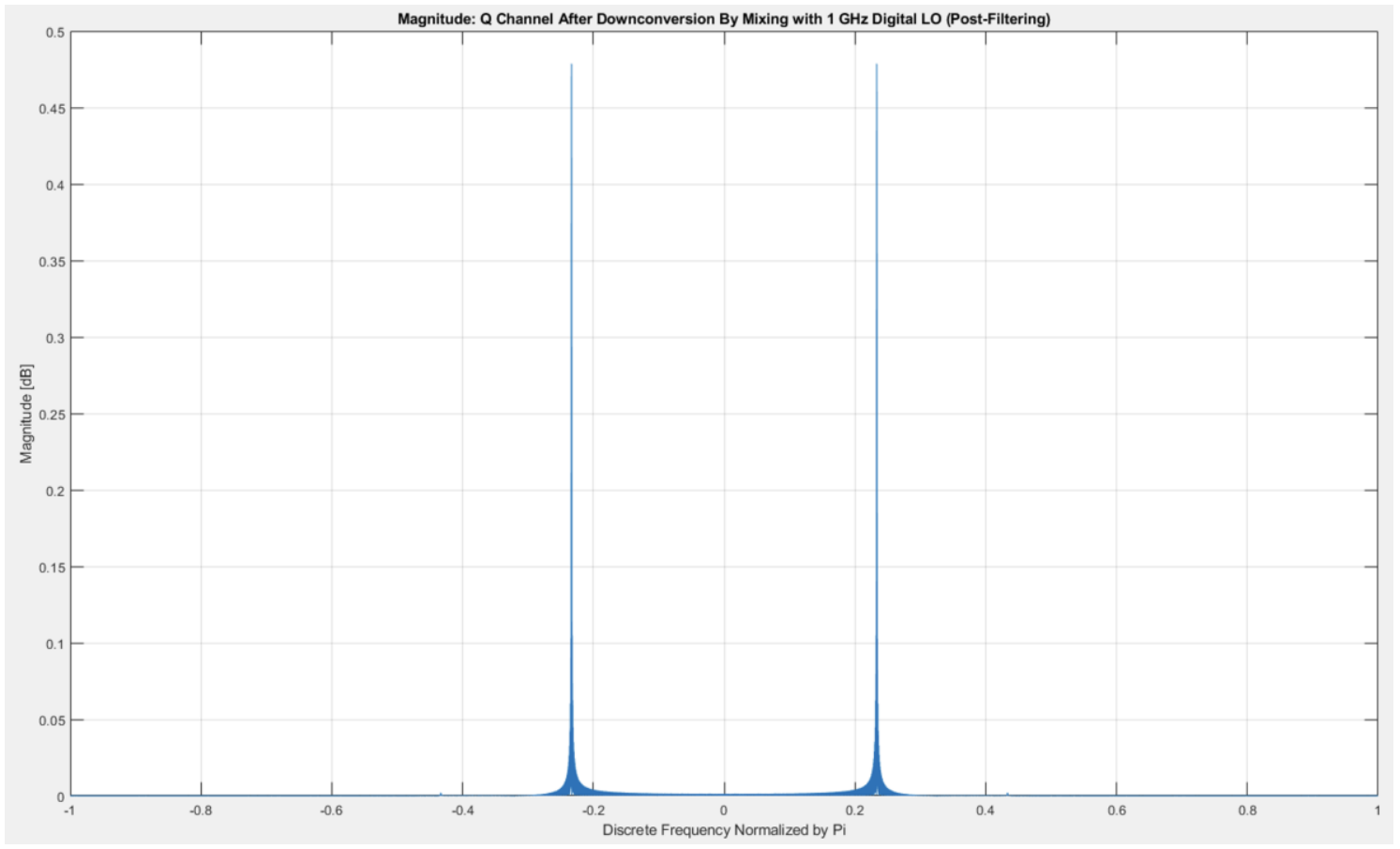

Figure 6.19: Magnitude spectrum of received (IF) $1.35 \mathrm{GHz}, 1 \mu$ s pulse, sampled at 3 GSPS, mixed with the quadrature digital LO at $1 \mathrm{GHz}$ to create the quadrature(Q) channel. Following filtering using the FIR Type 1 Kaiser Windowed LPF ( $\beta=$ 3.3953). 


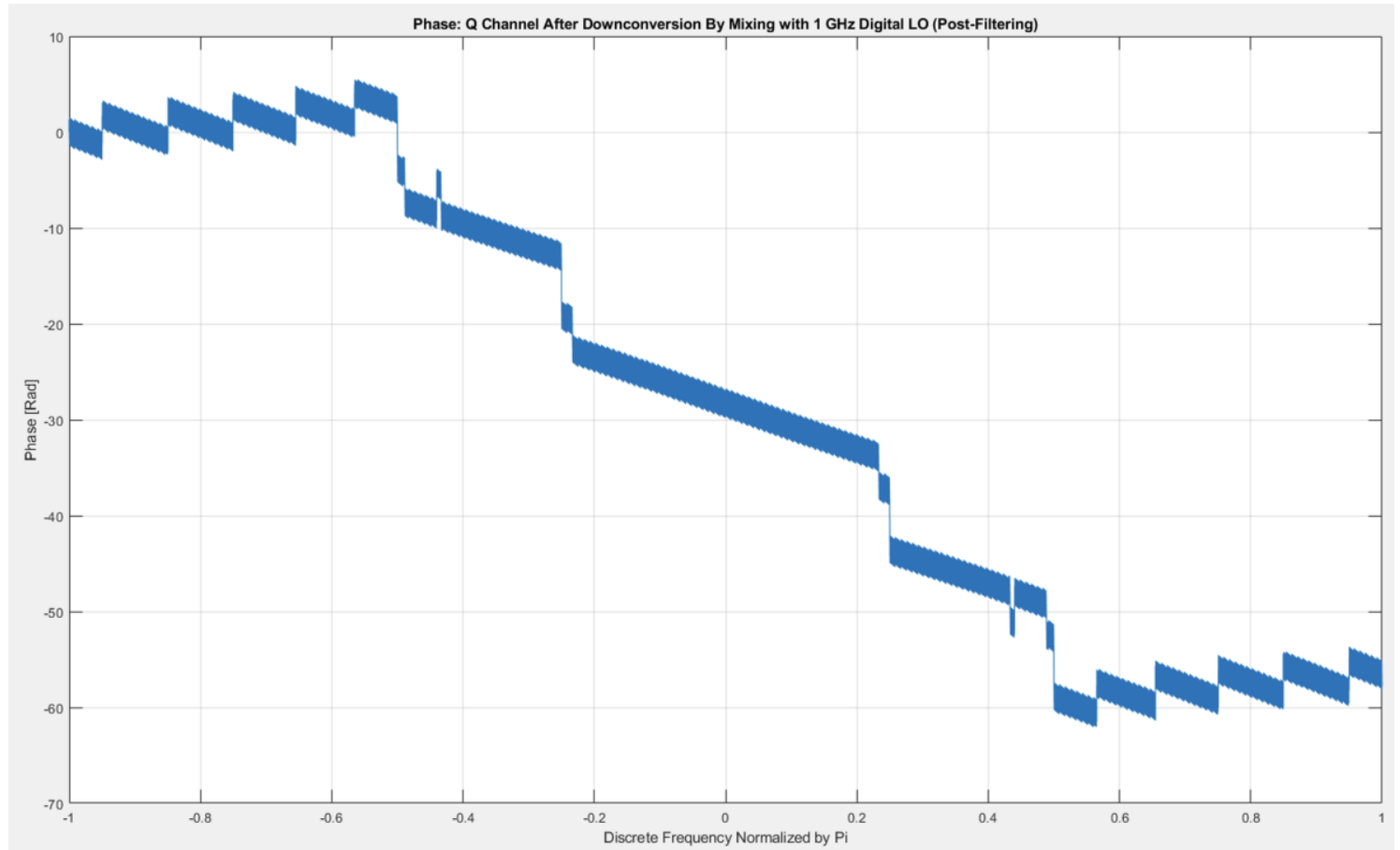

Figure 6.20: Phase spectrum of received (IF) $1.35 \mathrm{GHz}, 1 \mu$ s pulse, sampled at 3 GSPS, mixed with the quadrature digital LO at $1 \mathrm{GHz}$ to create the quadrature(Q) channel. Following filtering using the FIR Type 1 Kaiser Windowed LPF ( $\beta=$ 3.3953).

Table 6.4 outlines the steps a received signal undergoes, and the continuous and discrete frequency at each stage in the I/Q demodulation process. The formula used in frequency computation are applicable to the range of frequencies for assumed radar operation. The example frequency chosen is a received signal at an IF of $1.35 \mathrm{GHz}$ prior to sampling.

The signals are downsampled by a factor of four and magnitude and phase spectra of the I and Q channels are displayed in Figure 6.21 to 6.24. It is evident that downsampling has reduced the sampled spectral bandwidth from $3 \mathrm{GHz}$ to $750 \mathrm{MHz}$. On the spectrum the total bandwidth is visible in the frequency range of $-375 \mathrm{MHz}$ to +375 $\mathrm{MHz}$. 
Table 6.4: The continuous and discrete frequencies of a received signal through the stages of the $I / Q$ demodulation system.

\begin{tabular}{|c|c|c|c|}
\hline Stage & Formula & $\begin{array}{l}\text { Continuous } \\
\text { Frequency }\end{array}$ & Discrete Frequency \\
\hline Prior to Sampling & - & $f_{A, I F}=1.35 \mathrm{GHz}$ & - \\
\hline Post-Sampling & $\begin{array}{l}\omega_{A, I F, \text { sampled }} \\
=\frac{2 \pi f_{A, I F}}{F_{S}}\end{array}$ & $f_{A, I F}=1.35 \mathrm{GHz}$ & $\begin{array}{l}\omega_{A, I F, \text { sampled }} \\
=0.9 \pi\left[\frac{\mathrm{rad}}{\text { sample }}\right]\end{array}$ \\
\hline Digital LO & $\omega_{D, L O}=\frac{2 \pi f_{D, L O}}{F_{S}}$ & $f_{D, L O}=1 \mathrm{GHz}$ & $\begin{array}{l}\omega_{D, L O} \\
=\frac{2 \pi}{3}\left[\frac{\text { rad }}{\text { sample }}\right]\end{array}$ \\
\hline $\begin{array}{l}\text { Following Mixing } \\
\text { For I/Q }\end{array}$ & $\begin{array}{l}f_{D, I F} \\
=\frac{1}{2}\left\{\cos \left(f_{A, I F}\right.\right. \\
\left.-f_{D, L O}\right) \\
+\cos \left(f_{A, I F}\right. \\
\left.\left.+f_{D, L O}\right)\right\} \\
\omega_{D, I F}=\frac{2 \pi f_{D, I F}}{F_{S}}\end{array}$ & $\begin{array}{c}f_{D, I F, \text { sum }} \\
=2.35 \mathrm{GHz} \\
f_{D, I F, \text { difference }} \\
=350 \mathrm{MHz}\end{array}$ & $\begin{array}{l}\omega_{D, I F, \text { sum }} \\
=1.567 \pi\left[\frac{\text { rad }}{\text { sample }}\right] \\
\omega_{D, I F, \text { sum }, \text { ALIAS }} \\
=0.433 \pi\left[\frac{\text { rad }}{\text { sample }}\right] \\
\omega_{D, I F, \text { difference }} \\
=0.233 \pi\left[\frac{\text { rad }}{\text { sample }}\right]\end{array}$ \\
\hline Post-LPF & $\begin{array}{l}f_{D, I F, L P F} \\
=\left\{\cos \left(f_{A, I F}\right.\right. \\
\left.\left.-f_{D, L O}\right)\right\} \\
\omega_{D, I F, L P F} \\
=\frac{2 \pi f_{D, I F, L P F}}{F_{S}}\end{array}$ & $\begin{array}{l}f_{D, I F, L P F} \\
=350 \mathrm{MHz}\end{array}$ & $\begin{array}{l}\omega_{D, I F, L P F} \\
=0.233 \pi\left[\frac{\mathrm{rad}}{\text { sample }}\right]\end{array}$ \\
\hline $\begin{array}{l}\text { Post- } \\
\text { Downsampling } \\
(\mathrm{D}=4)\end{array}$ & $\begin{array}{l}\omega_{D, I F, L P F, D S} \\
=\frac{2 \pi f_{D, I F, L P F}}{F_{S} / D}\end{array}$ & $\begin{array}{l}f_{D, I F, L P F} \\
=350 \mathrm{MHz}\end{array}$ & $\begin{array}{l}\omega_{D, I F, L P F, D S} \\
=0.933 \pi\left[\frac{\mathrm{rad}}{\text { sample }}\right]\end{array}$ \\
\hline
\end{tabular}




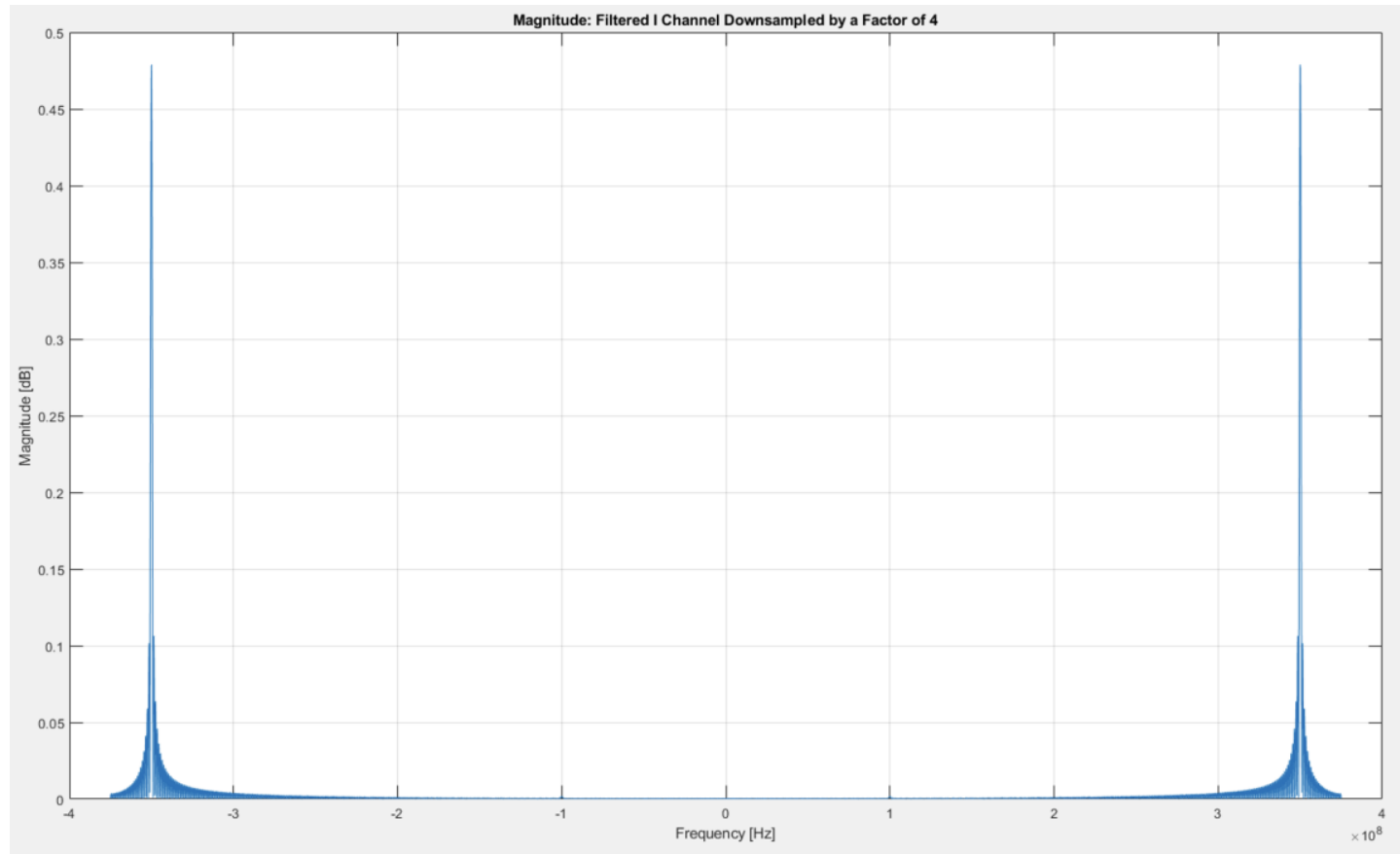

Figure 6.21: Filtered Magnitude spectrum of received (IF) $1.35 \mathrm{GHz}, 1 \mu \mathrm{s}$ pulse, sampled at 3 GSPS, mixed with the in-phase digital $\mathrm{LO}$ at $1 \mathrm{GHz}$ to create the inphase (I) channel. The signal has been downsampled by a factor of 4.

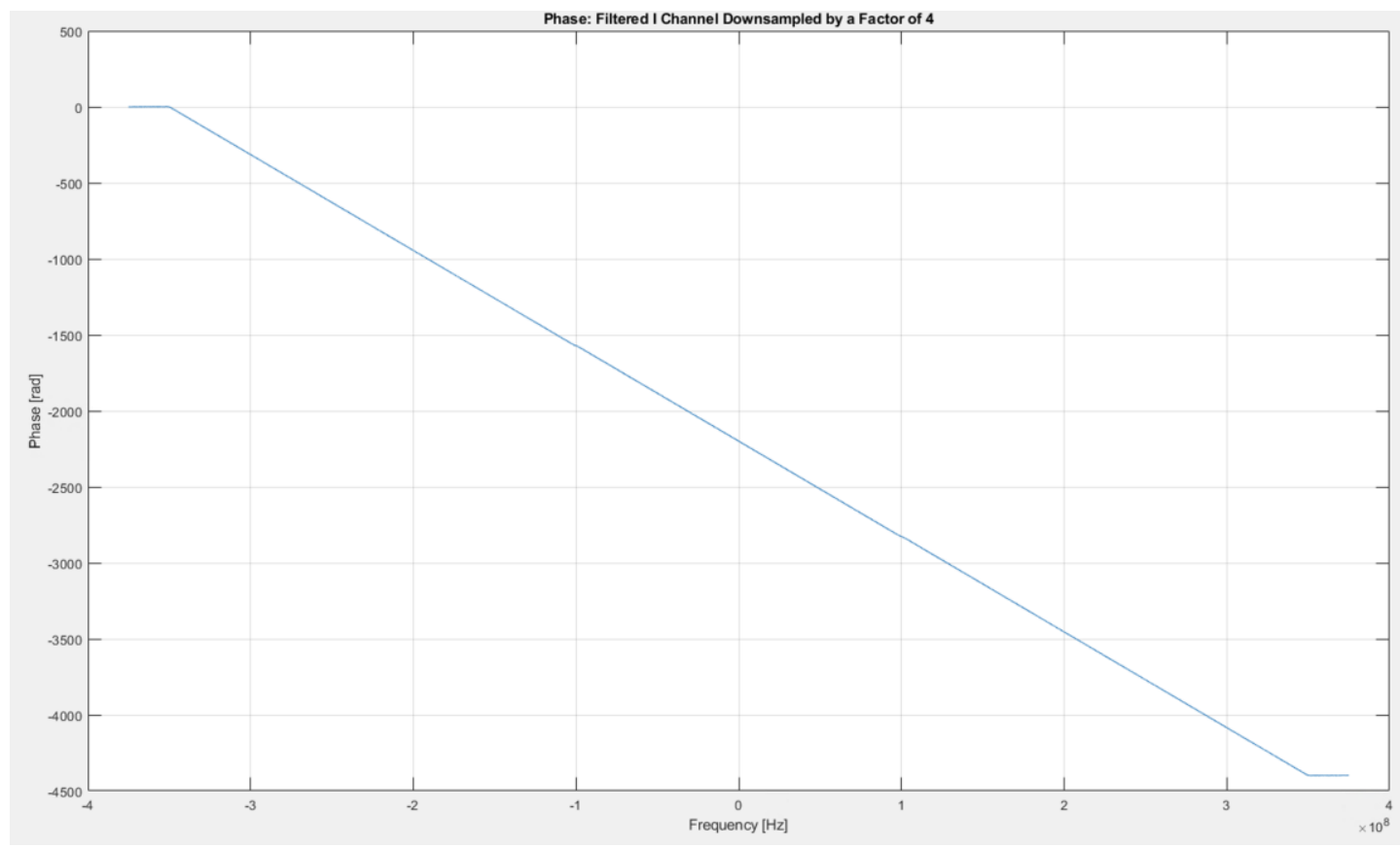

Figure 6.22: Filtered Phase spectrum of received (IF) $1.35 \mathrm{GHz}, 1 \mu$ s pulse, sampled at 3 GSPS, mixed with the in-phase digital LO at 1 GHz to create the in-phase (I) channel. The signal has been downsampled by a factor of 4 . 


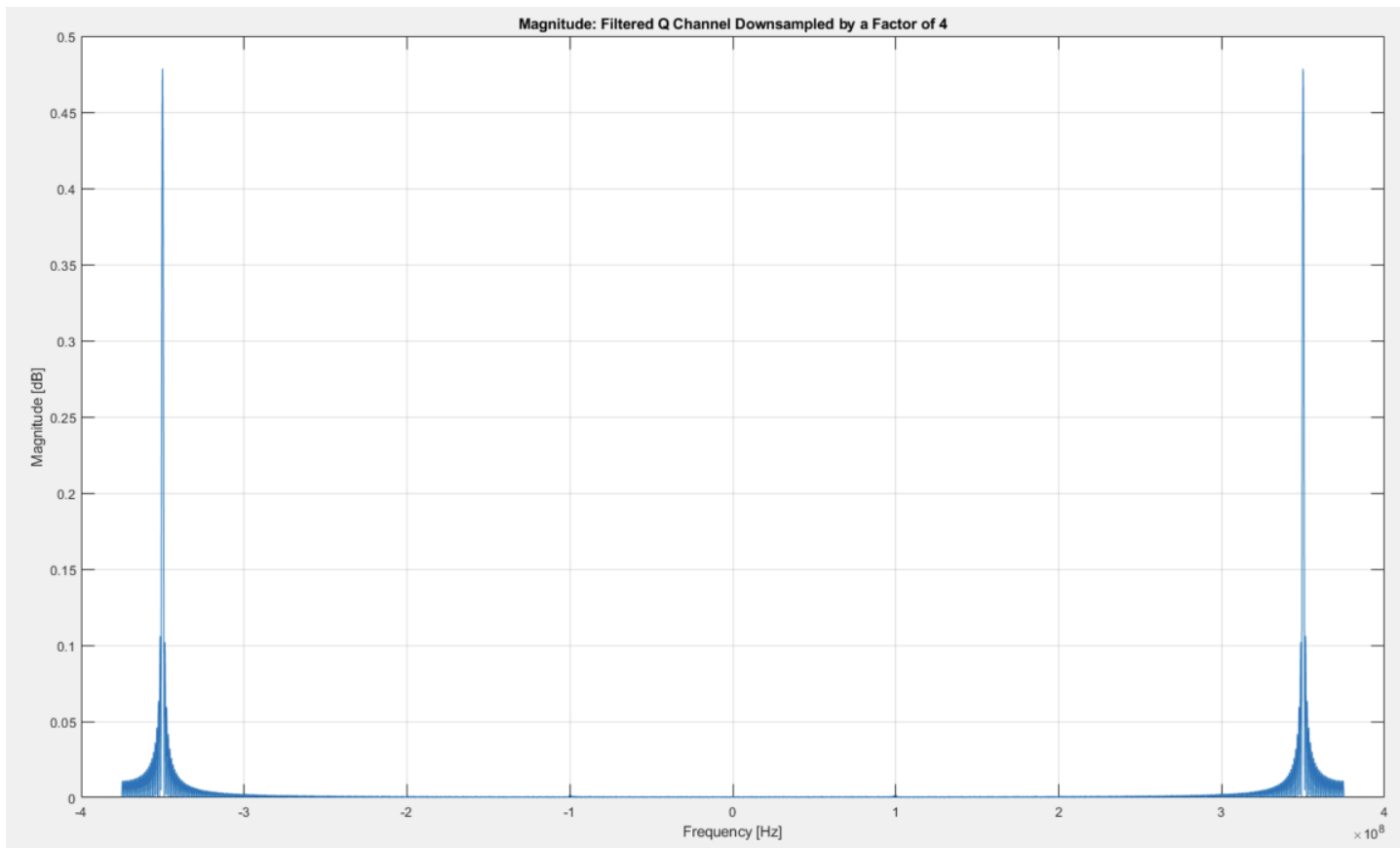

Figure 6.23: Filtered Magnitude spectrum of received (IF) $1.35 \mathrm{GHz}, 1 \mu$ s pulse, sampled at 3 GSPS, mixed with the quadrature digital $\mathrm{LO}$ at $1 \mathrm{GHz}$ to create the quadrature (Q) channel. The signal has been downsampled by a factor of 4 .

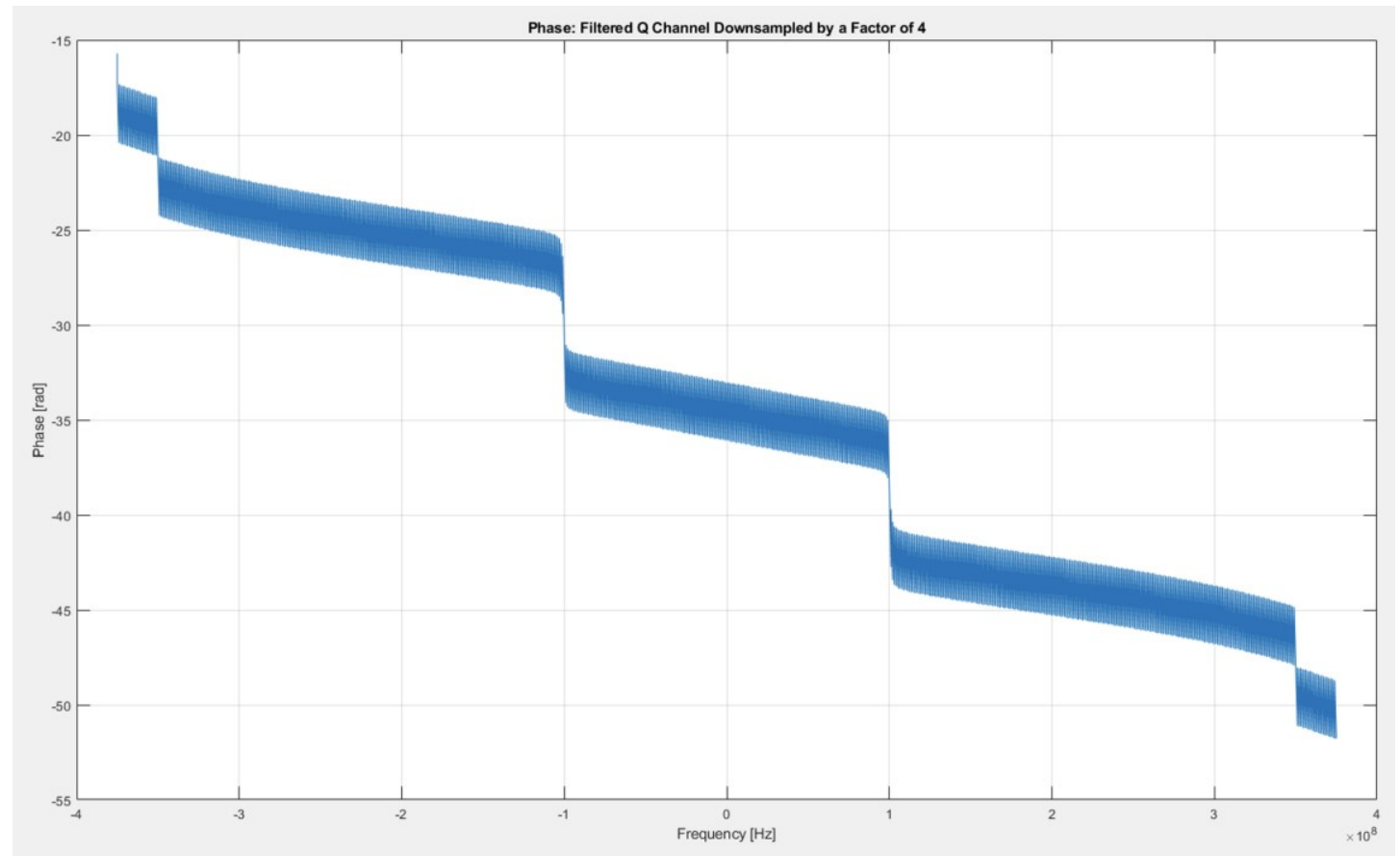

Figure 6.24: Filtered Phase spectrum of received (IF) $1.35 \mathrm{GHz}, 1 \mu$ s pulse, sampled at 3 GSPS, mixed with the quadrature digital $\mathrm{LO}$ at $1 \mathrm{GHz}$ to create the quadrature (Q) channel. The signal has been downsampled by a factor of 4 . 
The function of the digital system design in processing a constant frequency pulse from a stepped-frequency radar is displayed from Figure 6.11 to 6.24. This system is designed to counter both stepped-frequency, and wideband chirp radar systems. Verification of the digital system designed processes chirp signals, with the same design specifications as outlined in the beginning of this chapter, is shown in Appendix B. Through observation of the figures in Appendix B it is evident that the digital system design can be used to counter different types of wideband radar systems without introducing distortion in the magnitude or phase response of the baseband envelope.

\subsection{Digital Signal Modulation Application}

The modulation parameters to compose an echo signal have already been described in detail, and the computation of the parameters is discussed in chapter 5 . The equation representing the modulation on an echo signal, as the coherent summation of contributing scatterers is re-stated below.

$$
\begin{aligned}
\operatorname{Echo}(t)=\sum_{i=1}^{N} & A_{i}\left(t-\frac{2 r_{i}}{c}\right) \times \exp \left(-j\left(\frac{4 \pi}{\lambda}\right) r_{i}\right) \times \exp \left(+j 2 \pi\left(\frac{2 v_{i}}{\lambda}\right) t\right) \\
& \times \exp \left(j\left(2 \pi F_{c} t+\varphi_{0}\right)\right)
\end{aligned}
$$

The Doppler and phase modulation can be recombined into the carrier exponential term to represent the echo signal as it is transmitted. This is shown in equation 6.2.

$$
\operatorname{Echo}(t)=\sum_{i=1}^{N} A_{i}\left(t-\frac{2 r_{i}}{c}\right) \times \exp \left[j\left(2 \pi\left(F_{c}\left(\frac{2 v_{i}}{\lambda}\right)\right) t+\varphi_{0}-\left(\frac{4 \pi}{\lambda}\right) r_{i}\right)\right]
$$

Extraction of the modulating components from the carrier frequency term as in equation 6.1 allows the parameters to be applied to the baseband envelope of the echo pulse. This is the motivation for demodulation to I/Q baseband. This subsection addresses the application of modulating parameters in the following order: amplitude, time delay, phase, and Doppler. 


\subsubsection{Time Delay}

The amplitude envelope term in equation 6.1 is time delayed according to the range of the $i^{\text {th }}$ scattering from the reference scatterer. The computed values are on the order of microseconds or less, since the distance between scattering locations is relatively small compared to the propagation of waves at the speed of light.

Delay in a digital system is represented as a number of samples, as found by determining the group delay from a system transfer function [46]-[49]. The modulation parameters utilized do not require filter implementation, thus, group delay cannot be exploited to create a delay. The delay time is converted into a number of samples delay based on the sampling rate of the system. Equation 6.3 is used to compute the number of delay samples.

$$
n_{\text {delay }}=\left\lfloor\frac{\left(\frac{2 r_{i}}{c}\right)}{F_{S, D S}}\right\rfloor
$$

The variable $F_{S, D S}$, is the sampling rate of the digital system following downsampling. In this system design $F_{S, D S}=750 \mathrm{MHz}$. The \ 」, operator indicates rounding the computed value down to the nearest integer value [46]. The baseband signal is zero-padded at the front of the signal for the number of samples corresponding to the delay.

\subsubsection{Amplitude \& Phase Modulation}

The amplitude modulation is proportional to the RCS of the scattering location selected in the resolution cell. The phase modulation is proportional to the distance between the $\mathrm{i}^{\text {th }}$ scattering location and the reference scatterer. The formulation of the amplitude and phase values is discussed in the Target Signature Synthesis Software section. The amplitude and phase modulation values are constants. These modulation parameters can be applied in one of three methods.

The first method is to directly apply the amplitude modulation to each of the I/Q baseband channels. The phase modulation is applied to each channel as well through multiplication by a constant value of $\cos \left[\left(\frac{4 \pi}{\lambda}\right) r_{i}\right]$. This is implemented as a 
multiplication operation of all samples in the signal by a constant value. In combining the I/Q channels the amplitude modulation will be visible on the synthesized echo signal. The phase modulation is visible in the sample sequence but is not as overt as amplitude modulation.

The second method requires directly applying the amplitude and phase modulation constant to the sampled echo signal. Implying that I/Q baseband demodulation is not performed. A benefit of $I / Q$ demodulation is the reduction in the number of samples, and thus the number of multiplication operations required. Thus, this method is not viable in a system implementing I/Q demodulation.

The final method involves amplitude scaling the Doppler modulating signal which is applied to the I/Q baseband channels. The Doppler modulating signal is also phase shifted by the phase modulation constant. This is the chosen method of applying the modulation parameters to the complex baseband signal. Doppler modulating the echo signal is discussed in the following section.

\subsubsection{Doppler Modulation}

As mentioned, each dominant scattering location contributes a time delay, phase, amplitude, and Doppler modulation to the target echo signal.

The application of the Doppler modulation is a multi-step process described below.

1. A timer is created, starting at time 0 seconds from reception of the first pulse from the hostile radar system.

2. The Doppler shift of a scattering location is read into the program, and the number of samples required to represent one period of the Doppler signal is computed. Equation 6.4 shows how the number of samples required to represent a Doppler signal is computed.

$$
n_{\text {Doppler }}=\left\lceil\frac{f_{D S}}{f_{D, i}}\right\rceil
$$

In equation $6.4 f_{D S}$ is the sampling rate after downsampling. The variable $f_{D, i}$ represents the Doppler shift for the $\mathrm{i}^{\text {th }}$ scattering location. The $\lceil\mid$ operator indicates that the computed value is rounded up to the nearest integer. In general, the number 
of samples required to represent a Doppler shift is greater than the number of samples in the received waveform.

3. The scattering location phase and amplitude modulation parameters are included in the Doppler signal formulation. A Doppler signal for one period is created for a single scattering location. The modulating signal is shown in equation 6.5. This is effective since all modulation factors are included in a single multiplication operation, rather than performing a multiplication operation for each modulation factor individually.

$$
\text { ModSignal }_{i}=A_{i} \times \exp \left(+j\left[2 \pi\left(\frac{2 v_{i}}{\lambda}\right) t-\left(\frac{4 \pi}{\lambda}\right) r_{i}\right]\right)
$$

4. Repeat steps 2 and 3 for all scattering locations in the profile.

5. For every pulse after the $1^{\text {st }}$ pulse, based on the time elapsed determine the initial sample position for the Doppler modulation signal. The number of samples to represent a single Doppler signal is the maximum sample position. If the time elapsed is greater than a single Doppler signal period, then find the modulo of the sample position with the maximum number of samples for a single Doppler period. This is stated in equation 6.6 below.

$$
n_{\text {mod,start }}=\left(\frac{t_{\text {elapsed }}}{\frac{1}{f_{D S}}}\right) \bmod \left(n_{\text {Doppler }}\right)
$$

This is shown graphically in Figures 6.25 and 6.26 on the following pages. 

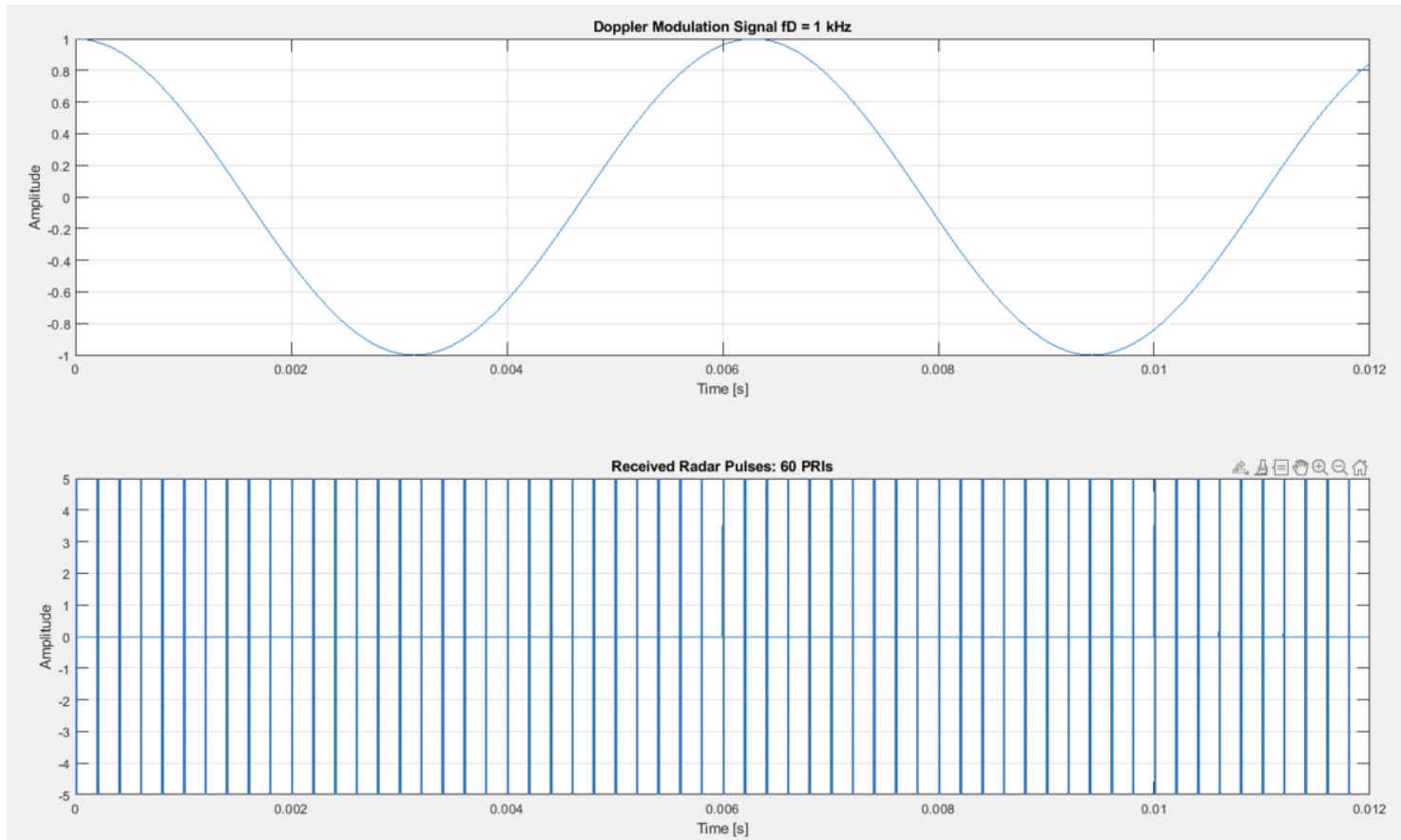

Figure 6.25: Doppler Modulation Signal for a single scatterer overlayed with 60 PRIs of the received hostile radar signal.

The Doppler signal is at a frequency of $1 \mathrm{kHz}$. The received radar pulses are $15 \mu \mathrm{s}$ in pulse width, with a PRF of $5 \mathrm{kHz}$ and a downconverted carrier frequency of $200 \mathrm{MHz}$. These signals are represented by the sampling rate of $750 \mathrm{MHz}$.

Across a total of 60 PRIs, it is observed that each pulse matches a different portion of the Doppler modulation signal. It is the overlapping section between the Doppler signal and the received pulse which are multiplied together to create the modulating effect across multiple PRIs. If each radar pulse is considered to be a sample of the Doppler signal, then by performing a DFT of the sampled signal the original Doppler spectrum is recreated. A subsection of two pulses is shown in Figure 6.26. It is observed that each pulse overlaps with a subsection of the Doppler signal. 

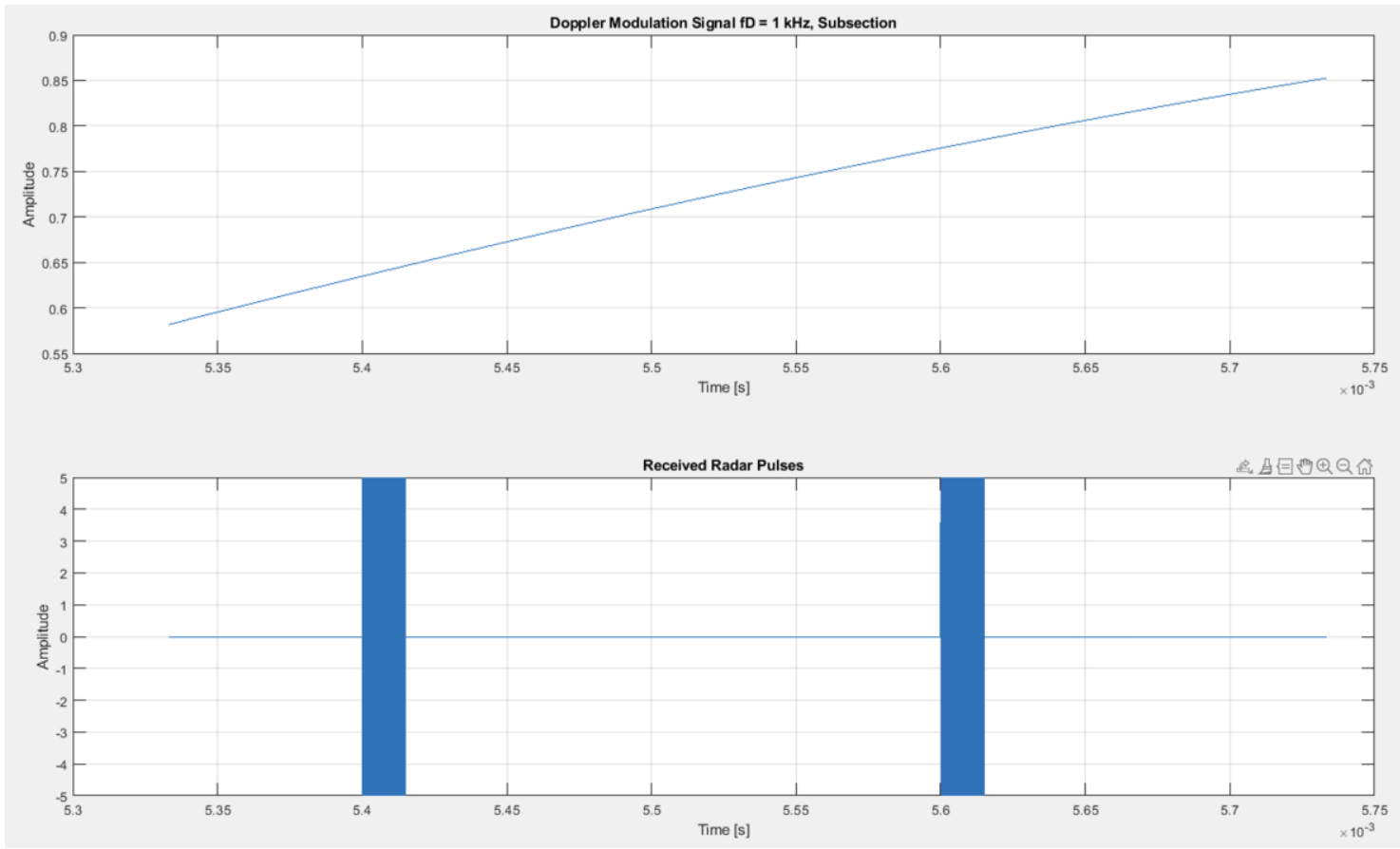

Figure 6.26: Subsection of Doppler Modulation Signal for a single scatterer overlayed with two PRIs of the received hostile radar signal.

6. With the new sample start position, the modulation equation from step 3 becomes the new equation below.

$$
\text { ModSignal }_{i}=A_{i} \times \exp \left(+j\left[2 \pi\left(\frac{2 v_{i}}{\lambda}\right) n_{\text {mod,start }}-\left(\frac{4 \pi}{\lambda}\right) r_{i}\right]\right)
$$

7. The modulating signal is multiplied by the samples of the received waveform. The value of $n_{\text {delay }}$ determines the sample position in the received waveform where the Doppler signal multiplication begins.

8. Steps 2-7 are repeated for all scattering locations contributing to the synthesized echo.

The Doppler modulation process is tested in the Digital System design for a single scattering location with a Doppler frequency of $3.5 \mathrm{kHz}$. The hostile radar has a pulse width of $1 \mu \mathrm{s}$, a PRF of $10 \mathrm{kHz}$, and a down-converted carrier frequency of $200 \mathrm{MHz}$. The Doppler signal is sampled over 286 total PRIs. The baseband complex envelope of the modulated slow-time series for the scattering location history is shown in Figure 6.27. 


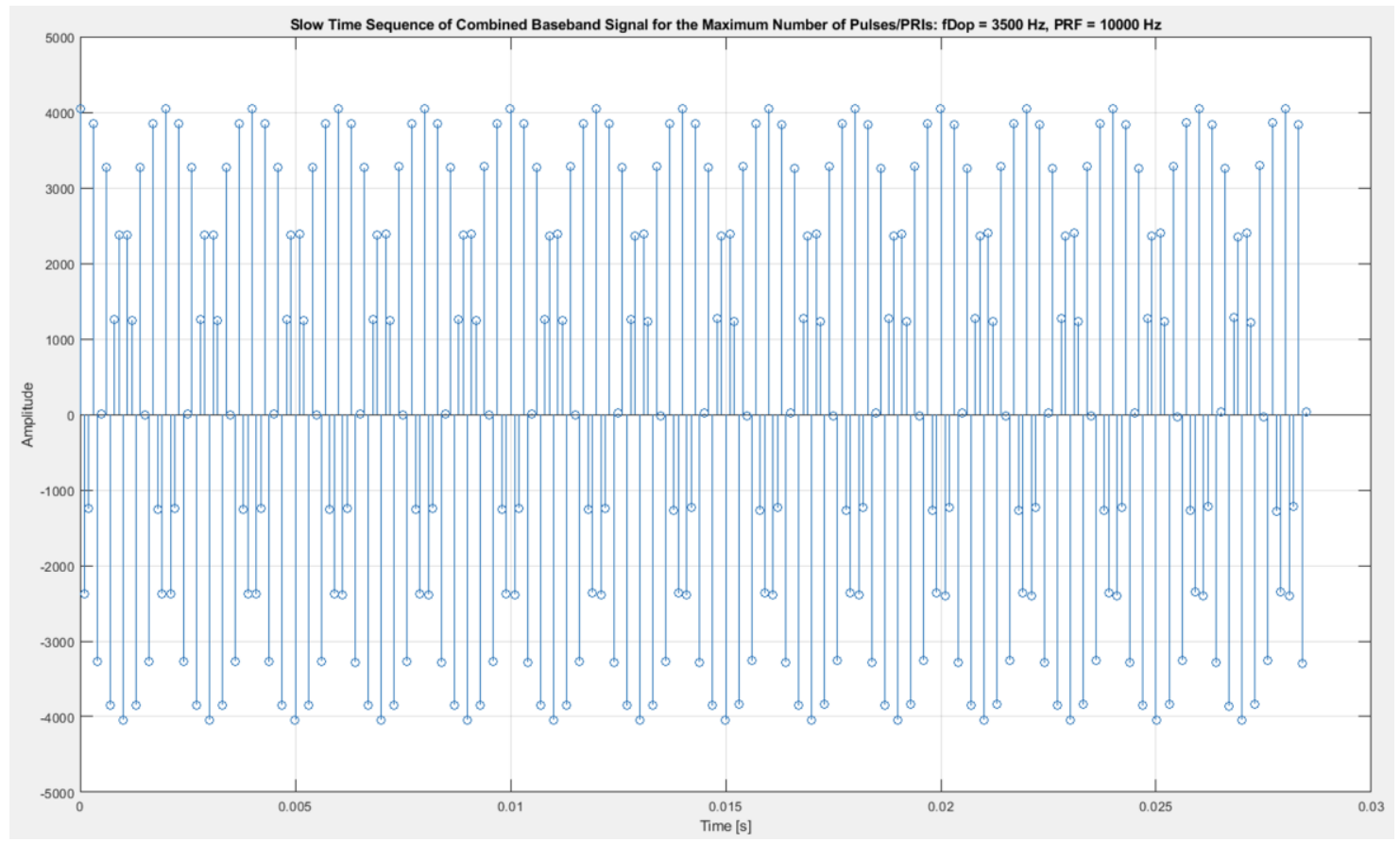

Figure 6.27: Baseband complex samples of slow-time sequence of a scattering location with a Doppler frequency of $3.5 \mathrm{kHz}$.

Taking the DFT of the slow-time samples will result in a magnitude spectrum with peaks at the Doppler frequency. This is shown in Figure 6.28 below.

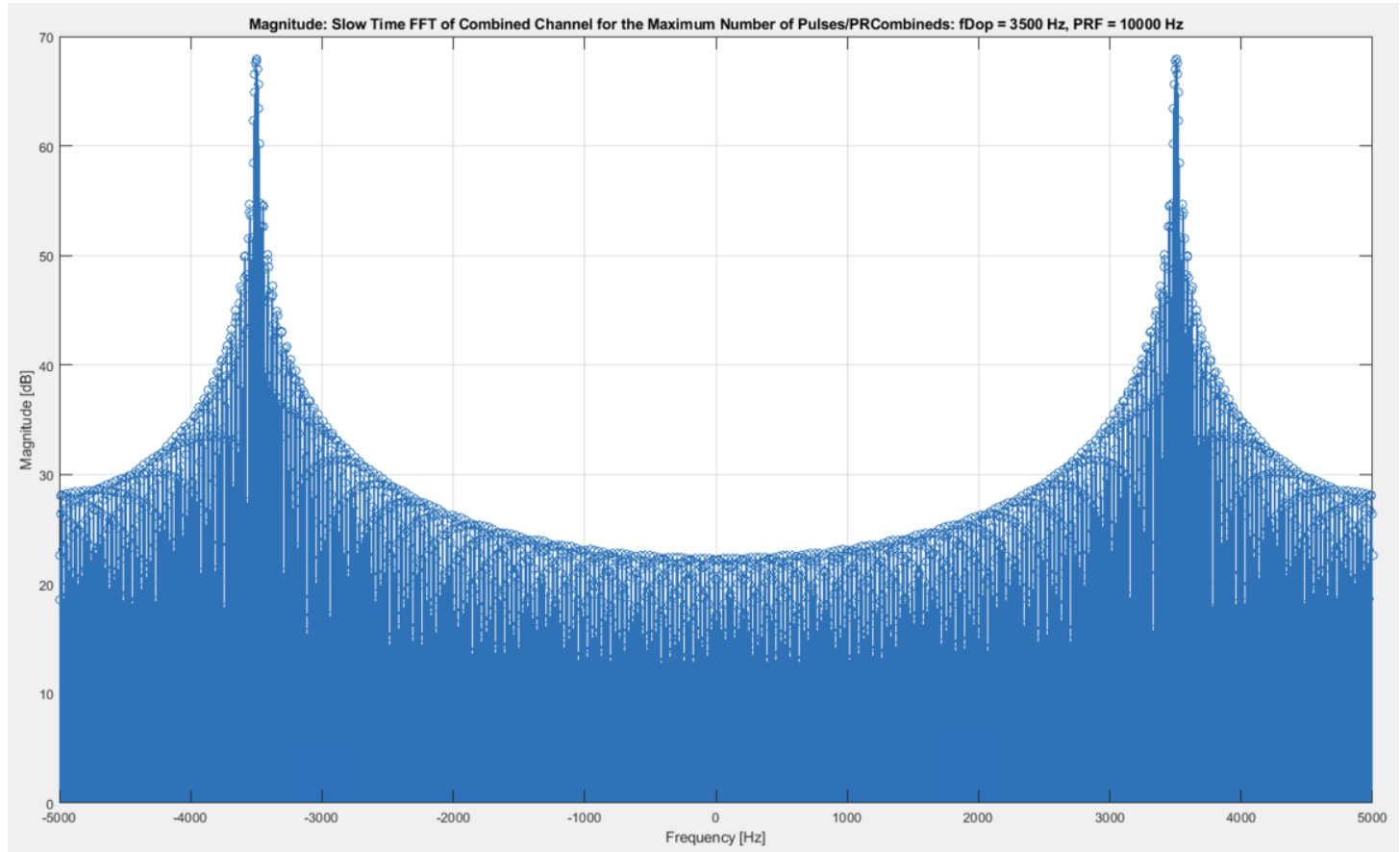

Figure 6.28: DFT of slow-time complex samples in Figure 6.27. Note the peaks at the Doppler frequency of $3.5 \mathrm{kHz}$. 
In target echo synthesis multiple scattering locations with different modulating parameters are coherently combined in the echo signal. Additionally, the Doppler frequency of a scattering location will only be constant until the profile decorrelation time is reached. This results in significant constructive and destructive interference in the slow-time history of a single range bin. The interference will result in a spread of the Doppler energy across the spectrum in an unpredictable manner. Another viewpoint of Doppler modulation is to consider it as a slow-time amplitude modulation on a portion of the echo signal. Thus, if the Amplitude modulation factor is not carefully considered in each profile echo synthesis the Doppler modulation can be negatively impacted. Amplitude modulation factor is directly tied to the Electromagnetic backscattering physics of the scattering location. Thus, in Range-Doppler imaging and phase histories significant spectral blurring is observed.

\subsection{Conclusion}

This chapter presented the digital system design which can be used to synthesize a target echo signal to counter stepped frequency or chirp radar signals. The system specifications are outlined, along with the sampling rate and downsampling factor. A quadrature demodulation system is designed, along with a digital lowpass filter implementation. The use of this system to apply the modulation parameters to a signal to synthesize the target echo is discussed as well. The effectiveness of this theoretical system design, as well as the TSS program from Chapter 5 is evaluated through simulations done in MATLAB presented in Chapter 7. 


\section{Chapter 7: Target Signature Synthesis System Simulation}

\subsection{Introduction}

The digital system designed in chapter 6 to synthesize target echoes is simulated in MATLAB to verify performance capabilities. This chapter uses the same radar design parameters outlined in the beginning of chapter 6 .

The first subsection of this chapter addresses the top-level simulation program setup in MATLAB for both stepped frequency and chirp radar systems. The second subsection provides more depth into the stepped frequency simulations and data processing to produce synthetic high-resolution range profiles and phase histories. The third subsection addresses simulation of range profile and phase history generation when countering chirp radar systems. The final subsection presents simulation data of RangeDoppler image processing for both radar types.

\subsection{Simulation Setup}

The Target Signature Synthesis system designed in this thesis is meant to operate on a ship, which is being tracked or observed by a hostile radar system. The real ship will produce an echo return to the hostile radar, however, with the target signature synthesis program, ideally a second realistic target echo return is transmitted. This would result in the hostile radar system observing two targets, one real and one 'false' target created by the program, rather than a single real target.

Figure 7.1 shows a block diagram of the operation of the system to synthesize a target echo. 


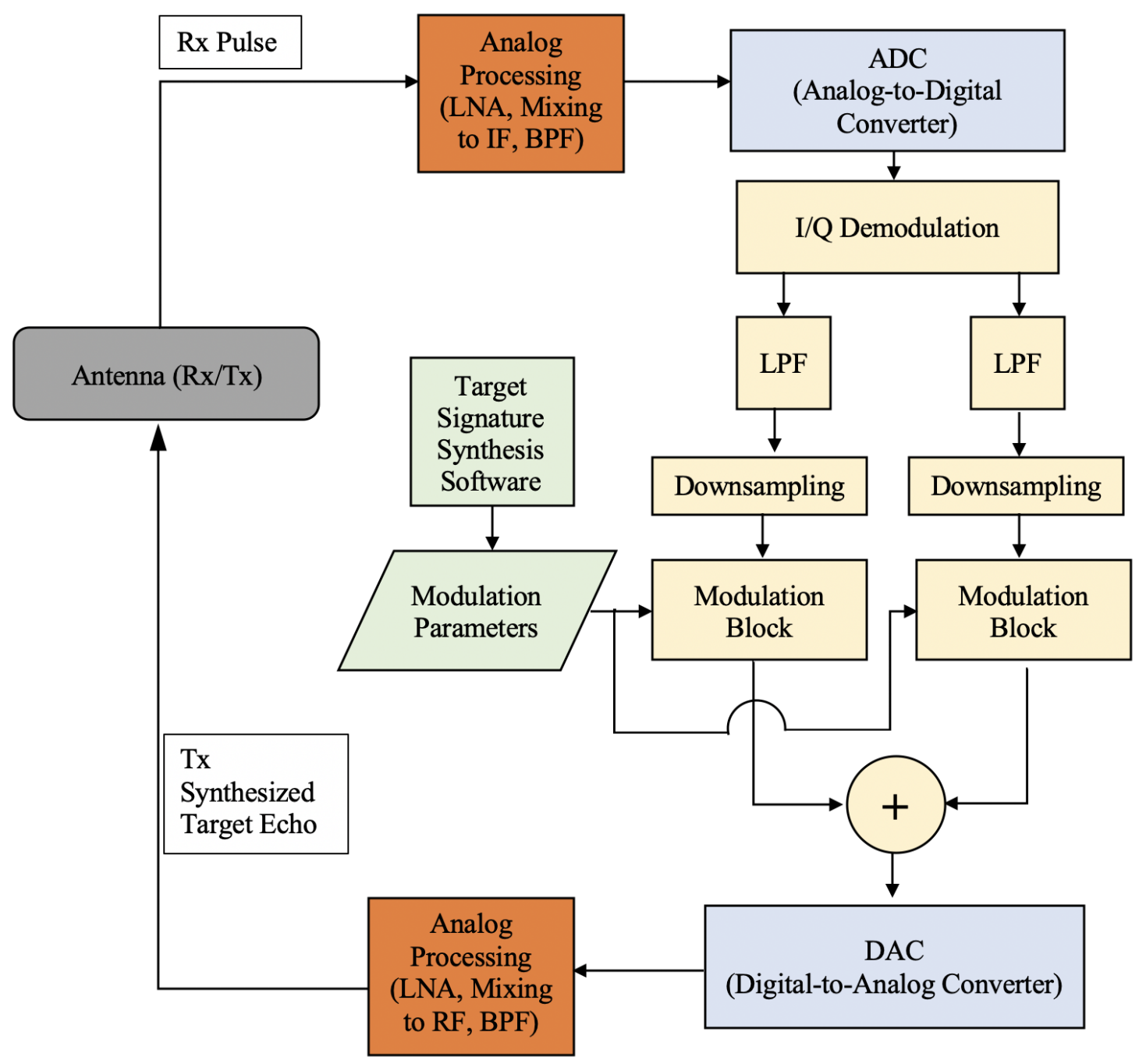

Figure 7.1: Top-Level Block Diagram of Target Signature Synthesis System.

The blocks highlighted in light green indicate offline software processing stages presented in chapter 5. The blocks highlighted in a light yellow in Figure 7.1 are to be implemented in the FPGA of a DRFM (Digital RF Memory) chip. These blocks perform the online, or real-time, processing to create the false target echo signal as designed in Chapter 6.

In the MATLAB simulations it is assumed the analog processing has been performed, and the target echo synthesis simulation occurs for the blocks highlighted in light yellow in Figure 7.1. Additionally, the resolution of the digital data represented in MATLAB is not limited by the 12-bit dynamic range of the hardware specified in 
Chapter 6. Instead the resolution of this data is represented by the default 32-bit representation of values in MATLAB.

Figure 7.2 shows a detailed process diagram of the digital system simulated in MATLAB.

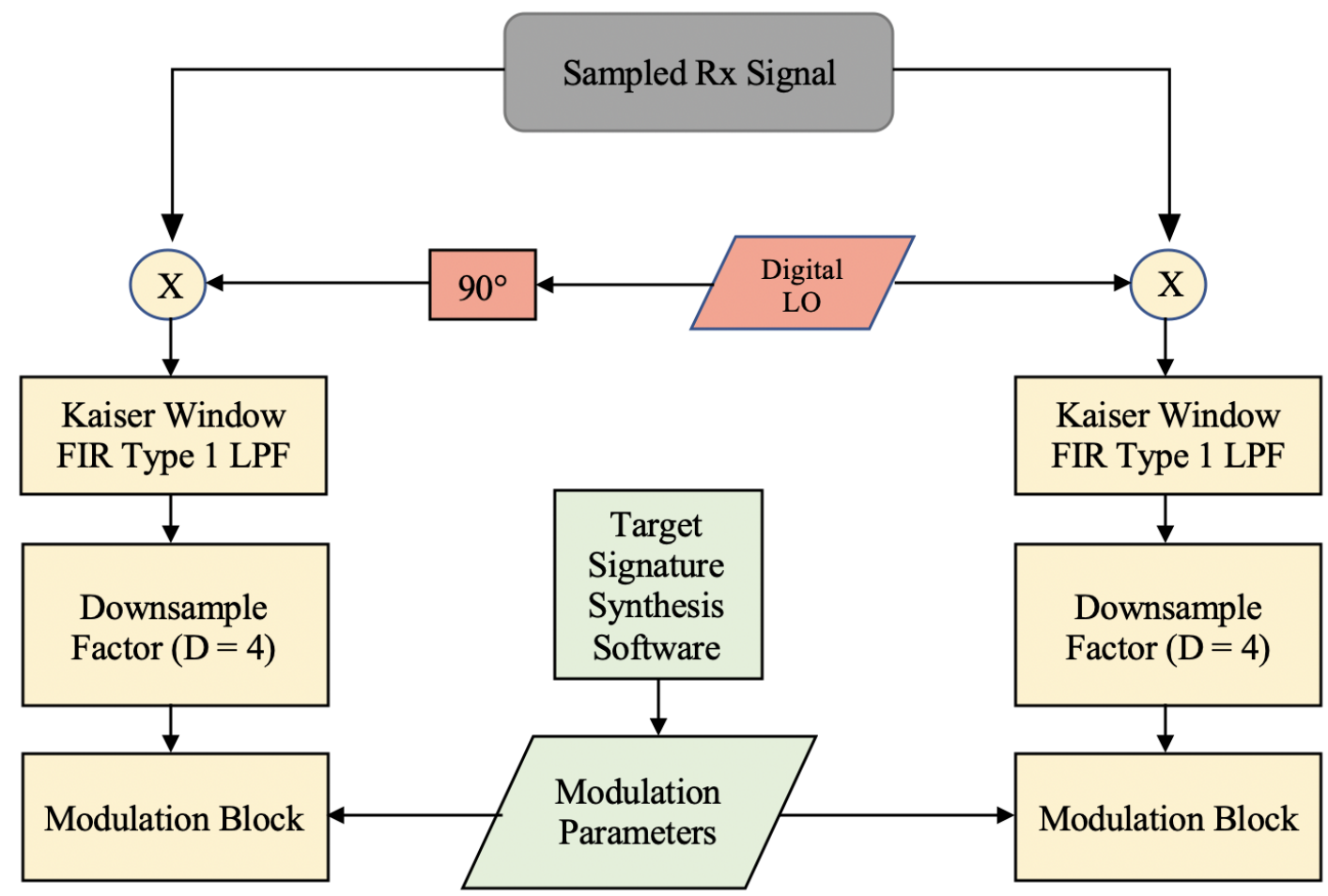

Figure 7.2: System Block Diagram of Target Signature Synthesis Digital System Design up to the modulation block.

The modulation block simulation in MATLAB follows the method outlined in chapter 6 for the application of Doppler modulation. The process of applying the modulation parameters can have multiple variations in a hardware implementation. Analysis of the design variations is beyond the scope of this research, which is focused on successful simulation of the conceptual digital system design.

The system block diagram of the modulation block as simulated in MATLAB is shown in Figure 7.3. Note that this block diagram outlines the process of applying the modulation parameters on the in-phase channel of the I/Q branches. The same process is applied to the quadrature channel simultaneously to create the modulated complex baseband envelope echo. 


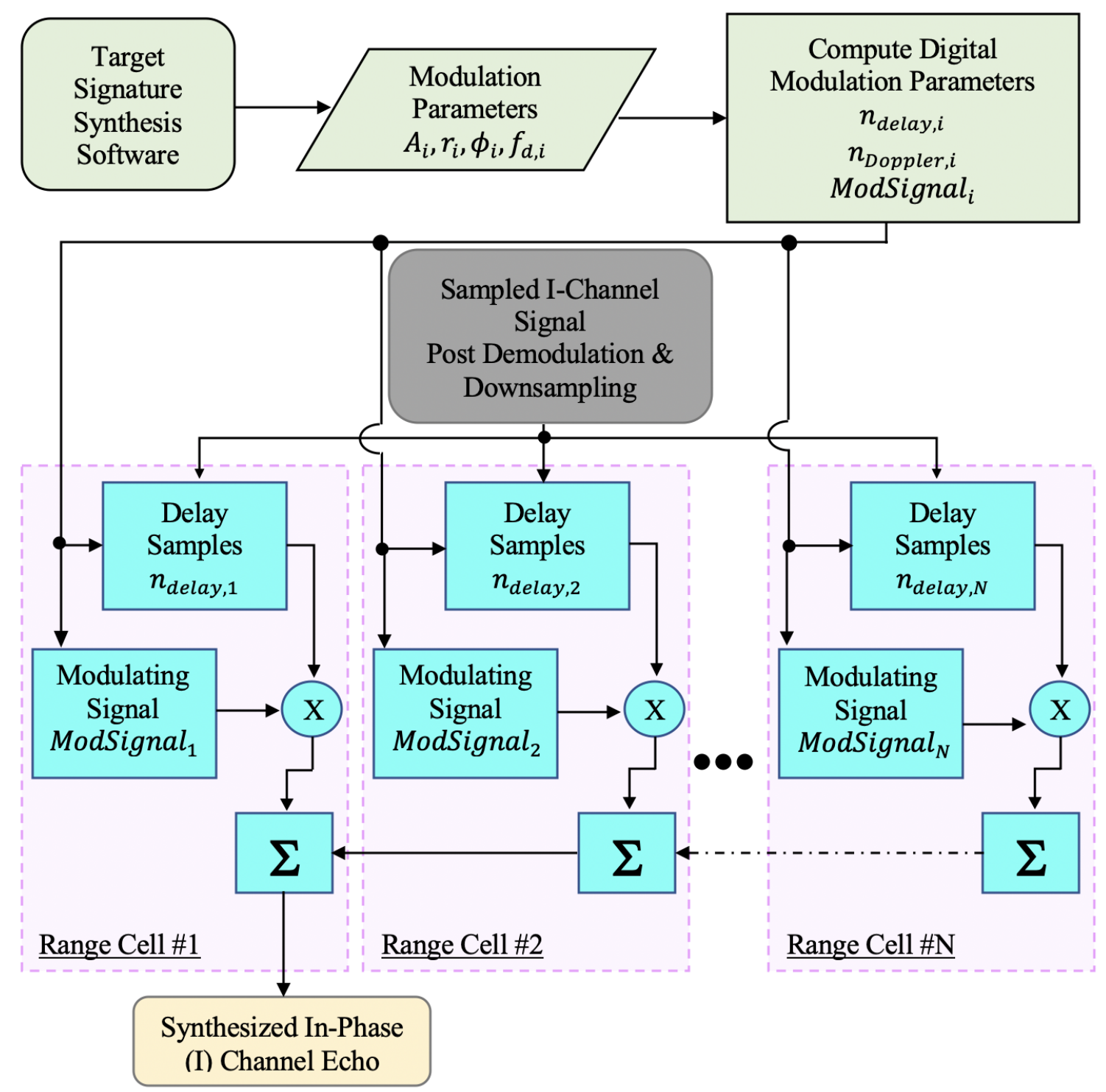

Figure 7.3: System Block Diagram of Signal Modulation Scheme in the In-Phase channel as implemented in MATLAB simulations. The same block diagram is applicable to the Quadrature channel.

After collecting the In-Phase and Quadrature Channel synthesized echo signals, the two channels are summed to create the complex baseband envelope signal. This signal is then converted to an analog signal through a digital-to-analog converter (DAC), and analog processing upconverts and performs any necessary filtering and amplifications prior to transmission of the synthesized echo. 
The data processing steps outlined for stepped frequency and chirp radar in chapters two and three are applied to generate the range profiles, phase histories, and Range-Doppler images for the simulation cases. The data processing is performed on the complex baseband synthesized echo signal. This is because the signal processing capabilities of the hostile radar are unknown. Thus, simulating data processing on the synthesized complex profile will verify the applied modulation's effectiveness in producing the range profile, phase history and Range-Doppler image. The hostile radar parameters are designed based on literature from Wehner, Skolnik and Richards [43][45][49][50].

Including all user defined variables, and possibilities for hostile radar parameters there are over 10 million different simulation cases. From these a random few are selected to verify system design and functionality. For each case, the motion parameters and the hostile radar LOS position is unique. The Target Signature Synthesis program's resolution is varied to view the impact of including more scattering locations in profile synthesis. The resolution of the hostile radar system is not a factor in selection of the resolution of the synthesized target signature in the TSS program. This means a lowresolution target signature can be chosen, through the TSS program, to counter a highresolution incoming radar signal, although this is not recommended.

Figure 7.4 summarizes the MATLAB simulation program in a flowchart. The functionality of each script is described in further detail in Table 7.1. 


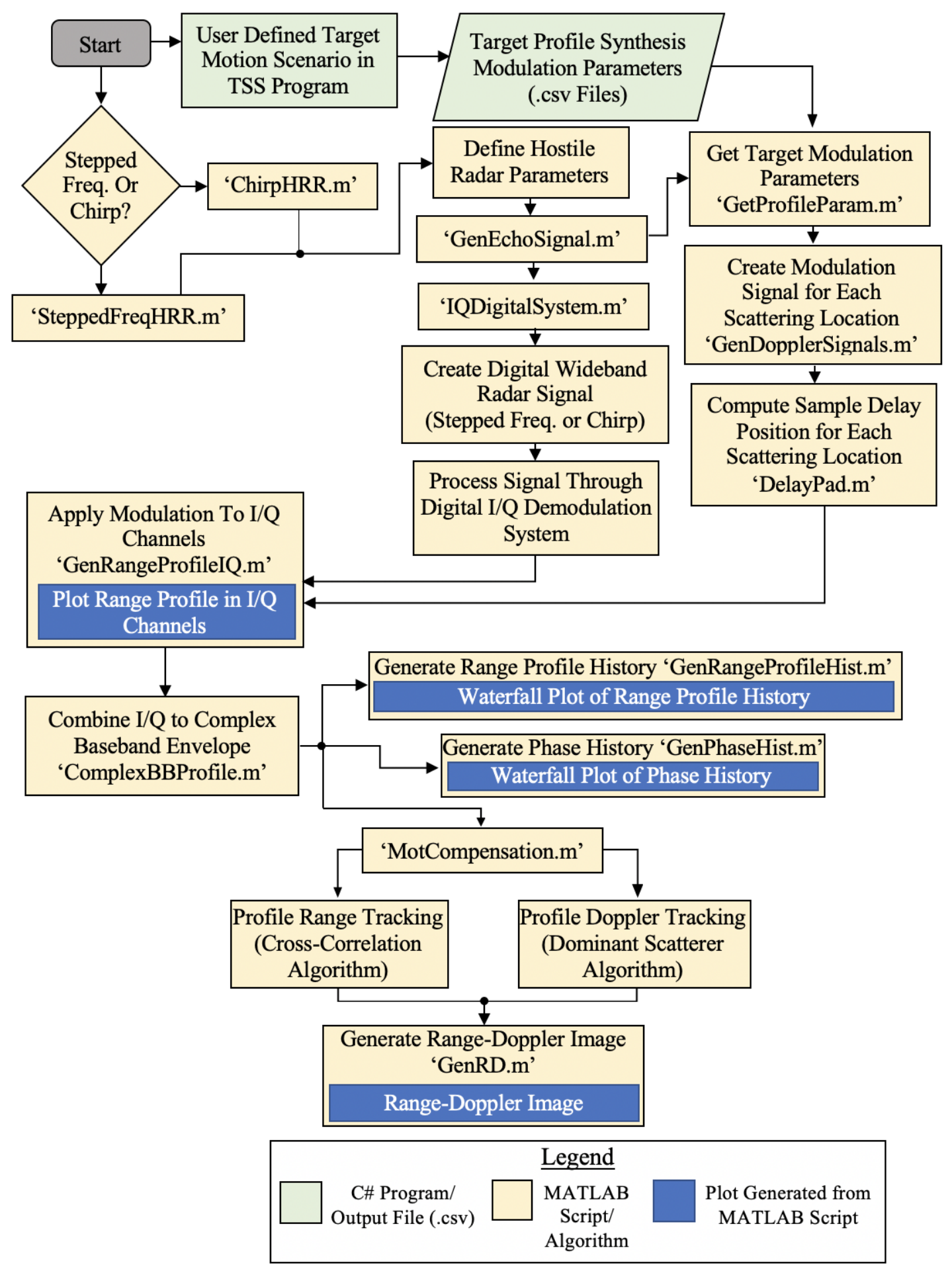

Figure 7.4: Flow Chart of MATLAB simulation program setup. 
Table 7.1: Description of MATLAB scripts used in system simulation.

\begin{tabular}{|c|c|}
\hline MATLAB Script/File & Functionality \\
\hline $\begin{array}{l}\text { SteppedFreqHRR.m } \\
\text { OR } \\
\text { ChirpHRR.m }\end{array}$ & $\begin{array}{l}\text { Common Variables: } \\
\text { Slant-Range/Cross-Range Window Size and Resolution, } \\
\text { Bandwidth, carrier frequency, PRF, decorrelation time, } \\
\text { initial phase, amplitude, pulse width. } \\
\text { Stepped Frequency Unique Variables: } \\
\mathrm{n}=\text { number of pulses in a burst } \\
\mathrm{N}=\text { number of bursts } \\
\Delta \mathrm{f}=\text { frequency step size } \\
\text { Chirp Unique Variables: } \\
\mathrm{K}=\text { chirp slope }\end{array}$ \\
\hline GenEchoSignal.m & $\begin{array}{l}\text { A Master Script which contains all function calls for other } \\
\text { scripts. }\end{array}$ \\
\hline $\begin{array}{l}\text { Profile Synthesis } \\
\text { Modulation Parameters } \\
\text { (.csv files) }\end{array}$ & $\begin{array}{l}\text { A table with } 4 \text { columns; delay (in seconds), phase (rad), } \\
\text { Doppler (Hz), and Amplitude Scaling Factor. } \\
\text { Each row in the table represents a single scattering location. }\end{array}$ \\
\hline GetProfileParam.m & Extract the modulation parameters from the .csv files. \\
\hline GenDopplerSignals.m & $\begin{array}{l}\text { Create the Modulation Signal for each scattering location. } \\
\text { As defined in equation } 6.7 \text {. }\end{array}$ \\
\hline DelayPad.m & Compute $n_{\text {delay }}$, using equation 6.3 . \\
\hline IQDigitalSystem.m & $\begin{array}{l}\text { Create the radar pulse and simulate processing through the } \\
\text { digital I/Q demodulation system designed in chapter } 6 \text {. }\end{array}$ \\
\hline GenRangeProfileIQ.m & $\begin{array}{l}\text { Apply the modulation signals to the I and Q Channel signals. } \\
\text { The synthesized echo for each channel (I and Q) is plotted. }\end{array}$ \\
\hline ComplexBBProfile.m & $\begin{array}{l}\text { Combine the I and Q Channels to create the complex } \\
\text { baseband echo signal. }\end{array}$ \\
\hline GenRangeProfileHist.m & $\begin{array}{l}\text { Stepped Frequency Radar: } \\
\text { For each burst generate the complex synthetic High- } \\
\text { Resolution Range Profile using the IDFT of the discrete } \\
\text { frequency signature samples. } \\
\text { Chirp Radar: } \\
\text { Generate the complex High-Resolution Range Profile by } \\
\text { performing digital pulse compression on the complex } \\
\text { baseband echo. } \\
\text { Using Waterfall plot display magnitude of Range Profile } \\
\text { history. }\end{array}$ \\
\hline GenPhaseProfileHist.m & Using Waterfall plot display phase of Range Profile history. \\
\hline MotCompensation.m & $\begin{array}{l}\text { Apply Range Tracking Using the Cross-Correlation } \\
\text { Algorithm. } \\
\text { Apply Doppler Tracking Using the Dominant Scatterer } \\
\text { Algorithm. }\end{array}$ \\
\hline GenRD.m & Create a 3D surface plot of the Range-Doppler image. \\
\hline
\end{tabular}




\subsection{Range Profile \& Phase History Simulation}

This subsection presents range profile and phase histories for processing the synthesized target echo when countering stepped frequency and chirp radar systems. Table 7.2 lists the variables and the corresponding meaning used for the simulation parameters.

Table 7.2: Meaning of variables used in simulation scenarios.

\begin{tabular}{|c|l|}
\hline Variable & Meaning \\
\hline$\mu$ & $\begin{array}{l}\text { Wave Heading Relative to Ship Bow (i.e. wave incident upon ship bow } \\
\text { is } 0^{\circ} \text { ). Angle increases moving towards ship portside (i.e. counter- } \\
\text { clockwise). }\end{array}$ \\
\hline $\mathrm{U}$ & Ship Velocity in Knots (Nautical Miles per Hour) \\
\hline$f_{\text {center }}$ & Center frequency of hostile radar signal. \\
\hline$\beta$ & Bandwidth of hostile radar signal. \\
\hline $\mathrm{n}$ & Stepped Frequency: Number of pulses in a burst. \\
\hline $\mathrm{N}$ & $\begin{array}{l}\text { Stepped Frequency: Number of Bursts. } \\
\text { Chirp Radar: Total number of pulses. }\end{array}$ \\
\hline$\Delta f$ & Frequency step size in stepped-frequency radar systems. \\
\hline$T_{B u r s t}$ & Period of a pulse burst in stepped-frequency radar systems. \\
\hline PRF & Pulse Repetition Frequency. \\
\hline$\tau$ & Pulse width/duration. \\
\hline$T_{\text {obs }}$ & Total observation period for simulation scenario. \\
\hline & \\
\hline
\end{tabular}

\subsubsection{Countering Stepped-Frequency Radar Systems}

Processing steps to obtain a synthetic high-resolution profile and Doppler history are discussed in chapter 2 . Table 7.3 defines the simulation cases for which data is produced using the TSS Software and MATLAB scripts, for countering a steppedfrequency radar system using the target model. 
Table 7.3: Simulation scenarios for countering a Stepped Frequency Radar System.

\begin{tabular}{|c|c|c|}
\hline $\begin{array}{l}\text { Simulation } \\
\text { Case }\end{array}$ & $\begin{array}{l}\text { Target Motion Parameters } \\
\text { and Resolution (TSS Program } \\
\text { Inputs) }\end{array}$ & $\begin{array}{l}\text { Stepped-Frequency Radar } \\
\text { Parameters } \\
\text { (MATLAB Script Inputs) }\end{array}$ \\
\hline 1 & $\begin{array}{l}\text { Sea State: } 7 \\
\mu=60^{\circ} \\
\mathrm{U}=25 \mathrm{Kts} \\
\text { Hostile Radar LOS: } \\
\text { Azimuth }=0^{\circ} \\
\text { Elevation }=30^{\circ} \\
\text { Resolution ( } \# \text { of resolution } \\
\text { cells): } \\
\text { Slant-Range }=4 \text { bins } \\
\text { Cross-Range }=4 \text { bins } \\
\text { Height }=4 \text { bins }\end{array}$ & $\begin{array}{l}\text { Slant-Range Window: } 220 \mathrm{~m} \\
\text { Slant-Range Resolution: } 1 \mathrm{~m} \\
\text { Cross-Range Window: } 40 \mathrm{~m} \\
\text { Cross-Range Resolution: } 1 \mathrm{~m} \\
f_{\text {center }}=10 \mathrm{GHz} \\
\beta=150 \mathrm{MHz} \\
\text { Number of Pulses per Burst (n): } 221 \\
\text { Number of Bursts (N): } 40 \\
\Delta f=678.733 \mathrm{kHz} \\
T_{B u r s t}=0.01 \mathrm{~s} \\
\mathrm{PRF}=22 \mathrm{kHz} \\
\tau=1.5 \mu \mathrm{s} \\
T_{\text {obs }}=0.4 \mathrm{~s}\end{array}$ \\
\hline 2 & $\begin{array}{l}\text { Sea State: } 2 \\
\mu=135^{\circ} \\
\mathrm{U}=15 \mathrm{Kts} \\
\text { Hostile Radar LOS: } \\
\text { Azimuth }=270^{\circ} \\
\text { Elevation }=0^{\circ} \\
\text { Resolution (\# of resolution } \\
\text { cells): } \\
\text { Slant-Range }=32 \text { bins } \\
\text { Cross-Range }=4 \text { bins } \\
\text { Height }=4 \text { bins }\end{array}$ & $\begin{array}{l}\text { Slant-Range Window: } 220 \mathrm{~m} \\
\text { Slant-Range Resolution: } 0.5 \mathrm{~m} \\
\text { Cross-Range Window: } 220 \mathrm{~m} \\
\text { Cross-Range Resolution: } 0.5 \mathrm{~m} \\
f_{\text {center }}=10 \mathrm{GHz} \\
\beta=300 \mathrm{MHz} \\
\text { Number of Pulses per Burst (n): } 441 \\
\text { Number of Bursts (N): } 440 \\
\Delta f=680.270 \mathrm{kHz} \\
T_{B u r s t}=0.0044 \mathrm{~s} \\
\mathrm{PRF}=100 \mathrm{kHz} \\
\tau=1.5 \mu \mathrm{s} \\
T_{\text {obs }}=1.936 \mathrm{~s}\end{array}$ \\
\hline
\end{tabular}




\begin{tabular}{|c|c|c|}
\hline 3 & $\begin{array}{l}\text { Sea State: } 2 \\
\mu=90^{\circ} \\
\mathrm{U}=10 \mathrm{Kts} \\
\text { Hostile Radar LOS: } \\
\text { Azimuth }=200^{\circ} \\
\text { Elevation }=50^{\circ} \\
\text { Resolution (\# of resolution } \\
\text { cells): } \\
\text { Slant-Range }=8 \text { bins } \\
\text { Cross-Range }=8 \text { bins } \\
\text { Height }=8 \text { bins }\end{array}$ & $\begin{array}{l}\text { Slant-Range Window: } 220 \mathrm{~m} \\
\text { Slant-Range Resolution: } 1 \mathrm{~m} \\
\text { Cross-Range Window: } 100 \mathrm{~m} \\
\text { Cross-Range Resolution: } 0.5 \mathrm{~m} \\
f_{\text {center }}=10 \mathrm{GHz} \\
\beta=150 \mathrm{MHz} \\
\text { Number of Pulses per Burst (n): } 221 \\
\text { Number of Bursts (N): } 200 \\
\Delta f=678.730 \mathrm{kHz} \\
T_{B u r s t}=0.0074 \mathrm{~s} \\
\text { PRF }=30 \mathrm{kHz} \\
\tau=1.5 \mu \mathrm{s} \\
T_{\text {obs }}=1.48 \mathrm{~s}\end{array}$ \\
\hline 4 & $\begin{array}{l}\text { Sea State: } 3 \\
\mu=165^{\circ} \\
\mathrm{U}=5 \mathrm{Kts} \\
\text { Hostile Radar LOS: } \\
\text { Azimuth }=320^{\circ} \\
\text { Elevation }=10^{\circ} \\
\text { Resolution (\# of resolution } \\
\text { cells): } \\
\text { Slant-Range }=8 \text { bins } \\
\text { Cross-Range }=4 \text { bins } \\
\text { Height }=4 \text { bins }\end{array}$ & $\begin{array}{l}\text { Slant-Range Window: } 220 \mathrm{~m} \\
\text { Slant-Range Resolution: } 0.5 \mathrm{~m} \\
\text { Cross-Range Window: } 100 \mathrm{~m} \\
\text { Cross-Range Resolution: } 1 \mathrm{~m} \\
f_{\text {center }}=10 \mathrm{GHz} \\
\beta=300 \mathrm{MHz} \\
\text { Number of Pulses per Burst (n): } 441 \\
\text { Number of Bursts (N): } 100 \\
\Delta f=680.270 \mathrm{kHz} \\
T_{\text {Burst }}=0.0088 \mathrm{~s} \\
\mathrm{PRF}=50 \mathrm{kHz} \\
\tau=1.5 \mu \mathrm{s} \\
T_{\text {obs }}=0.88 \mathrm{~s}\end{array}$ \\
\hline 5 & $\begin{array}{l}\text { Sea State: } 1 \\
\mu=150^{\circ} \\
\mathrm{U}=10 \mathrm{Kts} \\
\text { Hostile Radar LOS: } \\
\text { Azimuth }=130^{\circ} \\
\text { Elevation }=40^{\circ} \\
\text { Resolution (\# of resolution } \\
\text { cells): } \\
\text { Slant-Range }=16 \text { bins } \\
\text { Cross-Range }=8 \text { bins } \\
\text { Height }=4 \text { bins }\end{array}$ & $\begin{array}{l}\text { Slant-Range Window: } 220 \mathrm{~m} \\
\text { Slant-Range Resolution: } 2 \mathrm{~m} \\
\text { Cross-Range Window: } 100 \mathrm{~m} \\
\text { Cross-Range Resolution: } 2 \mathrm{~m} \\
f_{\text {center }}=10 \mathrm{GHz} \\
\beta=75 \mathrm{MHz} \\
\text { Number of Pulses per Burst (n): } 111 \\
\text { Number of Bursts (N): } 50 \\
\Delta f=675.680 \mathrm{kHz} \\
T_{\text {Burst }}=0.0222 \mathrm{~s} \\
\mathrm{PRF}=5 \mathrm{kHz} \\
\tau=1.5 \mu \mathrm{s} \\
T_{\text {obs }}=1.11 \mathrm{~s}\end{array}$ \\
\hline
\end{tabular}


For simulation case one the synthetic high-resolution range profile in the I and Q channels are shown in Figure 7.5 and 7.6, respectively. The range profile is generated from a single burst of 221 discrete frequency pulses stepped in frequency increments of $678.733 \mathrm{kHz}$.

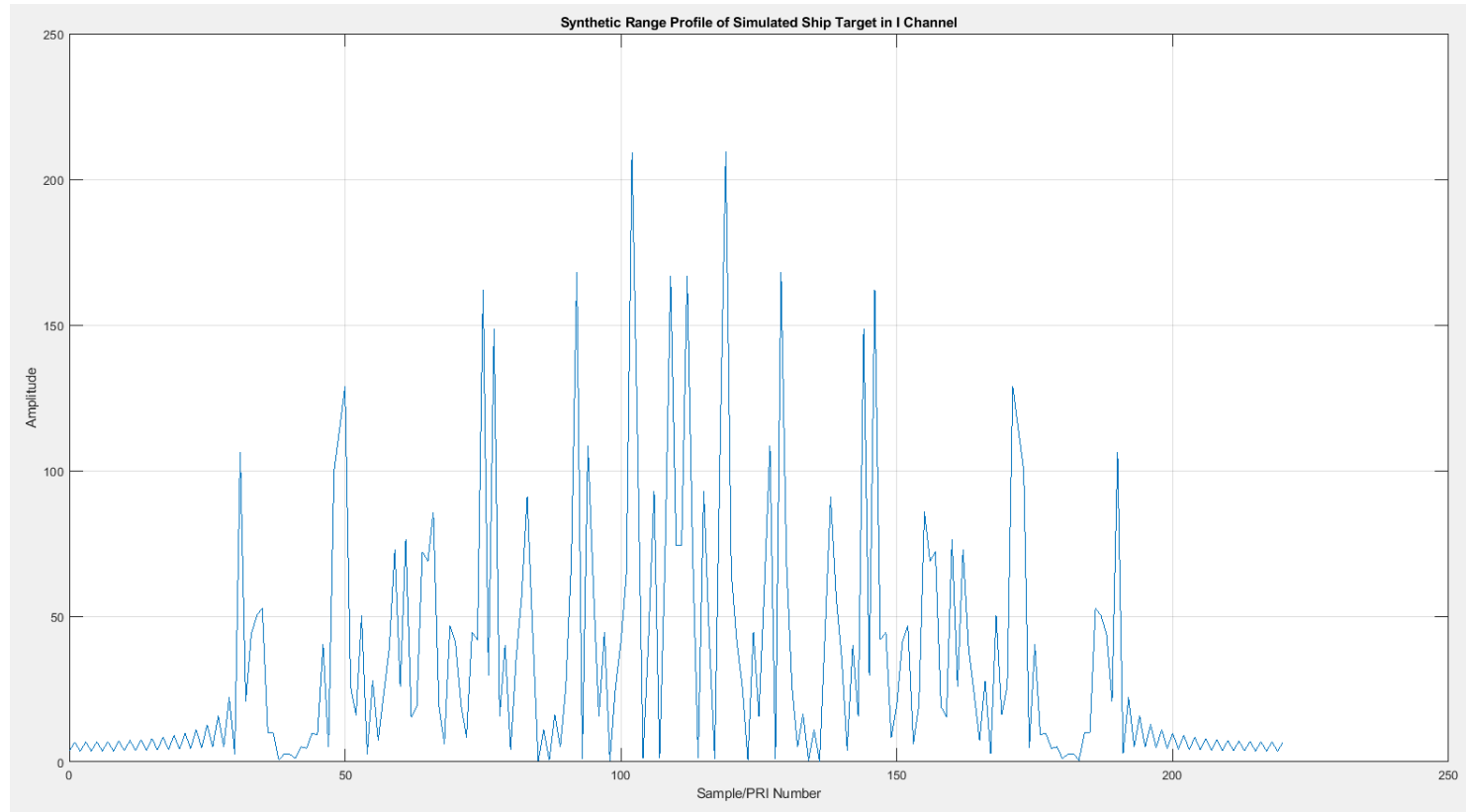

Figure 7.5: The synthetic range profile of the target in the I channel.

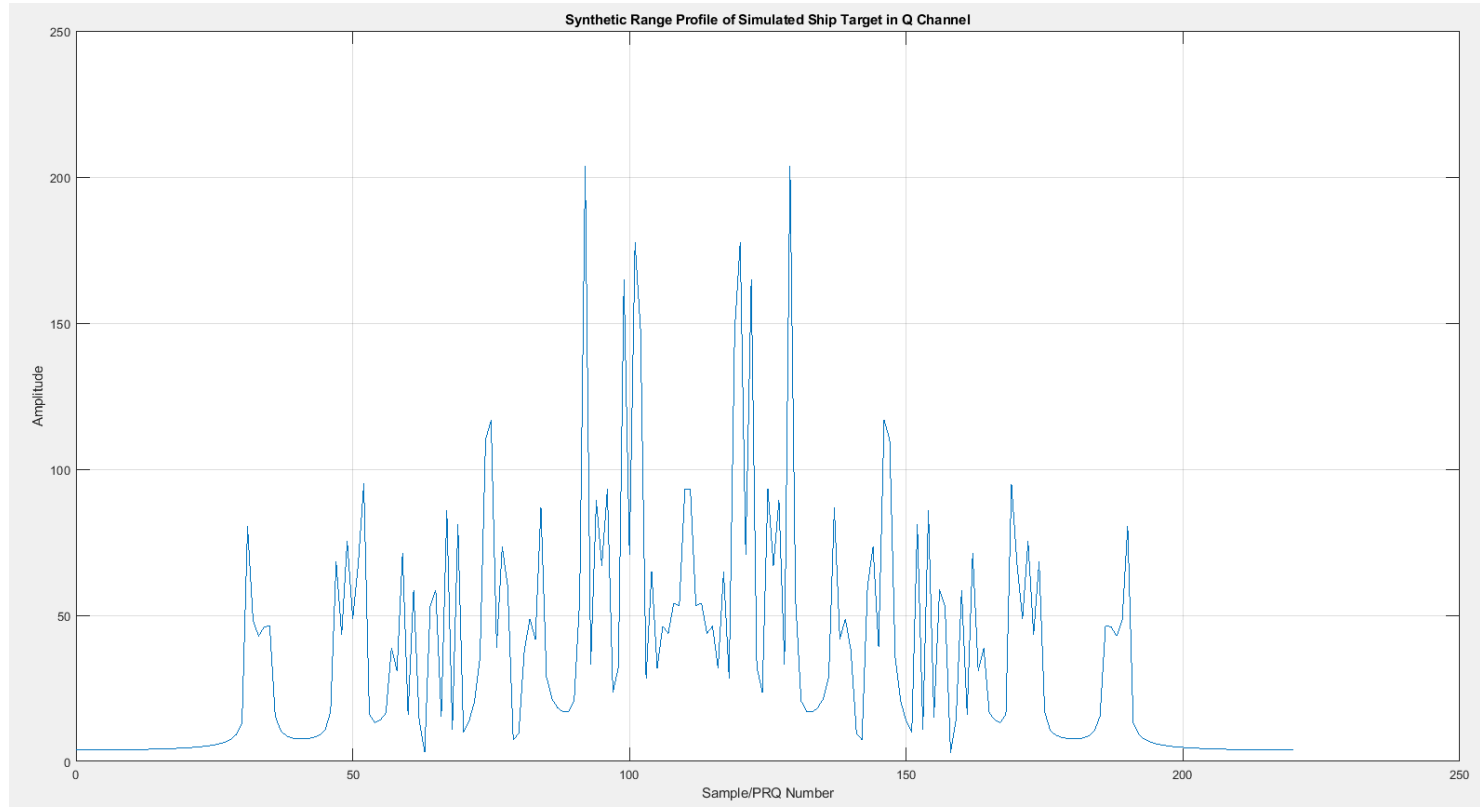

Figure 7.6: The synthetic range profile of the target in the $Q$ channel. 
The combined complex baseband signal is processed using an IDFT operation to produce a complex-valued synthetic high-resolution range profile, shown in Figure 7.7. The highlighted peaks in the complex synthetic range profile correspond to the dominant scattering locations for which the TSS program's modulation parameters are generated. In the synthetic range profile, the sample number (i.e. pulse number in the burst) is related to the range as per equation 7.1 .

$$
R_{d}=\frac{c \cdot n_{\text {sample \# }}}{2 \beta}
$$

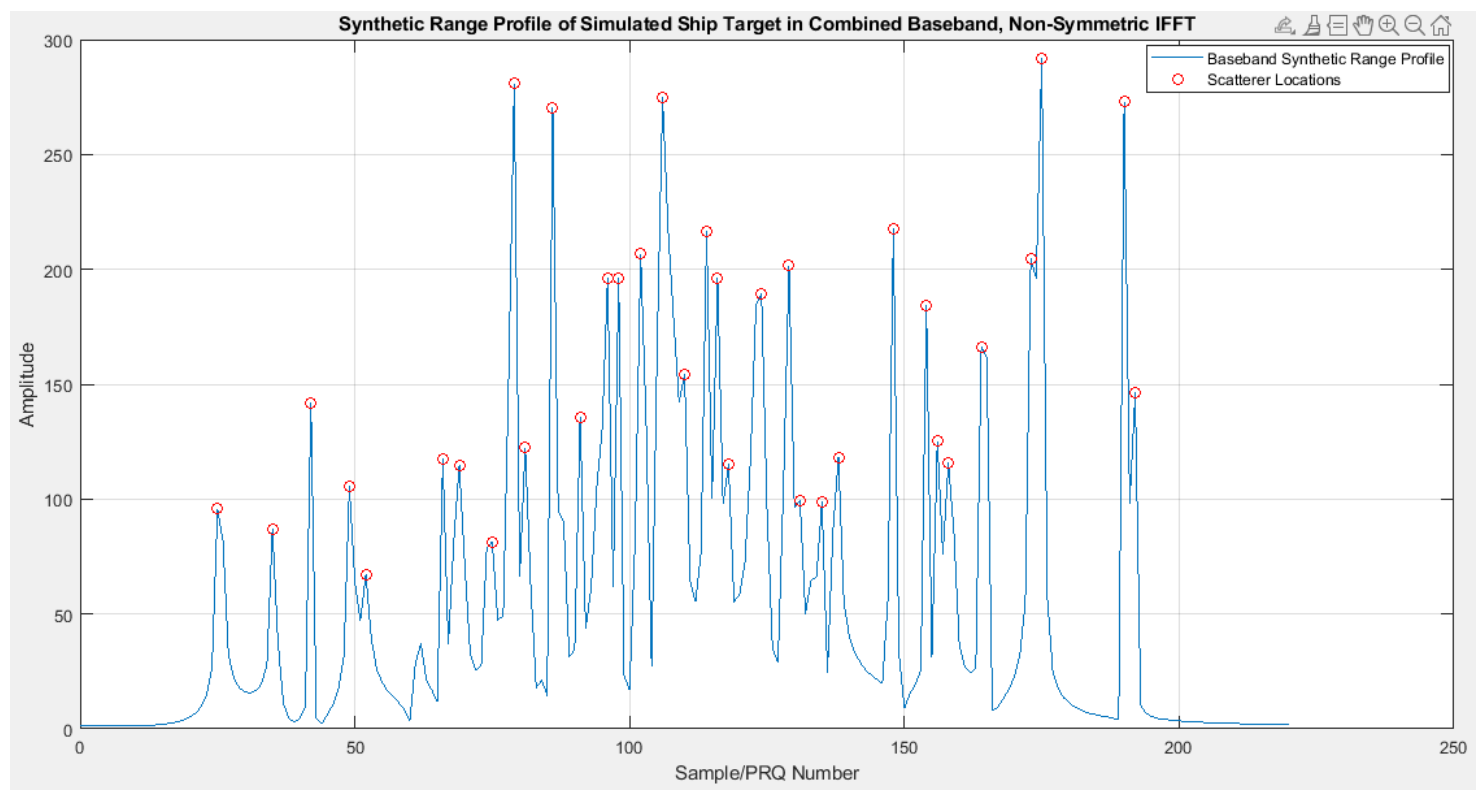

Figure 7.7: The complex valued synthetic high-resolution range profile for simulation case 1 .

In simulation case one the angle of incidence is directly upon the ship bow. This produces a range profile equivalent to a broadside perspective of the target. Comparing the range profile in Figure 7.7 to the aspect image of the target in Figure 7.8, the peak locations in the profile can be seen to correspond to key backscattering locations on the target. However, the magnitude of scattering locations is not always consistent due to the RCS statistics and electromagnetic interaction phenomena of the target not being included in the model. 


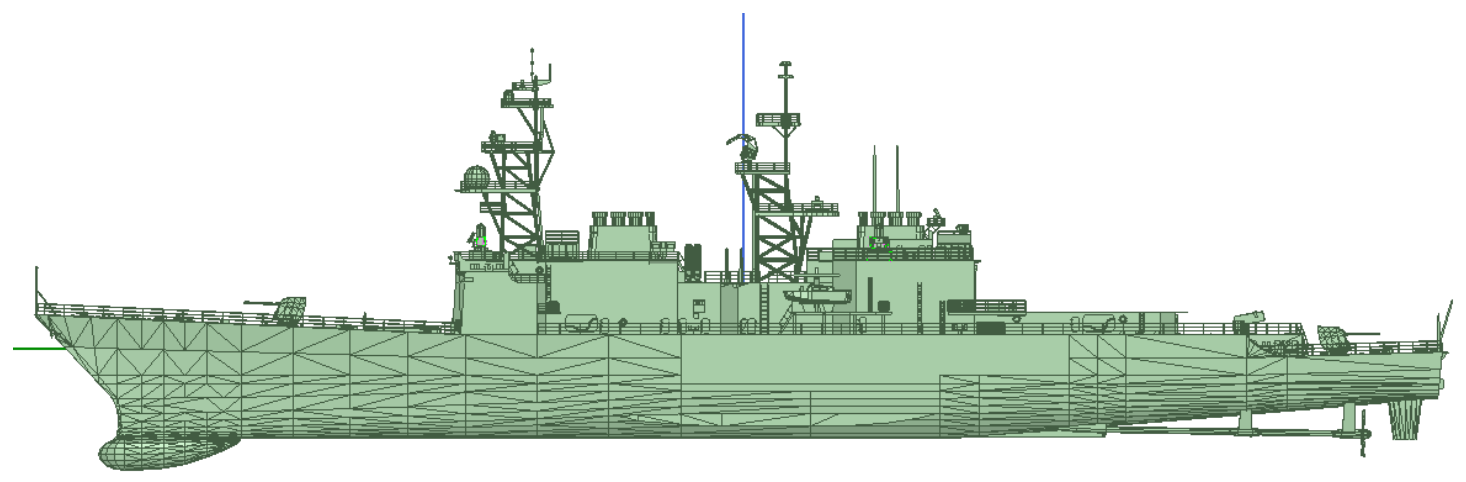

Figure 7.8: Target model perspective for which the range profile is produced in case one.

The profile synthesis file produced in the Target Signature Synthesis program for simulation case one includes 64 contributing scattering locations based on position in slant-range, cross-range, and height. It is evident in Figure 7.7 that there are only 34 scattering locations highlighted in the synthetic range profile. The inability to see 64 peaks is attributed to dominant scattering locations being within the same range cell, even if the scattering location has a unique cross-range or height position. When multiple scatterers exist within the same range bin, the delay to both scattering locations is similar, thus the echo from each scattering location is coherently combined and the ability to resolve these scattering locations in the slant range dimension is lost [28][29][41][42]. This is observed in Figure 7.9 which shows the dominant scattering locations selected in the TSS program for profile synthesis in simulation case one. 


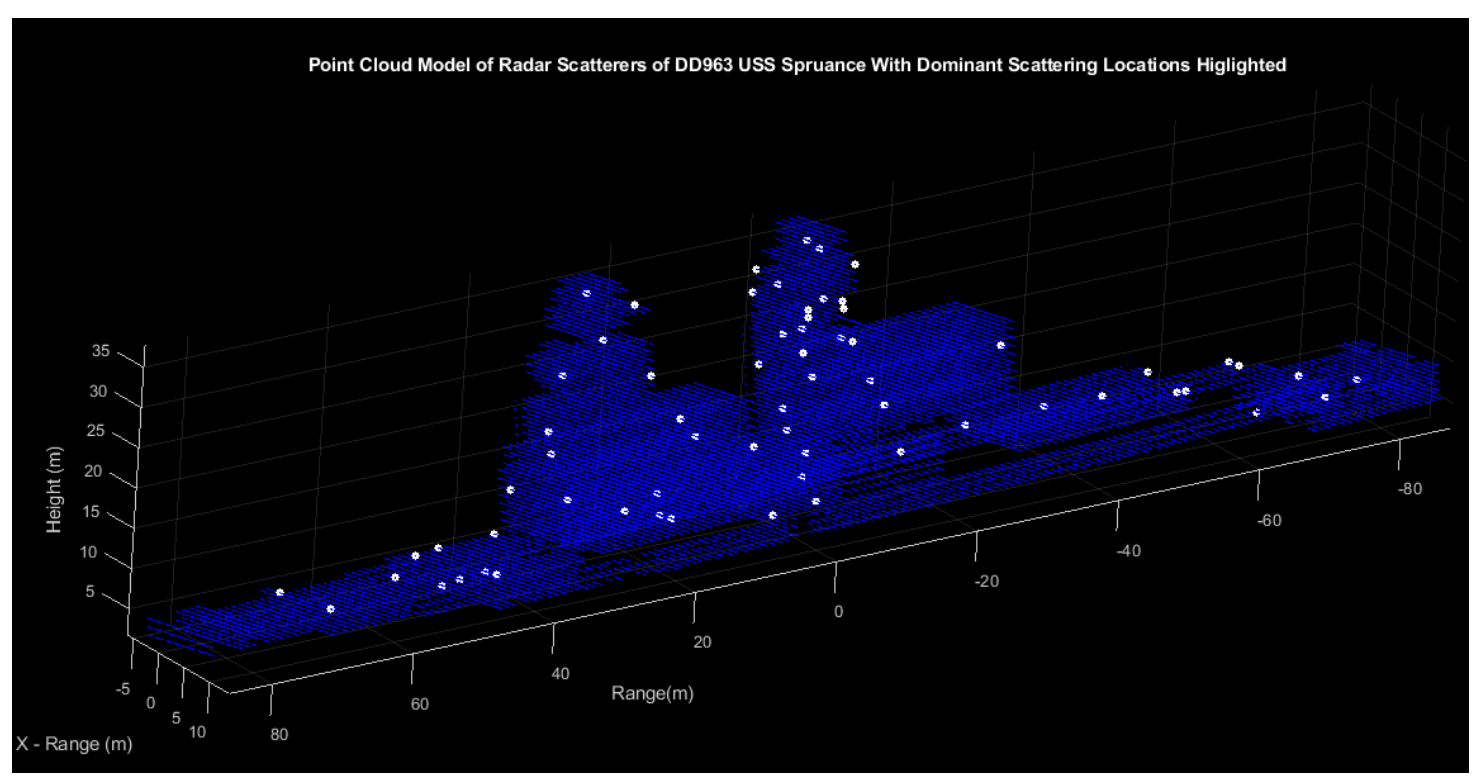

Figure 7.9: Target point cloud model with dominant scattering locations highlighted as chosen in the TSS program for case one.

The simulation profile is defined to decorrelate every 0.01 seconds, as per the discussion presented in chapter five. Thus, all pulses processed within a 0.01 second interval have the same modulation parameters applied. Defining a small decorrelation time results in visible variation in the range profile history and phase history of the target. These variations are often corrected using the motion compensation algorithms discussed in chapter three.

For the simulated observation period in case one, a total of 40 stepped frequency bursts are processed in the MATLAB simulation program with the profile synthesis parameters from the TSS program. The range profile history for simulation case one is shown in Figure 7.10. 


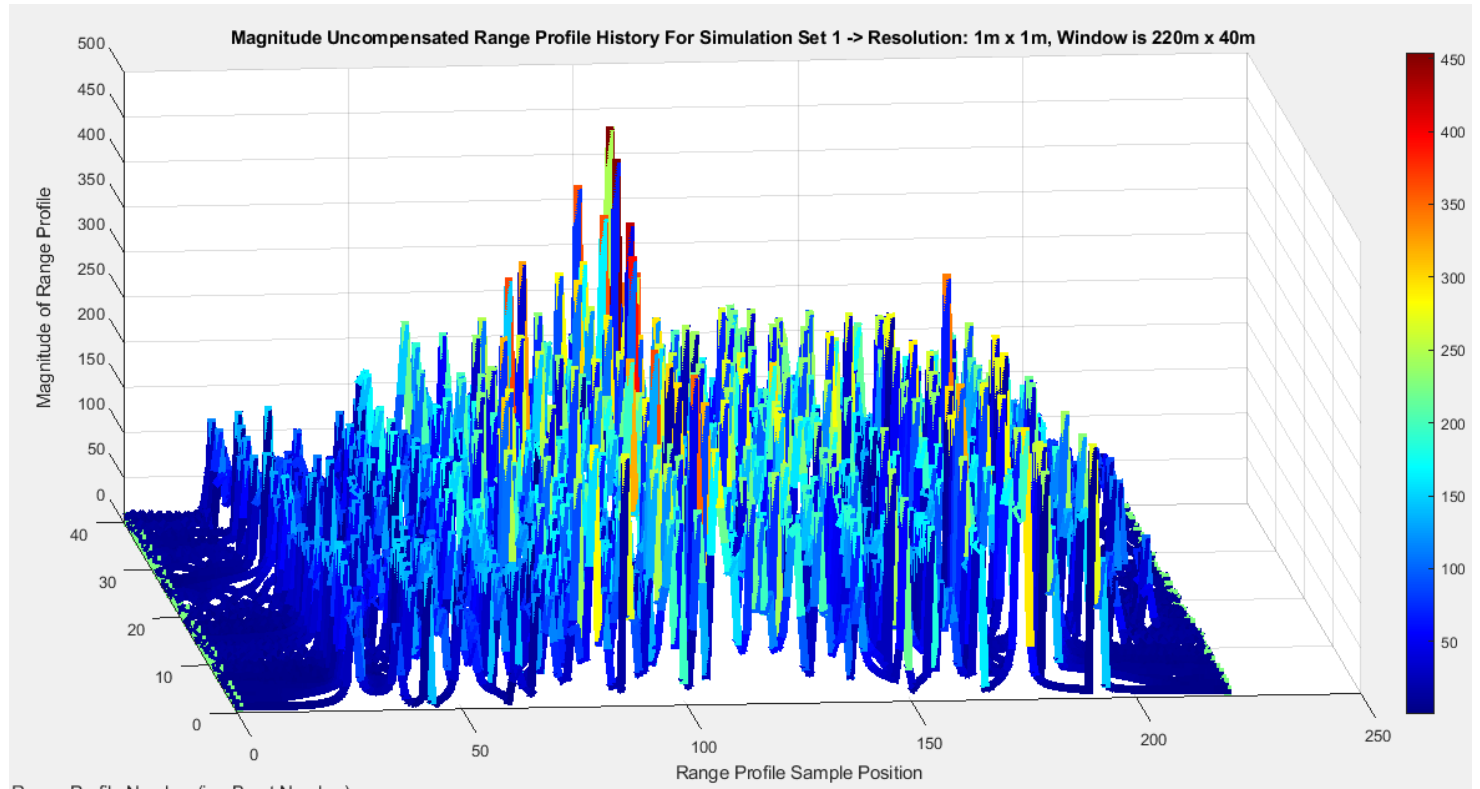

Figure 7.10: Waterfall plot of the magnitude of the synthetic range profile generated from each burst.

In Figure 7.10 observe that the range position of the dominant scattering locations in the range profile change over time showing range offset. This is a result of the ship's motion over the observation time, and the profile decorrelation time following which the change in delay position and Doppler frequency of scattering locations is evident. The magnitude of the range profile history plot is shown from a different perspective in Figure 7.11 . 


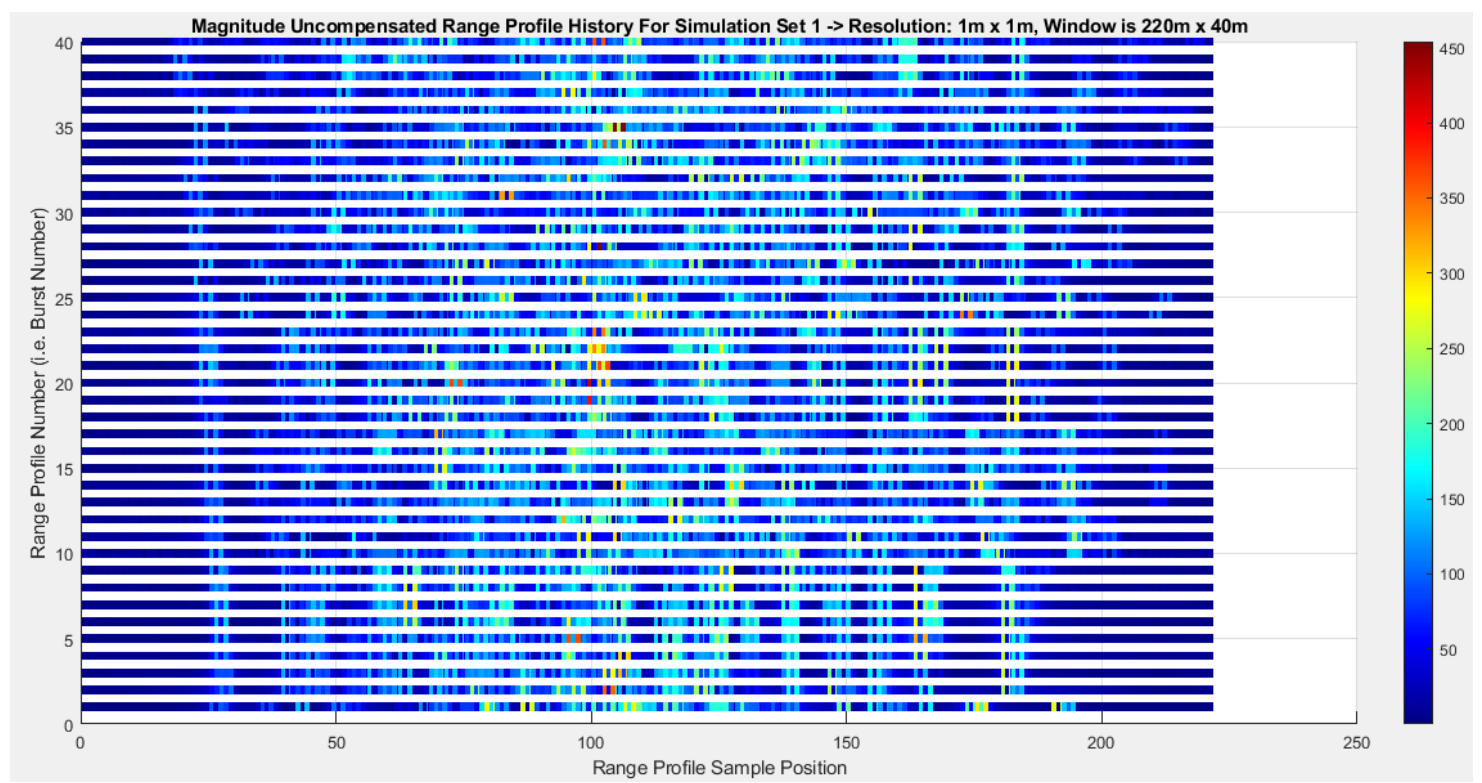

Figure 7.11: A bird's eye view of the waterfall plot of the magnitude of the synthetic range profile generated from each burst.

Figure 7.11 demonstrates that range profile modulation parameters remain constant for at most one burst, matching the bust period shown in Table 7.3. The range profile changes because of new dominant scattering locations being selected in the TSS program in each decorrelation period. A similar pattern is observed in the phase history of the synthetic range profiles. Phase variations are far more significant than magnitude variations as evidenced in Figure 7.12. 


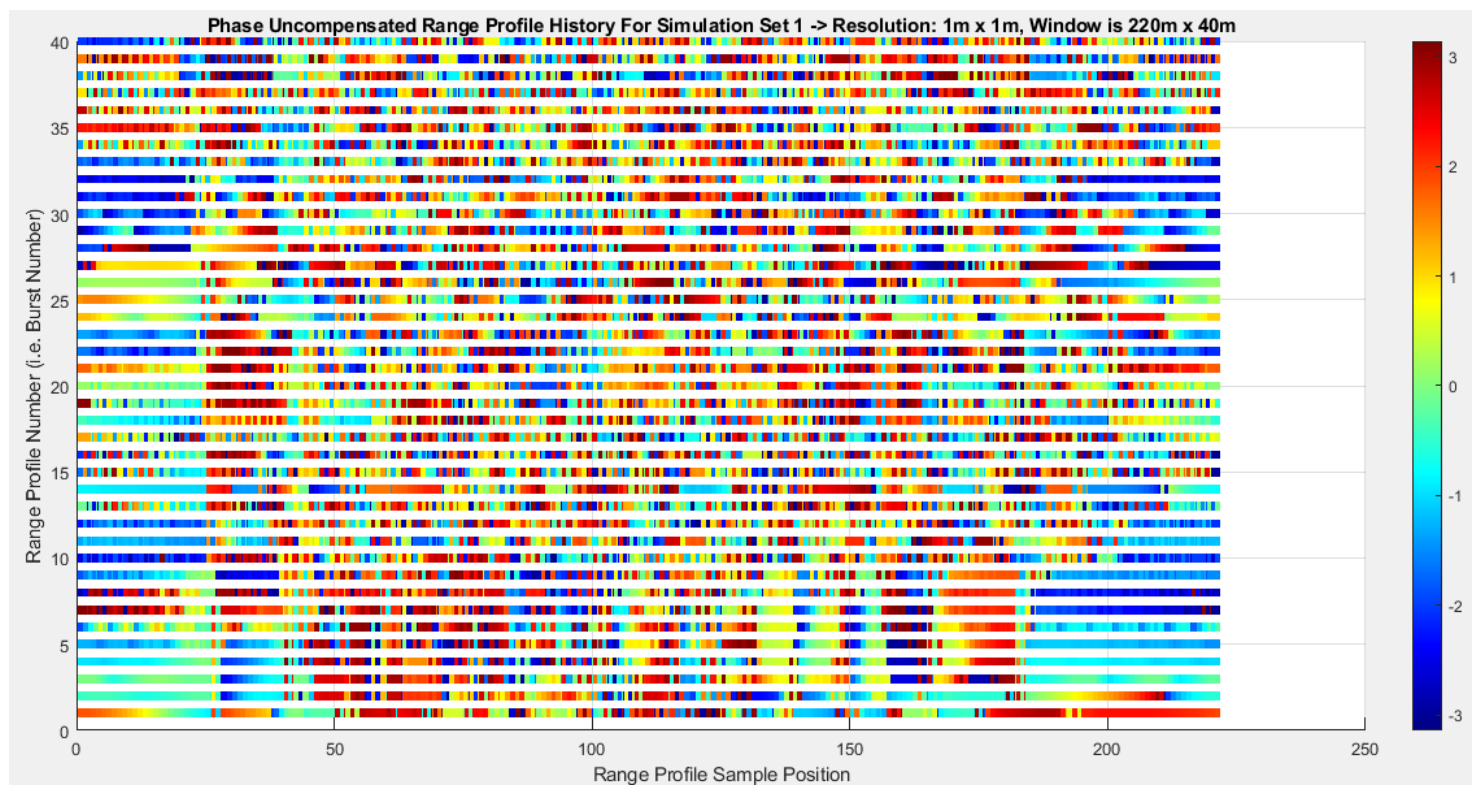

Figure 7.12: A bird's eye view of the waterfall plot of the phase of the synthetic range profile generated from each burst.

The simulation data for cases two and three can be found in Sub-Appendix C.1.

The complex synthetic range profile for simulation case four is presented in Figure 7.13. Target model perspective produced in the range profile is shown in Figure 7.14. 


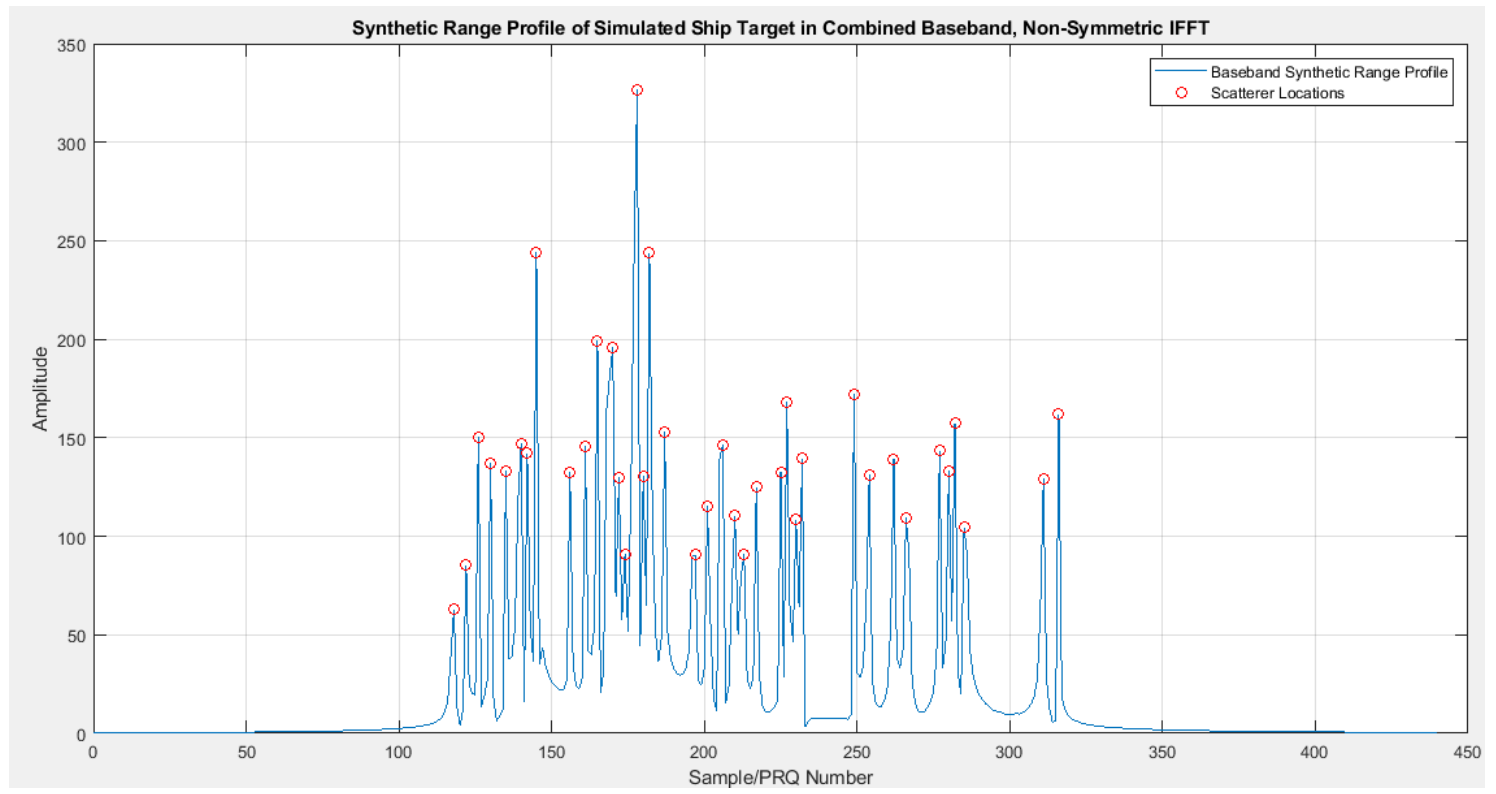

Figure 7.13: The complex valued synthetic high-resolution range profile for simulation case four.

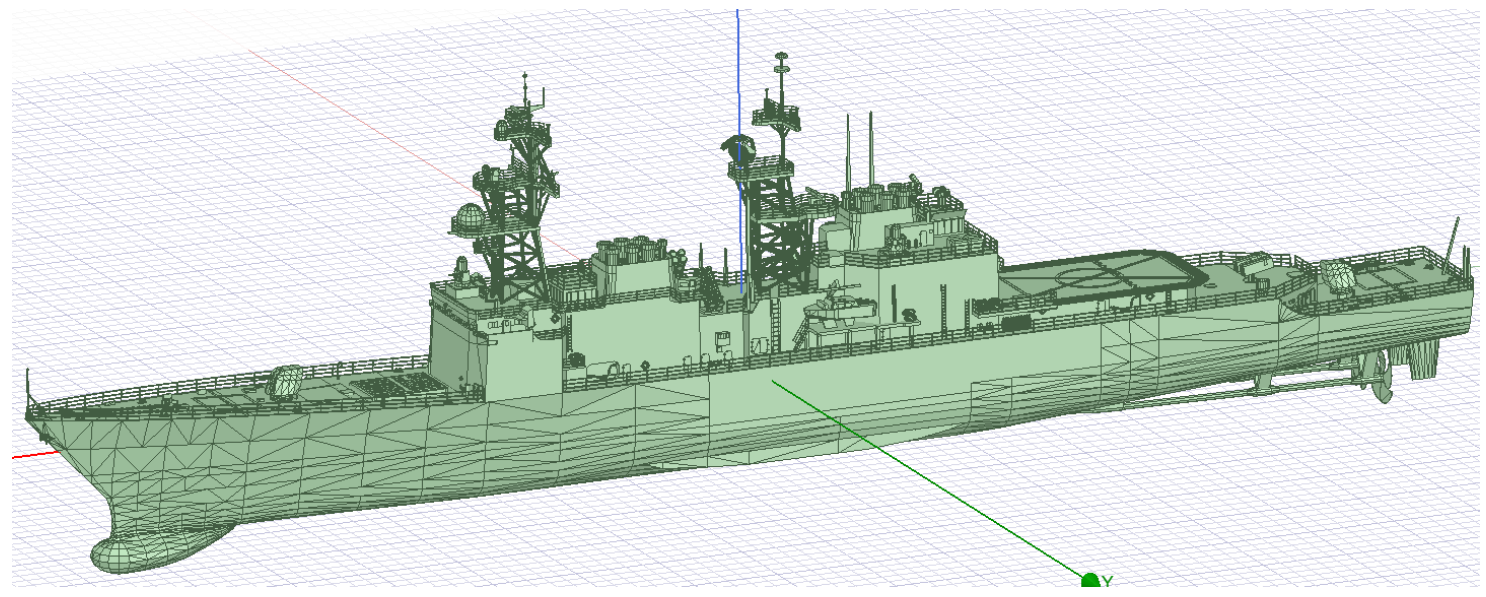

Figure 7.14: Target model perspective for which the range profile is produced in case four. 


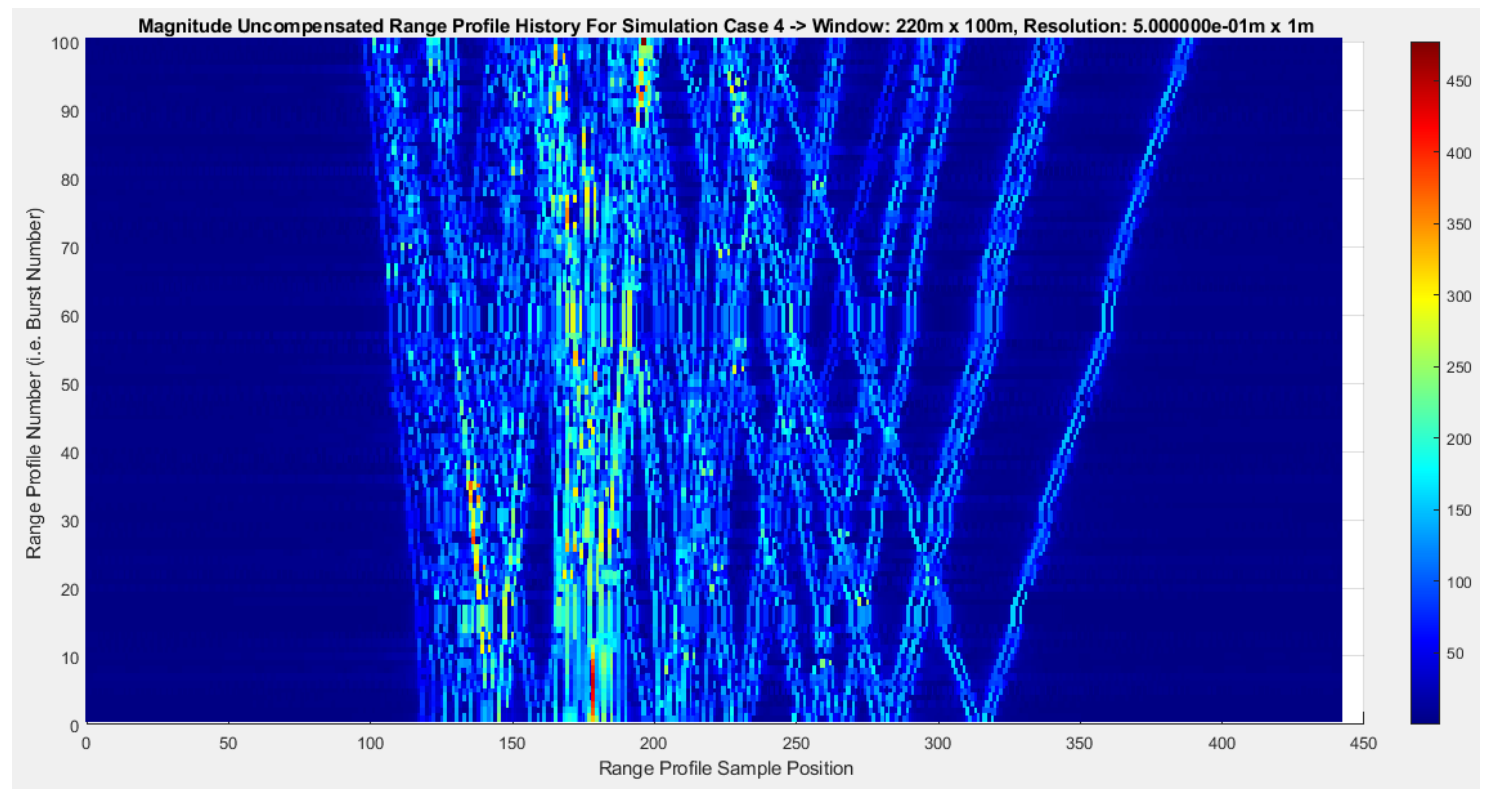

Figure 7.15: Simulation Case 4 Complex baseband magnitude range profile history.

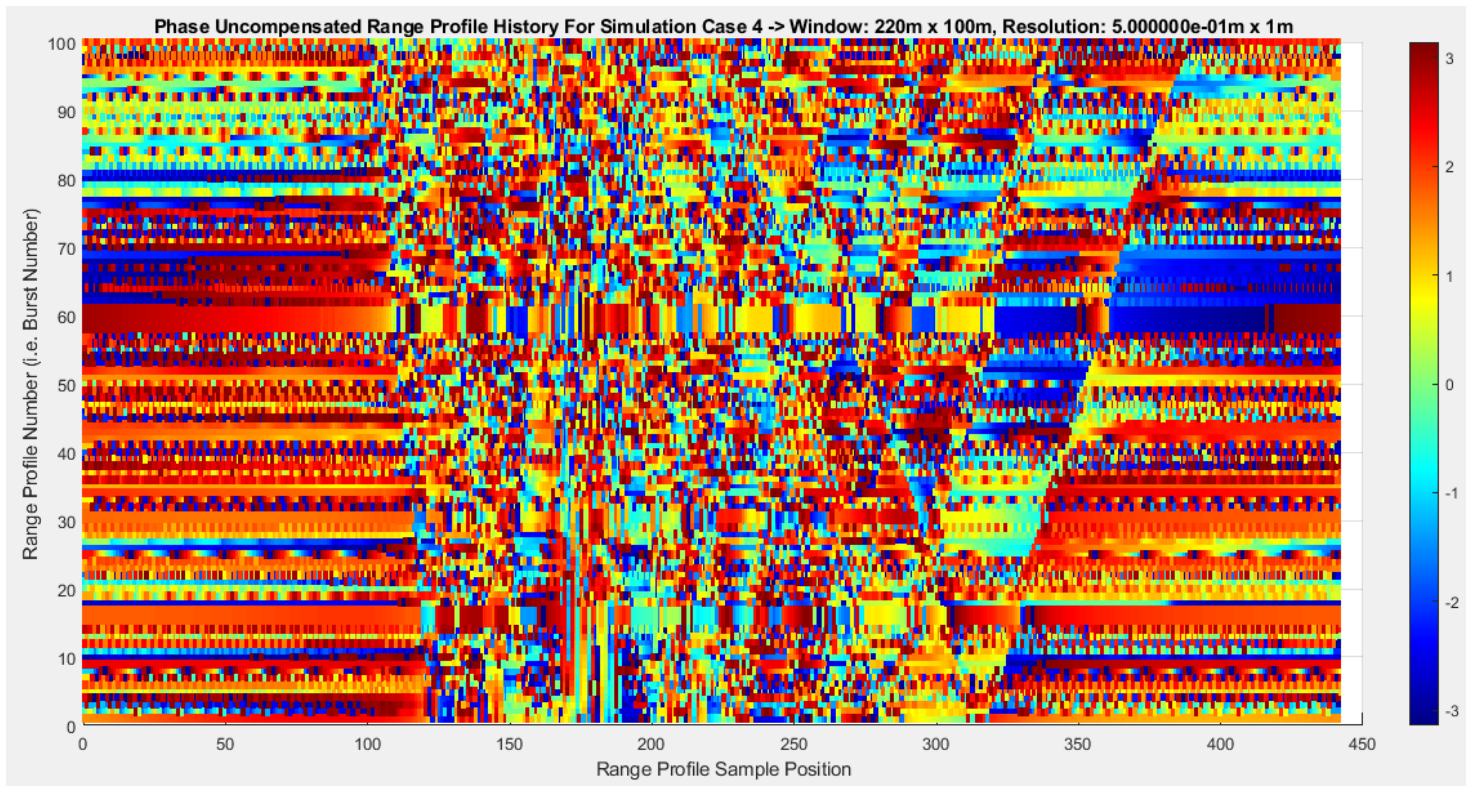

Figure 7.16: Simulation Case 4 Complex baseband phase history.

The magnitude and phase histories for simulation case four show clear migration of the dominant scattering locations across multiple range cells. This effect occurs in all simulation cases but is exaggerated in simulation case four due to the reduced ship forward velocity. At a lower forward velocity, the motion experienced by the ship target is majorly due to ship interaction with waves. This increases the total angular motion experienced by the ship, resulting in greater range migration of scattering locations. 


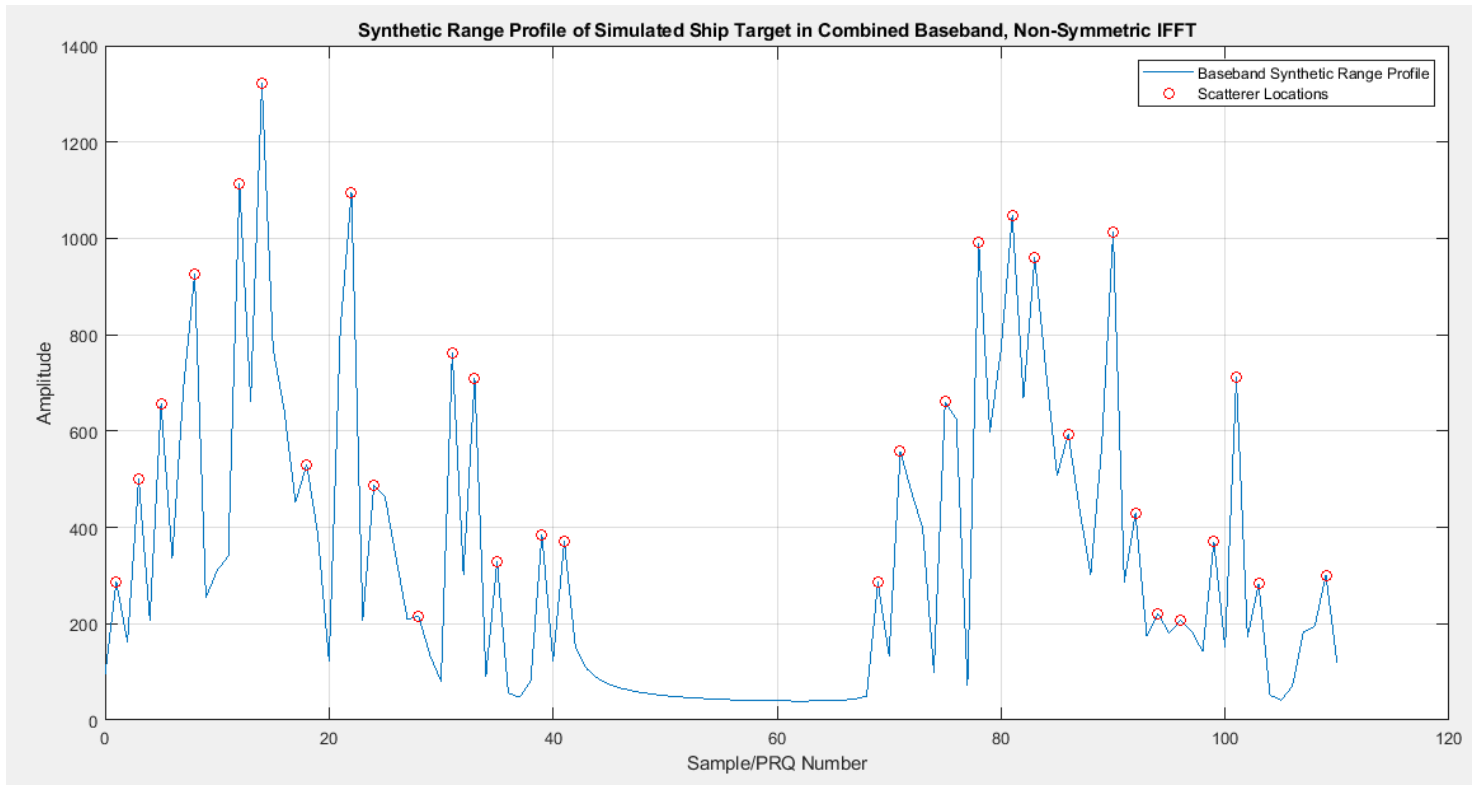

Figure 7.17: The complex valued synthetic high-resolution range profile for simulation case 5 .

Simulation case five presents a common issue encountered in radar operation when observing unknown targets. The selected operating PRF is low, and thus the entire target profile is not produced in a single window. The target profile has aliased, or folded over, in the time domain because of the low PRF and low-resolution parameter selection. Correction of the range aliasing can be done by changing the radar PRF. Another method of range aliasing correction is to simply re-arrange the synthetic range profile data, as shown in Figure 7.27. 


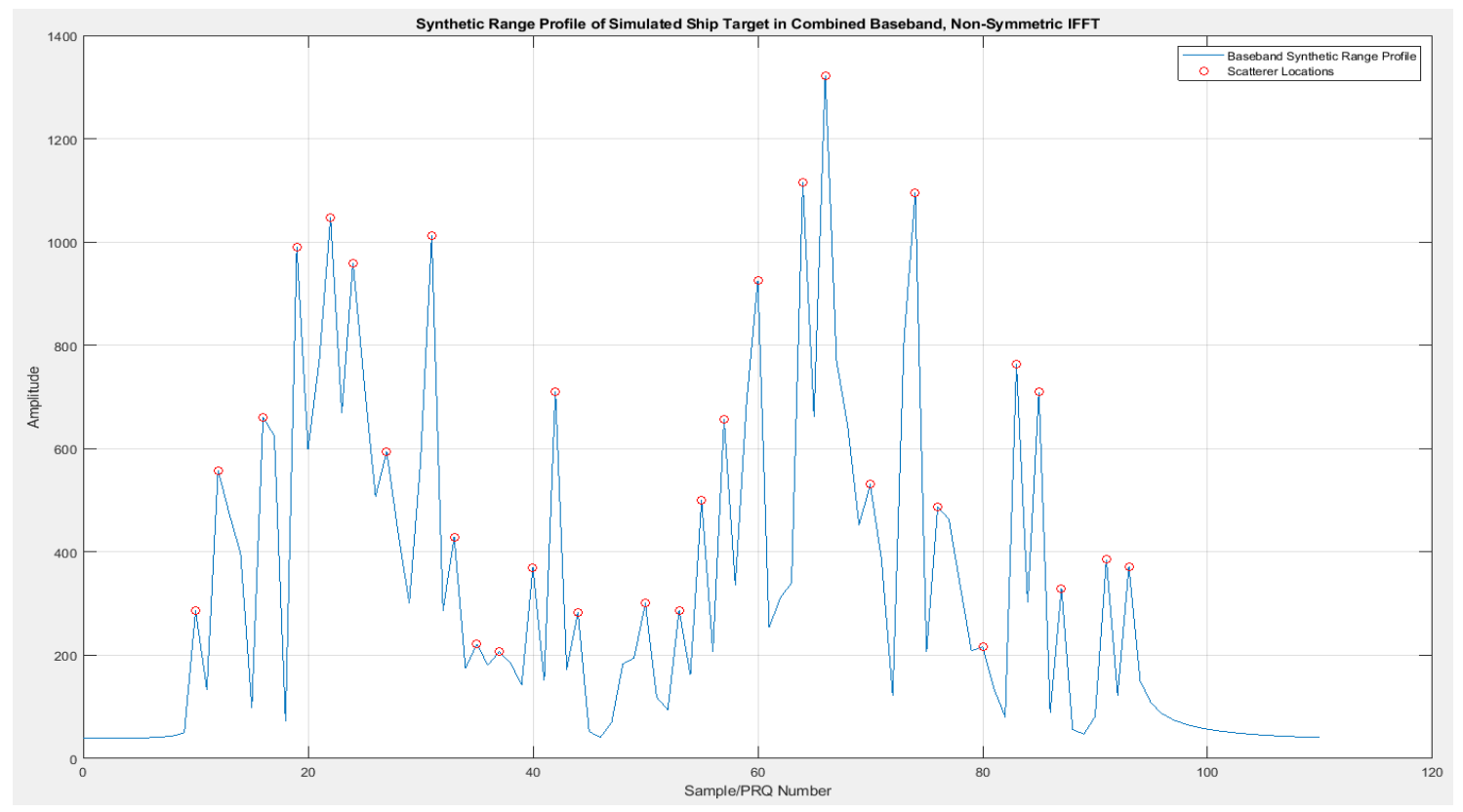

Figure 7.18: The complex valued synthetic high-resolution range profile for simulation case 5 , corrected for range aliasing.

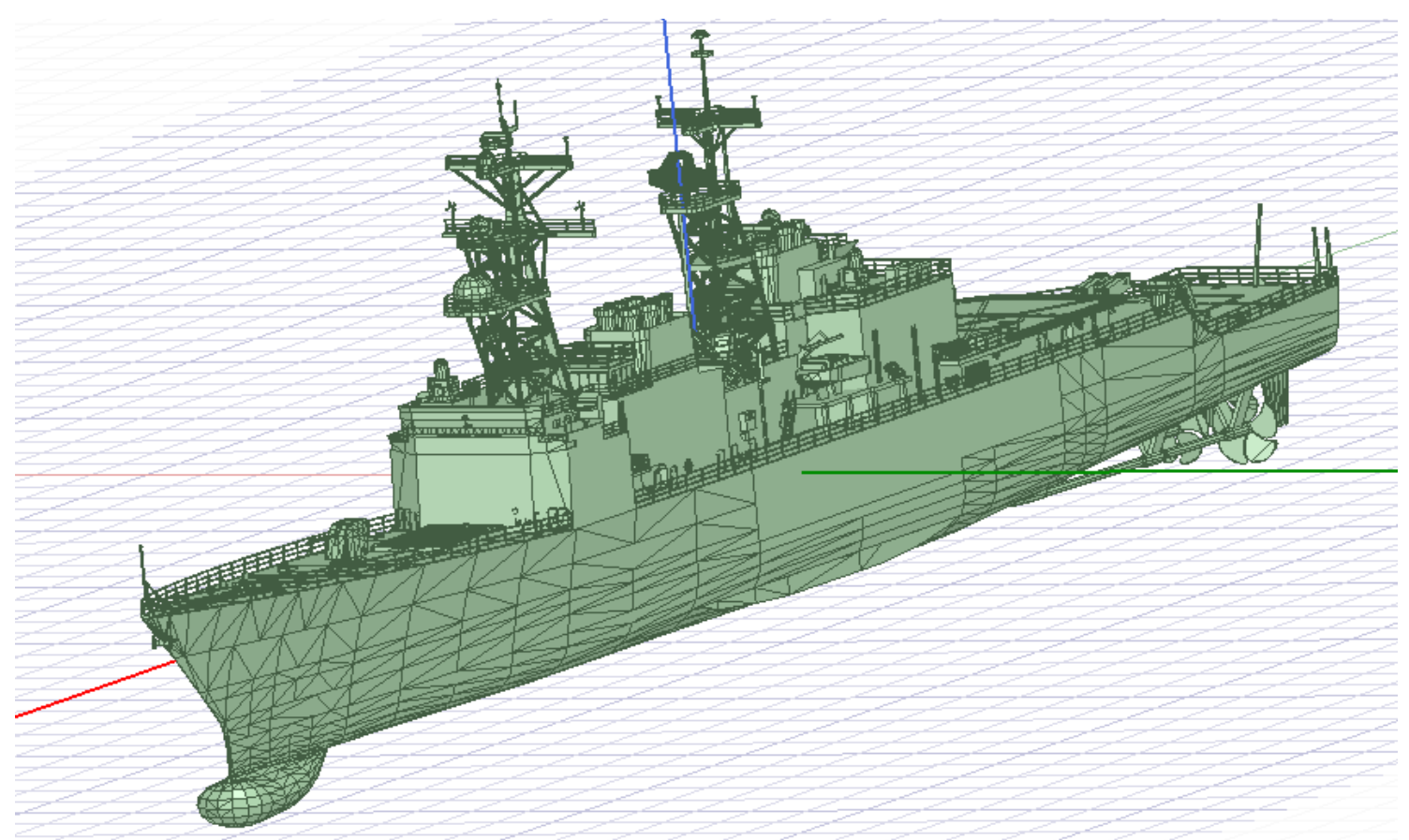

Figure 7.19: Target model perspective for which the range profile is produced in case five. 
The magnitude and phase history of the simulated range profile are shown in the figures below. This data has been corrected accounting for the range aliasing present in the original profile data.

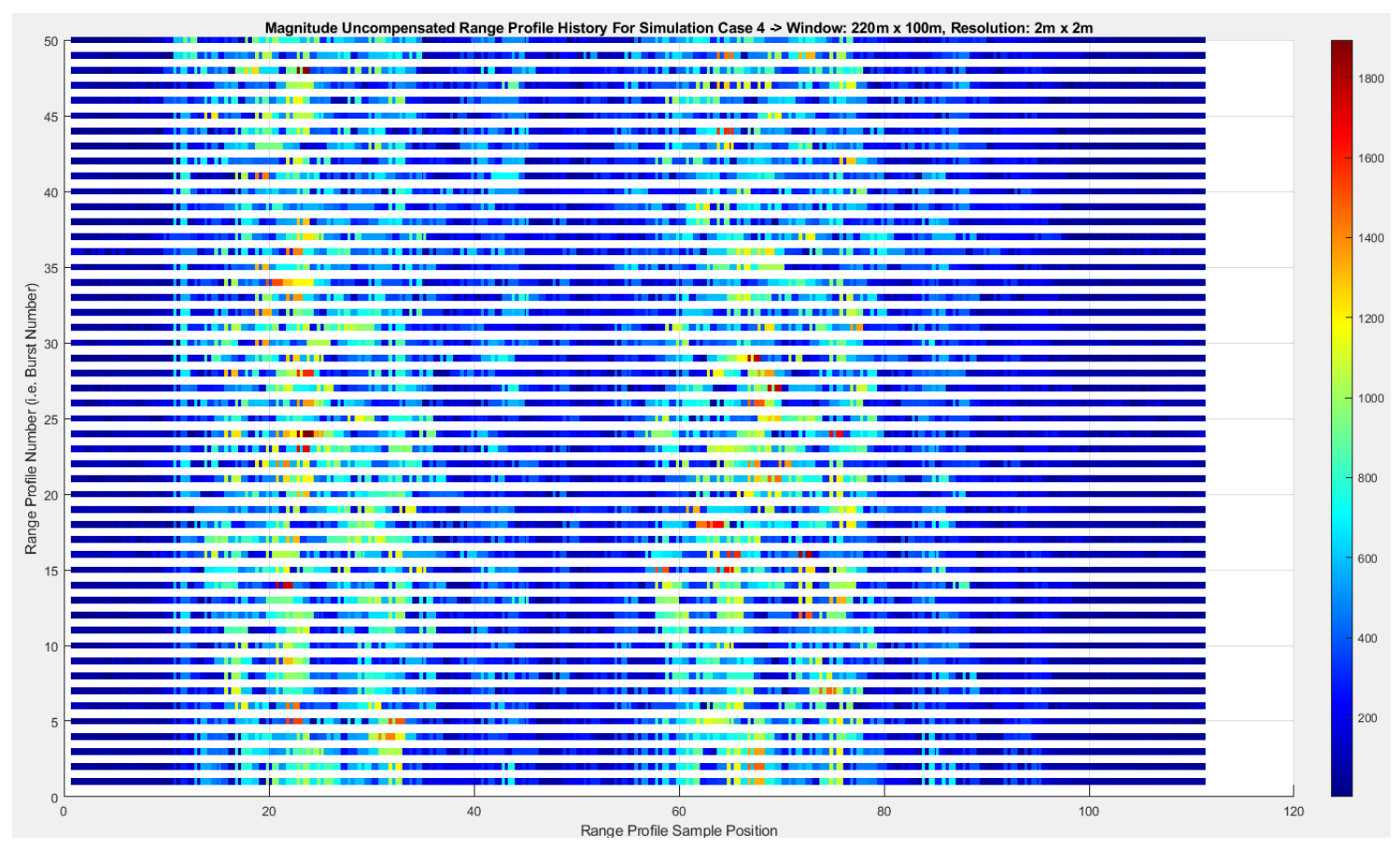

Figure 7.20: Simulation Case 5 Complex baseband magnitude range profile history.

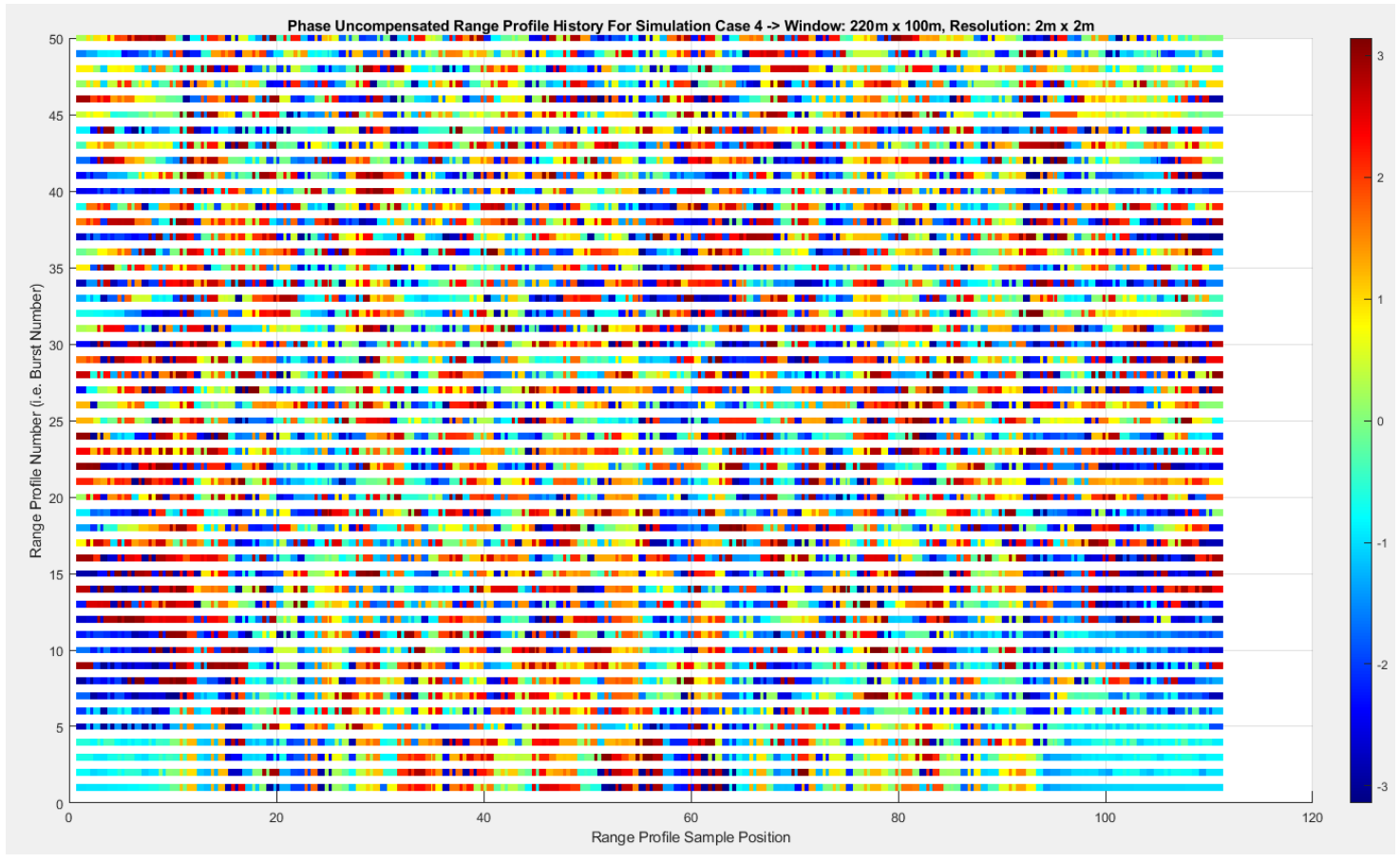

Figure 7.21: Simulation Case 5 Complex baseband phase history. 


\subsubsection{Countering Chirp Radar Systems}

Processing steps to obtain a high-resolution profile and Doppler history when processing chirp radar signals is discussed in chapter two. Table 7.4 defines the simulation cases for which data is produced using the TSS Software and MATLAB scripts.

The effect of changing PRF is more clearly visible when processing chirp radar data. This is observed by creating two variations of simulation cases in which in the PRF of the simulated radar is changed. The change in the phase history is difficult to observe due to the high variation in phase data. However, the effects of varied PRF are clearly observed in the Range-Doppler images, this will be presented in the next subsection.

Table 7.4: Simulation scenarios for countering a Chirp Radar System.

\begin{tabular}{|c|c|c|}
\hline $\begin{array}{l}\text { Simulation } \\
\text { Case }\end{array}$ & $\begin{array}{l}\text { Target Motion Parameters and } \\
\text { Resolution (TSS Program } \\
\text { Inputs) }\end{array}$ & $\begin{array}{l}\text { Chirp Radar Parameters } \\
\text { (MATLAB Script Inputs) }\end{array}$ \\
\hline 1a & $\begin{array}{l}\text { Sea State: } 7 \\
\mu=60^{\circ} \\
\mathrm{U}=25 \mathrm{Kts} \\
\text { Hostile Radar LOS: } \\
\text { Azimuth }=0^{\circ} \\
\text { Elevation }=30^{\circ} \\
\text { Resolution (\# of resolution } \\
\text { cells): } \\
\text { Slant-Range }=4 \text { bins } \\
\text { Cross-Range }=4 \text { bins } \\
\text { Height }=4 \text { bins }\end{array}$ & $\begin{array}{l}\text { Slant-Range Window: } 220 \mathrm{~m} \\
\text { Slant-Range Resolution: } 1 \mathrm{~m} \\
\text { Cross-Range Window: } 40 \mathrm{~m} \\
\text { Cross-Range Resolution: } 1 \mathrm{~m} \\
f_{\text {center }}=10 \mathrm{GHz} \\
\beta=150 \mathrm{MHz} \\
\mathrm{PRF}=4 \mathrm{kHz} \\
\tau=2 \mu \mathrm{s} \\
T_{\text {obs }}=0.01 \mathrm{~s}\end{array}$ \\
\hline $1 \mathrm{~b}$ & $\begin{array}{l}\text { Sea State: } 7 \\
\mu=60^{\circ} \\
\mathrm{U}=25 \mathrm{Kts} \\
\text { Hostile Radar LOS: } \\
\text { Azimuth }=0^{\circ} \\
\text { Elevation }=30^{\circ} \\
\text { Resolution (\# of resolution } \\
\text { cells): } \\
\text { Slant-Range }=4 \text { bins } \\
\text { Cross-Range }=4 \text { bins } \\
\text { Height }=4 \text { bins }\end{array}$ & $\begin{array}{l}\text { Slant-Range Window: } 220 \mathrm{~m} \\
\text { Slant-Range Resolution: } 1 \mathrm{~m} \\
\text { Cross-Range Window: } 40 \mathrm{~m} \\
\text { Cross-Range Resolution: } 1 \mathrm{~m} \\
f_{\text {center }}=10 \mathrm{GHz} \\
\beta=150 \mathrm{MHz} \\
\mathrm{PRF}=1 \mathrm{kHz} \\
\tau=2 \mu \mathrm{s} \\
T_{\text {obs }}=0.04 \mathrm{~s}\end{array}$ \\
\hline
\end{tabular}




\begin{tabular}{|c|c|c|}
\hline $2 a$ & $\begin{array}{l}\text { Sea State: } 2 \\
\mu=135^{\circ} \\
\mathrm{U}=15 \mathrm{Kts} \\
\text { Hostile Radar LOS: } \\
\text { Azimuth }=270^{\circ} \\
\text { Elevation }=0^{\circ} \\
\text { Resolution ( } \# \text { of resolution } \\
\text { cells): } \\
\text { Slant-Range }=32 \text { bins } \\
\text { Cross-Range }=4 \text { bins } \\
\text { Height }=4 \text { bins }\end{array}$ & $\begin{array}{l}\text { Slant-Range Window: } 220 \mathrm{~m} \\
\text { Slant-Range Resolution: } 0.5 \mathrm{~m} \\
\text { Cross-Range Window: } 220 \mathrm{~m} \\
\text { Cross-Range Resolution: } 0.5 \mathrm{~m} \\
f_{\text {center }}=10 \mathrm{GHz} \\
\beta=300 \mathrm{MHz} \\
\mathrm{PRF}=2 \mathrm{kHz} \\
\tau=2 \mu \mathrm{s} \\
T_{\text {obs }}=0.22 \mathrm{~s}\end{array}$ \\
\hline $2 \mathrm{~b}$ & $\begin{array}{l}\text { Sea State: } 2 \\
\mu=135^{\circ} \\
\mathrm{U}=15 \mathrm{Kts} \\
\text { Hostile Radar LOS: } \\
\text { Azimuth }=270^{\circ} \\
\text { Elevation }=0^{\circ} \\
\text { Resolution ( } \# \text { of resolution } \\
\text { cells): } \\
\text { Slant-Range }=32 \text { bins } \\
\text { Cross-Range }=4 \text { bins } \\
\text { Height }=4 \text { bins }\end{array}$ & $\begin{array}{l}\text { Slant-Range Window: } 220 \mathrm{~m} \\
\text { Slant-Range Resolution: } 0.5 \mathrm{~m} \\
\text { Cross-Range Window: } 220 \mathrm{~m} \\
\text { Cross-Range Resolution: } 0.5 \mathrm{~m} \\
f_{\text {center }}=10 \mathrm{GHz} \\
\beta=300 \mathrm{MHz} \\
\mathrm{PRF}=5 \mathrm{kHz} \\
\tau=2 \mu \mathrm{s} \\
T_{\text {obs }}=0.088 \mathrm{~s}\end{array}$ \\
\hline 3 & $\begin{array}{l}\text { Sea State: } 2 \\
\mu=90^{\circ} \\
\mathrm{U}=10 \mathrm{Kts} \\
\text { Hostile Radar LOS: } \\
\text { Azimuth }=200^{\circ} \\
\text { Elevation }=50^{\circ} \\
\text { Resolution ( } \# \text { of resolution } \\
\text { cells): } \\
\text { Slant-Range }=8 \text { bins } \\
\text { Cross-Range }=8 \text { bins } \\
\text { Height }=8 \text { bins }\end{array}$ & $\begin{array}{l}\text { Slant-Range Window: } 220 \mathrm{~m} \\
\text { Slant-Range Resolution: } 1 \mathrm{~m} \\
\text { Cross-Range Window: } 100 \mathrm{~m} \\
\text { Cross-Range Resolution: } 0.05 \mathrm{~m} \\
f_{\text {center }}=10 \mathrm{GHz} \\
\beta=150 \mathrm{MHz} \\
\mathrm{PRF}=6 \mathrm{kHz} \\
\tau=2 \mu \mathrm{s} \\
T_{\text {obs }}=0.333 \mathrm{~s}\end{array}$ \\
\hline 4 & $\begin{array}{l}\text { Sea State: } 3 \\
\mu=165^{\circ} \\
U=5 \mathrm{Kts} \\
\text { Hostile Radar LOS: } \\
\text { Azimuth }=320^{\circ} \\
\text { Elevation }=10^{\circ} \\
\text { Resolution ( } \# \text { of resolution } \\
\text { cells): } \\
\text { Slant-Range }=8 \text { bins } \\
\text { Cross-Range }=4 \text { bins } \\
\text { Height }=4 \text { bins }\end{array}$ & $\begin{array}{l}\text { Slant-Range Window: } 220 \mathrm{~m} \\
\text { Slant-Range Resolution: } 0.5 \mathrm{~m} \\
\text { Cross-Range Window: } 100 \mathrm{~m} \\
\text { Cross-Range Resolution: } 0.5 \mathrm{~m} \\
f_{\text {center }}=10 \mathrm{GHz} \\
\beta=300 \mathrm{MHz} \\
\mathrm{PRF}=10 \mathrm{kHz} \\
\tau=2 \mu \mathrm{s} \\
T_{\text {obs }}=0.02 \mathrm{~s}\end{array}$ \\
\hline
\end{tabular}




\begin{tabular}{|l|l|l|}
\hline 5 & Sea State: 1 & Slant-Range Window: $220 \mathrm{~m}$ \\
& $\mu=150^{\circ}$ & Slant-Range Resolution: $0.5 \mathrm{~m}$ \\
& $\mathrm{U}=10 \mathrm{Kts}$ & Cross-Range Window: $220 \mathrm{~m}$ \\
& Hostile Radar LOS: & Cross-Range Resolution: $0.05 \mathrm{~m}$ \\
& Azimuth $=130^{\circ}$ & $f_{\text {center }}=10 \mathrm{GHz}$ \\
& Elevation $=40^{\circ}$ & $\beta=300 \mathrm{MHz}$ \\
& Resolution (\# of resolution & $\mathrm{PRF}=12 \mathrm{kHz}$ \\
& cells): & $\tau=2 \mu \mathrm{s}$ \\
& Slant-Range $=16$ bins & $T_{\text {obs }}=0.366 \mathrm{~s}$ \\
& Cross-Range $=8 \mathrm{bins}$ & \\
& Height $=4$ bins & \\
\hline
\end{tabular}

Figure 7.22 shows the processed high-resolution range profile generated for simulation case one. The data is processed through a digital matched filter, resulting in a total number of samples which is double the original echo pulse duration. The peak data is observed to occur after the number of samples corresponding to the pulse duration. The true range position of scattering locations in the profile is obtained using equation 7.2 from [45].

$$
R_{d}=\frac{c\left(t_{d}-\tau\right)}{2}
$$

The variable $t_{d}$ indicates the time at which the peak is measured in the profile output from the matched filter. The variable $\tau$ represents the pulse duration in units of time or as a number of samples. The time values can be converted to number of samples based on the sampling rate when processing digital samples of the range profile. 


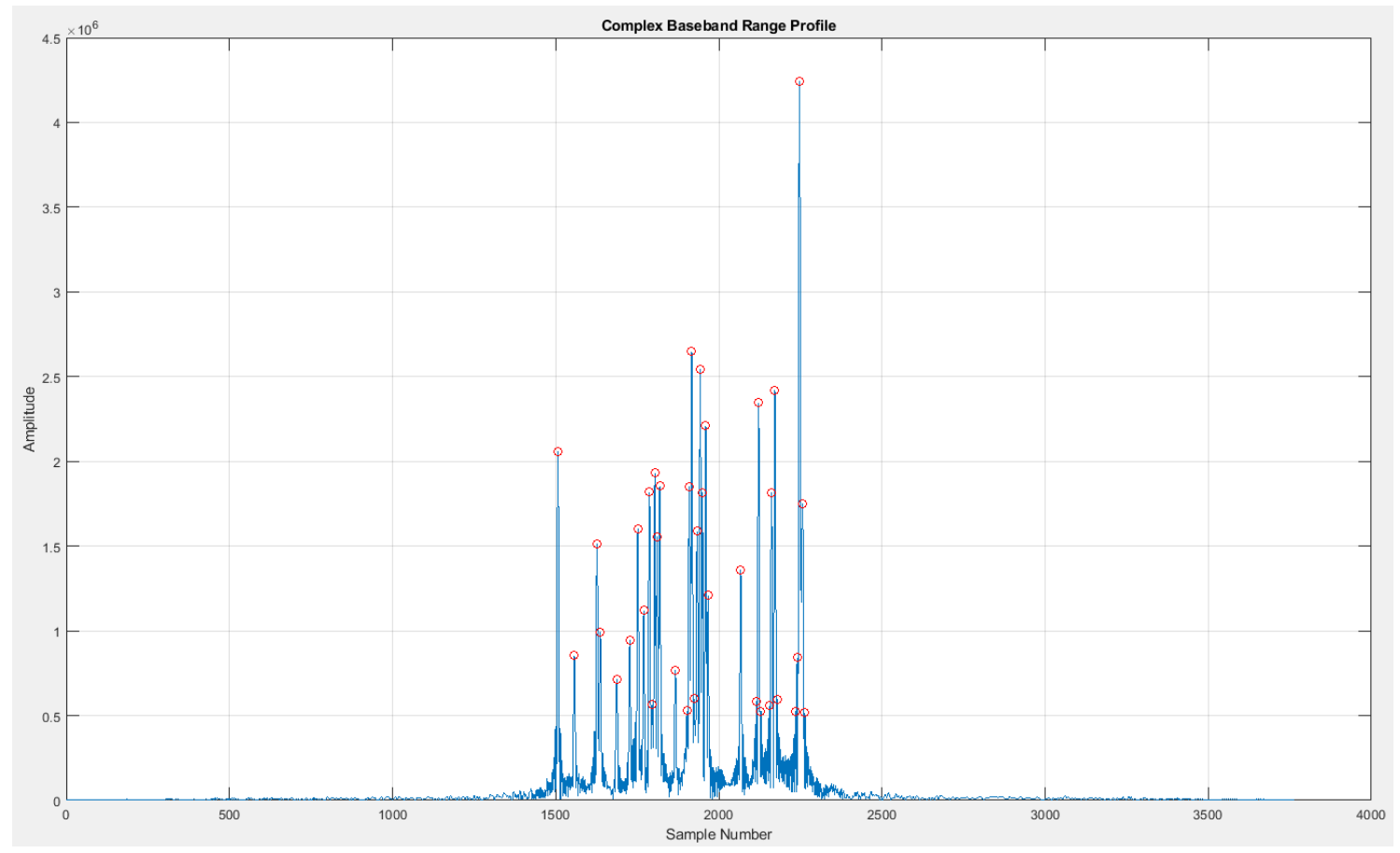

Figure 7.22: Chirp Radar simulation case 1a/1b, complex baseband range profile.

Figure 7.32 shows the zoomed in section of the range profile for better resolution.

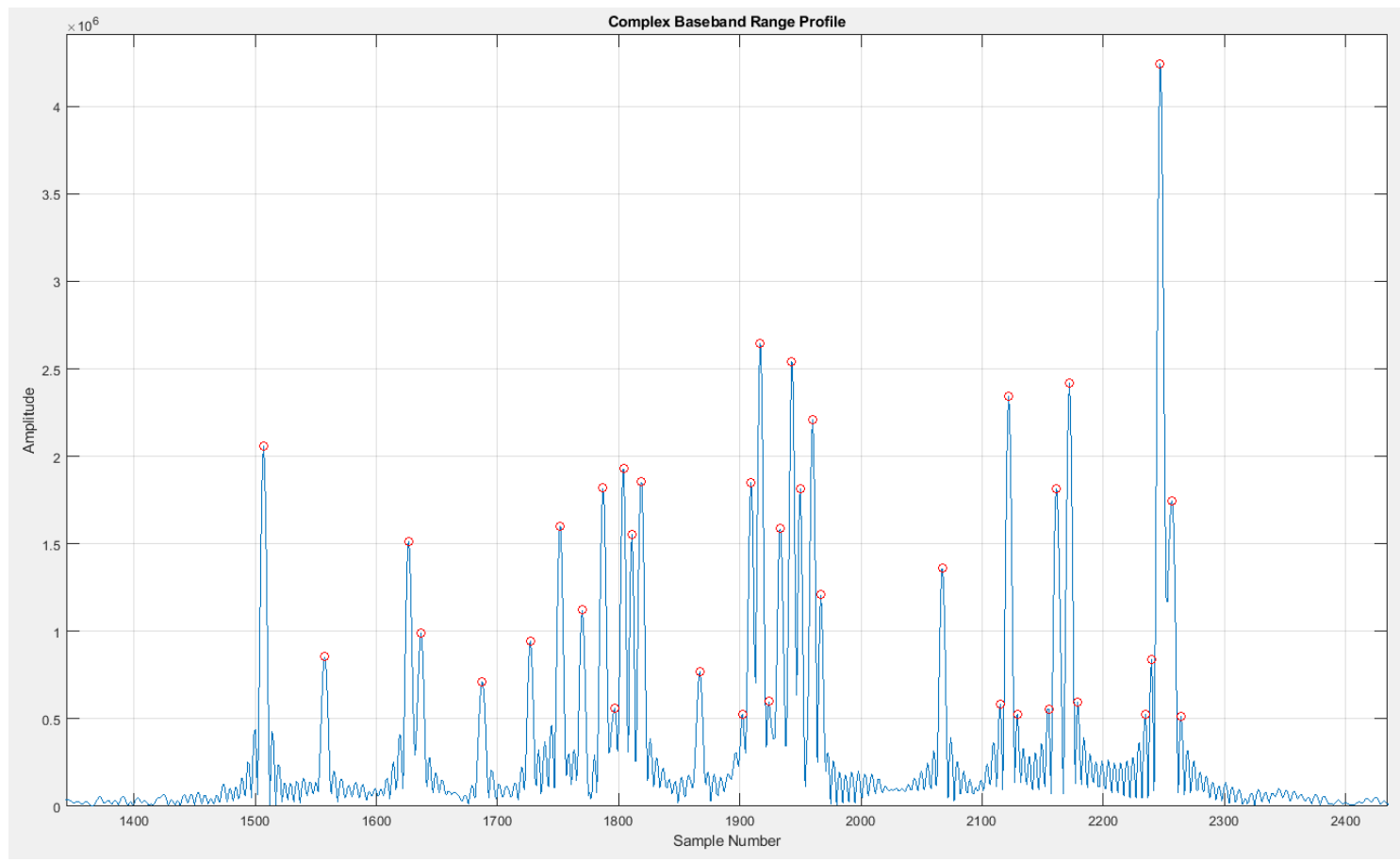

Figure 7.23: Chirp Radar simulation case 1a/1b, complex baseband range profile, zoomed in. 


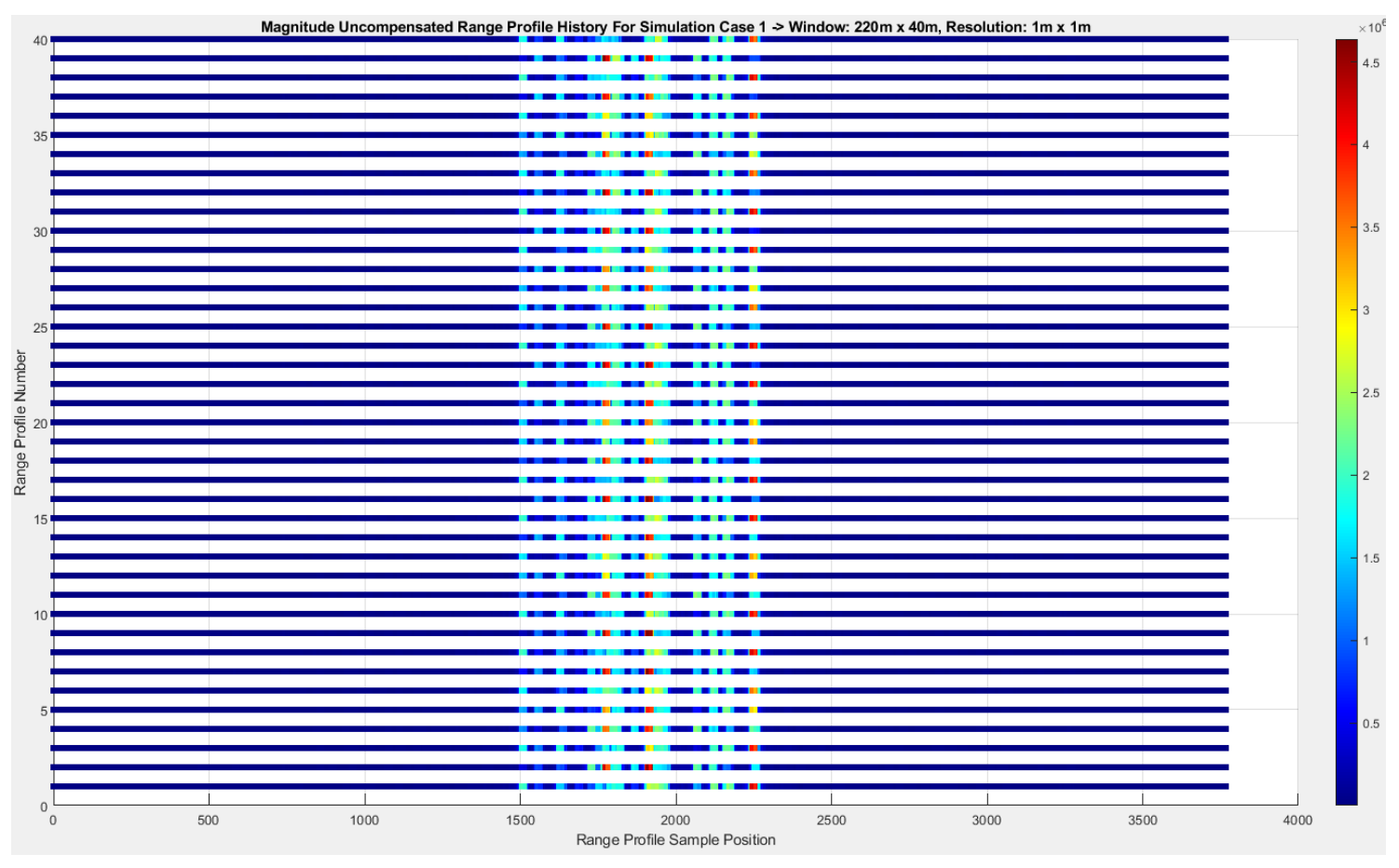

Figure 7.24: Simulation case 1a, magnitude of complex baseband range profile history.

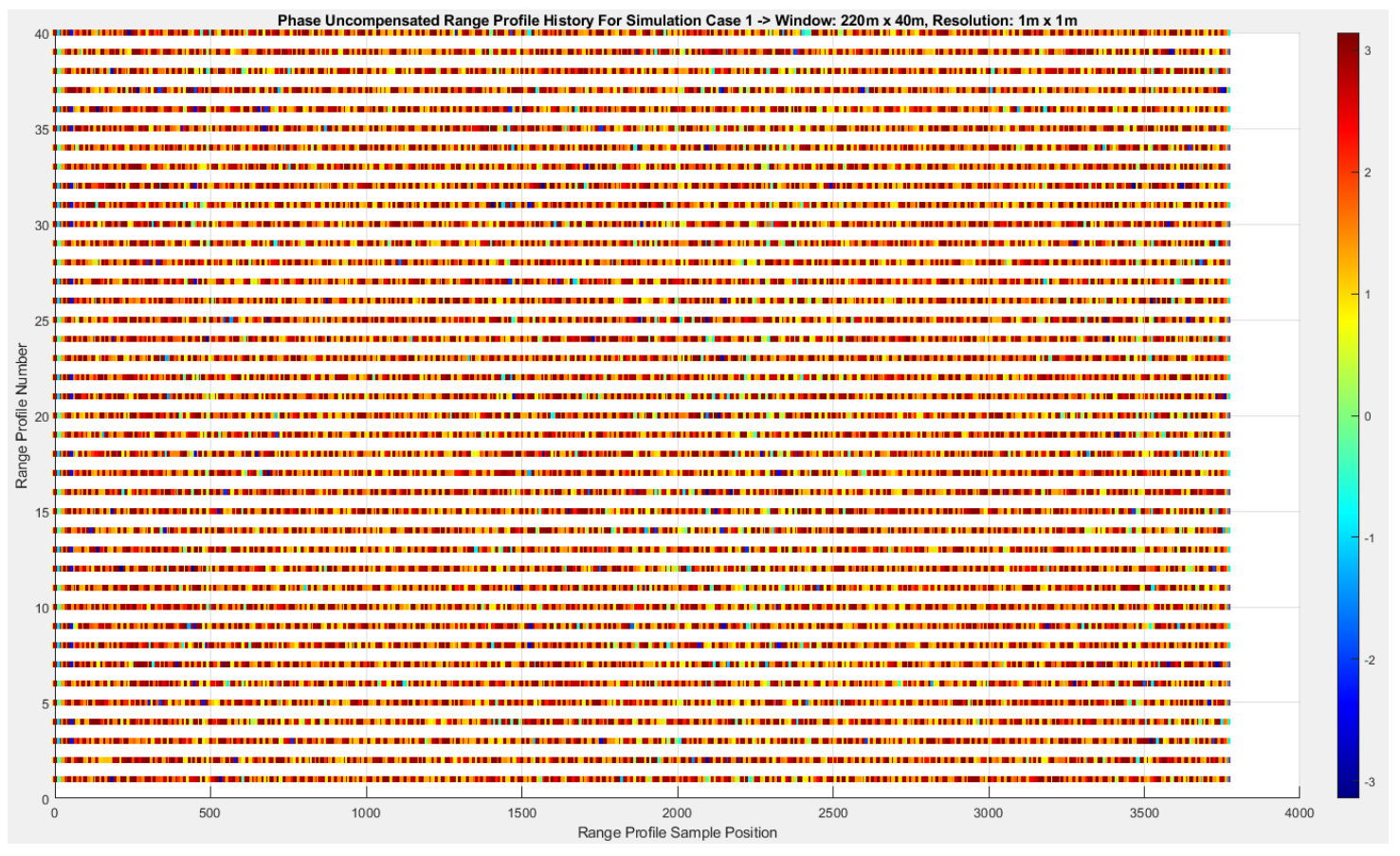

Figure 7.25: Simulation case 1a complex baseband phase history. 


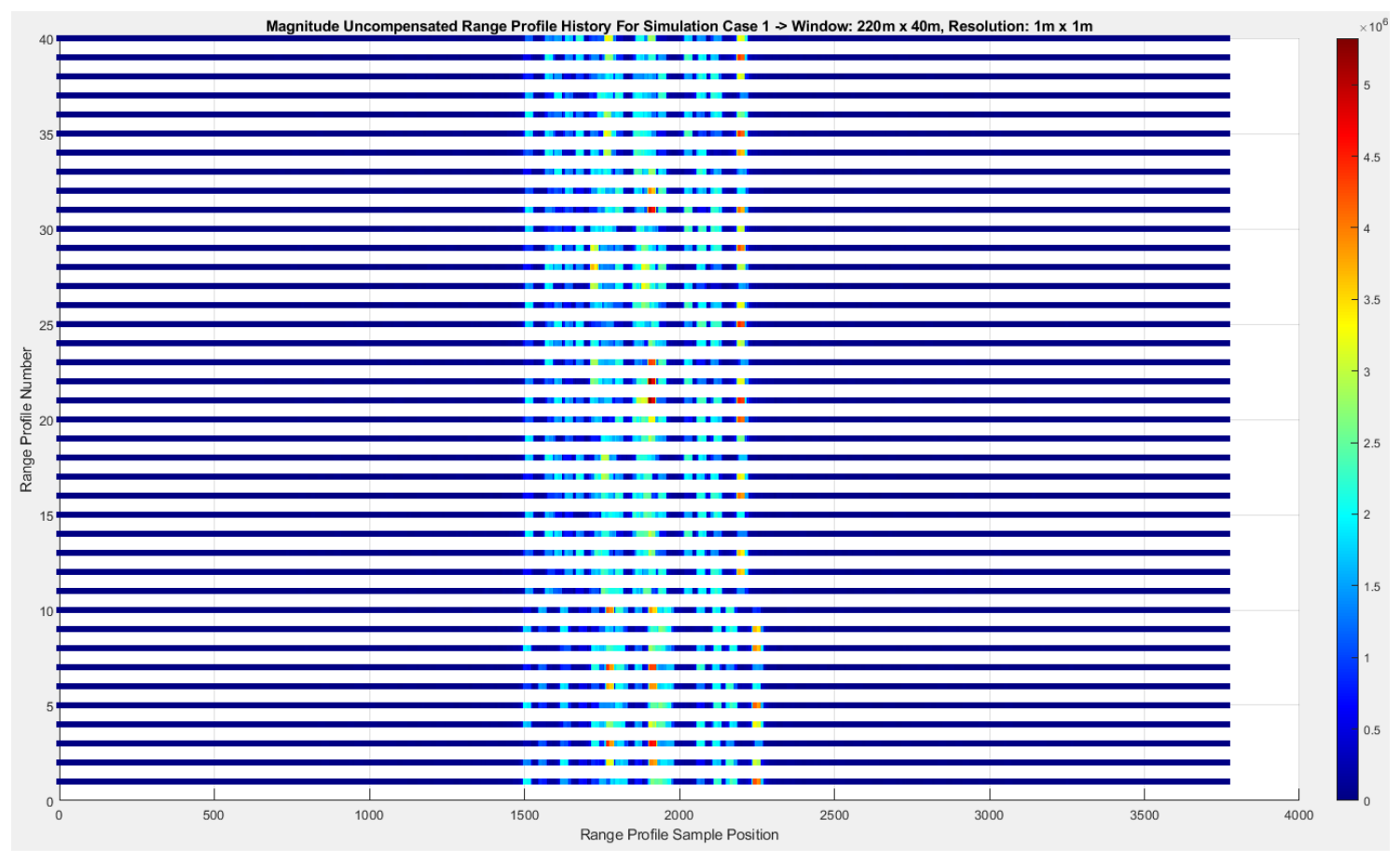

Figure 7.26: Simulation case 1b, magnitude of complex baseband range profile history.

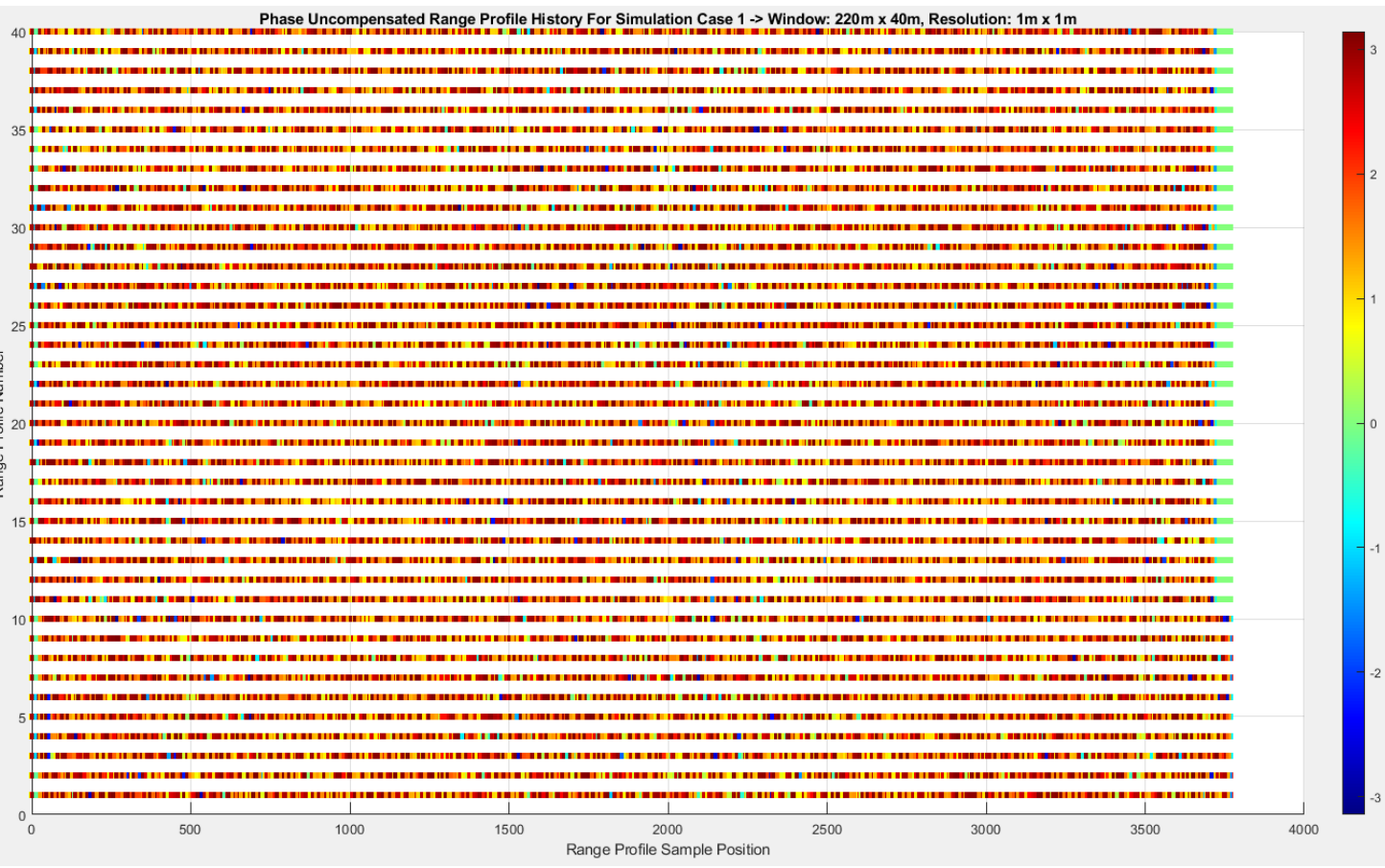

Figure 7.27: Simulation case 1b complex baseband phase history. 
Through comparison of the magnitude and phase histories of case $1 \mathrm{a}$ and $1 \mathrm{~b}$, it is observed that the difference in profile data due to the change in PRF is not overtly visible in the phase data. The variation in the magnitude history is visible if carefully observed. The range profile magnitude and phase history for simulation cases $2 \mathrm{a}, 2 \mathrm{~b}, 3$ and 5 are shown in Sub-Appendix C.2.

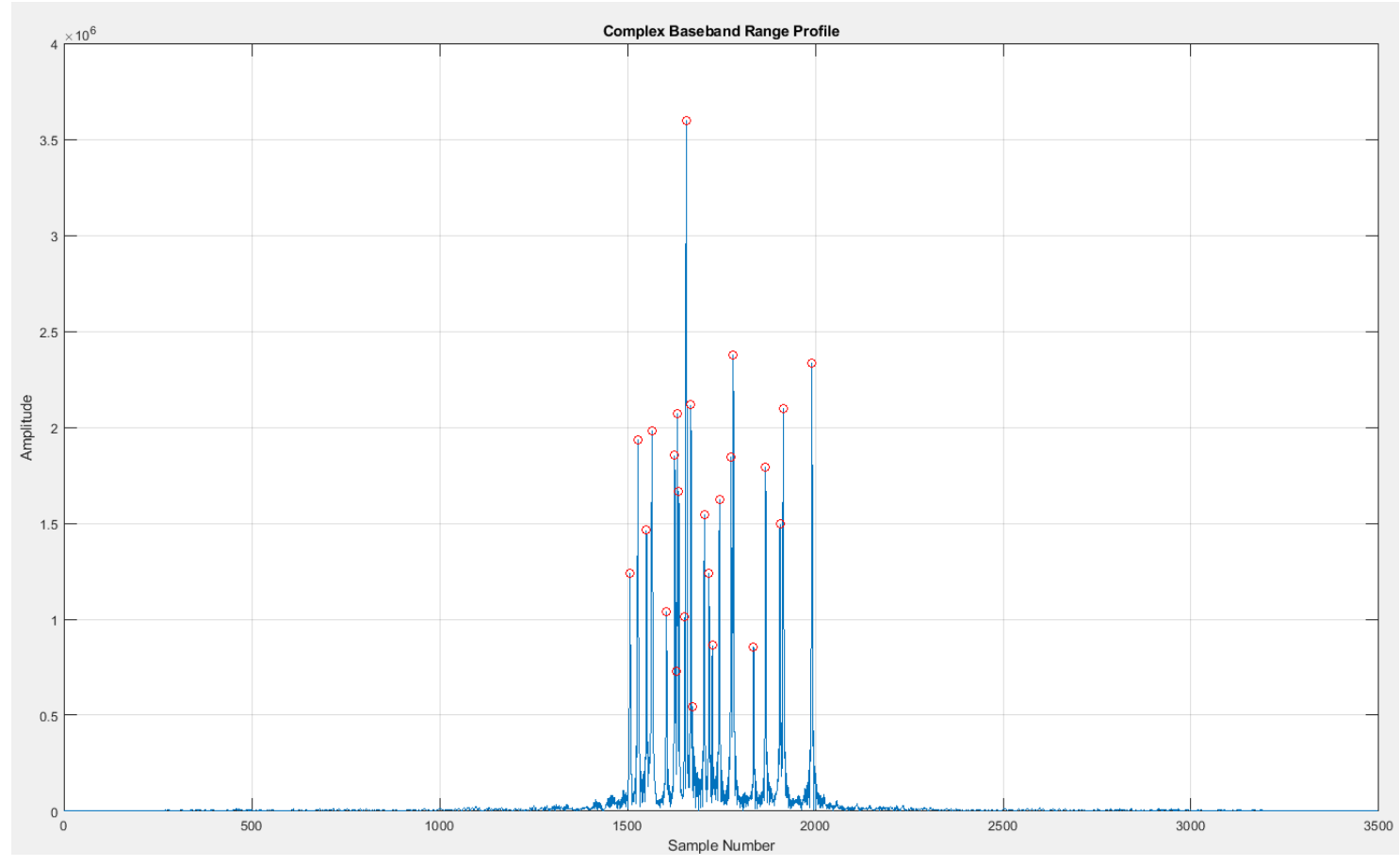

Figure 7.28: Complex baseband range profile Simulation Case 4. 


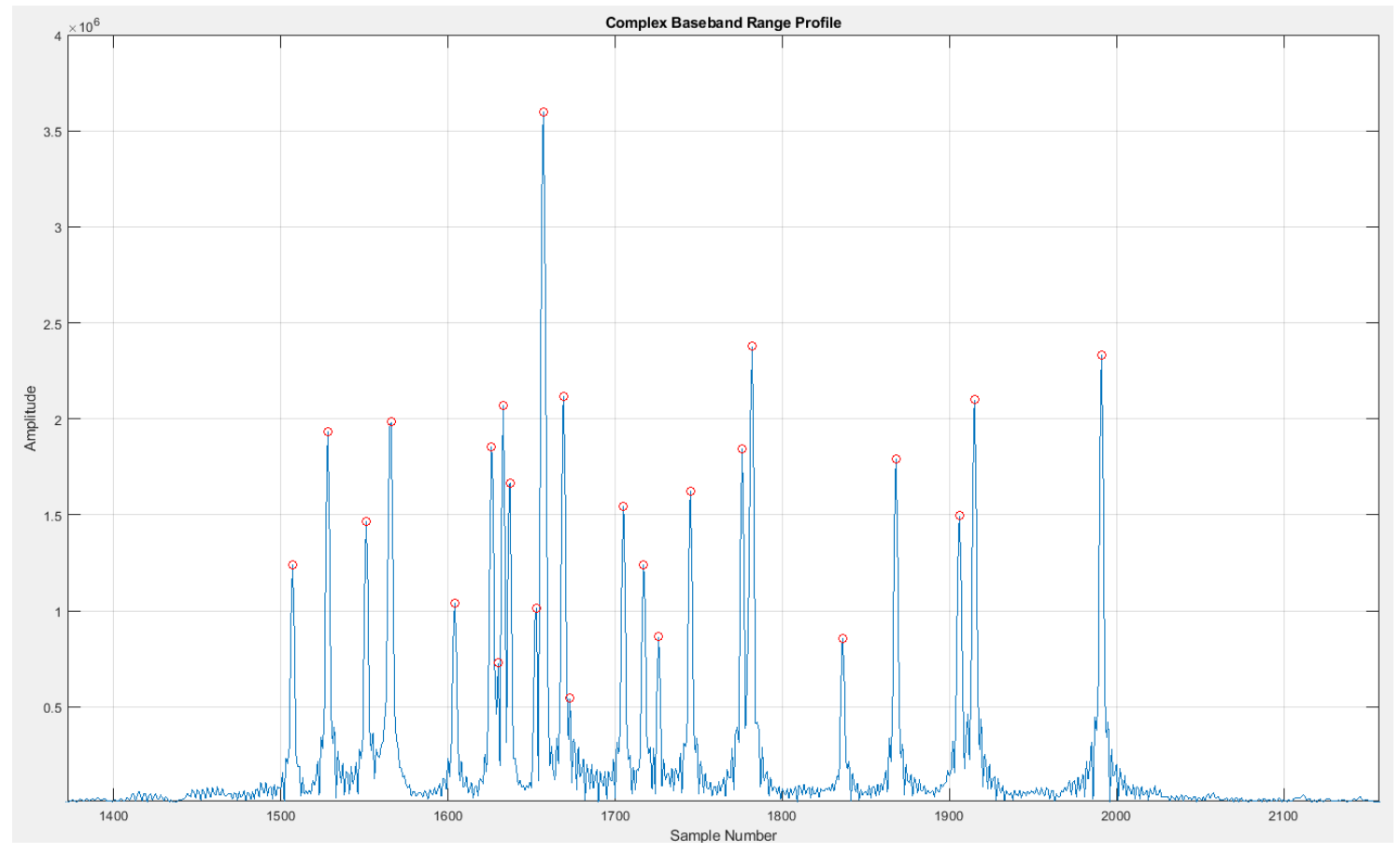

Figure 7.29: Complex baseband range profile Simulation Case 4, zoomed in.

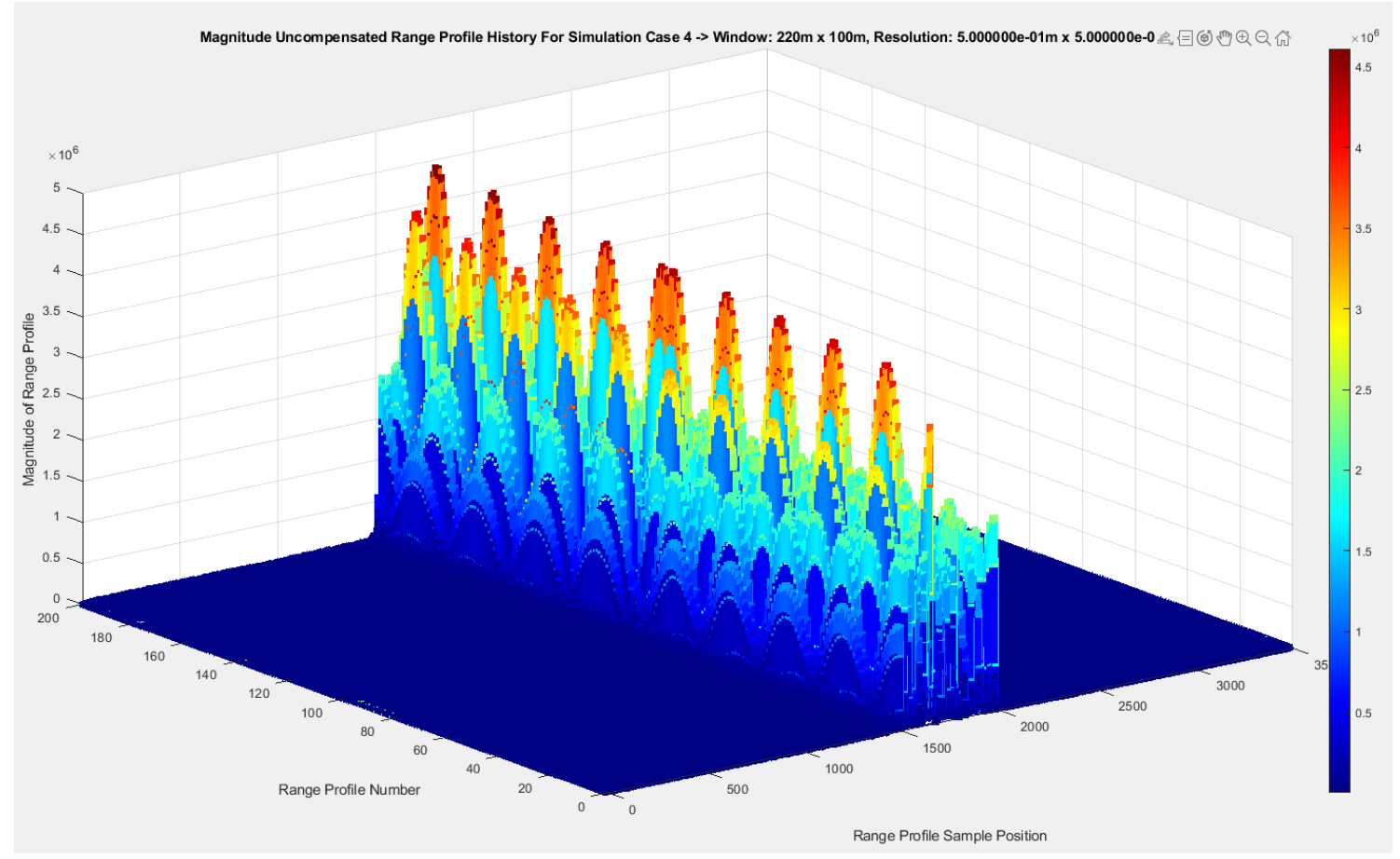

Figure 7.30: Chirp Radar simulation case 4, magnitude of complex baseband range profile history. 
In Figure 7.30 the Doppler history of scattering locations across multiple profiles can be observed clearly. The magnitude response of a scattering location is amplitude modulated by the Doppler shift of the scatterer. This Doppler response is constant for the profile until the decorrelation time is reached, at which point the Doppler and phase response of the target change. This is observed in Figure 7.30 in the middle of the profile data, where a double peak is present representing the time at which the profile has decorrelated and new dominant scattering modulation parameters are utilized in profile synthesis.

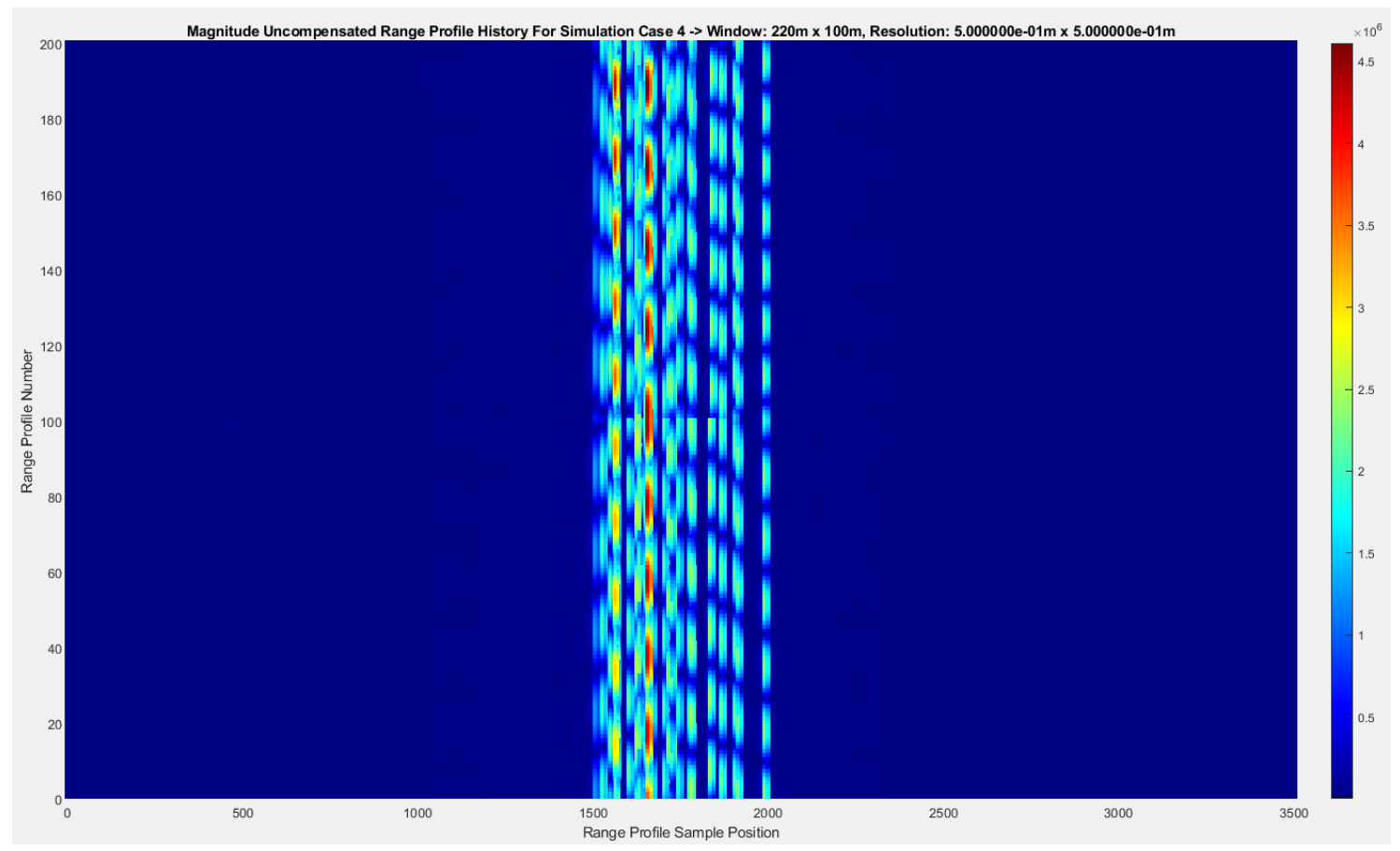

Figure 7.31: Chirp Radar simulation case 4, magnitude of complex baseband range profile history. 


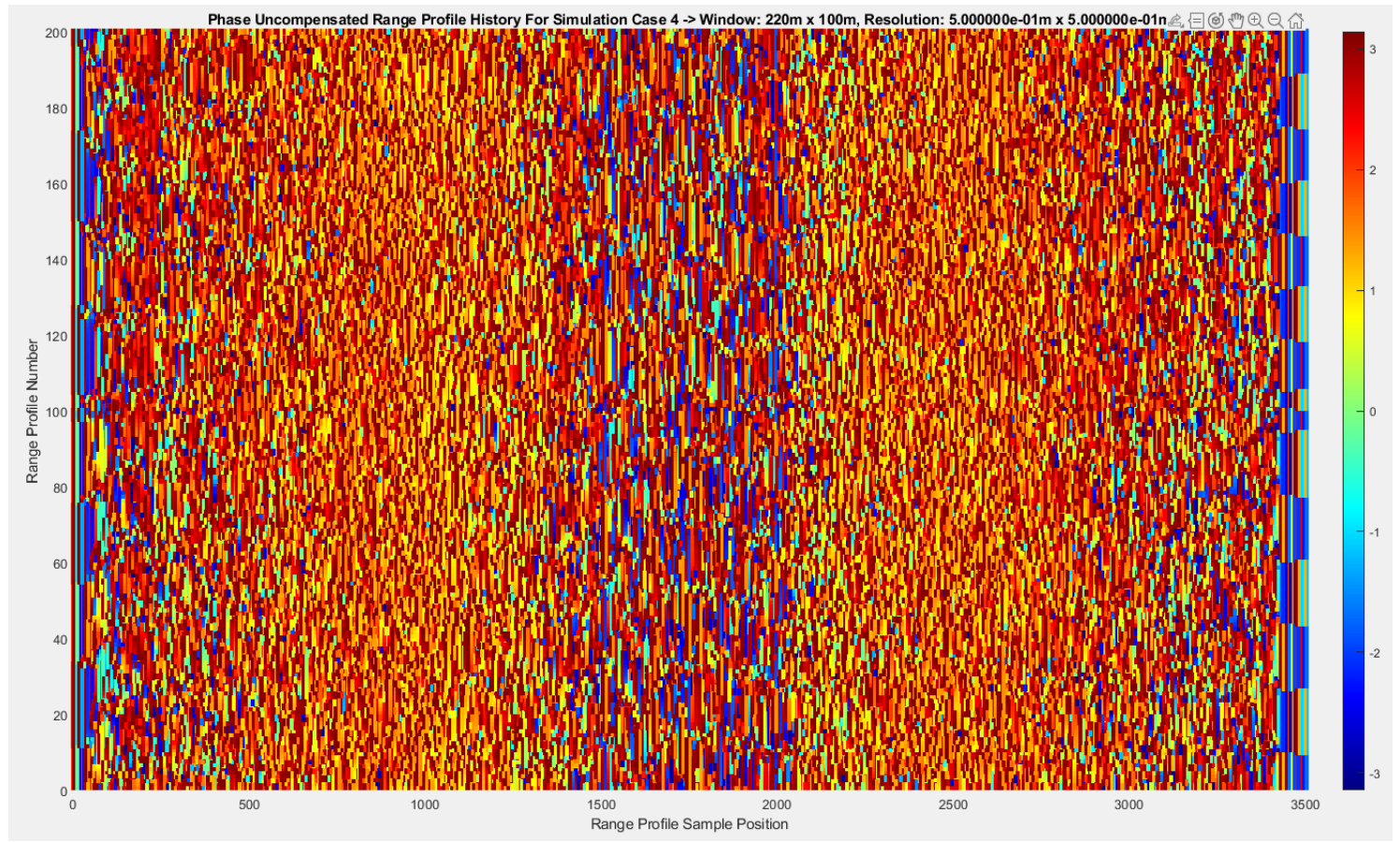

Figure 7.32: Chirp Radar simulation case 4, complex baseband phase history.

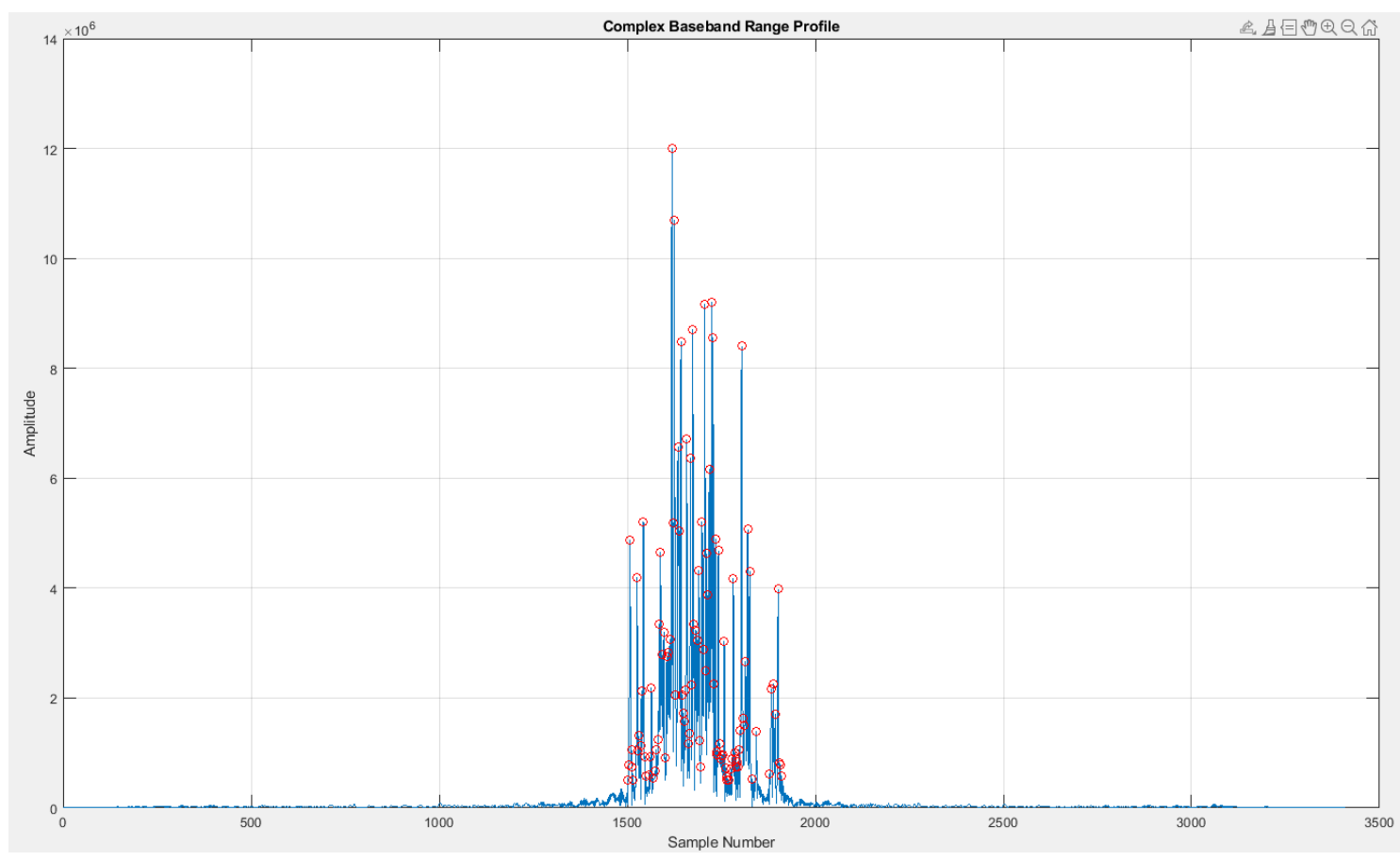

Figure 7.33: Complex baseband range profile Simulation Case 5. 


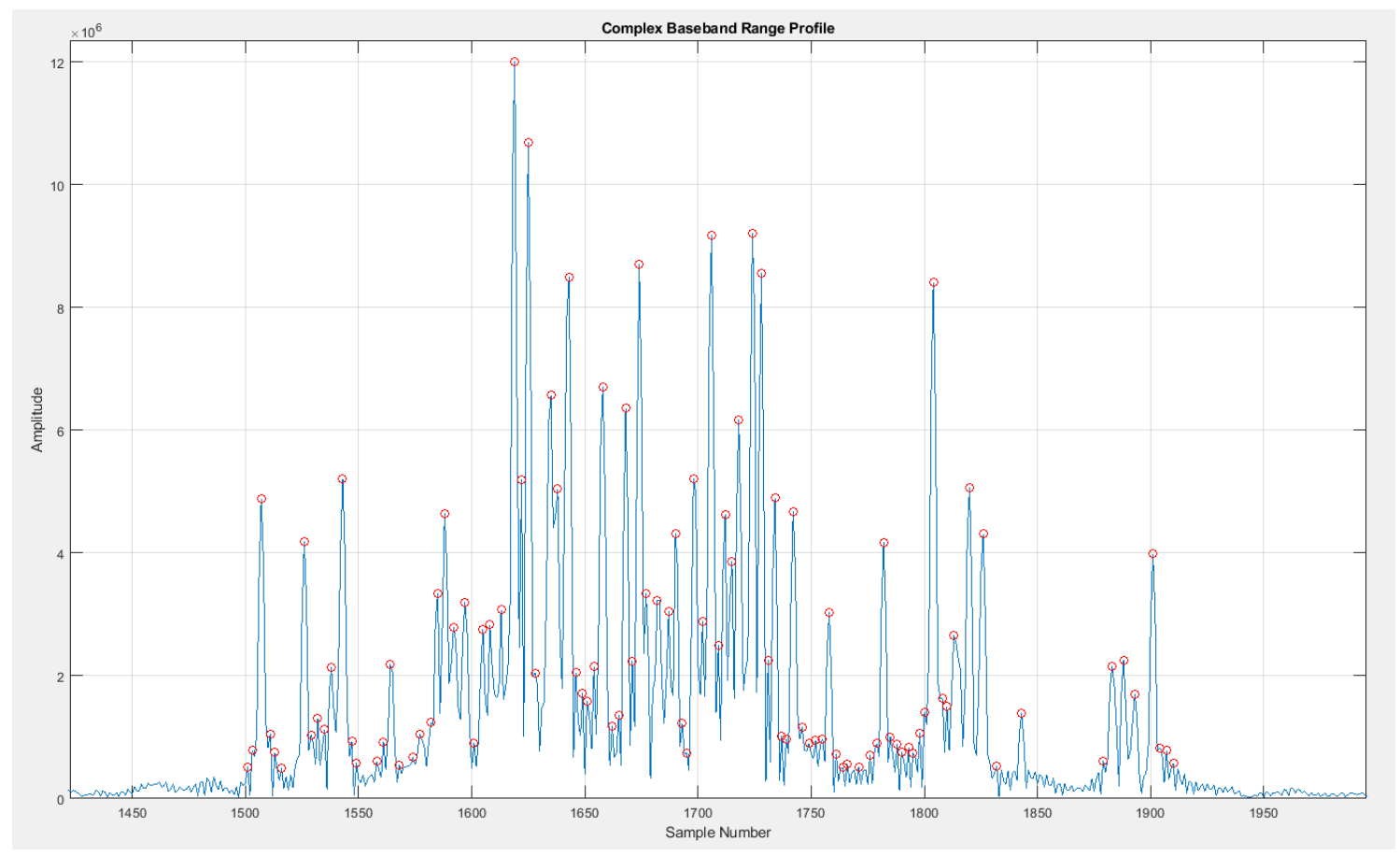

Figure 7.34: Complex baseband range profile Simulation Case 5, zoomed in.

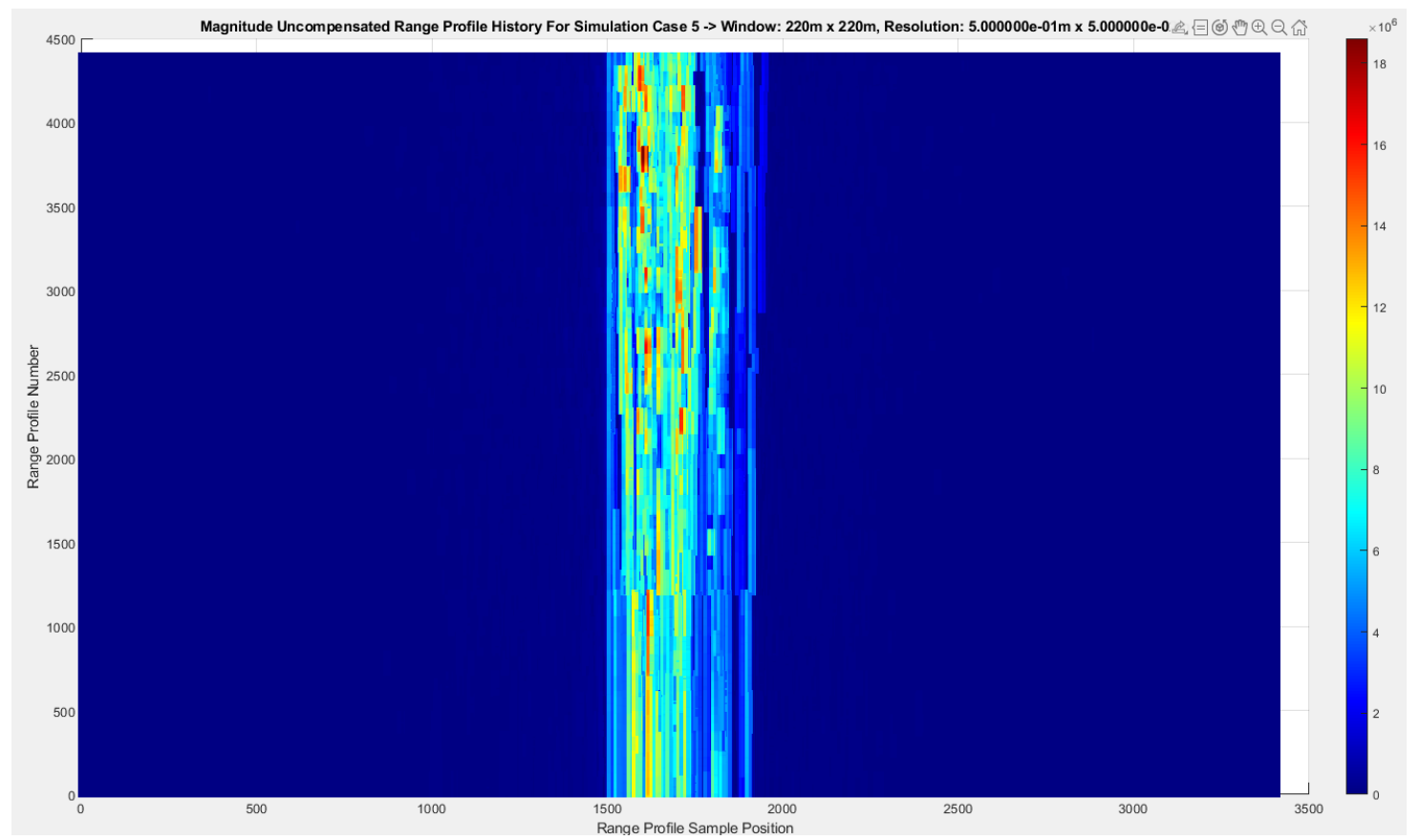

Figure 7.35: Chirp Radar simulation case 5, magnitude of complex baseband range profile history. 


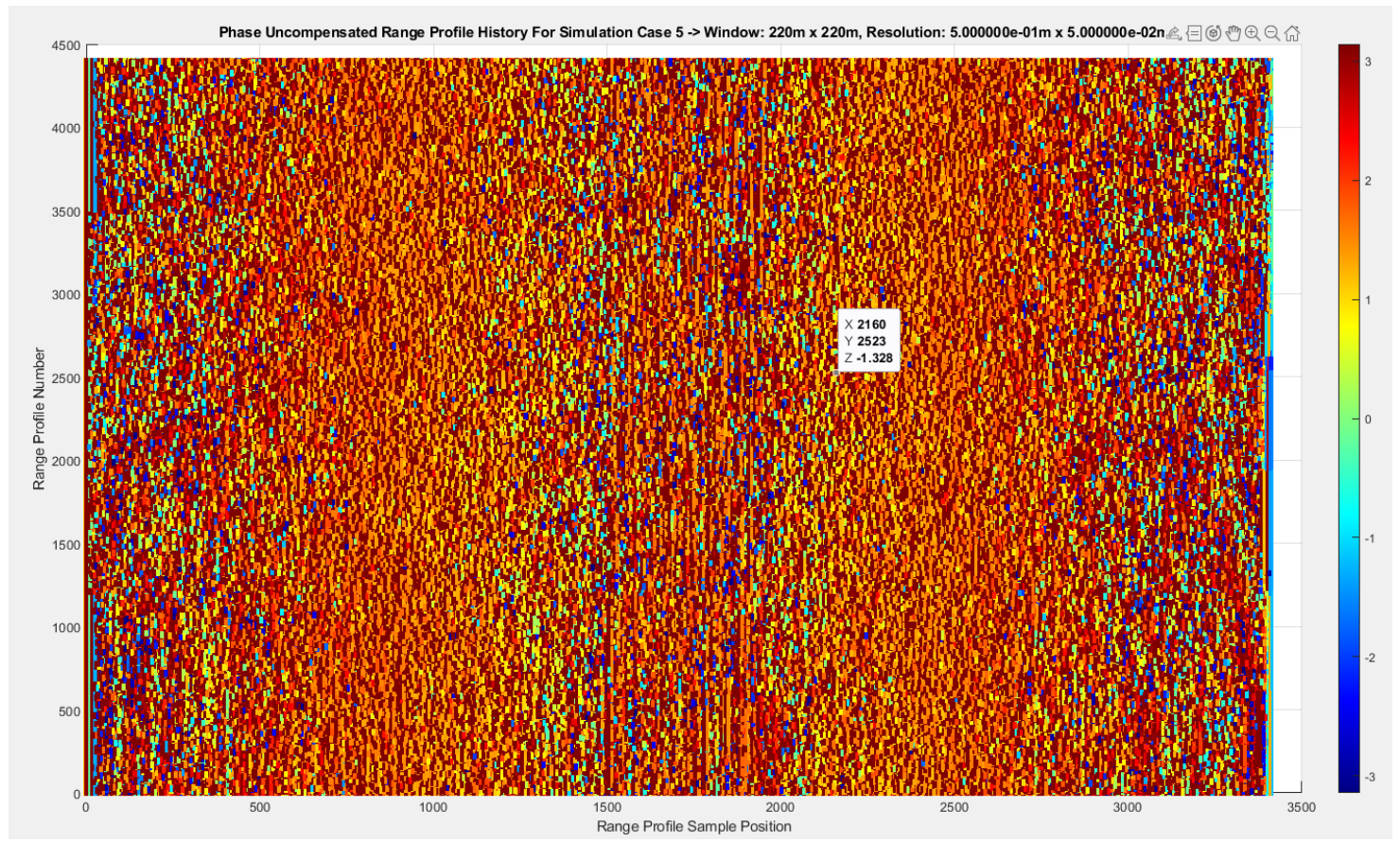

Figure 7.36: Chirp Radar simulation case 5, complex baseband phase history.

\subsection{Range-Doppler Image Simulation}

The Range-Doppler images are produced using the range profile history datasets for the simulation cases in countering both stepped-frequency and chirp radar systems. The data processing methods to obtain Range-Doppler images are discussed in chapter three. Chapter three also discusses the motion compensation algorithms used to correct the Range-Doppler data to improve image quality.

\subsubsection{Image Generation from Stepped-Frequency Data}

The figures on the following pages provide the uncompensated and motion compensated Range-Doppler images produced for simulation cases one and two in the stepped-frequency radar datasets. 


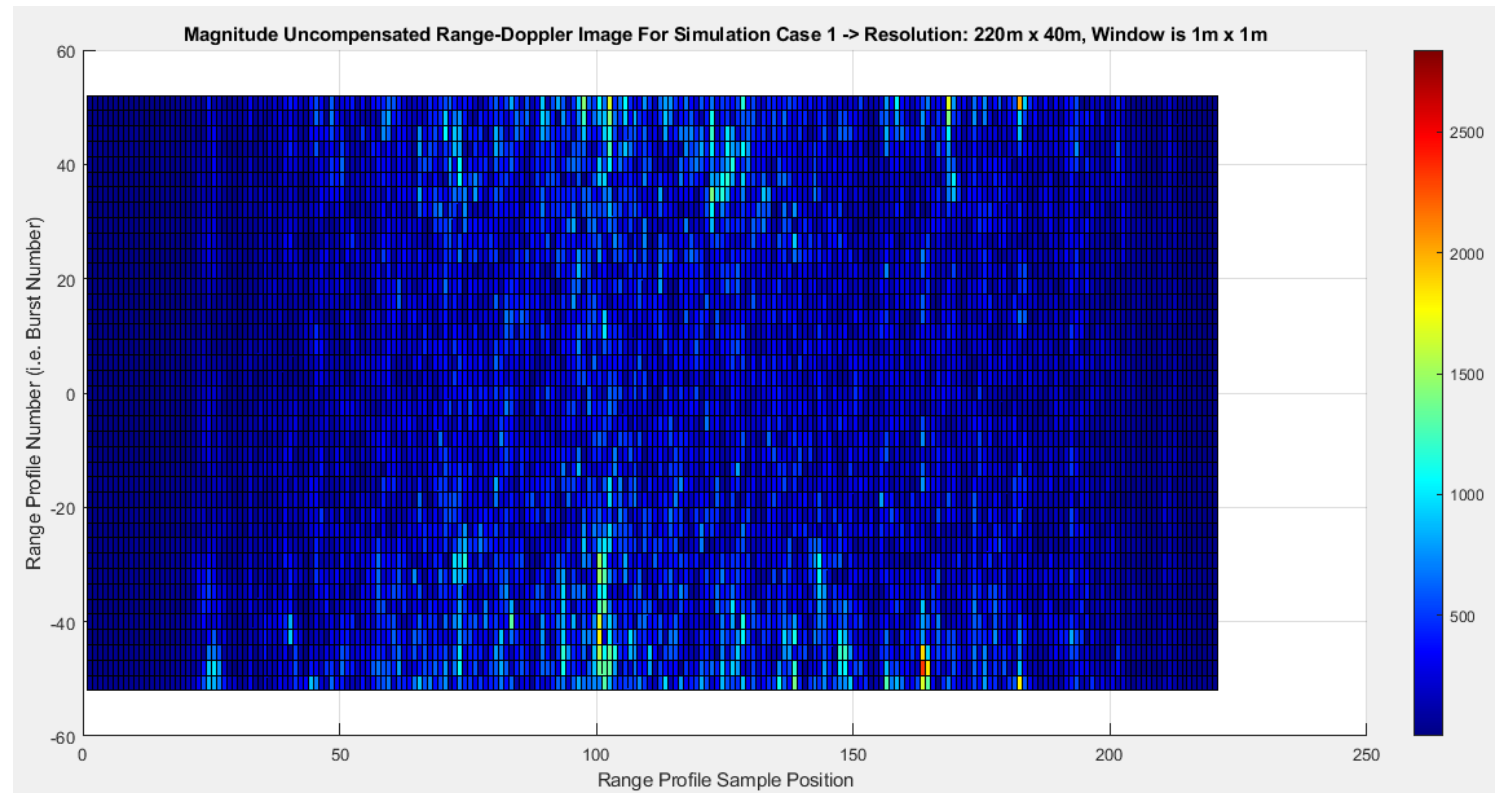

Figure 7.37: Simulation Case 1 Range Doppler image, uncompensated complex baseband data.

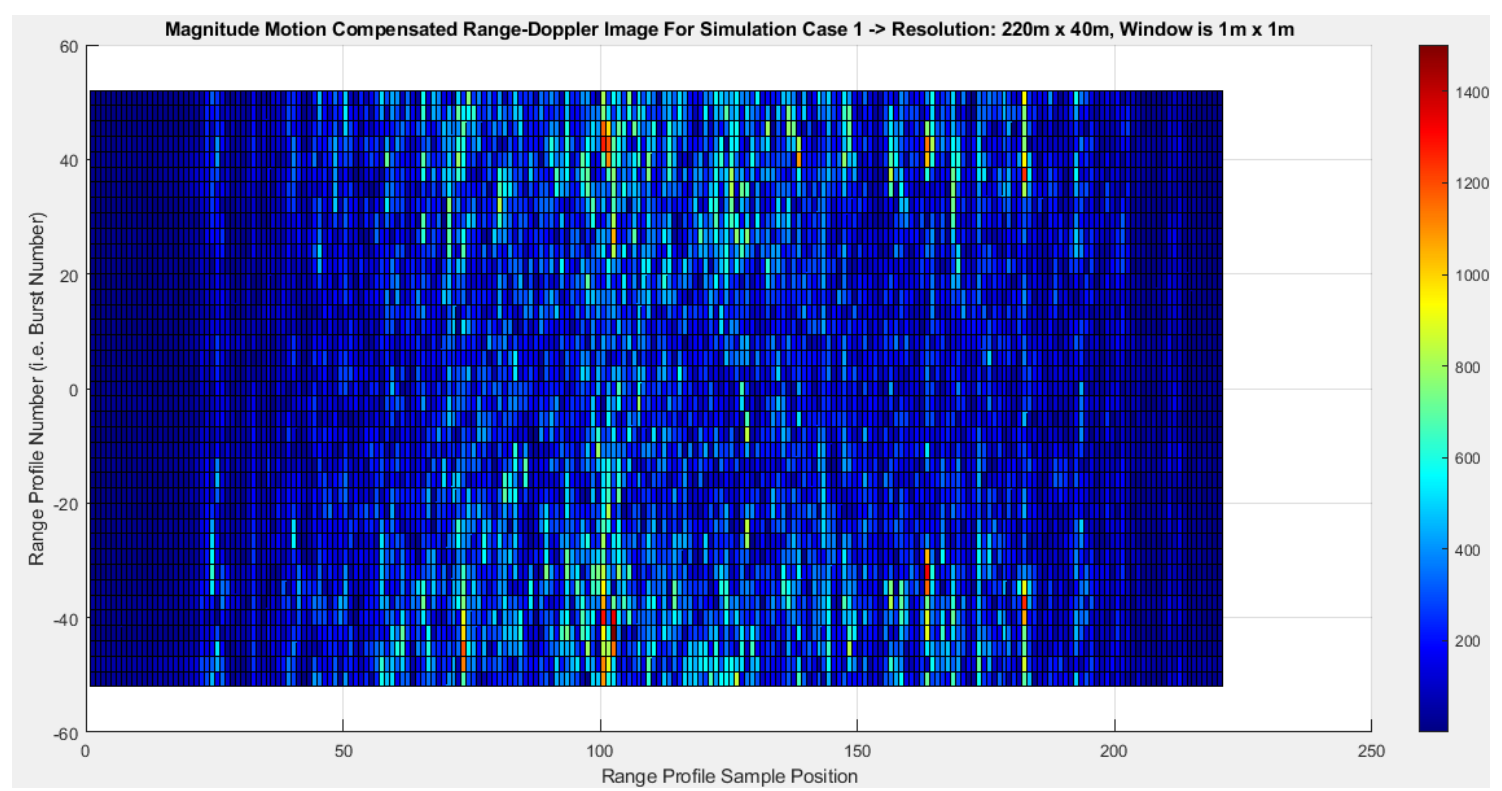

Figure 7.38: Simulation Case 1 Range Doppler image, motion compensated complex baseband data.

The compensated data image looks similar to the broadside profile of the ship. This image is flipped for positive frequency. However, significant blurring is also 
observed due to the inaccuracy in the amplitude estimation of scattering locations. This increases the energy spread in frequency creating the blurring effect.

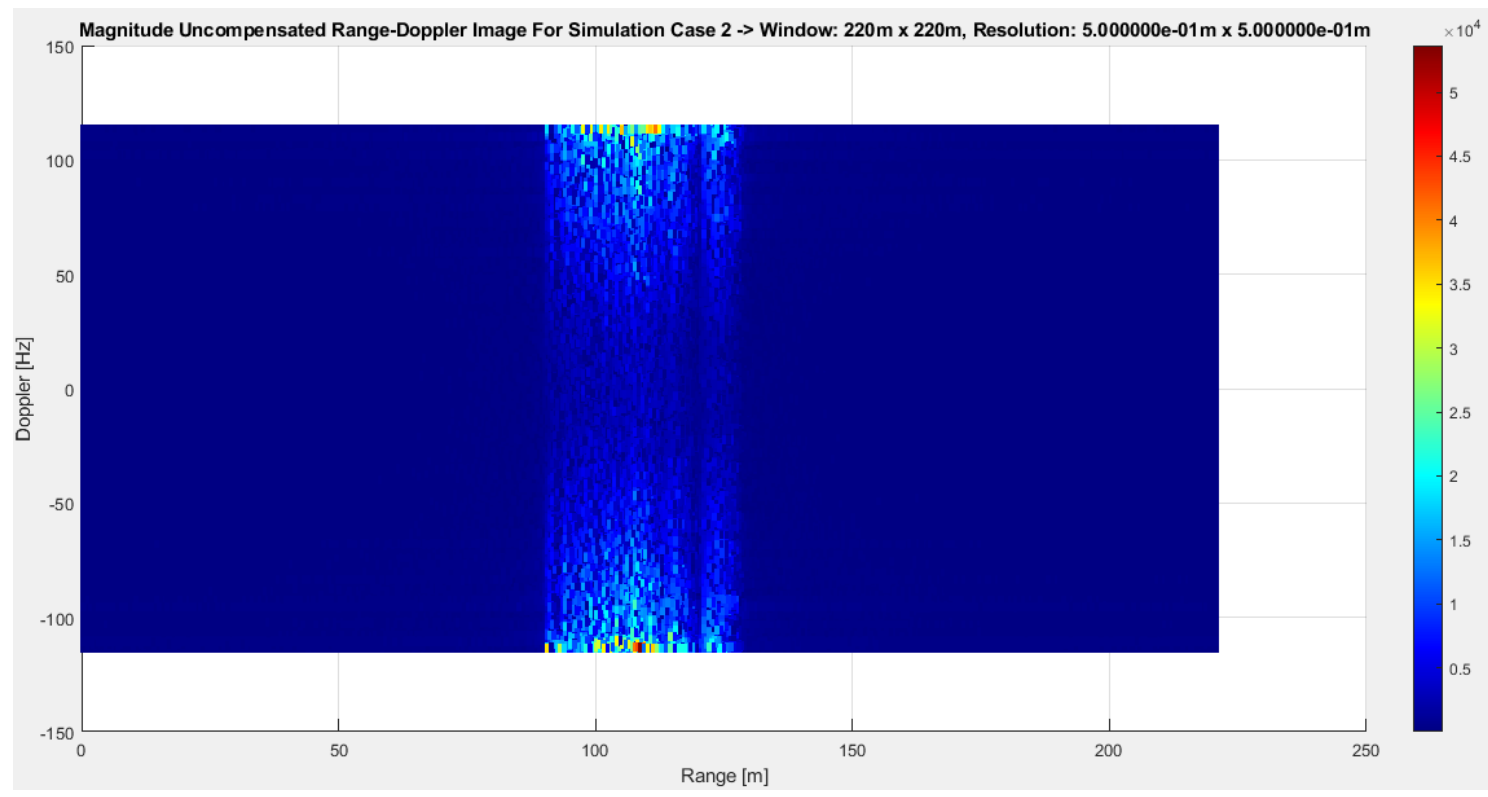

Figure 7.39: Simulation Case 2 Range Doppler image, uncompensated complex baseband data.

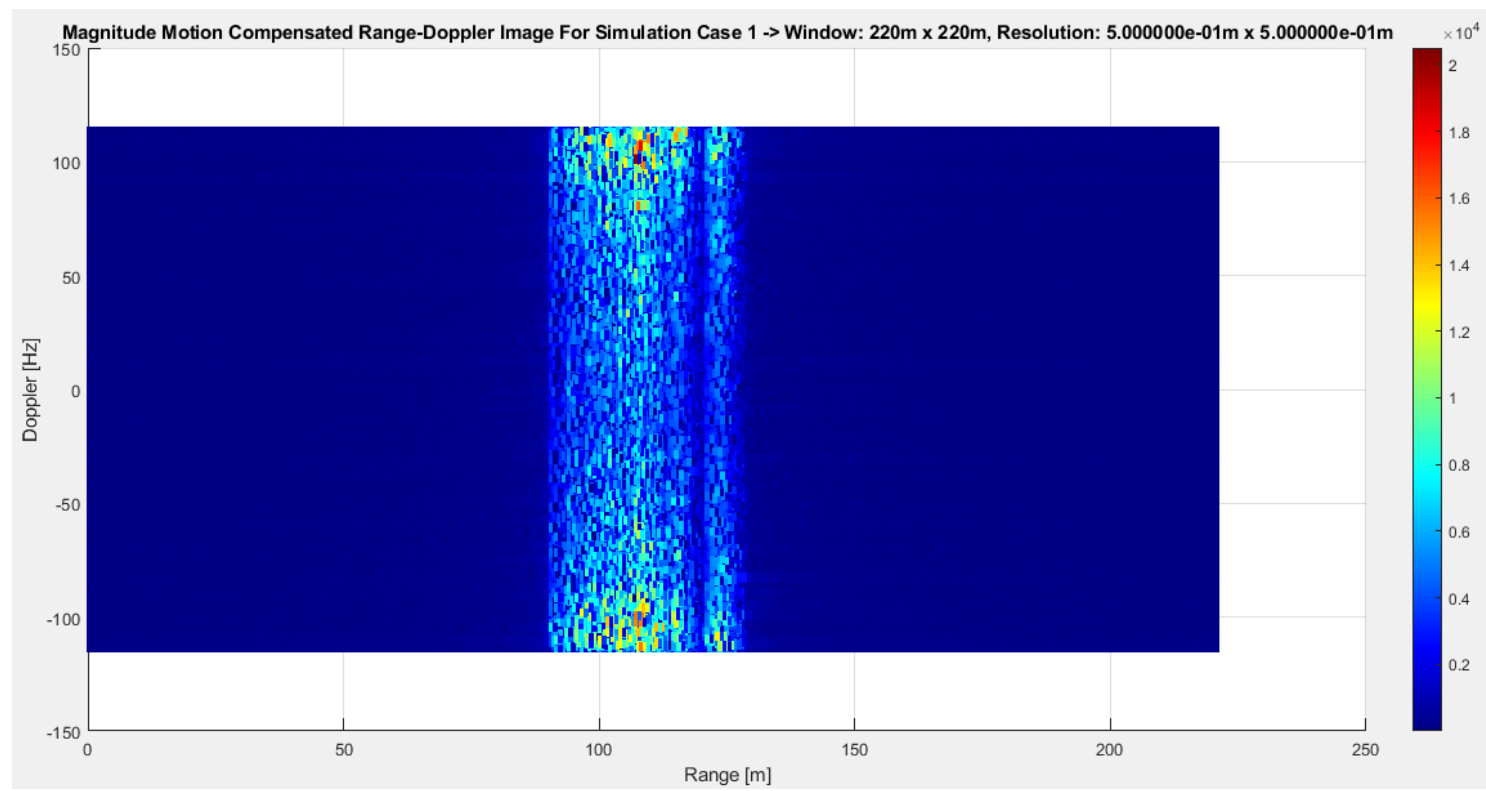

Figure 7.40: Simulation Case 2 Range Doppler image, range and Doppler tracked complex baseband data.

The Range-Doppler image data is observed in the negative frequency portion to be similar to observing the target from the bow of the ship. The Dominant-Scattering 
algorithm has in-fact reduced the visibility of the target image by introducing much noise. Research into proper amplitude estimation of scattering locations, and possible use of other motion compensation algorithms can increase the image's resolution.

\subsubsection{Image Generation from Chirp Data}

For simulation cases $1 \mathrm{a}, 1 \mathrm{~b}, 2 \mathrm{a}$ and $2 \mathrm{~b}$ the variation in the PRF is evident in the Range-Doppler images produced. The Doppler resolution is limited by the PRF, which is the Doppler sampling rate [45][49][50]. At a lower PRF spectral aliasing is present for scattering locations which have a Doppler frequency greater than the Nyquist sampling requirement. This is present in both the uncompensated and motion compensated RangeDoppler images.

The Range-Doppler images for chirp systems have a wider Doppler bandwidth since the Doppler is sampled at the PRF, and not the burst period as in steppedfrequency. This captures the entire target Doppler spectrum without aliasing the higher frequency motion of the ship. However, this also leads to compressed images in the Doppler dimension as the Doppler bandwidth of the target is a fraction of the Doppler bandwidth of the image. To correct this an estimate of the average Doppler of the target is obtained from the motion compensated Range-Doppler image. This Doppler estimate is used as a correction factor to reduce the target's Doppler frequency closer to $0 \mathrm{~Hz}$ in the image to provide greater image focus. The Doppler correction is multiplied into the quadrature channel data, which is already motion compensated, and then the RangeDoppler images are recomputed.

The following pages show the uncompensated, motion compensated, and Doppler corrected Range-Doppler images produced for simulation cases one and four in the chirp radar datasets. The plots begin on the following page. The data for the remaining cases can be found in Sub-Appendix C.4. 


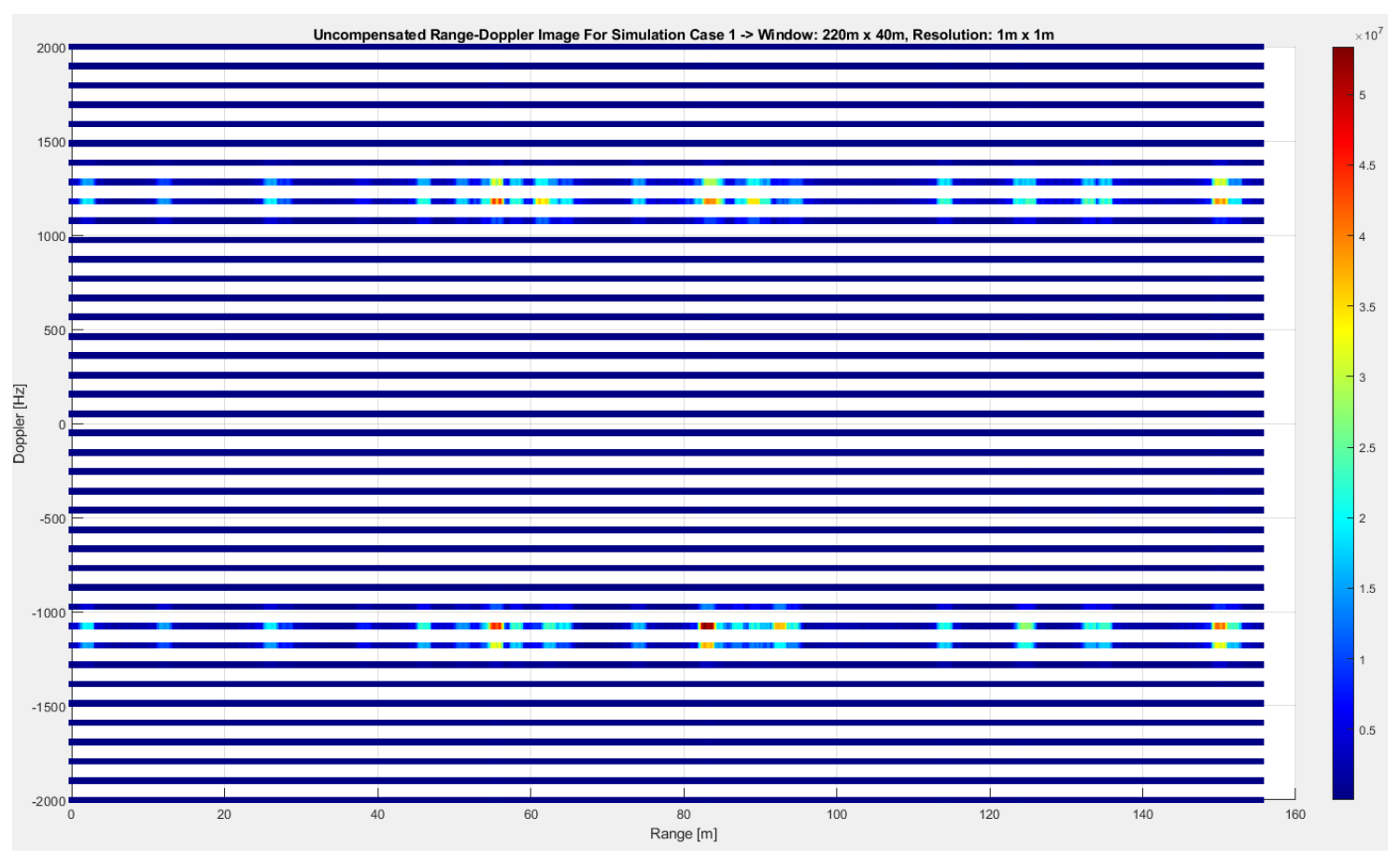

Figure 7.41: Uncompensated Range Doppler image for Simulation case 1a.

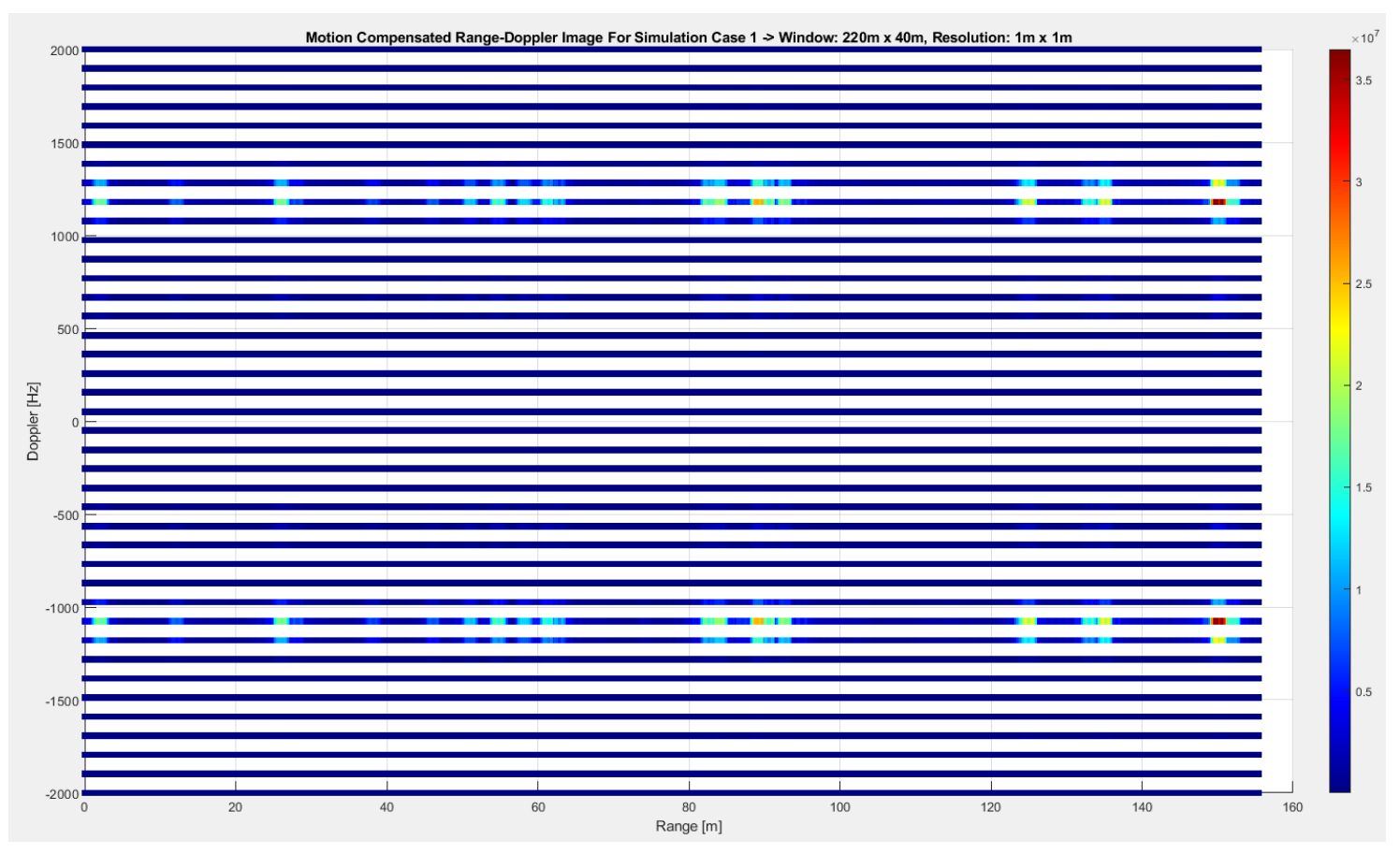

Figure 7.42: Motion compensated Range-Doppler image for Simulation case 1a. 


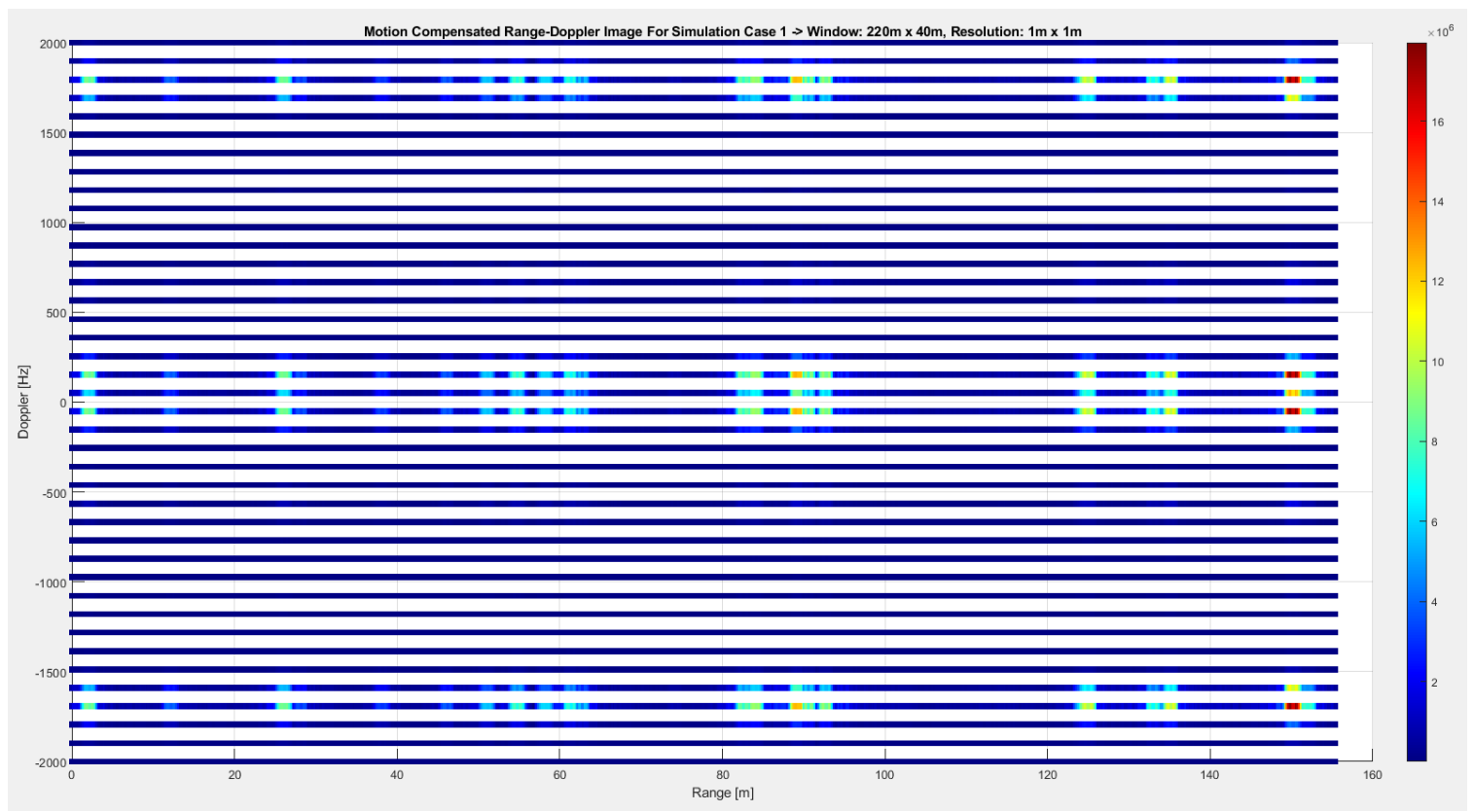

Figure 7.43: Range-Doppler image for case 1a, Doppler corrected by $1200 \mathrm{~Hz}$.

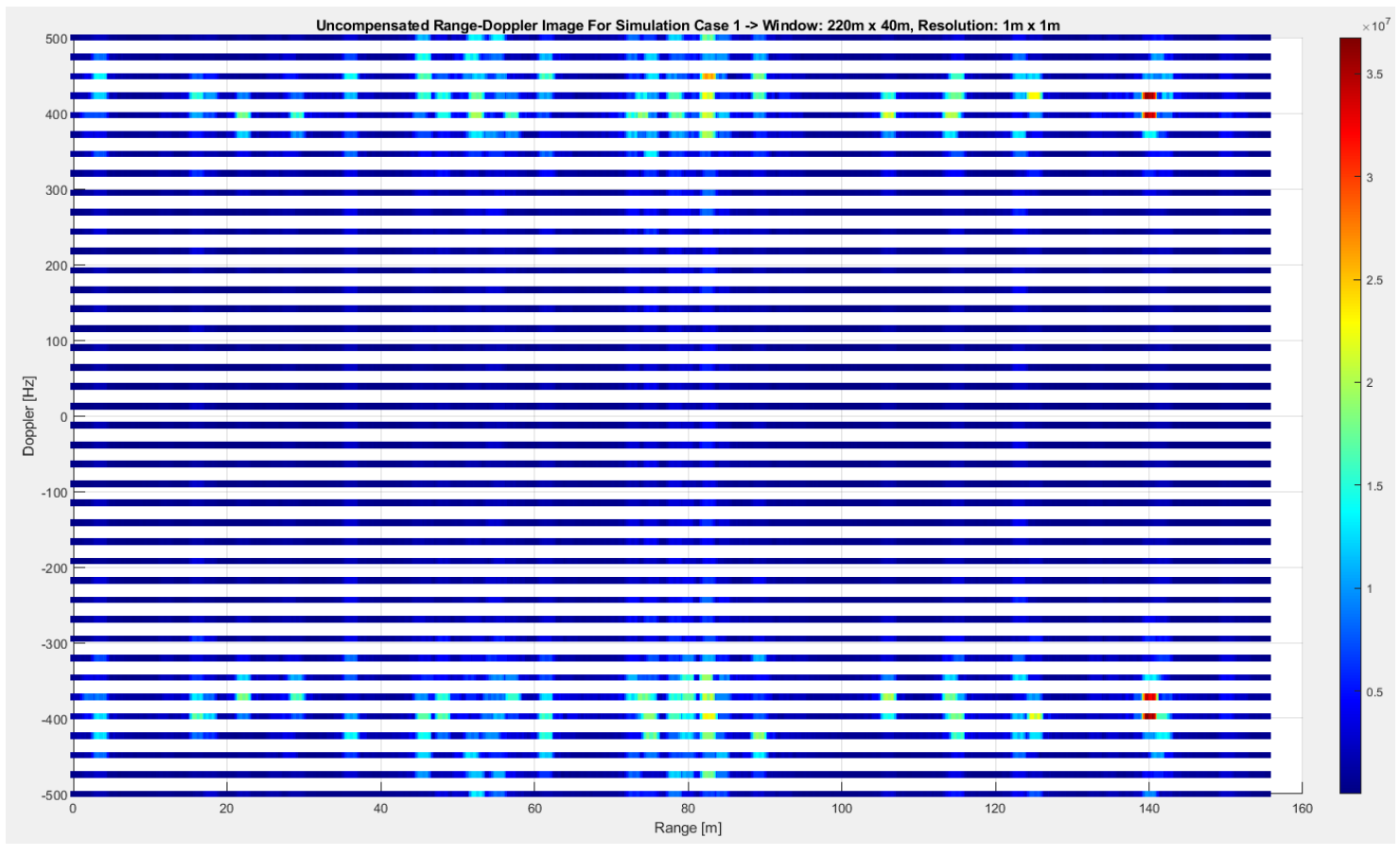

Figure 7.44: Uncompensated Range Doppler image for Simulation case $1 \mathrm{~b}$. 


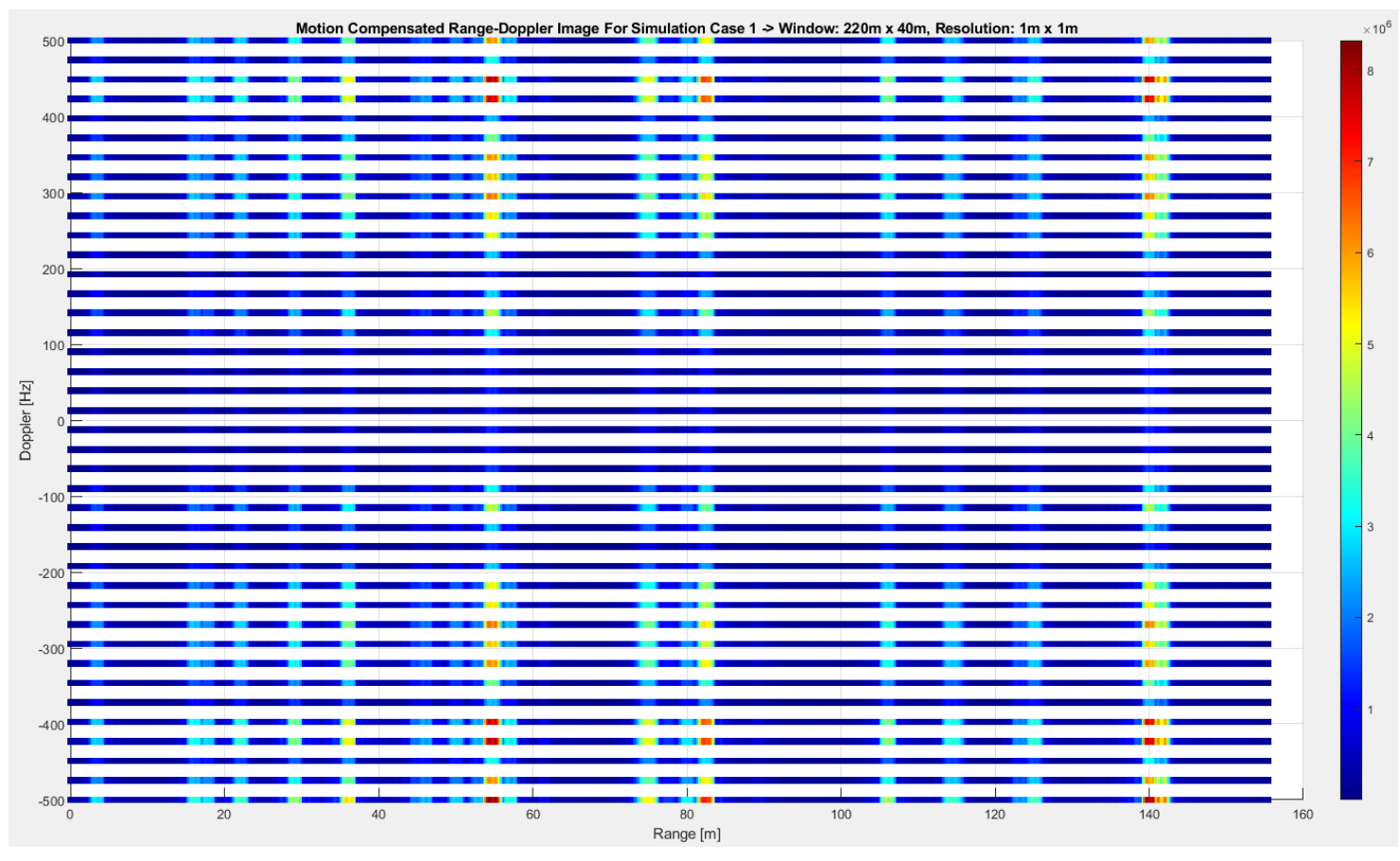

Figure 7.45: Motion compensated Range-Doppler image for Simulation case 1b.

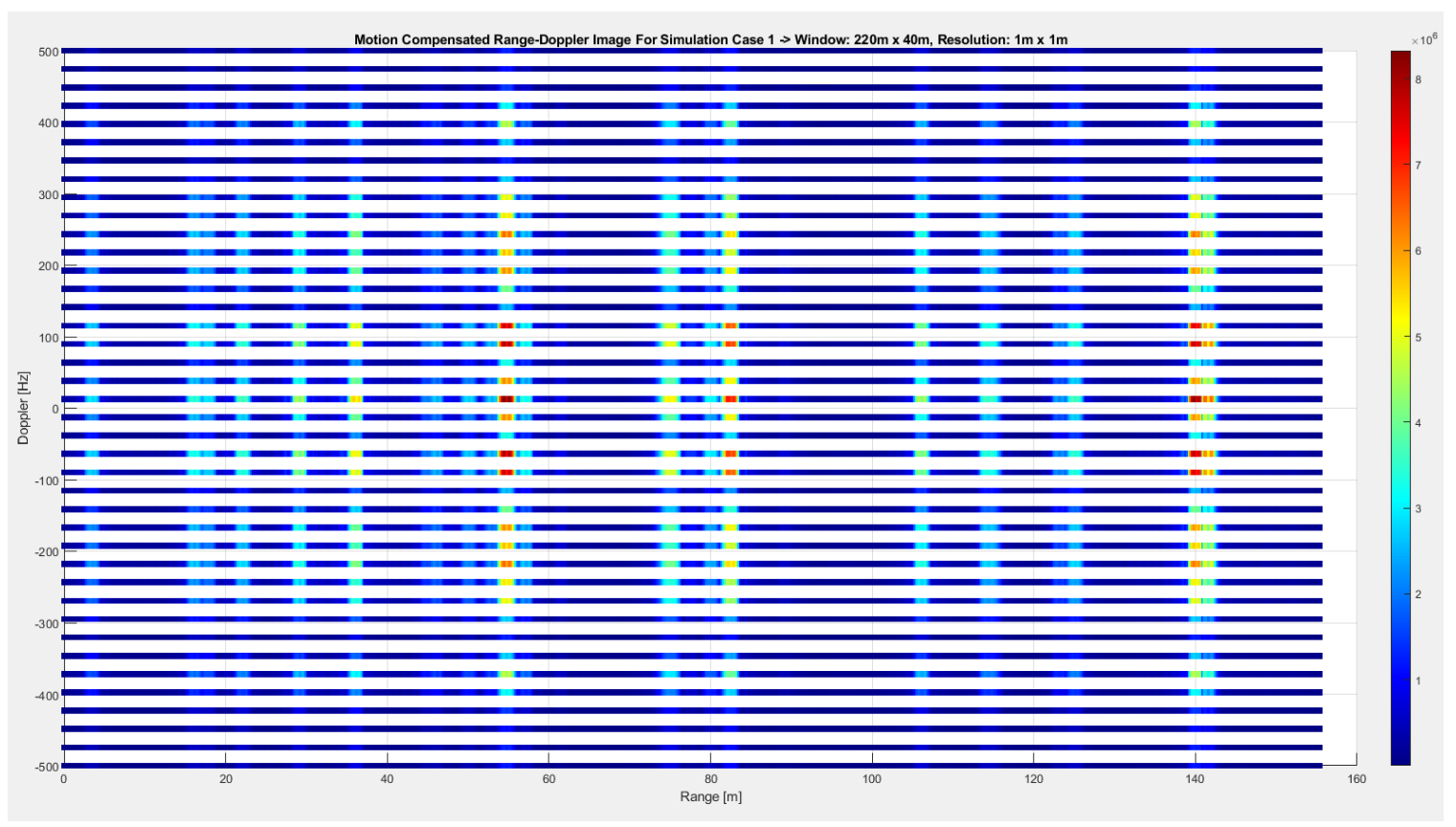

Figure 7.46: Range-Doppler image for case 1b, Doppler corrected by $500 \mathrm{~Hz}$. 


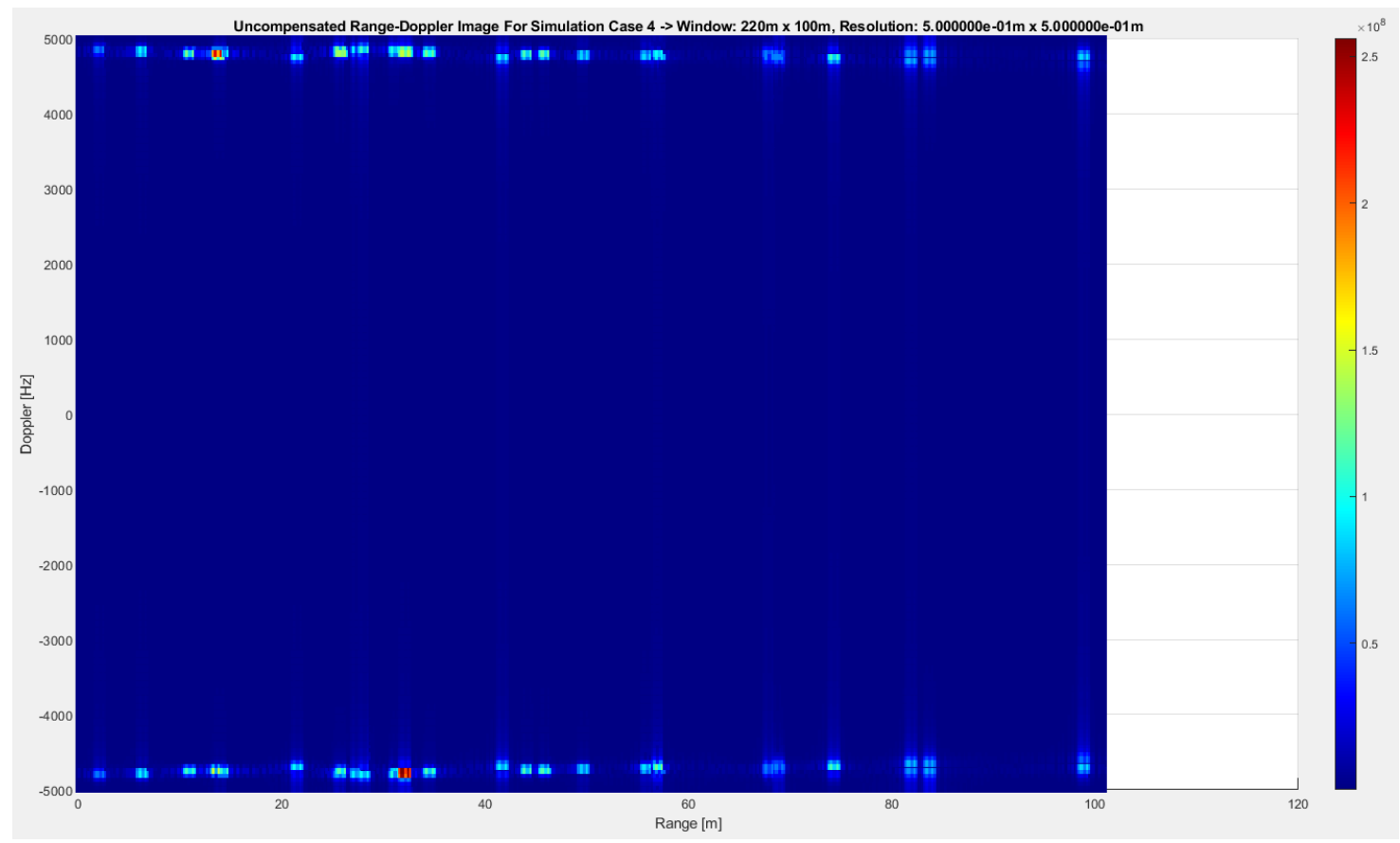

Figure 7.47: Uncompensated Range-Doppler image for Simulation case 4.

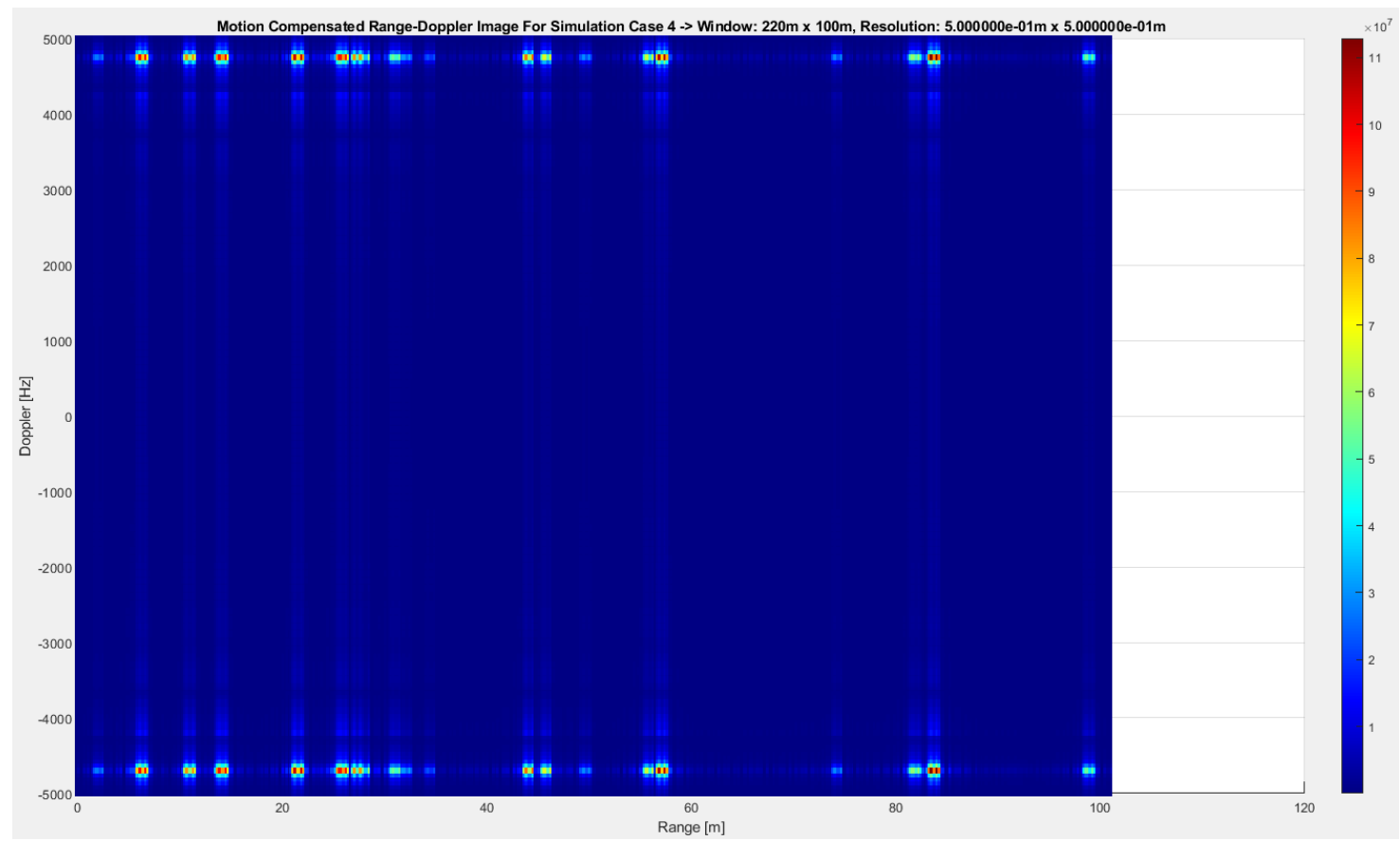

Figure 7.48: Motion compensated Range-Doppler image for Simulation case 4. 


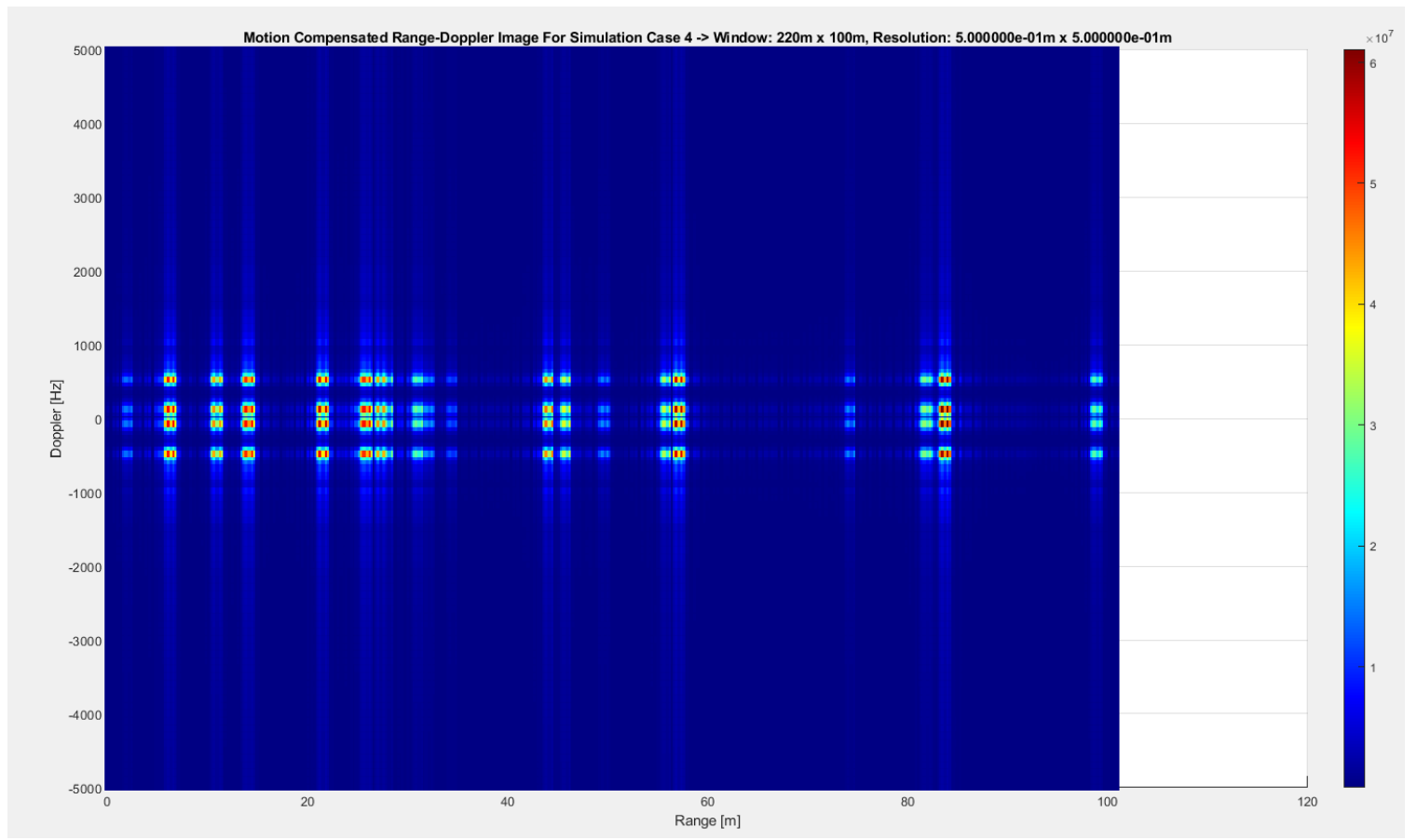

Figure 7.49: Range-Doppler image for case 4, Doppler corrected by $4800 \mathrm{~Hz}$.

The Range-Doppler images do not provide a clear image of the target for a specific pose. Rather they present the dominant scattering locations selected in the TSS program for synthesis of the target echo signal. After motion compensation significant spectral aliasing is observed in the image for both the stepped-frequency and chirp simulations. This is attributed to the use of the Doppler tracking algorithm implemented, specifically the Dominant-Scatterer Algorithm. As mentioned, this algorithm measures the variance in amplitude over the history of each range cell in the dataset. The phase variation across the history of the range cell which has the minimum amplitude variance determines the phase correction factor for each profile.

However, as mentioned, the focus of this research was to develop a program which implements a realistic motion model of the target. The amplitude of scattering locations, specifically the statistical RCS variations with frequency, and aspect angle are not included in the target model. This is a significant error source in the production of Range-Doppler images of the simulated target. The TSS program selects the largest amplitude within a resolution cell and assigns this value to the scattering location used to synthesize the target echo. 
Thus, as the target undergoes motion, and the scattering locations change after a decorrelation period the Doppler and phase of the scattering location change correspondingly. When multiple scattering locations are combined without the true amplitude and RCS statistics of the scattering locations accounted for the coherent combination of the modulation parameters results in an accumulation of error for each scattering location. Although the scattering location is amplitude modulated by the Doppler signal, the summation of significant amplitude responses from other scattering locations corrupts the Doppler modulation present from a single scattering location with amplitude noise from nearby scattering locations. The highly noisy dataset is then processed using a DFT operation which, even when windowed, cannot resolve a unique frequency for the range cell due to the amplitude inaccuracy over the range profile history.

Thus, through the research and development of the TSS program and digital modulation system as well as simulations in MATLAB, the results of this research are observed in the HRRPs and phase histories produced. The program and system design are shown to synthesize a target echo signal which can be used to counter wideband highresolution radar systems.

\subsection{Conclusion}

This chapter presented the simulation cases and results as programmed in MATLAB to test the motion algorithm, Target Signature Synthesis software, and the digital system design covered from chapters four to six. The complex range profile and phase histories are shown, as well as uncompensated and motion compensated RangeDoppler images for the simulation cases. Simulations were performed for two types of waveforms, stepped frequency and chirp, using the radar system parameters outlined in the beginning of chapter six. The next chapter will conclude this thesis and provide topics for future research and development to progress this work past a proof-of-concept to a practical system implementation. 


\section{Chapter 8: Conclusions and Contributions}

\subsection{Conclusion}

This thesis presented an innovative program developed in C\# which is capable of simulating realistic target motion to synthesize false target echo signals for countering wideband high-resolution radar systems. The target motion model can simulate ship motion in irregular sea conditions based on principles of Ocean Engineering and Naval Architecture which have been studied for decades. This motion model also provides the flexibility to simulate the motion of a variety of targets in various sea conditions if the appropriate ship response parameters and ocean wave parameters are input into the program.

An additional development as a part of this research is implementing a custom point matching algorithm that is used to generate target point cloud models. The algorithm provides the flexibility to control the number of data points included in the cloud which relates to the processing speed of the target signature synthesis program. The point cloud and the motion algorithm are collectively implemented in the Target Signature Synthesis program developed in C\#.

The Target Signature Synthesis software can be described as the primary focus of this research. The program is created to allow a user to define a target and motion scenario which will be used to create a target signature to implement deception jamming techniques towards hostile radars. The program is designed to compute the modulation parameters which are used in a digital modulation system which captures and modulates hostile radar signals to create false target echoes. These modulation parameters are output to a file which is used to communicate with the digital modulation system.

The final aspect of this thesis is designing the digital modulation system that would use the modulation parameters to synthesize the target echo signal. The system in theory would capture hostile wideband radar signals and apply the required modulation to create a target echo signal. Thus, this thesis presents a program which can create a target signature with emphasis on simulating realistic target motion and Doppler signatures of Naval targets. However, this is not a complete system, and future research and 
development is required in both software and hardware before this would be ready for industry.

\subsection{Future Work}

Further research to develop the Target Signature Synthesis system can be categorized into software and hardware development programs. In software development the primary research topic could be the inclusion of electromagnetic scattering physics in the target point cloud model. Specifically, the effects of shadowing, physical optics, radar cross-section statistics, and the inclusion of creeping wave and multipath effects in the target model would require significant research and development. An ideal scenario would be the design of algorithms based on ray-tracing or physical optics which would be applicable to a wide variety of target models rather than a program which customizes the point cloud model for each unique target.

The hardware development of the digital modulation system is also a key area of research. Since the application of this program would require high-speed and almost realtime processing research would be focused on the use of digital RF memory (DRFM) systems. Future work would have to evaluate the errors involved in applying modulation at high speeds, and optimization algorithms to reduce the total number of computations required to synthesize the target echo signal. Also, an investigation into the effectiveness of the simulated target model with varying resolution, as an increased resolution would require more computations which results in increased system latency. Thus, research into optimization of the speed of the hardware target synthesis system in conjunction with the resolution of the synthesized target echo can provide insight into potential countermeasures which would be used against this system. These countermeasures involve identifying patterns within the synthesized target echo that a hostile radar may exploit to differentiate between real and false targets. Therefore, there is still a significant amount of research required to develop a full system. This would require contribution from many specialized engineers and technicians to help this system achieve its full potential. 
This research can also be integrated with existing research in false target generation systems. This can enhance the existing research which focuses on unique aspects of target modelling such as appropriate definition of the EM signature of targets, inclusion of micro-Doppler effects or synthesizing multiple false targets echo signals simultaneously. This research provides an algorithm for motion simulation which can be ported to other languages besides $\mathrm{C} \#$ for use in a variety of existing research applications. 


\section{Appendices}

\section{Appendix A}

In this Appendix the screenshots of a ship target undergoing motion as programmed using the motion algorithm in Chapter 4 are shown. The motion is complex and is shown from two perspectives with different motion conditions to verify the effectiveness of the algorithm. The motion of the targets is shown over a total 15 second observation period, with snapshots taken at every 1 second interval.

The first data set is shown in Sub-Appendix 1, where the broadside profile of the target is shown such that the ship can be seen to have pitching and heave motion characteristics. The motion conditions for the data in Sup-Appendix 1 is Sea State 4, a wave heading of 0 degrees relative to the ship stern, and a ship velocity of 5 knots.

The second data set is shown in Sub-Appendix 2. In these screenshots, the ship is shown from the bow aspect, so the ship's roll and sway motions can be clearly seen as the motion algorithm simulates the target motion over 15 seconds. The motion conditions here are Sea State 6, the wave heading is 90 degrees relative to the stern. This means the waves are incident directly on the broadside of the ship, and the ship velocity is 5 knots. 


\section{A.1 Sub-Appendix}

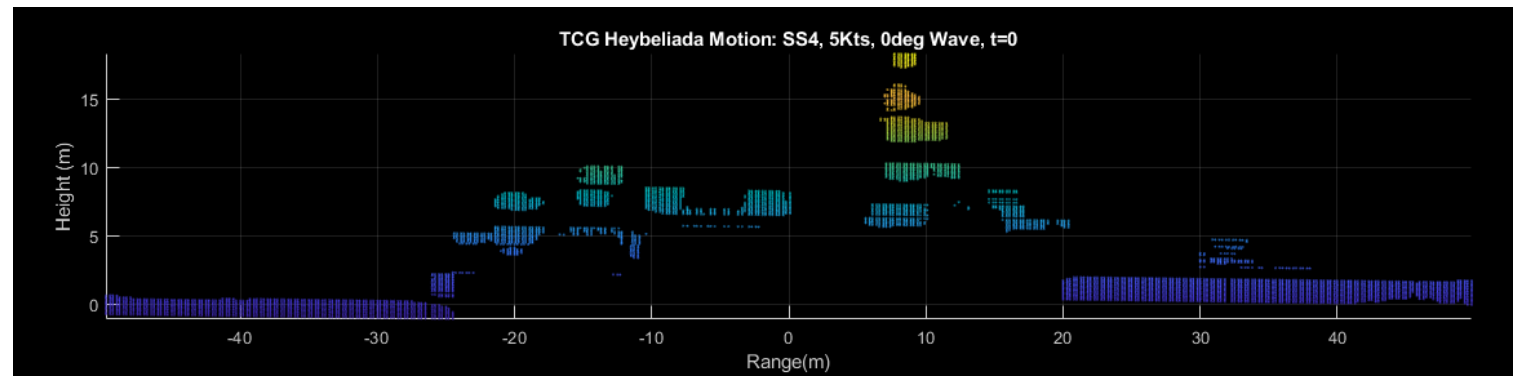

Figure A-1. 1: Target Pitch and Heave Motion, $t=0$ second.

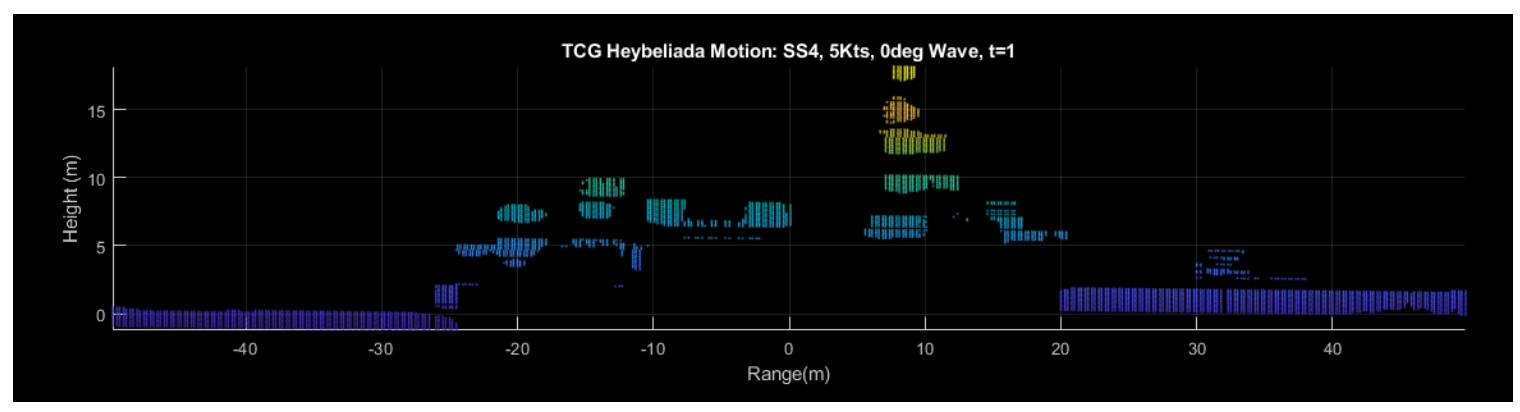

Figure A-1. 2: Target Pitch and Heave Motion, $t=1$ second.

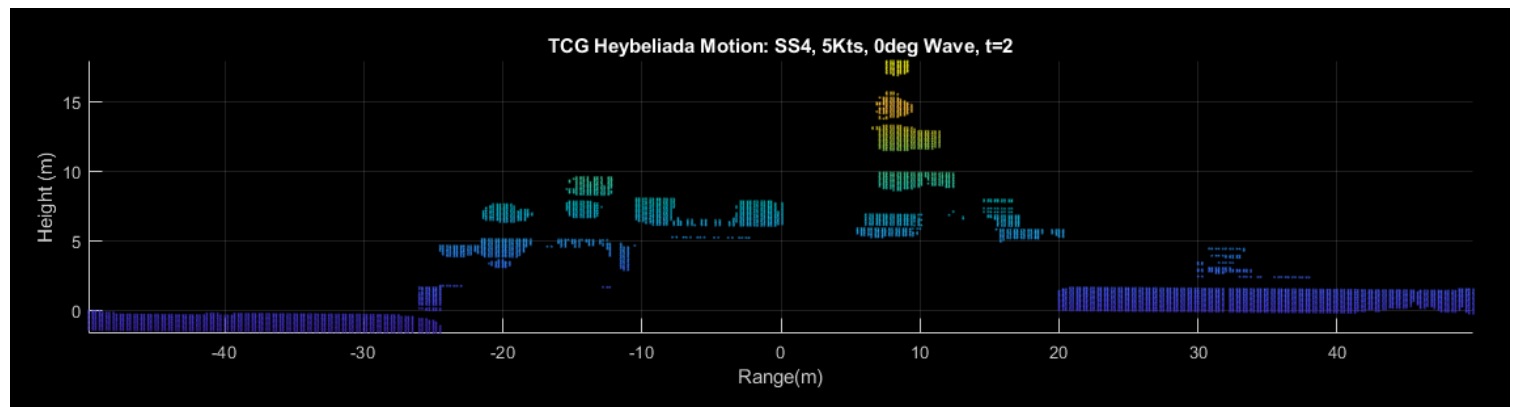

Figure A-1. 3: Target Pitch and Heave Motion, $t=2$ second.

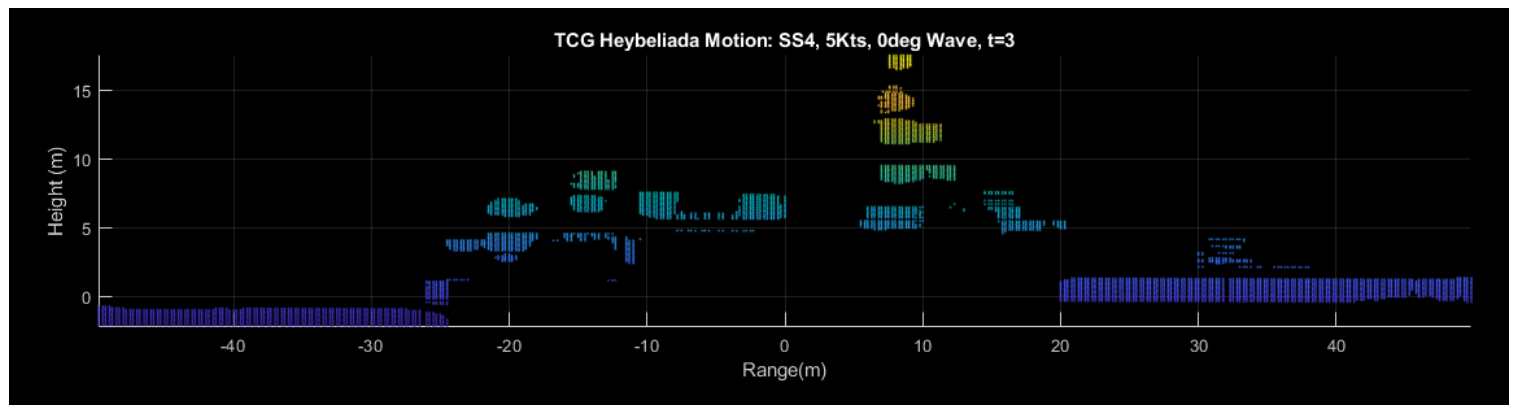

Figure A-1. 4: Target Pitch and Heave Motion, $t=3$ second. 


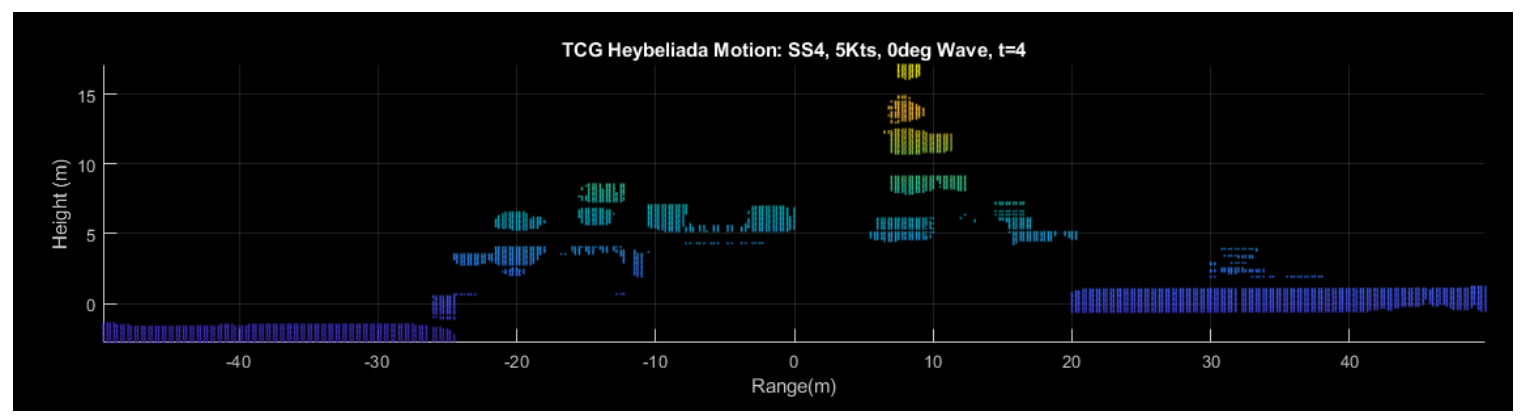

Figure A-1. 5: Target Pitch and Heave Motion, $t=4$ second.

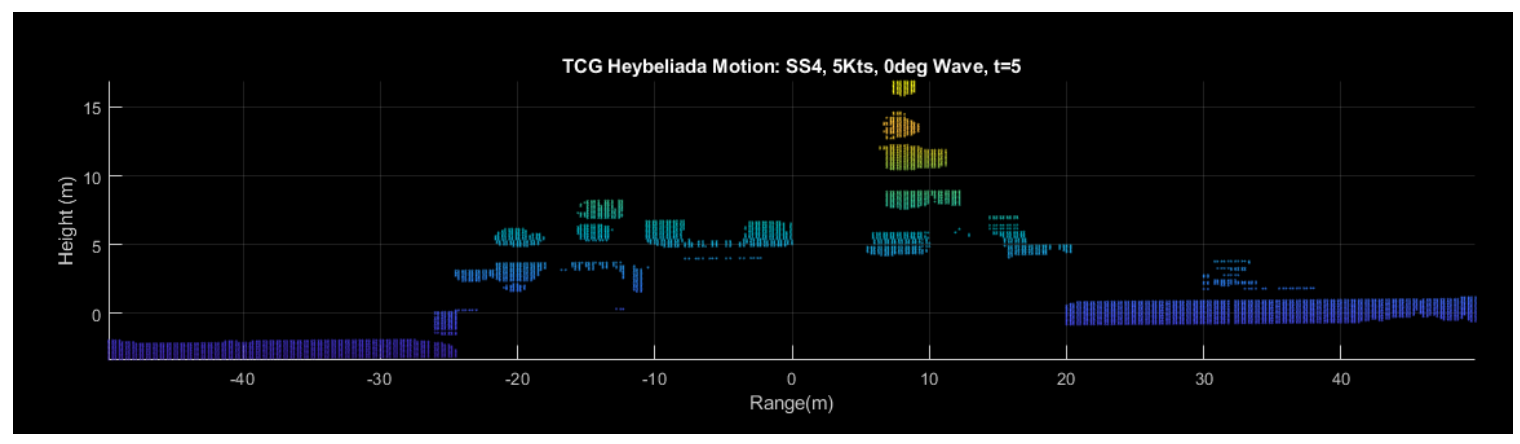

Figure A-1. 6: Target Pitch and Heave Motion, $t=5$ second.

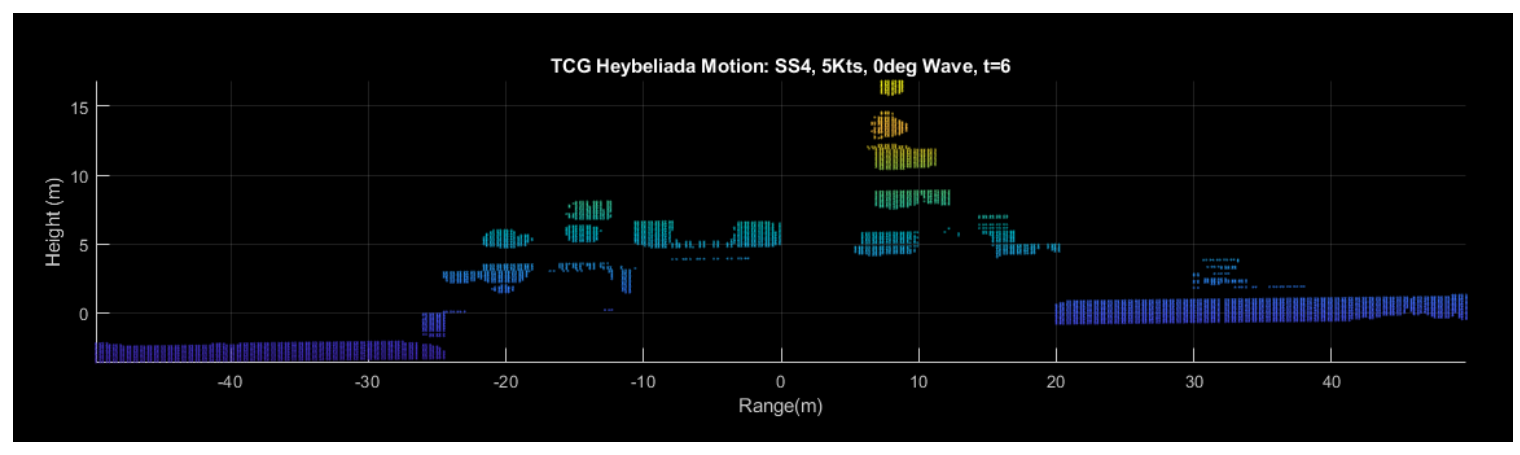

Figure A-1. 7: Target Pitch and Heave Motion, $t=6$ second.

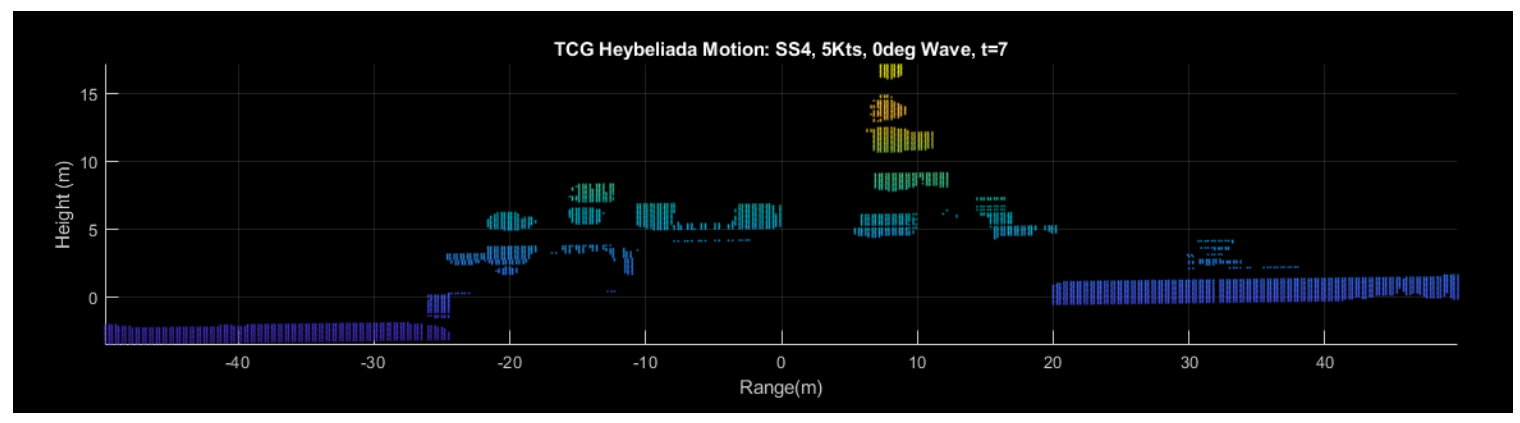

Figure A-1. 8: Target Pitch and Heave Motion, $t=7$ second. 


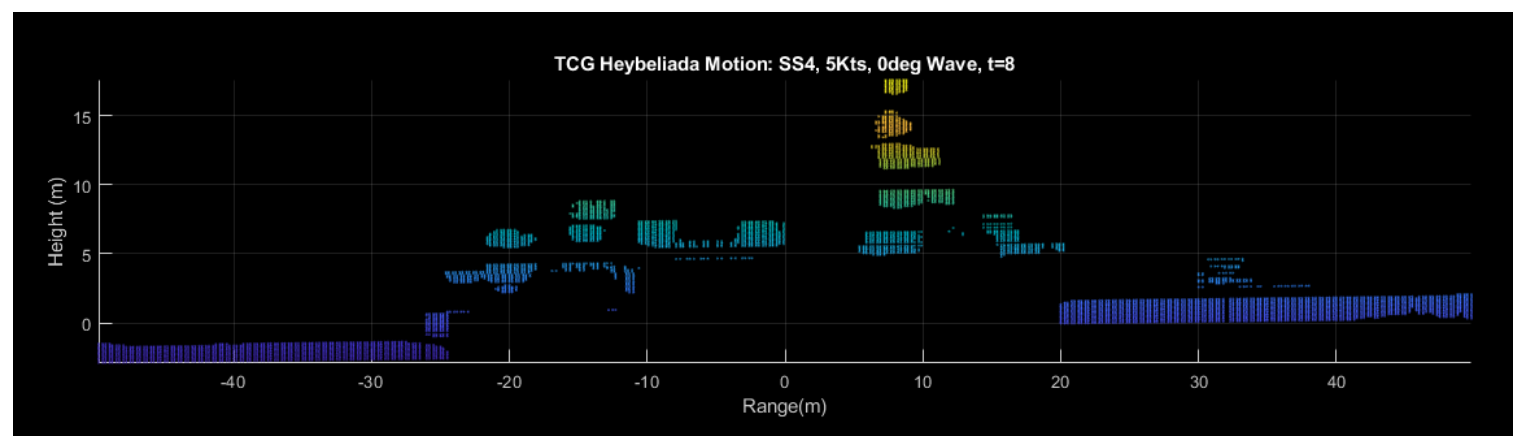

Figure A-1. 9: Target Pitch and Heave Motion, $t=8$ second.

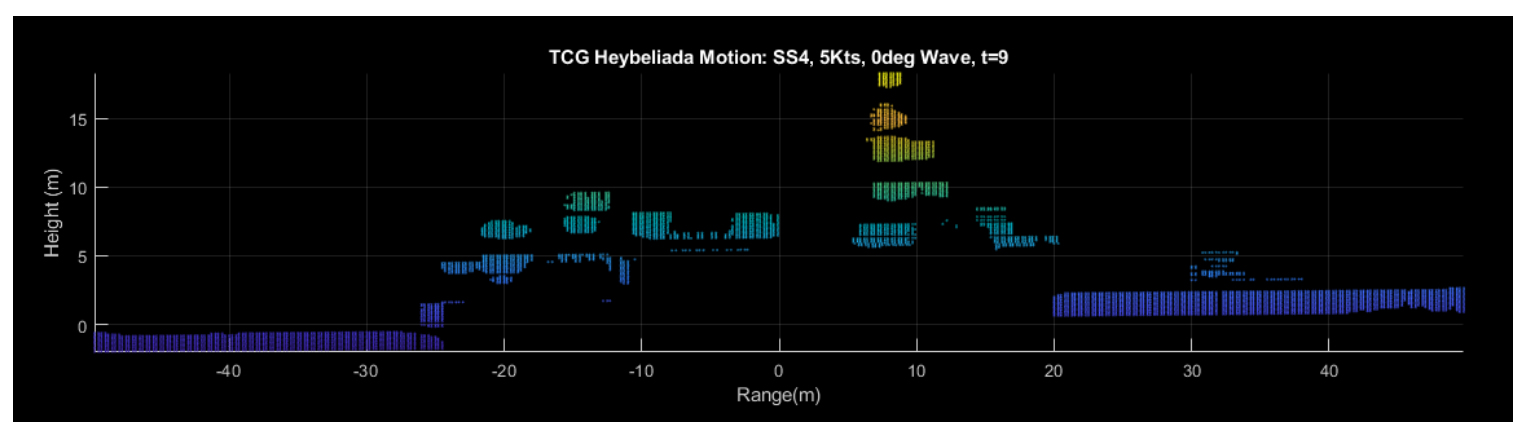

Figure A-1. 10: Target Pitch and Heave Motion, $t=9$ second.

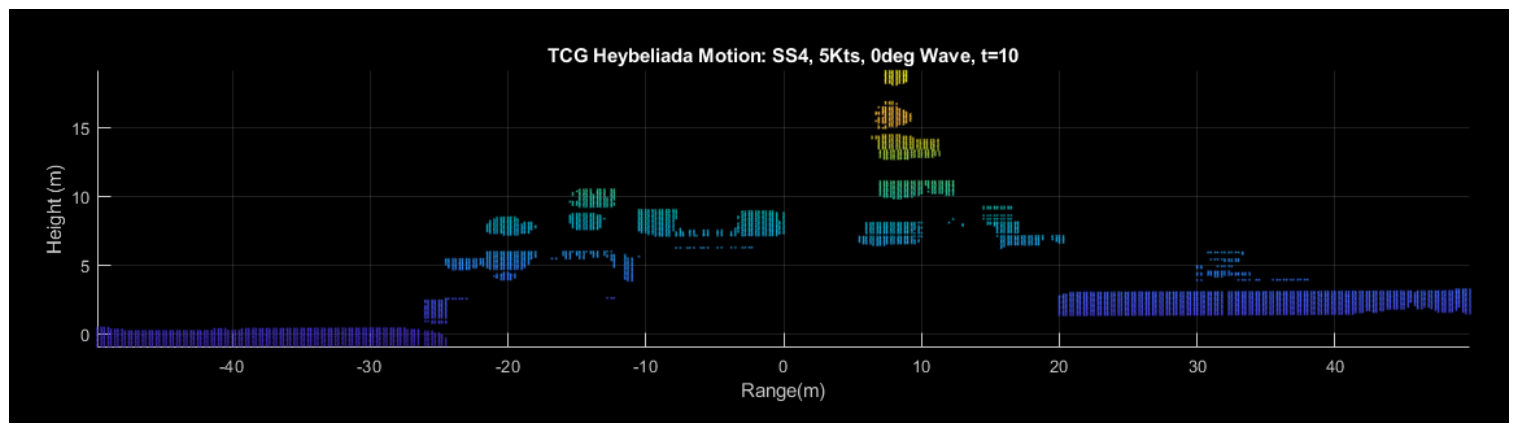

Figure A-1. 11: Target Pitch and Heave Motion, $t=10$ second.

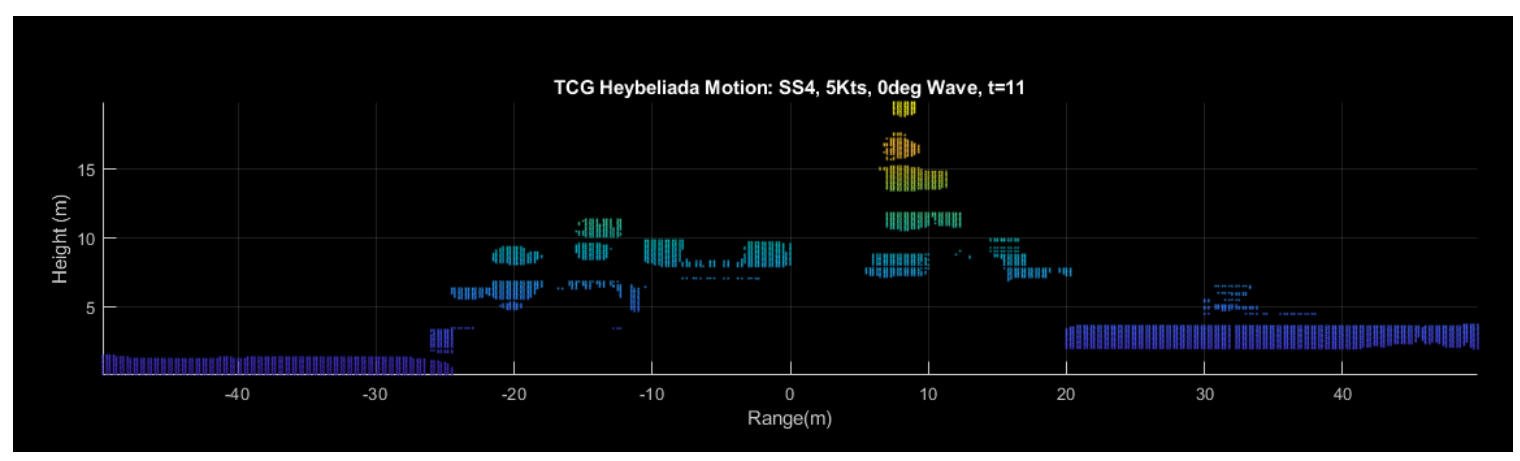

Figure A-1. 12: Target Pitch and Heave Motion, $t=11$ second. 


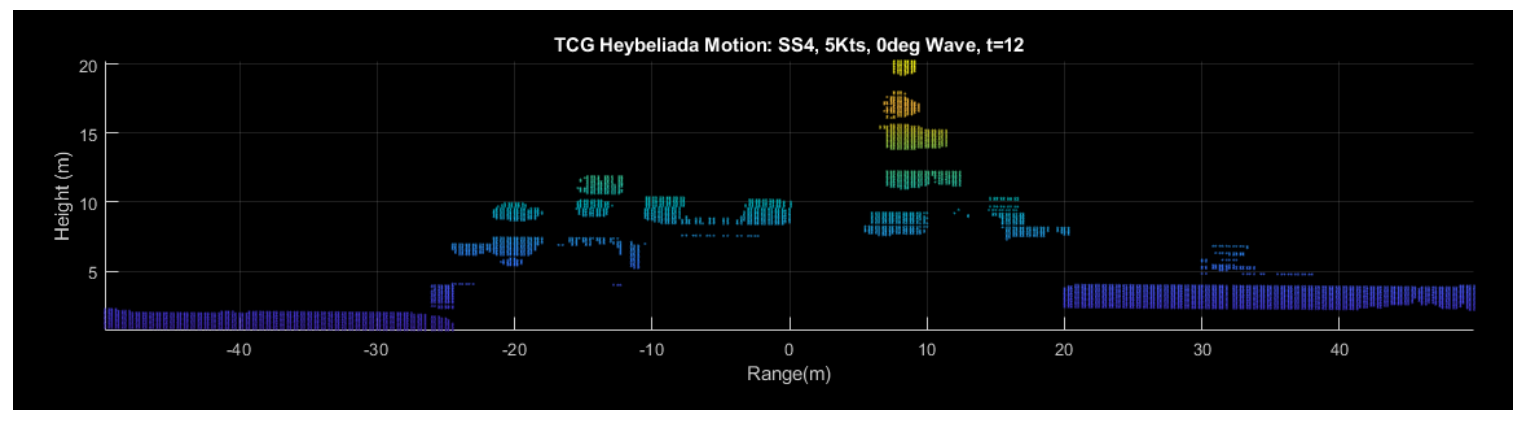

Figure A-1. 13: Target Pitch and Heave Motion, $t=12$ second.

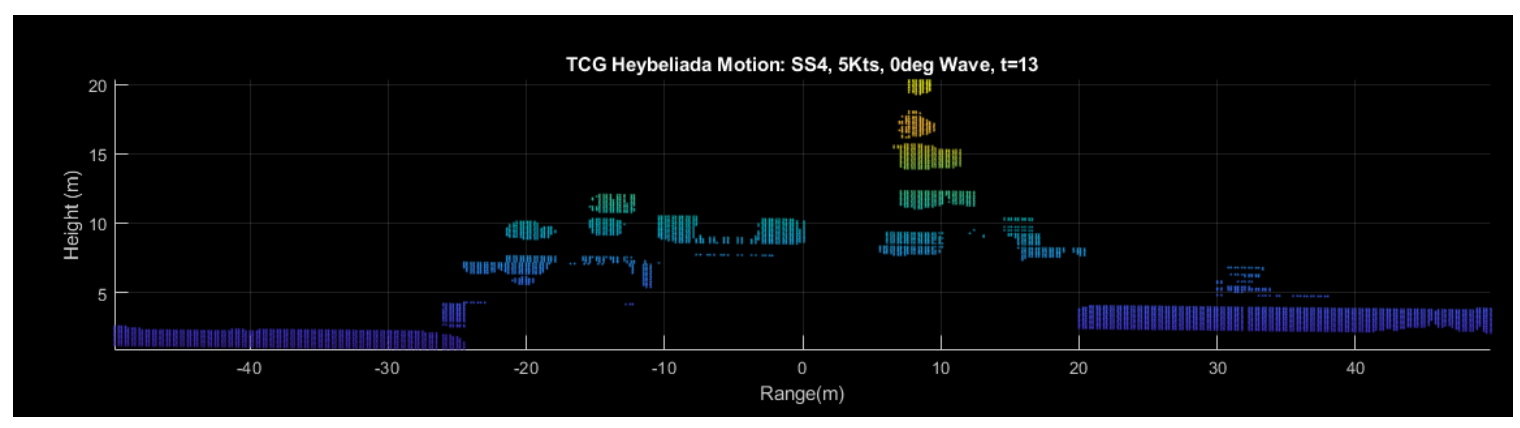

Figure A-1. 14: Target Pitch and Heave Motion, $t=13$ second.

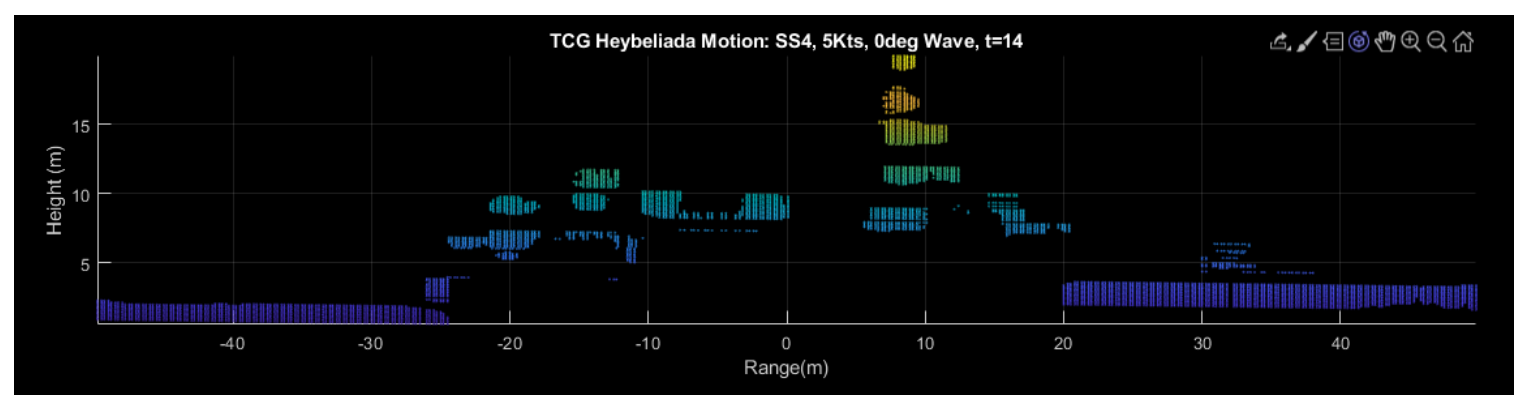

Figure A-1. 15: Target Pitch and Heave Motion, $t=14$ second.

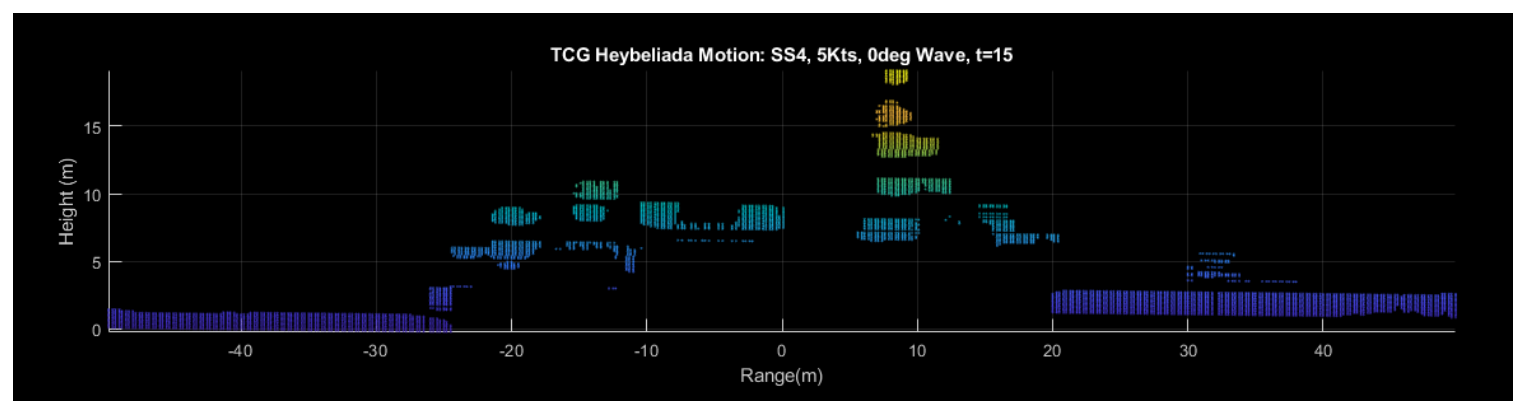

Figure A-1. 16: Target Pitch and Heave Motion, $t=15$ second. 


\section{A.2 Sub-Appendix}

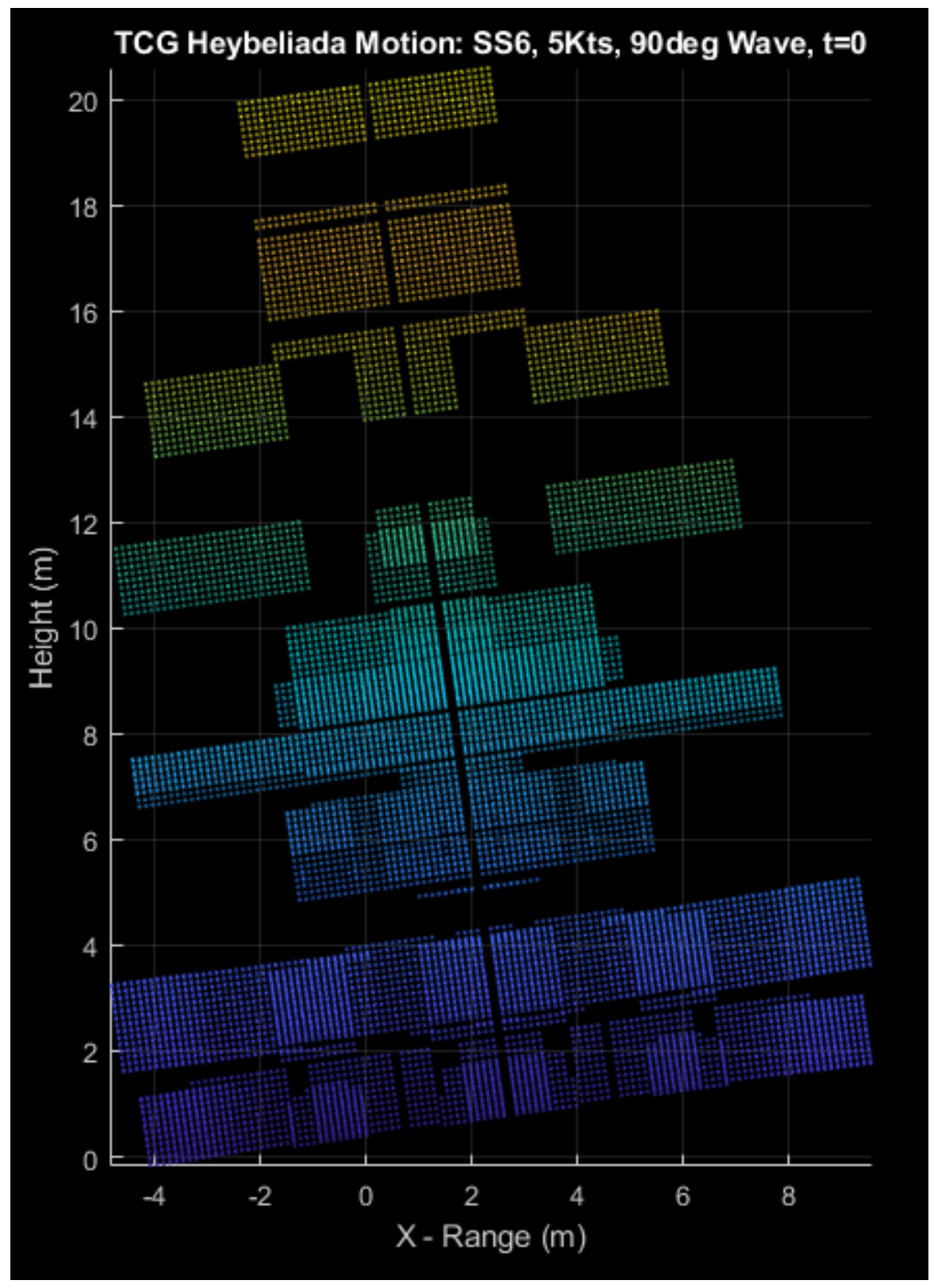

Figure A-2. 1: Target Roll \& Sway Motion, $t=0$ seconds. 


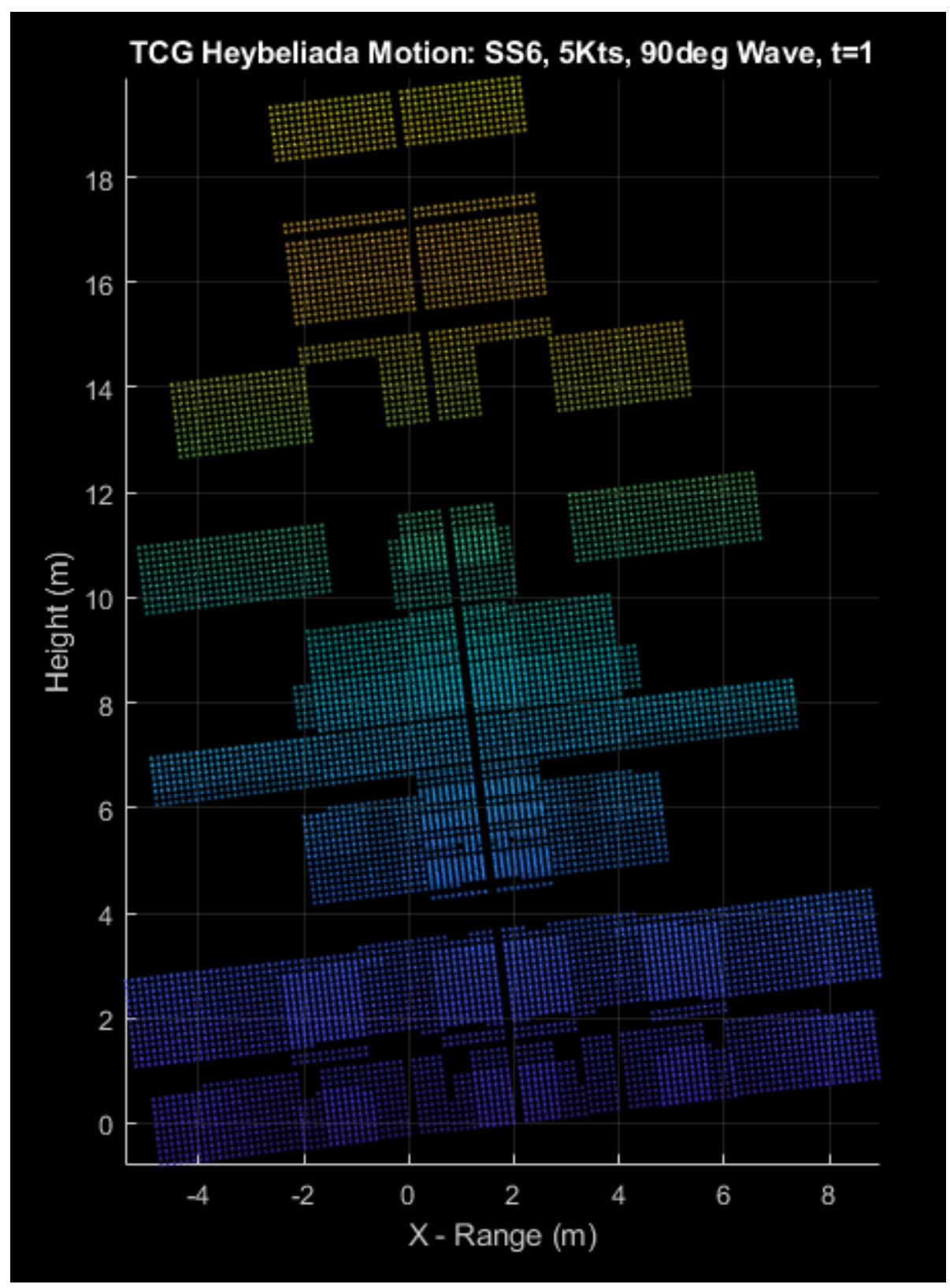

Figure A-2. 2: Target Roll \& Sway Motion, $t=1$ seconds. 


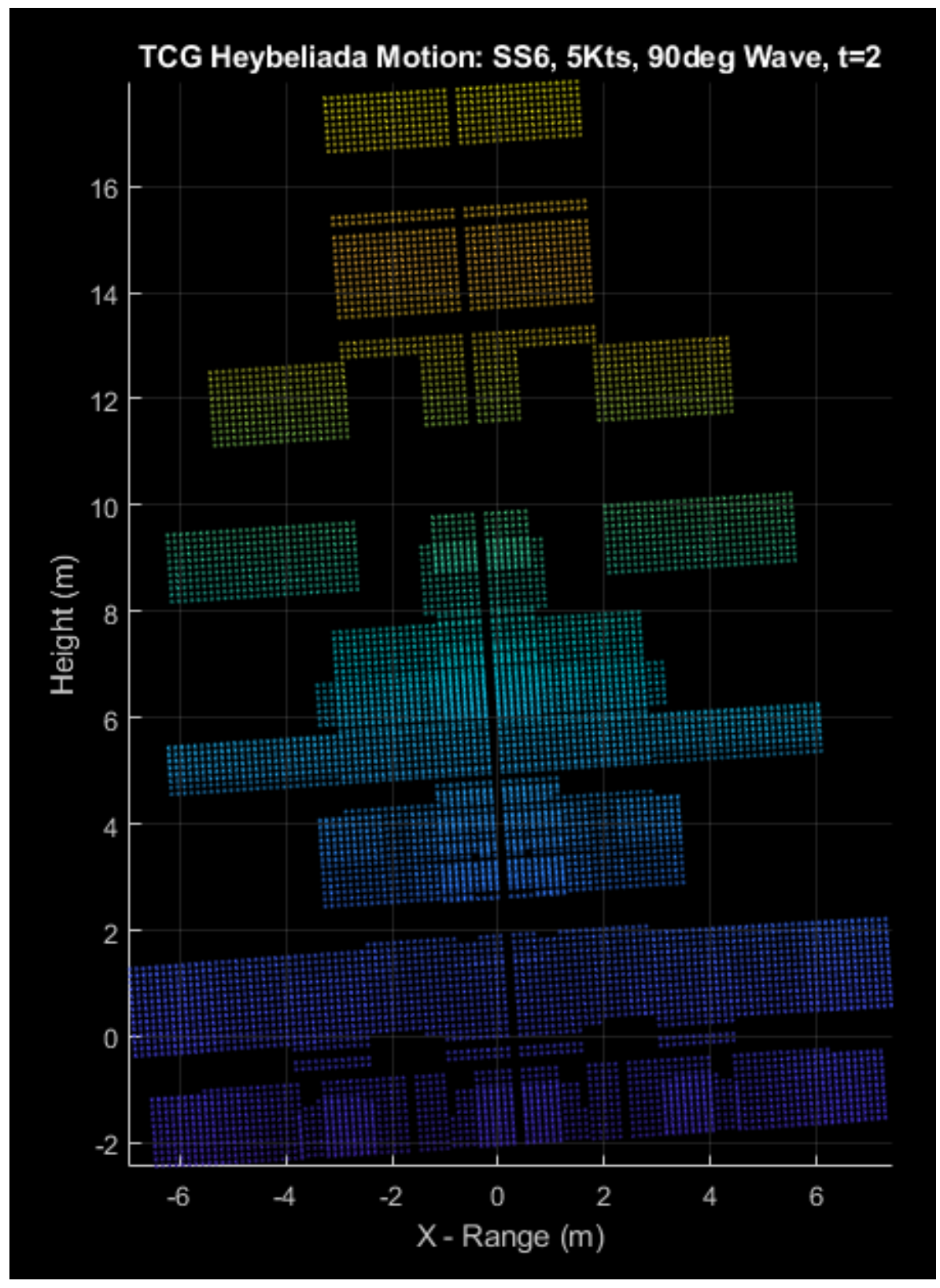

Figure A-2. 3: Target Roll \& Sway Motion, $t=2$ seconds. 


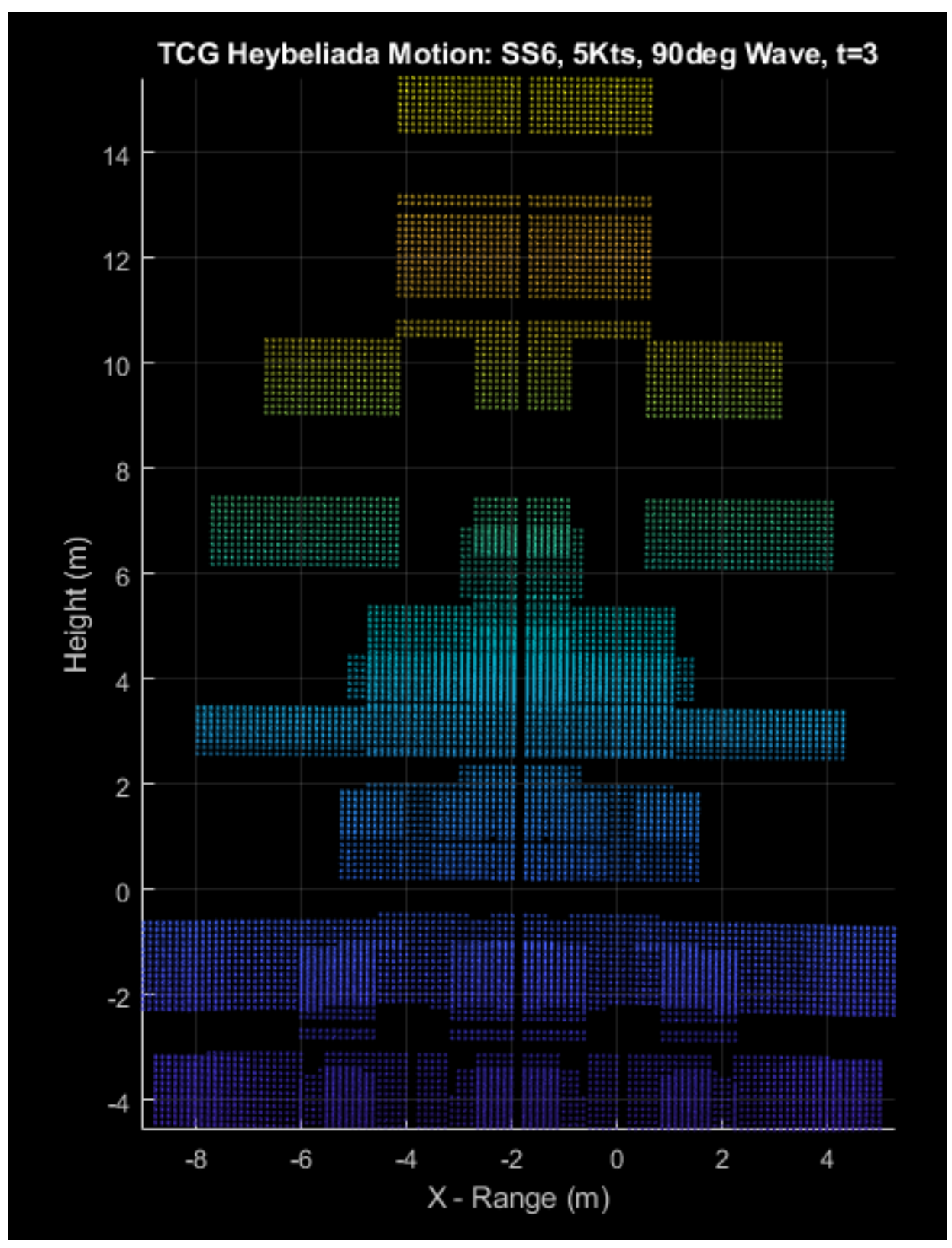

Figure A-2. 4: Target Roll \& Sway Motion, $t=3$ seconds. 


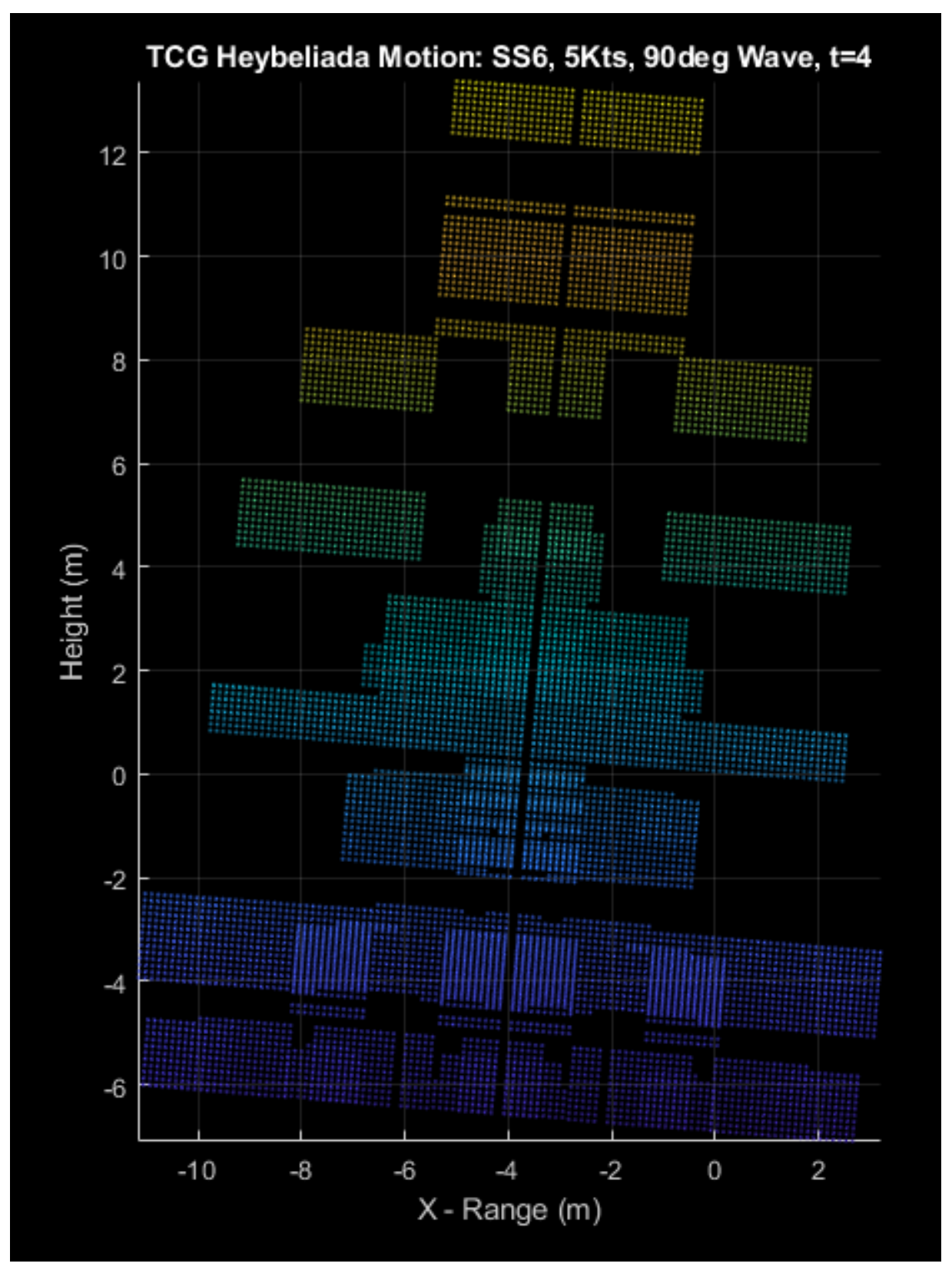

Figure A-2. 5: Target Roll \& Sway Motion, $t=4$ seconds. 


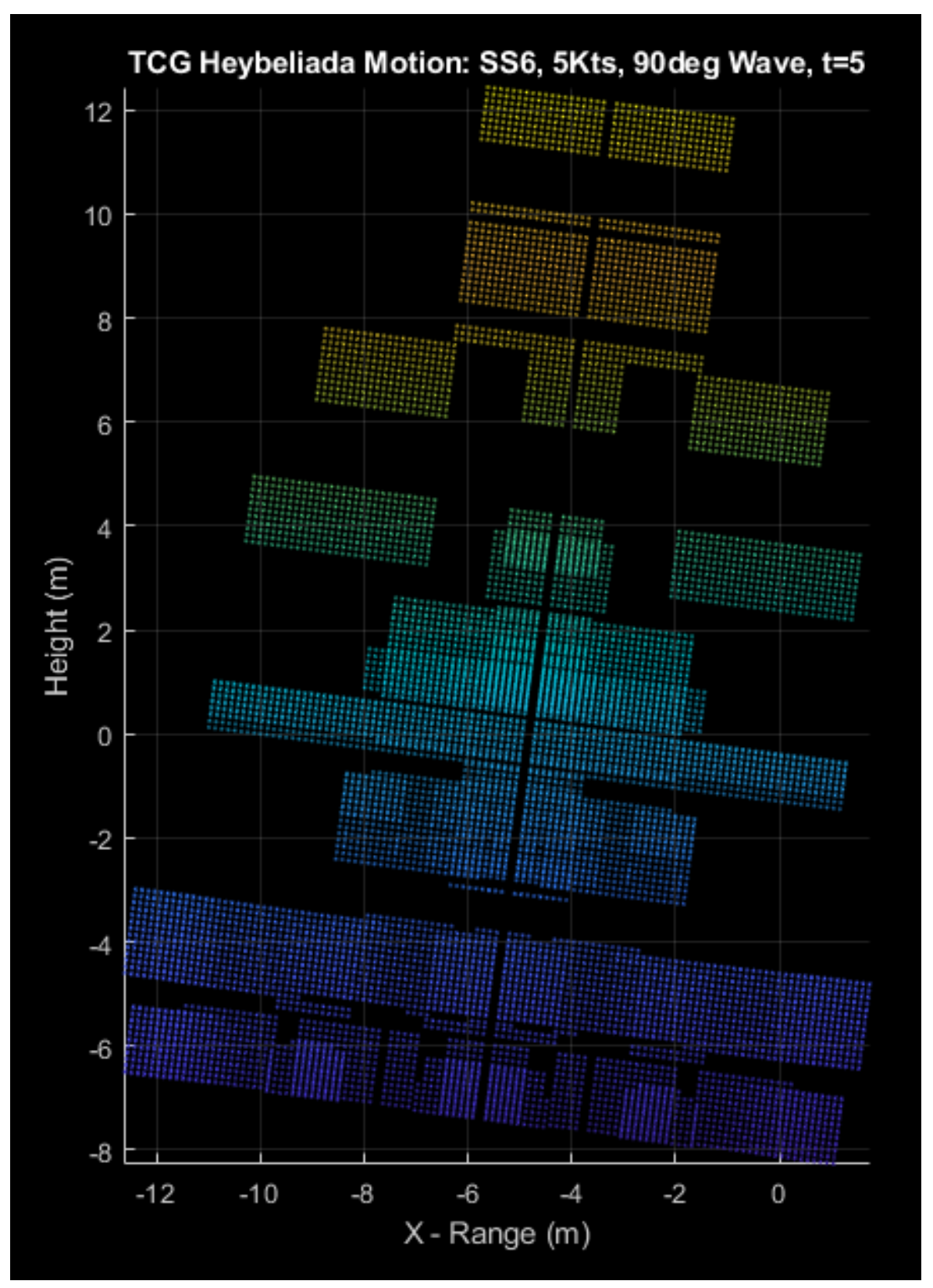

Figure A-2. 6: Target Roll \& Sway Motion, $t=5$ seconds. 


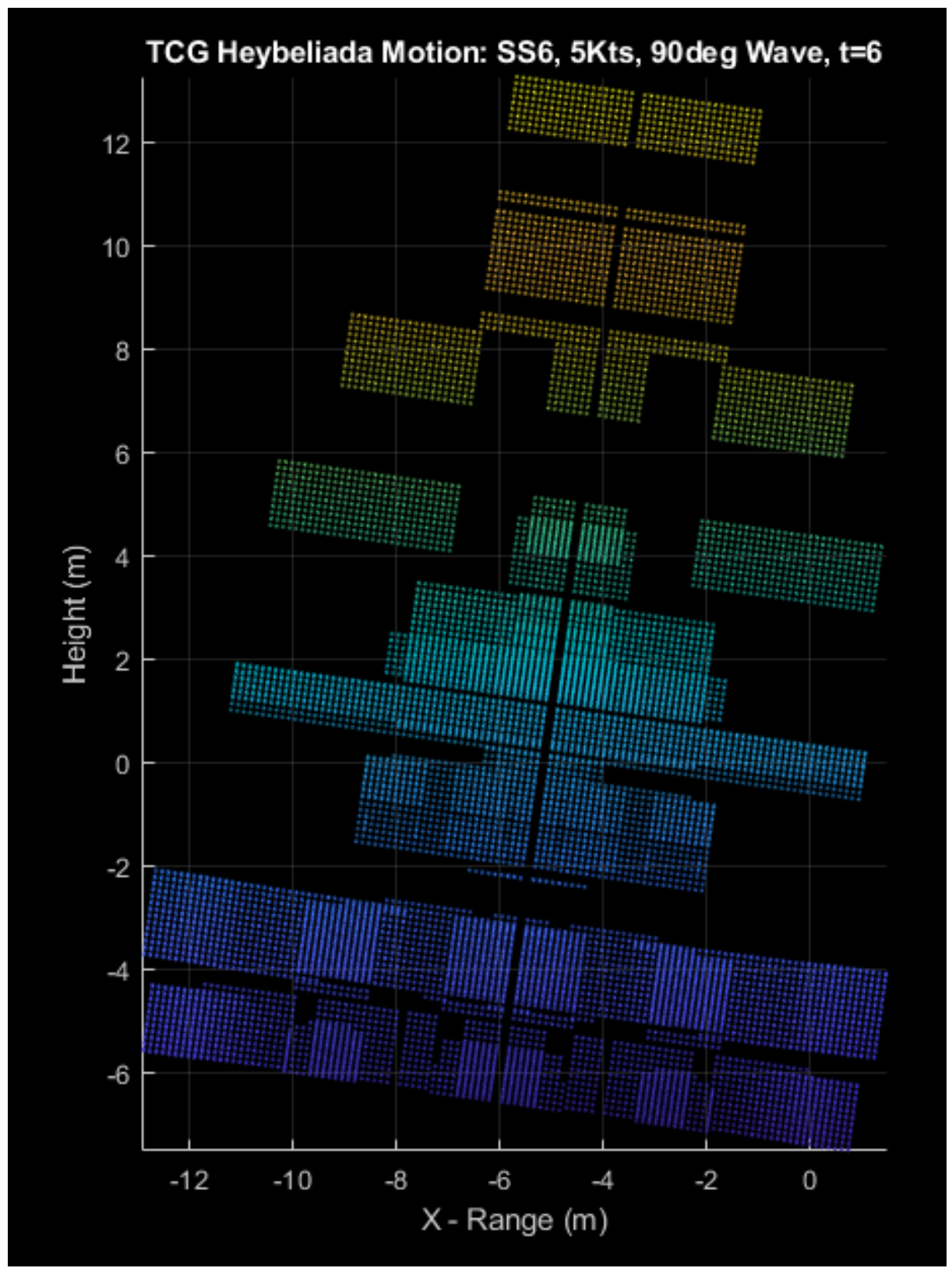

Figure A-2. 7: Target Roll \& Sway Motion, $t=6$ seconds. 


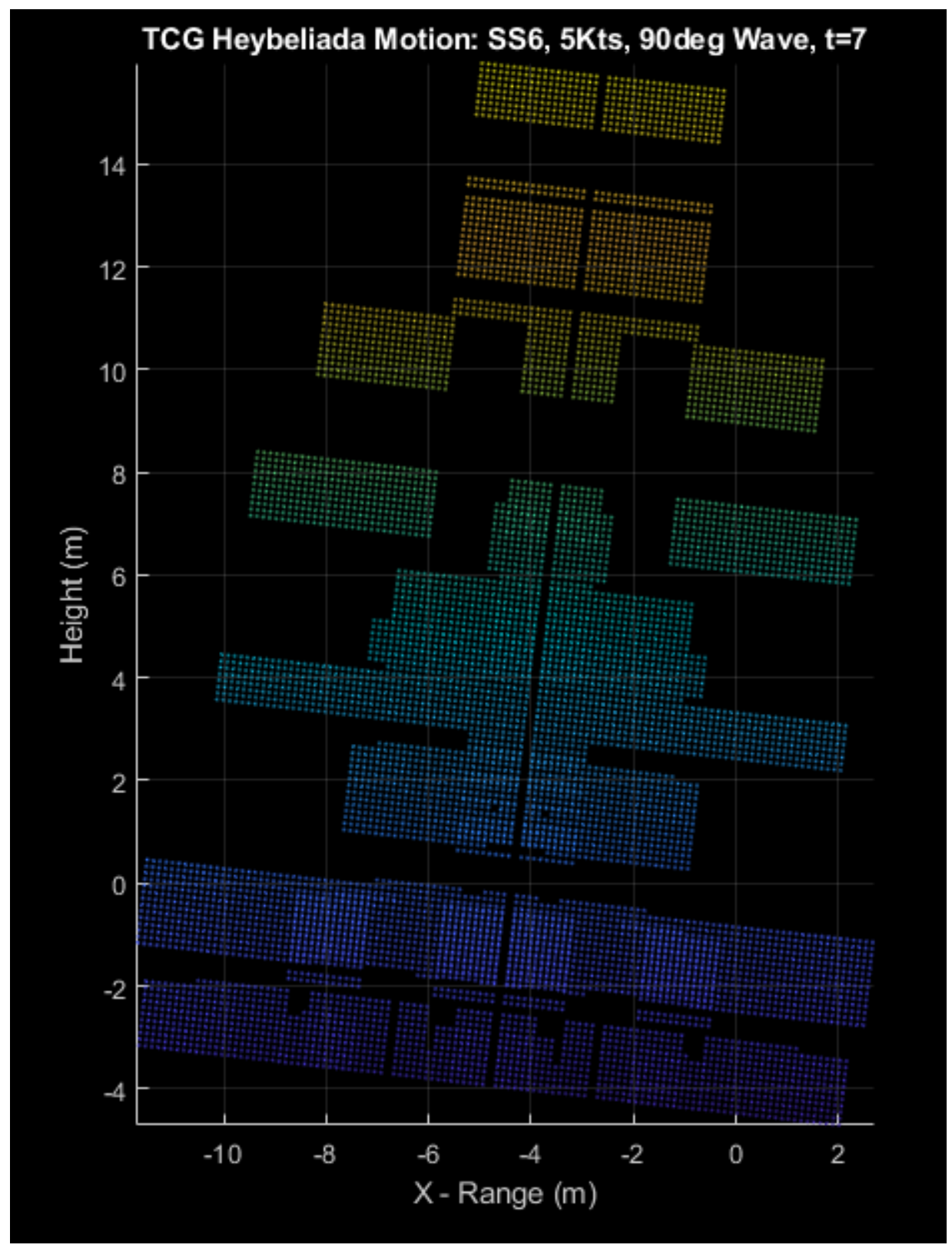

Figure A-2. 8: Target Roll \& Sway Motion, $t=7$ seconds. 


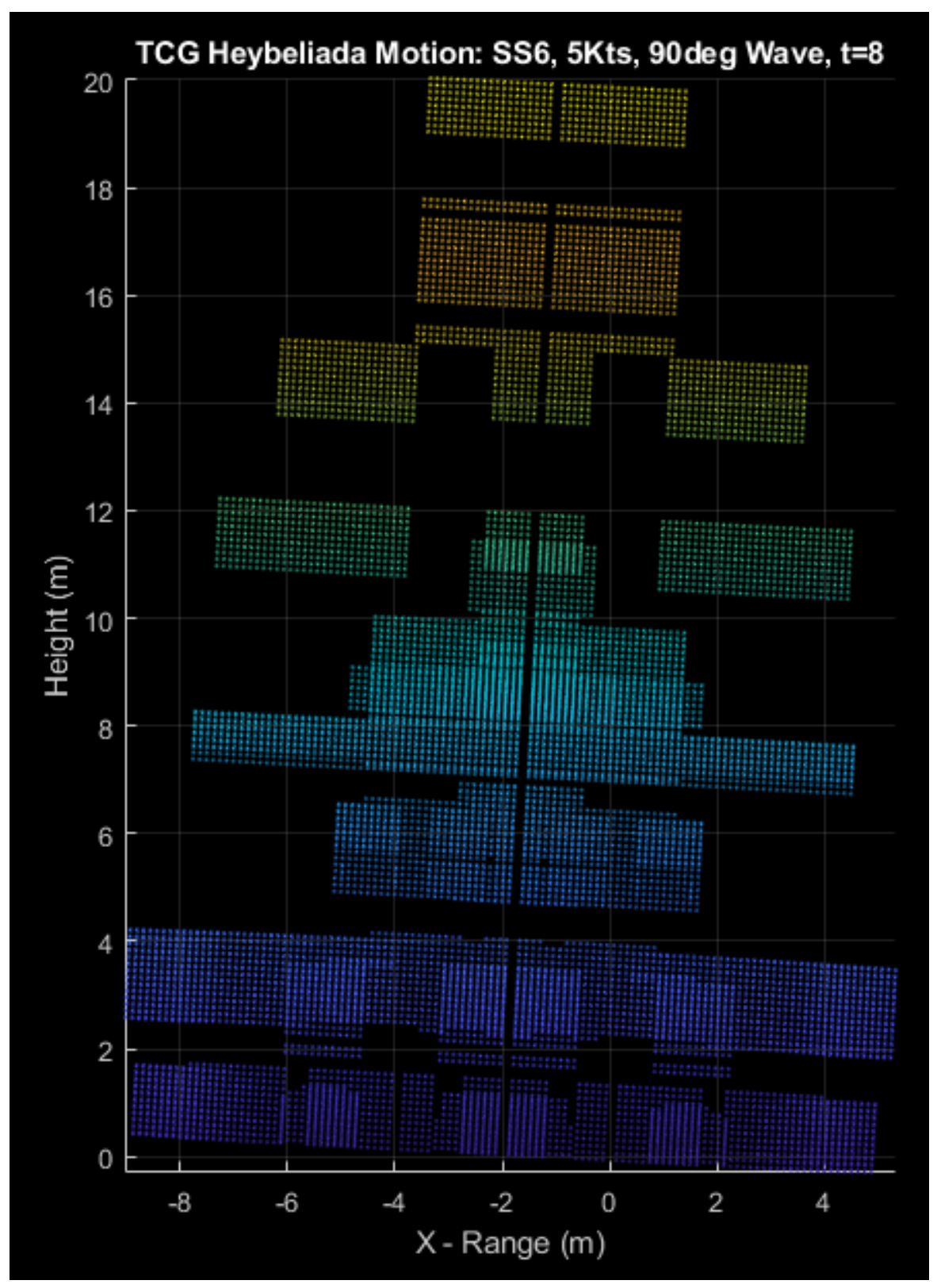

Figure A-2. 9: Target Roll \& Sway Motion, $t=8$ seconds. 


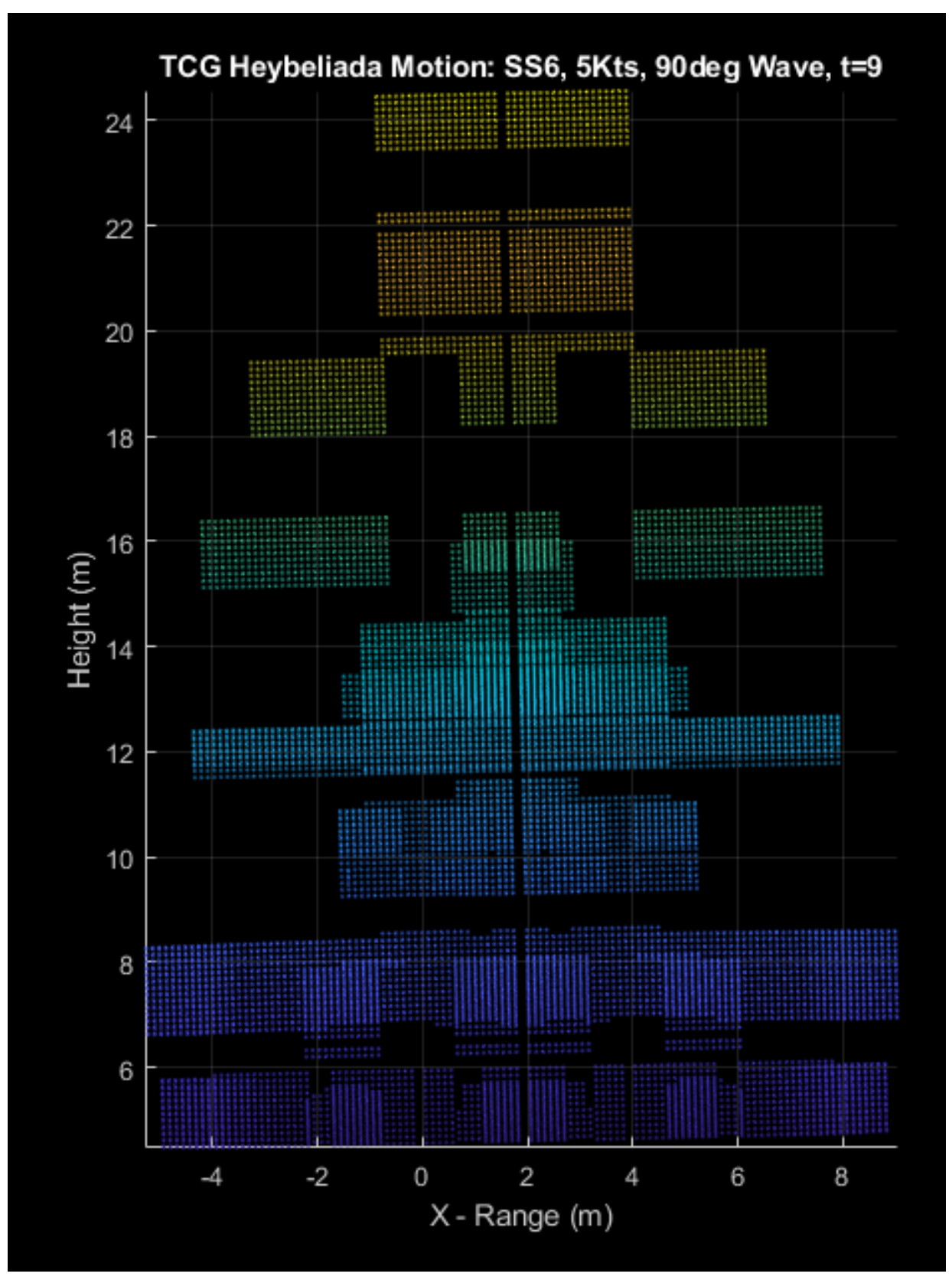

Figure A-2. 10: Target Roll \& Sway Motion, $t=9$ seconds. 


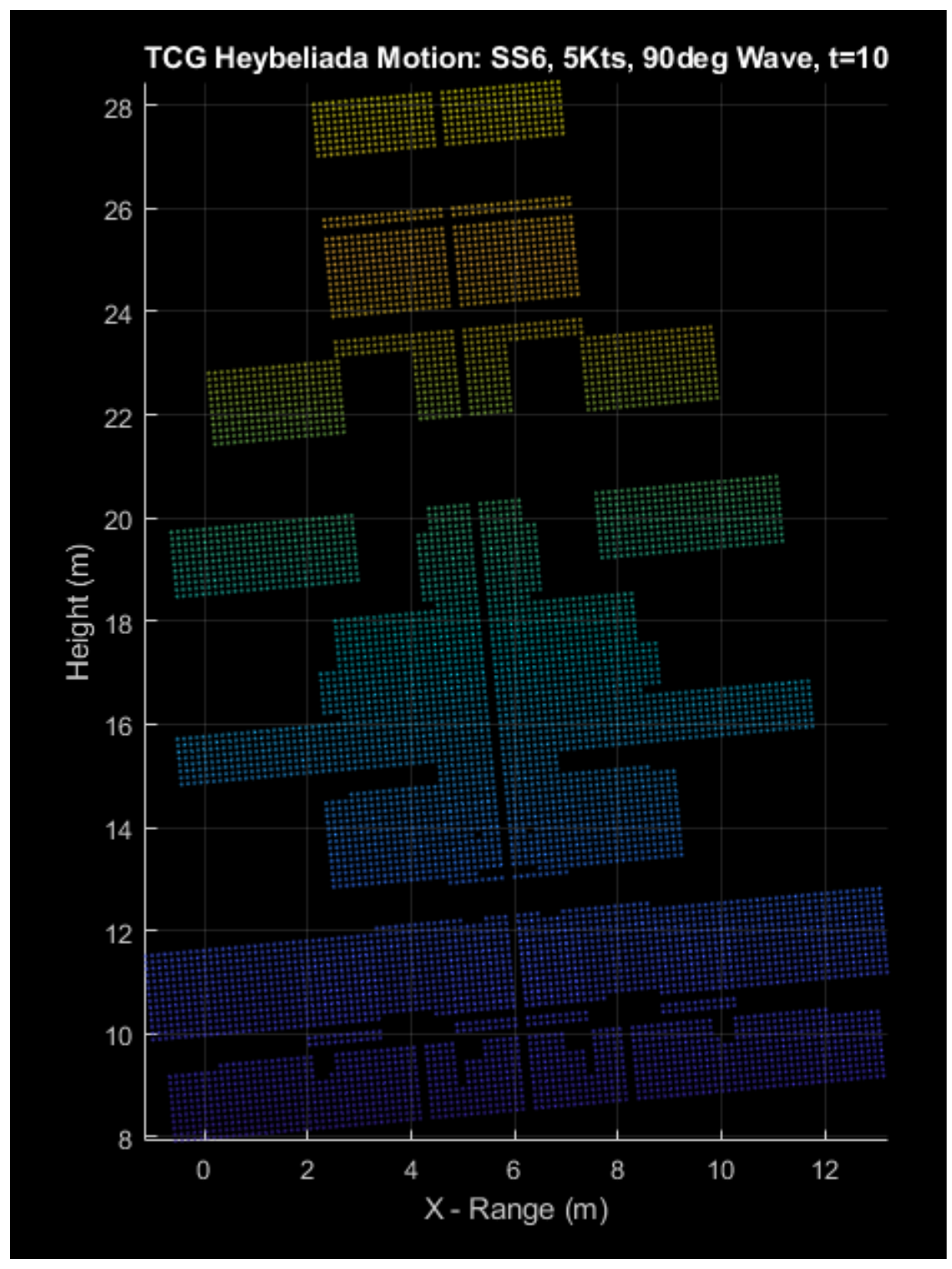

Figure A-2. 11: Target Roll \& Sway Motion, $t=10$ seconds. 


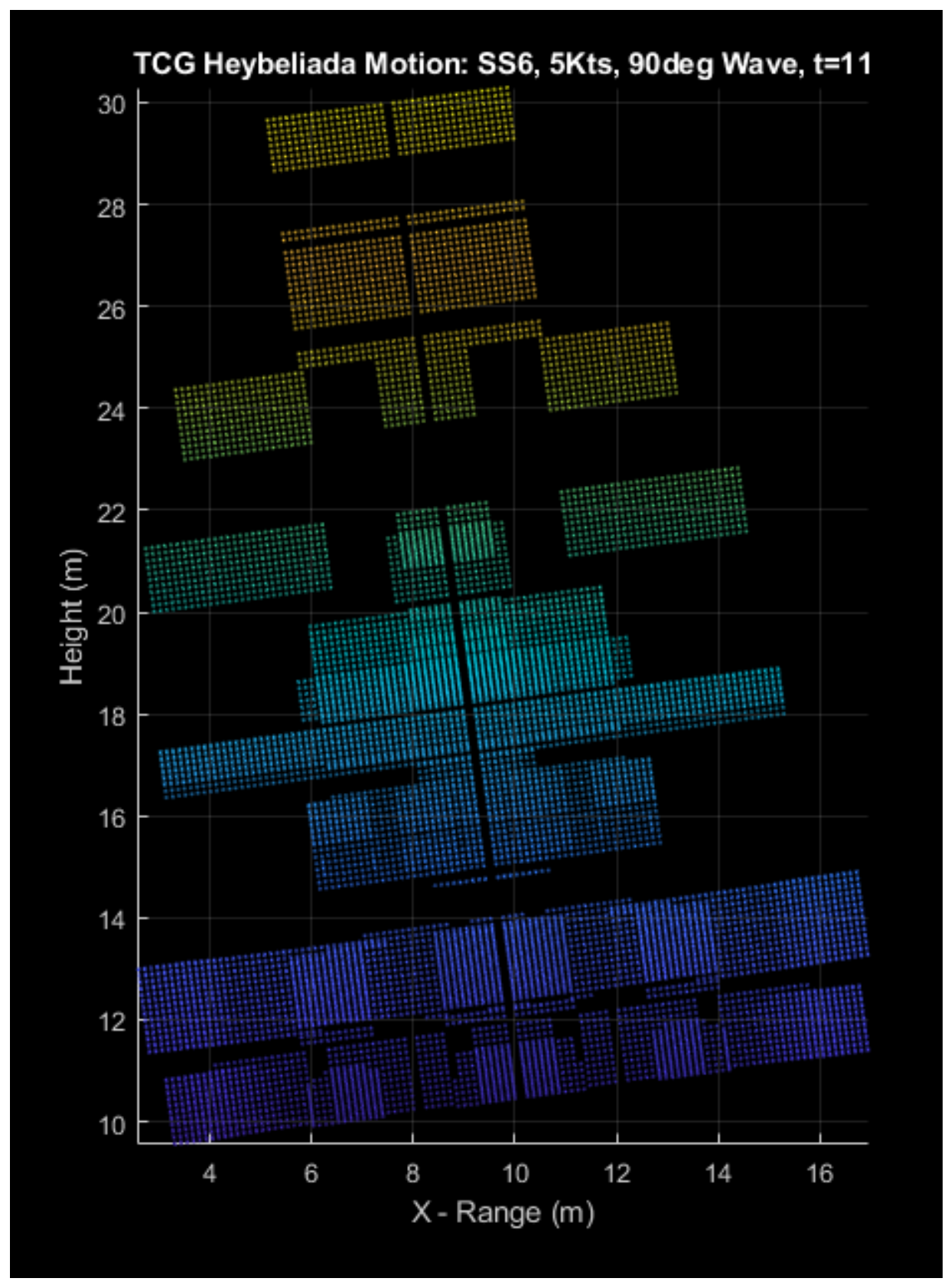

Figure A-2. 12: Target Roll \& Sway Motion, $t=11$ seconds. 


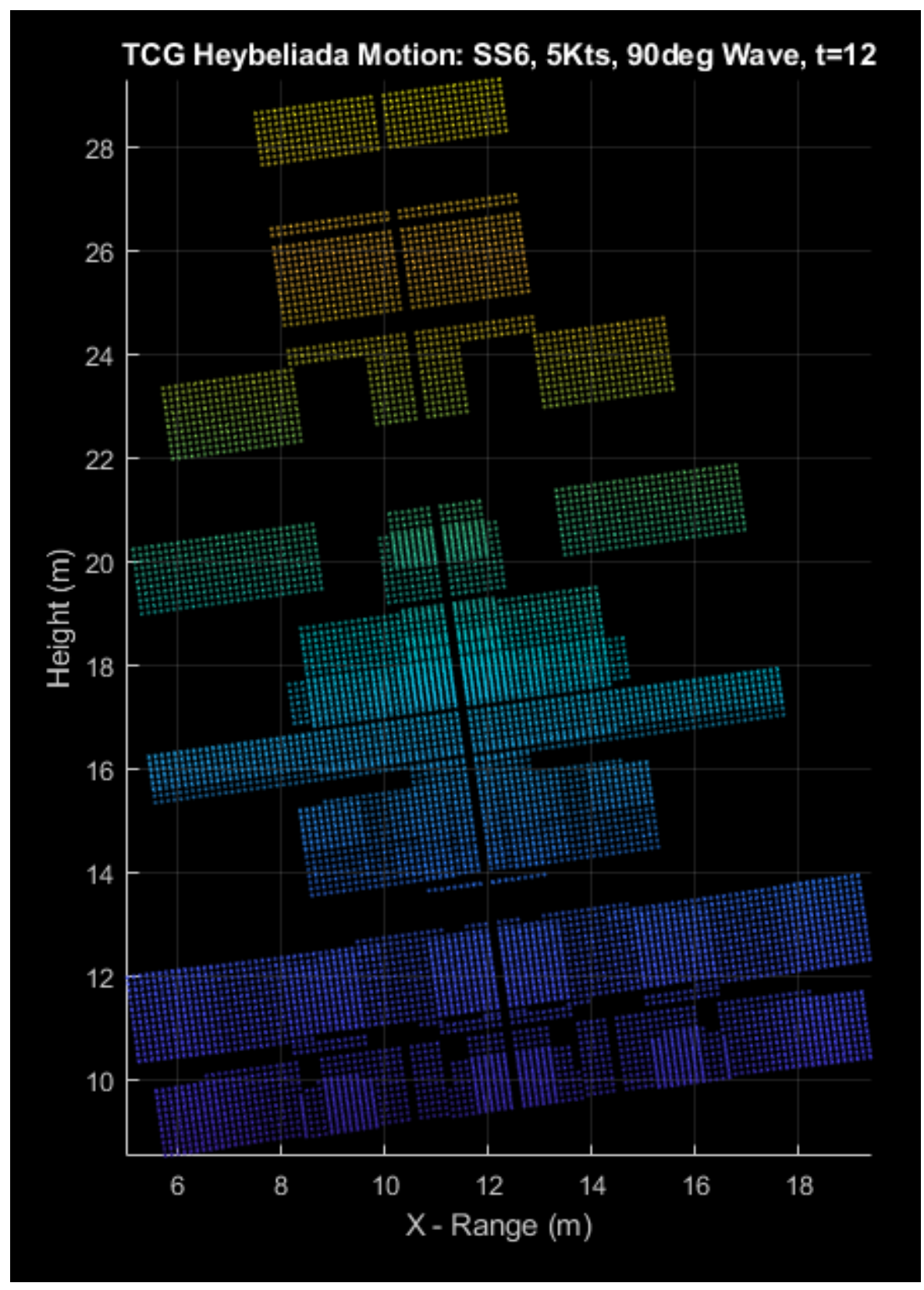

Figure A-2. 13: Target Roll \& Sway Motion, $t=12$ seconds. 


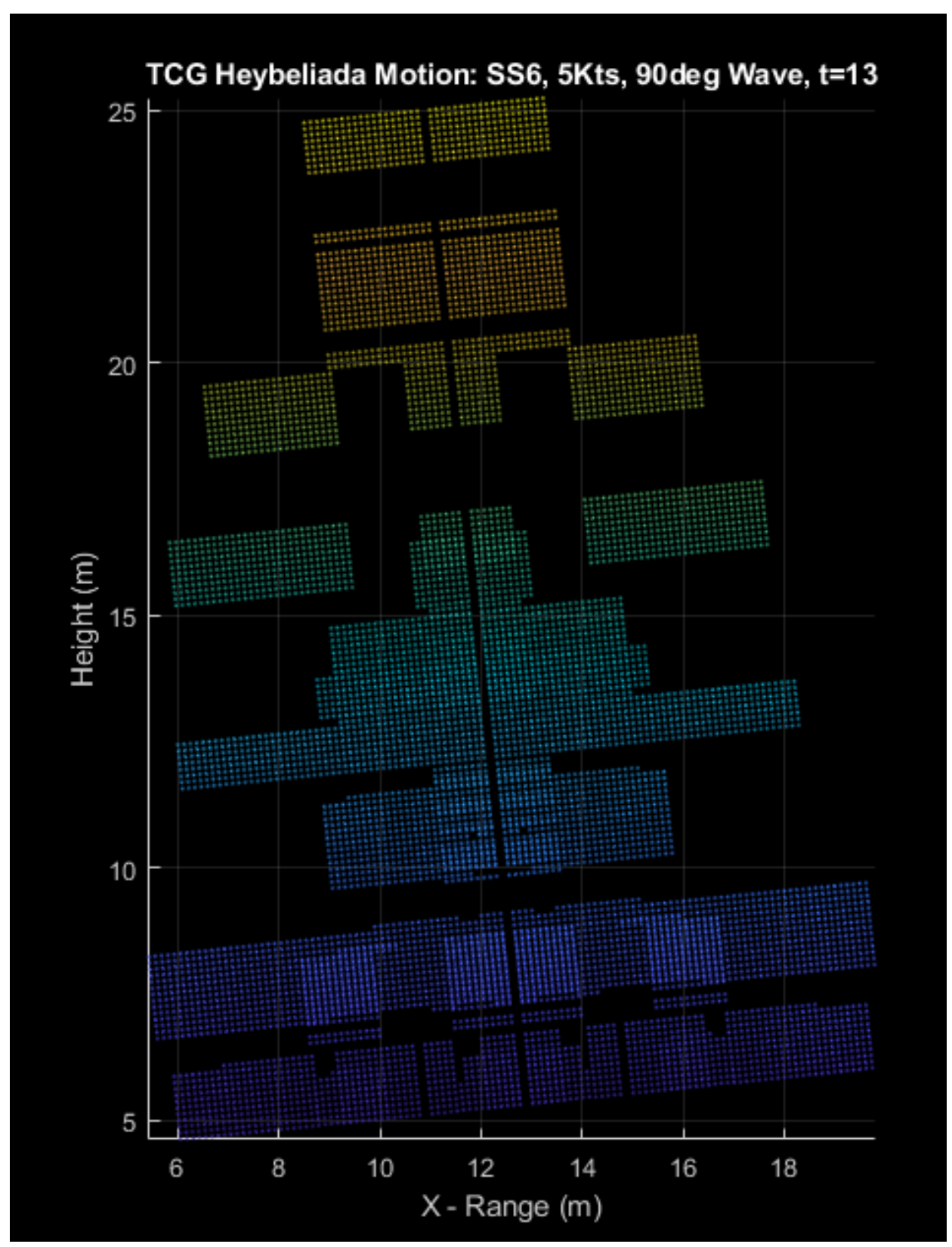

Figure A-2. 14: Target Roll \& Sway Motion, $t=13$ seconds. 


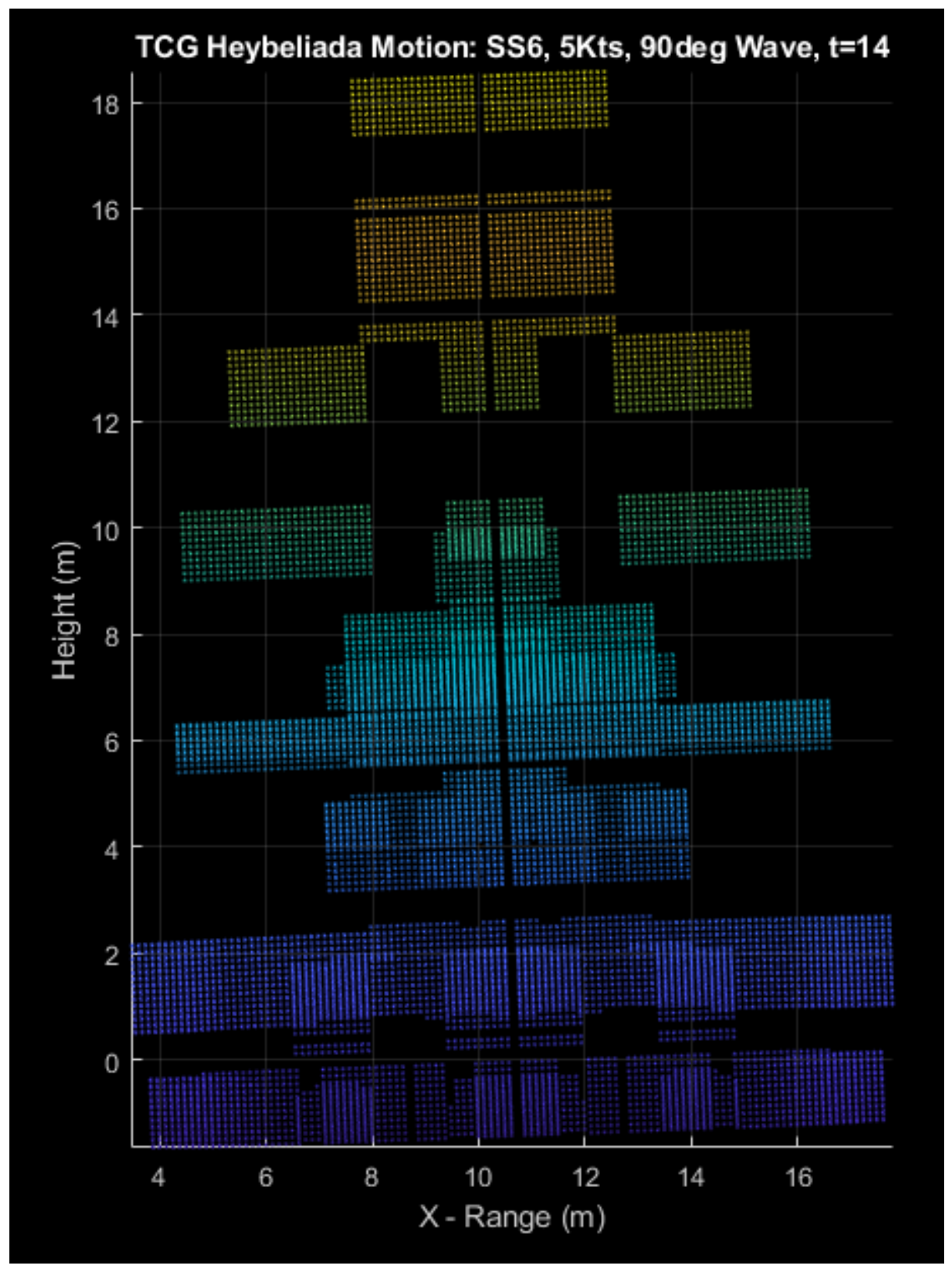

Figure A-2. 15: Target Roll \& Sway Motion, $t=14$ seconds. 


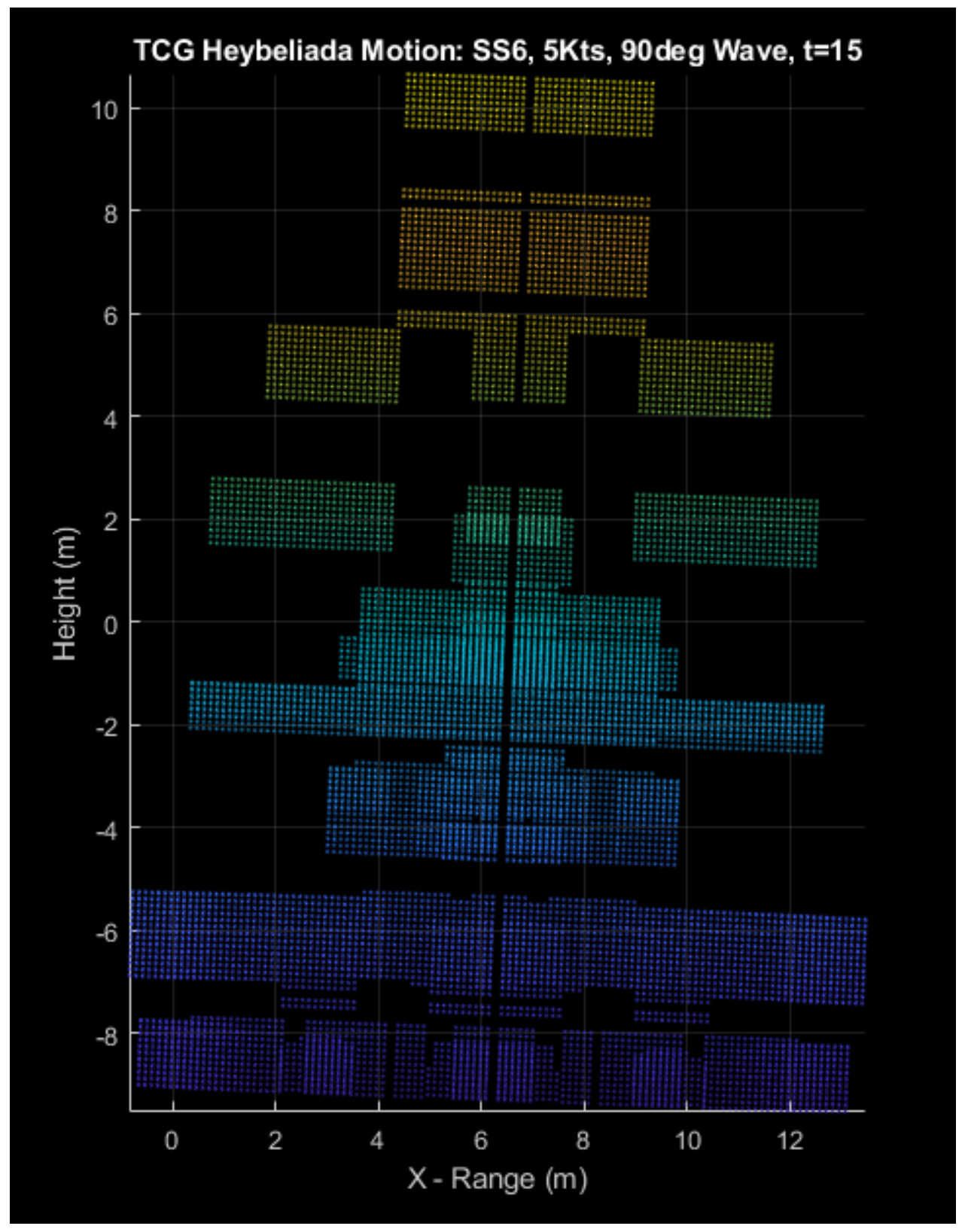

Figure A-2. 16: Target Roll \& Sway Motion, $t=15$ seconds. 


\section{Appendix B}

Beginning on the following page are the screen captures of the simulation of a chirp signal being processed through the designed digital quadrature modulation system. The data shown is the magnitude and phase spectrum of the sampled chirp signal after it has already been mixed to the IF band. The bandwidth of the chirp is $150 \mathrm{MHz}$, and the pulse duration is 1.5 microseconds. 


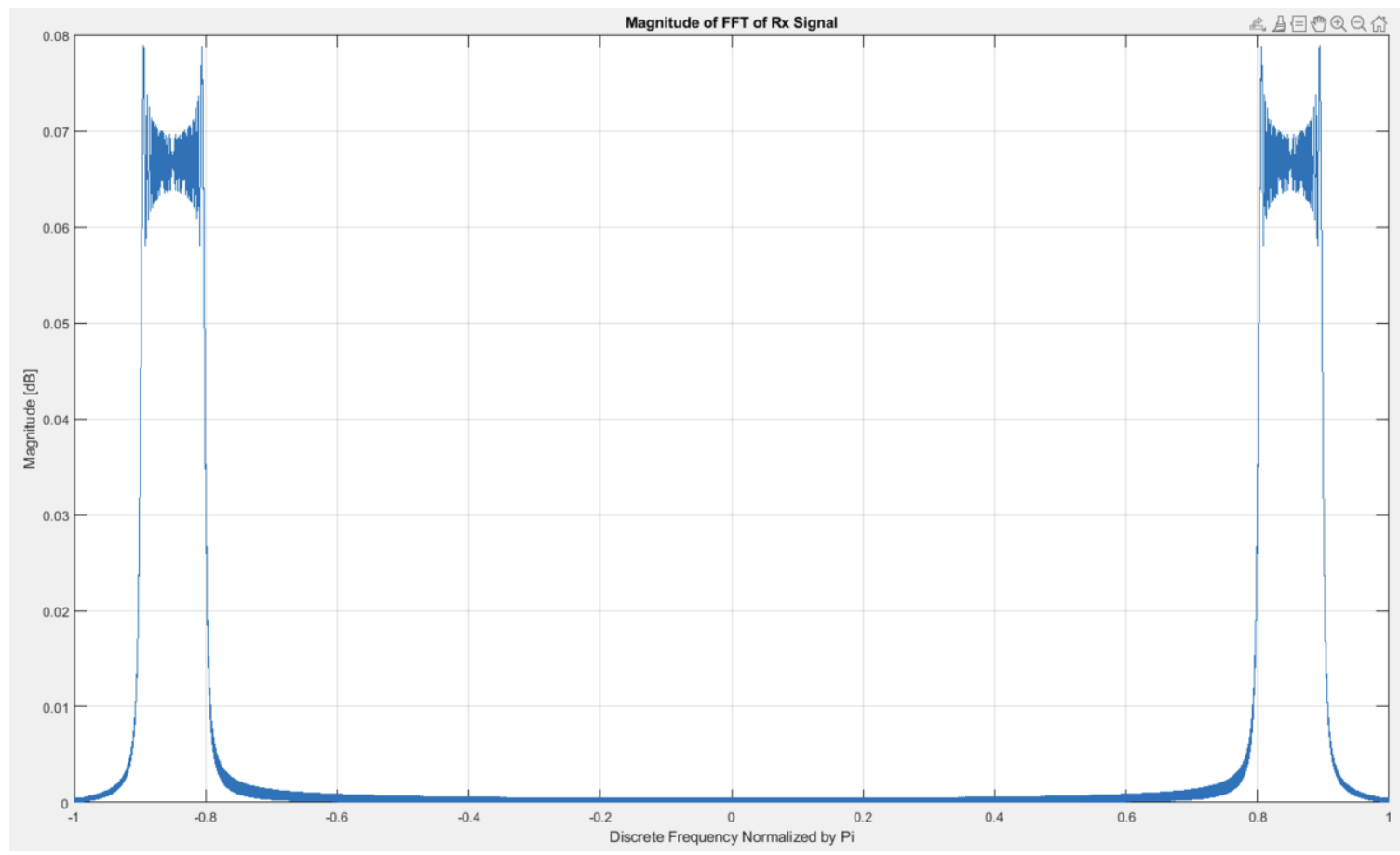

Figure B. 1: Magnitude Spectrum of received chirp signal at IF.

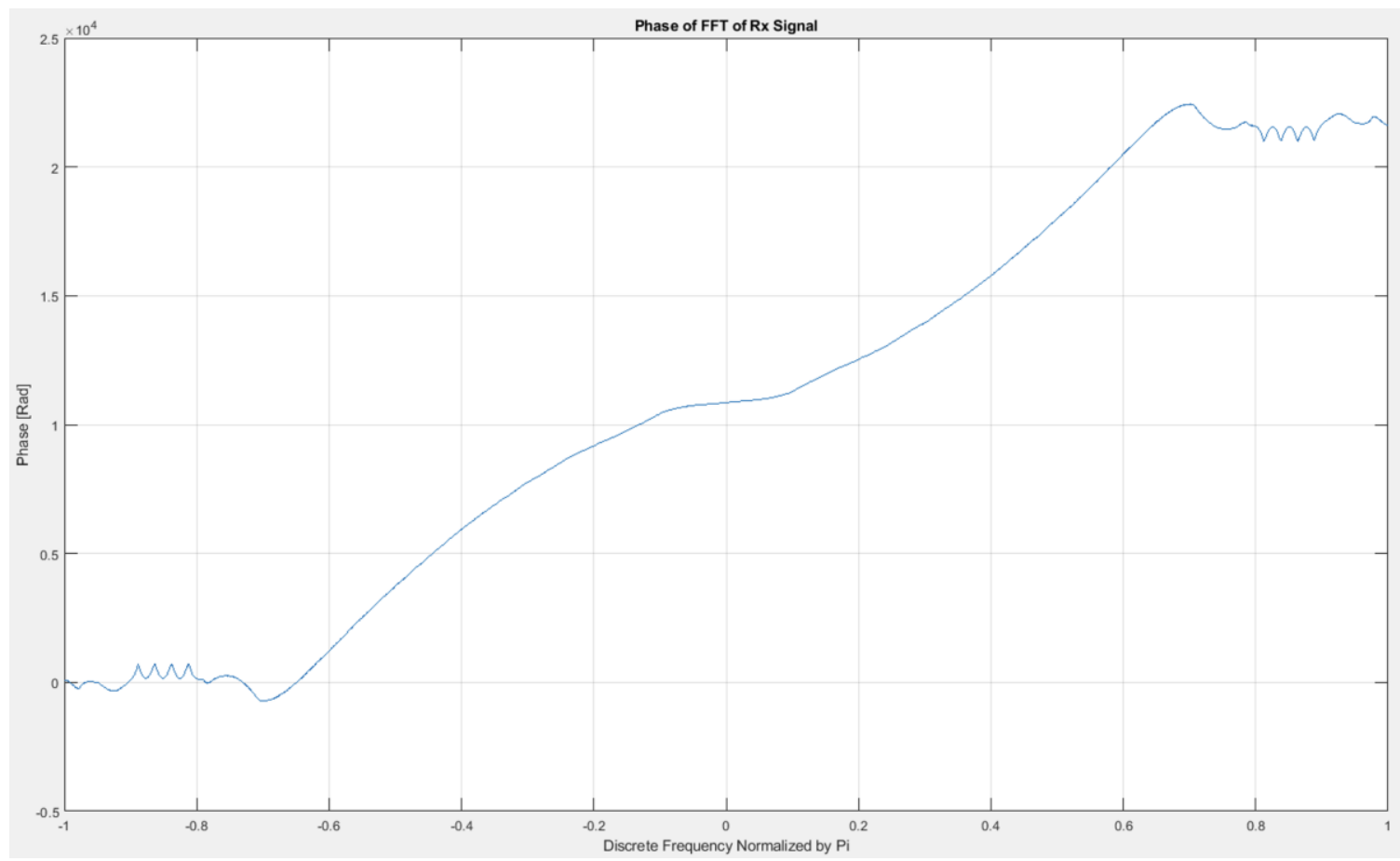

Figure B. 2: Phase Spectrum of received chirp signal at IF. 


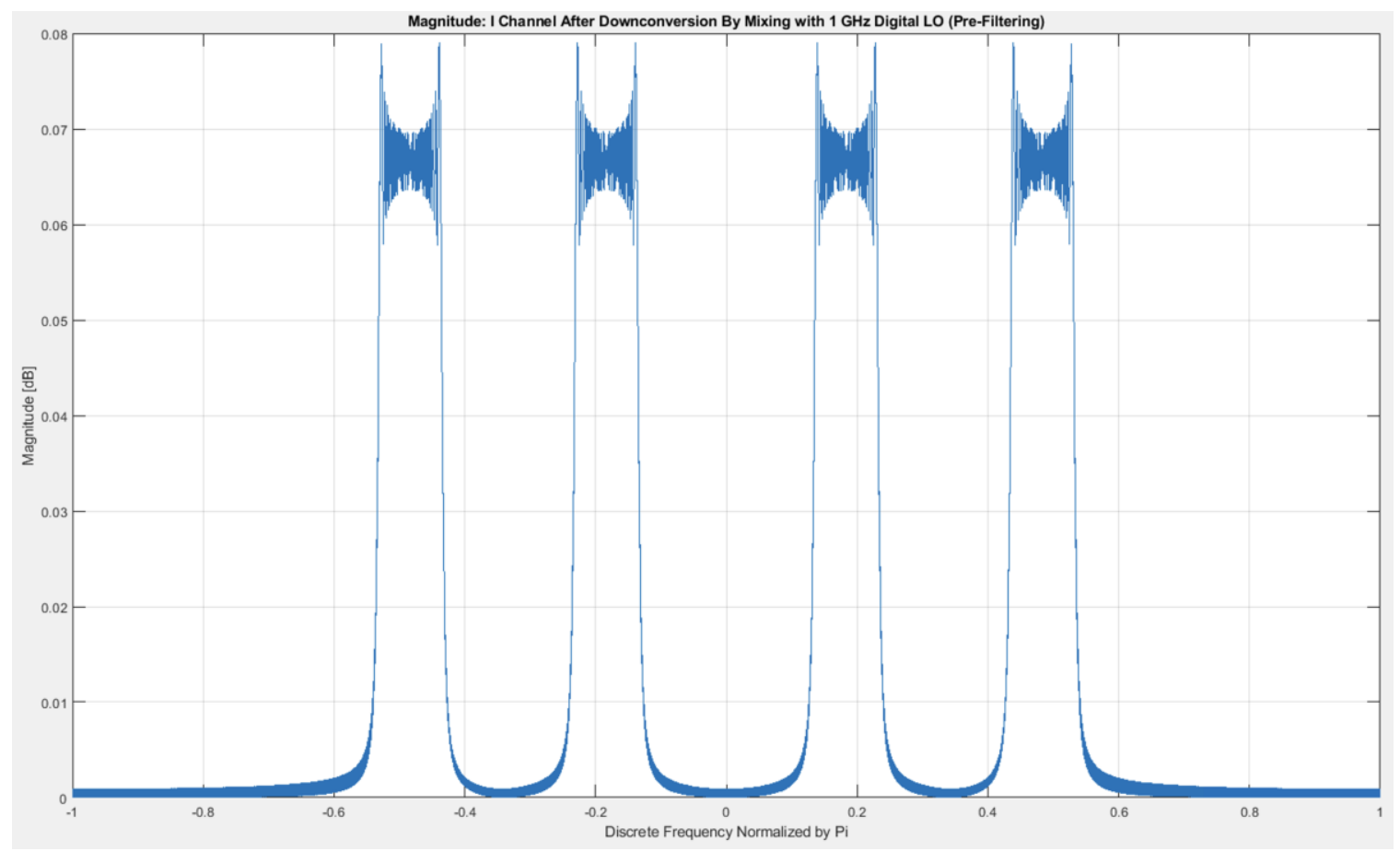

Figure B. 3: I Channel chirp signal magnitude spectrum after digital mixing, prior to filtering.

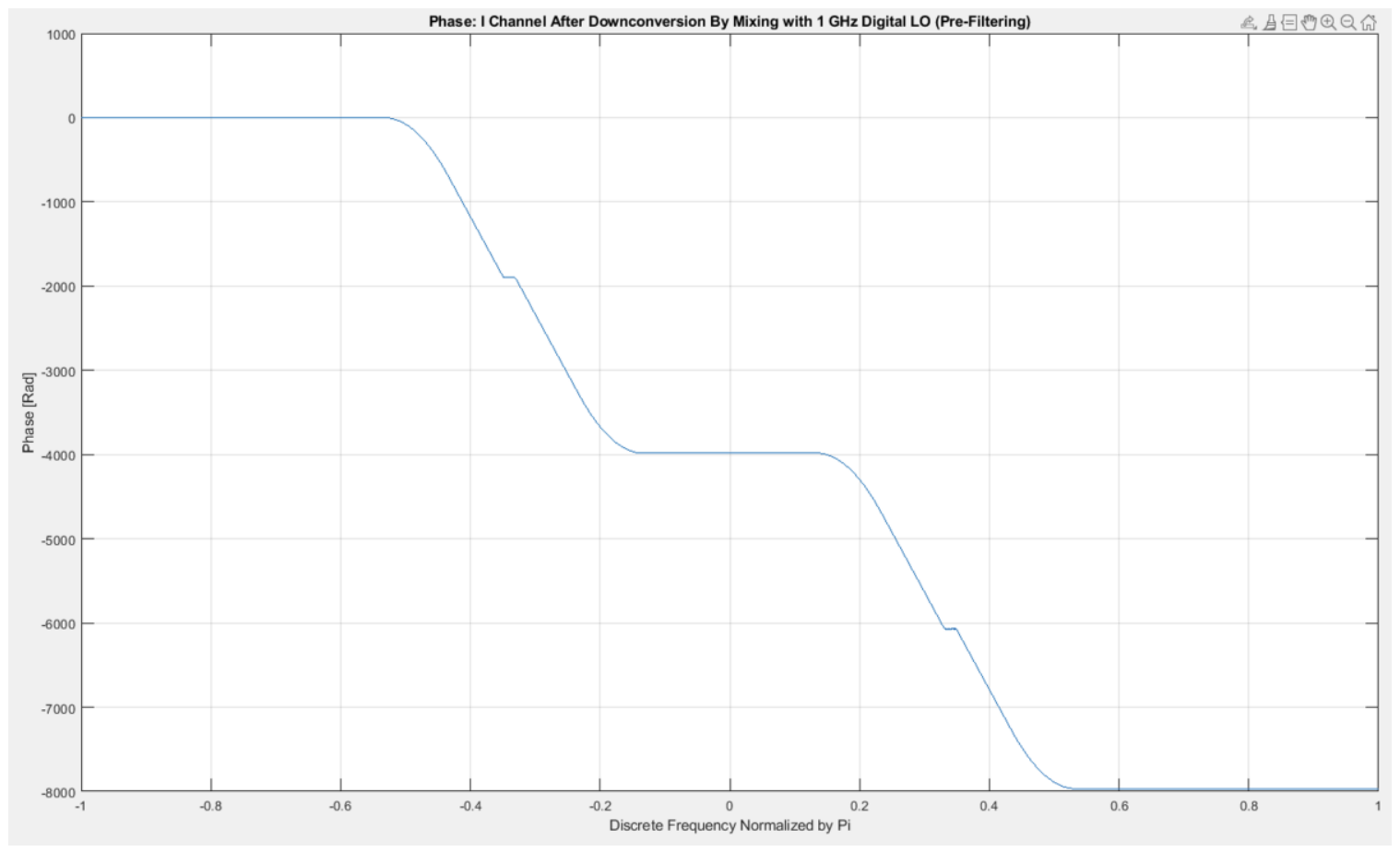

Figure B. 4: I Channel chirp signal phase spectrum after digital mixing, prior to filtering. 


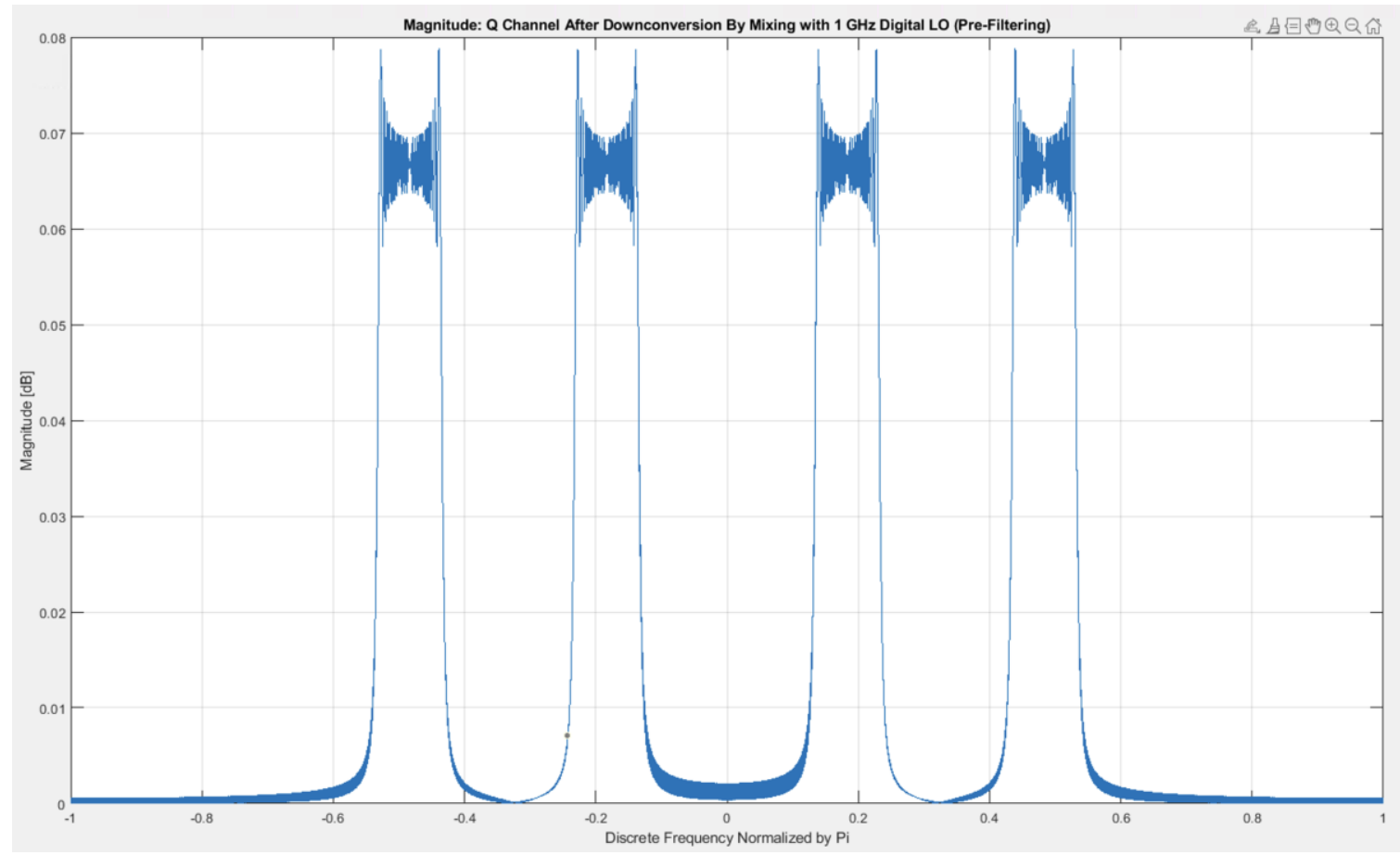

Figure B. 5: Q Channel chirp signal magnitude spectrum after digital mixing, prior to filtering.

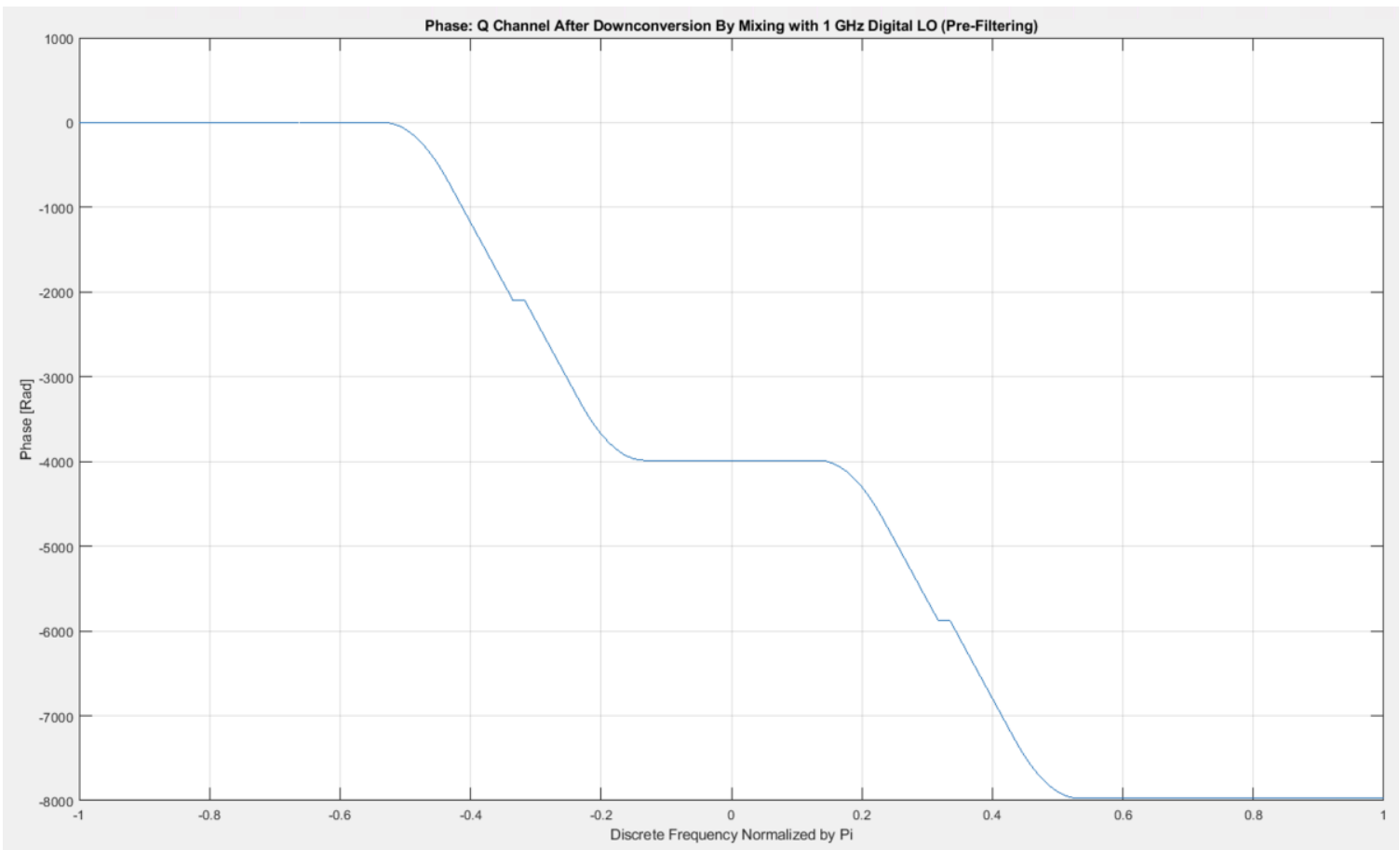

Figure B. 6: Q Channel chirp signal phase spectrum after digital mixing, prior to filtering.

226 


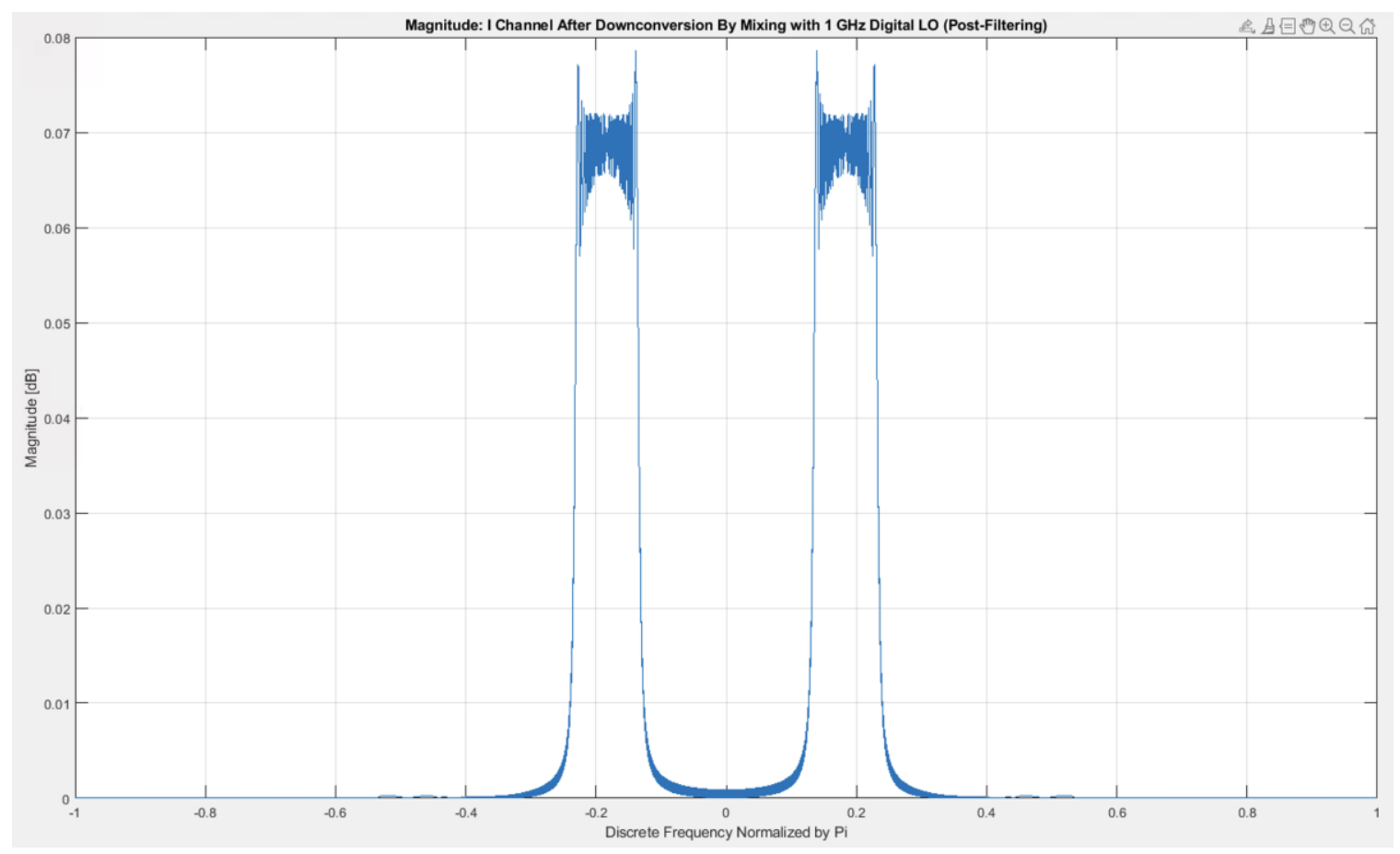

Figure B. 7: I Channel chirp signal magnitude spectrum after digital mixing, post-filtering.

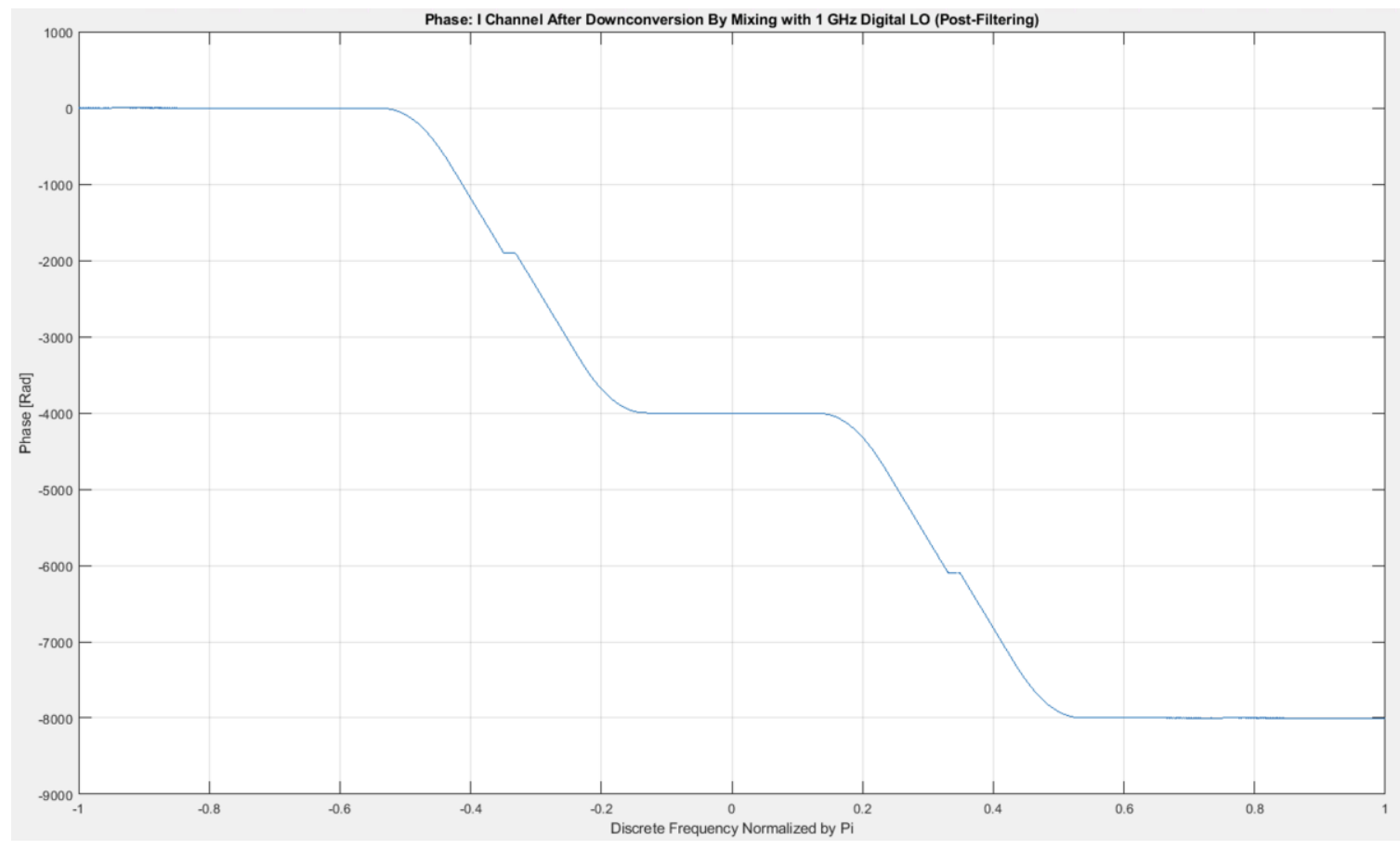

Figure B. 8: I Channel chirp signal phase spectrum after digital mixing, post-filtering. 


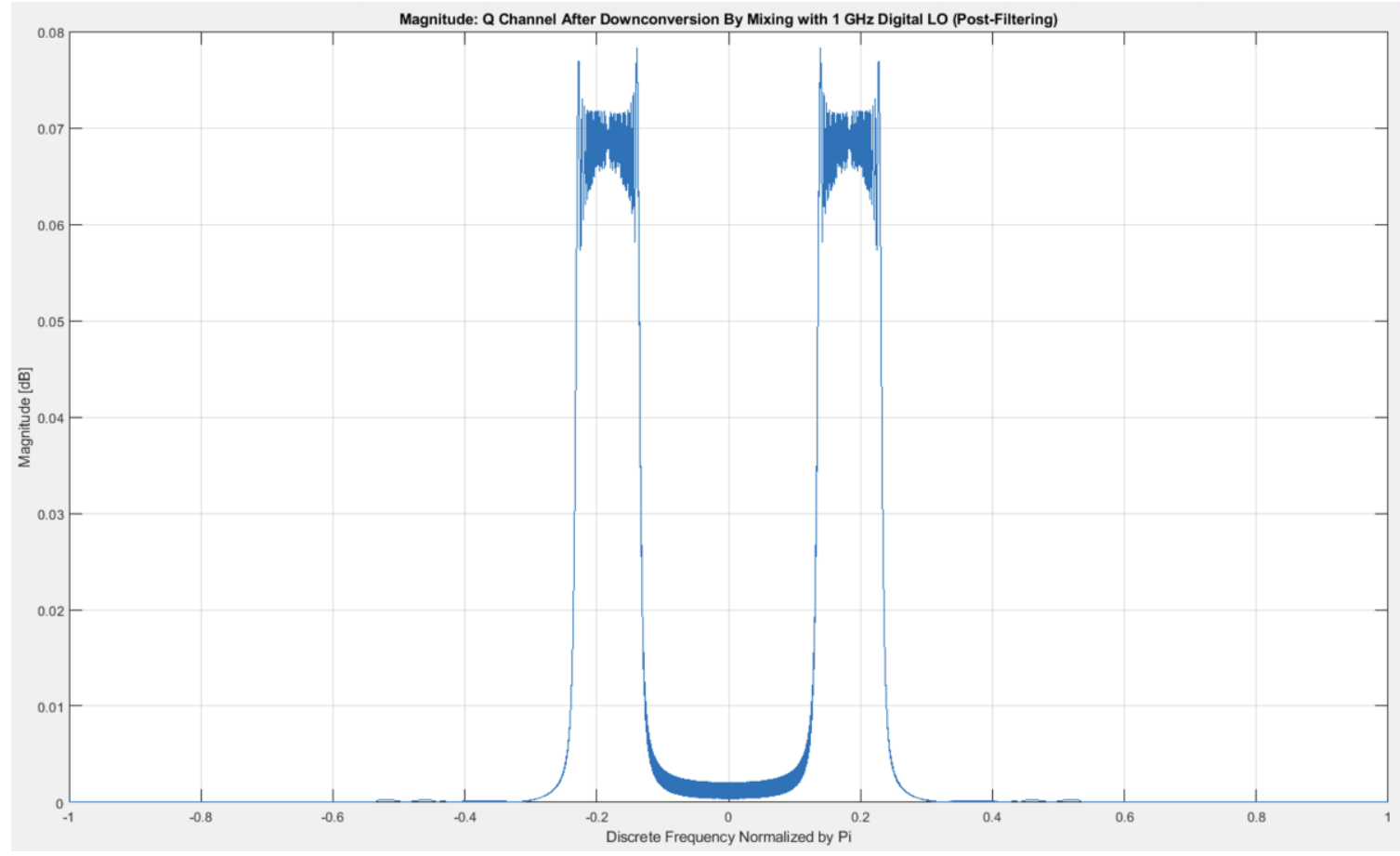

Figure B. 9: Q Channel chirp signal magnitude spectrum after digital mixing, post-filtering.

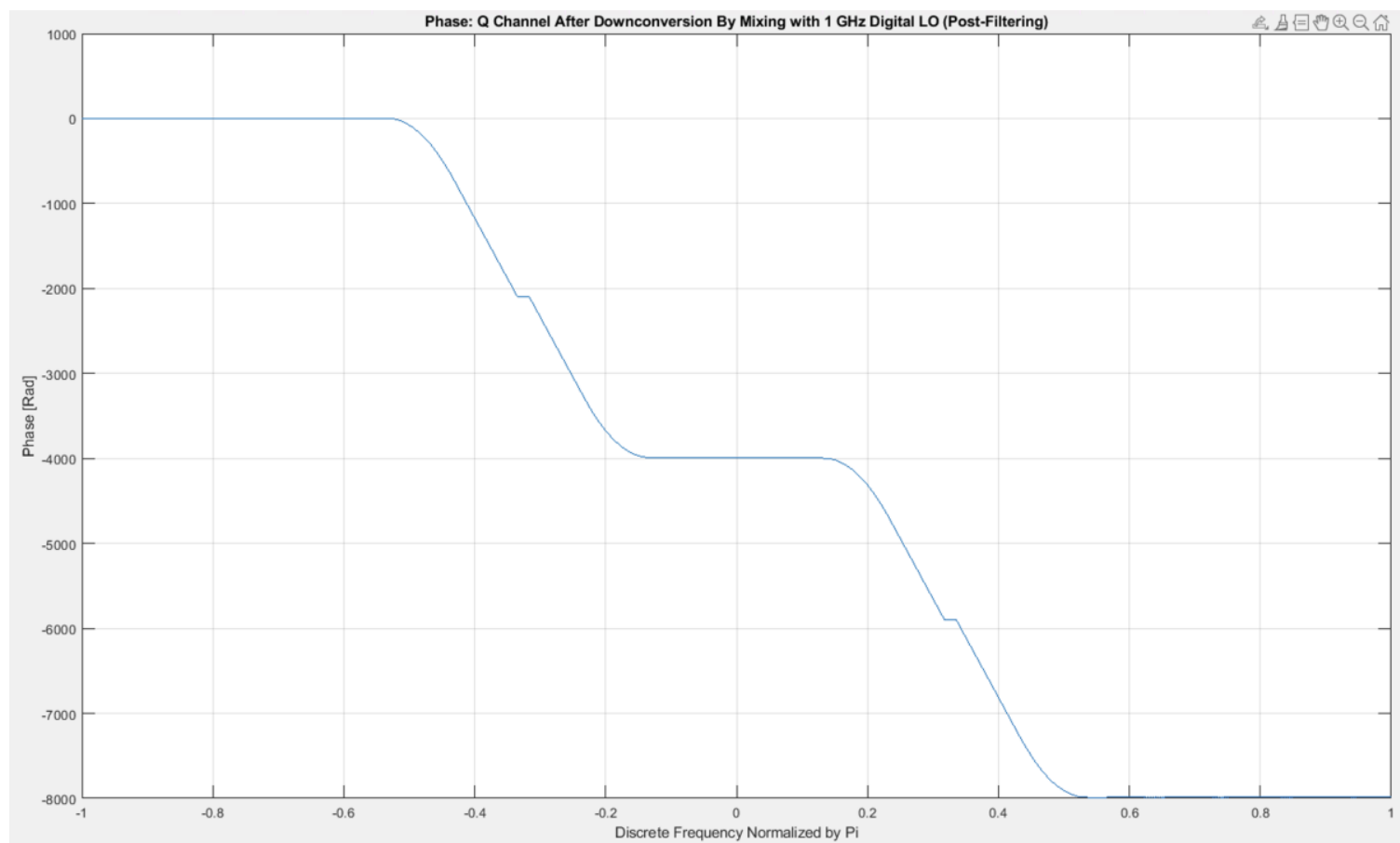

Figure B. 10: Q Channel chirp signal phase spectrum after digital mixing, post-filtering. 


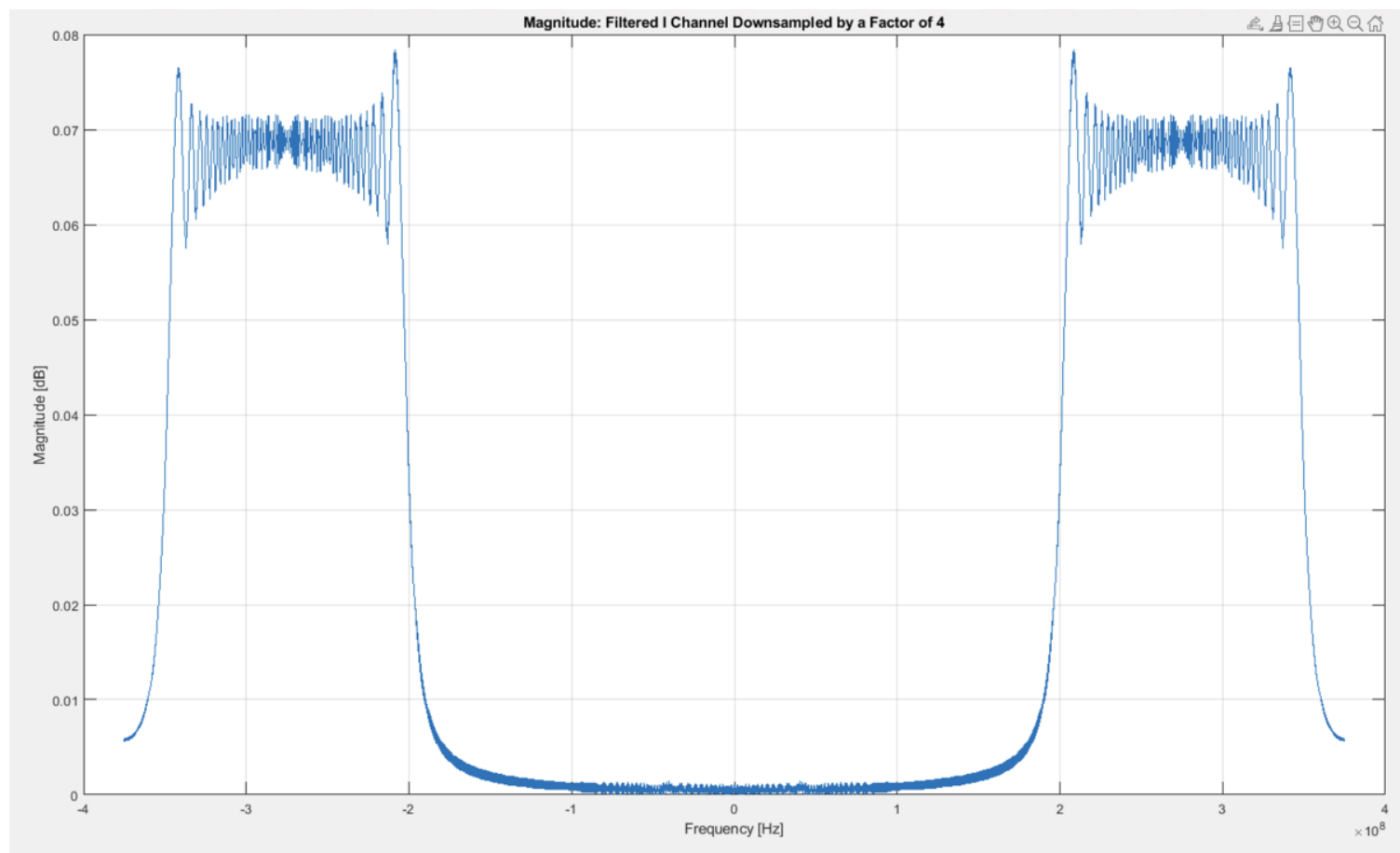

Figure B. 11: I Channel chirp signal magnitude spectrum after digital mixing, post-filtering \& downsampling.

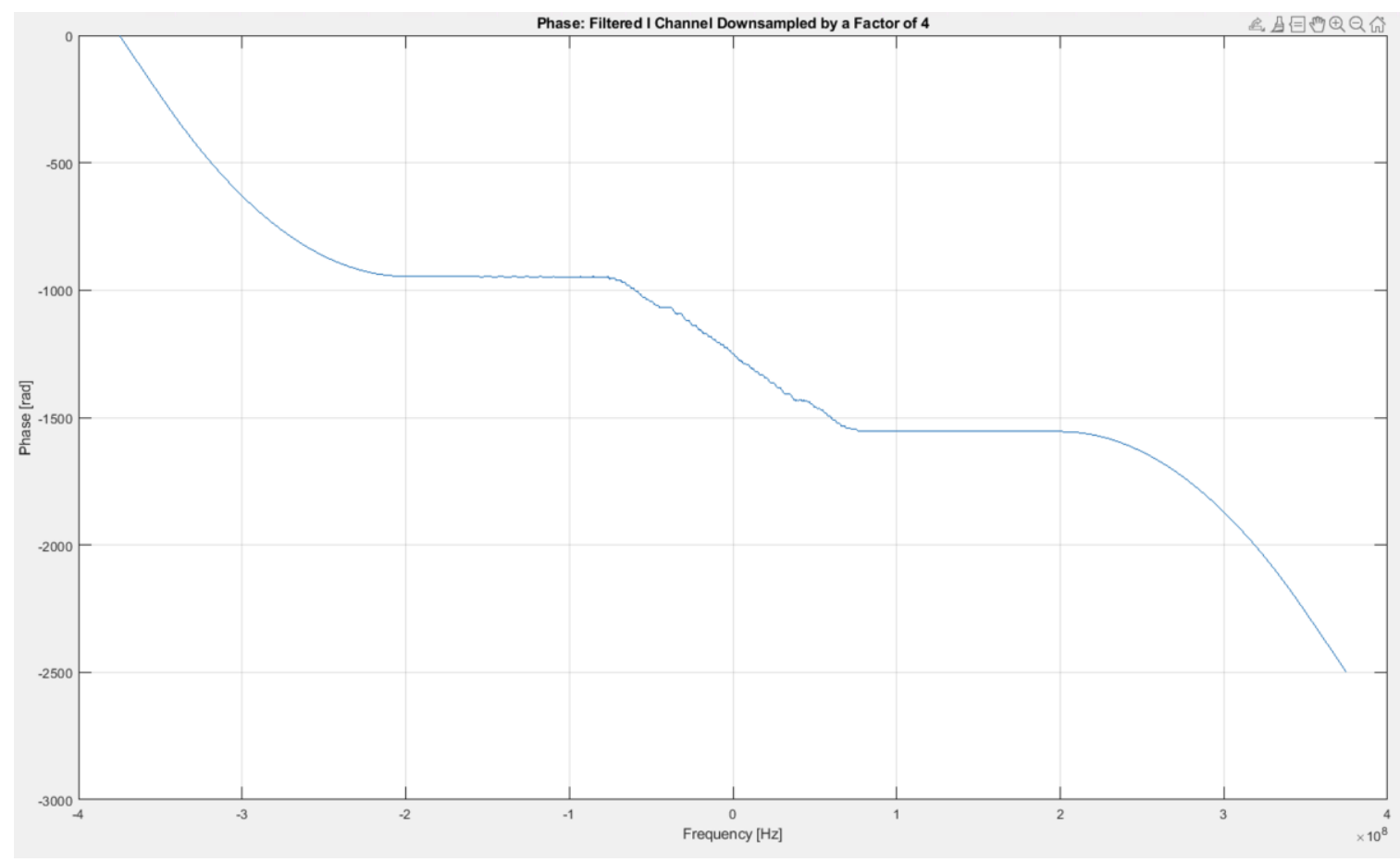

Figure B. 12: I Channel chirp signal phase spectrum after digital mixing, post-filtering \& downsampling. 


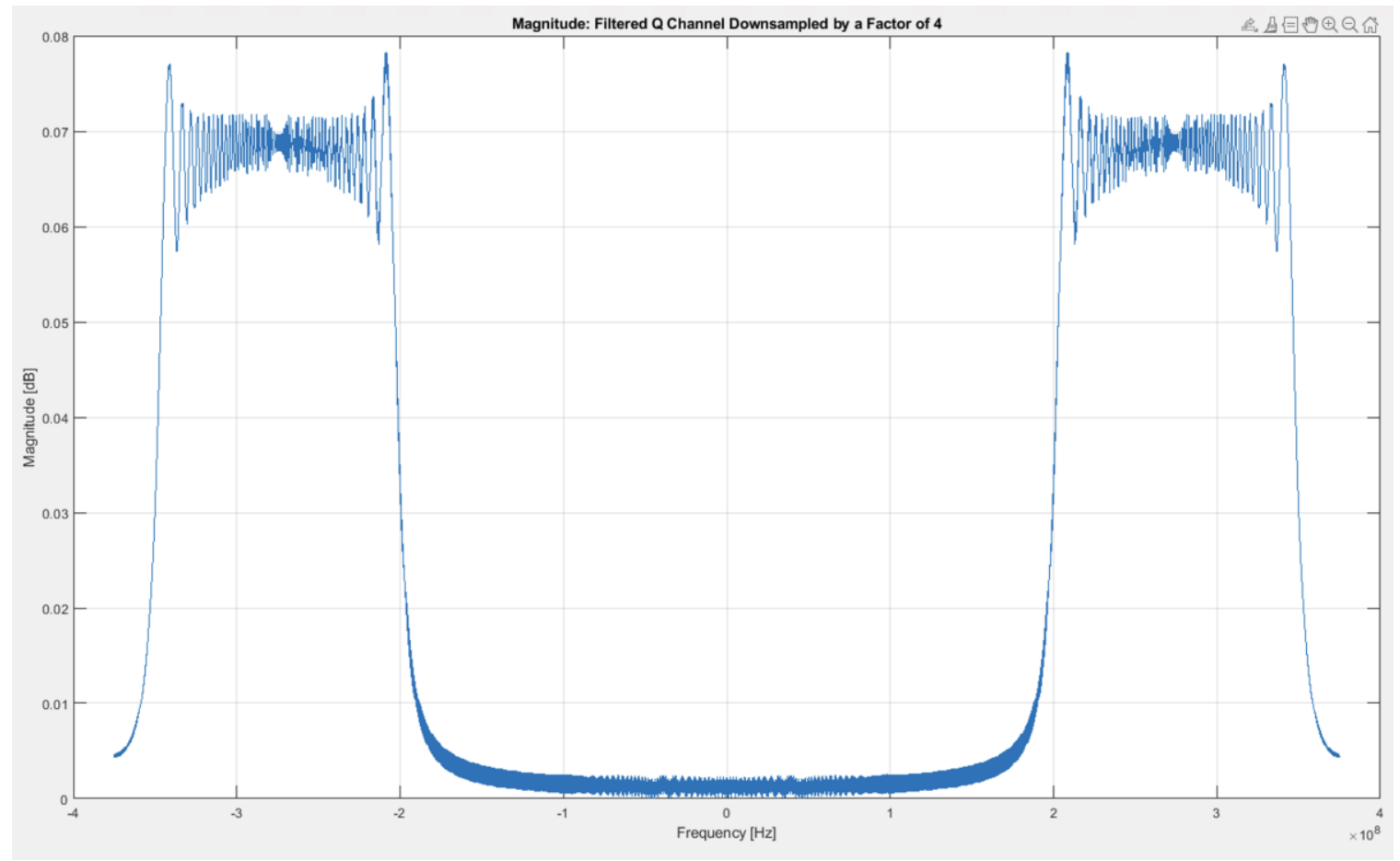

Figure B. 13: Q Channel chirp signal magnitude spectrum after digital mixing, post-filtering \& downsampling.

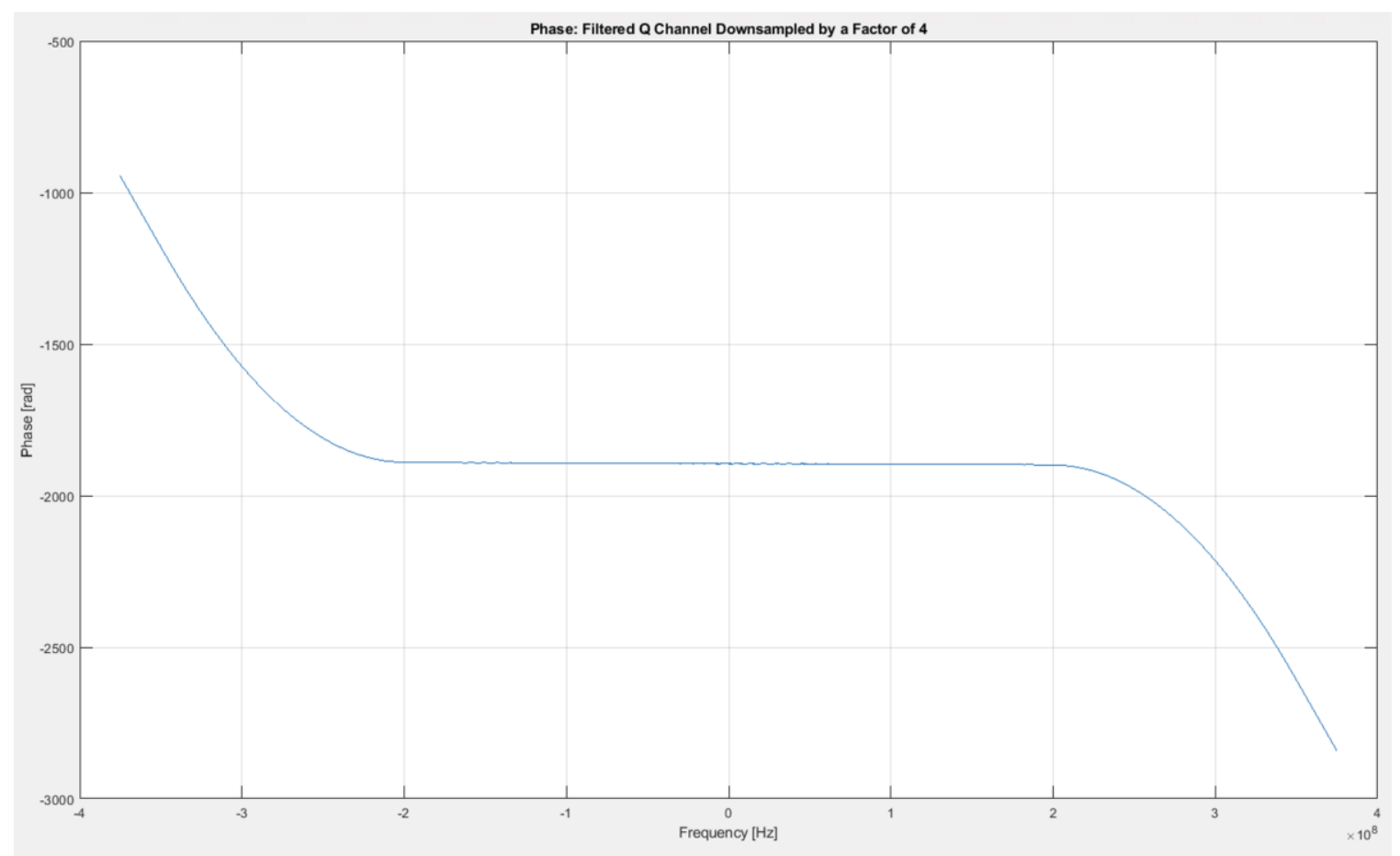

Figure B. 14: Q Channel chirp signal phase spectrum after digital mixing, post-filtering \& downsampling. 


\section{Appendix C}

In this section the HRRPs, phase histories and Range-Doppler images for the simulation cases not presented in chapter 7 are shown. Sub-appendix C.1 contains the HRRPs and phase histories for simulation cases 2 and three for the stepped-frequency simulations. In sub-appendix C. 2 the HRRPs and phase histories for countering chirp radar cases two and three are shown. In sub-appendix C.3 the Range-Doppler images generated using stepped frequency data is shown for cases 3,4 and 5. Finally, in subappendix C.4 the Range-Doppler images generated from chirp data is shown for cases 2,3 and 5 . 


\section{C.1 Sub-Appendix}

HRRPs and phase histories from stepped-frequency simulations.

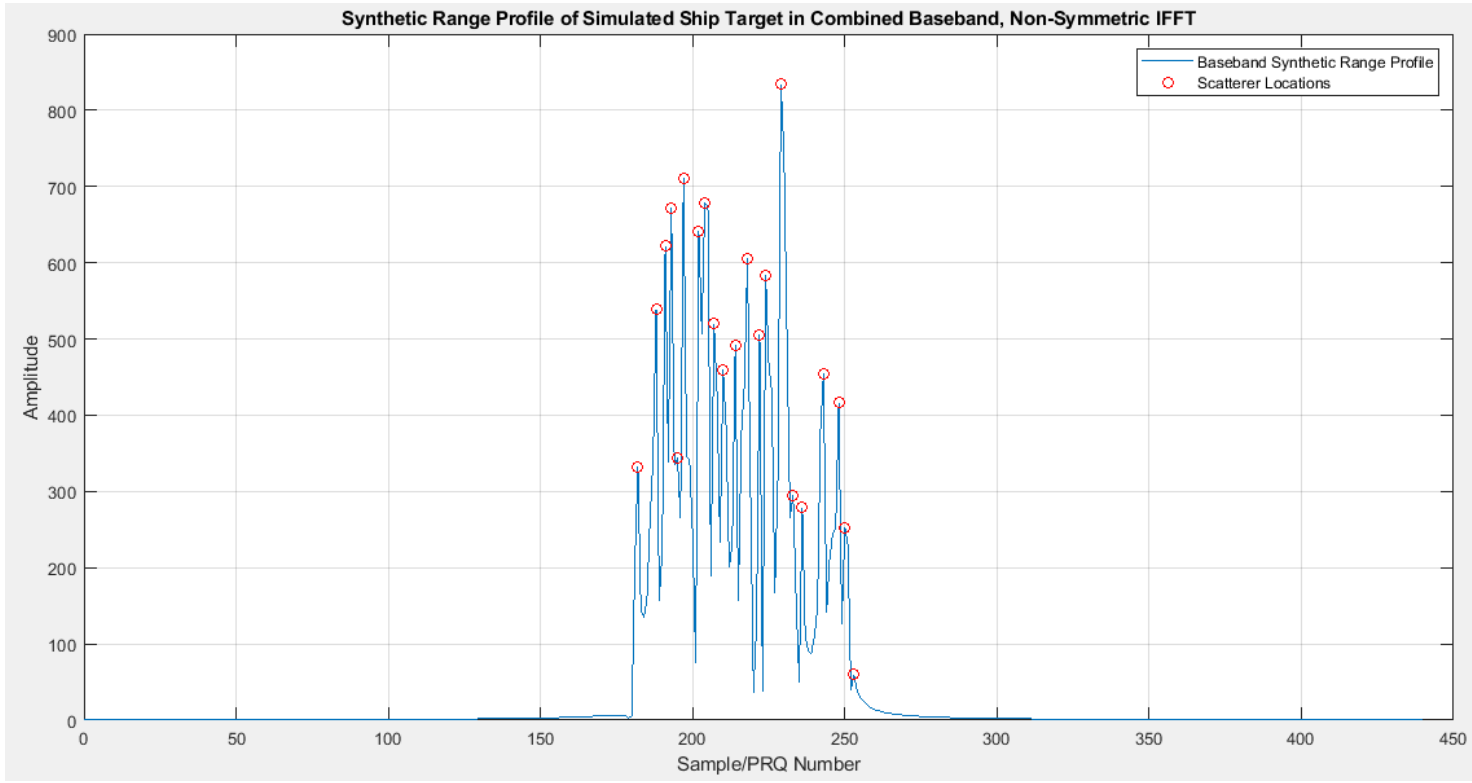

Figure C-1. 1: Complex baseband synthetic HRRP for simulation case 2.

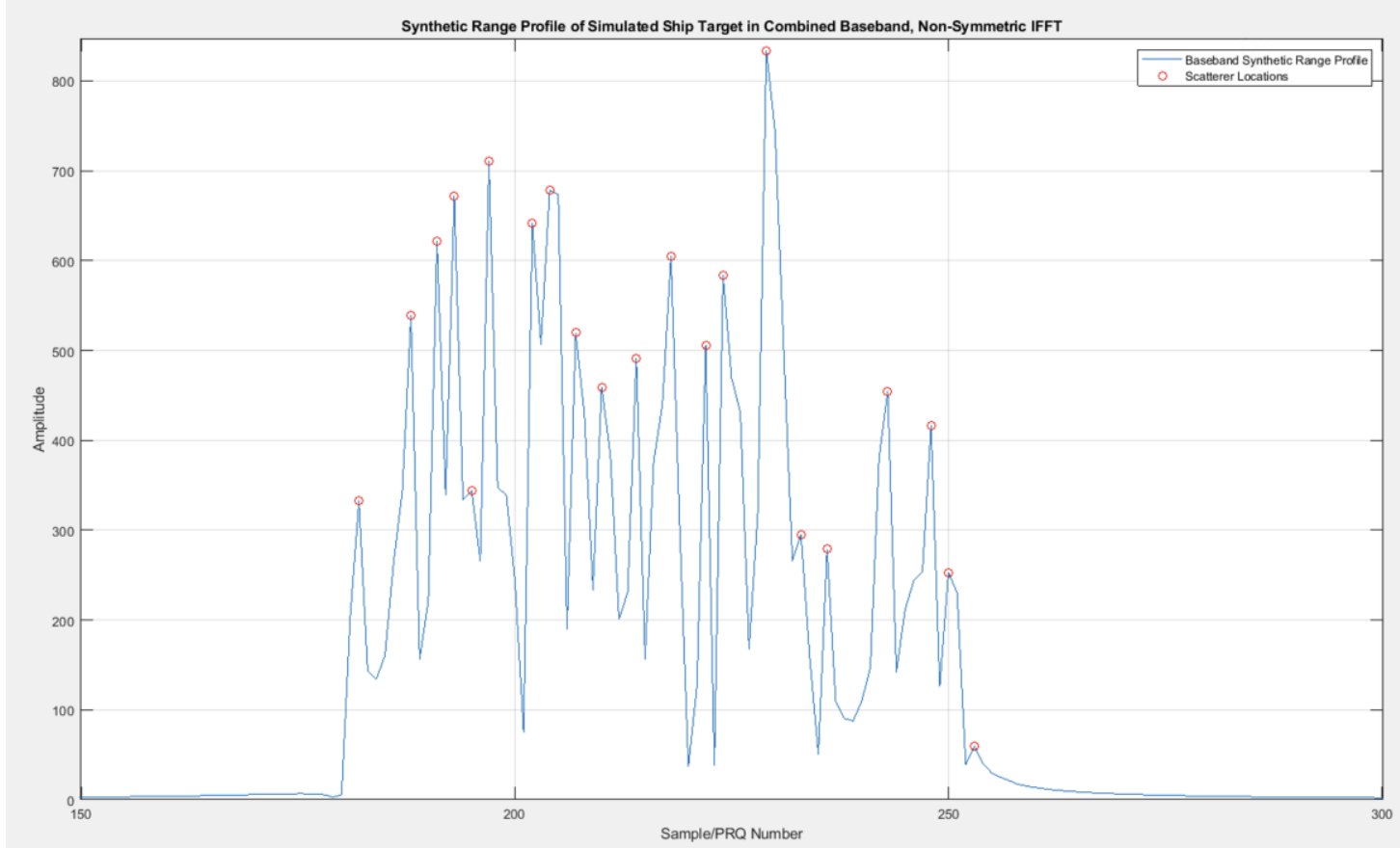

Figure C-1. 2: Zoomed in section of synthetic high-resolution range profile for simulation case 2. 


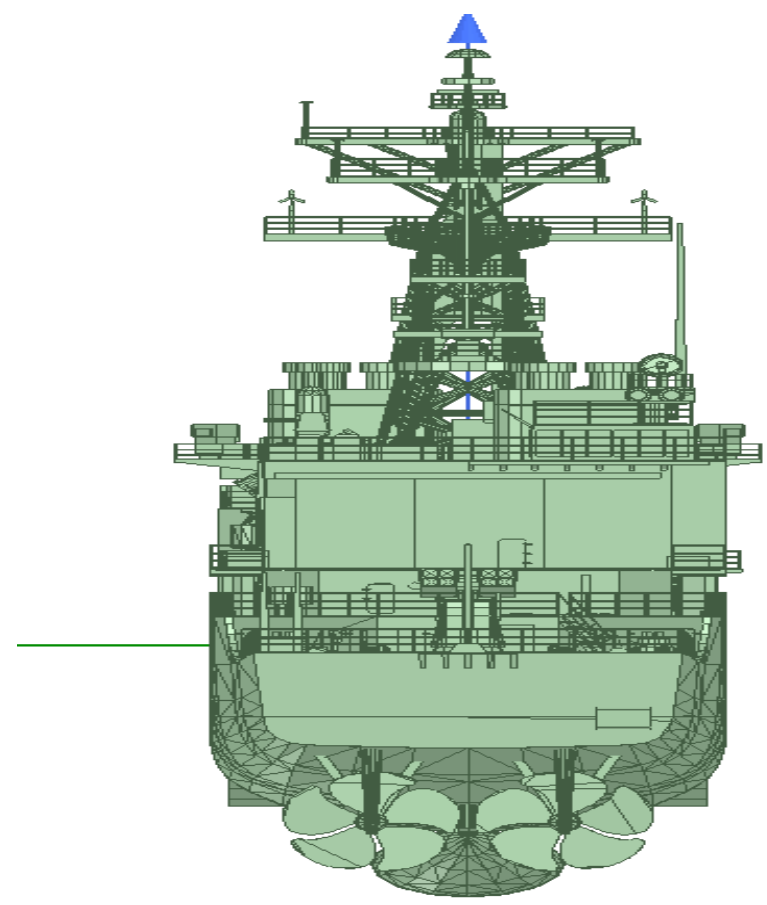

Figure C-1. 3: Target model perspective for which the range profile is produced in case two.

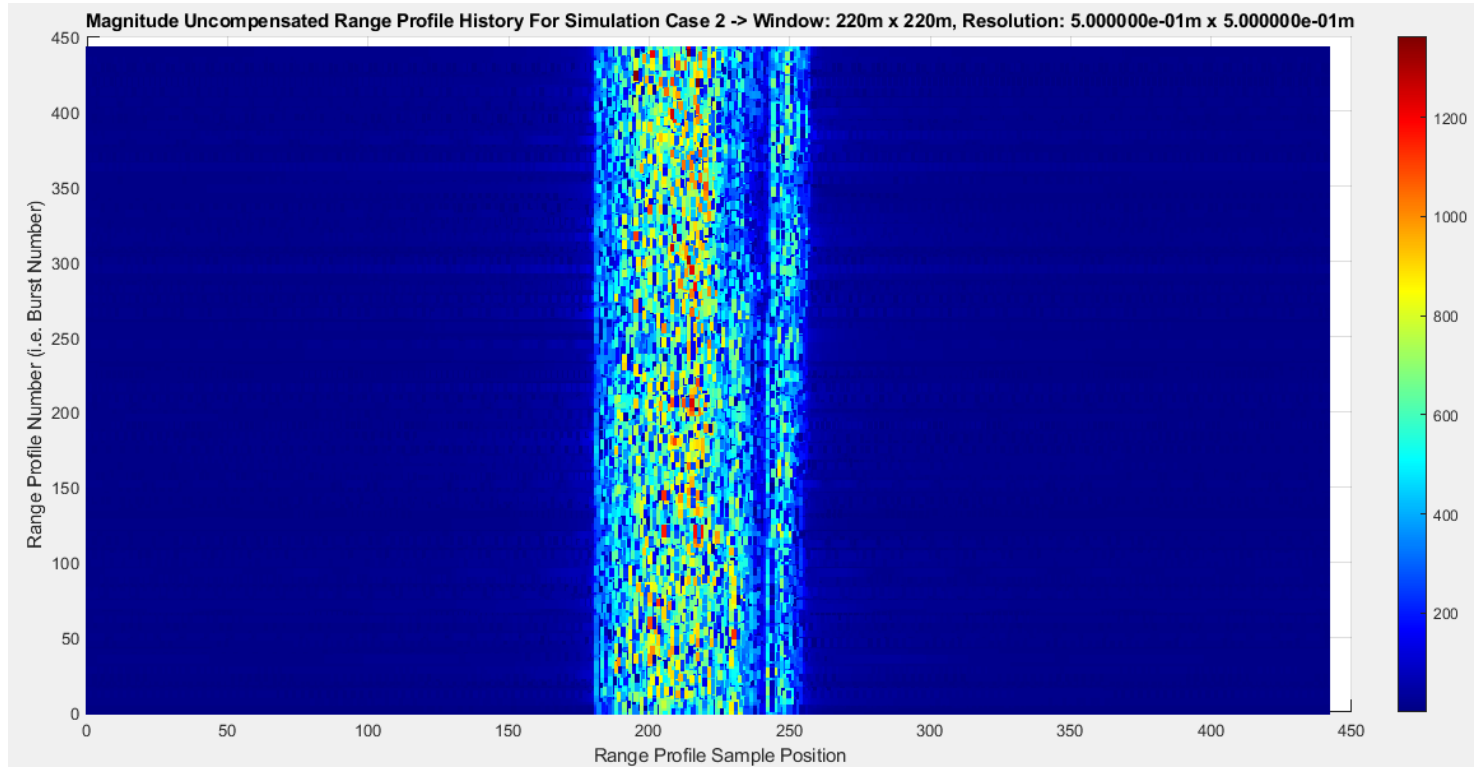

Figure C-1. 4: Simulation Case 2 Complex baseband magnitude range profile history. 


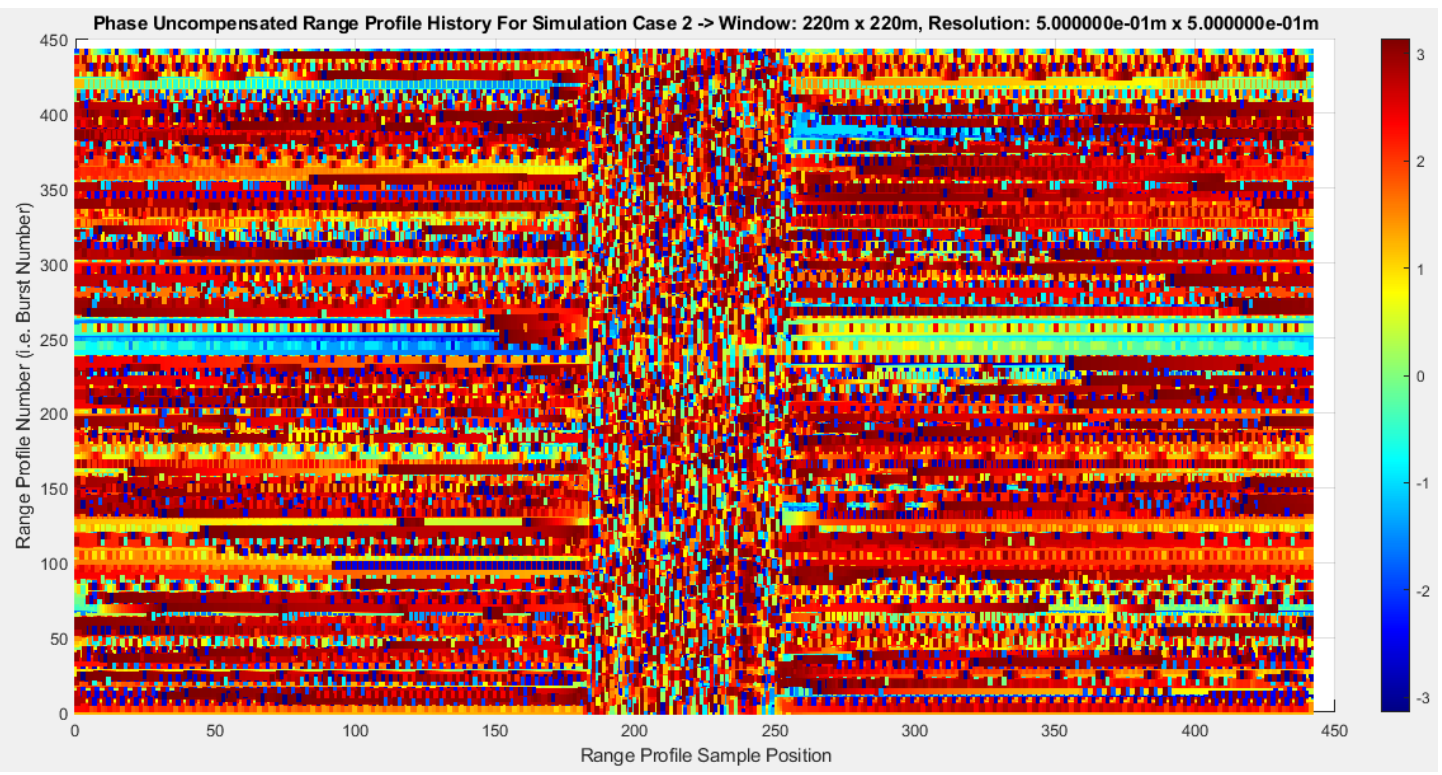

Figure C-1. 5: Simulation Case 2 Complex baseband phase history. 


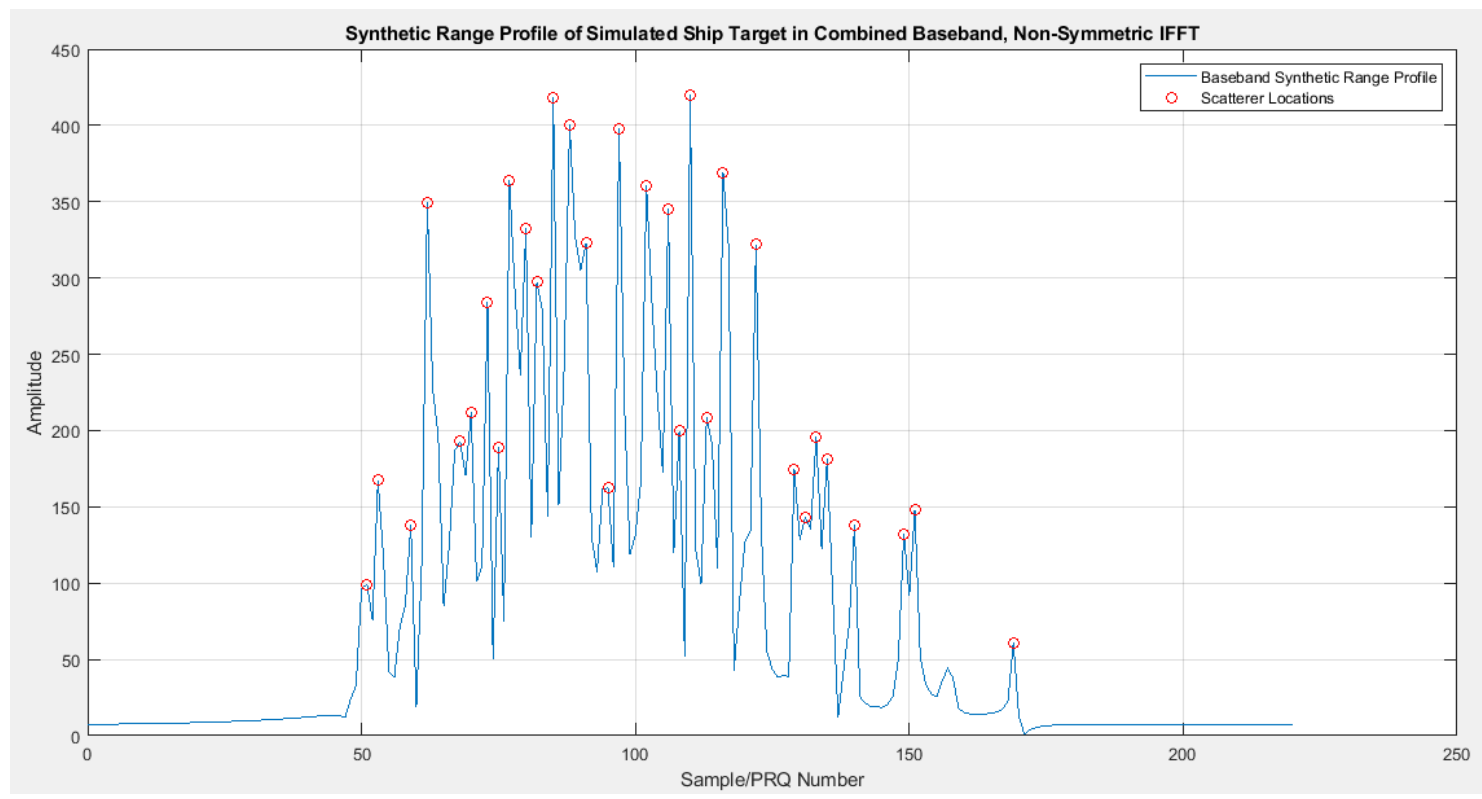

Figure C-1. 6: The complex valued synthetic high-resolution range profile for simulation case 3 .

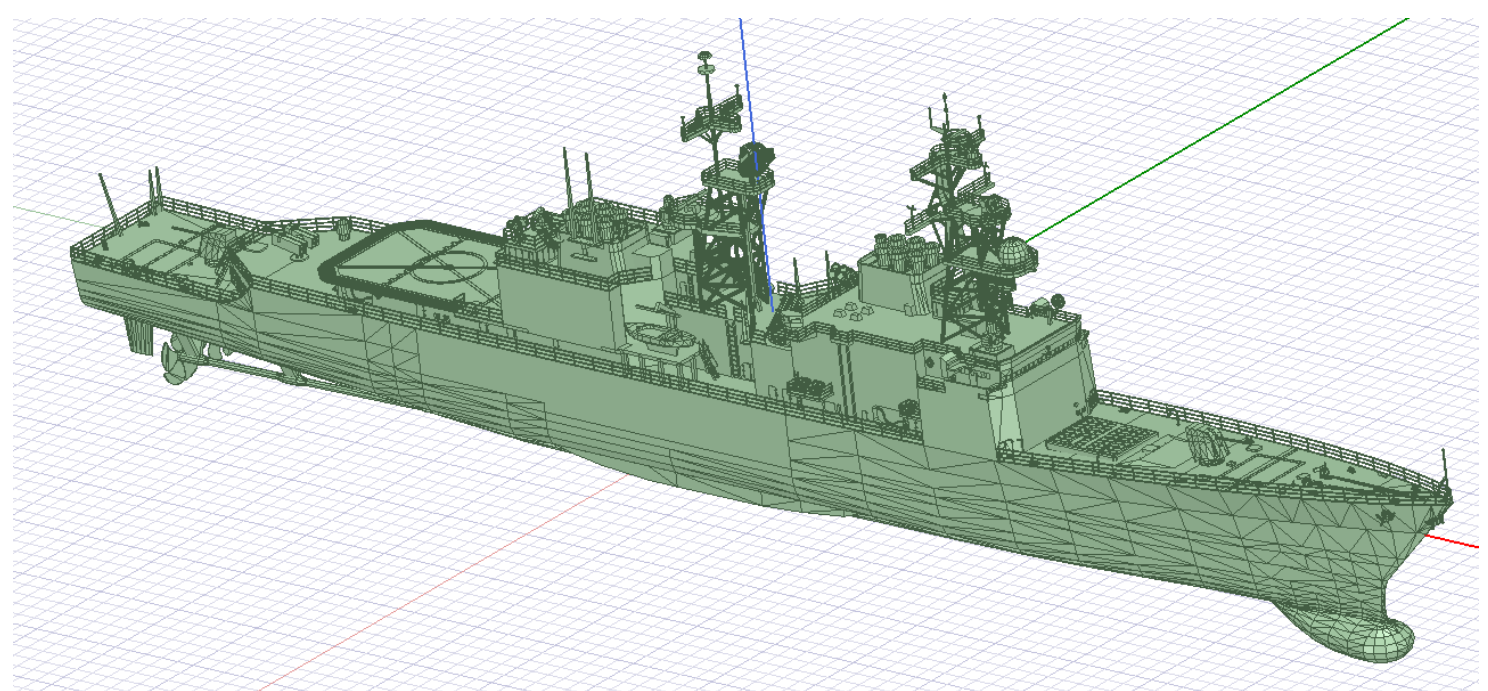

Figure C-1. 7: Target model perspective for which the range profile is produced in case three. 


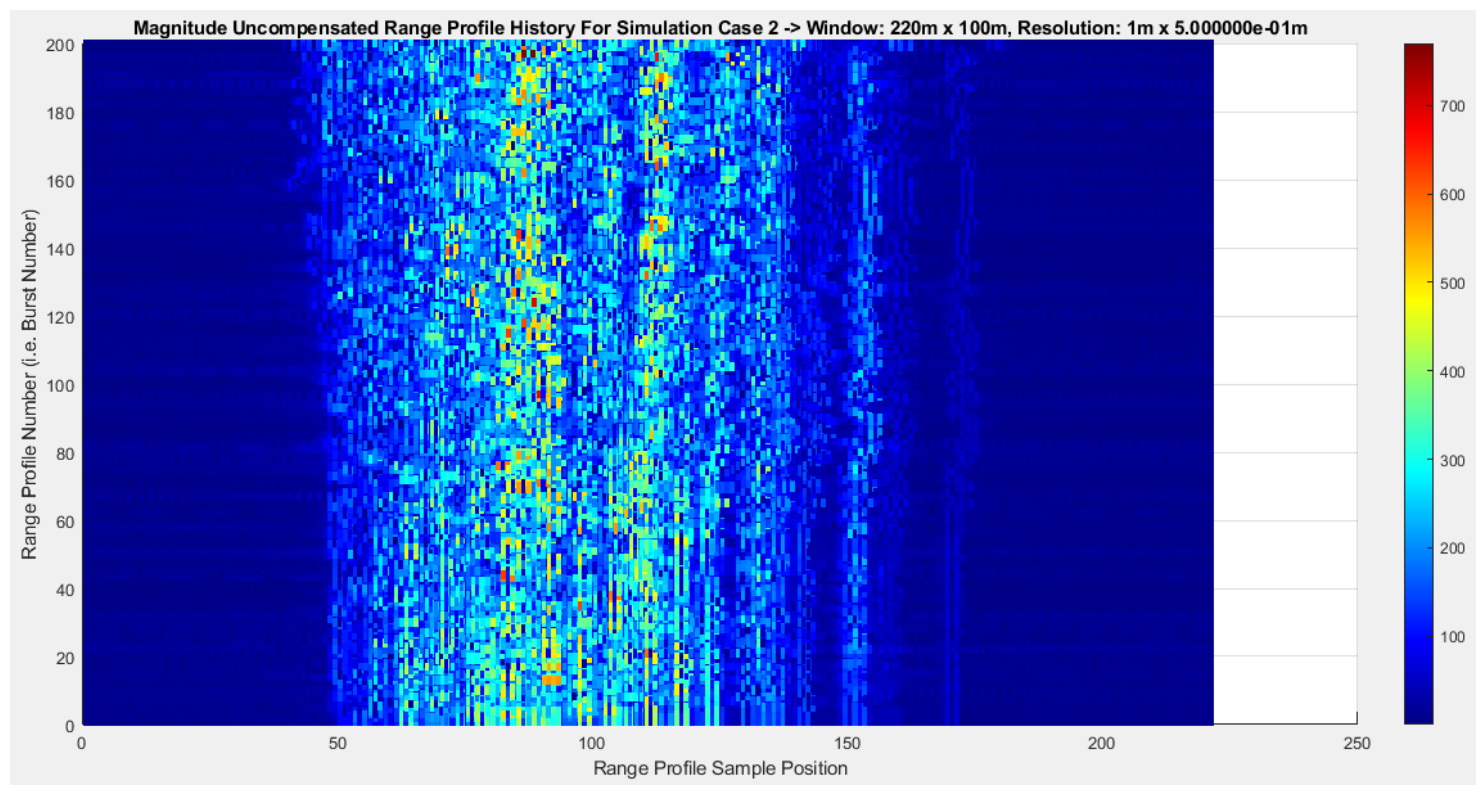

Figure C-1. 8: Simulation Case 3 Complex baseband magnitude range profile

\section{history.}

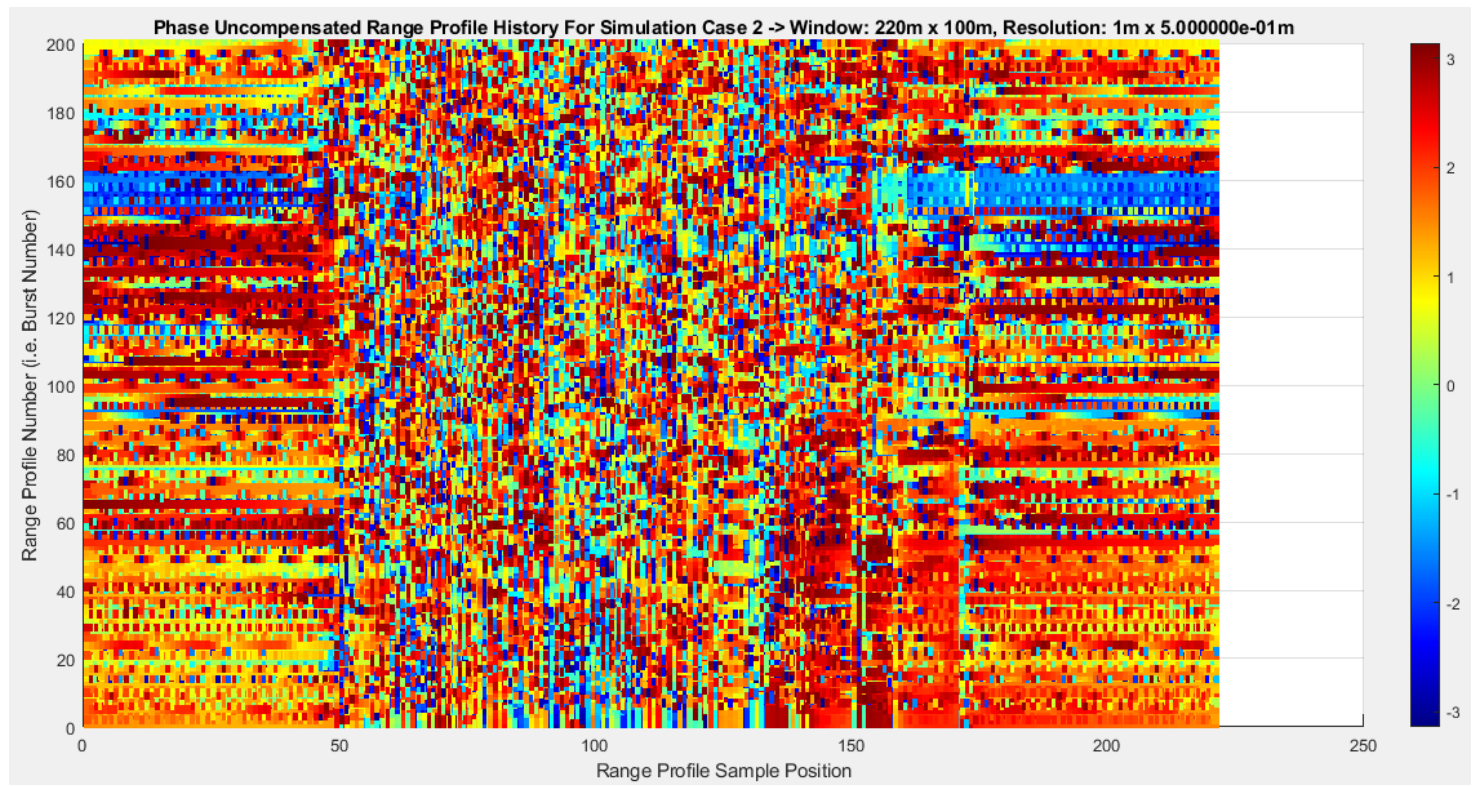

Figure C-1. 9: Simulation Case 3 Complex baseband phase history. 


\section{C.2 Sub-Appendix}

HRRPs and phase histories from chirp simulations.

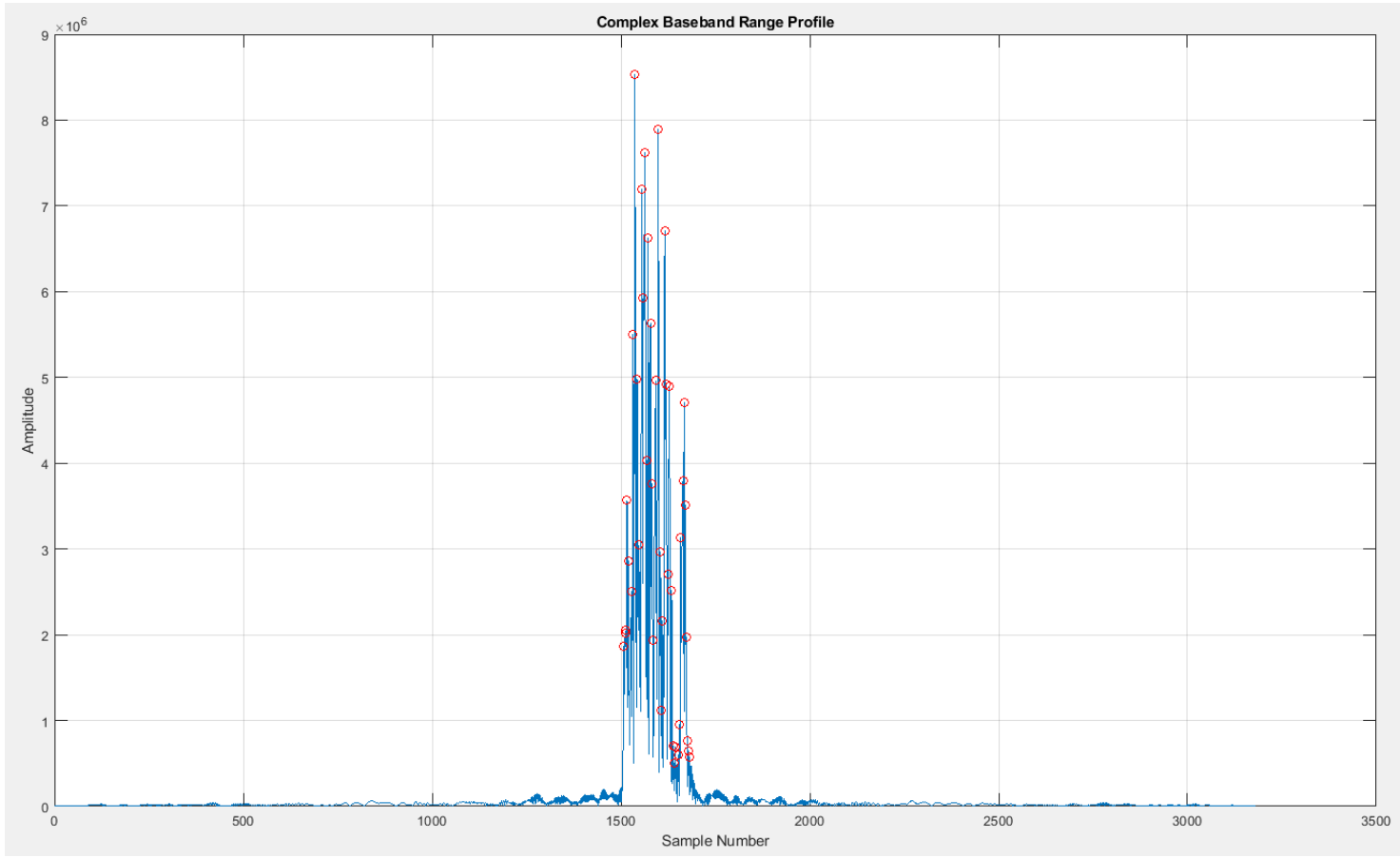

Figure C-2. 1: Chirp Radar simulation case 2 a/b, complex baseband range profile. 


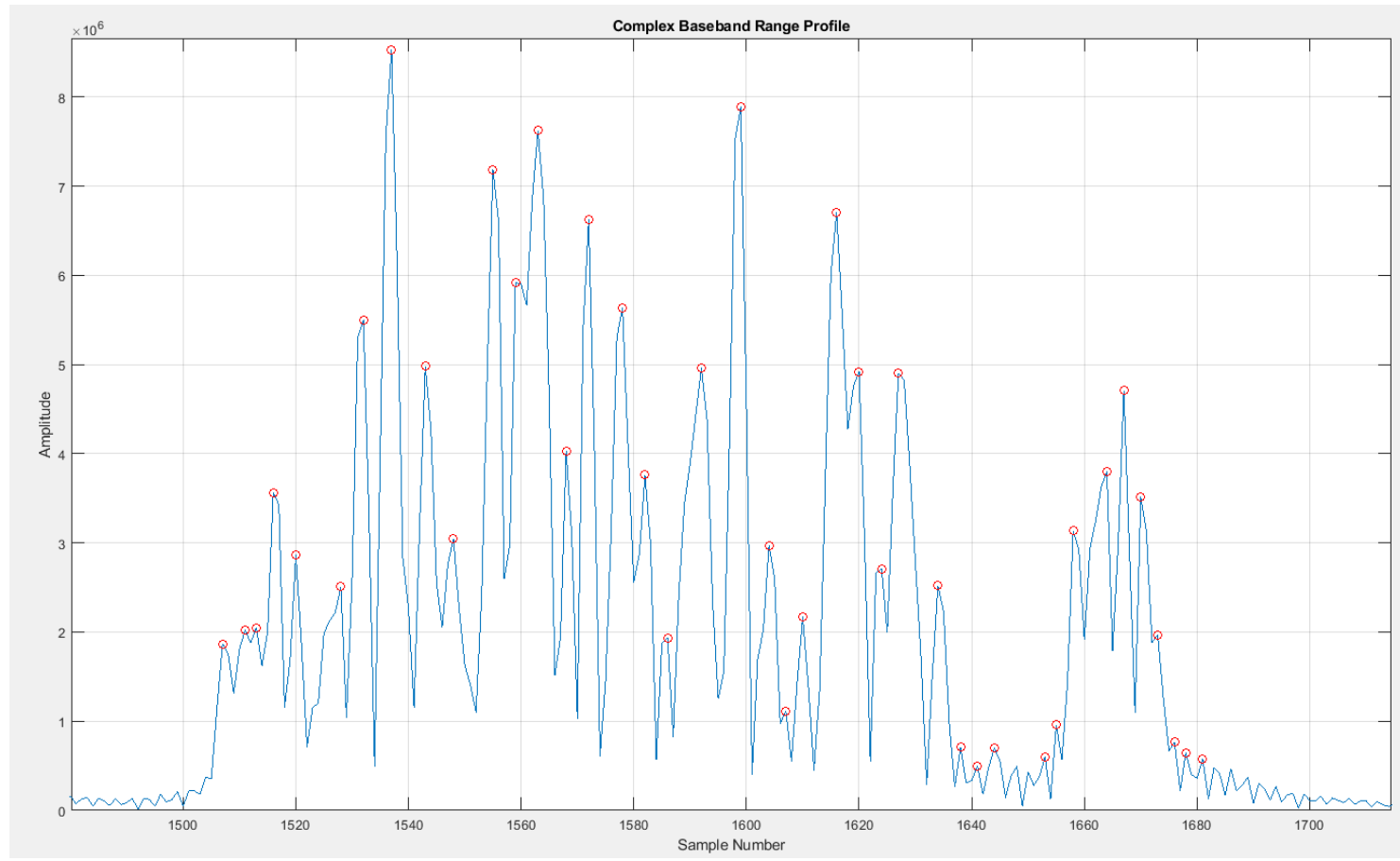

Figure C-2. 2: Chirp Radar simulation case 2 a/b, complex baseband range profile, zoomed in.

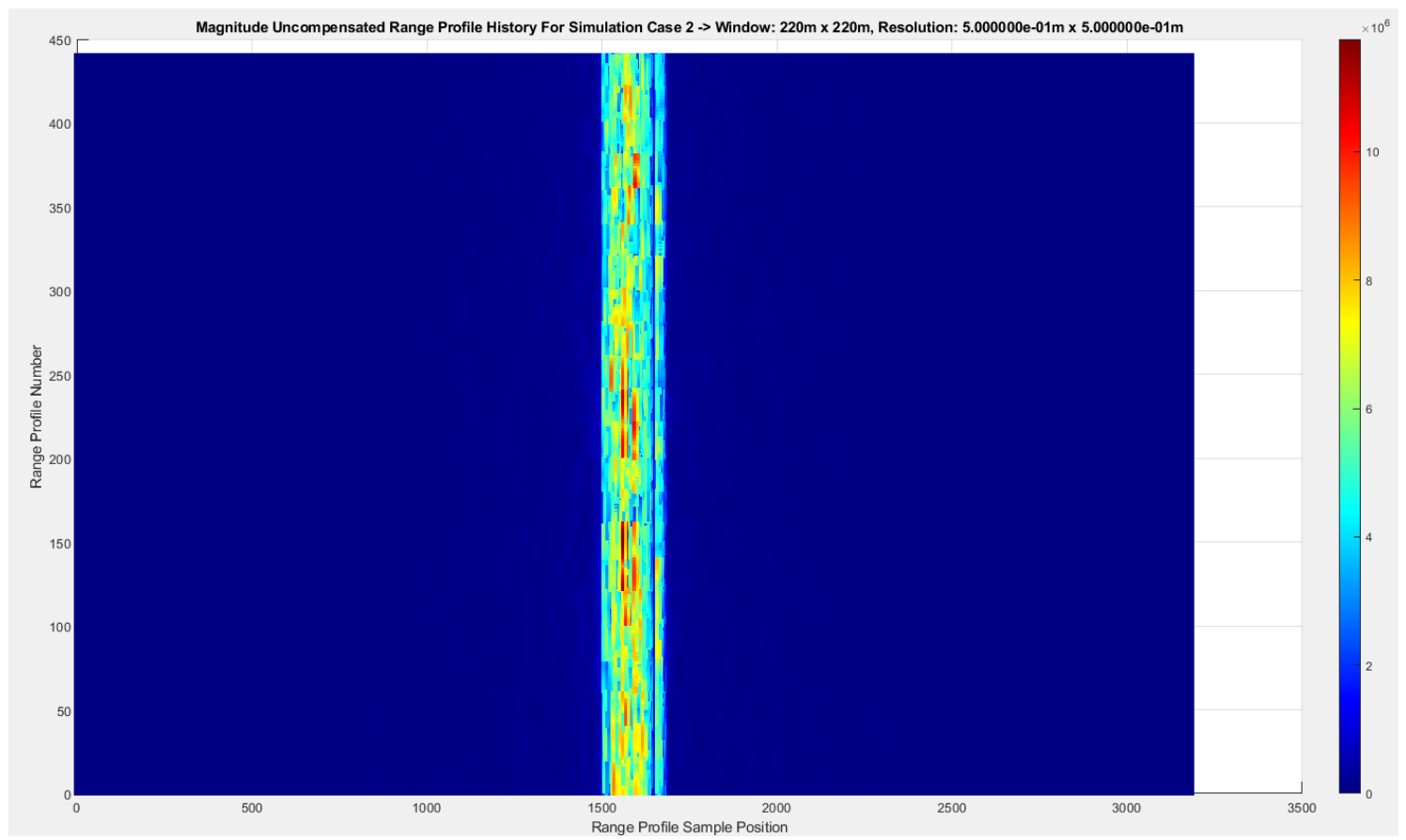

Figure C-2. 3: Chirp Radar simulation case 2a, magnitude of complex baseband range profile history. 


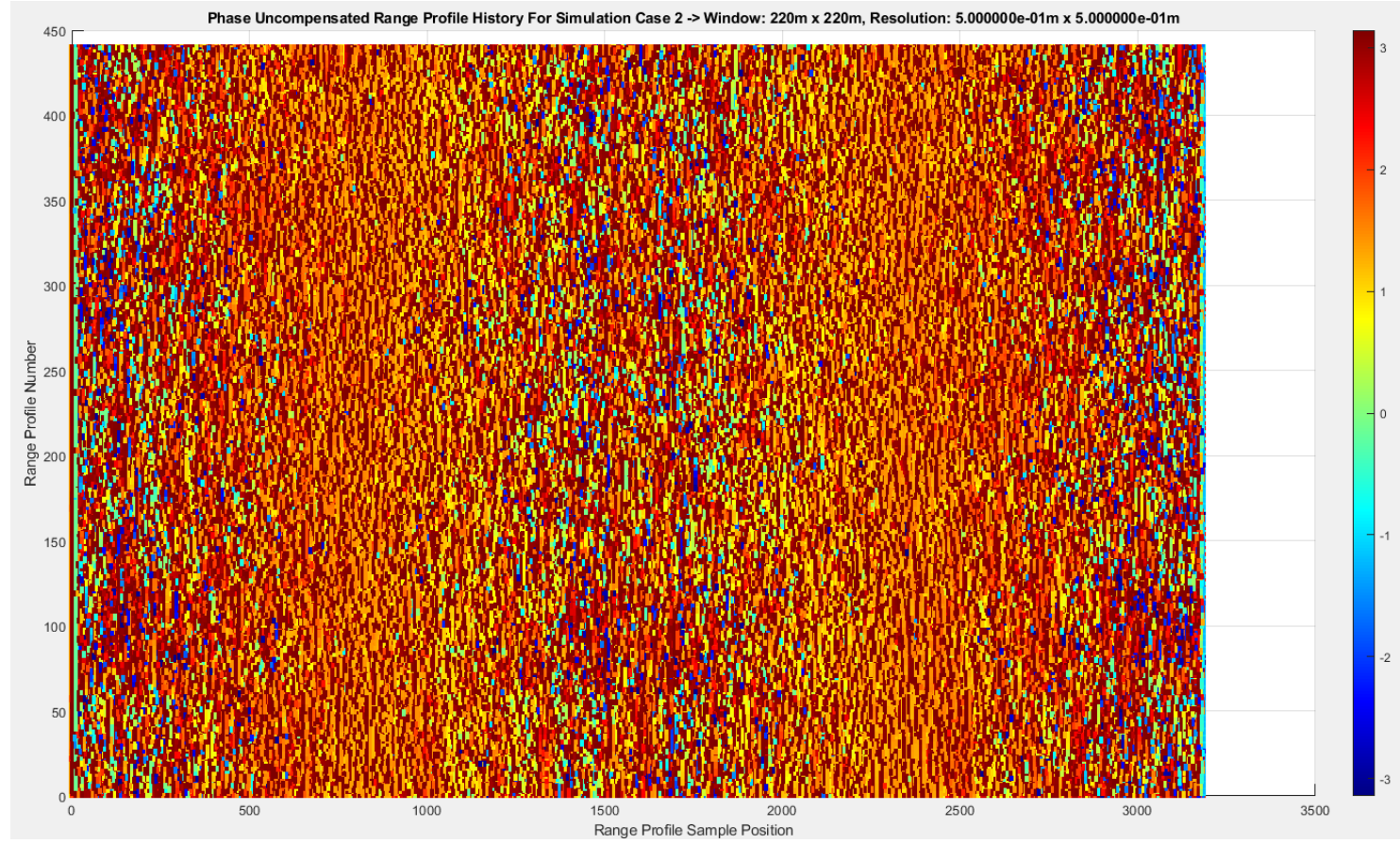

Figure C-2. 4: Chirp Radar simulation case 2a, complex baseband phase history.

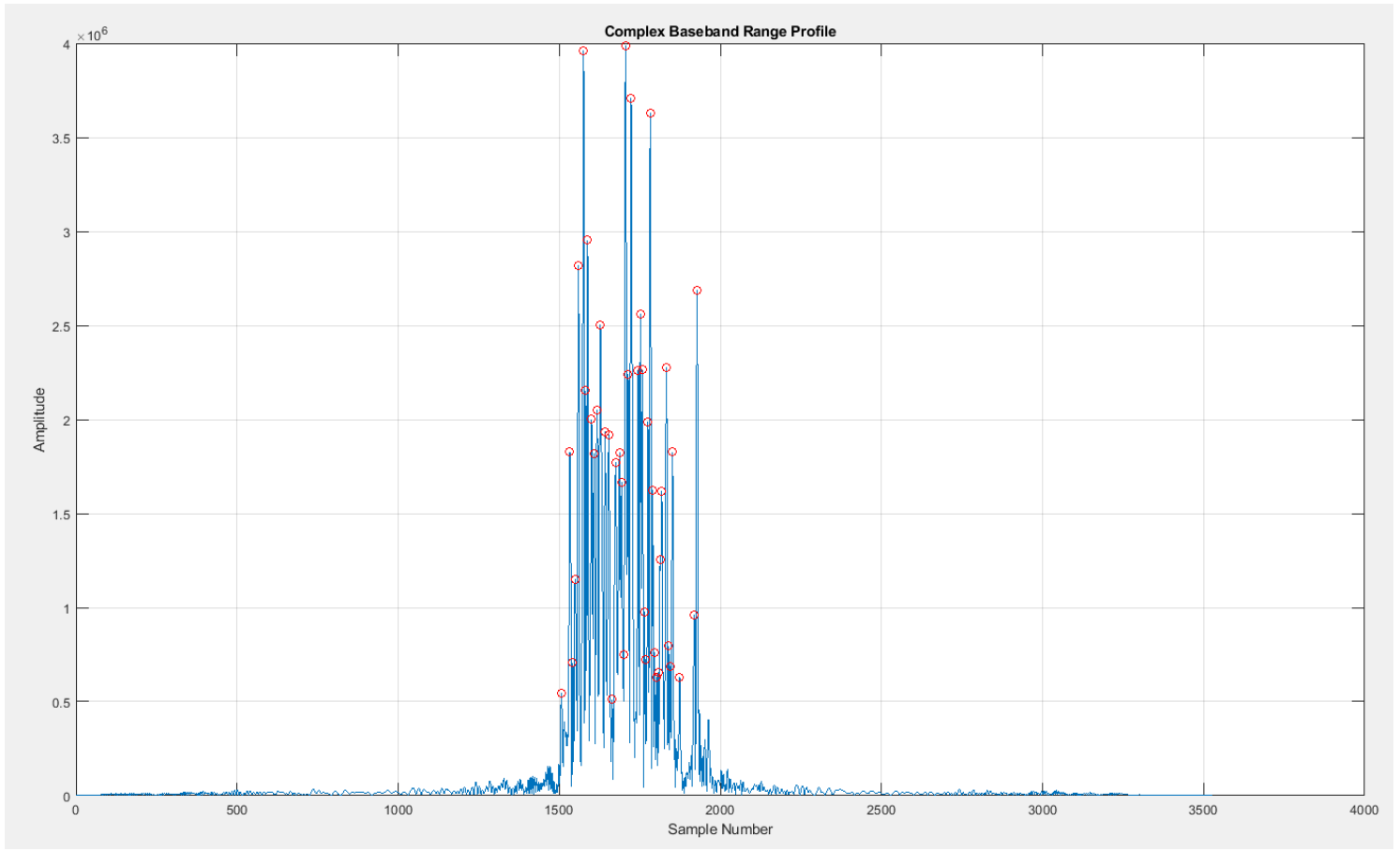

Figure C-2. 5: Complex baseband range profile Simulation Case 3. 


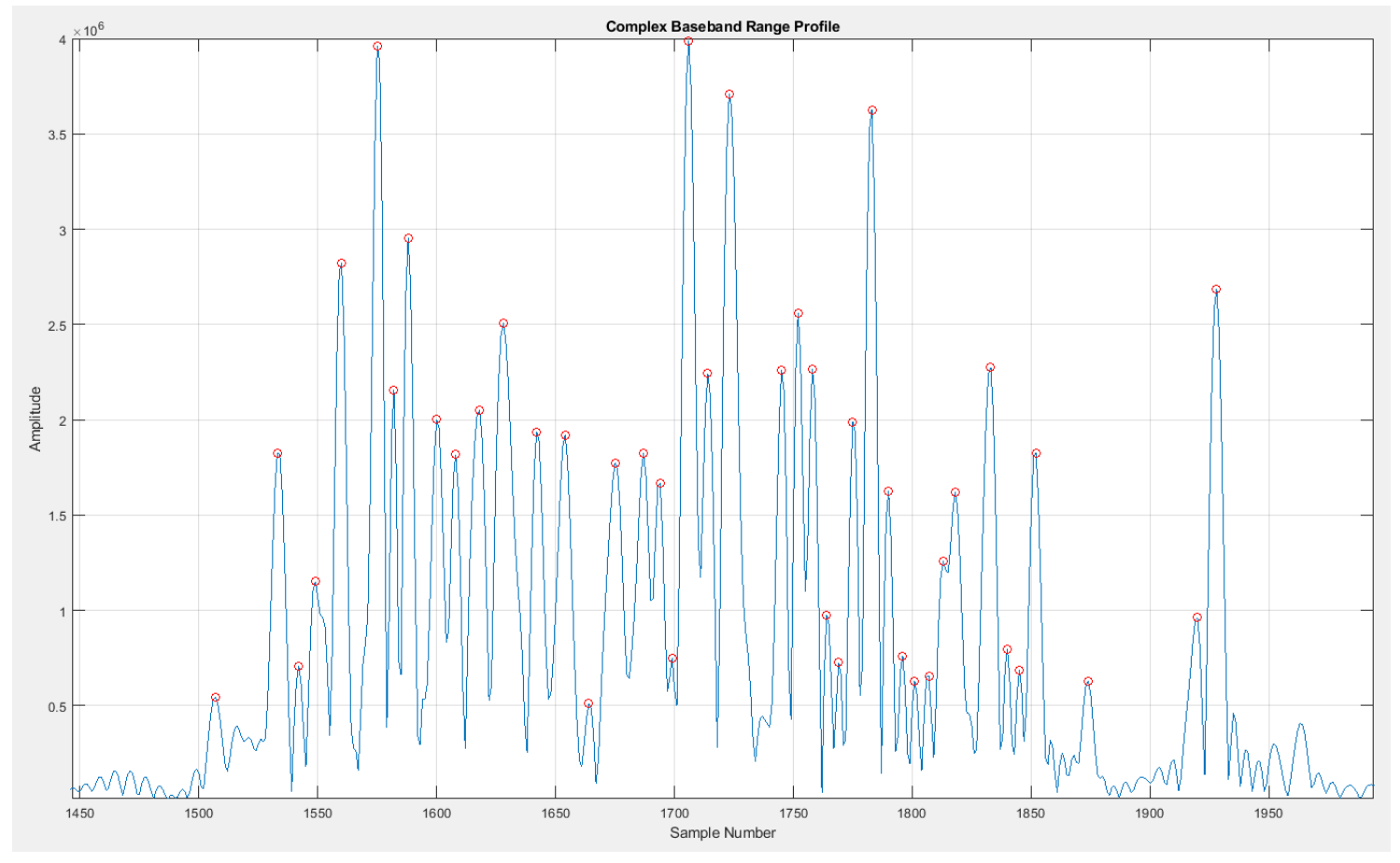

Figure C-2. 6: Complex baseband range profile Simulation Case 3, zoomed in.

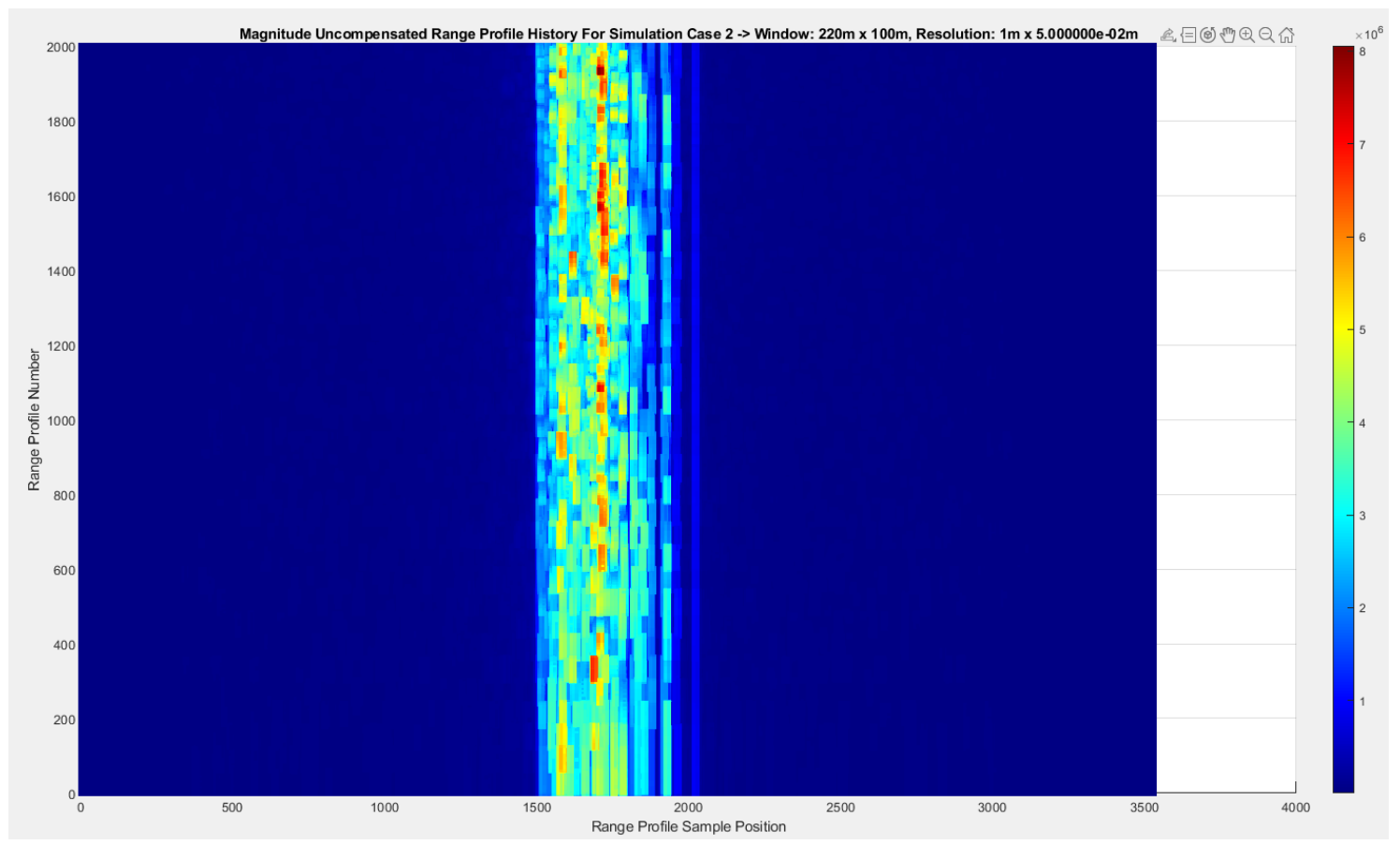

Figure C-2. 7: Chirp Radar simulation case 3, magnitude of complex baseband range profile history. 


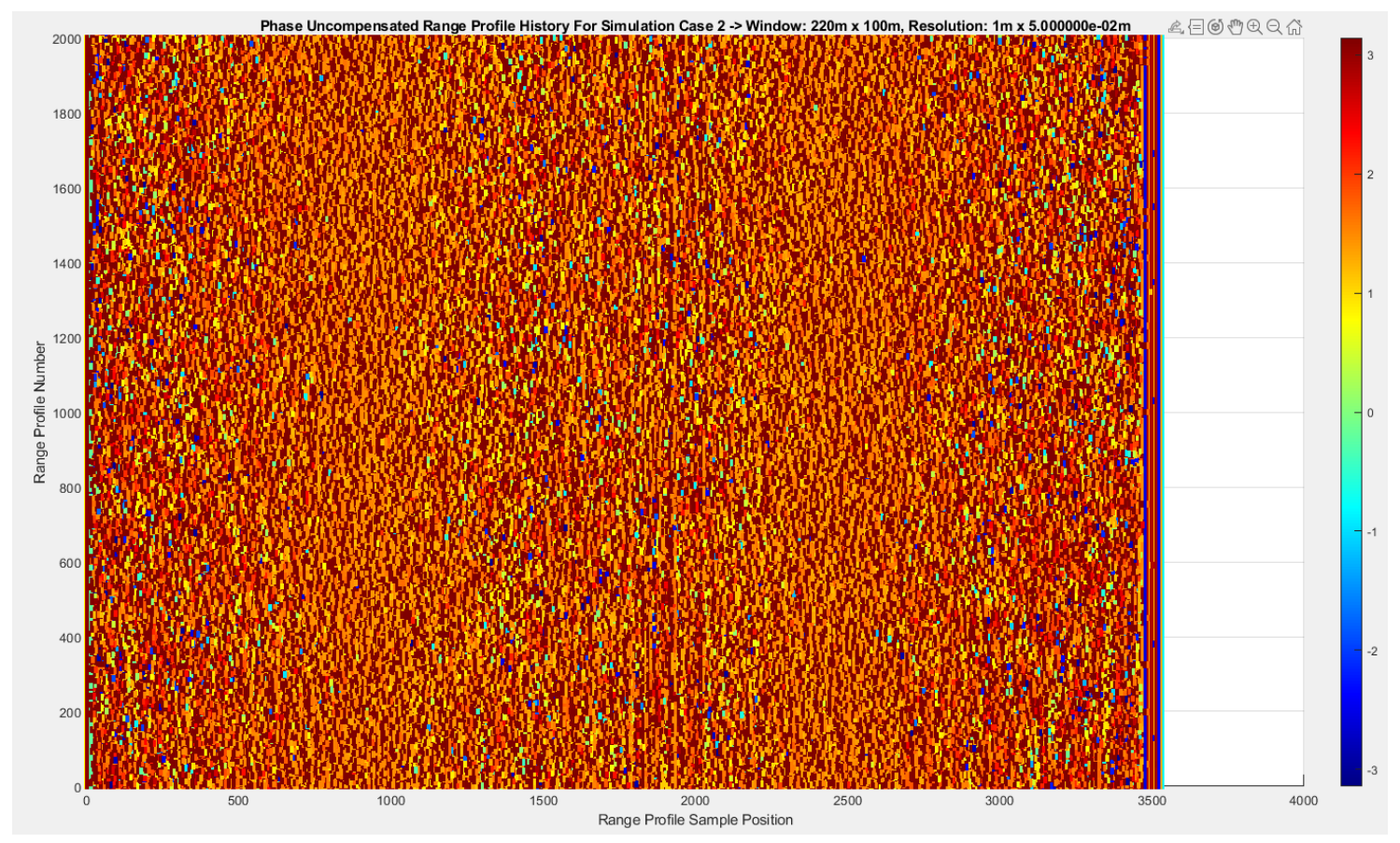

Figure C-2. 8: Chirp Radar simulation case 3, complex baseband phase history. 


\section{C.3 Sub-Appendix}

Range-Doppler images from stepped-frequency data.

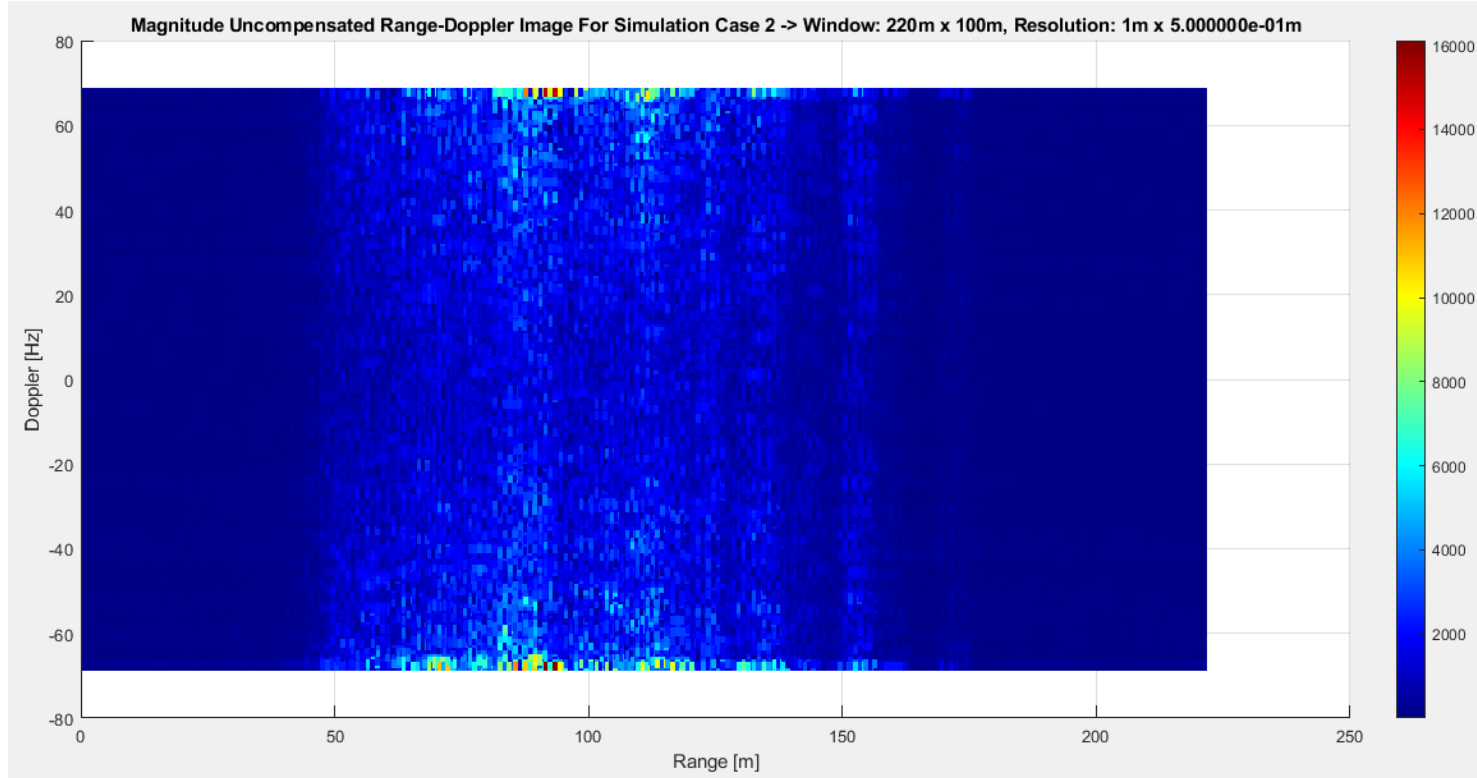

Figure C-3. 1: Stepped-frequency simulation Case 3 Range Doppler image, uncompensated complex baseband data.

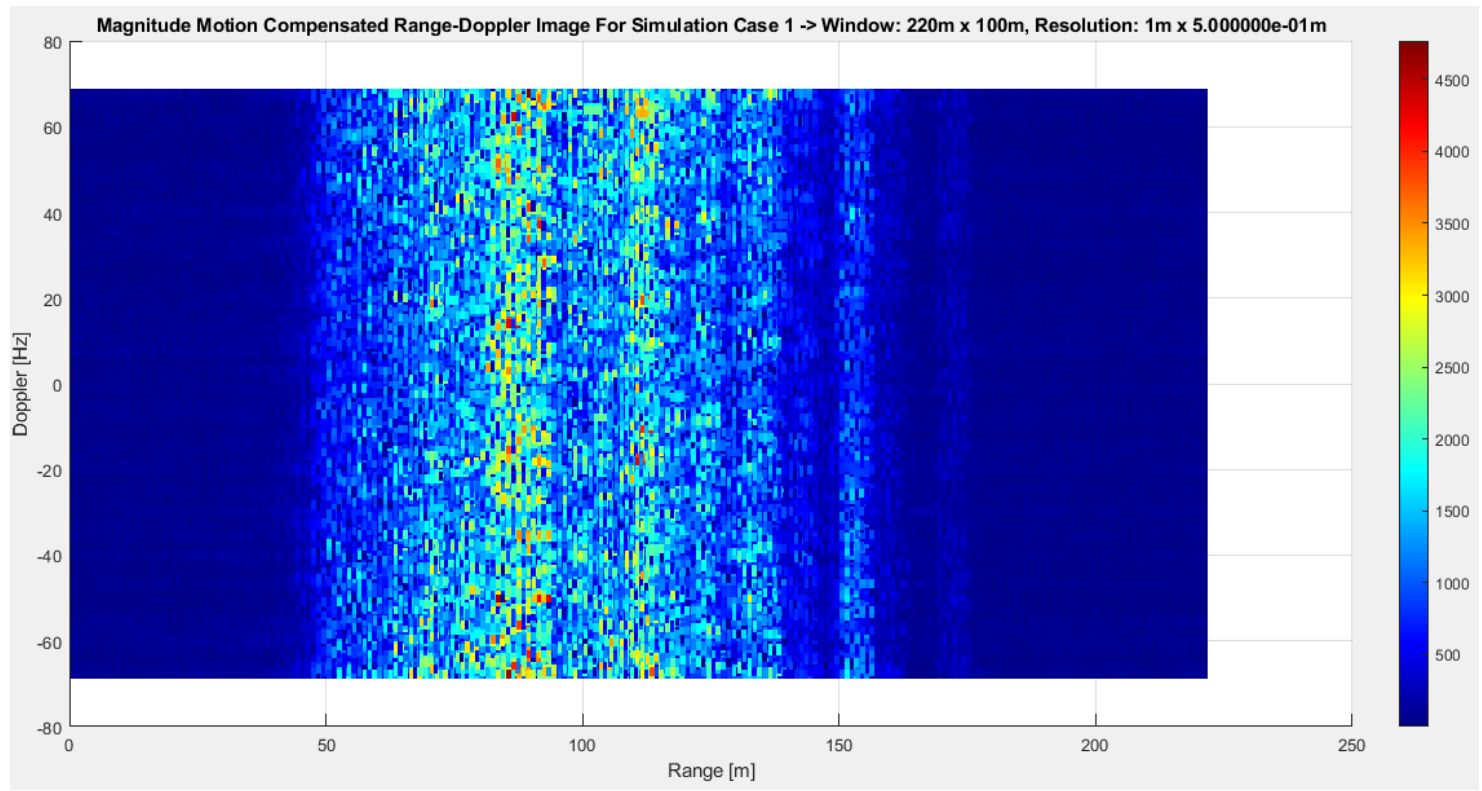

Figure C-3. 2: : Stepped-frequency simulation Case 3 Range Doppler image, range and Doppler tracked complex baseband data. 


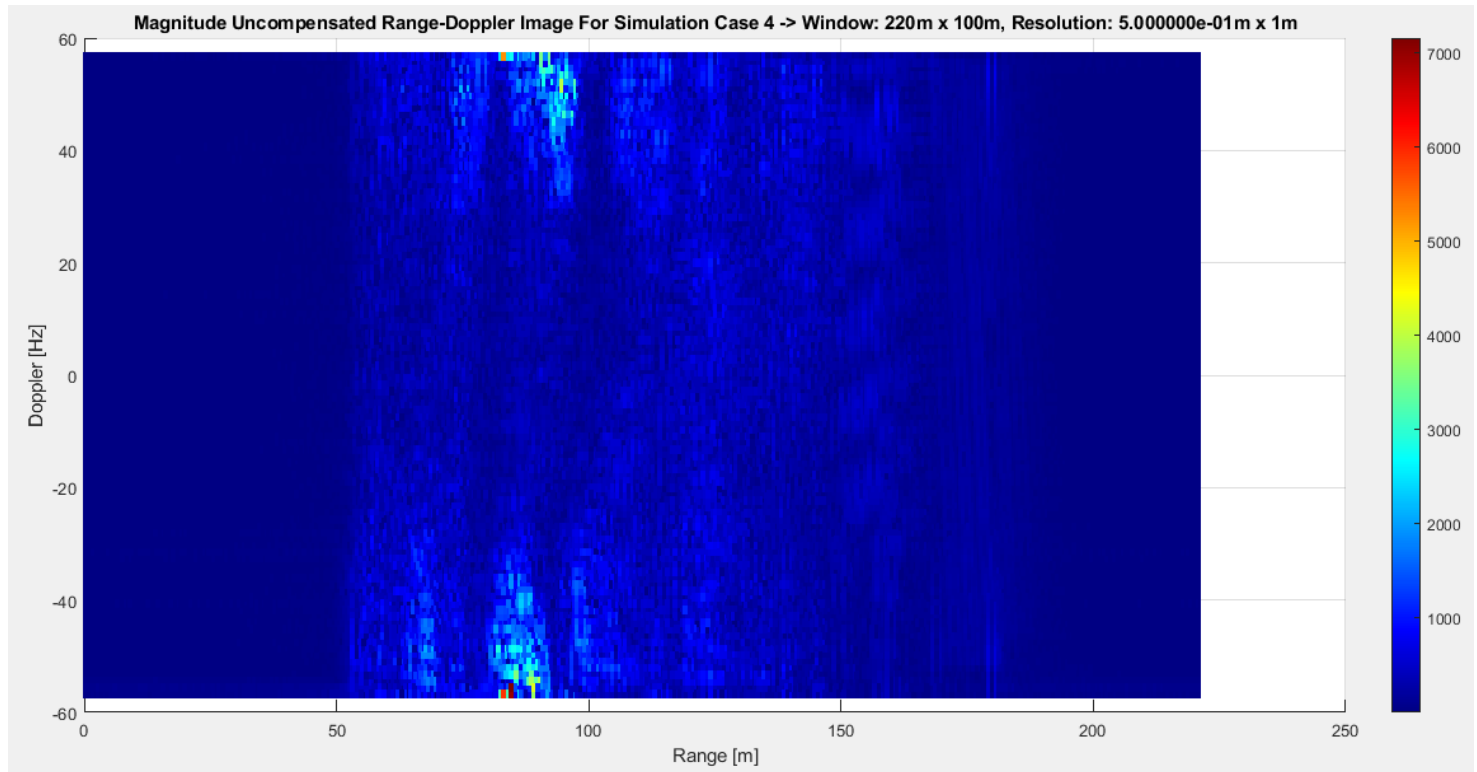

Figure C-3. 3: Stepped-frequency simulation Case 4 Range Doppler image, uncompensated complex baseband data.

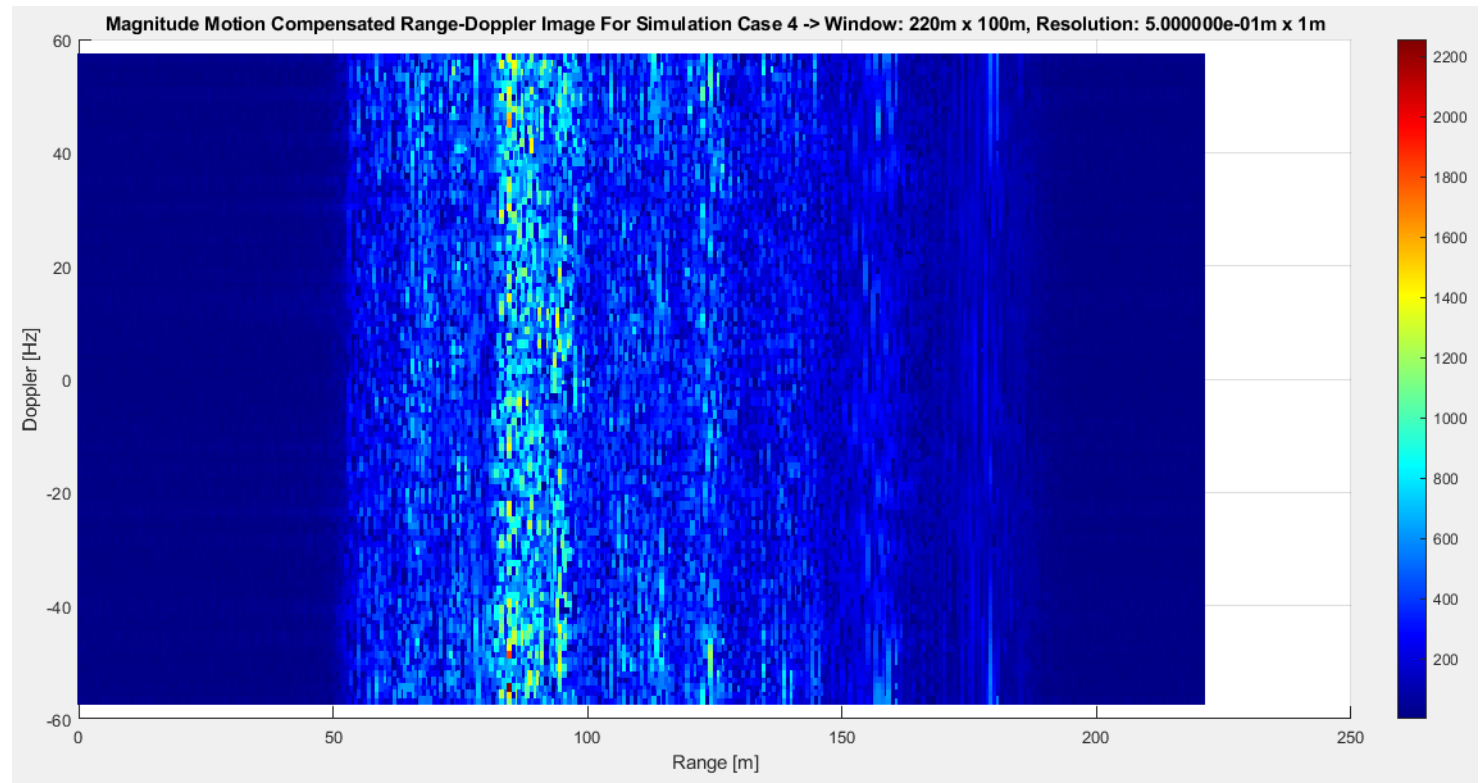

Figure C-3. 4: Stepped-frequency simulation Case 4 Range Doppler image, range and Doppler tracked complex baseband data. 


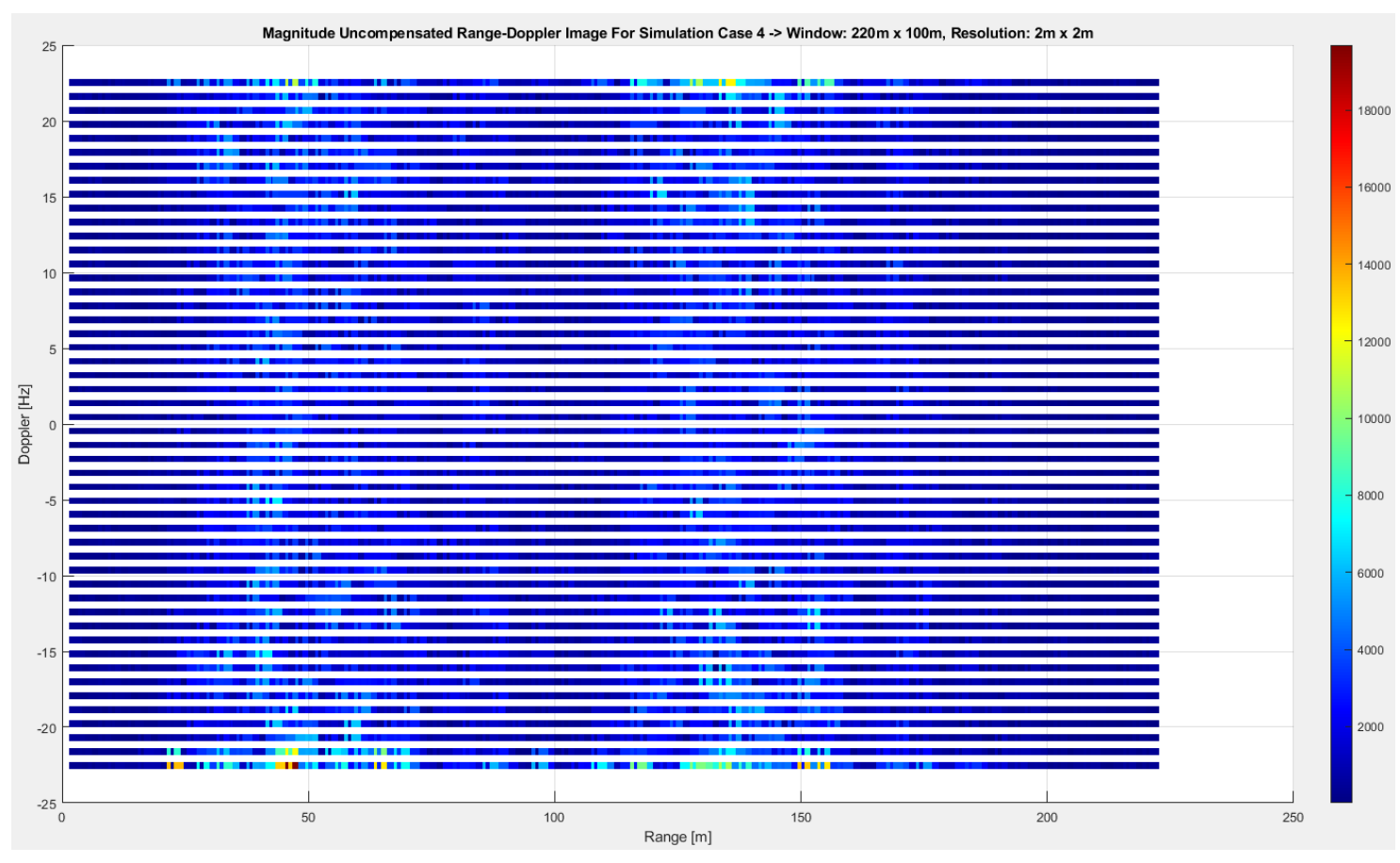

Figure C-3. 5: Stepped-frequency simulation Case 5 Range Doppler image,

uncompensated complex baseband data.

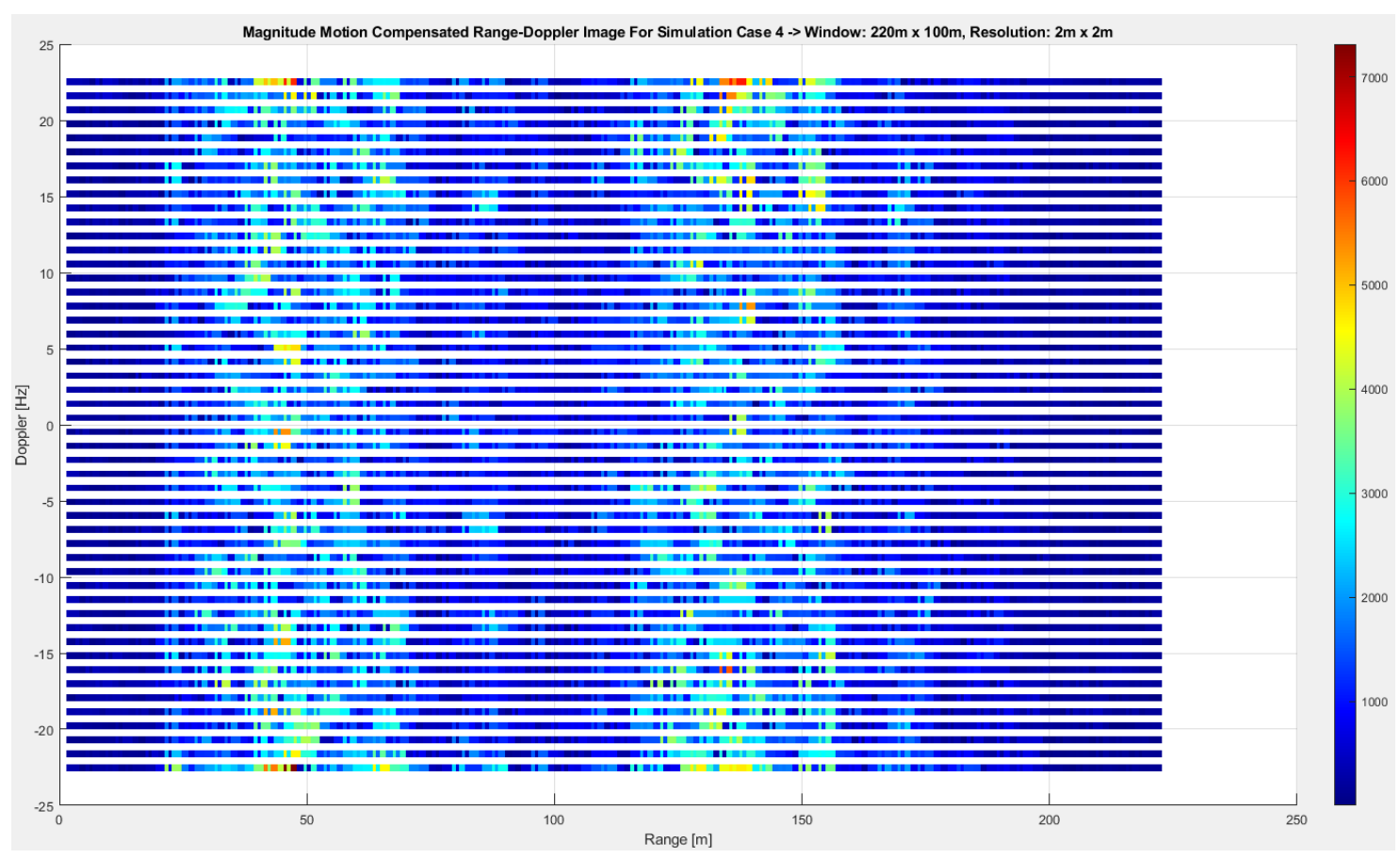

Figure C-3. 6: Stepped-frequency simulation Case 5 Range Doppler image, range and Doppler tracked complex baseband data. 


\section{C.4 Sub-Appendix}

Range-Doppler images from chirp data.

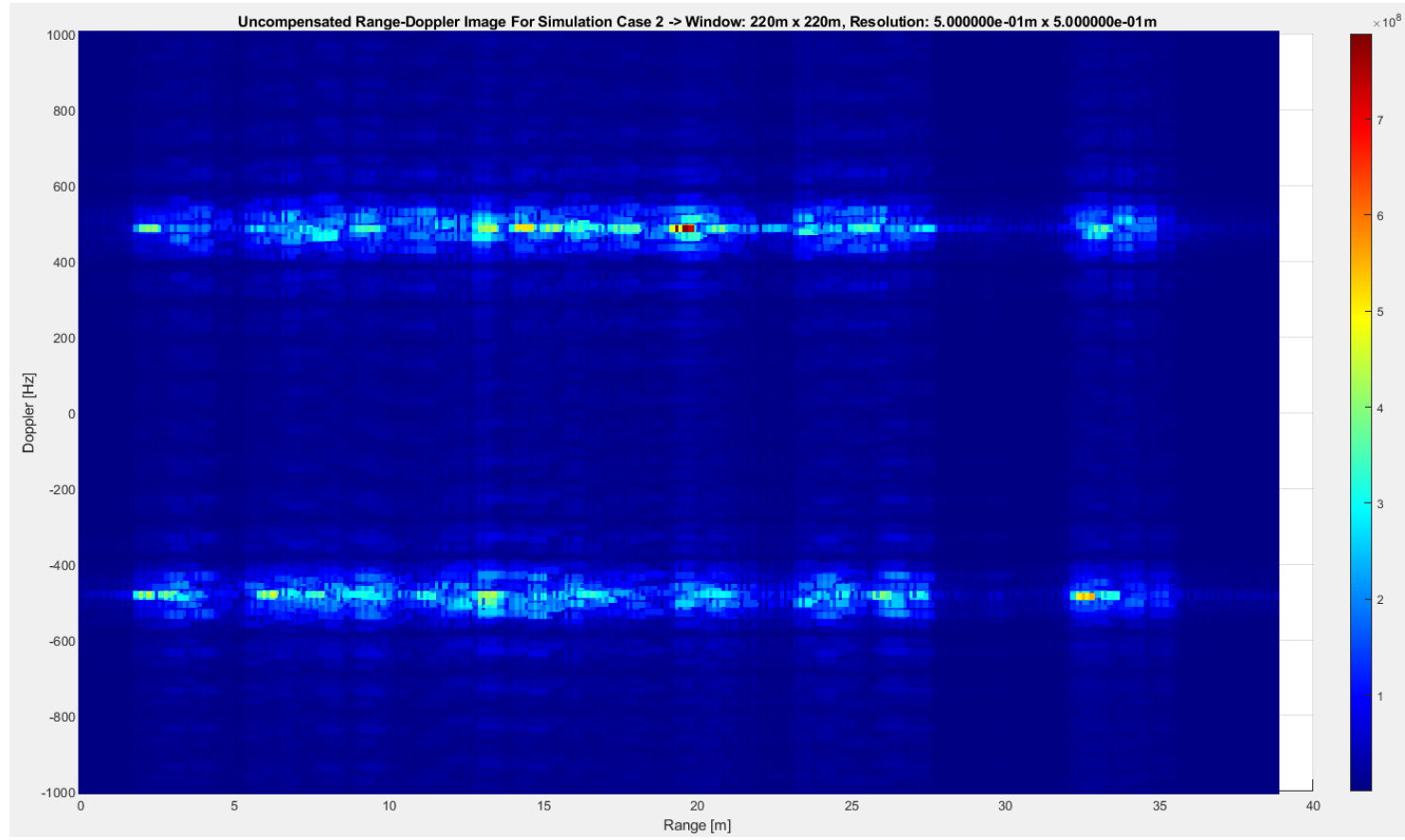

Figure C-4. 1: Uncompensated Range-Doppler image for Chirp Simulation case 2a. 


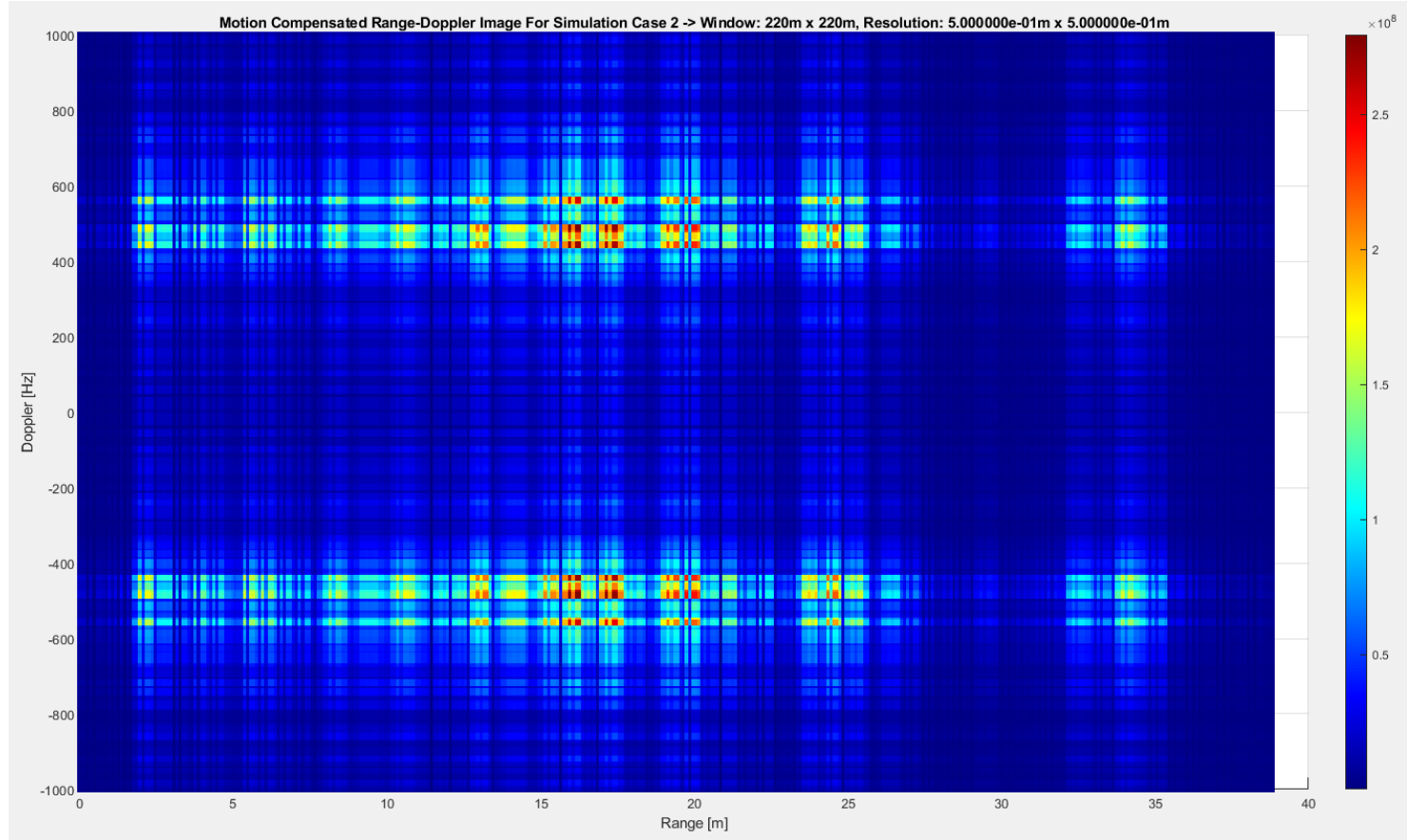

Figure C-4. 2: Motion Compensated Range-Doppler image for Chirp Simulation case $2 \mathbf{a}$.

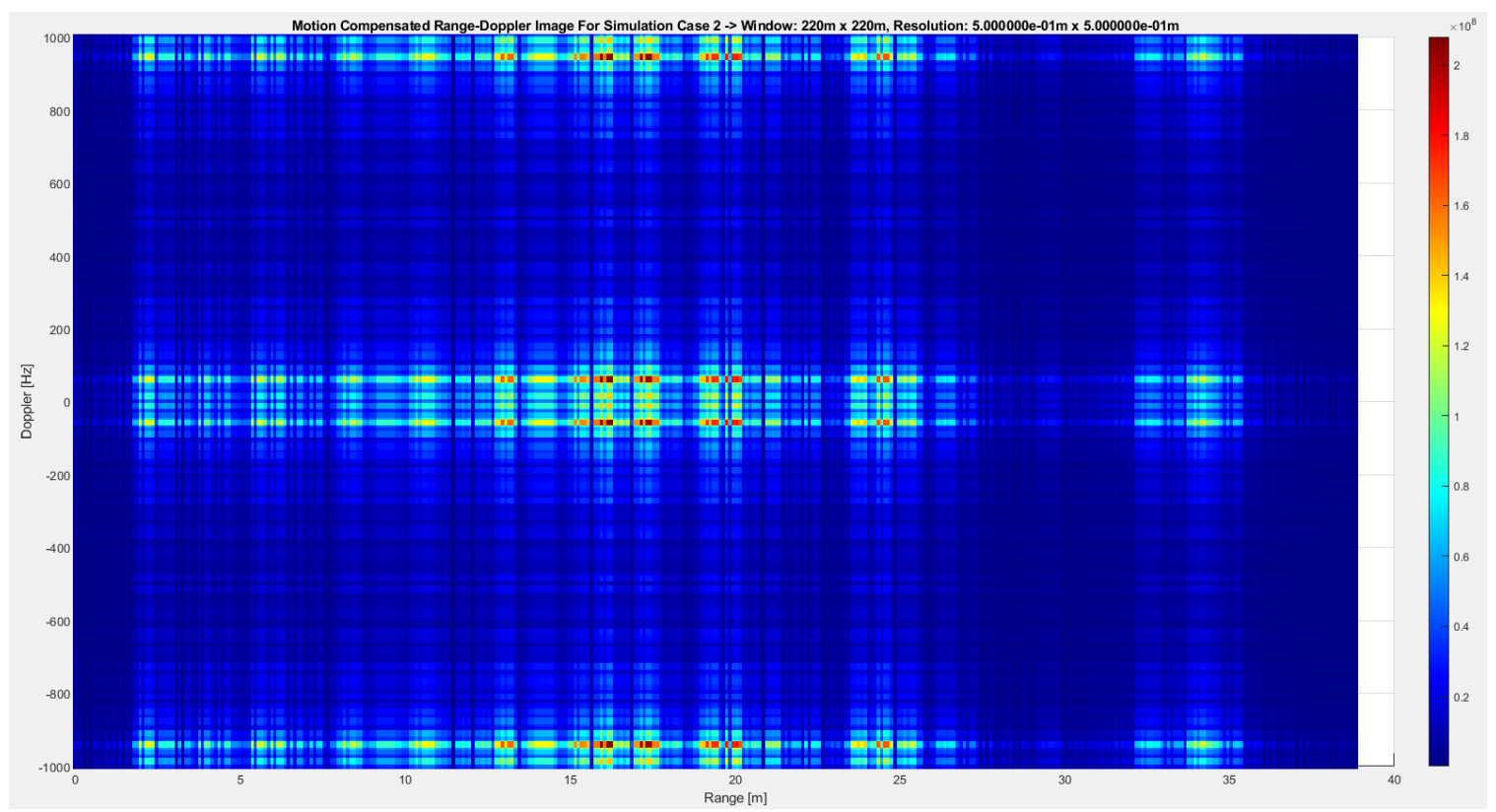

Figure C-4. 3: Range-Doppler image for Chirp case 2a, Doppler corrected by 500

Hz. 


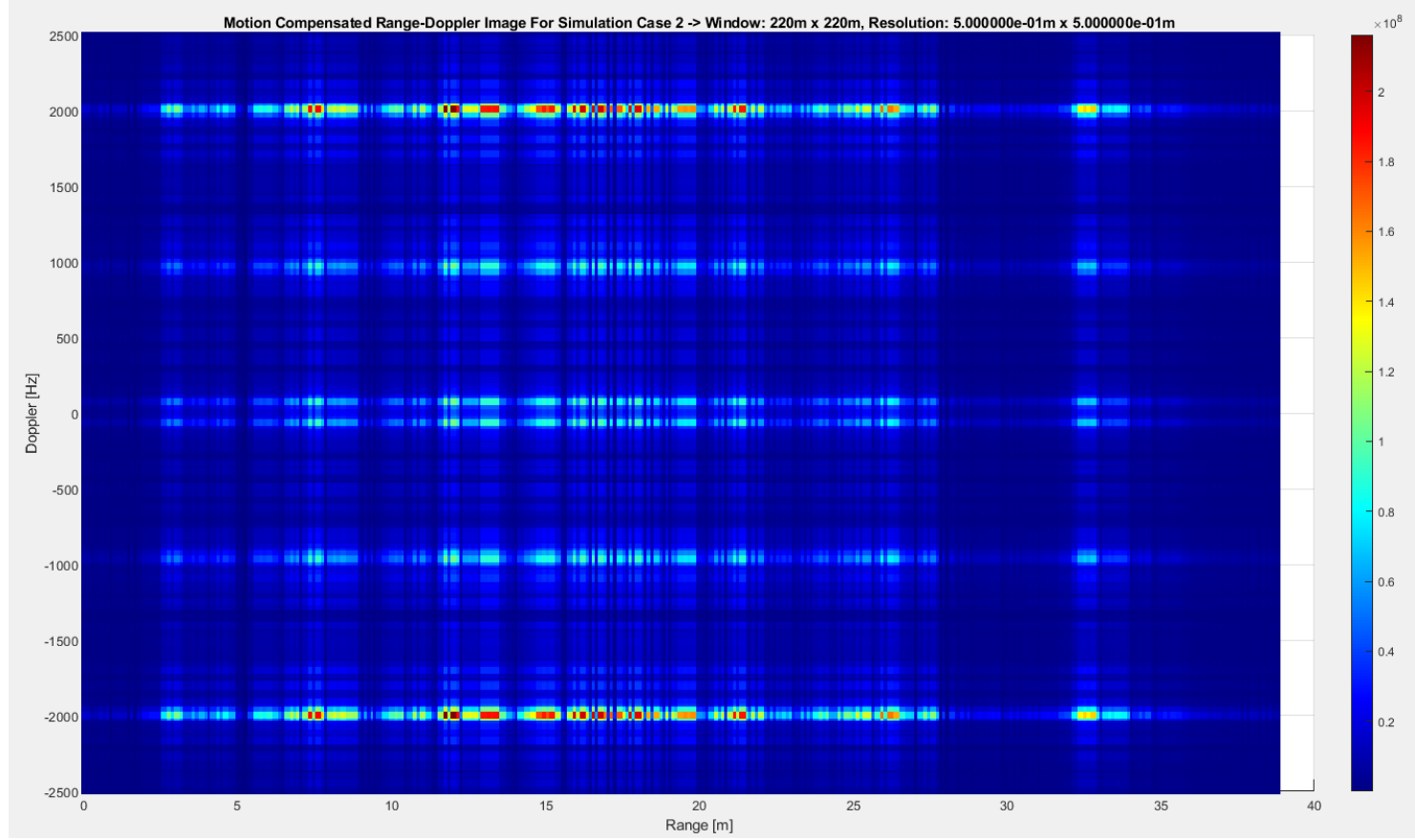

Figure C-4. 4: Motion Compensated Range-Doppler image for Chirp Simulation case $\mathbf{2 b}$.

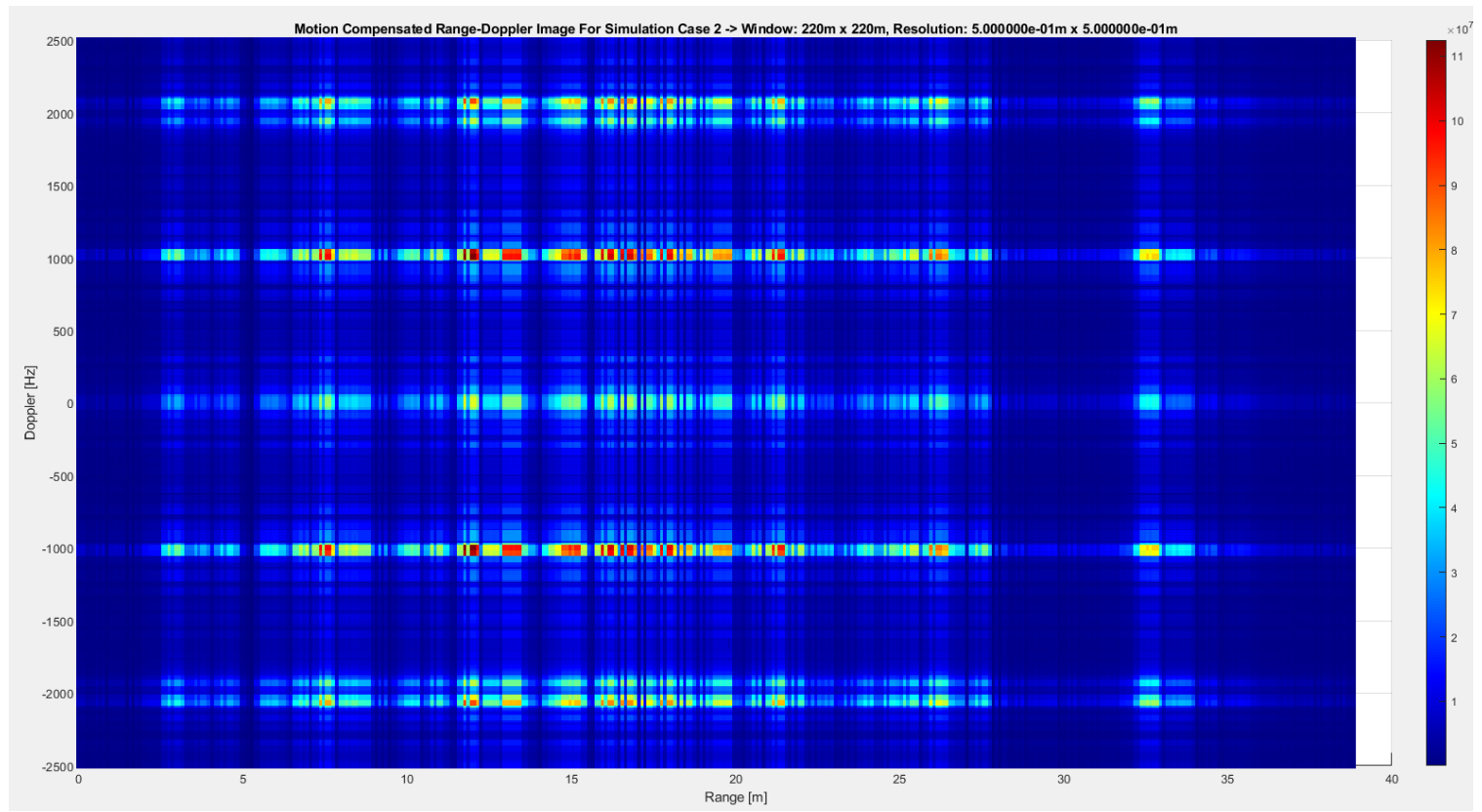

Figure C-4. 5: Range-Doppler image for Chirp case 2b, Doppler corrected by 2000

Hz. 


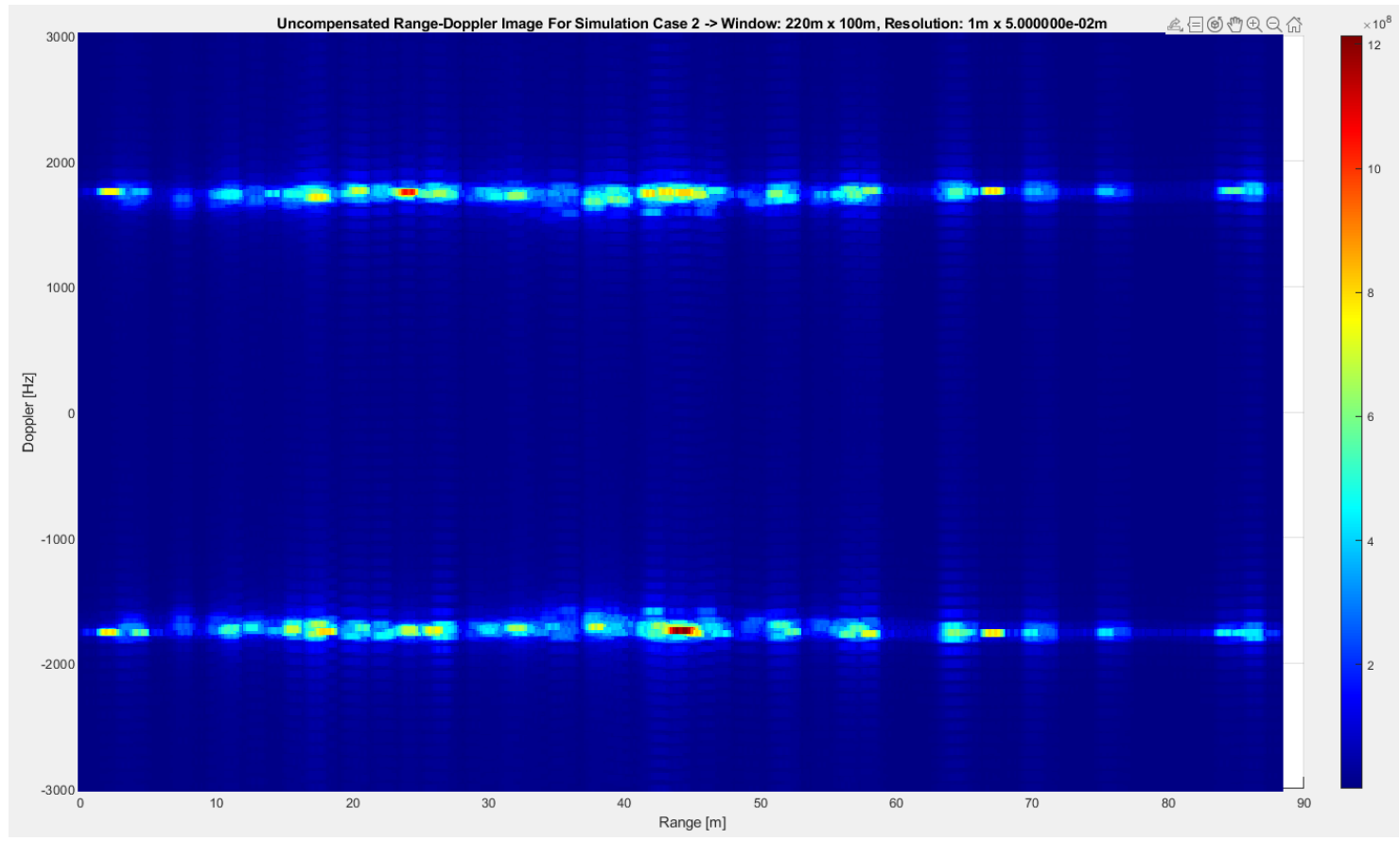

Figure C-4. 6: Uncompensated Range-Doppler image for Simulation case 3.

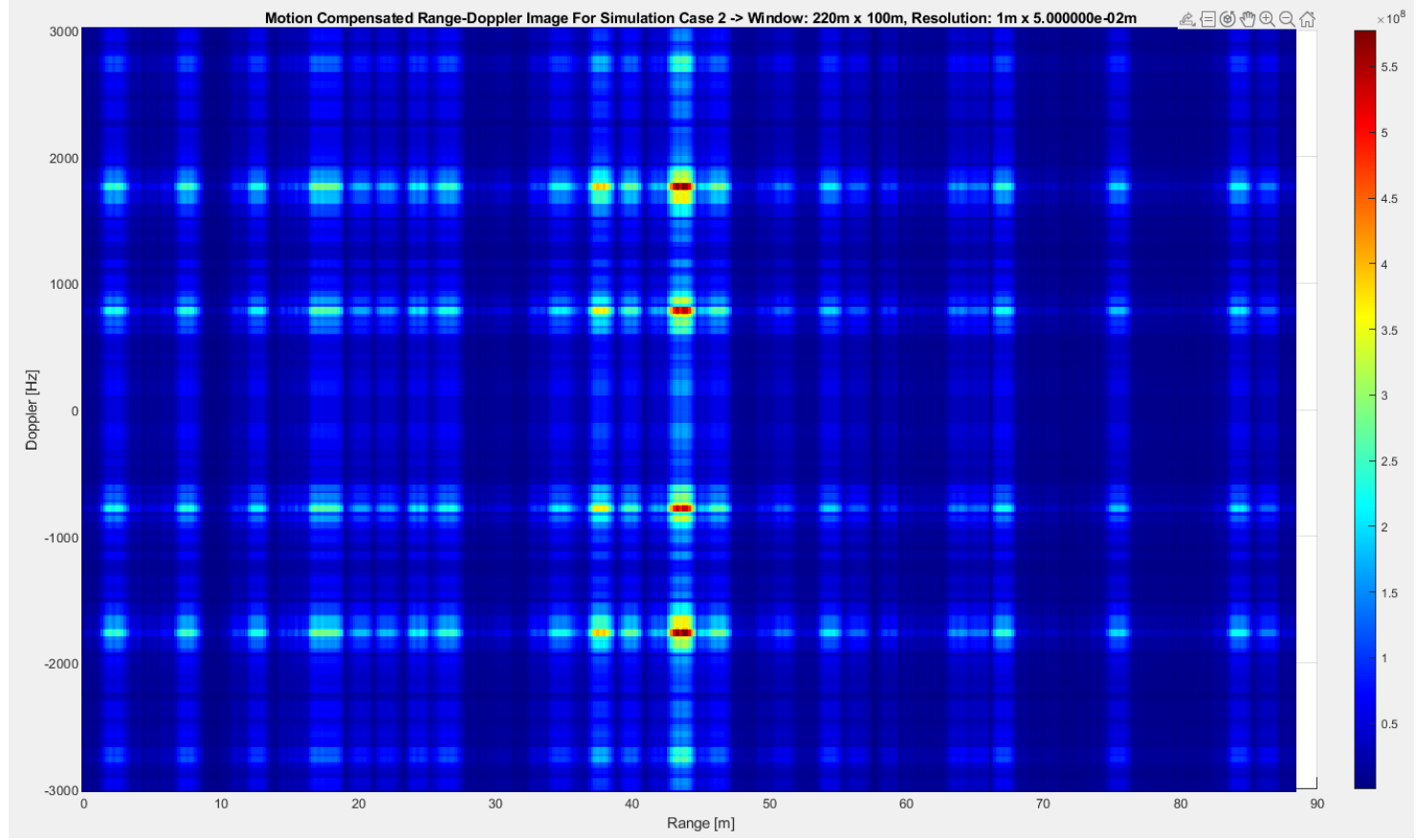

Figure C-4. 7: Motion compensated Range-Doppler image for Chirp Simulation case 3. 


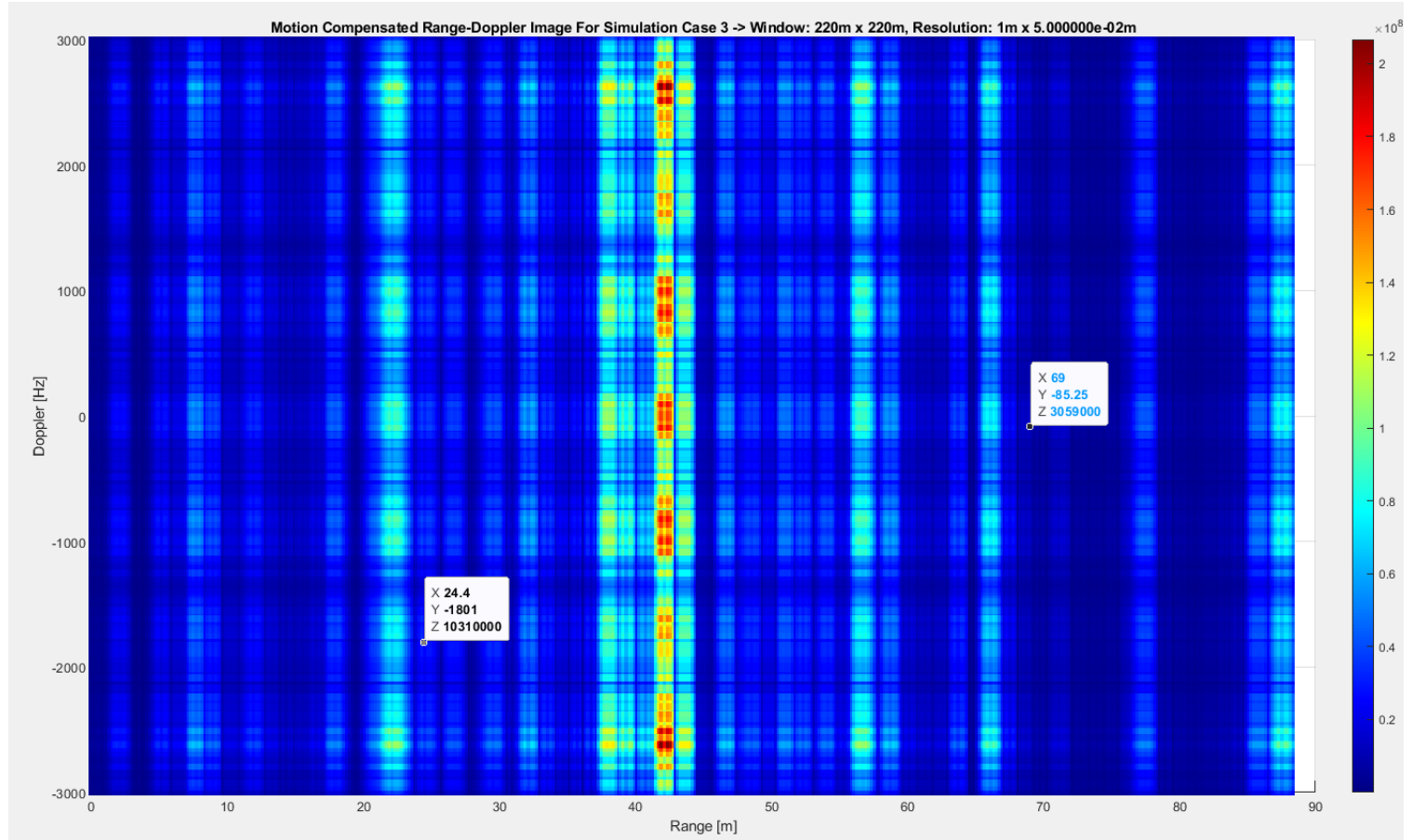

Figure C-4. 8: Range-Doppler image for Chirp case 3, Doppler corrected by $800 \mathrm{~Hz}$.

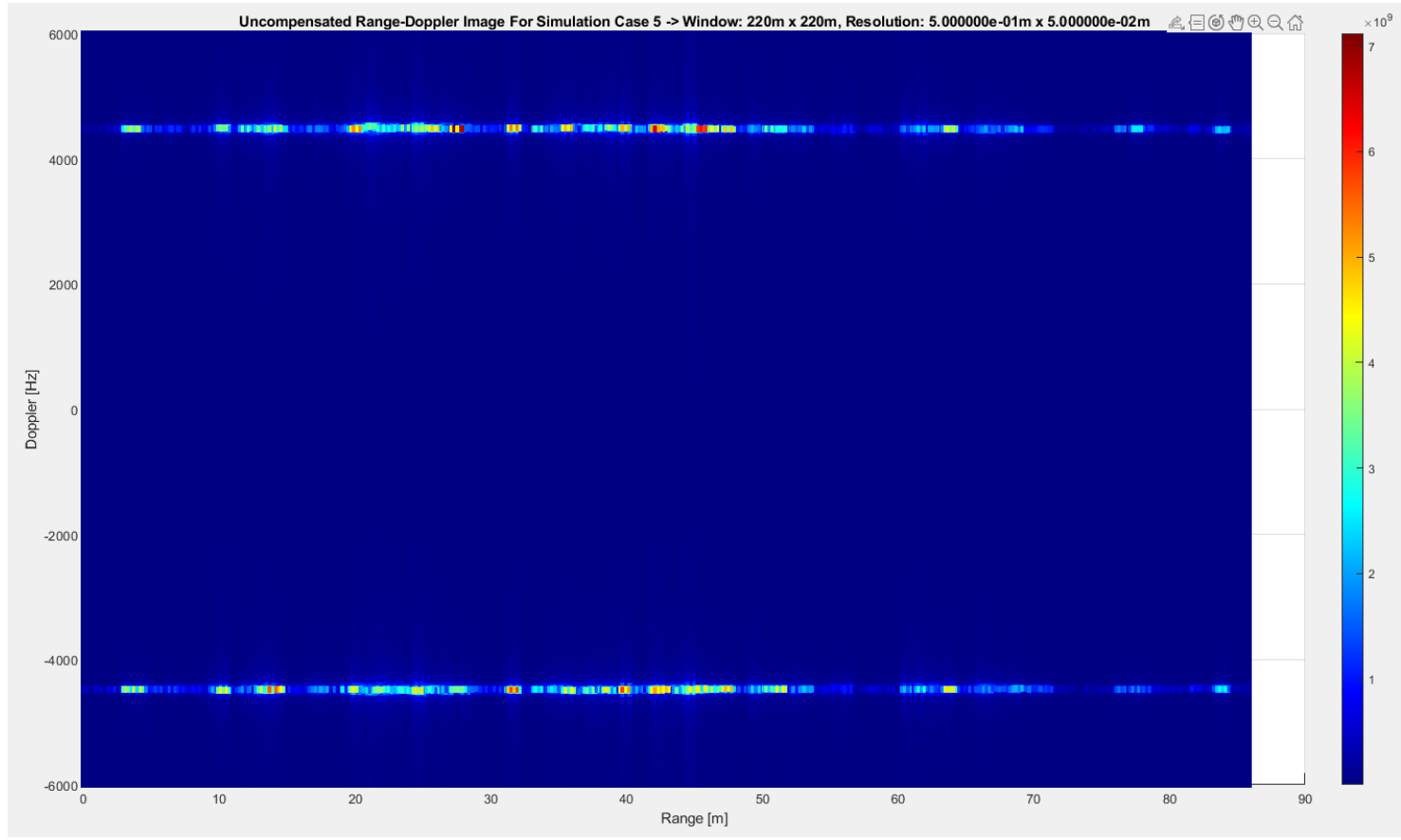

Figure C-4. 9: Uncompensated Range-Doppler image for Chirp Simulation case 5. 


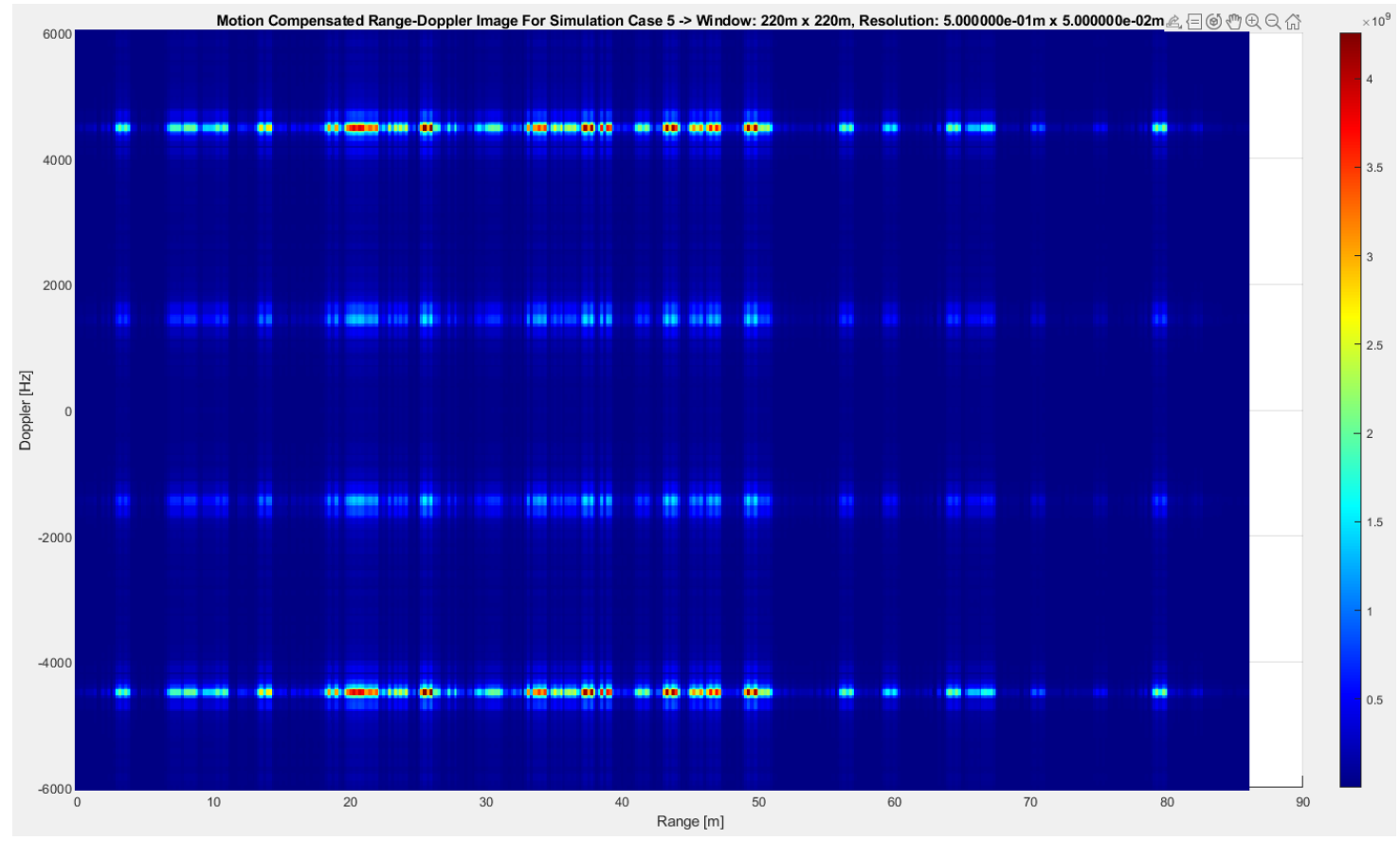

Figure C-4. 10: Motion compensated Range-Doppler image for Chirp Simulation

case 5.

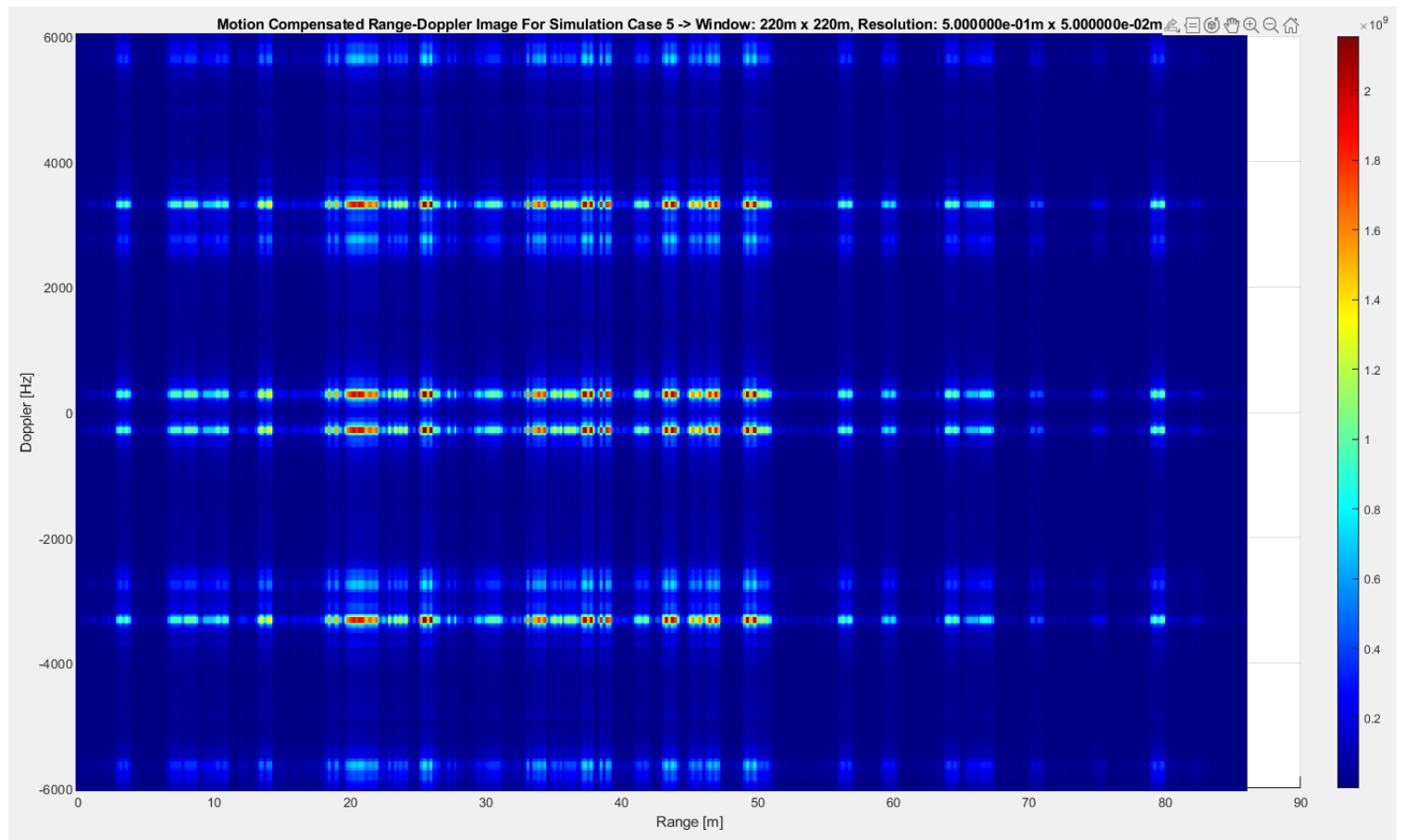

Figure C-4. 11: Range-Doppler image for Chirp case 5, Doppler corrected by 4200

Hz. 


\section{References}

[1] D. Gibbins, J. Symons and B. Haywood, "Ship motion estimation from ISAR data" ISSPA '99. Proceedings of the Fifth International Symposium on Signal Processing and its Applications (IEEE Cat. No.99EX359), Brisbane, Queensland, Australia, vol.1, pp. 333-336, 1999. Accessed 30-July-2020. [Online] Available: doi:

10.1109/ISSPA.1999.818180.

[2] A. Doerry, "Ship Dynamics for Maritime ISAR Imaging", U.S. Department of Energy Office of Scientific and Technical Information, 2020. Accessed: 13-Nov-2019. [Online]. Available: https://www.osti.gov/biblio/929523-ship-dynamics-maritimeisar-imaging.

[3] M. McDonald, "SHF SATCOM Terminal Ship-Motion Study", Naval Command Control and Ocean Surveillance Center, San Diego, 1993. Accessed: 13-Nov-2019. [Online] Available:

https://www.google.com/url?sa=t\&rct=j\&q=\&esrc=s\&source=web\&cd=\&cad=rja\&u act $=8 \& v e d=2$ ahUKEwj5qO-

8iYbwAhVMRqwKHRxuCEUQFjAAegQIBhAD\&url=https\%3A\%2F\%2Fapps.dtic .mil\%2Fdtic\%2Ftr\%2Ffulltext\%2Fu2\%2Fa267884.pdf\&usg=AOvVaw3FOcs8QkRmb1ITTxM8ZnX

[4] T. Li-bo, L. Dao-jing, H. Wen and C. Fang, "High Resolution SAR Imaging of Moving Ships," Proceedings. 2005 IEEE International Geoscience and Remote Sensing Symposium. IGARSS '05., Seoul, 2005, pp. 3329-3332, 2005. Accessed 30July-2020. [Online] Available: doi: 10.1109/IGARSS.2005.1526554.

[5] G. Hajduch, J. M. Le Caillec and R. Garello, "Airborne High-Resolution ISAR Imaging of Ship Targets at Sea," in IEEE Transactions on Aerospace and Electronic Systems, vol. 40, no. 1, pp. 378-384, Jan. 2004. Accessed 30-July-2020. [Online] Available: doi: 10.1109/TAES.2004.1292177.

[6] J. A. Given and W. R. Schmidt, "Generalized ISAR - Part I: An Optimal Method for Imaging Large Naval Vessels," in IEEE Transactions on Image Processing, vol. 14, no. 11, pp. 1783-1791, Nov. 2005. Accessed 30-July-2020. [Online] Available: doi: 10.1109/TIP.2005.857283. 
[7] D. Pastina, A. Montanari and A. Aprile, "Motion Estimation and Optimum Time Selection for Ship ISAR Imaging," Proceedings of the 2003 IEEE Radar Conference (Cat. No. 03CH37474), Huntsville, AL, USA, pp. 7-14. 2003. Accessed 30-July2020. [Online] Available: doi: 10.1109/NRC.2003.1203371.

[8] D. Pastina, C. Spina and A. Aprile, "A Slope-Based Technique for Motion Estimation and Optimum Time Selection for ISAR Imaging of Ship Targets," 2006 14th European Signal Processing Conference, Florence, pp. 1-5, 2006. Accessed 30-July2020. [Online] Available: https://ieeexplore.ieee.org/document/7071176.

[9] T. G. Kostis, "Simulator Implementation of an Inverse Synthetic Aperture Radar System for an Extended Naval Target in a Three Dimensional Synthetic Environment," Tenth International Conference on Computer Modeling and Simulation (uksim 2008), Cambridge, UK, pp. 366-371, 2008. Accessed 31-July2020. [Online] Available: doi: 10.1109/UKSIM.2008.14.

[10] M. St. Denis and W. Pierson, "On The Motions of Ships in Confused Seas", Armed Services Technical Information Agency, Dayton, 1953. Accessed: 16-July-2019. [Online]. Available:

https://www.google.com/url? sa=t\&rct=j\&q=\&esrc=s\&source=web\&cd=\&cad=rja\&u act=8\&ved=2ahUKEwiohZaji4bwAhUBYKwKHSz0C44QFjAAegQIBhAD\&url=ht tps $\% 3 \mathrm{~A} \% 2 \mathrm{~F} \% 2 \mathrm{Fapps}$.dtic.mil\%2Fdtic\%2Ftr\%2Ffulltext $\% 2 \mathrm{Fu} 2 \% 2 \mathrm{~F} 045880$.pdf\&us $\mathrm{g}=\mathrm{AOvV}$ Vaw0vDBltVTBxujuM-dLgQwdd

[11] A. Baitis, W. Meyers, D. Woolaver and C. Lee, "A Seakeeping Comparison Between Three Monohulls, Two Swaths, And A Column-Stabilized Catamaran Designed For The Same Mission", National Technical Information Service - U.S. Department of Commerce, Bethesda, 1975. Accessed: 16-July-2019. [Online]. Available:

https://www.google.com/url?sa=t\&rct=j\&q=\&esrc=s\&source=web\&cd=\&cad=rja\&u act=8\&ved=2ahUKEwjOgf27i4bwAhUPA6wKHXSGCkEQFjAAegQIBhAD\&url= https $\% 3 \mathrm{~A} \% 2 \mathrm{~F} \% 2$ Fapps.dtic.mil\%2Fdtic\%2Ftr\%2Ffulltext\%2Fu2\%2Fa023606.pdf\& usg=AOvVaw35ciy55FxWKVrw13q1108Z 
[12] A. Lloyd, Seakeeping: Ship Behavior in Rough Weather, 2nd ed. Hampshire: Ellis Horwood Ltd, 1998, pp. 9-226.

[13] E. Lewis, "The Principles of Naval Architecture: Volume III", The Society of Naval Architects and Marine Engineers, Jersey City, 1988.

[14]"EN455 - Chapter 1: An Introduction to Seakeeping", U.S. Naval Academy Website.

[15]"EN455 - Chapter 3: The Input: Waves", U.S. Naval Academy Website.

[16]"EN455 - Chapter 4: The System: Ship Dynamics", U.S. Naval Academy Website.

[17]"EN455 - Chapter 5: The Output: Ship Motions in Waves", U.S. Naval Academy Website.

[18]"EN455 - Chapter 8: Seakeeping", U.S. Naval Academy Website.

[19] S. Bales, W. Lee and J. Voelker, "Standardized Wave and Wind Environments For NATO Operational Areas", David W. Taylor Naval Ship Research and Development Center, Bethesda, 1981. Accessed: 16-July-2019. [Online]. Available: https://www.google.com/url?sa=t\&rct=j\&q=\&esrc=s\&source=web\&cd=\&cad=rja\&u act=8\&ved=2ahUKEwj1nODzi4bwAhUD26wKHYwwDN0QFjAAegQIBhAD\&url $=$ https $\% 3 \mathrm{~A} \% 2 \mathrm{~F} \% 2 \mathrm{Fapps}$.dtic.mil\%2Fdtic\%2Ftr\%2Ffulltext $\% 2 \mathrm{Fu} 2 \% 2 \mathrm{Fa} 105414 . p d f$ \&usg=AOvVaw3SRAEnvu1SrJRI82nDIw67

[20] W. Johnson, "Analysis Of Aircraft Carrier Motions In A High Sea State", Systems Technology Inc., Hawthorne, 1969. Accessed: 20-June-2019. [Online]. Available: https://www.google.com/url?sa=t\&rct=j\&q=\&esrc=s\&source=web\&cd=\&cad=rja\&u act=8\&ved=2ahUKEwjZp6aOjIbwAhUIKqwKHWUYBi8QFjAAegQIBBAD\&url= https\%3A\%2F\%2Fapps.dtic.mil\%2Fdtic\%2Ftr\%2Ffulltext\%2Fu2\%2F694049.pdf\& usg=AOvVaw2pP1DkVrhtM1lvGSyuXh-d

[21] U. Nielsen, "Response Prediction of Vessel Motions and Sea State Estimation from Ships", UC Berkeley, 2017. Accessed: 22-June-2019. [Online]. Available: https://www.google.com/url?sa $=\mathrm{t} \& \mathrm{rct}=\mathrm{j} \& \mathrm{q}=\& \mathrm{esrc}=\mathrm{s} \&$ source $=$ web\&cd $=\& \mathrm{ved}=2 \mathrm{ahU}$ KEwj3rmVjIbwAhXShK0KHWvhBFoQFjABegQIBRAD\&url=https\%3A\%2F\%2Forbit.dtu .dk\%2Ffiles\%2F139769933\%2FResponse_prediction_and_SSE_UCB_Nov._2017_. pdf\&usg=AOvVaw1UElSpHwEPSup6RxEBtFGw 
[22] M. Prat, Overview of Ocean Wave Statistics. 2008, pp. 31-40.

[23] "Rayleigh Probability Distribution Applied To Random Wave Heights", United States Naval Academy, 1983.

[24] P. Kopp and T. Applebee, "Documentation for Program SHIPMO: A Database For Ship Motions", Naval Surface Warfare Center, Carderock Division Code 5500, Seakeeping Department, West Bethesda, 1998. Accessed: 22-June-2019. [Online]. Available:

https://www.google.com/url?sa=t\&rct=j\&q=\&esrc=s\&source=web\&cd=\&cad=rja\&u act $=8 \&$ ved $=2$ ahUKEwiR7LLjIbwAhUCCc0KHSh2DxAQFjACegQIAxAD\&url=https\%3A\%2F\%2Fapps.dtic .mil\%2Fsti\%2Fcitations\%2FADA359837\&usg=AOvVaw0p0g7syQ0fcPDIDTsRF80 4

[25] A. Baitis, W. Meyers and T. Applebee, "A Non-Aviation Ship Motion Database For The DD 963, CG 26, FF 1052, And The FF 1040 Ship Classes", Ship Performance Department, David W. Taylor Naval Ship R\&D Center, Bethesda, 1976. Accessed: 22-June-2019. [Online]. Available: https://www.google.com/url?sa=t\&rct=j\&q=\&esrc=s\&source=web\&cd=\&cad=rja\&u act=8\&ved=2ahUKEwio7eXZjIbwAhVDWs0KHSa9D7sQFjAAegQIAhAD\&url=ht tps\%3A\%2F\%2Ftrid.trb.org\%2Fview\%2F50932\&usg=AOvVaw3SC_VD14bSDzOd sSHzwCHL

[26] CalQlata, Vessel 6-Degrees of Movement. 2011.

[27] Peng. Zhou, Yachao. Li, Long. Zhang and M. Xing, "A Novel Method for ISAR Imaging of Ship Target," 2009 IET International Radar Conference, Guilin, pp. 1-4, 2009. Accessed: 22-May-2019. [Online]. Available: doi: 10.1049/cp.2009.0289.

[28] T. G. Kostis, K. G. Galanis and S. K. Katsikas, "Simulator Implementation of an IFISAR System for Studies in Target Glint," 2008 Panhellenic Conference on Informatics, Samos, pp. 140-144, 2008. Accessed: 22-May-2019. [Online]. Available: doi: 10.1109/PCI.2008.41. 
[29] T. G. Kostis, "Simulator Implementation of an Inverse Synthetic Aperture Radar System for an Extended Naval Target in a Three Dimensional Synthetic Environment," Tenth International Conference on Computer Modeling and Simulation (uksim 2008), Cambridge, UK, pp. 366-371, 2008. Accessed 31-July2020. [Online] Available: doi: 10.1109/UKSIM.2008.14.

[30] T. G. Kostis, "Simulator Implementation of an Inverse Synthetic Aperture Radar System for an Extended Naval Target for Electronic Warfare Applications," Tenth International Conference on Computer Modeling and Simulation (uksim 2008), Cambridge, UK, pp. 366-371, 2008. Accessed 31-July-2020. [Online] Available: doi: 10.1109/UKSIM.2008.14.

[31] A. Jain and I. Patel, "SAR/ISAR imaging of a nonuniformly rotating target," in IEEE Transactions on Aerospace and Electronic Systems, vol. 28, no. 1, pp. 317-321, Jan. 1992. Accessed 08-August-2019. [Online] Available: doi: 10.1109/7.135457.

[32] J. A. Given and W. R. Schmidt, "Generalized ISAR-part II: interferometric techniques for three-dimensional location of scatterers," in IEEE Transactions on Image Processing, vol. 14, no. 11, pp. 1792-1797, Nov. 2005, doi: 10.1109/TIP.2005.857285.

[33] D. Zhu, L. Wang, Y. Yu, Q. Tao and Z. Zhu, "Robust ISAR Range Alignment via Minimizing the Entropy of the Average Range Profile," IEEE Geoscience and Remote Sensing Letters, vol. 6, no. 2, pp. 204-208, April 2009. Accessed 08-August2019. [Online] Available: doi: 10.1109/LGRS.2008.2010562.

[34] B. Haywood, W. C. Anderson, J. T. Morris and R. Kyprianou, "Generation of Point Scatterer Models for Simulating ISAR Images of Ships," Radar 97 (Conf. Publ. No. 449), Edinburgh, UK, pp. 700-704, 1997. Accessed 13-August-2019. [Online] Available: doi: 10.1049/cp:19971766.

[35] L. Hongya and J. Xin, "Methods to Recognize False Target Generated by DigitalImage-Synthesiser," 2008 International Symposium on Information Science and Engineering, Shanghai, pp. 71-75, 2008. Accessed 10-August-2019. [Online] Available: doi: 10.1109/ISISE.2008.238. 
[36] L. Xu, D. Feng, X. Pan, Q. Liu and X. Wang, "An Improved Digital False-Target Image Synthesizer Method for Countering Inverse Synthetic Aperture Radar," IEEE Sensors Journal, vol. 15, no. 10, pp. 5870-5877, Oct. 2015. Accessed 08-August2019. [Online] Available: doi: 10.1109/JSEN.2015.2453163.

[37] G. V. Morris and T. A. Kastle, "Trends In Electronic Counter-Countermeasures," NTC '91 - National Telesystems Conference Proceedings, Atlanta, GA, USA, pp. 265-269, 1991. Accessed 08-April-2020. [Online] Available: doi: 10.1109/NTC.1991.148028.

[38] L. Neng-Jing and Z. Yi-Ting, "A Survey of Radar ECM and ECCM," in IEEE Transactions on Aerospace and Electronic Systems, vol. 31, no. 3, pp. 1110-1120, July 1995. Accessed 08-April-2020. [Online] Available: doi: 10.1109/7.395232.

[39] D. Xue, X. Liu, R. Shen, Z. Liu and X. Wei, "Micro-Motion False Target Generation in Deception Jammer Against Pulse Doppler Radar," 2017 IEEE 2nd Advanced Information Technology, Electronic and Automation Control Conference (IAEAC), Chongqing, pp. 1057-1060, 2017. Accessed 08-October-2019. [Online] Available: doi: 10.1109/IAEAC.2017.8054175.

[40] Li Yuan, L. Gao-huan and L. Xue-mei, "A Method for Synthesizing Multi-falsetargets Signal for Countering Stepped-Frequency ISAR," 2008 Global Symposium on Millimeter Waves, Nanjing, pp. 1-4, 2008. Accessed 08-October-2019. [Online] Available: doi: 10.1109/GSMM.2008.4534667.

[41] S. Ekestrom and C. Karow, "An All-Digital Image Synthesizer for Countering HighResolution Imaging Radars", Master's Thesis, Naval Postgraduate School, USA, 2000. Accessed 08-October-2019. [Online] Available:

https://www.google.com/url?sa $=t \& r c t=j \& q=\& e s r c=s \&$ source $=$ web\&cd $=\& v e d=2 a h U$ KEwj64tmqjobwAhUVVs0KHUoLDPgQFjAAegQIAhAD\&url=https\%3A\%2F\%2F calhoun.nps.edu\%2Fhandle\%2F10945\%2F7620\&usg=AOvVaw2_xRmfJoP8jtV7w Aab4UzK 
[42] S. Yeo, "A Digital Image Synthesizer for ISAR Counter-Targeting", Master's Thesis, Naval Postgraduate School, USA, 1998. Accessed 08-October-2019. [Online] Available:

https://www.google.com/url?sa=t\&rct=j\&q=\&esrc=s\&source=web\&cd=\&cad=rja\&u act=8\&ved=2ahUKEwjyr6m1jobwAhXBVs0KHfx0BIcQFjAAegQIBRAD\&url=htt ps\%3A\%2F\%2Fwww.researchgate.net\%2Fpublication\%2F3357710_Digital_falsetarget_image_synthesiser_for_countering_ISAR\&usg=AOvVaw0sFUyfErxBUmPA kYENzIbz

[43] M. Richards, Fundamentals of Radar Signal Processing, 2nd ed. McGraw-Hill Education, 2014, pp. 1-510.

[44] B. Mahafza, Radar Systems Analysis and Design Using MATLAB Third Edition, 3rd Edition, 3rd ed. Chapman and Hall/CRC, 2013, pp. 3-735.

[45] D. Wehner, High-Resolution Radar, 2nd ed. Artech House Inc., 1987, pp. 1-545.

[46] J. Proakis, Digital Signal Processing Principles, Algorithms and Applications, 4th ed. Upper Saddle River: Pearson Education Inc., 2007, pp. 1-823.

[47] A. Oppenheim and R. Schafer, Discrete-Time Signal Processing, 3rd ed. Upper Saddle River: Pearson Education Inc., 2009, pp. 9-980.

[48] L. Couch, M. Kulkarni and U. Acharya, Digital And Analog Communication Systems, 8th ed. Harlow, Essex: Pearson Education Inc., 2013, pp. 34-569.

[49] M. Richards, J. Scheer and W. Holm, Principles of Modern Radar: Volume 1: Basic Principles. Scitech Publishing, 2010, pp. 247-893.

[50] M. Skolnik, Introduction to radar systems, 2nd ed. New York: McGraw-Hill, 1980, pp. 1-571. 
[51] V. Bäckström and A. Skårbratt, "Maritime ISAR Imaging With Airborne Radar", Master's Thesis, Chalmers University of Technology, Sweden, 2010. Accessed 13October-2019. [Online] Available:

https://www.google.com/url?sa=t\&rct=j\&q=\&esrc=s\&source=web\&cd=\&cad=rja\&u act=8\&ved=2ahUKEwihm97gjobwAhVGJKwKHdf0DywQFjACegQIBBAD\&url=h ttps\%3A\%2F\%2Fodr.chalmers.se $\% 2$ Fhandle $\% 2 F 20.500 .12380 \% 2 F 129953 \&$ usg=A OvVaw3iwy0LC1tu4wWA7r9jV1KT

[52] B. Haywood, R. Kyprianou, C. Fantarella and J. McCarthy, "ISARLAB - Inverse Synthetic Aperture Radar Simulation and Processing Tool”. Ft. Belvoir: Defense Technical Information Center, pp. 1-56, 1999. Accessed 13-November-2019. [Online] Available:

https://www.google.com/url?sa=t\&rct=j\&q=\&esrc=s\&source=web\&cd=\&cad=rja\&u act=8\&ved=2ahUKEwjzj8L3jobwAhXYB50JHU4_DX4QFjAAegQIAxAD\&url=htt ps\%3A\%2F\%2Fapps.dtic.mil\%2Fsti\%2Fpdfs\%2FADA368762.pdf\&usg=AOvVaw2 ZA2fpoKXCkL7kFGPYytQ1

[53] T. Küçükkiliç, "ISAR Imaging and Motion Compensation", Master's Thesis, Middle East Technical University, Turkey, 2006. Accessed 13-November-2019. [Online] Available:

https://www.google.com/url? sa=t\&rct=j\&q=\&esrc=s\&source=web\&cd=\&cad=rja\&u act=8\&ved=2ahUKEwih5qGSj4bwAhUJWK0KHfQhCxwQFjAFegQICRAD\&url= http\%3A\%2F\%2Fetd.lib.metu.edu.tr\%2Fupload\%2F12608102\%2Findex.pdf\&usg= AOvVaw1q-cCOxywxmquyAzmtVXh5

[54] G. Margarit and J. Mallorqui, "Scattering-Based Model of the SAR Signatures of Complex Targets for Classification Applications", International Journal of Navigation and Observation, vol. 2008, pp. 1-11, 2008. Accessed 31-July-2020. [Online] Available: https:/www.hindawi.com/journals/ijno/2008/426267/. 
[55] Y. Xiong, H. Gu, B. Tang and Y. Zhang, "An Investigation of Range-Velocity Deception Jamming Modeling", in International Conference on Advanced Computer Science and Electronics Information, Beijing, China, pp. 59-63, 2013. Accessed 31July-2020. [Online] Available: https://www.atlantis-press.com/proceedings/icacsei$13 / 7495$

[56] A. Sligar, "RadarPre: ACT Toolkit for ANSYS Electronics Desktop (AEDT)", 2018. Accessed 15-August-2020. [Online] Available: https://appstorecdn.ansys.com/imgs/ANSYSACTAppRadarPre_2018.pdf.

[57] A. Sligar, "RadarPost: ACT Toolkit for ANSYS Electronics Desktop (AEDT)", 2018. Accessed 15-August-2020. [Online] Available: https://appstorecdn.ansys.com/imgs/ANSYSACTAppRadarPost_2018.pdf.

[58] T. A. Carpenter, "DD-963 Spruance Class Destroyer 3D Model - 3D CAD Browser", 3dcadbrowser.com, 2008. Accessed: 03- Feb- 2020. [Online]. Available: https://www.3dcadbrowser.com/3d-model/dd-963-spruance-class-destroyer.

[59] P. Gothro, "USS Spruance (DD-963) (+2006)", Wrecksite, 2012. Accessed: 05- Mar2020. [Online]. Available: https://wrecksite.eu/wreck.aspx?183293.

[60] "Zynq UltraScale+ RFSoC ZCU111 Evaluation Kit", Xilinx, 2020. Accessed: 16Jan- 2020. [Online]. Available: https://www.xilinx.com/products/boards-andkits/zcu111.html\#specifications.

[61] "Introduction to Filter Designer- MATLAB \& Simulink Example", Mathworks.com, 2020. Accessed: 04- Oct- 2020. [Online]. Available: https://www.mathworks.com/help/signal/ug/introduction-to-filterdesigner.html;jsessionid=1fb0ee4297589cfed2ccff79f6ad.

[62] R. Pierce, “Cross Product” Accessed: 18-Dec-2020 [Online]. Available: https://www.mathsisfun.com/algebra/vectors-cross-product.html.

[63] "Electronic warfare,” Tech. Rep. JP 3-13.1, Joint Chiefs of Staff, 012007.

[64] L. Van Brunt, Applied ECM, vol. 1. EW Engineering Inc, 1978. 
[65] J. m. Guo, J. x. Li, and Q. Lv, "Survey on Radar ECCM Methods and Trends In Its Developments," in 2006 CIE International Conference on Radar, pp. 1-4, Oct 2006. Accessed: 14-Jan-2020. [Online]. Available: doi: 10.1109/ICR.2006.343210.

[66] J. Schuerger and D. Garmatyuk, "Deception Jamming Modeling in Radar Sensor Networks,” in MILCOM 2008 - 2008 IEEE Military Communications Conference, pp. 1-7, Nov 2008. Accessed: 14-Jan-2020. [Online]. Available: doi: 10.1109/MILCOM.2008.4753118.

[67] G. V. Morris and T. A. Kastle, "Trends in Electronic Counter-Countermeasures," in Telesystems Conference, 1991. Proceedings. Vol.1., NTC '91., National, pp. 265269, Mar 1991. Accessed: 21-Jan-2020. [Online]. Available: doi: 10.1109/NTC.1991.148028

[68] B.D. Steinberg, "Microwave Imaging of Aircraft", Proceedings of the IEEE, vol. 76, no. 12, December 1988. Accessed: 21-Jan-2020. [Online]. Available: doi: $10.1109 / 5.16351$.

[69] D.A.Ausherman, A. Kozma, J.L.Walker, “Developments in Radar Imaging”, IEEE Transactions on Aerospace and Electronic Systems, Vol. AES-20, No.4, pp. 363400, July 1984. Accessed: 21-Jan-2020. [Online]. Available: doi: 10.1109/TAES.1984.4502060.

[70] T.M. Calloway, et al. "Subaperture Autofocus for Synthetic Aperture Radar”, IEEE Trans. Aerospace Electronic Systems, Vol. AES-30, pp. 617-621, April 1994. Accessed: 23-Nov-2020. [Online]. Available: doi: 10.1109/7.272285.

[71] D.E.Wahl, "Phase Gradient Autofocus - A Robust for High Resolution SAR Phase Correction”, IEEE Transactions on Aerospace and Electronic Systems, Vol. AES-30, pp 827-835, July 1994. Accessed: 23-Nov-2020. [Online]. Available: doi: $10.1109 / 7.303752$. 
[72] W.G.Carrara, R.S.Goodman, R.M.Majevski, Spotlight Synthetic Aperture Radar: Signal Processing Algorithms, Artech House, 1995.

[73] S.Werness, W.Carrara, L.Joyce, D.Franczak "Moving Target Imaging Algorithm for SAR Data", IEEE Transactions on Aerospace and Electronic Systems, Vol. AES-26, pp 57-67, January 1990. Accessed: 30-Nov-2020. [Online]. Available: doi: http://www.geo.uzh.ch/microsite/rsldocuments/research/SARlab/GMTILiterature/Ver09/PDF/WCJF90.pdf

[74] B.Haywood, R.J.Evans, "Motion compensation for ISAR imaging", Proceedings of Australian Symposium on Signal Processing and Applications, pp. 112-117, 1989.

[75] T. Itoh, H. Sueda and Y. Watanabe, "Motion compensation for ISAR via centroid tracking," in IEEE Transactions on Aerospace and Electronic Systems, vol. 32, no. 3, pp. 1191-1197, July 1996. Accessed: 24-Dec-2020. [Online]. Available: doi: $10.1109 / 7.532283$.

[76] T. Young, “A Miniaturized Delay-Line Discriminator," Master's Thesis, Carleton University, Ottawa, 2018. Accessed 08-October-2019. [Online].

[77] "Warship Costs," New Wars, 21-Aug-2010. Accessed: 03-Feb-2021 [Online]. Available: https://newwars.wordpress.com/warship-costs/.

[78] Congressional Budget Office, E. J. Labs, D. Mosher, and E. G. Keating, Washington, DC: Congressional Budget Office, pp. 1-11, 2020. Accessed: 03-Feb2021 [Online].

[79] W. P. Hughes, "Naval warfare," Encyclopcedia Britannica, 1999. Accessed: 19-Feb2020 [Online]. Available: https://www.britannica.com/topic/naval-warfare. 